\title{
Computational study of heterogeneous catalytic systems. Kinetic and structural insights from Density Functional Theory
}

\author{
Author: Reisel Millán Cabrera \\ Doctoral Advisor: Dr. Mercedes Boronat Zaragozá
}

November 2020

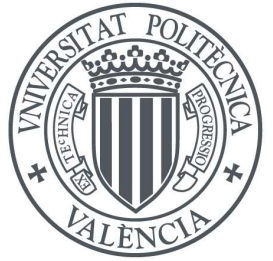

UNIVERSITAT POLITÈCNICA DE VALÈNCIA 



\title{
Computational study of heterogeneous catalytic systems. Kinetic and structural insights from Density Functional Theory
}

\author{
Author: Reisel Millán Cabrera \\ Doctoral Advisor: Dr. Mercedes Boronat Zaragozá
}

A dissertation submitted in partial fulfillment of the

requirements for the degree of Doctor of Philosophy

EDITORIAL

UNIVERSITAT POLITĖCNICA DE VALÈNCIA
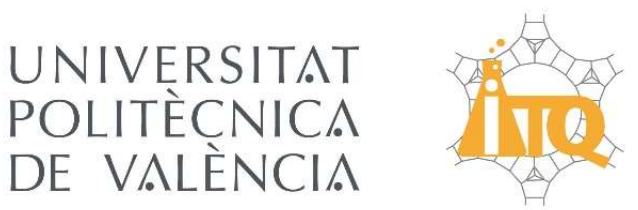



\section{Contents}

Acknowledgements ...........................................................................................................9

List of symbols and abbreviations ........................................................................ 11

1 Basic concepts of heterogenous catalysis .............................................................. 13

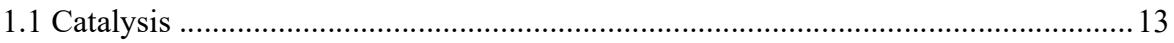

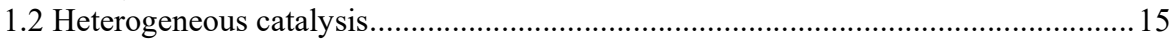

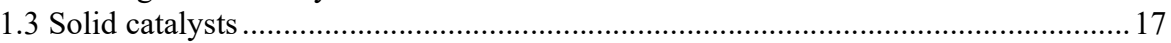

1.3.1 Crystal lattices...................................................................................................... 17

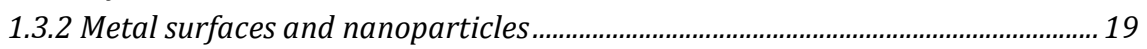

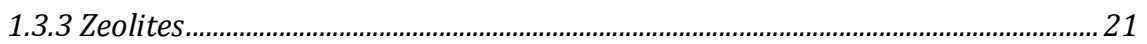

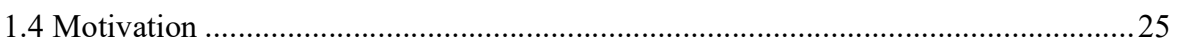

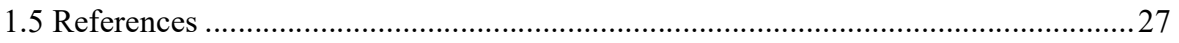

2 Methods and models .................................................................................................... 31

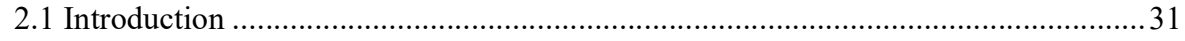

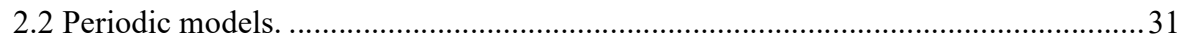

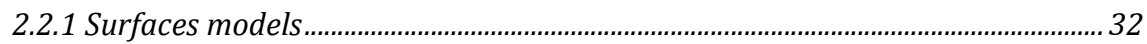

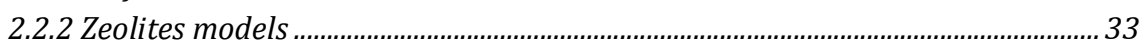

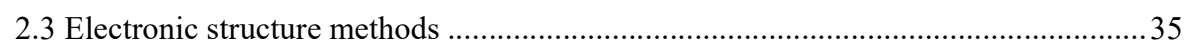

2.3.1 Basic concepts..................................................................................................... 35

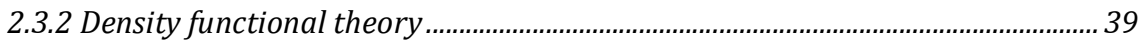

2.3.3 The Kohn-Sham approach................................................................................. 41

2.3.4 The search for approximations to the exchange-correlation functional........... 42

2.3.5 Dispersion corrections.......................................................................................... 44

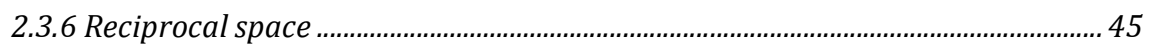

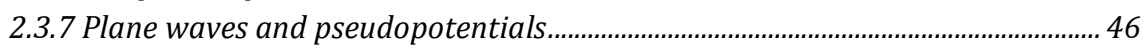

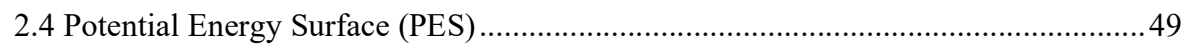

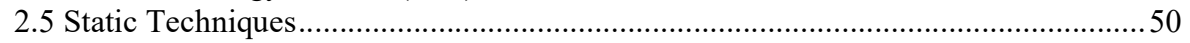

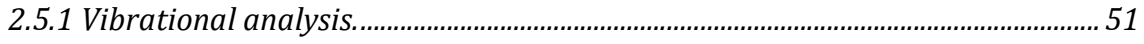

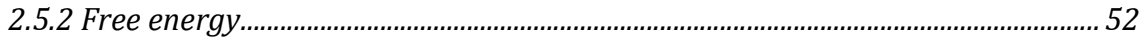

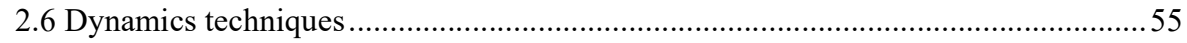

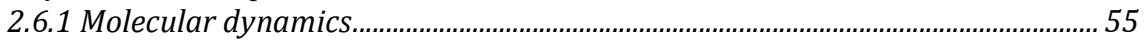

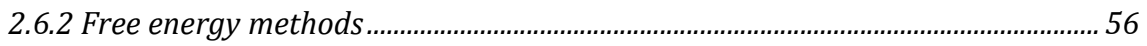

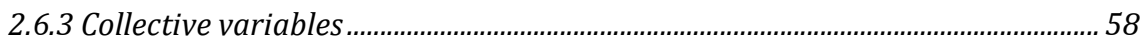

2.6.4 Vibrational frequencies from ab-initio molecular dynamics. ................................ 61

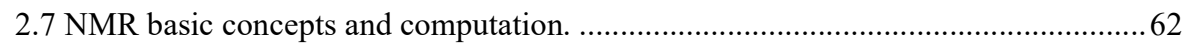


3 Chemoselective reduction of nitrostyrene with non-noble metals...................75

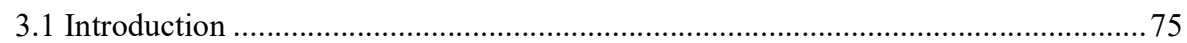

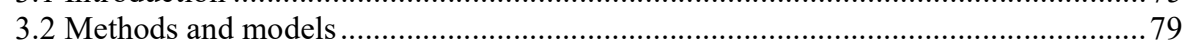

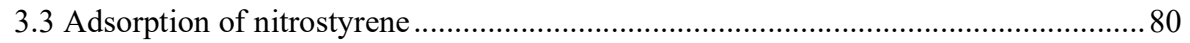

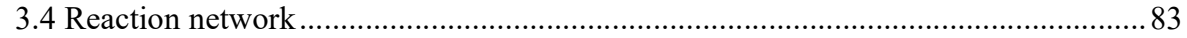

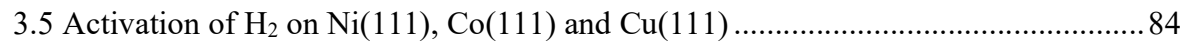

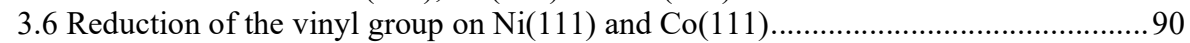

3.7 Reduction of the nitro group on $\mathrm{Ni}(111)$. Direct Route............................................92

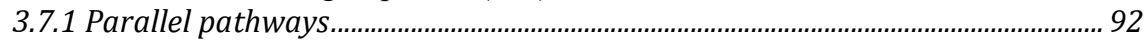

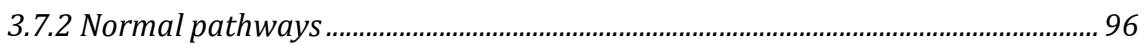

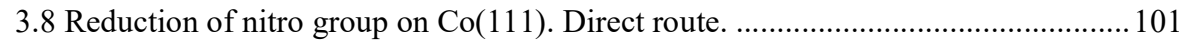

3.8.1 Parallel pathways................................................................................................101

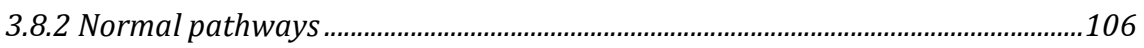

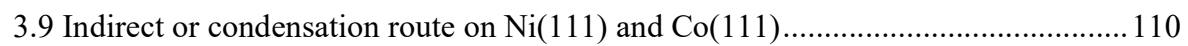

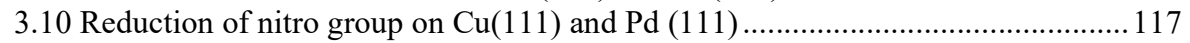

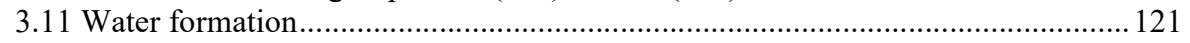

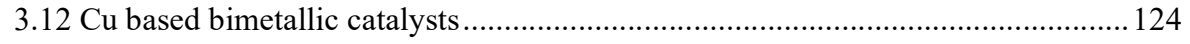

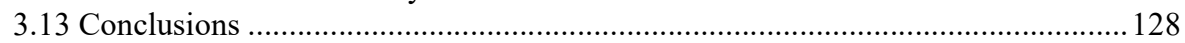

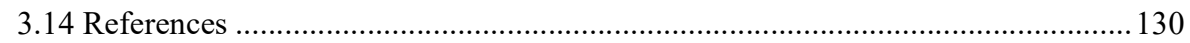

\section{Insights on the $\mathrm{NH}_{3}-\mathrm{SCR}-\mathrm{NOx}$ reaction with $\mathrm{Cu}-\mathrm{CHA}$ catalysts using static}

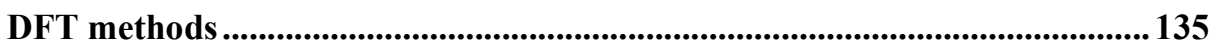

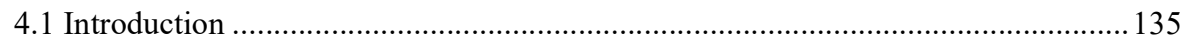

4.2 Methods and models .................................................................................... 138

4.3 Identification of $\mathrm{Cu}$ species in $\mathrm{Cu}-\mathrm{CHA}$ by IR spectroscopy and theoretical modeling

.

4.3.1 Stability of Cu species from DFT calculations......................................................140

4.3.2 Identification of the Cu species through IR analysis..........................................142

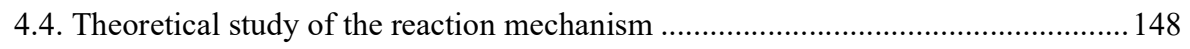

4.4.1. Oxidation half-cycle: formation of nitrites and nitrates on Cu-CHA catalysts

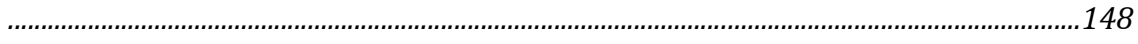

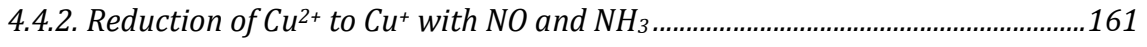

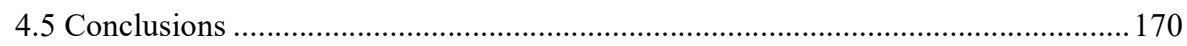

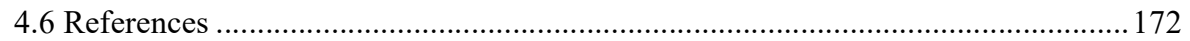

5 Insights on the NH3-SCR-NOx reaction from Molecular Dynamics

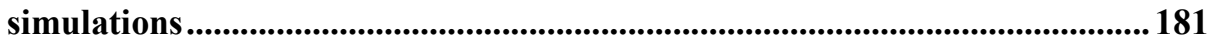

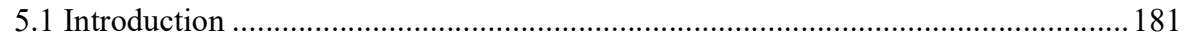

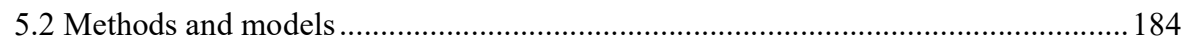


5.3 Adsorption of reactant molecules on the copper cations with AIMD ..................... 185

5.3.1 Interaction of the reactant molecules with $\mathrm{Cu}^{2+}$.................................................187

5.3.2 Interaction of the reactant molecules with $\mathrm{Cu}^{+}$..................................................192

5.4 Stability of $\mathrm{Cu}^{+}$complexes at low temperature with umbrella sampling .................201

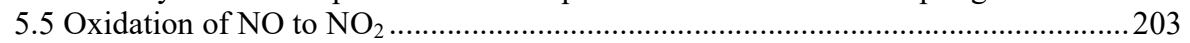

5.5.1 Oxidation of $\mathrm{NO}$ to $\mathrm{NO}_{2}$ at low temperature with umbrella sampling...............208

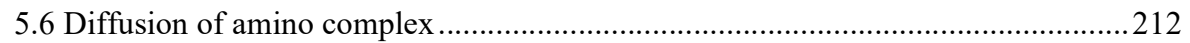

5.7 IR analysis. Comparison of static DFT and AIMD computed frequencies..............219

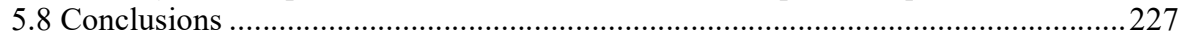

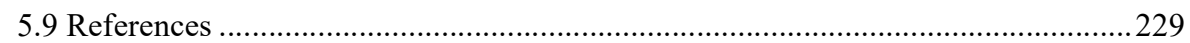

6 Ab-initio simulation of NMR parameters: location of the $F^{-}$in the as-made pure silica RTH .................................................................................................... 233

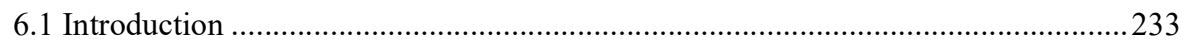

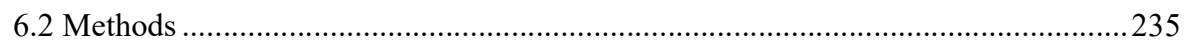

6.3 Elucidation of Fluoride location in the as-made RTH .......................................2236

6.3.1 Linear fit for the prediction of the ${ }^{19} \mathrm{~F}$ isotropic chemical shifts ..........................237

6.3.2 Simulation of the ${ }^{19} \mathrm{~F}$ chemical shift assuming a monoclinic unit cell...............239

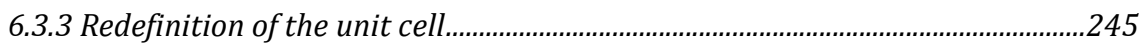

6.3.4 Simulation of the ${ }^{19} \mathrm{~F}$ chemical shift with the triclinic unit cell ...........................247

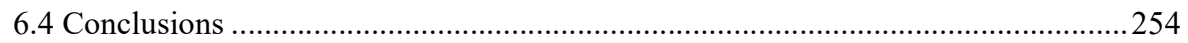

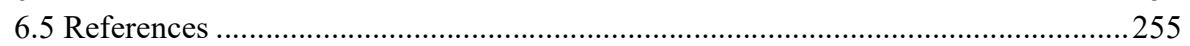

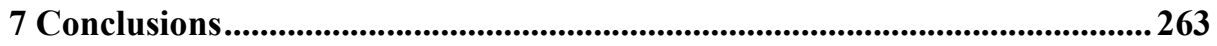

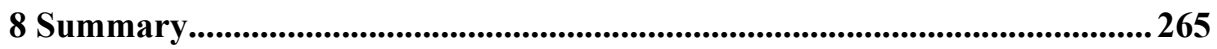

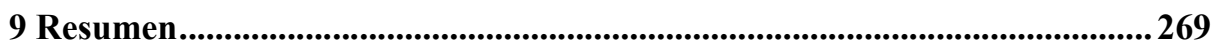

10 Resum ...............................................................................................2 273

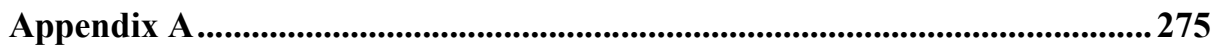

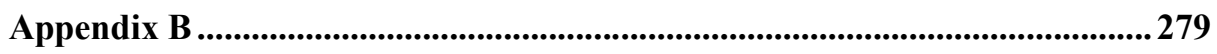

B.1 Calculated absolute ${ }^{29} \mathrm{Si}$ isotropic shieldings for the RTH framework with a

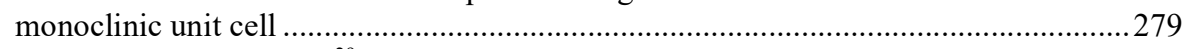
B.2 Calculated absolute ${ }^{29} \mathrm{Si}$ isotropic shieldings for the RTH framework with a triclinic unit cell 



\section{Acknowledgements}

It is with great pleasure that I dedicate these lines to recognize all those who have contributed to the development of this work. First and foremost, I want to thank Dr. Mercedes Boronat for her priceless scientific and professional guidance. It has been a privilege to work with you. Many thanks to Prof. Avelino Corma for his motivation and for the learned lessons during the scientific discussions. I also have to acknowledge Dra. Teresa Blasco, Dr. Fernando Rey and Dra. Patricia Concepción for being involved and contribute to the better quality of this work. Of course, to Dr. Germán Sastre and to my former and current colleagues from office, Dr. Tirso Lopez, Dr. Jordi Toda, Pau Ferri, Santiago León, Mario Gallego, Dr. Yohana Seminóvski, and Joaquín Martínez for their support and for creating an amenable work environment. In particular, to Dr. Estefanía Fernández, with whom I shared many conversations about DFT, Hartree-Fock, programming, politics, religion, society, and so on.

A part of this investigation was developed in collaboration with the Center for Molecular Modelling (CMM) of Ghent University, where I spent three months doing a research stay. I want to thank Prof. Veronique Van Speybroeck for allowing me to be part of their group and for her good scientific advice, and to all the staff of the CMM for bearing with me while I shot them with unending questions about molecular dynamics, in particular, Dr. Louis Vanduyfhuys, Dr. Peter Cnudde, Alexander Hoffman and Dr. Julianna Hajek.

Moreover, my acknowledgements to "La Caixa foundation" for the financial support through "La Caixa-Severo Ochoa" International PhD Fellowships (call 2015), to the Spanish Supercomputing Network (RES), to the Centre de Càlcul de la Universitat de València, to the Flemish Supercomputer Center (VSC) of Ghent University for the computational resources and technical support, and to the Spanish Government through the MAT2017-82288-C2-1-P programme. 
On a more personal note, I must thank my parents and my brother for their support and motivation. It was my brother, after all, who encouraged me to enroll in the national contest program of chemistry in high school, where I developed the habit of reading scientific books. My relation with chemistry started right there. I also feel grateful for Alechania, who stormed in very recently and has been an incredible help in many ways during this last stage. 


\section{List of symbols and abbreviations}

$\begin{array}{ll}\text { AIMD } & \text { Ab-initio Molecular Dynamics } \\ \text { CHA } & \text { Chabazite } \\ \text { CN } & \text { Coordination Number } \\ \text { DFT } & \text { Density Functional Theory } \\ \text { d6r } & \text { double six ring unit of zeolite frameworks } \\ \text { Eact } & \text { Activation Energy } \\ \text { Ereac } & \text { Reaction Energy } \\ \text { eV } & \text { Electronvolts } \\ \text { FF } & \text { Force field } \\ \text { FFT } & \text { Fast Fourier Transform } \\ \text { FT } & \text { Fourier Transform } \\ \text { GGA } & \text { Generalised Gradient Approximation } \\ \text { GIPAW } & \text { Gauge Included Projector Augmented Wave } \\ \text { HF } & \text { Hartree-Fock } \\ \text { IR } & \text { Infrared } \\ \text { IZA } & \text { International Zeolite Association } \\ \text { kcal/mol } & \text { Kilocalories per mole } \\ \text { LDA } & \text { Local Density Approximation } \\ \text { M } & \text { Metal } \\ \text { MD } & \text { Molecular Dynamics } \\ \text { MM } & \text { Molecular Mechanics } \\ \text { NEB } & \text { Nudge Elastic Band } \\ \text { NMR } & \text { Nuclear Magnetic Resonance } \\ & \end{array}$




$\begin{array}{ll}\text { NOx } & \text { Nitrogen oxides } \\ \text { PAW } & \text { Projector Augmented Wave } \\ \text { PBE } & \text { Perdew-Burke-Ernzerhof } \\ \text { PW91 } & \text { Perdew-Wang 91 } \\ \text { QM } & \text { Quantum Mechanics } \\ \text { revPBE } & \text { Revised Perdew-Burke-Ernzerhof } \\ \text { RMSD } & \text { Root Mean Square Deviation } \\ \text { SAPO } & \text { Silico-Aluminophospate } \\ \text { SCF } & \text { Self Consistent Field } \\ \text { SCR } & \text { Selective Catalytic Reduction } \\ \text { SSZ } & \text { Socal Silica Zeolite } \\ \text { TS } & \text { Transition State } \\ \text { TST } & \text { Transition State Theory } \\ \text { US } & \text { Umbrella Sampling } \\ \text { VASP } & \text { Vienna Ab-initio Simulation Package } \\ \text { WHAM } & \text { Weighted Histogram Analysis Method } \\ \text { XRD } & \text { X-ray Diffraction }\end{array}$




\section{Chapter 1 \\ Basic concepts of heterogenous catalysis}

\subsection{Catalysis}

Catalysis is a chemical phenomenon in which the rate of a chemical reaction is increased by a substance called catalyst. This latter operates by destabilizing the internal bonds of the reactants, making them more reactive so that their conversion to products is accelerated. In the presence of a catalyst, chemical reactions follow different mechanisms with lower activation energies compared with the equivalent uncatalyzed reaction. This is illustrated in the hypothetical energy diagram of Figure 1.1. Notice also that the catalyst does not affect the energy change of the reaction, i.e, it alters the kinetics, not the thermodynamics.

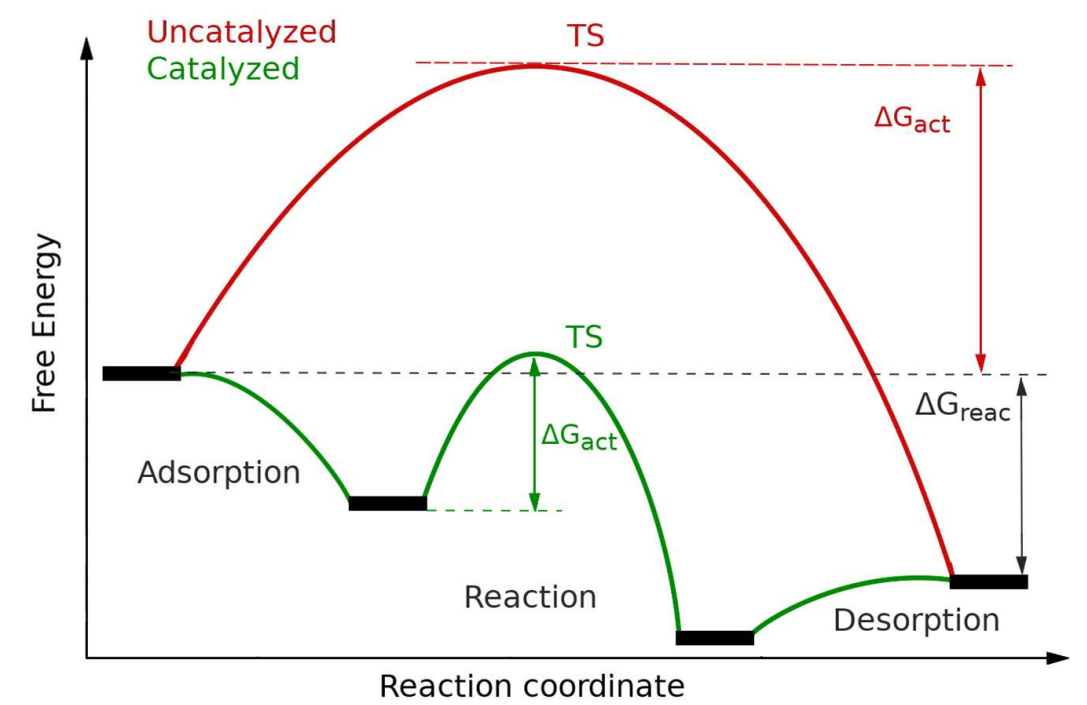

Figure 1.1 Potential energy diagram of a hypothetical catalyzed and uncatalyzed reaction. 
The activation free energy, $\Delta \mathrm{G}_{\text {act, }}$, is the difference between the absolute free energy of the transition state (TS) and the absolute free energy of the reactants. The transition state is the point of maximum free energy along the reaction coordinate (e.g. a bond vibration). The TS, also called activated complex, is a key concept in the transition state theory (TST) in which reactants reach the transition state (or are activated) through the collisions with the surrounding molecules. This theory was developed by Henry Eyring and independently by M.G. Evans and Michael Polanyi ${ }^{1,2}$, and is the fundamental model with which the kinetics of chemical reactions are explained. In the TST, the reaction rate constant is related to the activation free energy by the Eyring-Polanyi equation:

$$
k=\frac{k_{B} T}{h} e^{-\Delta G_{a c t} / R T}
$$

which by decomposing the free energy according to

$$
\Delta G=\Delta H-T \Delta S
$$

yields

$$
k=\frac{k_{B} T}{h} e^{\Delta S_{a c t} / R} e^{-\Delta H_{a c t} / R T}
$$

Expression 1.3 explicitly relates the rate constant with the entropy and the enthalpy change between the activated complex and the reactants. The influence of catalysts can be observed even if they are present in small amounts. The enhancing capacity of a catalyst is called activity. The activity is usually expressed as turnover frequency (TOF), measured as the ratio between the moles of reactant converted per moles of catalyst per second. Because catalysts change the mechanisms of chemical reactions, when more than one product can be formed from the same reactants, the ratio of the products is also altered. The relative amount of specific products can increase in catalyzed reactions because the pathway (sequence of elementary steps) of the mechanism leading to these products are favored over other pathways. The capacity of a catalyst to drive a chemical reaction toward a specific product is called selectivity. Even though catalysts participate in the reaction mechanism, they are not consumed in the reaction but are regenerated at the end of it, after which they can be reused. 
Catalysis has a huge impact on modern society being present in around $85-90 \%$ of today's chemical processes ${ }^{3}$. As examples of such processes we can cite the catalytic cracking of crude oil with zeolites ${ }^{4,5}$, the chemoselective reduction of nitroarenes with noble and non-noble metal based catalysts ${ }^{6}$, the synthesis of ammonia ${ }^{3}$, and the selective oxidation of hydrocarbons ${ }^{7,8}$. Catalyzed chemical processes are usually carried out under more efficient thermodynamics conditions, at significantly lower temperatures and pressures. This obviously reduces costs, time, and pollution compared with the uncatalyzed counterparts. The environmental impact of catalysis does not only stem from the minimization of waste in chemical processes, catalysis is also applied in the abatement of environmental pollution ${ }^{9,10}$. A classic example of this is the selective catalytic reduction of nitrogen oxides (SCR-NOx) aiming at cleaning the gas emissions of power plants and automotive exhaust ${ }^{11,12}$. This reaction has been investigated in this work and more details are given in chapters four and five. Catalysis is not only present in human-induced processes but occurs naturally as well, for example, in our own bodies. Actually, the most efficient catalysts known are enzymes, responsible for the regulation of our metabolism ${ }^{13,14}$. Without them we could just not live.

Being a very wide discipline, catalysis is commonly divided into homogeneous, heterogeneous and biocatalysis. In homogeneous catalysis all the chemical species, including the catalyst, are in the same phase, i.e, all in solid, gas or liquid phase. The latter two are the most common. In heterogeneous catalysis, the catalyst is present as a different phase from that of the reactant molecules and biocatalysis basically deals with reactions catalyzed by enzymes, such as the replication or transcription of the DNA.

\subsection{Heterogeneous catalysis}

In heterogeneous catalysis, reactions occur in the interface between a solid phase and a liquid or gas phase, where the solid phase acts as the catalyst. A heterogeneous catalytic cycle consists of the following steps: adsorption of the reactant molecules, reaction, and desorption of the products (Figure 1.1).

Adsorption can be the result of weak intermolecular interactions of the adsorbate with the catalyst with no chemical bond formation, this is called physisorption. 
Conversely, the adsorption where chemical bonds are formed is called chemisorption. This is a crucial step where internal bonds of the reactant molecules are weakened because of their interaction with the catalyst, we say then that they have been activated. Activation can also imply the dissociation of chemical bonds of the adsorbates, in which case we talk about dissociative adsorption. For example, a $\mathrm{H}_{2}$ molecule dissociates into two $\mathrm{H}$ atoms as a result of its interaction with the active sites. The term "active sites" was coined by Hugh Stott Taylor to refer to the groups of atoms of the catalyst responsible for the enhancement of chemical reactions ${ }^{15}$. They could be for example the atoms of a metal surface, Brønsted and Lewis centers in zeolites, etc.

The interaction of the adsorbates with the active sites has to be strong enough to activate the reactant molecules, but a too strong interaction of the chemical species with the active sites might prevent the products from desorbing. There is then an optimum interaction energy for which the reaction rate is maximized. The volcanoshaped ${ }^{16}$ dependence of the reaction rate on the adsorption energy of adsorbates is known as Sabatier's principle. After desorption of the products the active sites become again available for the next catalytic cycle.

There are two general mechanisms that explain how heterogeneous reactions occur, the Langmuir-Hinshelwood and Eley-Rideal mechanisms. The former mechanism assumes that species A and B chemisorb first before any reaction between them can take place. In the latter, one chemisorbed species reacts with another species which is not adsorbed but comes directly from the gas phase. The difference between both mechanisms is that in the Eley-Rideal mechanism the reaction can still take place without the need of free active sites for one of the reactants. However, direct reaction from the gas phase usually implies a considerable loss of entropy and consequently, Eley-Rideal kinetics is rarely observed (see eq 1.3). A general set of elementary steps (reaction network) consistent with the Langmuir-Hinshelwood mechanism can be laid out as follows:

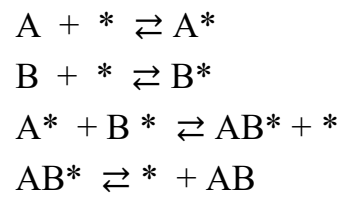


where $*$ represents the empty active site, and $\mathrm{A}^{*}, \mathrm{~B}^{*}$ and $\mathrm{AB}^{*}$ represent the chemisorbed state of species $\mathrm{A}, \mathrm{B}$ and $\mathrm{AB}$. In a similar way, a reaction network consistent with the Eley-Rideal mechanism can be laid out as follows
$\mathrm{A}+* \rightleftarrows \mathrm{A}^{*}$
chemisorption
$\mathrm{A}^{*}+\mathrm{B} \rightleftarrows \mathrm{AB}+*$
reaction and desorption

\subsection{Solid catalysts}

In principle there is no limitation in the nature of solid catalysts, any type of material could be a catalyst. However, a good catalyst should have certain properties. They should have an extensive surface area, the larger the surface area the greater the number of active sites that will be exposed and available for the reactant molecules. They should also be stable against deactivation or easy to regenerate if deactivation is fast, and thermally stable against sintering and structural change. In the following sections some comments are devoted to the structure of common solid catalysts.

\subsubsection{Crystal lattices.}

Solid catalysts are usually crystalline materials, which means that their structure is defined as a periodic arrangement of atoms. The unit cell is the smallest repeating unit that contains the full symmetry of the crystal structure. In a three-dimensional space, unit cells are specified by three vectors $(a, b$, and $c)$ and three angles $(\alpha, \beta$ and $\gamma)$. This is illustrated in Figure 1.2. Any point in the three-dimensional space can be generated by discrete translation operations along the unit cell vectors according to:

$\vec{T}=n_{1} a+n_{2} b+n_{3} c$

where $n_{1}, n_{2}, n_{3}$ are integers, and $a, b$ and $c$ are the unit cell vectors.

Seven lattice systems can be defined by taking different combinations of unit cell vectors and angles, namely, cubic, hexagonal, rhombohedral, tetragonal, orthorhombic, monoclinic and triclinic (Table 1.1). Different arrangements of particles inside unit cells give rise to different possible centering: primitive $(\mathrm{P})$, facecentered (A, B and C), all-face-centered (F), body-centered (I), and rhombohedral (R). The combination of the seven lattice systems with the lattice centering yields the so-called 14 Bravais lattices (Table 1.1). 
Table 1.1 Definition of the seven lattice systems, each of which can have different centering.

\begin{tabular}{cccc}
\hline Name & vectors & angles & Lattice centering \\
\hline Cubic & $a=b=c$ & $\alpha=\beta=\gamma=90^{\circ}$ & $\mathrm{P}, \mathrm{F}, \mathrm{I}$ \\
Hexagonal & $a=b$ & $\alpha=\beta=90^{\circ}, \gamma=120^{\circ}$ & $\mathrm{P}$ \\
Rhombohedral & $a=b=c$ & $\alpha=\beta=\gamma \neq 90^{\circ}$ & $\mathrm{R}$ \\
Tetragonal & $a=b \neq c$ & $\alpha=\beta=\gamma=90^{\circ}$ & $\mathrm{P}, \mathrm{I}$ \\
Orthorhombic & $a \neq b \neq c$ & $\alpha=\beta=\gamma=90^{\circ}$ & $\mathrm{P}, \mathrm{C}, \mathrm{F}, \mathrm{I}$ \\
Monoclinic & $a \neq c$ & $\alpha=\gamma=90^{\circ}, \beta \neq 90^{\circ}$ & $\mathrm{P}, \mathrm{C}$ \\
Triclinic & $a \neq b \neq c$ & $\alpha \neq \beta \neq \gamma \neq 90^{\circ}$ & $\mathrm{P}$ \\
\hline
\end{tabular}

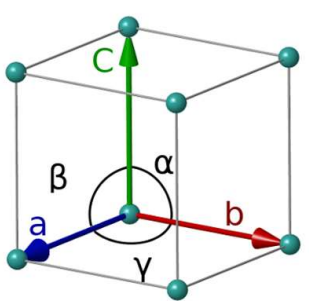

Primitive (P)

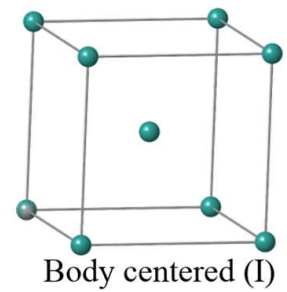

a) Unit cell

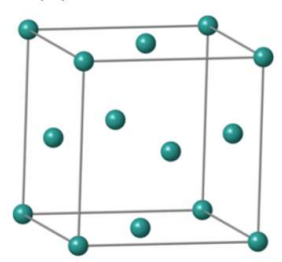

Face centered $(\mathrm{F})$

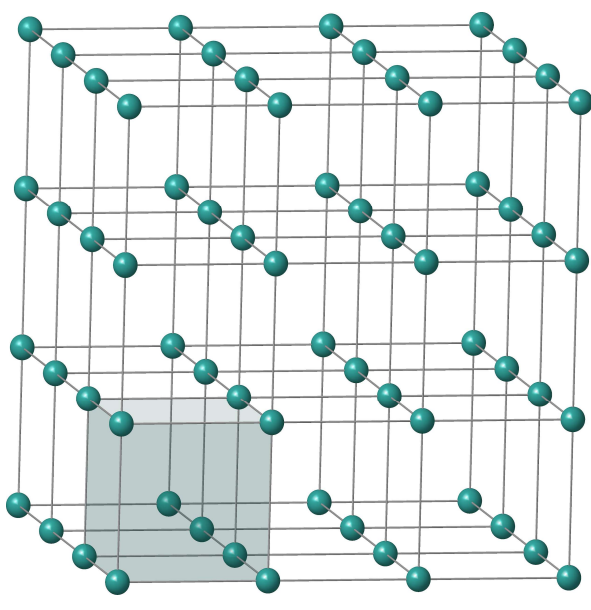

b) Crystal Lattice

Figure 1.2 Representation of a) unit cell and b) crystal lattice generated by repetition of the unit cell. 


\subsubsection{Metal surfaces and nanoparticles}

Solid surface atoms have lower coordination than those of the bulk because the creation of surfaces always involves the rupture of chemical bonds and a consequent gain in free energy. The surface free energy $\gamma$, which is always positive, is related to the cohesive energy, $\Delta H_{c o h}$, and to the number of broken bonds between nearest neighbor atoms, given as

$$
\gamma=\Delta H_{\operatorname{coh}} \frac{Z_{s}}{Z} N_{s}
$$

where $Z$ is the coordination number of an atom in the bulk, $Z_{s}$ the number of missing coordinated atoms of a surface atom and $N_{s}$ the surface atom density. The fewer the missing neighbors of the surface atoms the lower the surface free energy and the more stable the surface is. Solid materials tend to expose the most thermodynamically stable surfaces. Examples of possible surfaces of the FCC crystal system are shown in Figure 1.3.

The surface free energy lowers through the spontaneous adsorption of chemical species on the surface. Depending on how the species interact with the surface atoms they can occupy different adsorption sites. The most common include on-top, bridge and hollow sites (Figure 1.3).

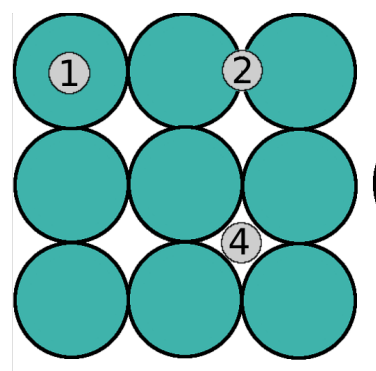

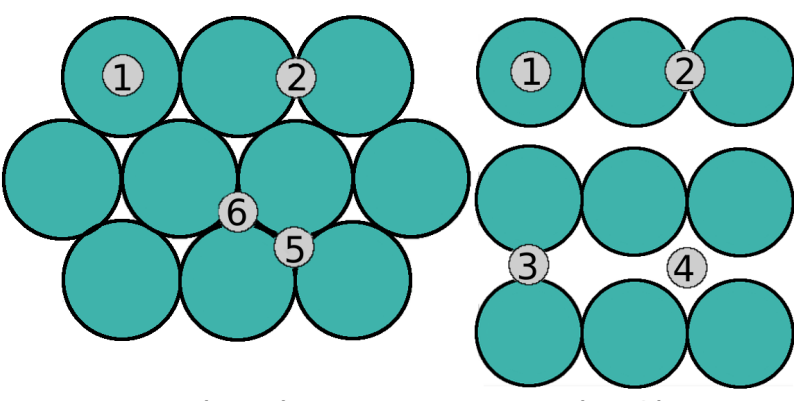

(100)

(111)

(110)

1 - On top

2 - Bridge
3 - Long bridge 4 - Four fold hollow
5 - Three fold hollow FCC 6 - Three fold hollow HCP

Figure 1.3 Representation of adsorption sites on several surfaces of an FCC crystal. 
Metals and metal oxides are commonly used catalysts because they exhibit high surface free energies. An example is the Raney Nickel catalyst, with a high surface area, $\sim 100 \mathrm{~m}^{2}$ per gram of catalyst ${ }^{17}$, used for the hydrogenation of a wide range of organic compounds, such as benzene, nitroaromatics, nitriles and olefins ${ }^{6,17}$.

As the particle size decreases more atoms from the bulk will be exposed at the surface and more active sites will be available, resulting in an increase of the activity. Nanoparticles are particles with nanoscale sizes, ranging from $\sim 1-100 \mathrm{~nm}^{18}$. The origin of the higher activity of nanoparticles does not stem only from the greater number of surface atoms compared with bigger particles. These smaller particles exhibit geometric features such as corners, terraces and edges where the atoms have an even lower coordination compared with the atoms on the surface plane (Figure 1.4). Consequently, these atoms are more reactive, often being the main active sites.

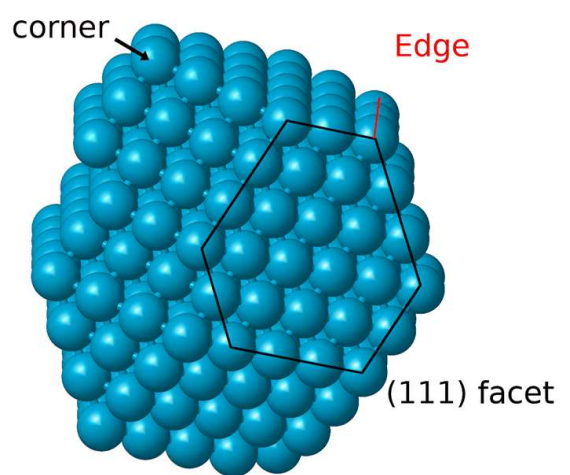

a)

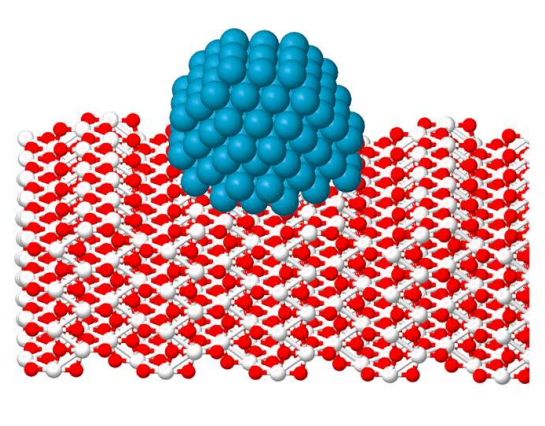

b)

Figure 1.4 Illustration of a nanoparticle a) isolated and b) supported.

Because of their higher surface free energy, small metal particles are unstable and tend to fuse together forming solid mass of material, a process known as sintering. The usual way to avoid this is by stabilizing them on a thermally stable and chemically inert support (Figure 1.4). Supports are usually metal oxides such as 
silica, alumina, titania and even zeolites, which estabilize the metal nanoparticles and, in some cases, participate in the reaction mechanism.

\subsubsection{Zeolites}

Zeolites are crystalline, micro-porous aluminosilicates composed of corner-sharing tetrahedra, $\mathrm{TO}_{4}(\mathrm{~T}=\mathrm{Si}, \mathrm{Al})$, that can be synthesized or found naturally ${ }^{19}$. The threedimensional networks formed by the sequence of $\mathrm{TO}_{4}$ units possess channels and cavities of molecular dimensions, $\sim 3-12 \AA$ (Figure 1.5).

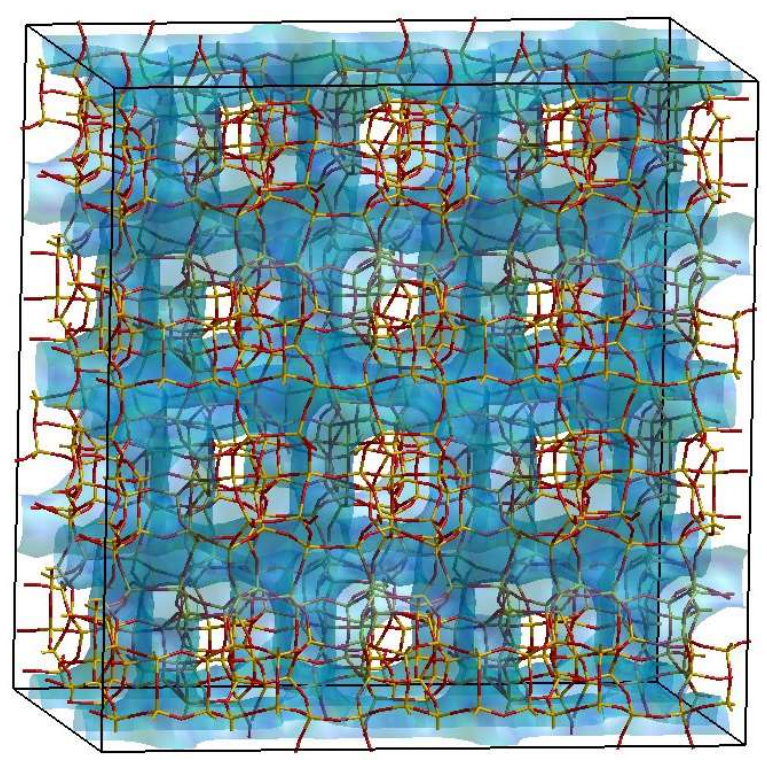

Figure 1.5 Schematic representation of the channel systems of the MFI framework.

The $\mathrm{TO}_{4}$ unit is just the first level of structural complexity in zeolites commonly referred to as primary building units (PBUs). These tetrahedra connect with each other in various geometric arrangements to form the so-called secondary building units (SBUs) with which the whole three-dimensional framework of a zeolite can be generated. Some examples including squares, single and double rings, and even more complex polyhedra are shown in Figure 1.6. 


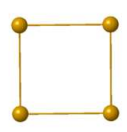

4

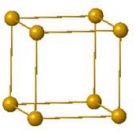

4-4

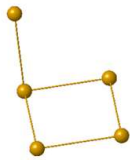

4-1

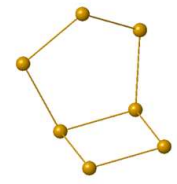

5-2

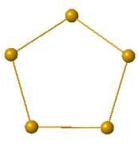

5

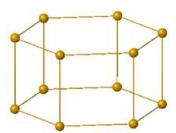

6-6

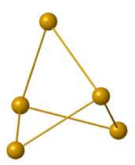

$4=1$

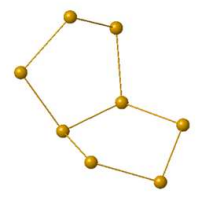

5-3

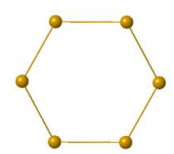

6

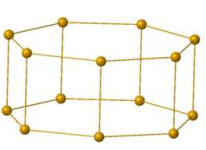

8-8

6-2

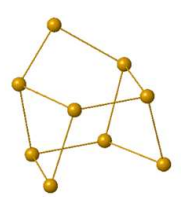

$4-4=1$

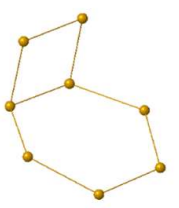

8

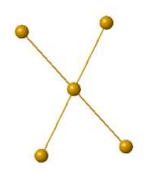

Spiro-5

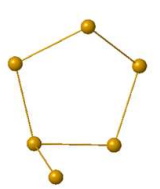

5-1

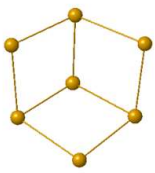

$6 \equiv 1$

Figure 1.6 Schematic representation of some secondary building units (SBUs).

Rings are named by specifying the number $n$ of $\mathrm{TO}_{4}$ units they are made up with, i.e $n$-ring. The size $n$ of rings controls the size of the pores, a fundamental characteristic of zeolites and one of the criteria used to classify them. Small-pore zeolites, such as LTA or CHA, contain rings of up to eight members $(8 r)$ with an approximate diameter of 3.4-4 $\AA$. Medium-pore zeolites possess rings of 9-11 members with diameters of $\sim 5-5.5 \AA$ A. Similarly, large and extra-large pore frameworks contain rings with 12 and more members respectively. Typical pore openings are shown in Figure 1.7. Zeolites are also classified according to the dimensionality of the channel systems. They can be one-, two- and three-dimensional when one, two and three 
channel systems respectively exist in the framework. These channels can be independent or can be directly connected or indirectly through cavities. Each unique topology that can be generated, regardless of the chemical composition, is assigned a three-letters code by the Structure Commission of the International Zeolite Association (IZA), and included in the IZA database ${ }^{20}$. So far more than 200 of these frameworks have been synthesized. In this work, we have studied zeolites corresponding to the CHA and RTH frameworks. Because of their particular channel systems and cavities, zeolites possess shape selectivity, which is widely exploited in industry. They can act as molecular sieves in refining and petrochemistry catalytic processes, isomerization and alkylation of aromatic compounds increasing the selectivity toward specific products ${ }^{21-24}$.

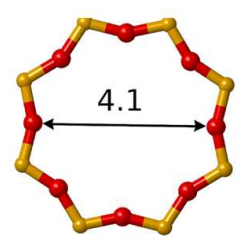

LTA

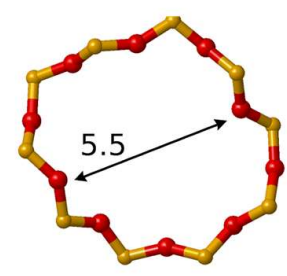

$\mathrm{MFI}$

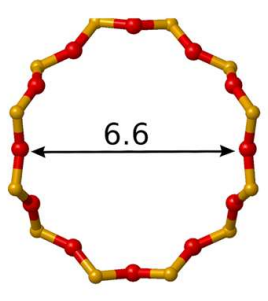

BEA

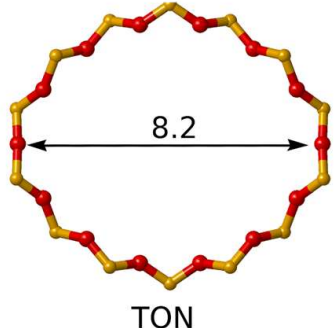

TON

Figure 1.7 Pore diameter of the several zeolite frameworks.

Zeolites can also be considered siliceous structures in which a fraction of the tetravalent $\mathrm{Si}$ atoms have been substituted by the trivalent Al. This substitution produces a negative charge on the $\mathrm{AlO}_{4}^{-}$unit that must be compensated with a cation. If the compensating cation is a proton, then, a Brønsted acid site (Figure 1.8a) is generated. Similarly, compensation of the negative charge with metal cations produces Lewis acid sites. Possible extra framework sitting positions of metal cations of the CHA topology is shown in figure 1.8b. According to Lowestein's rule the Al-O-Al bonds cannot exist in zeolite frameworks which implies that the ratio $\mathrm{Si} / \mathrm{Al}$ ranges from 1 to infinity ${ }^{25}$. The incorporated $\mathrm{Al}$ atoms must be stabilized by the existence of Si-O-Al-O-Si moieties with which the repulsive interaction of adjacent $\mathrm{AlO}_{4}^{-}$is avoided. 
<smiles>CO[Si](O)(O)O[Al](O)(O)O[Si](O)(O)OC</smiles>

a)

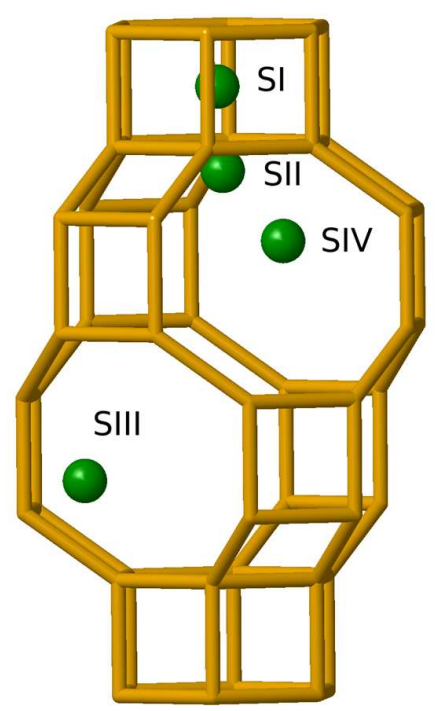

b)

Figure 1.8 Illustration of a) Brønsted acid site and b) extra-framework sitting positions of metal cations in a chabazite topology: SI in the center of the d6r, SII in the plane of the $6 r$, SIII next to a $4 r$ of a d6r inside the cavity, and SIV in the 8r.

Another important class of crystalline materials is made up of the microporous aluminophosphates, namely the ALPOs family ${ }^{26}$. In these materials, the framework is composed of $\mathrm{AlO}_{4}$ and $\mathrm{PO}_{4}$ units in alternate order forming topological 3D structures similar to those of zeolites. Substitution of a $\mathrm{P}^{5+}$ ion with a $\mathrm{Si}^{4+}$ also generates a negative charge that must be compensated, yielding the so-called silicoaluminophosphates (SAPOs). These also display properties such as Brønsted and Lewis acidities.

Since zeolites occupy a prominent place in heterogeneous catalysis their synthesis is a very active field of research. New ways of synthesis and new structures are constantly looked for. The synthesis of zeolites is usually carried in the presence of organic structure directing agents (OSDAs) or templates which have a significant influence on the size and shape of pores and channel systems ${ }^{27-32}$. The most common OSDAs are organic cations such as tetraalkylammonium and tetraalkylphosphonium. During crystallization, they remain trapped inside the cavities stabilizing the zeolite framework mainly through van der Waals interactions. 
This zeolite form where the OSDA is compensating the negatively charged fragments of the framework $\left(\mathrm{AlO}_{4}^{-}\right.$, defects, etc) is called as-made or as-synthesized. After synthesis, the OSDA is usually removed by calcination, and the negative charge in the calcined material is compensated by a proton.

\subsection{Motivation}

The design of efficient solid catalysts is in general very complicated and a mechanistic understanding of catalytic reactions is often very difficult to achieve. This is so because the performance of catalysts is determined by molecular processes occurring at the active sites, and a direct mapping between the macroscopic properties and the microscopic true nature of the active sites is usually very hard to lay out (structure-reactivity relationships). Especially, when the nature of the active sites changes under reaction conditions ${ }^{33}$. Because of its complexity, heterogeneous catalysis is a multidisciplinary field in which the combination of several techniques is mandatory. Spectroscopic techniques are routinely exploited for ex-situ characterization of solid catalysts but their interpretation is not always unambiguous and clear. In situ spectroscopy is increasingly used as well. Nonetheless, the lifetime of reaction intermediates is usually very short so that their detection is in general very difficult ${ }^{34}$. A further complication has to do with the fact that spectator species, not actively participating in the main reaction, are many times present in significant amounts.

Computational chemistry operates by definition at the molecular-level. In principle, although not trivial at all, it is possible to individually study the elementary steps and their relevance for catalytic reactions, and also to predict the spectroscopic properties of solid catalysts. Consequently, computational chemistry has become a valuable complementary tool in the study of catalytic processes and in the elucidation of the structure of catalysts, especially after the development and implementation of density functional theory (DFT). It became more popular among chemists, in the 1990s, due to the availability of more accurate functionals, new methods and computer codes that are constantly under development.

There are, however, lots of research areas within heterogeneous catalysis still awaiting for the aid that computational chemistry methods have to offer. We have spotted three of such subjects for which we have carried out computational studies 
and the results are presented in this work. The first is the chemoselective hydrogenation of nitroaromatics with non-noble metals included in chapter 1 . We have explored the reaction pathways involved in the reduction of nitrostyrene and propose a bimetallic $\mathrm{Cu}$-based catalyst whose surface is doped with atoms of an $\mathrm{H}_{2}$ activating metal, such as $\mathrm{Ni}$ or Pd. In chapters 4 and 5, we present the study of the SCR reaction with static DFT and molecular dynamics respectively. Previous studies have combined computational chemistry with experiments to study this particular reaction but it is not completely understood yet. Besides, to the best of our knowledge, enhanced methods of molecular dynamics have not been systematically used before. Finally, in chapter 6 , we present computational simulations of the NMR properties of the as-made RTH framework aiming at elucidating its structure. A more detailed context is given in the introduction of each chapter. 


\subsection{References}

(1) Eyring, H. The Activated Complex in Chemical Reactions. J. Chem. Phys. 1935, 3 (2), 107-115.

(2) Laidler, K. J.; King, M. C. Development of Transition-State Theory. J. Phys. Chem. 1983, 87 (15), 2657-2664.

(3) Introduction to Catalysis. In Concepts of Modern Catalysis and Kinetics; John Wiley \& Sons, Ltd, 2005; pp 1-21.

(4) Amghizar, I.; Vandewalle, L. A.; Van Geem, K. M.; Marin, G. B. New Trends in Olefin Production. Engineering 2017, 3 (2), 171-178.

(5) Ren, T.; Daniëls, B.; Patel, M. K.; Blok, K. Petrochemicals from Oil, Natural Gas, Coal and Biomass: Production Costs in 2030-2050. Resour. Conserv. Recycl. 2009, 53 (12), 653-663.

(6) Blaser, H.-U.; Steiner, H.; Studer, M. Selective Catalytic Hydrogenation of Functionalized Nitroarenes: An Update. ChemCatChem 2009, 1 (2), 210-221.

(7) Tolman, C. A.; Herron, N. The Selective Catalytic Oxidation of Hydrocarbons in Zeolites under Mild Conditions. Catal. Today 1988, 3 (2), 235-243.

(8) Liu, C.-C.; Ramu, R.; Chan, S. I.; Mou, C.-Y.; Yu, S. S.-F. Chemistry in Confined Space: A Strategy for Selective Oxidation of Hydrocarbons with High Catalytic Efficiencies and Conversion Yields under Ambient Conditions. Catal. Sci. Technol. 2016, 6 (20), 7623-7630.

(9) Armor, J. N. Environmental Catalysis. Appl. Catal. B Environ. 1992, 1 (4), 221-256.

(10) Iwamoto, M. Air Pollution Abatement through Heterogeneous Catalysis. In Studies in Surface Science and Catalysis; Corma, A., Melo, F. V., Mendioroz, S., Fierro, J. L. G., Eds.; 12th International Congress on Catalysis; Elsevier, 2000; Vol. 130, pp 23-47.

(11) Environmental Catalysis. In Concepts of Modern Catalysis and Kinetics; John Wiley \& Sons, Ltd, 2005; pp 377-400.

(12) Koebel, M.; Elsener, M.; Kleemann, M. Urea-SCR: A Promising Technique to Reduce NOx Emissions from Automotive Diesel Engines. Catal. Today 2000, 59 (3), 335-345.

(13) Radzicka, A.; Wolfenden, R. A Proficient Enzyme. Science 1995, 267 (5194), 90-93.

(14) Shevelev, I. V.; Hübscher, U. The 3'-5' Exonucleases. Nat. Rev. Mol. Cell Biol. 2002, 3 (5), 364-376.

(15) Taylor, H. S.; Armstrong, E. F. A Theory of the Catalytic Surface. Proc. R. Soc. Lond. Ser. Contain. Pap. Math. Phys. Character 1925, 108 (745), 105111.

(16) Ichikawa, S. Volcano-Shaped Curves in Heterogeneous Catalysis. Chem. Eng. 
Sci. 1990, 45 (2), 529-535.

(17) Ertl, G.; Weitkamp, J.; Knözinger, H. Preparation of Solid Catalysts; John Wiley \& Sons, 1999.

(18) Taylor, R.; Coulombe, S.; Otanicar, T.; Phelan, P.; Gunawan, A.; Lv, W.; Rosengarten, G.; Prasher, R.; Tyagi, H. Small Particles, Big Impacts: A Review of the Diverse Applications of Nanofluids. J. Appl. Phys. 2013, 113 (1), 011301.

(19) McCusker, L. B.; Liebau, F.; Engelhardt, G. Nomenclature of Structural and Compositional Characteristics of Ordered Microporous and Mesoporous Materials with Inorganic Hosts(IUPAC Recommendations 2001). Pure Appl. Chem. 2001, 73 (2), 381-394.

(20) Baerlocher, C.; McCusker, L. B. Database of Zeolite Structures http://www.iza-structure.org/databases/ (accessed Jun 21, 2020).

(21) Degnan, T. F. The Implications of the Fundamentals of Shape Selectivity for the Development of Catalysts for the Petroleum and Petrochemical Industries. J. Catal. 2003, 216 (1), 32-46.

(22) Marcilly, C. R. Where and How Shape Selectivity of Molecular Sieves Operates in Refining and Petrochemistry Catalytic Processes. Top. Catal. 2000, 13 (4), 357-366.

(23) Gao, S.; Liu, Z.; Xu, S.; Zheng, A.; Wu, P.; Li, B.; Yuan, X.; Wei, Y.; Liu, Z. Cavity-Controlled Diffusion in 8-Membered Ring Molecular Sieve Catalysts for Shape Selective Strategy. J. Catal. 2019, 377, 51-62.

(24) Ghysels, A.; Moors, S. L. C.; Hemelsoet, K.; De Wispelaere, K.; Waroquier, M.; Sastre, G.; Van Speybroeck, V. Shape-Selective Diffusion of Olefins in 8Ring Solid Acid Microporous Zeolites. J. Phys. Chem. C 2015, 119 (41), 23721-23734.

(25) Loewenstein, W. The Distribution of Aluminum in the Tetrahedra of Silicates and Aluminates. Am. Mineral. 1954, 39 (1-2), 92-96.

(26) Wilson, S. T.; Lok, B. M.; Messina, C. A.; Cannan, T. R.; Flanigen, E. M. Aluminophosphate Molecular Sieves: A New Class of Microporous Crystalline Inorganic Solids. J. Am. Chem. Soc. 1982, 104 (4), 1146-1147.

(27) Cundy, C. S.; Cox, P. A. The Hydrothermal Synthesis of Zeolites: Precursors, Intermediates and Reaction Mechanism. Microporous Mesoporous Mater. 2005, 82 (1), 1-78.

(28) Cundy, C. S.; Cox, P. A. The Hydrothermal Synthesis of Zeolites: History and Development from the Earliest Days to the Present Time. Chem. Rev. 2003, 103 (3), 663-702.

(29) Davis, M. E.; Lobo, R. F. Zeolite and Molecular Sieve Synthesis. Chem. Mater. 1992, 4 (4), 756-768.

(30) Martens, J. A.; Jammaer, J.; Bajpe, S.; Aerts, A.; Lorgouilloux, Y.; Kirschhock, 
C. E. A. Simple Synthesis Recipes of Porous Materials. Microporous Mesoporous Mater. 2011, 140 (1), 2-8.

(31) Rimer, J. D.; Fedeyko, J. M.; Vlachos, D. G.; Lobo, R. F. Silica Self-Assembly and Synthesis of Microporous and Mesoporous Silicates. Chem. - Eur. J. 2006, 12 (11), 2926-2934.

(32) Martínez, C.; Corma, A. Inorganic Molecular Sieves: Preparation, Modification and Industrial Application in Catalytic Processes. Coord. Chem. Rev. 2011, 255 (13), 1558-1580.

(33) Paolucci, C.; Khurana, I.; Parekh, A. A.; Li, S.; Shih, A. J.; Li, H.; Iorio, J. R. D.; Albarracin-Caballero, J. D.; Yezerets, A.; Miller, J. T.; Delgass, W. N.; Ribeiro, F. H.; Schneider, W. F.; Gounder, R. Dynamic Multinuclear Sites Formed by Mobilized Copper Ions in NOx Selective Catalytic Reduction. Science 2017, 357 (6354), 898-903.

(34) Weckhuysen, B. M. Snapshots of a Working Catalyst: Possibilities and Limitations of in Situ Spectroscopy in the Field of Heterogeneous Catalysis. Chem. Commun. 2002, No. 2, 97-110. 



\section{Chapter 2 Methods and models}

\subsection{Introduction}

Computational chemistry combines the fundamental laws of physics with mathematical algorithms to study relevant chemical processes. In this endeavor, and within the context of heterogeneous catalysis, we need two sets of specifications. First, we need a description of the physical system, that is, the number, nature and position of the particles. This set of information will be referred to as a model. In the next section, the models used for zeolites and metal surfaces are described. Secondly, we need the mathematical form of the forces acting among the particles in order to solve the equations of motion and compute the system's properties. An overview of the theory and most important approximations is provided in sections 2.3 to 2.7 .

\subsection{Periodic models.}

The success in predicting the rate of chemical reactions catalyzed by solids largely depends on how accurately the models describe the real catalytic environment. In the case of reactions on metal surfaces, the theoretical study is usually performed with supercell models that represent the metal surface. In this work, we are interested in understanding the influence of the nature of the metals on surface reaction mechanisms and we limit ourselves to the description of the most stable surface of $\mathrm{Ni}, \mathrm{Co}, \mathrm{Cu}$ and $\mathrm{Pd}$. Defects or size effects are out of the scope of the present investigation. The description of supercells is given in section 2.2.1.

For zeolites, there are two common models, cluster and periodic models. Zeolite cluster models are actually an approximation of periodic models. Clusters are constructed by cutting the catalytically relevant region out of the framework. The goal is to reduce the number of atoms shortening computation times and allowing 
the use of higher levels of theory. A serious drawback of this approach is that the topology of the zeolite framework, very often a relevant feature, is neglected. Besides, confinement effects and long-range interactions are better captured by considering the whole periodic structure. Periodic models consist of the unit cell of zeolite crystals repeated in the three dimensions. The downside is the greater number of atoms included in the zeolite unit cell (compared with clusters) because of which calculations are usually performed at lower levels of theory.

In this thesis, we have relied on periodic models and have performed the calculations at the GGA level of theory (see section 2.3.4). More details on zeolite models are given in section 2.2.2.

\subsubsection{Surfaces models}

Metal surfaces are described by the crystallographic planes of the bulk structure that is exposed. These crystallographic planes can be specified by a vector $H$ whose coefficients $(h, k, l)$ indicate where the crystallographic plane intersects the points $a / h, b / k$ and $c / l$ (eq 2.1 and Figure 2.1), being $a, b$ and $c$ the unit cell vectors. Negative values are denoted with a bar above the coefficient and zero means that the plane never intercepts the corresponding axis.

$$
H=h a+k b+l c
$$

The planes are referred to by writing the vector coefficient in parenthesis. For instance, the notation $\mathrm{Ni}(111)$ refers to the $\mathrm{Ni}$ surface corresponding to the (111) plane. This happens to be the most stable surface of the face-centered cubic unit cell and the one that we have studied in this work.

In order to create a supercell, we need to define the following parameters:

1) The exposed crystallographic plane.

2) Size of the supercell.

3) Thickness or number of atomic layers.

4) Vacuum region. 
(1 $\left.\begin{array}{lll}1 & 1 & 1\end{array}\right)$

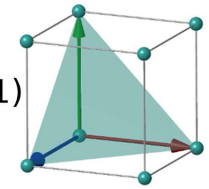

(1 110$)$

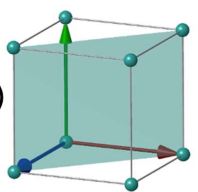

$\left(\begin{array}{lll}1 & 0 & 0\end{array}\right)$

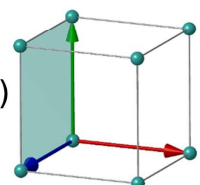

a

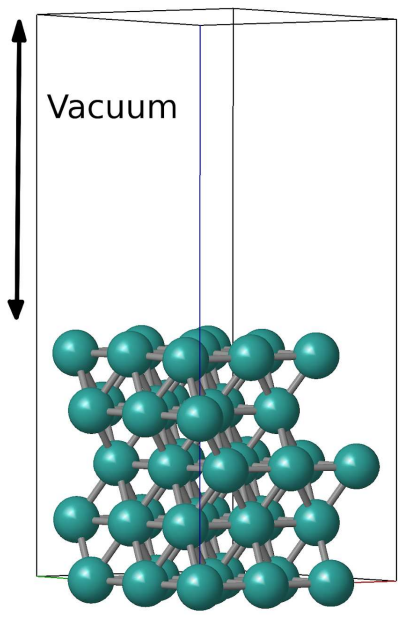

b
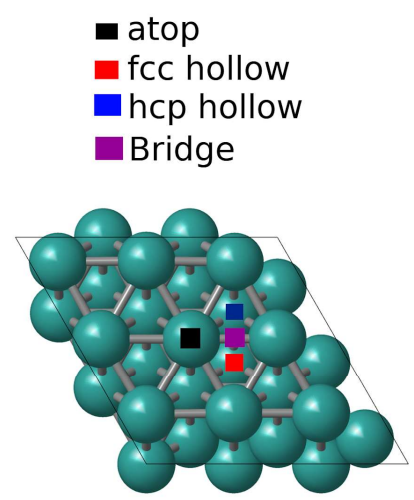

c

Figure 2.1 Schematic representation of a) crystallographic planes in the unit cell, b) five layer supercell of the (111) surface showing the vacuum region and c) five layer supercell showing different adsorptions sites.

Figure 2.1 shows a $3 \times 3$ supercell with five layers. The size $(\mathrm{NxN})$ must be carefully chosen so that there are no interactions between the periodic images. In this work, we have used $2 \times 2,3 \times 3$ and $4 \times 6$ supercells depending on the size and number of adsorbates. The number of layers is another important variable, because the utmost layers have to be optimized together with the adsorbed species while the lowest layers must be fixed to mimic the bulk behavior. It is usually sufficient to choose five layers, relax the two upmost and fix the lower three layers. Finally, it is also important to choose a sufficiently large vacuum region to avoid interactions between repeated images.

\subsubsection{Zeolites models}

Zeolites and SAPOs are crystalline materials and therefore can be represented with periodic models. In this work we have studied three microporous materials, the $\mathrm{Cu}$ SSZ-13 and Cu-SAPO-34 both with a CHA framework, and the RUB-13 with a RTH framework. 


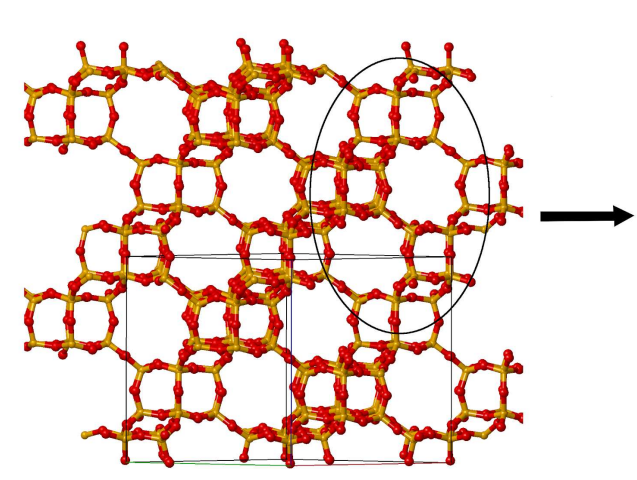

a

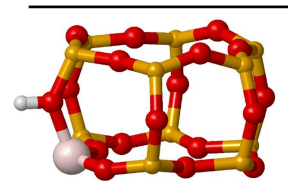

C

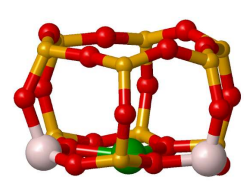

d

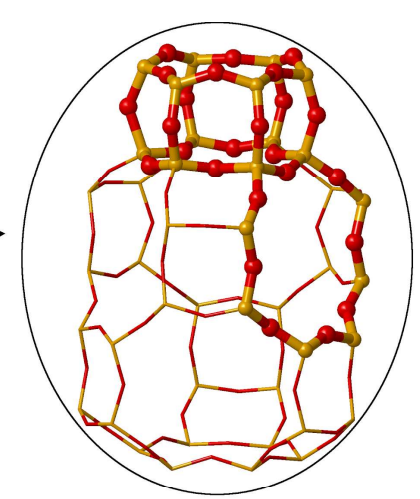

b

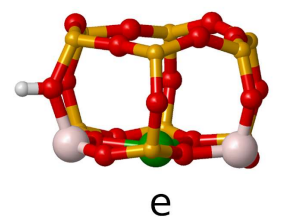

e

Figure 2.2. Representation of the CHA framework. a) Structure repeated along the three directions ( $\mathrm{a}, \mathrm{b}$, and $\mathrm{c})$. The unit cell is shown in black. b) the big CHA cavity. The $8 r$ and the $d 6 r$ units are shown with balls and sticks. c) one Al substitution compensated with a proton. d) two $\mathrm{Al}$ substitutions compensated with a $\mathrm{Cu}^{2+}$ cation. e) two $\mathrm{Al}$ substitutions compensated with a $\mathrm{Cu}^{+}$and a proton. $\mathrm{Si}, \mathrm{O}, \mathrm{Al}, \mathrm{H}$ and $\mathrm{Cu}$ atoms are orange, red, thatch, gray and green.

The chabazite structure was modeled by means of a hexagonal unit cell with lattice parameters $\mathrm{a}=\mathrm{b}=13.8026 \AA, \mathrm{c}=15.0753 \AA, \alpha=\beta=90^{\circ}$, and $\gamma=120^{\circ}$, containing $72 \mathrm{O}$ atoms and either $36 \mathrm{Si}$ or $18 \mathrm{Al}$ and $18 \mathrm{P}$ atoms. To generate the Cu-SSZ-13 and $\mathrm{Cu}-\mathrm{SAPO}-34$ models, one or two framework $\mathrm{Si}$ or $\mathrm{P}$ atoms were substituted by $\mathrm{Al}$ or $\mathrm{Si}$ atoms, respectively, $(\mathrm{Si} / \mathrm{Al}$ and $(\mathrm{Al}+\mathrm{P}) / \mathrm{Si}$ ratio $=17)$. When only one $\mathrm{Si}$ or $\mathrm{P}$ is substituted the negative charge is compensated with a $\mathrm{Cu}^{+}$cation in the $6 r$ or $8 r$. When two $\mathrm{Si}$ or $\mathrm{P}$ are substituted a $\mathrm{Cu}^{+}$cation with a Brönsted acid site nearby or a $\mathrm{Cu}^{2+}$ cation was placed in a $6 r$ or $8 r$ ring to keep the system neutral. This is shown in Figure 2.2. Details of the models for the RTH framework are given in the discussion of the results in chapter 6 . 


\subsection{Electronic structure methods}

The two most important approximations in computational chemistry to compute the forces on atoms are molecular mechanics (MM) and electronic structure methods. In MM, no explicit description of electrons is included; the system is described as a mechanically connected set of atoms. The pairwise interaction between particles is described through a set of potentials called force fields parametrized to fit experimental data or ab-initio calculations.

However, in order to study the formation and breaking of chemical bonds the motion of electrons must be properly described. Though force field methods are widely used to study the physical phenomena present in catalytic processes (e.g. adsorption and diffusion inside zeolites) they are not suitable to study the mechanism of a catalytic reaction (although reactive force fields with that aim are currently under development $)^{1,2}$.

\subsubsection{Basic concepts}

If we want to describe the motion of electrons it is mandatory to enter the realm of quantum mechanics whose fundamental equation is the Schrödinger equation:

$i \hbar \frac{\partial}{\partial t} \Psi(r, t)=\hat{H} \Psi(r, t)$

Where $r \equiv x_{1}, x_{2}, \ldots x_{N}$, with $x_{1} \equiv \overrightarrow{r_{1}}, \sigma_{1}$, being $\sigma_{1}$ the electron spin. By solving this equation, we have access, through the wavefunction $\Psi$, to all the information needed to define the state of the system. It means that once the wavefunction is known we can calculate the energy of the system and all the physical properties corresponding to that state. The wavefunction is the central object of the Quantum Theory and the main goal of quantum mechanical methods is to find accurate approximations to it. To this end, the time-independent, non-relativistic Schrödinger equation is usually solved instead:

$\hat{H}|\Psi\rangle=E|\Psi\rangle$ 
When solving this equation probably the firstly applied tweak is the BornOppenheimer approximation. The idea is to decouple the motion of electrons and nuclei. Because of the greater mass of nuclei, they move significantly slower than electrons, therefore, their kinetic energy is set to zero and the repulsion term between nuclei is set to a constant. We end up with the electronic Hamiltonian:

$\hat{H}_{\text {elect }}=-\frac{1}{2} \sum_{i=1}^{N} \nabla_{i}^{2}-\sum_{i=1}^{N} \sum_{A=1}^{M} \frac{Z_{A}}{r_{i A}}+\sum_{i=1}^{N} \sum_{j>i}^{N} \frac{1}{r_{i j}}=\hat{T}+\hat{V}_{N e}+\hat{V}_{e e}$

where, $\hat{T}$ is the kinetic energy operator, $\hat{V}_{N e}$ is the attractive potential between the nuclei and electrons and $\hat{V}_{e e}$ is the electronic repulsion term.

The Born-Oppenheimer approximation allows us to set up a simpler Hamiltonian for an arbitrary system, but it turns out that the Schrodinger equation cannot be solved for multi-electron systems. However, it is possible to start with a guess of the wavefunction and systematically approximate it to the ground state wavefunction. The permissibility of this approach is grounded on the variational principle which states that the energy computed according to equation 2.5 is an upper bound to the true energy of the system's ground state.

$$
E=\frac{\langle\Psi|\hat{H}| \Psi\rangle}{\langle\Psi \mid \Psi\rangle}
$$

In the above equation $\Psi$ is a guess wavefunction and $\hat{H}$ is the electronic Hamiltonian operator. Put in other words, the goal is to minimize the functional $E[\Psi]$.

The first reasonably accurate approach to solve this problem, used in real applications, was the Hartree-Fock method. In the Hartree-Fock method, the wavefunction of an $\mathrm{N}$-electron system is approximated as an antisymmetrized product of $\mathrm{N}$ one-electron wavefunctions $\chi_{i}$ called spin-orbitals, given as: 


$$
\Phi_{S D}=\frac{1}{\sqrt{N !}}\left|\begin{array}{cccc}
\chi_{1}\left(x_{1}\right) & \chi_{2}\left(x_{1}\right) & \ldots & \chi_{N}\left(x_{1}\right) \\
\chi_{1}\left(x_{2}\right) & \chi_{2}\left(x_{2}\right) & \ldots & \chi_{N}\left(x_{2}\right) \\
\vdots & \vdots & & \vdots \\
\chi_{1}\left(x_{N}\right) & \chi_{2}\left(x_{N}\right) & \ldots & \chi_{N}\left(x_{N}\right)
\end{array}\right|
$$

$\Phi_{S D}$ is known as Slater determinant. Determinants are naturally antisymmetric with respect to the exchange of rows and columns and consequently wavefunctions expressed in such a way fulfill Pauli's exclusion principle. By minimizing equation 2.5 with respect to the spin-orbitals of equation 2.6 and subject to the constraint that the spin-orbitals remain orthonormal, the Hartree-Fock equation (2.7) is obtained:

$\hat{f}_{i} \chi_{i}=\epsilon_{i} \chi_{i}$

where $\hat{f}_{i}$ is the one-electron Fock operator defined as:

$$
\hat{f}_{i}=-\frac{1}{2} \nabla_{i}^{2}-\sum_{A=1}^{M} \frac{Z_{A}}{r_{i A}}+V^{H F}(i)
$$

$V^{H F}$ is Hartree-Fock potential which represents the average potential on the electron $i$ caused by the presence of the remaining N-1 electrons. In turn, the Hartree-Fock potential $V^{H F}$ includes two terms as follows:

$$
v^{H F}\left(x_{1}\right)=\sum_{j}^{N}\left(\hat{J}_{j}\left(x_{1}\right)-\hat{K}_{j}\left(x_{1}\right)\right)
$$

where,

$\hat{J}_{j}\left(x_{1}\right)=\int\left|\chi_{j}\left(x_{2}\right)\right|^{2} \frac{1}{r_{12}} d x_{2}$

and 
$\hat{K}_{j}\left(x_{1}\right) \chi_{i}\left(x_{1}\right)=\int \chi_{j}^{*}\left(x_{2}\right) \frac{1}{r_{12}} \chi_{i}\left(x_{2}\right) d x_{2} \chi_{j}\left(x_{1}\right)$

The first term in equation 2.9 is the coulomb operator and represents the Coulombic potential experienced by an electron at position $\mathrm{x}_{1}$ due to the average distribution of another electron in spin-orbital $j$. The second operator, $K_{j}$, with no classical interpretation, is the exchange contribution to the Hartree energy which arises from the antisymmetric nature of the Slater determinant used to expand the wavefunction. It describes the effect of exchanging two electrons of parallel spin. As can be seen from equations 2.10 and 2.11 both operators depend on the spin-orbitals. Therefore, the Hartree-Fock equation has to be solved self-consistently. It means that a first guess of the spin-orbitals has to be defined to construct the Hartree-Fock operators. After solving the eigenvalue equations, a new set of spin-orbitals is obtained. This new set of spin-orbitals is used again to construct the coulomb and exchange operators and solve again the Hartree-Fock equations. New iterations are carried out until the input and output orbitals differ by less than a threshold. This procedure is called the self-consistent field (SCF) method.

The energy of the Hartree-Fock ground state, $E_{H F}$, is always higher than the energy of the ground state of the system $E_{0}$. This difference is called correlation energy,

$$
E_{c}^{H F}=E_{0}-E_{H F}
$$

and has two components, the static and dynamic correlation energies. The static correlation has its origin in the fact that the Slater determinant is just an approximation to the exact wavefunction, it captures the exchange effect but completely neglects the Coulomb correlation. The dynamic correlation energy is related to the instantaneous repulsion of electrons, which is not included in the Hartree-Fock formalism, because they only feel the average repulsion caused by the rest of electrons. Even though a very important part of the physics is captured by Hartree-Fock, the neglect of the electron correlation prevents it from providing chemical accuracy and thus is barely used today in real applications. However, we 
have devoted a couple pages to it because it constitutes the basis of most quantum mechanical wavefunction based methods. Most modern QM methods (such as Configuration Interactions, Coupled Cluster and Møller Plesset Perturbation theory) focus on finding better corrections to the electron correlation energy. We will not deal with them but an excellent discussion can be found in Szabo ${ }^{3}$.

\subsubsection{Density functional theory}

Despite the knowledge of the wave function enables us to access all the information of the state of the system, it depends on $4 \mathrm{~N}$ variables ( 3 spatial and 1 spin for each electron). It has what is called an exponential Wall ${ }^{4}$. Moreover, the wavefunction is not an observable, which means that it cannot be measured, but the electron density can in fact be measured by X-ray diffraction.

Here is where the electron density comes in as a fundamental quantity. The first attempt to use the electron density to access the information of a quantum system dates back to the work of Thomas and $\mathrm{Fermi}^{5-8}$. Based on the (fictitious) uniform electron gas model and treating the nuclear-electron and electron-electron interactions in a classical manner they derived the following equation for the energy:

$E[\rho(\vec{r})]=\frac{3}{10}\left(3 \pi^{2}\right)^{2 / 3} \int \rho^{5 / 3}(\vec{r}) d r-Z \int \frac{\rho(\vec{r})}{r}+\frac{1}{2} \iint \frac{\rho\left(\vec{r}_{1}\right) \rho\left(\vec{r}_{2}\right)}{r_{12}} d \vec{r}_{1} d \vec{r}_{2}$

In the above expression the energy is a function of the electron density, but the electron density is at the same time a function of the wave function,

$$
\rho(\vec{r})=N \int\left|\Psi\left(\vec{r} \sigma, x_{2}, \ldots, x_{N}\right)\right|^{2} d \sigma d x_{2}, \ldots, d x_{N}
$$

In mathematics, a function of a function is called a functional and hence the name "Density Functional Theory".

Those attempts did not achieve much popularity due to their limitations ${ }^{4}$. The foundations of a useful density functional theory formalism were laid by Hohenberg and Kohn with the two theorems they published in a paper entitled "Inhomogeneous Electron Gas" in 1964 . 
The first Hohenberg-Kohn theorem is the formal proof that the electron density can indeed be used to determine the properties of a particular quantum state. Let's quote from their original paper: "the external potential $V_{\text {ext }}(r)$ is (to within a constant) a unique functional of $\rho(\vec{r})$; since, in turn $V_{\text {ext }}(r)$ fixes $\hat{H}$ we see that the full many particle ground state is a unique functional of $\rho(\vec{r})$ ". Stated in other words, two different external potentials cannot produce the same ground state electron density. The second theorem helps us know when a particular density is the ground state density we are interested in. It states that the functional arrives at a minimum of energy if and only if the density is the true ground state density of the system. This statement is equivalent to the variational principle, that is, any trial electron density associated with an external potential will deliver an energy value that is an upper bound to the true energy of the ground state.

The energy of a system of particles in its ground state energy is a functional of the electron density. It is obvious that the same holds for the kinetic and the electronic interaction energy. Based on that, Hohenberg and Kohn introduced the following universal functional (which is valid for any system with any number of electrons):

$F[\rho]=T\left[\rho_{0}\right]+E_{e e}\left[\rho_{0}\right]$

where $\mathrm{T}$ is the kinetic energy and $E_{e e}$ is the electronic interaction. Thus, the energy can be thought of as consisting of two parts: one dependent and one independent of the system:

$E_{0}\left[\rho_{0}\right]=\int \rho_{0}(r) V_{N e} d r+F[\rho]$

where the first term of the right hand is system-dependent. The functional form of the second term is not known and the main goal of modern density functional theory is to find better approximations to this quantity.

The two previously mentioned theorems constituted a major breakthrough in the development of the modern density functional theory. However, none of them provide any clue on how to go about in practical terms finding the ground state density. Next section will describe how Kohn and Sham came up with a solution for this. 


\subsubsection{The Kohn-Sham approach}

Out of the three terms that make up the universal functional $F[\rho]$

$F[\rho]=T[\rho(\vec{r})]+J[\rho(\vec{r})]+E_{n c l}[\rho(\vec{r})]$

only the Coulomb interaction term $J[\rho(r)]$ is known. $E_{n c l}$ includes all non-classical interactions. However, the exact ground state wave function of a non-interacting system of electrons is a Slater determinant. Therefore, the kinetic energy of this system can be calculated exactly. This was realized by Kohn and Sham who proposed the idea of using a non-interacting reference system confined in an effective potential $V s$ through the following Hamiltonian:

$\hat{\mathcal{H}}=-\frac{1}{2} \sum_{i}^{N} \nabla_{i}^{2}+\sum_{i}^{N} V_{s}\left(\vec{r}_{i}\right)$

which generates the same density as the real interacting system. Notice that this Hamiltonian does not contain any electron-electron interaction term, and so, it is the true Hamiltonian of the non-interacting system. Thus, the spin orbitals can be obtained in a similar manner to the HF method as:

$\left(-\frac{1}{2} \nabla^{2}+V_{s}(\vec{r})\right) \phi_{i}=\epsilon_{i} \phi_{i}$

where,

$\hat{f}^{k s}=-\frac{1}{2} \nabla^{2}+V_{s}(\vec{r})$

is the Kohn-Sham one-electron operator. The kinetic energy of the real system is not the same as the fictitious system,

$T s[\rho(\vec{r})]=\sum\left\langle\Psi\left|-\frac{1}{2} \nabla^{2}\right| \Psi\right\rangle$ 
but the underlying idea is to split up the energy into all that can be calculated exactly and dump the rest into one term, that is, approximate the unknown contributions. So the universal functional is expressed as follows:

$F[\rho(\vec{r})]=T_{s}[\rho(\vec{r})]+J[\rho(\vec{r})]+E_{x c}[\rho(\vec{r})]$

where $E_{x c}$ is the exchange-correlation energy. This term contains the non-classical contributions from the self-interaction corrections, exchange and correlation, and the difference of the kinetic energy between the real and the fictitious system. Minimization of the former expression under the constraint that the Kohn-Sham spin-orbitals remain orthogonal (similar to Hartree-Fock) results in the Kohn-Sham equations:

$\left|-\frac{1}{2} \nabla^{2}+\int \frac{\rho\left(\vec{r}^{\prime}\right)}{\left|\vec{r}-\vec{r}^{\prime}\right|} d \vec{r}^{\prime}+V_{x c}(\vec{r})-\sum \frac{Z_{A}}{\left|\vec{r}-\vec{R}_{A}\right|}\right| \phi_{i}(\vec{r})=\epsilon_{i} \phi_{i}(\vec{r})$

which are solved self-consistently very similar to Hartree-Fock method. In fact, many DFT codes use routines already implemented for Hartree-Fock.

\subsubsection{The search for approximations to the exchange-correlation functional.}

The first approximation to the exchange-correlation functional was suggested by Kohn and Sham in the same paper where they proposed the self-consistent equations ${ }^{10}$. This approximation is based on the homogeneous electron gas model because it is the only system for which the exchange and correlation functionals are known. It consists in expressing the exchange energy $E_{x}$ as follows:

$E_{x}^{L D A}[\rho(\vec{r})]=-\frac{3}{4}\left(\frac{3}{\pi}\right)^{1 / 3} \int \rho(\vec{r})^{4 / 3} d \vec{r}$

which was derived by Dirac in $1930^{6}$. On the other hand, the correlation energy has been derived from Monte Carlo simulations of the uniform electron gas ${ }^{11}$. This first approximation is known as local density approximation (LDA) and was used mainly by physicists. Although reasonable geometries can be obtained using LDA it 
overbinds molecules. An example of these functionals is the VWN functional developed by Vosko, Wilk and Nusair in $1980^{12}$.

The next step toward a more robust functional was to include the gradient of the electron density $\nabla \rho(\vec{r})$ in order to describe the non-homogeneity of the true electron density. This improvement was introduced by Becke, Perdew, Langreth, and Parr in the 1980 s and is called generalized gradient approximation (GGA) ${ }^{13,14}$. In GGA, the exchange-correlation term $(E x c)$ is also split into the exchange $(E x)$ and correlation $(E c)$ contributions. The exchange term is expressed as:

$E_{x}^{G G A}[x, \rho]=\int \rho^{4 / 3} F(x) d \vec{r}$

where the argument $x$ of the function $F(x)$ is the reduced density gradient defined as:

$x=\frac{|\nabla \rho(\vec{r})|}{\rho^{4 / 3}(\vec{r})}$

The correlation part is much more complicated and is usually derived with Monte Carlo techniques. Among the most popular GGA functionals we encounter the $\mathrm{B} 88^{15}$, the PW9 $1{ }^{16}$ and $\mathrm{PBE}^{14}$ functionals. In this work, we have made ample use of the GGA functionals because they offer a reasonable compromise between accuracy and computational cost for periodic systems. In particular, we have used the PBE functional for which the exchange contribution is given by,

$E_{x}^{P B E}=-\int \rho^{4 / 3}(\vec{r})\left[\frac{3}{4}\left(\frac{3}{\pi}\right)^{1 / 3}+\frac{\mu x^{2}}{1+\mu x^{2} / \kappa}\right] d \vec{r}$

and also its revised version, the revPBE functional ${ }^{17}$.

The last two clear advances in DFT are probably the inclusion of the kinetic energy density, which resulted in the so-called meta-GGA ${ }^{18}$ functionals and the inclusion of a fraction of the Hartree-Fock exact exchange energy to generate the family of hybrid functionals. From the former group we have used the modified Becke-Johnson potential (TB-mBJ) ${ }^{19}$ and from the latter we have used the HSE06 hybrid functional ${ }^{20}$. 
This stepwise improvement in DFT is often called the Jacob's ladder ${ }^{18}$ of approximations to the exact exchange-correlation functional. This is a ladder that conveys the idea of ascending from the roughest approximations toward the "heaven" of chemical accuracy, $\sim 1 \mathrm{kcal} / \mathrm{mol}$. Details about functionals currently being developed can be found in recent reviews ${ }^{5,21}$

\subsubsection{Dispersion corrections.}

A big disadvantage of DFT is that the approximate functionals cannot capture the asymptotic $1 / R^{6}$ behaviour of long-range interactions such as the van der Waals forces. A method introduced by Grimme et al. ${ }^{22-24}$ allows to include these terms a posteriori. In this scheme the dispersion interactions are given by:

$$
E^{\text {disp }}=\sum_{i=1}^{N-1} \sum_{j=i+1}^{N}\left[\frac{C_{6}^{i j}}{R_{i j}^{6}}\right] f^{d a m p}\left(R_{i j}\right)
$$

where $\mathrm{C}_{6}$ is an empirical parameter and $f^{\text {damp }}$ is a damping function that varies smoothly from 0 to 1 defined as:

$f^{\text {damp }}(R)=\frac{1}{1-e^{-\propto\left(R / R_{0}-1\right)}}$

This pairwise correction incorporated into DFT is called DFT-D2. Notice that $E_{e x}{ }^{\text {disp }}$ is a functional of the nuclear geometry and not of the density.

The DFT-D3 correction is an extension of the above definition that includes higher dispersion orders, and modifications to the damping function:

$$
\begin{aligned}
& E^{d i s p}=\sum_{A B} \sum_{n=6,8,10} s_{n} \frac{C_{n, A B}}{r_{A B}^{n}} f_{d, n}\left(r_{A B}\right) \\
& f_{d, n}\left(r_{A B}\right)=\frac{1}{1+6\left(r_{A B} /\left(s_{n, r} R_{0}^{A B}\right)\right)^{-\alpha_{n}}}
\end{aligned}
$$

where $C_{n}{ }^{A B}$ are averaged $n$ th-order dispersion coefficient for $\mathrm{n}=6,8,10, \ldots$ for the atom pairs $\mathrm{AB}$ with internuclear distance $r_{A B}$. This approach is less empirical than $\mathrm{D} 2$, for example, the coefficients $\mathrm{C}_{6}{ }^{\mathrm{AB}}$ are no longer fixed but geometry dependent. 
Alternatively, the Becke-Johnson damping function can be used as well in this formulation:

$f_{d, n}\left(r_{A B}\right)=\frac{s_{n} r_{A B}^{n}}{r_{A B}^{n}+\left(a_{1} R_{0}^{A B}+a_{2}\right)^{n}}$

In this work, we have used the DFT-D3 with the damping function defined in equation 2.31 for calculations carried out with $\mathrm{CP} 2 \mathrm{~K}$ and with the damping defined in 2.32 for calculations carried with VASP unless otherwise specified. In all cases we have used the default parameters of the above definitions.

\subsubsection{Reciprocal space}

The description of crystal lattices in section 1.3.1 corresponds to real space representations of crystals. However, not all concepts in materials science can be adequately represented in real space. There is another way to describe lattices in solid state physics called "reciprocal space". This is a non-physical definition of space whose immediate application is the representation of diffraction phenomena and is an extremely useful frame to carry out electronic calculation in solids. The reciprocal space is the Fourier Transform (FT) of the real space and the reciprocal unit cell vectors $\left(a^{*}, b^{*}, c^{*}\right)$ are related to the real unit cell vectors $(a, b, c)$ as follows:

$a^{*}=2 \pi \frac{b \times c}{a(b \times c)}, \quad b^{*}=2 \pi \frac{a \times c}{b(a \times c)}, \quad c^{*}=2 \pi \frac{a \times b}{c(a \times b)}$

The reciprocal basis vectors are given in units of inverse of the length of the real basis vectors and the volume of the reciprocal unit cell is the inverse of the volume of the real unit cell. Notice that the reciprocal vectors are orthogonal to the real vectors (Figure 2.3). Similar to the real space, the reciprocal space vectors $G$ are given by,

$G=k_{1} a^{*}+k_{2} b^{*}+k_{3} c^{*}$

where $k_{i}$ are integers. The reciprocal space is also called $k$-space. Figure 2.3 illustrates in a 2-D representation the relations between real space and reciprocal space unit cells and also shows the first Brillouin zone. 

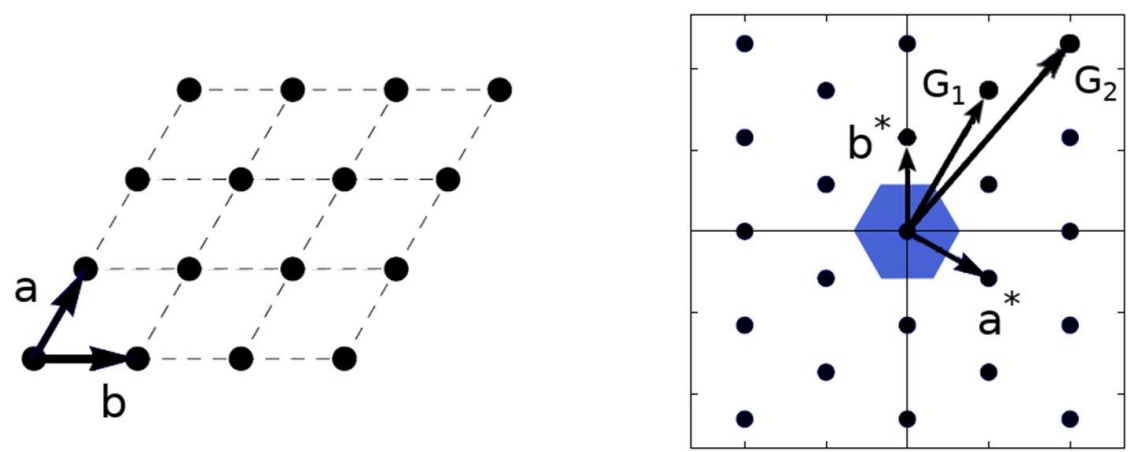

Figure 2.3 Hypothetical 2-dimensional lattice in real space (a) and its corresponding representation in the reciprocal space (b). The first Brillouin zone is delimited in blue.

The first Brillouin zone is a Wigner-Seitz in the reciprocal space, i.e, the closest set of points to the origin of the reciprocal lattice. The importance of the Brillouin zone stems from the fact that it completely accounts for the symmetry of the crystal so its properties can be studied by finding the solutions of the electronic wavefunctions within the first Brillouin zone.

\subsubsection{Plane waves and pseudopotentials}

In order to solve the Kohn-Sham equations we have to make a choice on how to express the solutions. In solid state physics, the best choice is to use plane waves which have the following mathematical form:

$\Psi(r)=e^{i k r}$

where $k=2 \pi / \lambda$ is the wave vector associated with the momentum $p=\hbar k$ and consequently to the energy of the wave, and $r$ is the position vector.

Plane waves are intimately connected with the Bloch theorem ${ }^{25-27}$ which is fundamental to describe periodic systems. The Bloch theorem states that in a periodic solid the one-electron wavefunctions can be expressed as the product of a plane wave and a function $u(r)$ with the same periodicity $t$ of the crystal, 


$$
\Psi(r)=e^{i k r} u(r)
$$

$u(r)=u(r+t)$

The periodic function $u(r)$ is commonly expanded on a basis set of plane waves for which the wave vectors are reciprocal lattice vectors $G$.

$u(r)=\sum_{G} c_{i, G} e^{i G r}$

Thus the solution of the wavefunction is expressed in terms of an infinite number of k-points in the first Brillouin zone. In practice, the energy is evaluated at a finite set of special k-points because the electronic wavefunction is almost identical for kpoints that are very close to each other.

One of the main advantages of plane waves is that they are naturally periodic so it is straightforward to use them to describe the periodicity of crystal structures. It is also possible to use highly efficient implementations of the fast Fourier transform (FFT) algorithms to switch between real and reciprocal space representations and the size of the basis set can be easily controlled by increasing the kinetic energy cut off,

$$
E_{c u t}=\frac{\hbar}{2 m}|G|^{2}
$$

However, a recent publication suggests that when large vacuum regions are required localized basis sets might be more efficient ${ }^{28}$.

Electronic wavefunctions oscillate very rapidly in the core region (Figure 2.4). As a result, the number of plane waves needed to describe this behavior is extremely large with which an impractical amount of computational time would be required. Here, a commonly used workaround is the pseudopotential approximation ${ }^{29-31}$. This approximation relies on the fact that most physical properties of solids depend mainly on the behavior of electrons in the valence region. Therefore, the core electrons are removed from the all-electron wavefunction and the strong ionic 
potential below a cutoff radius $R_{c}$ is replaced by a weaker pseudopotential. This is illustrated in the Figure 2.4
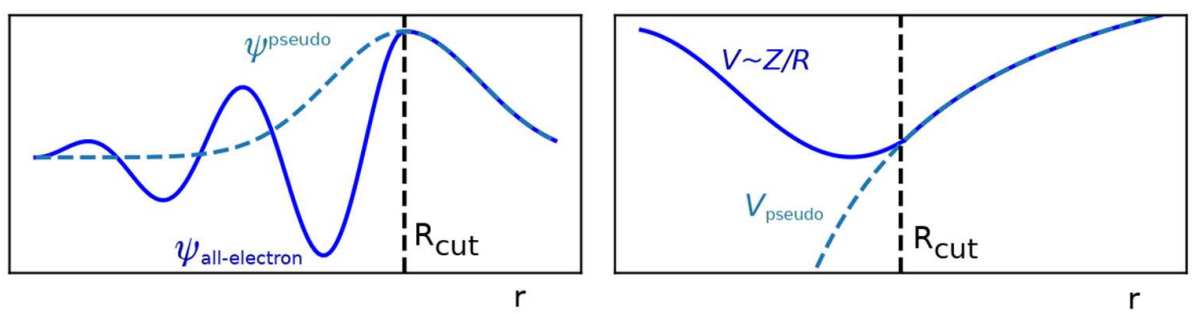

Figure 2.4 Schematic representation of potentials (right panel) and their corresponding wavefunctions (left panel) corresponding to all-electron (solid lines) and the pseudopotential approximations (dashed lines).

The most popular pseudopotentials are the ones defined by Martin-Troullier ${ }^{32}$, the ultrasoft pseudopotentials ${ }^{33}$ and the projector augmented wave $(\mathrm{PAW})^{34}$. The latter is the most accurate because it allows the reconstruction of the all-electron wavefunction but in turn the most demanding compared with the other pseudopotential methods. In the PAW scheme Blöchl introduces a linear transformation operator $\tau$ to map the valence pseudo wave functions onto the corresponding all-electron wave functions as follows:

$$
|\Psi\rangle=\tau|\tilde{\Psi}\rangle
$$

The transformation operator $\tau$ is defined as:

$$
\tau=1+\sum_{R, n}\left[\left|\phi_{R, n}\right\rangle-\left|\tilde{\phi}_{R, n}\right\rangle\right]\left\langle\tilde{p}_{R, n}\right|
$$

where $\left\langle\tilde{p}_{R, n}\right|$ are a set of projectors, $\left|\phi_{R, n}\right\rangle$ are the target all-electron partial waves obtained by applying $\tau$ on a set of pseudo partial waves $\left|\tilde{\phi}_{R, n}\right\rangle$. The projectors and pseudo partial waves are atom-centered functions with angular momentum quantum number $n$. The most important advantage of the PAW method is that it allows obtaining the expectation values of all-electron operators. In this work all electronic calculations have used this approach. 


\subsection{Potential Energy Surface (PES)}

The above methods allow us to compute the energy of a system of particles. However, we still need to specify a configuration for the set of nuclei, i.e, the atomic positions. Each distinct configuration will result in a different energy value. If we calculate the energy of a set of particles for all possible nuclei configurations of the set, we obtain what is called a potential energy surface (PES). The PES is multidimensional and the only way to visualize the surface is by plotting the energy as a function of only two variables (Figure 2.5). For computational chemists the most interesting regions of a PES are minima (local and global), and saddle points. Minima represent states of higher stability and are associated with reactants and products. First order saddle points are spots on the surface where the energy has maximum value along one coordinate, called reaction coordinate, and a minimum along all other coordinates. They always connect two minima along the reaction coordinate and are associated with transition states. The activation energy of a step of a reaction mechanism can be computed as the energy difference between the transition state and the minimum representing the reactants.
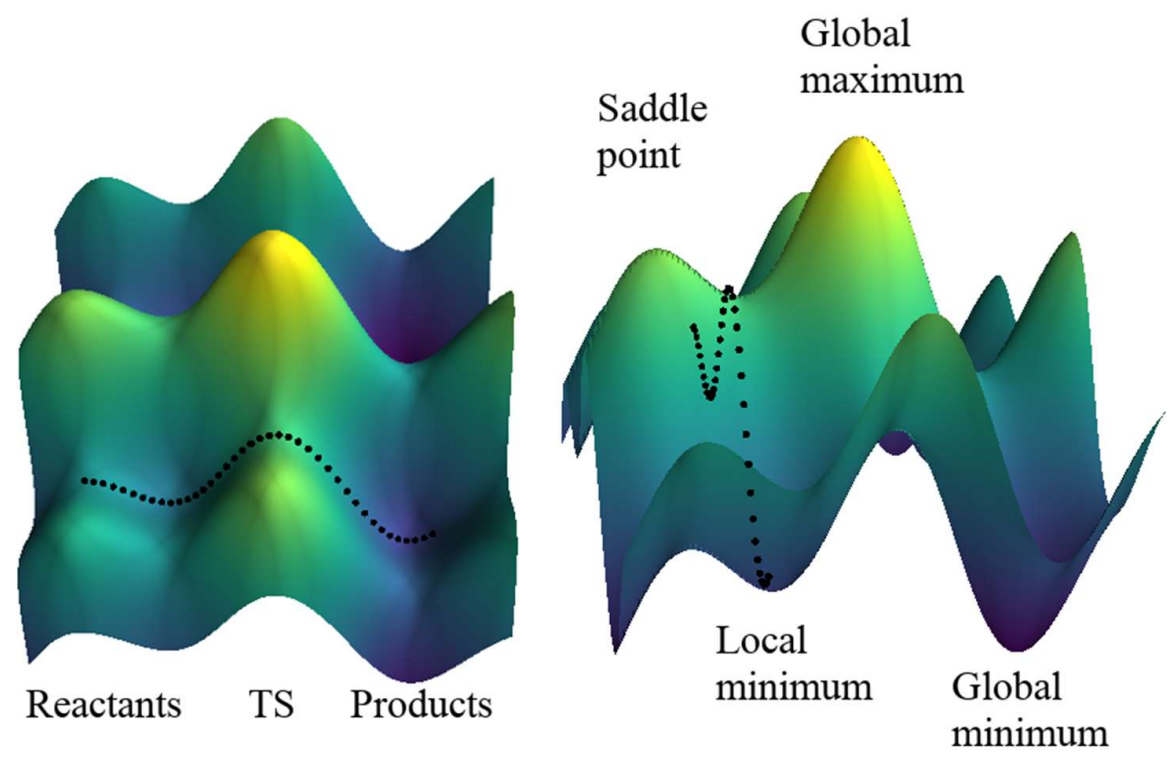

Figure 2.5 Representation of a potential energy surface. 


\subsection{Static Techniques}

The most common way to study chemical reactions is by exploring the PES using optimization techniques, since the derivative of the energy with respect to atomic positions is zero in minima and saddle points along all coordinates. By minimizing the energy with respect to the atomic positions we can find structures that in principle could be relevant for a chemical reaction. Popular optimization techniques include the conjugate gradient (CG), steepest descent (SD) and Limited-memory BroydenFletcher-Goldfarb-Shanno (LBFGS) algorithms. An overview of these and other methods, and aspects of their implementation in computer programs can be found in the book 35 "Numerical recipes: the art of scientific computing". We have mainly used the conjugate gradient as implemented in VASP because it is a very stable method and convergence is nearly always guaranteed.

Transition states are much more difficult to find. They have the particularity that the second derivative of the energy with respect to the atomic positions (curvature) is negative along the reaction coordinate and positive along all other coordinates, so the algorithms are slightly more complex and computationally expensive. In this work, we have used two methods implemented in VASP to find such saddle points, namely, the nudge elastic band (NEB) ${ }^{36}$ and dimer ${ }^{37,38}$ methods.

In the NEB method the two minima connected by the transition state have to be defined first. Then, a series of structures called images are created along the reaction coordinate between both minima and the optimization of all images runs in parallel. Spring (artificial) forces between contiguous images are introduced so that all images are equally spaced from each other resembling an elastic band. The number of images necessary to get a converged saddle point varies from $\sim 4$ to $\sim 20$ depending on the system. In the dimer method only an initial guess of the transition state structure and a guess of the reaction coordinate unit vector are needed. Two images (dimer) equally displaced from the initial guess structure along the reaction coordinate unit vector (dimer axis) are created. These two images are rotated around the dimer axis to maximize the curvature $\mathrm{C}$ along the dimer axis. The curvature is not calculated exactly but approximated according to equation 2.42 with which the computational cost is significantly reduced compared with methods in which the Hessian matrix (eq 2.48) is calculated. 
$C=\frac{\left(F_{2}-F_{1}\right) \cdot \hat{N}}{2 \Delta R}=\frac{E-2 E_{0}}{\Delta R^{2}}$

where $F_{1}$ and $F_{2}$ are forces acting on image 1 and 2, $\Delta R$ is the spacing between both images, $E$ is the sum of the both images' energy and $E_{0}$, the energy of the midpoint of the dimer. After rotation, the dimer is pushed uphill to the saddle point.

In the search for a transition state it is advisable to run first a NEB calculation to obtain an almost converged initial guess that will later be used in a dimer calculation. Of course, it all depends on the specific system in question. NEB calculations are efficient because images are optimized in parallel, but when the adequate number of cores are not affordable the NEB method might become very slow. Sometimes, when the reaction coordinate is not too complex, it is better to directly use the dimer method.

\subsubsection{Vibrational analysis.}

The standard way to confirm that we have obtained either a minimum or a transition state structure is by performing a vibrational analysis on the optimized geometry. For a minimum structure all calculated frequencies must be positive and for a transition state there must be only one imaginary frequency.

The calculation of the vibrational frequencies on an optimized structure is most commonly carried out by considering that the molecular vibrations can be described as those of a harmonic oscillator. The energy is expressed as a Taylor series:

$E(R)=E\left(R_{0}\right)+\frac{d E}{d R}\left(R-R_{0}\right)+\frac{1}{2} \frac{d^{2} E}{d R^{2}}\left(R-R_{0}\right)^{2}+\frac{1}{6} \frac{d^{3} E}{d R^{3}}\left(R-R_{0}\right)^{3}+\ldots$

where the third and higher order terms are neglected. The first order term is zero because the structure is a minimum and we end up with the following harmonic potential:

$E(R)=\frac{1}{2} \frac{d^{2} E}{d R^{2}}\left(R-R_{0}\right)^{2}=\frac{1}{2} k\left(R-R_{0}\right)^{2}$ 
Solving the Schrödinger equation for such an approximation we get the following vibrational energy levels:

$$
\begin{aligned}
& \varepsilon_{n}=\left(n+\frac{1}{2}\right) h \nu \\
& \nu=\frac{1}{2 \pi} \sqrt{\frac{k}{\mu}} \\
& \mu=\frac{m_{1} m_{2}}{m_{1}+m_{2}}
\end{aligned}
$$

where $n$ is a quantum number that takes up values from zero to infinity, $h$ is the Planck's constant, $v$ is the vibrational frequency, $k$ is the force constant and $\mu$ is the reduced mass. Notice that the energy levels are quantized and equally spaced (eq 2.45). The anharmonic corrections to the frequencies are performed by including higher other terms in the energy expansion (eq 2.43).

For a system with $\mathrm{N}$ atoms $(\mathrm{N}>2)$ the Hessian matrix (eq 2.48), has to be computed or approximated.

$$
H=\frac{\partial^{2} E}{\partial x_{1} \partial x_{2}}
$$

$H$ is a $3 \mathrm{Nx} 3 \mathrm{~N}$ symmetric matrix whose eigenvalues and eigenvectors are the force constants and the normal modes respectively of the $3 \mathrm{~N}-6$ frequencies. In VASP there are two methods to approximate the Hessian matrix: finite differences and density functional perturbation theory (DFPT) $)^{39}$.

\subsubsection{Free energy}

The methods described in the last section allow exploring the potential energy surface aiming at finding minima and saddle points. The structures thus obtained correspond to the $0 \mathrm{~K}$ and low pressure experimental conditions. However, real chemical systems are studied at much higher temperatures and pressures. They are not made up of isolated molecules but of a huge number of them, $\sim 10^{23}$. Besides, the change in free energy, and not just the electronic energy, is the thermodynamic gauge 
to predict spontaneity at constant temperature and pressure of such macroscopic systems. The free energy change between two states is given by the Gibbs equation:

$\Delta G=\Delta H-T \Delta S$

If the change in entropy tends to zero and enthalpy is approximated to the electronic energy, then the latter can serve as a fairly good approximation to describe the kinetics and thermodynamics of a chemical reaction. This is the case of some surface reactions following a Langmuir-Hinshelwood mechanism where the ratedetermining step occurs with all species adsorbed on the surface. Nonetheless, entropic effects are very often non-negligible and the activation free energy of a chemical reaction can significantly differ from the electronic energy.

The most straightforward way to get an approximate value of the free energy is to resort to statistical mechanics methods. In statistical mechanics, the macroscopic thermodynamics properties are calculated based on the partition function $\mathrm{Z}$ of an ensemble of particles. The partition function is the central quantity of statistical mechanics given as,

$Z=\sum_{i} e^{-E_{i} / k_{B} T}$

where the summation runs over all microstates $i$ of the ensemble. Similar to the wavefunction, the knowledge of the partition function provides access to the macroscopic thermodynamic properties, since the possible microstates in turn depend on the macroscopic constraints of the ensemble, i. e, number of particles, total energy, volume, pressure, etc.

In the following we will present the formulas used in this work to calculate the free energy when static DFT methods were used to study the PES. No derivation of the formulas will be given. For a detailed overview we refer the reader to the literature ${ }^{75}$

The absolute Gibbs free energies were calculated as follows:

$$
G=E_{\text {elec }}+E_{\text {zpe }}+R T+E_{\text {vib }}-T S_{\text {vib }}
$$


where $E_{\text {elec }}$ is the electronic energy obtained from the DFT calculation, $E_{z p e}$ is the zero point energy correction, $E_{v i b}$ is the vibrational thermal energy contribution and $\mathrm{S}_{\mathrm{vib}}$ is the vibrational entropy. The zero-point energy corrections and the vibrational contributions to the energy and entropy were calculated according to:

$$
\begin{aligned}
& E_{z p e}=\sum_{i=1}^{3 N-6} \frac{1}{2} h v_{i} \\
& E_{v i b}=R \sum_{i=1}^{3 N-6} \frac{h v_{i}}{k_{b}\left(e^{h v_{i} / k_{b} T}-1\right)} \\
& S_{v i b}=R \sum_{i=1}^{3 N-6}\left[\frac{h v_{i}}{k_{b}\left(e^{h v_{i} / k_{b} T}-1\right)}\right]-\ln \left(1-e^{h v_{i} / k_{b} T}\right)
\end{aligned}
$$

using the vibrational frequencies $v$ obtained from the DFT calculations. In the case of gaseous molecules, the rotation and translational contributions to the entropy and energy were also included in the calculation of the free energy as follows:

$$
G=E_{\text {tot }}+E_{z p e}+E_{v i b}+E_{\text {rot }}+E_{\text {trans }}+R T-T\left(S_{v i b}+S_{\text {rot }}+S_{\text {trans }}\right)
$$

where

$$
E_{\text {rot }}=E_{\text {trans }}=3 / 2 R T
$$

and the rotational and translational contributions to the entropy are given by:

$$
\begin{aligned}
& S_{\text {rot }}=R\left\{\ln \left[\sqrt{\frac{\pi I_{a} I_{b} I_{c}}{\sigma}}\left(\frac{2 \pi k_{b} T}{h^{2}}\right)^{3 / 2}\right]+\frac{3}{2}\right\} \\
& S_{\text {trans }}=R\left\{\ln \left[\left(\frac{2 \pi M k_{b} T}{h^{2}}\right)^{3 / 2} V\right]+\frac{3}{2}\right\}
\end{aligned}
$$


Finally, the equilibrium $\mathrm{K}_{\mathrm{eq}}$ and kinetic rate constants $K_{r}$ for the most relevant steps were derived from the calculated reaction and activation Gibbs free energies, as follows:

$$
\begin{aligned}
& K_{e q}=e^{-\Delta G / R T} \\
& K_{r}=\frac{k_{b} T}{h} e^{-\Delta G_{a c t} / R T}
\end{aligned}
$$

\subsection{Dynamics techniques}

The two most widely used techniques to naturally include entropic contributions are molecular dynamics (MD) ${ }^{40}$ and Monte Carlo (MC) ${ }^{41}$ simulations. In this work, we have used molecular dynamics to study the selective catalytic reduction of nitrogen oxides with $\mathrm{NH}_{3}\left(\mathrm{NH}_{3}-\mathrm{SCR}-\mathrm{NOx}\right)$ in CHA catalysts at different temperatures (chapter 5).

\subsubsection{Molecular dynamics}

The goal in molecular dynamics is to sample the phase space of a system of particles. In this technique atoms are considered classical particles and their positions are updated by integrating Newton's equations of motion (EOM). Velocity Verlet ${ }^{42,43}$ is probably the most widely used algorithm to integrate the EOM because it guarantees that the system will be time reversible. Time reversibility means that the same trajectory is reproduced exactly by iterating forward or backward in time. This important feature in principle guarantees the conservation of the energy over long simulation times. In velocity Verlet the time is discretized and at each time $t$ the velocities and positions of the particles are updated according to the following equations:

$\vec{x}(t+\Delta t)=\vec{x}(t)+\vec{v}(t) \Delta t+\frac{1}{2} \vec{a}(t) \Delta t^{2}$
$\vec{v}(t+\Delta t)=\vec{v}(t)+\frac{1}{2}(\vec{a}(t)+\vec{a}(t+\Delta t)) \Delta t$ 
The accelerations are computed from the forces acting on the atoms, which in turn are calculated as the first derivative of the potential energy with respect to the positions of the particles. In ab-initio molecular dynamics (AIMD) the forces are derived from electronic structure calculations at each time step which result in significantly expensive simulations. Thus, while in force field MD simulations it is possible to cover time scales of hundreds of nanoseconds, with AIMD simulations we are limited to a few hundreds of picoseconds for systems constituted by some hundreds of atoms with modern supercomputers.

All possible states of a system of particles subject to the same macroscopic constraints constitute an ensemble. The most basic is the microcanonical ensemble (NVE) in which the number of particles, the volume and the total energy of the system are constant. Real experiments are carried out at different conditions, therefore more realistic ensembles such as NVT or NPT are normally used. In NVT simulations the number of particles, the volume of the system and the temperature are constant. Similarly, the number of particles, the pressure and the temperature are constant for the NPT ensemble.

\subsubsection{Free energy methods}

In regular MD simulations the probability of sampling different states of the system is given by the Boltzman distribution ${ }^{43}$ and therefore, only low energy states are sampled. Since calculations of free energies from MD simulations rely on sufficiently sampled states, highly activated transitions cannot be properly studied. There are several techniques with which this limitation can be overcome, they are called enhanced sampling MD or free energy methods. Some of the most popular include potential of mean force (PMF), metadynamics (MTD) and umbrella sampling (US) ${ }^{44-47}$. In this work, we have used umbrella sampling to compute the Gibbs free energy of several processes (chapter 5).

The umbrella sampling method was introduced by Torrie and Valleau ${ }^{48}$ and it is routinely used in simulations with force fields. Its use in AIMD simulations of catalytic reactions is less common due to the computational cost. 
In order to apply umbrella sampling (and all the aforementioned free energy methods) we must first define the reaction coordinate or collective variable (CV) that best describes the transition in question. A CV can be any function of the particles' positions that unambiguously define the different states of the system during a transition. Common CVs are, for example, distances and angles between atoms, the volume of the system, center of mass, coordinations numbers, etc. Next section will cover in more detail the collective variables used in this work.

Once the collective variable $\xi$ is defined a bias potential term along $\xi$ is applied to drive the system through the different relevant states (e.g., reactants, transition states and products). The trajectory is split into several windows (points along the collective variable) for which individual MD simulations are performed to sample the region around each $\xi_{i}$ (Figure 2.6a).
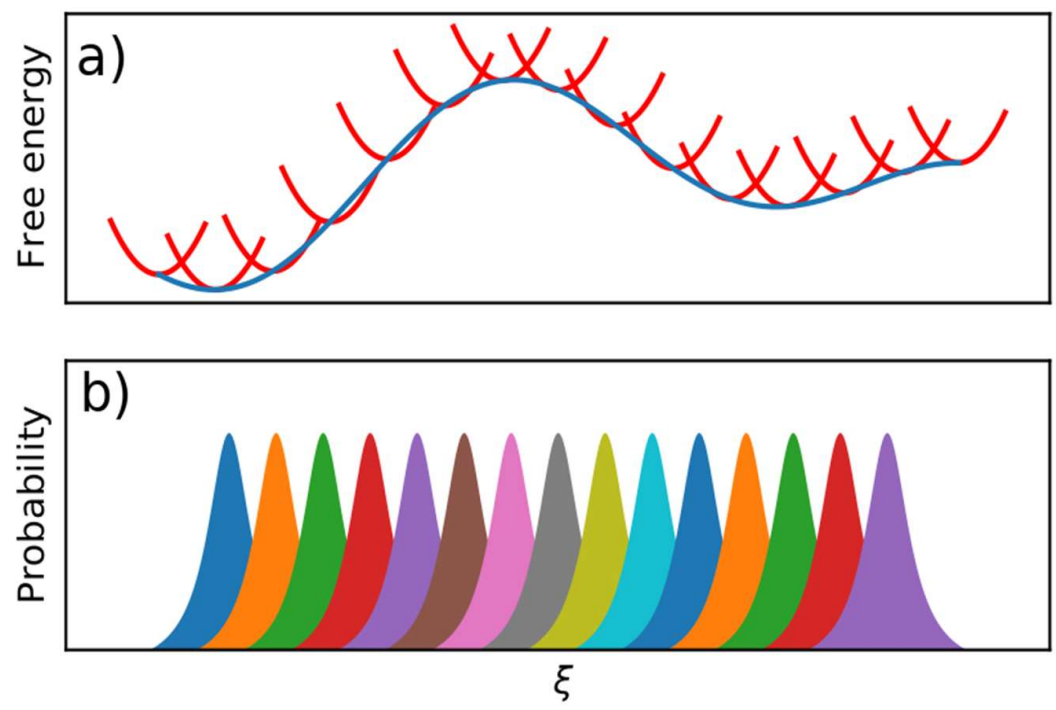

Figure 2.6 Representation of a) harmonic potentials for each window over an umbrella sampling free energy profile and b) overlapped histograms of individual windows.

A harmonic bias potential is applied to each window in order to restrict the sampling to a region centered at the value $\xi_{i}$ of the $\mathrm{CV}$ corresponding to the window $i$. Each bias potential $U_{i}(\xi)$ is defined as: 
$U_{i}(\xi)=\frac{K}{2}\left(\xi-\xi_{i}\right)^{2}$

where $\mathrm{K}$ is the bias strength. The choice of $K$ is the most important parameter. A sufficiently large value will guarantee the sampling of low probability regions. In turn, $K$ has to be sufficiently small to allow for overlapping between adjacent windows. Finally, the sampling obtained for each window is then analyzed altogether using a post-processing algorithm to construct the free energy profile as a function of $\xi$. To this end, we have used the weighted histogram analysis method $(\text { WHAM })^{49,50}$. The global distribution of probabilities is calculated by a weighted average of the distributions of the individual windows $P_{i}(\xi)$ according to equation 2.64,

$P(\xi)=\sum_{i}^{\text {windows }} p_{i}(\xi) P_{i}(\xi)$

where the weights $p_{i}(\xi)$ minimize the statistical error of $P(\xi)$.

$\frac{\partial \sigma^{2}(P)}{\partial p_{i}}=0$

Finally, the free energy $F$ is calculated from the global probability distributions as:

$F=-k_{B} T \ln P(\xi)+C$

The histograms of the CV of a sufficiently sampled umbrella sampling simulation should look like Figure $2.6 \mathrm{~b}$ where it can be observed that there is enough overlap between adjacent windows.

\subsubsection{Collective variables}

In this work, we have made use of several collective variables. One of them is the coordination number which measures the number of contacts between groups of atoms $\mathrm{A}$ and $\mathrm{B}$. It is defined as 
$C N=\sum_{i \in A} \sum_{j \in B} s_{i j}$

where $s_{i j}$ is 1 if contact between atoms $i$ and $j$ is formed, zero otherwise. In practice, to make sij continuous it is defined as a switching function as follows,

$$
s_{i j}=\frac{1-\left(R_{i j} / d_{0}\right)^{m}}{1-\left(R_{i j} / d_{0}\right)^{n}}
$$

where $R_{i j}$ is the distance between atoms $i$ and $j, d_{0}$ a switching cutoff distance that defines interatomic contact, $m$ and $n$ are the exponents of the switching function. In this work, $m$ and $n$ have been set to 6 and 12 respectively. In this way, $s_{i j}$ is zero if the distance of atom $i$ and $j$ is greater than $d_{0}, 0.5$ if the distance between atoms $i$ and $j$ equals $d_{0}$ and 1 if the distance between atom $i$ and $j$ is shorter than $d_{0}$. This is illustrated in Figure 2.7 where two switching functions have been defined $s 1$ and $s 2$ with cutoff distances of $\mathrm{d}_{0}=3$ and $\mathrm{d}_{0}=2$ respectively. The coordination number is defined as the summation of $s 1$ and $s 2$. Atoms inside the dotted circles (cutoff radii) belong to group A and those in the outside belong to group B. Groups A and B are not restricted to two atoms but any number of atoms can be included. Coordination numbers can also be normalized through division by the number of atoms so that they range from 0 to 1 .

We have used another collective variable, $\xi$, to study the diffusion of molecules through the $8 r$ of the CHA framework in chapter 5. We have defined $\xi$ as the projection of the center of mass of a molecule on the unit vector $\vec{n}$ normal to the average plane of the $8 r$. This is illustrated in Figure 2.8. The center of mass of $8 r$ represents the reference coordinate such that when the center of mass of the diffusing molecule is in the center of the $8 r$, $\xi$ is equal to 0 . Also, depending on the direction of the unit vector $\vec{n}, \xi$ takes up negative and positive values on the opposite sides of the $8 r$.

The absolute value of $\xi$ is the distance of the center of mass of a molecule in an arbitrary location to the average plane of the $8 r$. Notice that instead of the center of mass we might be interested in a specific fragment or atom of the molecule, or also 
be interested in another ring of the zeolite framework. In this sense, we have used the absolute value of $\xi$ with respect to the $6 r$ of the CHA to study the interaction of the $\mathrm{Cu}^{+}$and $\mathrm{Cu}^{2+}$ cations with negatively charged oxygen atoms of the $6 r$. This distance has been called $\mathrm{Cu}-6 r$ in this work.

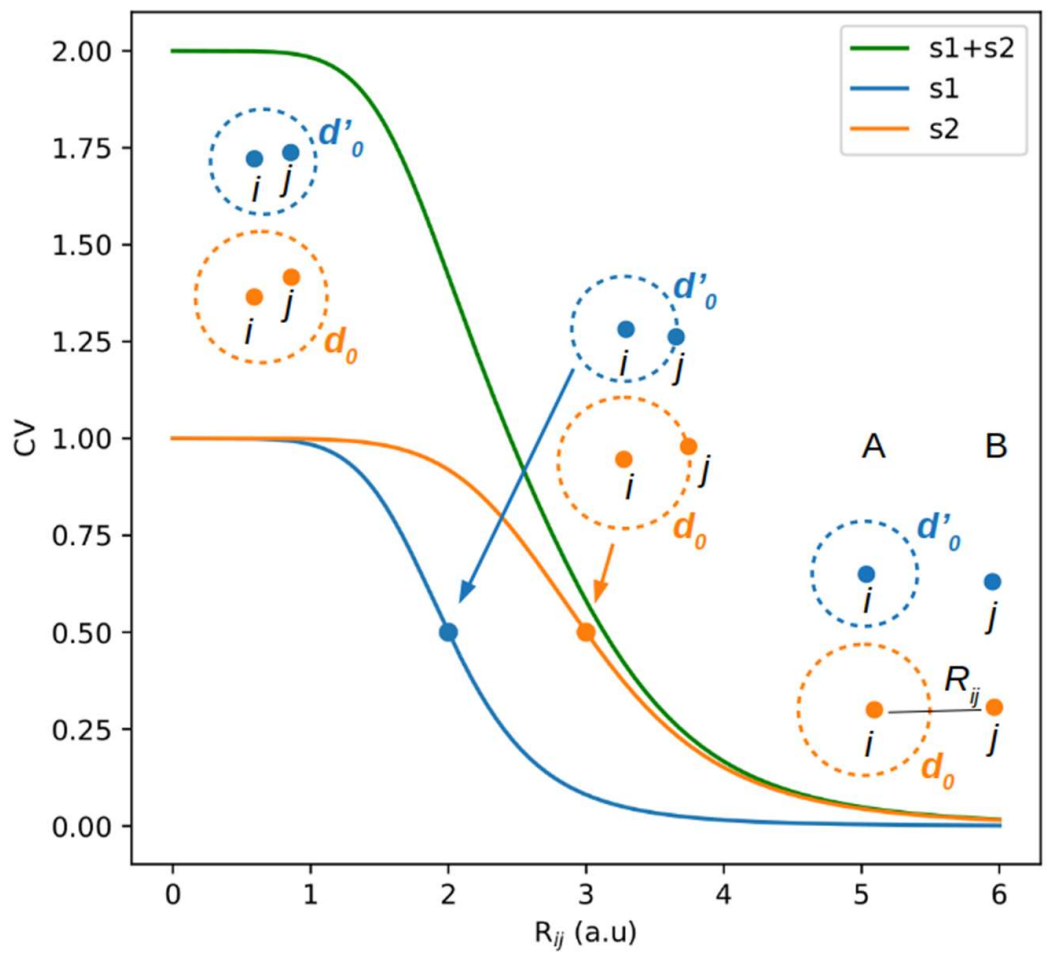

Figure 2.7 Illustration of the coordination number as a summation of two switching functions $\mathrm{s} 1$ and $\mathrm{s} 2$ with two switching cutoff $\mathrm{d}_{0}=3$ and $\mathrm{d}_{0}=2 . \mathrm{m}=6, \mathrm{n}=12$. Group $\mathrm{A}$ is made up of atoms $i$ (inside dotted circles) and group B is made up of atoms $j$ (outside dotted circles) 


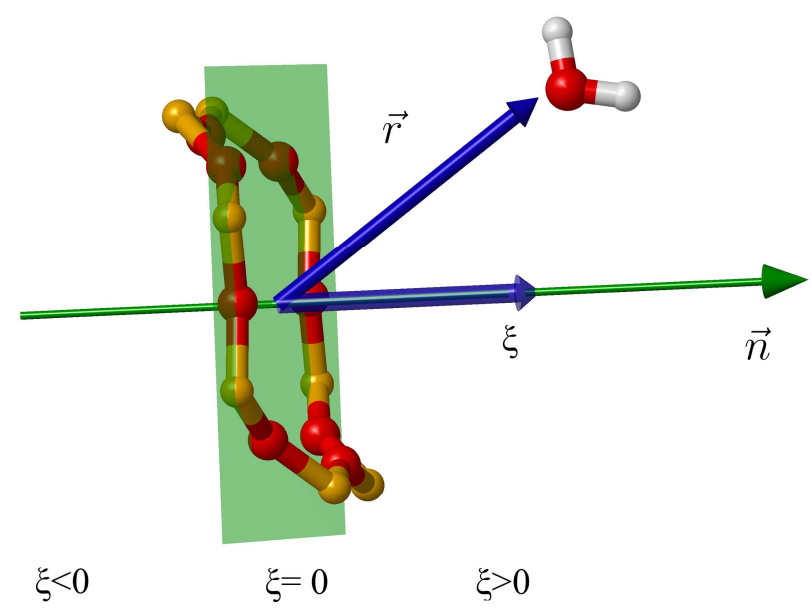

Figure 2.8. Representation of the diffusion of a water molecule through the $8 r$ window by means of the collective variable $\xi$ defined as the projection of the vector $\vec{r}$ on the unit vector $\vec{n}$ normal to the average plane of the $8 r$ (green plane). The vector $\vec{r}$ is the vector that represents the position of center of mass of the molecule with respect to the center of mass of the $8 r$. The $\mathrm{Si}$ and $\mathrm{O}$ atoms of the $8 r$ are shown in yellow and red, respectively.

\subsubsection{Vibrational frequencies from ab-initio molecular dynamics.}

The calculation of the vibrational frequencies using static techniques (section 2.5.1) suffers from other limitations apart from usually ignoring the anharmonic effects. The vibrational analysis is performed on optimized structures that correspond to $0 \mathrm{~K}$. Therefore, finite temperature effects are completely neglected. Besides, the models very often correspond to gas phase isolated molecules or clusters where intermolecular interactions like hydrogen bonds or other kinds of solvent effects are also completely neglected. These effects are included, however, in molecular dynamics simulations.

In AIMD simulations the vibrational spectra are derived by computing the Fourier transform (FT) of autocorrelation functions ${ }^{51,52}$. Thus, the FT of the particles velocities, the dipole moments and polarizabilities yield the power spectra, IR and Raman spectra respectively. The power spectrum is the easiest one because the velocities of nuclei are calculated as part of the integration of the EOM. It has peaks 
for each of vibrational normal modes but unfortunately the intensities are only of qualitative usefulness. Therefore, the usual method is to calculate the FT of the dipole moments, given as ${ }^{52,53}$ :

$I(\omega)=\frac{2 \pi \beta \omega^{2}}{3 c V} \int_{-\infty}^{\infty}\langle\delta \mu(t) \cdot \delta \mu(0)\rangle e^{i \omega t} d t$

where $\beta=1 / k_{B} T, \omega$ is the frequency of the adsorbed radiation, $V$ the system volume, $c$ the speed of light, and $\mu$ the dipole moment.

The dipole moments can be calculated at each time step with Berry phase method ${ }^{54,55}$ or with the maximally localized Wannier functions ${ }^{56-58}$. The former method is computationally less expensive but we only have access to the total dipole moment of the system. It means that it is not possible to analyze the individual contributions of different parts of the system, we cannot associate specific vibrations to functional groups. In the second approach the wavefunction is localized at the so-called Wannier centers at each time step with which the total dipole can be split into localized contributions. This method is nonetheless much more expensive than the Berry phase approach. We have computed the IR spectra from the dipole moments calculated using the Berry phase method implemented in $\mathrm{CP} 2 \mathrm{~K}$. The results are presented in chapter 5 .

\subsection{NMR basic concepts and computation.}

Nuclear Magnetic Resonance (NMR) spectroscopy is a widely used technique in the elucidation of chemical structures. It provides extremely valuable information about the electronic structure of materials because of its high sensitivity to the local environment of atoms. The central quantity of this technique is the chemical shift, $\delta$, which has its origin in the influence that an external magnetic field exerts on atoms.

When an external magnetic field $\vec{B}_{\text {ext }}$ is applied to a sample an electric current $\vec{J}$ arises due to orbital motions of electrons (for non-magnetic and insulating materials). This induced current $\vec{J}$ is proportional to $\vec{B}_{\text {ext }}$. In turn, the current $\vec{J}$ produces a nonuniform magnetic field ${ }^{59}$ defined as, 
$\vec{B}_{\text {ind }}=\frac{1}{c} \int d^{3} r^{\prime} \vec{j}\left(r^{\prime}\right) \times \frac{r-r^{\prime}}{\left|r-r^{\prime}\right|^{3}}$

The proportionality constant between the induced and the external uniform magnetic field is the shielding tensor:

$$
\vec{B}_{\text {ind }}=-\stackrel{\leftrightarrow}{\sigma} \vec{B}_{\text {ext }}
$$

The basic interactions in NMR are anisotropic, that is, dependent on the orientation with respect to the external magnetic field. Therefore, the shielding tensor is expressed in matricial form as follows ${ }^{60}$ :

$$
\overleftrightarrow{\sigma}=\left[\begin{array}{lll}
\sigma_{x x} & \sigma_{x y} & \sigma_{x z} \\
\sigma_{y x} & \sigma_{y y} & \sigma_{y z} \\
\sigma_{z x} & \sigma_{z y} & \sigma_{z z}
\end{array}\right]
$$

where the matrix elements $i j$ are the $i$-component of the shielding tensor along the $j$ axis. By diagonalization of the former matrix the three principal components (orientation dependent) of the shielding tensor can be obtained,

$$
\overleftrightarrow{\sigma}=\left[\begin{array}{ccc}
\sigma_{11} & 0 & 0 \\
0 & \sigma_{22} & 0 \\
0 & 0 & \sigma_{33}
\end{array}\right]
$$

By convention the three principal components are assigned in the following way ${ }^{61}$ :

$\sigma_{11} \leqslant \sigma_{22} \leqslant \sigma_{33}$

where $\sigma_{11}$ is the least shielded and $\sigma_{33}$ the most shielded component tensor.

The isotropic shielding is defined as the mean of the three principal axes of the shielding tensor (eq 2.74). In this way, the isotropic shielding is independent of the chosen reference frame ${ }^{59}$. 


$$
\sigma_{i s o}=\frac{1}{3} \operatorname{Tr}\{\stackrel{\leftrightarrow}{\sigma}\} \equiv \frac{1}{3}\left(\sigma_{11}+\sigma_{22}+\sigma_{33}\right)
$$

Other useful quantities to describe the shielding tensor are the span $(\Omega)$ and skew $(\kappa)$ defined by the Maryland group ${ }^{61,62}$ as:

$$
\begin{aligned}
& \Omega=\sigma_{33}-\sigma_{11}>0 \\
& \kappa=3\left(\sigma_{\text {iso }}-\sigma_{22}\right) /(\Omega)
\end{aligned}
$$

The span describes the degree of the chemical shift anisotropy and the skew the axial symmetry of the shielding tensor. A graphical representation of these concepts is provided in Figure 2.9.

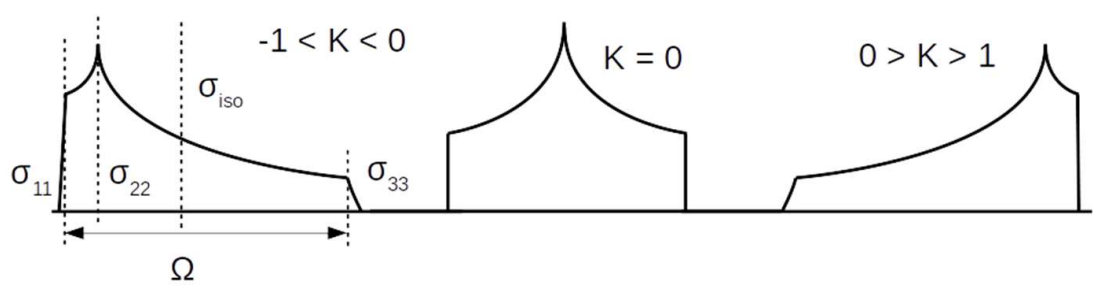

Figure 2.9 Representation of the shielding tensor components of a hypothetical powder sample NMR spectrum. Depending on the axial symmetry of the shielding tensor the skew can take up values from $-1\left(\sigma_{22}=\sigma_{33}\right)$ to $+1\left(\sigma_{11}=\sigma_{22}\right)$, see equation 2.76 .

The quantity that is accessible from experiments is the chemical shift, $\delta$, which is the isotropic shielding with respect to some reference compound:

$$
\delta=\frac{\sigma_{r e f}-\sigma}{1-\sigma_{r e f}}
$$

which is usually approximated $d^{59,60}$ as,

$$
\delta=\sigma_{r e f}-\sigma
$$


Similarly, the three components of the shielding tensor can be related to the three components of the chemical shift tensor,

$$
\delta_{i i}=\frac{\sigma_{r e f}-\sigma_{i i}}{1-\sigma_{r e f}}
$$

and so the isotropic chemical shift $\delta_{i s o}$ can also be defined as:

$\delta_{i s o}=\frac{1}{3} \operatorname{Tr}\{\stackrel{\leftrightarrow}{\delta}\} \equiv \frac{1}{3}\left(\delta_{11}+\delta_{22}+\delta_{33}\right)$

For liquid samples only the $\delta_{\text {iso }}$ is experimentally accessible because the random molecular tumbling only allows to detect a time average of the chemical shift tensor. Thus, NMR spectra of liquids are characterized by well-defined and narrow peaks. For powder samples, however, broad and featureless lines are observed because the static average of all possible orientations is measured, not the time average. The behavior of liquids can be mimicked by spinning the powder sample at speeds of up to $70 \mathrm{kHz}$ and at an angle $\theta_{m}$ of 54.74 with respect to the $B_{\text {ext }}$. This technique is called Magic Angle Spinning (MAS) $)^{63-65}$ and $\theta_{m}$ is called magic angle (Figure 2.10).
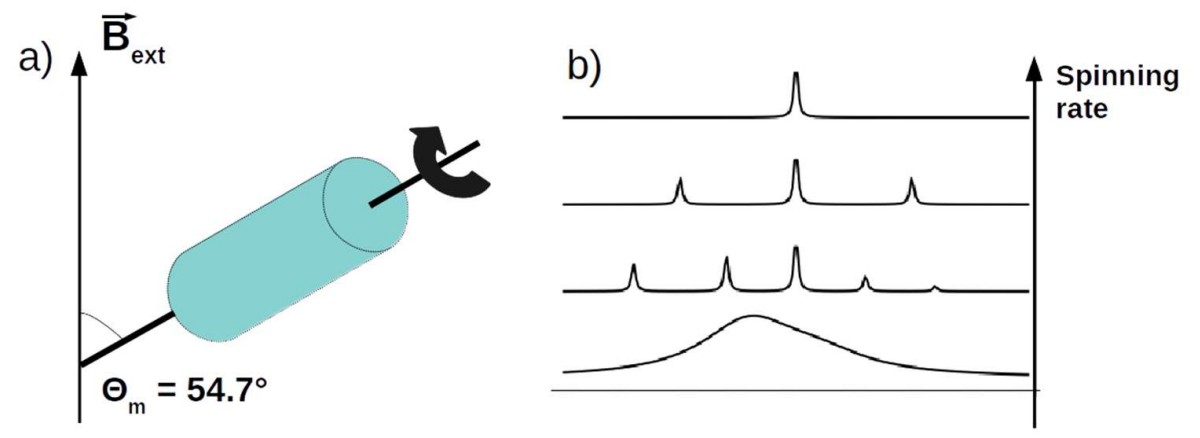

Figure 2.10. Representation of the magic angle spinning technique (MAS). a) Scheme showing the orientation and angle with respect to the external magnetic field $\vec{B}_{\text {ext }}$. b) Comparison of a NMR spectrum obtained with and without MAS.

In spite of its capabilities, the interpretation of the spectra is in many cases very complicated due to the complexity of the chemical systems (especially solid 
materials). It is there when the computational simulation of the chemical shift comes in as a complementary tool to aid in the elucidation of structures.

Most modern implementations of the computation of NMR properties are based on regular DFT functionals. These implementations suffer from the so-called "gaugeproblem" due to the use of finite one-electron basis sets. In the Kohn-Sham Hamiltonian, instead of the magnetic field $\vec{B}$, the related vector potential $\vec{A}$ enters the equation. They are related by the gradient of the field such that:

$$
\begin{aligned}
\vec{B} & =\nabla \times \vec{A} \\
H & =\frac{1}{2}\left(p+\frac{1}{c} \vec{A}(r)\right)^{2}+V
\end{aligned}
$$

The gauge origin problem arises because there can be different values of $A$ (different choices in the origin) that yield the same magnetic field. There is no unique definition of $A$. But the shielding tensor only depends on $\vec{B}$, so its expectation value must be independent of the choice of $\vec{A}$. Then it is said that the magnetic field is gauge invariant. In normal implementations using finite basis sets this condition is never fulfilled. Until the mid-90s two fundamental ways of coping with this problem were available: the individual gauge for localized orbitals (IGLO) $)^{66,67}$ and gauge including atomic orbital (GIAO) ${ }^{68,69}$. Unfortunately those methods are not applicable to periodic systems and the calculation of the NMR parameters was performed using clusters $^{70,71}$ as approximations to the periodic structure.

In 1996, Mauri et al. ${ }^{72,73}$, introduced a new theory with which the NMR shieldings of condensed matter systems could be calculated using periodic boundary conditions. This approach was formalized for all-electron Hamiltonians and it was not until 2001 with the introduction of the GIPAW method $^{73}$ (Gauge Including Projector Augmented Wave) that it was actually coded and applied. From its name it is easy to guess that it is related to the PAW approach described in section 2.3.6.

The first obvious drawback of pseudopotentials is that the form of the electronic wavefunction near the nucleus is completely neglected. NMR properties however require a detailed description of the electronic structure near the nucleus. This 
deficiency is dealt with by using the PAW method which reconstructs the all-electron wavefunction. Another drawback has to do with the aforementioned gauge problem, the PAW method does not preserve translational invariance in a uniform magnetic field. Analogous to the PAW method, a field dependent transformation operator $\tau_{B}$ is introduced in the GIPAW approach. This transformation operator imposes by definition the translational invariance as follows,

$\tau_{B}=1+\sum_{R, n} e^{(i / 2 c) r \cdot R \times \vec{B}}\left[\left|\phi_{R, n}\right\rangle-\left|\tilde{\phi}_{R, n}\right\rangle\right]\left\langle\tilde{p}_{R, n}\right| e^{(i / 2 c) r \cdot R \times \vec{B}}$

This approach is implemented in popular Quantum softwares like CASTEP, Wien2k, Quantum Espresso and VASP. We have used VASP because of its good performance.

The absolute chemical shielding, $\sigma_{i s o}$, is calculated directly with the above DFT codes. However, we have already mentioned that the experimentally available quantity is the chemical shift, $\delta_{i s o}$. Therefore, $\sigma_{i s o}$ has to be converted into $\delta_{i s o}$. One way to achieve this is to scale the calculated $\sigma_{\text {iso }}$ with a reference according to equation 2.77. It means that the absolute shielding of a reference compound must be computed as well. There are at least two sources of errors that affect the accuracy of the prediction of the chemical shift: inaccurate structural models and limitations in the used DFT functionals ${ }^{74}$. A more statistically accurate but more computationally demanding method is to fit the calculated shieldings to experimental chemical shifts for a series of compounds to a linear regression model, $m \sigma_{i s o}+n$. The predicted chemical shift is then given as

$\delta_{i s o}^{p r e d}=\frac{n-\sigma_{i s o}^{c a l c}}{m}$

The more compounds included in the regression the better the cancellation of errors. Ideally, the slope, $m$, should equal 1 so that by extrapolation to zero $\delta_{i s o}$ we get $\sigma_{\text {ref }}=n$ and

$\delta_{i s o}^{\text {pred }}=\sigma_{\text {ref }}-\sigma_{\text {iso }}^{\text {calc }}$ 


\subsection{References}

(1) van Duin, A. C. T.; Dasgupta, S.; Lorant, F.; Goddard, W. A. ReaxFF: A Reactive Force Field for Hydrocarbons. J. Phys. Chem. A 2001, 105 (41), 9396-9409. https://doi.org/10.1021/jp004368u.

(2) Senftle, T. P.; Hong, S.; Islam, M. M.; Kylasa, S. B.; Zheng, Y.; Shin, Y. K.; Junkermeier, C.; Engel-Herbert, R.; Janik, M. J.; Aktulga, H. M.; Verstraelen, T.; Grama, A.; van Duin, A. C. T. The ReaxFF Reactive Force-Field: Development, Applications and Future Directions. Npj Comput. Mater. 2016, 2 (1), 1-14. https://doi.org/10.1038/npjcompumats.2015.11.

(3) Szabo, A.; Oslund, N. S. Modern Quantum Chemistry: Introduction to Advanced Electronic Structure Theory; 1989.

(4) The Nobel Prize in Chemistry 1998 https://www.nobelprize.org/prizes/chemistry/1998/kohn/lecture/ (accessed Jun 23, 2020).

(5) Cohen, A. J.; Mori-Sánchez, P.; Yang, W. Challenges for Density Functional Theory. Chem. Rev. 2012, 112 (1), 289-320. https://doi.org/10.1021/cr200107z.

(6) Dirac, P. a. M. Note on Exchange Phenomena in the Thomas Atom. Math. Proc. Camb. Philos. Soc. 1930, 26 (3), 376-385. https://doi.org/10.1017/S0305004100016108.

(7) Slater, J. C. A Simplification of the Hartree-Fock Method. Phys. Rev. 1951, 81 (3), 385-390. https://doi.org/10.1103/PhysRev.81.385.

(8) March, N. H. Electron Density Theory of Atoms and Molecules. J. Phys. Chem. 1982, 86 (12), 2262-2267.

(9) Hohenberg, P.; Kohn, W. Inhomogeneous Electron Gas. Phys. Rev. 1964, 136 (3B), B864-B871. https://doi.org/10.1103/PhysRev.136.B864.

(10) Kohn, W.; Sham, L. J. Self-Consistent Equations Including Exchange and Correlation Effects. Phys. Rev. 1965, 140 (4A), A1133-A1138. https://doi.org/10.1103/PhysRev.140.A1133.

(11) Ceperley, D. M.; Alder, B. J. Ground State of the Electron Gas by a Stochastic Method. Phys. Rev. Lett. 1980, 45 (7), 566-569. https://doi.org/10.1103/PhysRevLett.45.566.

(12) Vosko, S. H.; Wilk, L.; Nusair, M. Accurate Spin-Dependent Electron Liquid Correlation Energies for Local Spin Density Calculations: A Critical Analysis. Can. J. Phys. 1980, 58 (8), 1200-1211. https://doi.org/10.1139/p80-159.

(13) Langreth, D. C.; Mehl, M. J. Beyond the Local-Density Approximation in Calculations of Ground-State Electronic Properties. Phys. Rev. B 1983, 28 (4), 1809-1834. https://doi.org/10.1103/PhysRevB.28.1809.

(14) Perdew, J. P.; Burke, K.; Ernzerhof, M. Generalized Gradient Approximation 
Made Simple. Phys. Rev. Lett. 1996, 77 (18), 3865-3868. https://doi.org/10.1103/PhysRevLett.77.3865.

(15) Becke, A. D. Density-Functional Exchange-Energy Approximation with Correct Asymptotic Behavior. Phys. Rev. A 1988, 38 (6), 3098-3100. https://doi.org/10.1103/PhysRevA.38.3098.

(16) Burke, K.; Perdew, J. P.; Wang, Y. Derivation of a Generalized Gradient Approximation: The PW91 Density Functional. In Electronic Density Functional Theory: Recent Progress and New Directions; Dobson, J. F., Vignale, G., Das, M. P., Eds.; Springer US: Boston, MA, 1998; pp 81-111. https://doi.org/10.1007/978-1-4899-0316-7_7.

(17) Zhang, Y.; Yang, W. Comment on "Generalized Gradient Approximation Made Simple". Phys. Rev. Lett. 1998, 80 (4), 890-890. https://doi.org/10.1103/PhysRevLett.80.890.

(18) Perdew, J. P.; Schmidt, K. Jacob's Ladder of Density Functional Approximations for the Exchange-Correlation Energy. AIP Conf. Proc. 2001, 577 (1), 1-20. https://doi.org/10.1063/1.1390175.

(19) Becke, A. D.; Johnson, E. R. A Simple Effective Potential for Exchange. J. Chem. Phys. 2006, 124 (22), 221101. https://doi.org/10.1063/1.2213970.

(20) Krukau, A. V.; Vydrov, O. A.; Izmaylov, A. F.; Scuseria, G. E. Influence of the Exchange Screening Parameter on the Performance of Screened Hybrid Functionals. J. Chem. Phys. 2006, 125 (22), 224106. https://doi.org/10.1063/1.2404663.

(21) Cramer, C. J.; Truhlar, D. G. Density Functional Theory for Transition Metals and Transition Metal Chemistry. Phys. Chem. Chem. Phys. 2009, 11 (46), 10757-10816. https://doi.org/10.1039/B907148B.

(22) Grimme, S. Semiempirical GGA-Type Density Functional Constructed with a Long-Range Dispersion Correction. J. Comput. Chem. 2006, 27 (15), 17871799. https://doi.org/10.1002/jcc.20495.

(23) Grimme, S.; Antony, J.; Ehrlich, S.; Krieg, H. A Consistent and Accurate Ab Initio Parametrization of Density Functional Dispersion Correction (DFT-D) for the 94 Elements $\mathrm{H}-\mathrm{Pu}$. J. Chem. Phys. 2010, 132 (15), 154104. https://doi.org/10.1063/1.3382344.

(24) Grimme, S.; Ehrlich, S.; Goerigk, L. Effect of the Damping Function in Dispersion Corrected Density Functional Theory. J. Comput. Chem. 2011, 32 (7), 1456-1465. https://doi.org/10.1002/jcc.21759.

(25) Bloch, F. Über die Quantenmechanik der Elektronen in Kristallgittern. Z. Für Phys. 1929, 52 (7), 555-600. https://doi.org/10.1007/BF01339455.

(26) Bohm, D. Note on a Theorem of Bloch Concerning Possible Causes of Superconductivity. Phys. Rev. 1949, 75 (3), 502-504. https://doi.org/10.1103/PhysRev.75.502. 
(27) Yamamoto, N. Generalized Bloch Theorem and Chiral Transport Phenomena. Phys. Rev. D 2015, $92 \quad$ (8), 085011. https://doi.org/10.1103/PhysRevD.92.085011.

(28) Adhikari, K.; Chakrabarty, A.; Bouhali, O.; Mousseau, N.; Becquart, C. S.; ElMellouhi, F. Benchmarking the Performance of Plane-Wave vs. Localized Orbital Basis Set Methods in DFT Modeling of Metal Surface: A Case Study for Fe-(110). J. Comput. Sci. 2018, 29, 163-167. https://doi.org/10.1016/j.jocs.2018.10.008.

(29) Hellmann, H. A New Approximation Method in the Problem of Many Electrons. J. Chem. Phys. 1935, 3 (1), 61-61. https://doi.org/10.1063/1.1749559.

(30) Gutsev, G. L. Numerical Pseudopotentials within DV-X $\alpha$ Framework. In Advances in Quantum Chemistry; Löwdin, P.-O., Sabin, J. R., Zerner, M. C., Brändas, E., Kövér, L., Kawai, J., Adachi, H., Eds.; Academic Press, 1998; Vol. 29, pp 137-157. https://doi.org/10.1016/S0065-3276(08)60267-1.

(31) Heine, V. The Pseudopotential Concept. In Solid State Physics; Ehrenreich, H., Seitz, F., Turnbull, D., Eds.; Academic Press, 1970; Vol. 24, pp 1-36. https://doi.org/10.1016/S0081-1947(08)60069-7.

(32) Troullier, N.; Martins, J. L. Efficient Pseudopotentials for Plane-Wave Calculations. Phys. Rev. B 1991, 43 (3), 1993-2006. https://doi.org/10.1103/PhysRevB.43.1993.

(33) Vanderbilt, D. Soft Self-Consistent Pseudopotentials in a Generalized Eigenvalue Formalism. Phys. Rev. B 1990, 41 (11), 7892-7895. https://doi.org/10.1103/PhysRevB.41.7892.

(34) Blöchl, P. E. Projector Augmented-Wave Method. Phys. Rev. B 1994, 50 (24), 17953-17979. https://doi.org/10.1103/PhysRevB.50.17953.

(35) Press, W. H. Numerical Recipes: The Art of Scientific Computing; Cambridge [Cambridgeshire]; New York : Cambridge University Press, 1986.

(36) Henkelman, G.; Uberuaga, B. P.; Jónsson, H. A Climbing Image Nudged Elastic Band Method for Finding Saddle Points and Minimum Energy Paths. J. Chem. Phys. 2000, 113 (22), 9901-9904. https://doi.org/10.1063/1.1329672.

(37) Henkelman, G.; Jónsson, H. A Dimer Method for Finding Saddle Points on High Dimensional Potential Surfaces Using Only First Derivatives. J. Chem. Phys. 1999, 111 (15), 7010-7022. https://doi.org/10.1063/1.480097.

(38) Heyden, A.; Bell, A. T.; Keil, F. J. Efficient Methods for Finding Transition States in Chemical Reactions: Comparison of Improved Dimer Method and Partitioned Rational Function Optimization Method. J. Chem. Phys. 2005, 123 (22), 224101. https://doi.org/10.1063/1.2104507.

(39) Gonze, X.; Lee, C. Dynamical Matrices, Born Effective Charges, Dielectric Permittivity Tensors, and Interatomic Force Constants from Density- 
Functional Perturbation Theory. Phys. Rev. B 1997, 55 (16), 10355-10368. https://doi.org/10.1103/PhysRevB.55.10355.

(40) Gunsteren, W. F. van; Berendsen, H. J. C. Computer Simulation of Molecular Dynamics: Methodology, Applications, and Perspectives in Chemistry. Angew. Chem. Int. Ed. Engl. 1990, 29 (9), 992-1023. https://doi.org/10.1002/anie.199009921.

(41) Dubbeldam, D.; Torres-Knoop, A.; Walton, K. S. On the Inner Workings of Monte Carlo Codes. Mol. Simul. 2013, 39 (14-15), 1253-1292. https://doi.org/10.1080/08927022.2013.819102.

(42) Verlet, L. Computer "Experiments" on Classical Fluids. I. Thermodynamical Properties of Lennard-Jones Molecules. Phys. Rev. 1967, 159 (1), 98-103. https://doi.org/10.1103/PhysRev.159.98.

(43) Frenkel, D.; Smit, B. Understanding Molecular Simulation: From Algorithms to Applications; Elsevier Ltd, London, 2001.

(44) Hansen, N.; van Gunsteren, W. F. Practical Aspects of Free-Energy Calculations: A Review. J. Chem. Theory Comput. 2014, 10 (7), 2632-2647. https://doi.org/10.1021/ct500161f.

(45) Christ, C. D.; Mark, A. E.; Gunsteren, W. F. van. Basic Ingredients of Free Energy Calculations: A Review. J. Comput. Chem. 2010, 31 (8), 1569-1582. https://doi.org/10.1002/jcc.21450.

(46) Abrams, C.; Bussi, G. Enhanced Sampling in Molecular Dynamics Using Metadynamics, Replica-Exchange, and Temperature-Acceleration. Entropy 2014, 16 (1), 163-199. https://doi.org/10.3390/e16010163.

(47) Pietrucci, F. Strategies for the Exploration of Free Energy Landscapes: Unity in Diversity and Challenges Ahead. Rev. Phys. 2017, 2, 32-45. https://doi.org/10.1016/j.revip.2017.05.001.

(48) Torrie, G. M.; Valleau, J. P. Nonphysical Sampling Distributions in Monte Carlo Free-Energy Estimation: Umbrella Sampling. J. Comput. Phys. 1977, 23 (2), 187-199. https://doi.org/10.1016/0021-9991(77)90121-8.

(49) Kumar, P.; Kim, D. W.; Rangnekar, N.; Xu, H.; Fetisov, E. O.; Ghosh, S.; Zhang, H.; Xiao, Q.; Shete, M.; Siepmann, J. I.; Dumitrica, T.; McCool, B.; Tsapatsis, M.; Mkhoyan, K. A. One-Dimensional Intergrowths in TwoDimensional Zeolite Nanosheets and Their Effect on Ultra-Selective Transport. Nat. Mater. 2020, 19 (4), 443-449. https://doi.org/10.1038/s41563019-0581-3.

(50) Souaille, M.; Roux, B. Extension to the Weighted Histogram Analysis Method: Combining Umbrella Sampling with Free Energy Calculations. Comput. Phys. Commun. 2001, 135 (1), 40-57. https://doi.org/10.1016/S00104655(00)00215-0.

(51) Thomas, M.; Brehm, M.; Fligg, R.; Vöhringer, P.; Kirchner, B. Computing 
Vibrational Spectra from Ab Initio Molecular Dynamics. Phys. Chem. Chem. Phys. 2013, 15 (18), 6608-6622. https://doi.org/10.1039/C3CP44302G.

(52) Galimberti, D. R.; Milani, A.; Tommasini, M.; Castiglioni, C.; Gaigeot, M.-P. Combining Static and Dynamical Approaches for Infrared Spectra Calculations of Gas Phase Molecules and Clusters. J. Chem. Theory Comput. 2017, 13 (8), 3802-3813. https://doi.org/10.1021/acs.jctc.7b00471.

(53) McQuarrie, D. A. Statistical Mechanics; University Science Books: Sausolito, CA, 2003.

(54) King-Smith, R. D.; Vanderbilt, D. Theory of Polarization of Crystalline Solids. Phys. Rev. B 1993, 47 (3), 1651-1654. https://doi.org/10.1103/PhysRevB.47.1651.

(55) Vanderbilt, D.; King-Smith, R. D. Electric Polarization as a Bulk Quantity and Its Relation to Surface Charge. Phys. Rev. B 1993, 48 (7), 4442-4455. https://doi.org/10.1103/PhysRevB.48.4442.

(56) Gaigeot, M.-P.; Sprik, M. Ab Initio Molecular Dynamics Computation of the Infrared Spectrum of Aqueous Uracil. J. Phys. Chem. B 2003, 107 (38), 1034410358. https://doi.org/10.1021/jp034788u.

(57) Marzari, N.; Souza, I.; Vanderbilt, D. An Introduction to Maximally-Localized Wannier Functions. 40.

(58) Marzari, N.; Vanderbilt, D. Maximally Localized Generalized Wannier Functions for Composite Energy Bands. Phys. Rev. B 1997, 56 (20), 1284712865. https://doi.org/10.1103/PhysRevB.56.12847.

(59) Haeberlen, U. High Resolution NMR in Solids Selective Averaging: Supplement 1 Advances in Magnetic Resonance; Elsevier, 2012.

(60) Widdifield, C. M.; Schurko, R. W. Understanding Chemical Shielding Tensors Using Group Theory, MO Analysis, and Modern Density-Functional Theory. Concepts Magn. Reson. Part A 2009, $34 A$ (2), 91-123. https://doi.org/10.1002/cmr.a.20136.

(61) Mason, J. Conventions for the Reporting of Nuclear Magnetic Shielding (or Shift) Tensors Suggested by Participants in the NATO ARW on NMR Shielding Constants at the University of Maryland, College Park, July 1992. Solid State Nucl. Magn. Reson. 1993, 2 (5), 285-288. https://doi.org/10.1016/0926-2040(93)90010-K.

(62) Jameson, C. J. Reply to 'conventions for Tensor Quantities Used in Nuclear Magnetic Resonance, Nuclear Quadrupole Resonance and Electron Spin Resonance Spectroscopy. Solid State Nucl. Magn. Reson. 1998, 11 (3), 265268. https://doi.org/10.1016/S0926-2040(98)00029-0.

(63) Andrew, E. R.; Eades, R. G. Possibilities for High-Resolution Nuclear Magnetic Resonance Spectra of Crystals. Discuss. Faraday Soc. 1962, 34, 38. https://doi.org/10.1039/df9623400038. 
(64) Lowe, I. J. Free Induction Decays of Rotating Solids. Phys. Rev. Lett. 1959, 2 (7), 285-287. https://doi.org/10.1103/PhysRevLett.2.285.

(65) Lesage, A. Recent Advances in Solid-State NMR Spectroscopy of Spin I = 1/2 Nuclei. Phys. Chem. Chem. Phys. 2009, 11 (32), 6876-6891. https://doi.org/10.1039/B907733M.

(66) Kutzelnigg, W.; van Wüllen, Ch.; Fleischer, U.; Franke, R.; v. Mourik, T. The IGLO Method. Recent Developments. In Nuclear Magnetic Shieldings and Molecular Structure; Tossell, J. A., Ed.; NATO ASI Series; Springer Netherlands: Dordrecht, 1993; pp 141-161. https://doi.org/10.1007/978-94011-1652-7_7.

(67) Gregor, T.; Mauri, F.; Car, R. A Comparison of Methods for the Calculation of NMR Chemical Shifts. J. Chem. Phys. 1999, 111 (5), 1815-1822. https://doi.org/10.1063/1.479451.

(68) Ditchfield, R. Self-Consistent Perturbation Theory of Diamagnetism. Mol. Phys. 1974, 27 (4), 789-807. https://doi.org/10.1080/00268977400100711.

(69) Schreckenbach, G. On the Relation between a Common Gauge Origin Formulation and the GIAO Formulation of the NMR Shielding Tensor. Theor. Chem. Acc. Theory Comput. Model. Theor. Chim. Acta 2002, 108 (4), 246253. https://doi.org/10.1007/s00214-002-0379-1.

(70) Schreckenbach, G.; Dickson, R. M.; Ruiz-Morales, Y.; Ziegler, T. The Calculation of NMR Parameters by Density-Functional Theory. In Chemical Applications of Density-Functional Theory; ACS Symposium Series; American Chemical Society, 1996; Vol. 629, pp 328-341. https://doi.org/10.1021/bk-1996-0629.ch023.

(71) Alkan, F.; Dybowski, C. Calculation of Chemical-Shift Tensors of Heavy Nuclei: A DFT/ZORA Investigation of $199 \mathrm{Hg}$ Chemical-Shift Tensors in Solids, and the Effects of Cluster Size and Electronic-State Approximations. Phys. Chem. Chem. Phys. 2014, 16 (27), 14298-14308.

(72) Mauri, F.; Pfrommer, B. G.; Louie, S. G. Ab Initio Theory of NMR Chemical Shifts in Solids and Liquids. Phys. Rev. Lett. 1996, 77 (26), 5300-5303.

(73) Pickard, C. J.; Mauri, F. All-Electron Magnetic Response with Pseudopotentials: NMR Chemical Shifts. Phys. Rev. B 2001, 63 (24), 245101.

(74) Ashbrook, S. E.; McKay, D. Combining Solid-State NMR Spectroscopy with First-Principles Calculations - a Guide to NMR Crystallography. Chem. Commun. 2016, 52 (45), 7186-7204.

(75) Cramer, C. J. Essentials of Computational Chemistry: Theories and Models; Wiley, 2005. 



\section{Chapter 3}

\section{Chemoselective reduction of nitrostyrene with non-noble metals}

\subsection{Introduction}

The industrial production of pharmaceuticals, agrochemicals, dyes and pigments makes use of functionalized anilines as starting materials. The preferred method for the production of such functionalized anilines is the chemoselective hydrogenation of nitroarenes with heterogeneous catalysts ${ }^{1,2}$. Chemoselectivity in this particular reaction means reducing the nitro group without reducing other functional groups of the nitroaromatic molecule such as $-\mathrm{CH}=\mathrm{CH}_{2},-\mathrm{Cl}$, - $\mathrm{CO}$, etc. Figure 3.1 shows the desired product out of three possible outcomes of the hydrogenation of nitrostyrene.

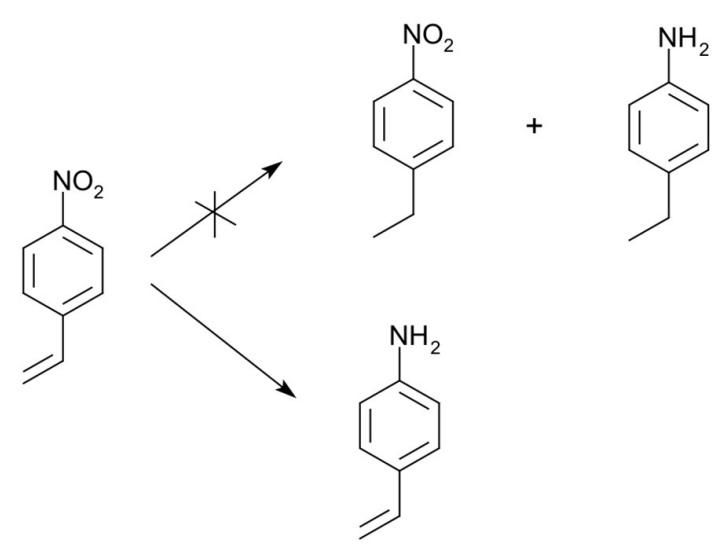

Figure 3.1 Chemoselective hydrogenation of 4-nitrostyrene. Only the nitro group is reduced, being aminostyrene the desired product. 
Catalysts based on Pt and Pd supported on active carbon, alumina and $\mathrm{CaCO}_{3}$ are probably the most frequently used. An example is the $1 \% \mathrm{Pt} / \mathrm{C}$ catalyst developed by Johnson-Matthey ${ }^{1}$ for halonitroarenes with which $99.3 \%$ selectivity at $100 \%$ conversion was achieved. Other catalysts such as Pt and Pd nanoparticles supported on carbon nanofibers ${ }^{3}$ performed well for chloronitrobenzenes but yielded somewhat lower selectivities for more challenging substituents such as $-\mathrm{CH}=\mathrm{CHCOOEt}$. In fact, functional groups containing double and triple bonds such as $\mathrm{C}=\mathrm{C}$, and $\mathrm{C}=\mathrm{O}$ are arguably the most demanding and, in most cases, require the catalysts to be tailored. On the one hand, organic or inorganic modifiers can be added to improve the selectivity or reduce the accumulation of undesirable intermediates like hydroxylamines. Typical modifiers to Pt-based catalysts are $\mathrm{H}_{3} \mathrm{PO}_{2}, \mathrm{~Pb}$ and $\mathrm{ZnX}_{2}$ $(\mathrm{X}=\mathrm{Be}, \mathrm{I})^{1,4}$. Probably the best example is the $\mathrm{Pt} / \mathrm{C}-\mathrm{H}_{3} \mathrm{PO}_{2}-\mathrm{V}$ catalytic system which exhibits high selectivities for carbon-carbon triple bonds ${ }^{5,6}$. On the other hand, catalysts can also be modified by reducing the metal particle size. In this regard, the most important contribution was probably made by Corma et al. ${ }^{7}$ who reported selectivities up to $99.6 \%$ at $96 \%$ conversion for the hydrogenation of 3-nitrostyrene with noble metal based catalysts. These catalysts were prepared by dispersing Au on reducible oxides such as $\mathrm{TiO}_{2}$ and $\mathrm{Fe}_{2} \mathrm{O}_{3}{ }^{8-11}$. By means of IR spectroscopy and computational methods ${ }^{12,13}$ it was shown that the high chemoselectivity achieved with $\mathrm{Au} / \mathrm{TiO}_{2}$ stems from the geometry of adsorption of the reactant nitroarene. It adsorbs perpendicular to the catalyst surface in such a way that only the nitro group interacts with the metal oxide support. In this way, the other reducible groups are not accessible for hydrogenation. An important drawback of the $\mathrm{Au} / \mathrm{TiO}_{2}$ catalysts is that gold hardly activates $\mathrm{H}_{2}$, which was overcome by introducing small amounts of $\mathrm{Pt}^{9}$. Platinum is highly active to dissociate the $\mathrm{H}_{2}$ bond so that the resulting bimetallic $1.5 \% \mathrm{Au} @ 0.01 \% \mathrm{Pt} / \mathrm{TiO}_{2}$ catalyst is also selective and an order of magnitude more active. Vilé et al. ${ }^{14}$ also reported high selectivities with ligand-capped platinum nanoparticles where the ligand controls the adsorption geometry favoring the interaction of the nitro group with the surface of the nanoparticle. The main drawback of these catalytic systems is that noble metals are expensive.

Nowadays, the focus has been redirected toward the less expensive non-noble metals expecting them to achieve the same performance and eventually replace the noble metal based catalysts. Nanoparticles of $\mathrm{Co}_{3} \mathrm{O}_{4}$ and $\mathrm{Fe}_{2} \mathrm{O}_{3}$ stabilized on carbon and Ndoped carbon supports ${ }^{15-18}$, and mono- and bimetallic nanoparticles containing $\mathrm{Co}$, 
$\mathrm{Ni}$ and Fe, usually supported on carbon-containing materials ${ }^{19-24}$ have been studied with promising results. It is clear that a great progress is taking place in the development of new catalysts for the chemoselective reduction of nitroarenes. Unfortunately, the same cannot be said when it comes to understanding the mechanism. Less attention has been paid to it and the few mechanistic studies on such materials usually rely on the classical macroscopic mechanism proposed by Haber in $1898^{25}$ (see Figure 3.2).

Haber's mechanism comprises two main routes to convert nitrobenzene into aniline: the direct and the condensation (or indirect) routes. In the direct route, three successive hydrogenation steps convert nitrobenzene $\left(\mathrm{Ph}-\mathrm{NO}_{2}\right)$ into aniline. In this process, nitrosobenzene ( $\mathrm{Ph}-\mathrm{NO})$ and phenylhydroxylamine $(\mathrm{Ph}-\mathrm{NHOH})$ are the key intermediates. In the condensation route, azoxybenzene $(\mathrm{Ph}-\mathrm{N}=\mathrm{NO}-\mathrm{Ph})$ forms by reaction between nitrosobenzene (Ph-NO) and hydroxylamine (Ph-NHOH). Subsequent hydrogenation steps lead to azo (R-N=N-R), hydrazo (R-NH-NH-R) and finally aniline.

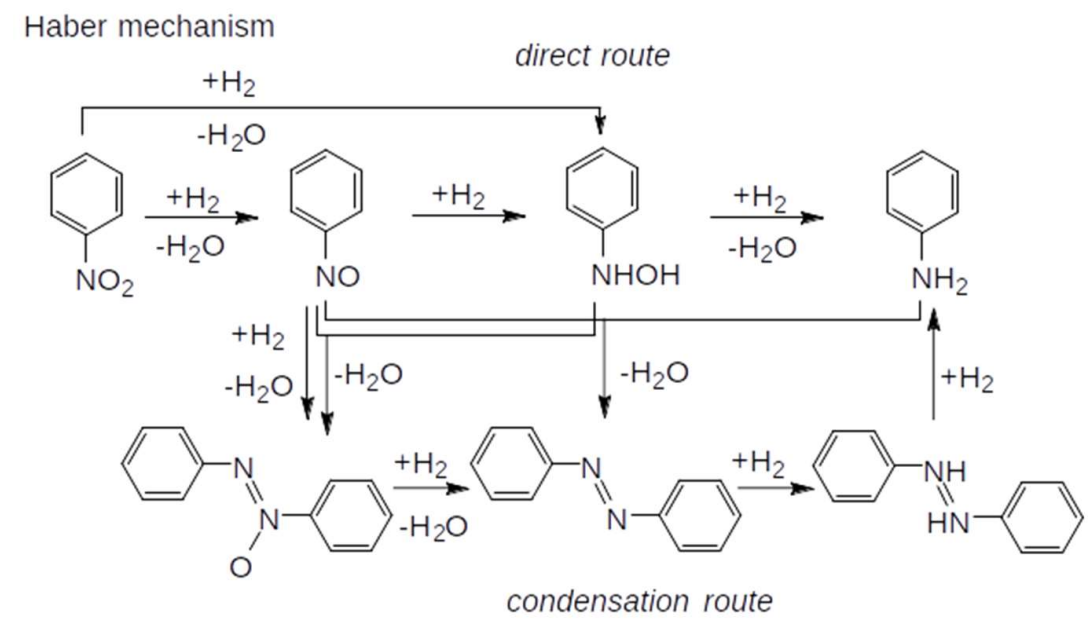

Figure 3.2 Proposed mechanisms for the hydrogenation of nitrobenzene. Direct and condensation routes described by Haber. 
Some research has been devoted to clarify aspects of the mechanism, always based or slightly modifying Haber's scheme. For example, Gelder et al. ${ }^{26}$ concluded that Haber's mechanism was incorrect since nitrosobenzene was not detected as a reaction intermediate in the direct route to aniline on Pd or Raney Ni catalysts.

They proposed instead that $\mathrm{Ph}-\mathrm{NOH}$ species was the true intermediate. On the other hand, Corma et al. ${ }^{27}$ showed that phenylhydroxylamine is formed when reducing nitrobenzene to aniline on $\mathrm{Au} / \mathrm{TiO}_{2}$. Another factor that complicates the investigation of the mechanism is that, obviously, different types of catalysts and different experimental conditions will probably drive the reaction through different pathways. For example, nitrosobenzene has been observed on Raney Ni catalyst at low $\mathrm{H}_{2}$ pressure $^{26}$. Besides, Haber's mechanism does not include all possible elementary steps.

Some attempts have also been made using a theoretical approach. Based on DFT calculations Sheng et al. ${ }^{28}$ proposed that the most demanding step on the $\operatorname{Pt}(111)$ surface was breaking the $\mathrm{N}-\mathrm{O}$ bond and that it had to be first activated by hydrogen transfer resulting in the following pathway: $\mathrm{Ph}-\mathrm{NO}_{2} \rightarrow \mathrm{Ph}-\mathrm{NOOH} \rightarrow \mathrm{Ph}-\mathrm{N}(\mathrm{OH})_{2} \rightarrow$ $\mathrm{Ph}-\mathrm{NOH} \rightarrow \mathrm{Ph}-\mathrm{NHOH} \rightarrow \mathrm{Ph}-\mathrm{NH} \rightarrow \mathrm{Ph}-\mathrm{NH}_{2}$. Zheng et al. ${ }^{29}$ studied the reduction of nitrobenzene on the bimetallic $\mathrm{Pd}_{3} \mathrm{Pt}$ system but only considered the adsorption of nitrobenzene through the nitro group. Namely, the molecule is oriented normal to the surface and adsorbs only through the nitro group. Other works were limited to only the adsorption of nitroarenes and no mechanistic inquiry was undertaken. Again, due to the difficulty of postulating a general mechanism for current catalysts, the mechanism is still unclear, especially for non-noble metals based catalysts.

In this chapter, we study the hydrogenation of nitrostyrene on different non noble metals surfaces aiming to shed light on the mechanism. The main purpose is to get a fundamental understanding of how the nature of the metal contributes or forces specific pathways. We will not take into account structural factors such as size, defects, etc. We begin with the adsorption of the reactant molecules and proceed with the detailed study of all possible elementary steps on $\mathrm{Ni}$ and $\mathrm{Co}$. The best routes found are also studied on $\mathrm{Cu}$ and $\mathrm{Pd}$. Finally, a presumably more efficient bimetallic catalyst is proposed based on the results obtained. 


\subsection{Methods and models}

DFT calculations were performed using the PBE exchange-correlation functional within the generalized gradient approach (GGA) ${ }^{30,31}$ as implemented in the VASP $\operatorname{code}^{32}$. The valence density was expanded in a plane wave basis set with a kinetic energy cutoff of $450 \mathrm{eV}$, and the effect of the core electrons in the valence density was taken into account by means of the projected augmented wave (PAW) formalism $^{33}$. All calculations are spin polarized. The atomic positions were optimized by means of the conjugate-gradient algorithm until atomic forces were smaller than $0.01 \mathrm{eV} / \AA$. Transition state structures were located using the DIMER method $^{34,35}$, and vibrational frequencies were calculated numerically. Dispersion energies were evaluated using the D3 Grimme's method ${ }^{36,37}$ with the Becke-Johnson damping $^{38}$. All energies reported in this chapter are the electronic energies with the D3 correction. The zero point energy correction (ZPE) has not been applied. However, the calculated Gibs free energies are summarized in appendix A.

The catalyst surface was simulated by means of a supercell slab model. Previous to the construction of the supercells the bulk lattice constants were optimized so that the three lowest layers that are kept fixed represent the bulk material. The optimized unit cell lattice constants of the fcc structure corresponding to the bulk Ni, Co, Cu and Pd are shown in Table 3.1.

Table 3.1. Experimental and optimized bulk lattice constants $(\AA)$ of the fcc unit cell.

\begin{tabular}{cccccc}
\hline & $\mathrm{Ni}$ & $\mathrm{Co}$ & $\mathrm{Cu}$ & $\mathrm{Pd}$ & $\mathrm{Pt}$ \\
\hline Optimized & 3.52 & 3.51 & 3.63 & 3.94 & 3.96 \\
Experimental & $3.50^{39}$ & $3.55^{40,41}$ & $3.60^{39}$ & $3.86^{39}$ & $3.91^{39}$ \\
\hline
\end{tabular}

The slab model for each metal consists of five atomic layers oriented along the (111) plane, which is the most stable and preferentially exposed, separated by a vacuum region of $10 \AA$ to avoid interaction between periodically repeated slabs. The size of the supercell slab was also large enough to avoid interaction between the periodically repeated adsorbates. Thus, nitrostyrene adsorption on the $\mathrm{Co}(111), \mathrm{Ni}(111), \mathrm{Cu}(111)$ and $\operatorname{Pd}(111)$ surfaces, the complete reaction mechanism for nitrobenzene hydrogenation, and the reaction of adsorbed oxygen and hydrogen atoms to form water, were investigated using a large $4 \times 6$ supercell slab containing 120 metal atoms 
as catalyst model. In this case, the integration in the reciprocal space was carried out at the $\Gamma \mathrm{k}$-point of the Brillouin zone. Smaller $2 \times 2$ and $3 \times 3$ supercell slabs were used to model $\mathrm{H}_{2}$ dissociation on $\mathrm{Co}(111), \mathrm{Ni}(111), \mathrm{Cu}(111)$ surfaces, using a converged Monkhorst-Pack mesh of $8 \times 8 \times 1 k$-points. During the geometry optimizations the atomic positions of the adsorbates and of the metal atoms in the two uppermost layers were allowed to fully relax, while the metal atoms in the three bottom layers were kept fixed at their bulk optimized positions.

For each system, adsorption ( $\left.E_{\text {ads }}\right)$, activation $\left(E_{\text {act }}\right)$ and reaction $\left(E_{\text {reac }}\right)$ energies were calculated as:

$$
\begin{aligned}
& E_{a d s}=E(\text { slab }+ \text { adsorbate })-E(\text { slab })-E(\text { adsorbate }) \\
& E_{a c t}=E(T S)-E(R) \\
& E_{\text {reac }}=E(P)-E(R)
\end{aligned}
$$

where $\mathrm{E}($ slab+adsorbate), $\mathrm{E}(\mathrm{slab})$ and $\mathrm{E}$ (adsorbate) are the total energies of the optimized M(l 111$)$ +adsorbate complex, clean $\mathrm{M}\left(\begin{array}{lll}1 & 1 & 1\end{array}\right)$ surface model and isolated adsorbate molecule, respectively. For each elementary step considered, E(R), E(TS) and $\mathrm{E}(\mathrm{P})$ are the total energies of the corresponding reactant complex, transition state and product, respectively.

\subsection{Adsorption of nitrostyrene}

Nitrostyrene can adsorb on the metal surface with two different orientations: parallel $(\mathrm{P})$ and normal $(\mathrm{N})$ to the surface. In the parallel adsorbed state, all the functional groups are able to interact with the catalyst surface while in the normal orientation only the nitro group is close enough to form chemical bonds with the surface metal atoms. The optimized structures of the adsorption states of nitrostyrene on $\mathrm{Ni}(111)$, $\mathrm{Co}(111), \mathrm{Cu}(111)$ and $\mathrm{Pd}(111)$ are shown in Figure 3.3.

In the parallel adsorption on $\mathrm{Ni}(111)$ and $\mathrm{Co}(111)$ the molecule is located above the surface plane with optimized Ni-O and Co-O distances of $\sim 2.0 \AA$, and Ni-C and Co$\mathrm{C}$ distances between 2.00 and $2.32 \AA$. The $\mathrm{M}-\mathrm{C}$ interactions lead to an expansion of the aromatic ring, with the $\mathrm{C}=\mathrm{C}$ bond lengths increasing by $\sim 0.05 \AA$. The $\mathrm{C}=\mathrm{C}$ bond 
of the vinyl group is also elongated $0.1 \AA$, and both N-O distances increase by $0.9 \AA$ as compared with the gas phase or isolated molecule, and the $\mathrm{H}$ atoms are tilted away from the aromatic ring plane by $\sim 23^{\circ}$. On $\operatorname{Pd}(111)$, however, the nitro group does not interact with the metal and the shortest Pd-O distances are as long as 3.20 and $3.46 \AA$. The $\mathrm{C}=\mathrm{C}$ bond lengths of the aromatic ring and of the vinyl group undergo a similar increase of $0.05-0.10 \AA$, and the $\mathrm{H}$ atoms are tilted $\sim 22^{\circ}$ from the aromatic ring plane. These modifications in the geometry indicate a change of hybridization of the vinyl and phenyl $\mathrm{C}$ atoms from $\mathrm{sp} 2$ to $\mathrm{sp} 3$ in the chemisorbed state. The calculated interaction energies are large in all cases, $-74 \mathrm{kcal} / \mathrm{mol}$ on $\mathrm{Ni}(111)$ and $\mathrm{Co}(111)$, and $-61 \mathrm{kcal} / \mathrm{mol}$ on $\mathrm{Pd}(111)$ (Table 3.2). The behavior of $\mathrm{Cu}(111)$ is different, and it was not possible to obtain a parallel adsorption.

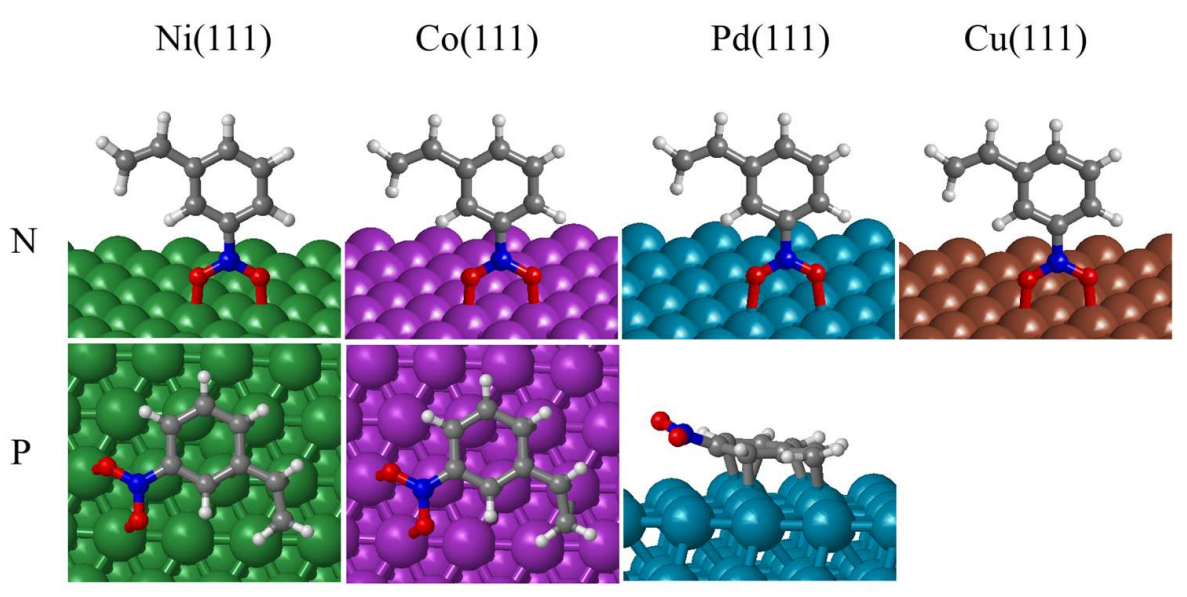

Figure 3.3 Optimized geometry of nitrostyrene adsorbed on $\mathrm{M}(111)$ surfaces in normal $(\mathrm{N})$ and parallel $(\mathrm{P})$ orientation. $\mathrm{Ni}, \mathrm{Co}, \mathrm{Pd}, \mathrm{Cu}, \mathrm{C}, \mathrm{N}, \mathrm{O}$ and $\mathrm{H}$ atoms are depicted as green, purple, eastern blue, maroon, gray, blue, red and white balls, respectively.

In the normal geometry, the molecule adsorbs on-top site with both $\mathrm{O}$ atoms of the nitro group bonded to two surface metal atoms (Figure 3.3). The M-O distances are somewhat shorter than in the parallel geometry, $1.96 \AA$ on $\mathrm{Ni}(111)$ and $\mathrm{Co}(111), 2.0$ $\AA$ on $\mathrm{Cu}(111)$ and $2.31 \AA$ on $\mathrm{Pd}(111)$. The geometrical parameters of the aromatic ring and the vinyl group do not change as compared with the isolated molecule but 
the $\mathrm{N}-\mathrm{O}$ bond length increases $0.05 \AA$ on $\mathrm{Ni}(111), \mathrm{Co}(111)$ and $\mathrm{Cu}(111)$, and $0.02 \AA$ on $\mathrm{Pd}$. The calculated interaction energies for the normal adsorption are clearly lower than for the parallel geometry, $\sim-30 \mathrm{kcal} / \mathrm{mol}$ on $\mathrm{Ni}(111)$ and $\mathrm{Co}(111)$ and $\sim-20$ $\mathrm{kcal} / \mathrm{mol}$ on $\mathrm{Cu}(111)$ and $\mathrm{Pd}(111)$ (Table 3.2). The significant difference in the calculated adsorption energies for the horizontal and normal configurations reflect the fact that in the latter geometry only the formation of $\mathrm{M}-\mathrm{O}$ bonds contributes to the adsorption energy. However, in the parallel geometry three types of interactions contribute, namely, the formation of M-O bonds (except in $\operatorname{Pd}(111)$ ), the formation of $\mathrm{C}-\mathrm{M}$ bond (aromatic and vinyl, except in $\mathrm{Cu}(111)$ ), plus the dispersion interactions.

Table 3.2 Adsorption energies of nitrostyrene on clean, O-covered and H-covered $\mathrm{M}(111)$ surfaces in parallel and normal orientation. DFT-D3 interaction energies are given in $\mathrm{kcal} / \mathrm{mol}$.

\begin{tabular}{ccccccc}
\hline Orientation & $\mathbf{H}$ atoms & $\mathbf{O}$ atoms & $\mathbf{N i}$ & $\mathbf{C o}$ & $\mathbf{C u}$ & $\mathbf{P d}$ \\
\hline \multirow{4}{*}{ Parallel } & $\mathbf{0}$ & $\mathbf{0}$ & -74.0 & -73.9 & - & -61.1 \\
& $\mathbf{0}$ & $\mathbf{6}$ & -63.5 & -69.9 & - & - \\
& $\mathbf{0}$ & $\mathbf{1 2}$ & -39.3 & -45.1 & - & - \\
& $\mathbf{6}$ & $\mathbf{0}$ & -69.8 & -70.7 & - & - \\
& $\mathbf{1 2}$ & $\mathbf{0}$ & -59.2 & -62.4 & - & - \\
\hline \multirow{4}{*}{ Normal } & $\mathbf{0}$ & $\mathbf{0}$ & -29.9 & -33.1 & -23.7 & -20.8 \\
& $\mathbf{0}$ & $\mathbf{6}$ & -31.9 & -34.6 & -27.1 & - \\
& $\mathbf{0}$ & $\mathbf{1 2}$ & -33.1 & -35.9 & - & - \\
& $\mathbf{6}$ & $\mathbf{0}$ & -30.1 & -32.3 & -23.4 & - \\
& $\mathbf{1 2}$ & $\mathbf{0}$ & -29.8 & -33.2 & -24.1 & - \\
\hline
\end{tabular}

Taking into account the oxophylic nature of the non-noble metals and the possibility that they are partly oxidized even under reaction conditions, we have considered the possible effect of the $\mathrm{H}^{*}$ and $\mathrm{O}^{*}$ adatoms on the adsorption of nitrostyrene. The presence of additional $\mathrm{O}$ atoms on the catalyst surface weakens the chemisorption of the aromatic ring and, as a consequence, the calculated interaction energies for the 
parallel adsorption continuously decrease in $\mathrm{Co}(111)$ and $\mathrm{Ni}(111)$ with increasing number of co-adsorbed $\mathrm{O}$ atoms. In contrast, the interaction energies obtained for the normal orientation remain constant in $\mathrm{Co}(111), \mathrm{Ni}(111)$ and $\mathrm{Cu}(111)$, indicating that this adsorption geometry might become competitive at large degree of $\mathrm{O}$ coverage. A similar effect is observed for the H-covered surfaces, the calculated interaction energies in the parallel adsorption decrease in $\mathrm{Co}(111)$ and $\mathrm{Ni}(111)$ as the number of co-adsorbed $\mathrm{H}$ atoms increase and remain practically constant for the normal adsorption in $\mathrm{Co}(111), \mathrm{Ni}(111)$ and $\mathrm{Cu}(111)$. The decrease of the interaction energy in the parallel adsorption due to $\mathrm{H}$ adatoms is less marked, however, compared with the $\mathrm{O}$ adatoms.

\subsection{Reaction network}

By taking nitrobenzene and styrene as model molecules we have studied the reduction of the nitro group and the $\mathrm{C}=\mathrm{C}$ bond separately on $4 \times 6$ supercell models. The first step in the reaction network, step 1, is the initial dissociation of $\mathrm{H}_{2}$, described in Section 3.5. Steps 2 and 3 represent the hydrogenation of the $\mathrm{C}=\mathrm{C}$ bond of styrene, studied in section 3.6. The main elementary steps in the mechanism of nitrobenzene hydrogenation to aniline catalyzed by metal $\mathrm{M}(111)$ surfaces are summarized in reactions 4-18, and the results obtained on $\mathrm{Ni}(111), \mathrm{Co}(111), \mathrm{Pd}(111)$ ad $\mathrm{Cu}(111)$ are discussed in sections 3.7, 3.8 and 3.10. Some relevant steps $(19,20$ and 21) of the condensation route on $\mathrm{Ni}(111)$ and $\mathrm{Co}(111)$ are studied in section 3.9, and the formation of water (steps 22 and 23) is studied in section 3.11 .

$$
\begin{aligned}
& \mathrm{H}_{2}{ }^{*} \leftrightarrow 2 \mathrm{H}^{*} \\
& \mathrm{Ph}-\mathrm{CH}=\mathrm{CH}_{2}{ }^{*}+\mathrm{H}^{*} \leftrightarrow \mathrm{Ph}-\mathrm{CH}-\mathrm{CH}_{3}{ }^{*} \\
& \mathrm{Ph}-\mathrm{CH}-\mathrm{CH}_{3}{ }^{*}+\mathrm{H}^{*} \leftrightarrow \mathrm{Ph}-\mathrm{CH}_{2}-\mathrm{CH}_{3}{ }^{*} \\
& \mathrm{Ph}-\mathrm{NO}_{2}{ }^{*}+\mathrm{H}^{*} \leftrightarrow \mathrm{Ph}-\mathrm{NOOH}^{*} \\
& \mathrm{Ph}-\mathrm{NOOH}^{*}+\mathrm{H}^{*} \leftrightarrow \mathrm{Ph}-\mathrm{N}(\mathrm{OH})_{2}{ }^{*}
\end{aligned}
$$

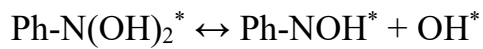

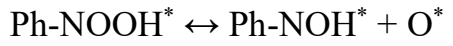

$$
\begin{aligned}
& \mathrm{Ph}-\mathrm{NOH}^{*} \leftrightarrow \mathrm{Ph}-\mathrm{N}^{*}+\mathrm{OH}^{*} \\
& \mathrm{Ph}-\mathrm{NOH}^{*}+\mathrm{H}^{*} \leftrightarrow \mathrm{Ph}^{-\mathrm{NHOH}^{*}}
\end{aligned}
$$




$$
\begin{aligned}
& \mathrm{Ph}-\mathrm{N}^{*}+\mathrm{H}^{*} \leftrightarrow \mathrm{Ph}^{-\mathrm{NH}^{*}} \\
& \mathrm{Ph}-\mathrm{NH}^{*}+\mathrm{H}^{*} \leftrightarrow \mathrm{Ph}^{-\mathrm{NH}_{2}}{ }^{*} \\
& \mathrm{Ph}^{-\mathrm{NHOH}^{*}} \leftrightarrow \mathrm{Ph}^{-\mathrm{NH}^{*}}+\mathrm{OH}^{*} \\
& \mathrm{Ph}-\mathrm{NO}_{2}{ }^{*} \leftrightarrow \mathrm{Ph}-\mathrm{NO}^{*}+\mathrm{O}^{*} \\
& \mathrm{Ph}-\mathrm{NO}^{*}+\mathrm{H}^{*} \leftrightarrow \mathrm{Ph}-\mathrm{NOH}^{*} \\
& \mathrm{Ph}-\mathrm{NO}^{*}+\mathrm{H}^{*} \leftrightarrow \mathrm{Ph}-\mathrm{NHO}^{*} \\
& \mathrm{Ph}-\mathrm{NO}^{*} \leftrightarrow \mathrm{Ph}-\mathrm{N}^{*}+\mathrm{O}^{*} \\
& \mathrm{Ph}-\mathrm{NHO}^{*} \leftrightarrow \mathrm{Ph}-\mathrm{NH}^{*}+\mathrm{O}^{*} \\
& \text { Ph-NHO }{ }^{*}+\mathrm{H}^{*} \leftrightarrow \mathrm{Ph}^{-\mathrm{NHOH}^{*}} \\
& \mathrm{Ph}-\mathrm{NO}^{*}+\mathrm{Ph}-\mathrm{N}^{*} \leftrightarrow \mathrm{Ph}-\mathrm{N}-\mathrm{NO}-\mathrm{Ph}^{*} \\
& \mathrm{Ph}-\mathrm{N}^{*}+\mathrm{Ph}-\mathrm{N}^{*} \leftrightarrow \mathrm{Ph}-\mathrm{N}-\mathrm{N}-\mathrm{Ph}^{*} \\
& \mathrm{Ph}-\mathrm{N}-\mathrm{N}-\mathrm{Ph}^{*}+\mathrm{O}^{*} \leftrightarrow \mathrm{Ph}-\mathrm{N}-\mathrm{NO}-\mathrm{Ph}^{*} \\
& \mathrm{O}^{*}+\mathrm{H}^{*} \leftrightarrow \mathrm{OH}^{*} \\
& \mathrm{OH}^{*}+\mathrm{H}^{*} \leftrightarrow \mathrm{H}_{2} \mathrm{O}^{*}
\end{aligned}
$$

Every time we refer to a step, mechanism or optimized structure as parallel, the intermediates involved are interacting with the surface through all functional groups. Similarly, normal (to the surface) means that the structures involved are interacting with the surface only through the nitro group (M-N or M-O bonds). The asterisk superscript means that the species is adsorbed on the metal (111) surface. These steps will be used for all metals and the numbering will be the same throughout the chapter, that is, step 4 will always refer to $\mathrm{Ph}-\mathrm{NO}_{2}+\mathrm{H}^{*} \leftrightarrow \mathrm{Ph}-\mathrm{NOOH}$ regardless of the metal surface and the orientation of the molecule (parallel or normal). Moreover, transition states structures will be labeled according to the elementary steps they belong to. The letter P means parallel and $\mathrm{N}$ means normal. For example, P-TS1 is the transition state of step 1 with parallel orientation, N-TS2 the transition state of step 2 with normal orientation and so on.

\subsection{Activation of $\mathrm{H}_{2}$ on $\mathrm{Ni}(111), \mathrm{Co}(111)$ and $\mathrm{Cu}(111)$}

As described in the literature, adsorption of $\mathrm{H}_{2}$ on $\mathrm{Pt}$ and $\mathrm{Pd}$ catalysts is assumed to be dissociative ${ }^{42-44}$, and therefore we have only calculated the reaction pathway for 
$\mathrm{H}_{2}$ dissociation on $\mathrm{Co}(111), \mathrm{Ni}(111)$ and $\mathrm{Cu}(111)$ surface models. The activation and reaction energies at different coverages are summarized in Table 3.3. The coverage is calculated as the number of adsorbates divided by the number of metal atoms on the surface.

The activation of the $\mathrm{H}_{2}$ molecule on the $\mathrm{Ni}(111)$ and $\mathrm{Co}(111)$ surfaces at low coverage $(\theta=0.22 \mathrm{ML})$ is a two-step mechanism: adsorption and subsequent dissociation into two $\mathrm{H}$ adatoms. The most stable adsorption site of the molecular $\mathrm{H}_{2}$ is on-top position for $\mathrm{Ni}(111)$ and $\mathrm{Co}(111)$ as shown in Figure 3.4. Every attempt to find a minimum structure on an hcp, fcc, or bridge site failed; $\mathrm{H}_{2}$ was always dragged to the nearest $\mathrm{Ni}$ or $\mathrm{Co}$ atom. The $\mathrm{H}-\mathrm{H}$ bond length increases from $0.78 \AA$ in the gas phase to $0.88 \AA$ on $\mathrm{Ni}(111)$ and to $0.91 \AA$ on $\mathrm{Co}(111)$ in the physisorbed state, indicating a certain degree of activation. Conversely, on $\mathrm{Cu}(111)$ the $\mathrm{H}_{2}$ molecule remains physisorbed at a distance of $3.5 \AA$ from the surface with the same $\mathrm{H}-\mathrm{H}$ bond length as in the gas phase.

Table 3.3 Calculated adsorption $\left(E_{a d s}\right)$, activation $\left(E_{\text {act }}\right)$ and reaction $\left(E_{\text {reac }}\right)$ energies for the dissociation of $\mathrm{H}_{2}$ on $\mathrm{M}(111)$ surfaces at four coverages $(\theta)$. Energies are given in $\mathrm{kcal} / \mathrm{mol}$.

\begin{tabular}{|c|c|c|c|c|c|}
\hline$\theta(\mathrm{ML})$ & 0.22 & 0.44 & 0.66 & 0.88 & 1.1 \\
\hline \multicolumn{6}{|c|}{$\mathbf{N i}$} \\
\hline $\mathbf{E}_{\text {ads }}\left(\mathbf{H}_{2}\right)$ & -9.6 & -8.3 & -8.7 & -6.3 & - \\
\hline $\mathbf{E}_{\text {act }}$ & 1.4 & 1.3 & 0.9 & 0.6 & 4.2 \\
\hline Ereac & -19.3 & -20.3 & -20.3 & -28.9 & -11.3 \\
\hline \multicolumn{6}{|c|}{ Co } \\
\hline $\operatorname{Eads}\left(\mathrm{H}_{2}\right)$ & -10.9 & -8.7 & -9.1 & -4.8 & - \\
\hline $\mathbf{E}_{\text {act }}$ & 0.6 & 0.6 & 0.5 & 7.4 & 4.1 \\
\hline $\mathbf{E}_{\text {reac }}$ & -19.6 & -19.7 & -20.4 & -20.3 & -1.3 \\
\hline \multicolumn{6}{|c|}{$\mathrm{Cu}$} \\
\hline $\mathbf{E}_{\text {ads }}\left(\mathbf{H}_{2}\right)$ & -1.7 & - & -0.6 & - & - \\
\hline $\mathbf{E}_{\text {act }}$ & 6.9 & 7.1 & 7.8 & 16.2 & 21.7 \\
\hline Ereac & -13.5 & -13.7 & -12.7 & -4.8 & 21.4 \\
\hline
\end{tabular}




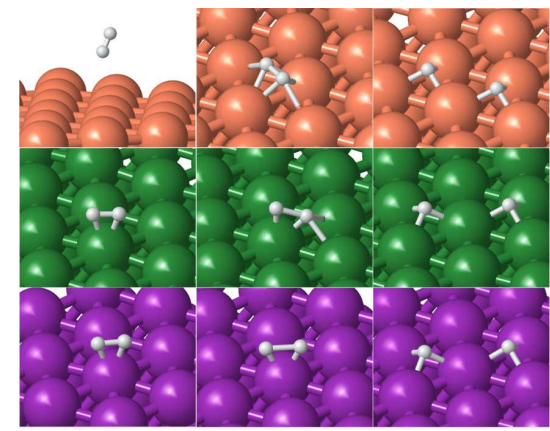

a)

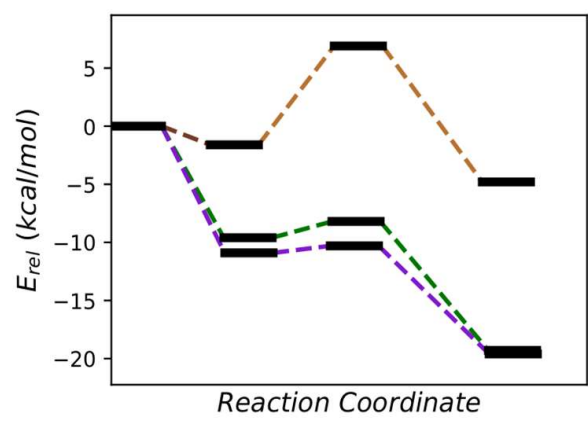

b)

Figure 3.4. Optimized geometry of reactant (R), transition state (TS) and product (P) structures (a) and calculated energy profile (b) for $\mathrm{H}_{2}$ adsorption and dissociation on clean $\mathrm{Co}(111)$ (purple), $\mathrm{Ni}(111)$ (green) and $\mathrm{Cu}(111)$ (orange) surfaces at 0.22 coverage.

The dissociation of the $\mathrm{H}-\mathrm{H}$ bond occurs with activation energies of $\sim 1 \mathrm{kcal} / \mathrm{mol}$ on both $\mathrm{Ni}(111)$ and $\mathrm{Co}(111)$ (Figure 3.4 and Table 3.3) through a transition state where the $\mathrm{H}-\mathrm{H}$ bond length is elongated to $1.1 \AA$ on $\mathrm{Ni}(111)$ and to $1.2 \AA$ on $\mathrm{Co}(111)$. On $\mathrm{Cu}(111)$, however, the dissociation occurs on a bridge site with a $\mathrm{H}-\mathrm{H}$ bond length of $0.94 \AA$ and the barrier required to reach this transition state is $6.9 \mathrm{kcal} / \mathrm{mol}$. This higher value compared with $\mathrm{Ni}(111)$ and $\mathrm{Co}(111)$ is, at least in part, a consequence of the lack of activation of the H-H bond in the physisorbed state.

After dissociation on $\mathrm{Ni}(111)$ and $\mathrm{Co}(111), \sim 20 \mathrm{kcal} / \mathrm{mol}$ are released and the two $\mathrm{H}$ atoms occupy hep and fcc hollow sites (Figure 3.4). On $\mathrm{Cu}(111)$, this step is less exothermic, only $-4.9 \mathrm{kcal} / \mathrm{mol}$ are released and the hcp and fcc hollows are also the preferred adsorption site for atomic $\mathrm{H}$ (Figure 3.4). We could not find minima corresponding to the adsorption of atomic $\mathrm{H}$ on the bridge or top sites for any of the three M(111) surfaces. The relative energies of the hollow fcc and hep sites, summarized in Table 3.4, show that the interaction of $\mathrm{Cu}(111)$ with $\mathrm{H}$ is significantly less favorable than $\mathrm{Ni}(111)$ and $\mathrm{Co}(111)$. The diffusion of adsorbed atomic $\mathrm{H}$ to an empty hcp or fcc site is very fast on all the three surfaces because the diffusion barrier is only $2.3 \mathrm{kcal} / \mathrm{mol}$. 
We have also considered the effect of coverage on the chemisorption and activation energies (Table 3.3). The energy of adsorption of $\mathrm{H}_{2}$ on top site is very similar at coverage 0.22 and 0.66 and then decreases at coverage 0.88 on $\mathrm{Ni}(111)$ and $\mathrm{Co}(111)$. At coverage 1.1 the $\mathrm{H}_{2}$ molecule chemisorbs neither on $\mathrm{Ni}(111)$ nor on $\mathrm{Co}(111)$. The activation energies are always higher on $\mathrm{Cu}(111)$ compared with $\mathrm{Ni}(111)$ and $\mathrm{Co}(111)$, see Table 3.3 and Figure $3.5 \mathrm{a}$. It increases significantly to $21.7 \mathrm{kcal} / \mathrm{mol}$ after being constant at around $\sim 7 \mathrm{kcal} / \mathrm{mol}$ until coverage 0.66 . On Ni(111) and $\mathrm{Co}(111)$ the activation energies remain nearly constant up to coverage 0.66 , then it slightly increases on $\mathrm{Co}(111)$. Notice how the $\mathrm{E}_{\text {act }}$ finally increases on $\mathrm{Ni}(111)$ at coverage 1.1 while decreases on $\operatorname{Co}(111)$. The reason why these two values are similar at coverage 1.1 is because the transition states are also similar on $\mathrm{Ni}(111)$ and $\mathrm{Co}(111)$. The $\mathrm{H}_{2}$ molecule dissociates on top of a metal atom coming directly from the gas phase. This step at coverage 1.1 is significantly less exothermic, especially on $\operatorname{Co}(111)$, see Figure 3.5 b.

a)
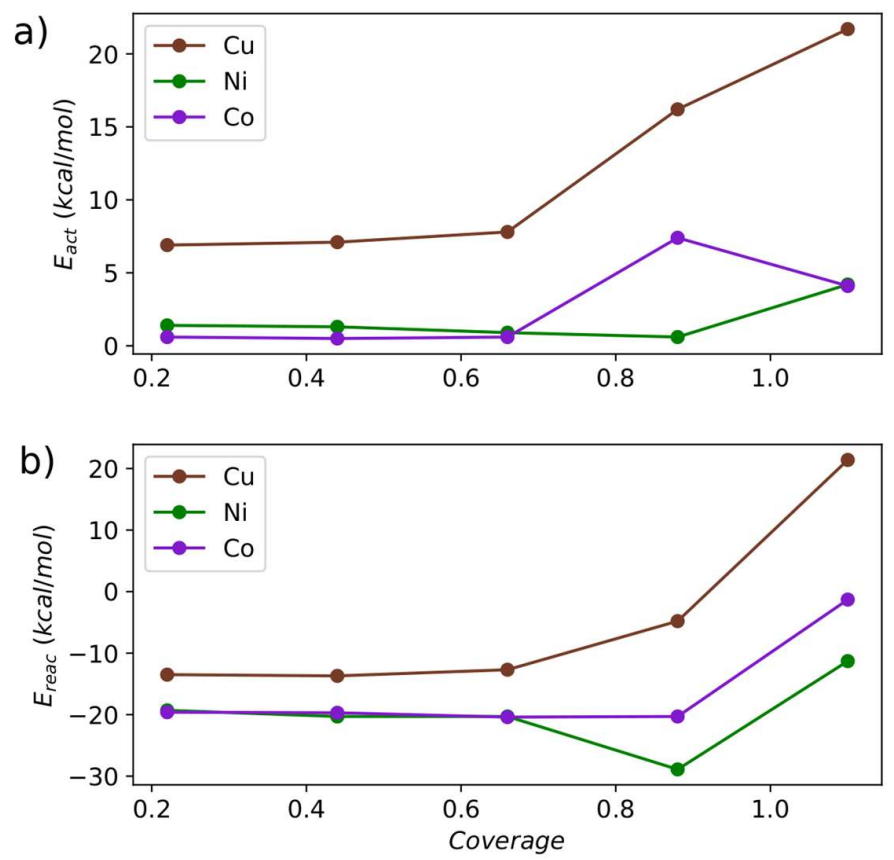

Figure 3.5 Dependence of the activation a) and reaction b) electronic energies with coverage. Energy values are included in Table 3.3. 


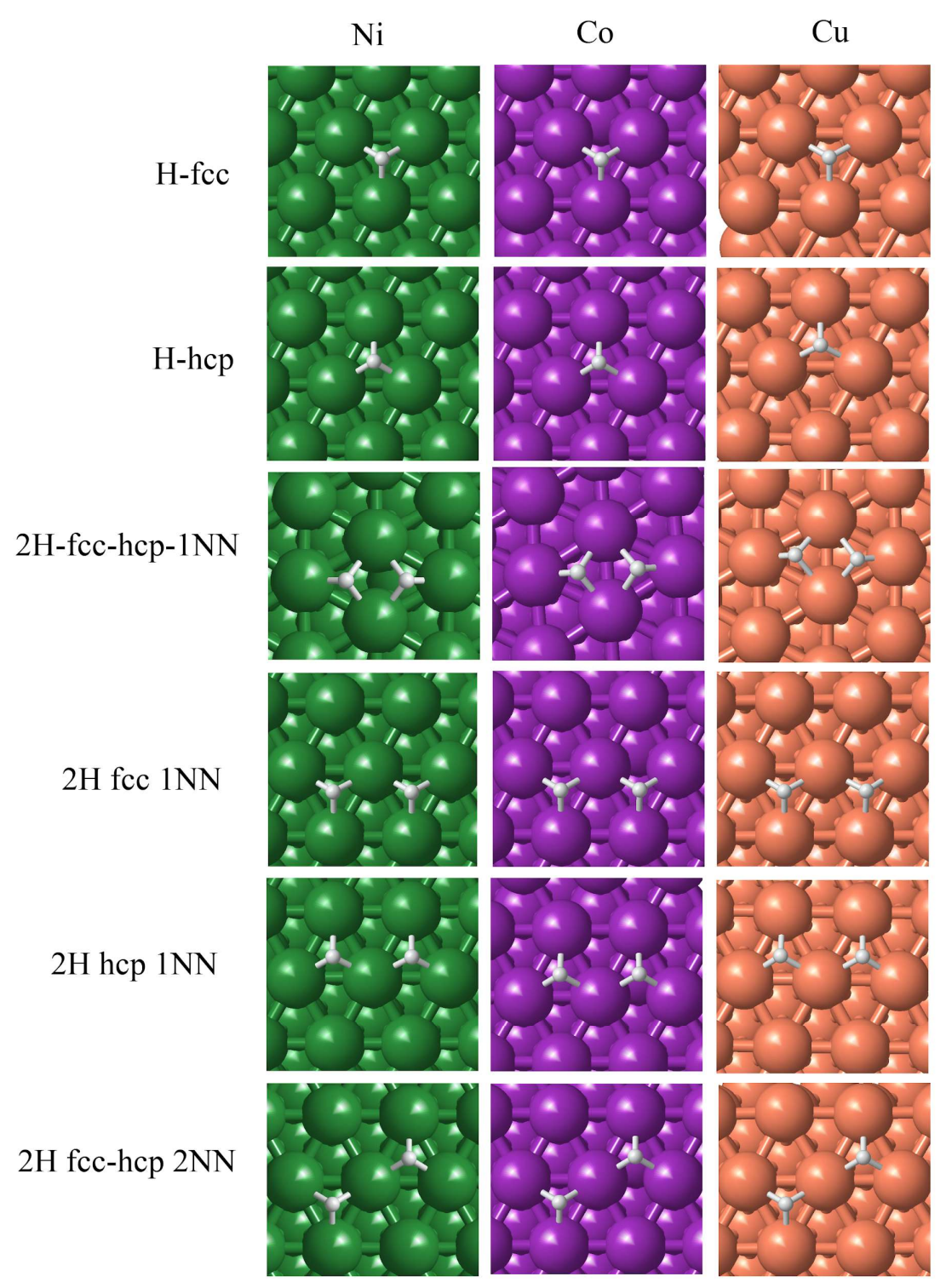

Figure 3.6. Optimized structures of the most stable adsorption sites and some H-pairs representing lateral interactions. Interaction energies are shown in Tables 3.4 and 3.5. 
Table 3.4 Relative energies of the most stable adsorption sites with respect to the energy of $1 \mathrm{slab}+1 / 2 \mathrm{H}_{2}$. The optimized geometries are shown in Figure 3.6.

\begin{tabular}{|cccc}
\hline Species & Ni & Co & Cu \\
\hline H-fcc & -13.7 & -13.7 & -4.3 \\
H-hcp & -13.4 & -13.0 & -3.8 \\
\hline
\end{tabular}

Table 3.5 Interaction energies of H-pairs representing lateral interactions on the M(111) surface. The interaction energies are calculated as the difference between the optimized pair and the sum of the relative energies of the two corresponding H-fcc and/or H-hcp structures (Table 3.4). Geometries are shown in Figure 3.6.

\begin{tabular}{cccc}
\hline Species & Ni & Co & Cu \\
\hline 2H fcc-hcp 1NN & 4.6 & 7.9 & 8.0 \\
2H fcc 1NN & -0.8 & 1.4 & 1.7 \\
2H hcp 1NN & -0.2 & 1.6 & 1.8 \\
2H fcc-hcp 2NN & -0.9 & 0.2 & 1.1 \\
\hline
\end{tabular}

Finally, the lateral interactions of $\mathrm{H}$ pairs, shown in Table 3.5, help rationalize why the activation energy increases so much on $\mathrm{Cu}(111)$ compared with $\mathrm{Ni}(111)$ and $\operatorname{Co}(111)$. Figure 3.6 shows the optimized structures. Notice how the interaction of two $\mathrm{H}$ atoms in first-nearest-neighbor hep and fcc sites (2H-1NN-fec-hep) is not favorable for any metal. As the two $\mathrm{H}$ atoms separate from each other the interaction becomes favorable for $\mathrm{Ni}(111)$ but still unfavorable for $\mathrm{Co}(111)$ and $\mathrm{Cu}(111)$. The interaction energies are similar for $\mathrm{Co}(111)$ and $\mathrm{Cu}(111)$ and always less favorable than $\mathrm{Ni}(111)$. As a consequence, an increase of the coverage of the $\mathrm{Cu}(111)$ and $\operatorname{Co}(111)$ surfaces will result in an increase of the system energy. Notice that dissociation of $\mathrm{H}_{2}$ on $\mathrm{Cu}(111)$ at coverage $>1$ is very endothermic (Table 3.3). These results indicate that $\mathrm{Ni}(111)$ and $\mathrm{Co}(111)$ surfaces activate molecular $\mathrm{H}_{2}$ very effectively while $\mathrm{Cu}(111)$ is expected to be significantly less active. Also notice, that at high coverage Co will probably perform slightly worse than $\mathrm{Ni}$. 


\subsection{Reduction of the vinyl group on $\mathrm{Ni}(111)$ and $\mathrm{Co}(111)$}

The reduction of the vinyl $\mathrm{C}=\mathrm{C}$ bond is the main competitor of the selective hydrogenation of the nitro group. To investigate this reaction styrene was used as substrate model, as an approximation to using nitrostyrene. The calculated reaction and activation energies are summarized in Table 3.6 and the energy profile is shown in Figure 3.7. The optimized geometries of all structures involved in the mechanism are shown in Figure 3.8. It is obvious that styrene only adsorbs parallel to the surface and thus this is the orientation for the mechanism in this section. Both $\mathrm{Ni}(111)$ and $\mathrm{Co}(111)$ are included. This reaction was not studied on $\mathrm{Cu}(111)$ because nitrostyrene does not adsorb parallel to the surface (see section 3.3).

Table 3.6 Calculated reaction and activation energies of the reduction of styrene on the $\mathrm{Ni}(111)$ and $\mathrm{Co}(111)$ surfaces. All energies are given in $\mathrm{kcal} / \mathrm{mol}$.

\begin{tabular}{cccccc}
\hline \multirow{2}{*}{ No } & \multirow{2}{*}{ Step } & \multicolumn{2}{c}{$\mathrm{Ni}$} & \multicolumn{2}{c}{ Co } \\
\cline { 3 - 6 } & & $\mathrm{E}_{\text {reac }}$ & $\mathrm{E}_{\text {act }}$ & $\mathrm{E}_{\text {reac }}$ & $\mathrm{E}_{\text {act }}$ \\
\hline 2 & $\mathrm{Ph}-\mathrm{CH}=\mathrm{CH}_{2}{ }^{*}+\mathrm{H}^{*} \leftrightarrow \mathrm{Ph}-\mathrm{CH}-\mathrm{CH}_{3}{ }^{*}$ & 6.1 & 15.5 & 8.5 & 16.8 \\
3 & $\mathrm{Ph}-\mathrm{CH}-\mathrm{CH}_{3}{ }^{*}+\mathrm{H}^{*} \leftrightarrow \mathrm{Ph}-\mathrm{CH}_{2}-\mathrm{CH}_{3}{ }^{*}$ & 6.8 & 15.5 & 2.4 & 11.3 \\
\hline
\end{tabular}

The initial reactant system consists of one styrene molecule adsorbed on the metal surface together with two $\mathrm{H}$ atoms occupying hollow positions. This is a fairly simple reaction with only two elementary steps, two hydrogen transfers to the $\mathrm{C}=\mathrm{C}$ carbon atoms (steps 2 and 3). In all optimized structures, both minima and transition states, the aromatic ring remains approximately with the same adsorption geometry, with all $\mathrm{C}$ atoms directly interacting with surface metal atoms. On both $\mathrm{Ni}(111)$ and $\mathrm{Co}(111)$ the $\mathrm{C}=\mathrm{C}$ bond length of the vinyl group increases from $1.42 \AA$ in the adsorbed styrene to $1.45 \AA$ in TS2. Subsequently, it increases even more to $1.52 \AA$ in $\mathrm{Ph}_{-} \mathrm{CHCH}_{3}$ as well as in TS3 and in the ethylbenzene product. The optimized length of the C-H bond being formed in TS2 is $1.39 \AA$, and $1.50 \AA$ in TS3. Both steps are energetically similar, slightly endothermic ( $\sim-7 \mathrm{kcal} / \mathrm{mol}$ for both $\mathrm{H}$ transfers) and involving activation energy barriers of $\sim 15 \mathrm{kcal} / \mathrm{mol}$ on both metals. 


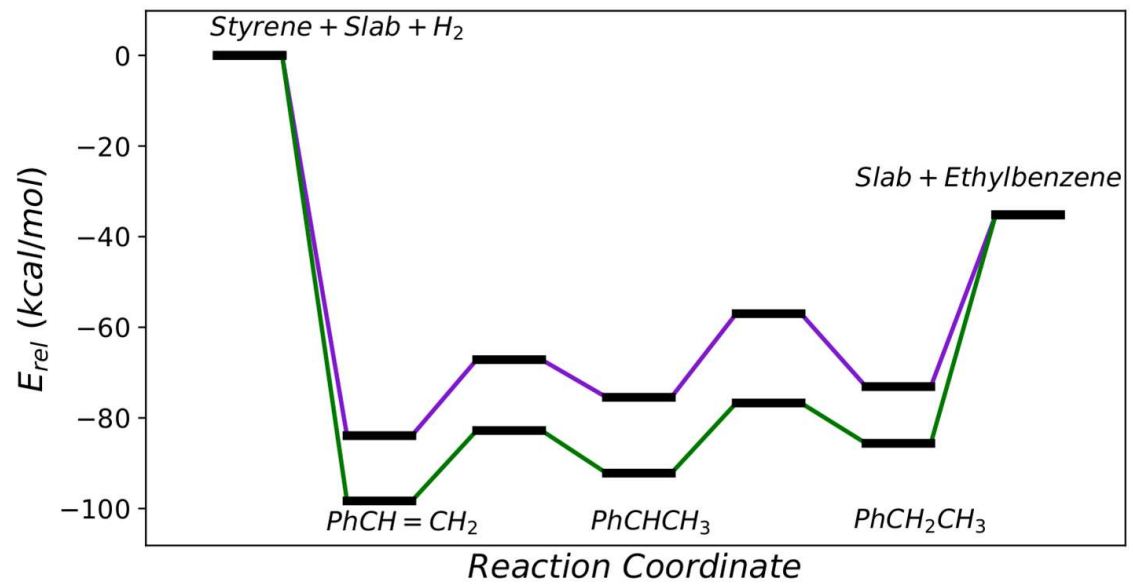

Figure 3.7 Calculated energy profile for the hydrogenation of the $\mathrm{C}=\mathrm{C}$ bond in styrene on the $\mathrm{Ni}(111)$ (green) and $\mathrm{Co}(111)$ (purple) surfaces. The zero energy level corresponds to the sum of the electronic energies of the $\mathrm{Ni}$ slab $+\mathrm{H}_{2}+$ Styrene.

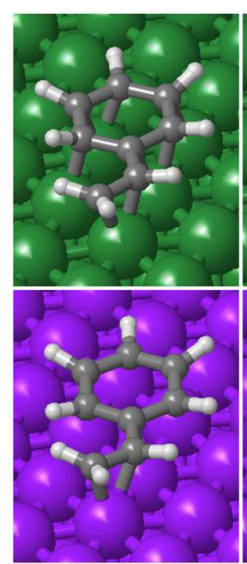

$\mathrm{Ph}-\mathrm{CH}=\mathrm{CH} 2$

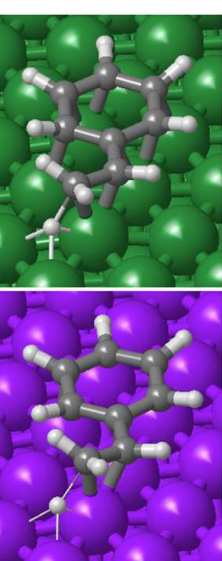

TS1
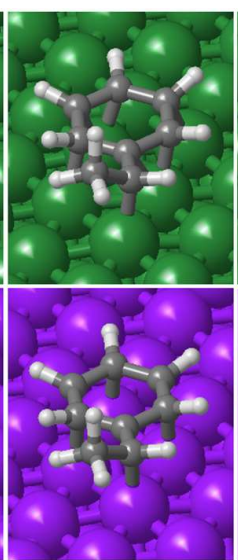

Ph-CH-CH3
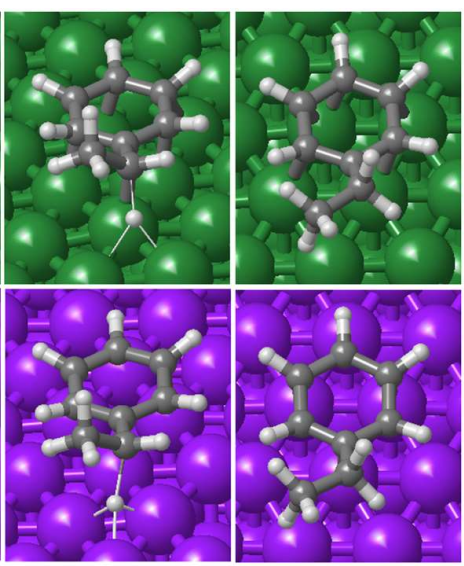

TS2

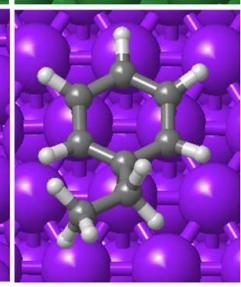

ethylbenzene

Figure 3.8 Optimized geometries of minima and transition states involved in the hydrogenation of styrene on $\mathrm{Ni}(111)$ (top) and $\mathrm{Co}(111)$ (bottom) surfaces. Ni, Co, C, N, 
$\mathrm{O}$ and $\mathrm{H}$ atoms are depicted as green, purple, gray, blue, red and white balls.

The main difference found between $\mathrm{Ni}(111)$ and $\mathrm{Co}(111)$ is that the adsorption and relative energies of all intermediates are lower on $\mathrm{Co}(111)$ by $\sim 10 \mathrm{kcal} / \mathrm{mol}$. Desorption of the ethylbenzene product from the Co(111) surface is endothermic by $37.9 \mathrm{kcal} / \mathrm{mol}$ and by $50.4 \mathrm{kcal} / \mathrm{mol}$ from the $\mathrm{Ni}(111)$ surface. Therefore, once formed, ethylbenzene will desorb faster from $\operatorname{Co}(111)$.

\subsection{Reduction of the nitro group on Ni(111). Direct Route.}

The reduction of the nitro group is the target reaction and the one that should be favored in order to obtain high selectivity. The study of this reaction was carried out using nitrobenzene as reactant molecule. We will see that the effect of the oxophilicity of $\mathrm{Ni}$ is so significant that the conclusions might as well be valid for other arenes with similar functional groups.

\subsubsection{Parallel pathways}

The calculated activation and reaction energies for the reduction of nitrobenzene adsorbed in a parallel orientation are summarized in Table 3.7. The calculated energy profiles are shown in Figure 3.9. The optimized geometries of the intermediate and transition state structures involved are depicted in Figure 3.10.

Table 3.7 Calculated reaction and activation energies for all elementary steps of the reduction of nitrobenzene on $\mathrm{Ni}(111)$ surface with parallel $(\mathrm{P})$ geometries. All energies are given in $\mathrm{kcal} / \mathrm{mol}$.

\begin{tabular}{|c|c|c|c|}
\hline \multirow{2}{*}{ step } & \multirow{2}{*}{ Reaction } & \multicolumn{2}{|c|}{ Electronic Energy } \\
\hline & & $E_{\text {reac }}$ & $\mathrm{E}_{\mathrm{act}}$ \\
\hline 4 & $\mathrm{Ph}-\mathrm{NO}_{2}+\mathrm{H}^{*} \leftrightarrow \mathrm{Ph}-\mathrm{NOOH}$ & 0.1 & 20.0 \\
\hline 5 & $\mathrm{Ph}-\mathrm{NOOH}^{*}+\mathrm{H}^{*} \leftrightarrow \mathrm{Ph}-\mathrm{N}(\mathrm{OH})_{2}$ & 9.5 & 20.2 \\
\hline 6 & 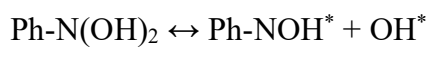 & -34.2 & 4.6 \\
\hline 7 & $\mathrm{Ph}-\mathrm{NOOH}^{*} \leftrightarrow \mathrm{Ph}-\mathrm{NOH}+\mathrm{O}^{*}$ & -26.2 & 9.2 \\
\hline 8 & $\mathrm{Ph}-\mathrm{NOH}^{*} \leftrightarrow \mathrm{Ph}-\mathrm{N}+\mathrm{OH}^{*}$ & -34.5 & 4.4 \\
\hline 9 & $\mathrm{Ph}-\mathrm{NOH}+\mathrm{H}^{*} \leftrightarrow \mathrm{Ph}-\mathrm{NHOH}^{*}$ & -1.2 & 24.4 \\
\hline 10 & $\mathrm{Ph}-\mathrm{N}^{*}+\mathrm{H}^{*} \leftrightarrow \mathrm{Ph}-\mathrm{NH}^{*}$ & -5.9 & 23.1 \\
\hline
\end{tabular}


3.7 Reduction of the nitro group on Ni(111). Direct Route.

Table 3.7 Calculated reaction and activation energies for all elementary steps of the reduction of nitrobenzene on $\mathrm{Ni}(111)$ surface with parallel (P) geometries. All energies are given in $\mathrm{kcal} / \mathrm{mol}$.

\begin{tabular}{|c|c|c|c|}
\hline \multirow{2}{*}{ step } & \multirow{2}{*}{ Reaction } & \multicolumn{2}{|c|}{ Electronic Energy } \\
\hline & & $E_{\text {reac }}$ & $E_{\text {act }}$ \\
\hline 11 & $\mathrm{Ph}-\mathrm{NH}^{*}+\mathrm{H}^{*} \leftrightarrow \mathrm{Ph}-\mathrm{NH}_{2}^{*}$ & -1.4 & 23.5 \\
\hline 12 & $\mathrm{Ph}-\mathrm{NHOH}^{*} \leftrightarrow \mathrm{Ph}-\mathrm{NH}^{*}+\mathrm{OH}^{*}$ & -39.3 & 10.4 \\
\hline 13 & $\mathrm{Ph}-\mathrm{NO}_{2} \leftrightarrow \mathrm{Ph}-\mathrm{NO}^{*}+\mathrm{O}^{*}$ & -32.2 & 10.6 \\
\hline 14 & $\mathrm{Ph}-\mathrm{NO}^{*}+\mathrm{H}^{*} \leftrightarrow \mathrm{Ph}-\mathrm{NOH}^{*}$ & 6.1 & 16.9 \\
\hline 15 & $\mathrm{Ph}-\mathrm{NO}^{*}+\mathrm{H}^{*} \leftrightarrow \mathrm{Ph}-\mathrm{NHO}^{*}$ & -1.4 & 19.7 \\
\hline 16 & $\mathrm{Ph}-\mathrm{NO}^{*} \leftrightarrow \mathrm{Ph}-\mathrm{N}^{*}+\mathrm{O}^{*}$ & -30.0 & 11.7 \\
\hline 17 & 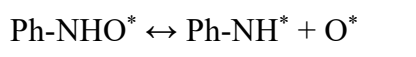 & -34.5 & 8.6 \\
\hline 18 & $\mathrm{Ph}^{-\mathrm{NHO}^{*}}+\mathrm{H}^{*} \leftrightarrow \mathrm{Ph}-\mathrm{NHOH}^{*}$ & 6.3 & 18.4 \\
\hline
\end{tabular}

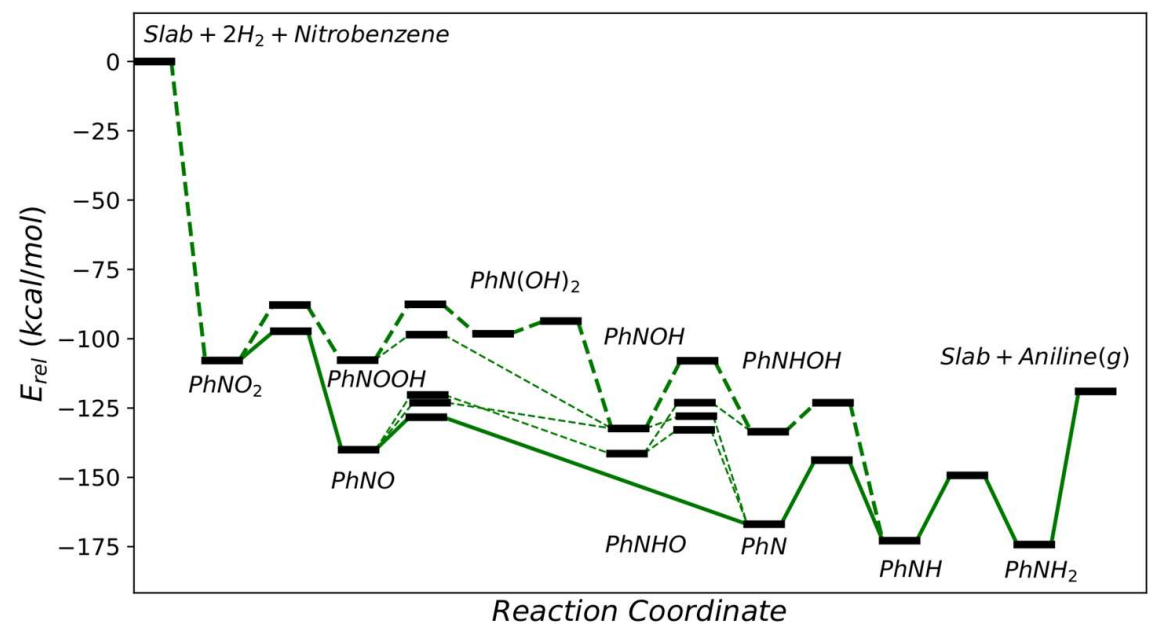

Figure 3.9 Calculated energy profile of the parallel pathways for nitrobenzene reduction over $\mathrm{Ni}(111)$ surface. The zero energy level corresponds to the sum of the absolute energies of the $\mathrm{Ni} \mathrm{slab}+3 \mathrm{H}_{2}+$ Nitrobenzene. 


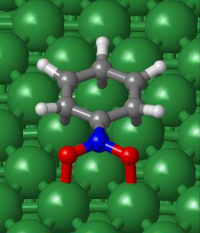

$\mathrm{Ph}-\mathrm{NO}_{2}$

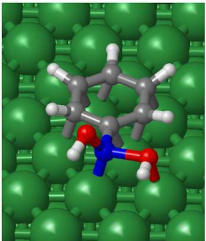

P-TS6

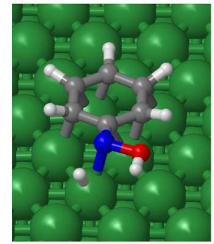

P-TS9

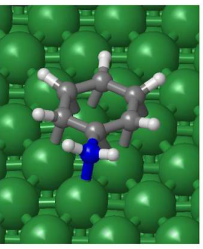

Ph-NH 2

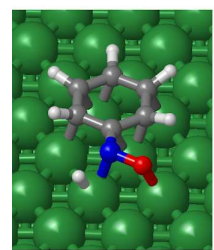

P-TS15

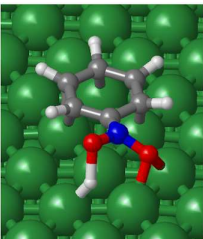

P-TS4

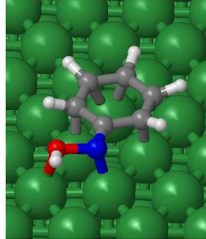

$\mathrm{Ph}-\mathrm{NOH}$

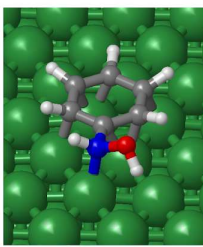

$\mathrm{Ph}-\mathrm{NHOH}$

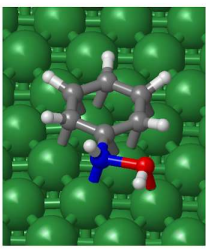

P-TS12

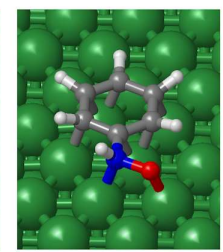

$\mathrm{Ph}-\mathrm{NHO}$

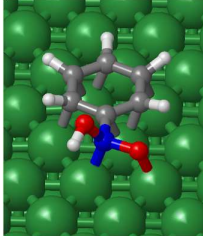

$\mathrm{Ph}-\mathrm{NOOH}$

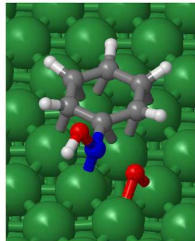

P-TS7

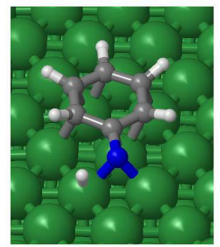

P-TS10

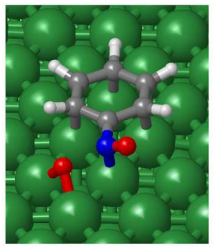

P-TS13

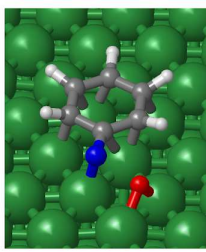

P-TS16

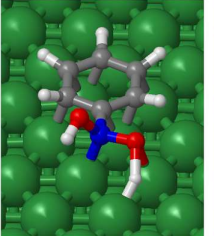

P-TS5

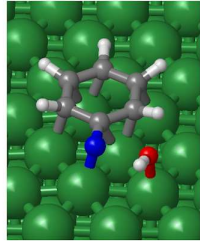

P-TS8

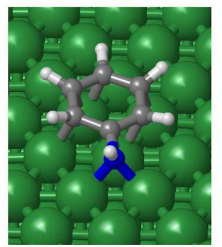

$\mathrm{Ph}-\mathrm{HN}$

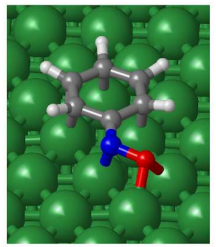

$\mathrm{Ph}-\mathrm{NO}$

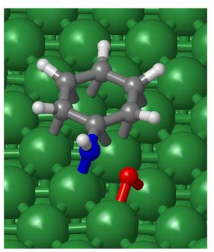

P-TS17

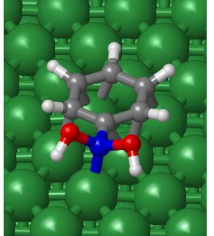

$\mathrm{Ph}-\mathrm{N}(\mathrm{OH})_{2}$

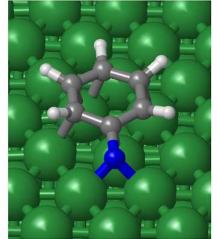

$\mathrm{Ph}-\mathrm{N}$

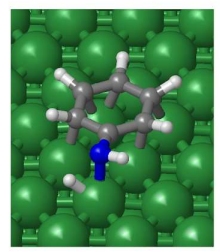

P-TS11

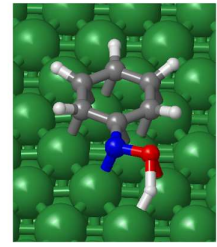

P-TS14

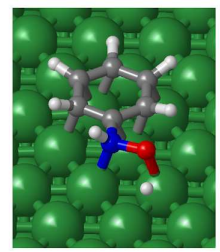

P-TS18

Figure 3.10 Optimized geometries of minima and transition states involved in the parallel pathway of nitrobenzene hydrogenation on $\mathrm{Ni}(111)$ surface. $\mathrm{Ni}, \mathrm{C}, \mathrm{N}, \mathrm{O}$ and $\mathrm{H}$ atoms are depicted as green, gray, blue, red and white balls. 
The reaction starts with $\mathrm{H}$ transfer to one of the $\mathrm{O}$ atoms of the nitro group leading to the formation of $\mathrm{Ph}-\mathrm{NOOH}$ (step 4). The $\mathrm{O}-\mathrm{H}$ distance in the transition state structure is $1.49 \AA$ and the other N-O bond is elongated from 1.32 to $1.37 \AA$. The calculated activation and reaction energies are 20.0 and $0.1 \mathrm{kcal} / \mathrm{mol}$ respectively. In principle, there are three possible steps starting from $\mathrm{Ph}-\mathrm{NOOH}$; $\mathrm{H}$ transfer to the second $\mathrm{O}$ atom of the nitro group to form $\mathrm{Ph}-\mathrm{N}(\mathrm{OH})_{2}$ (step 5), direct $\mathrm{N}-\mathrm{OH}$ bond breaking to form nitrosobenzene, and $\mathrm{N}-\mathrm{O}$ bond breaking to form $\mathrm{Ph}-\mathrm{NOH}$ (step 7).

The activation and reaction energies for the second $\mathrm{H}$ transfer to $\mathrm{Ph}-\mathrm{NOOH}$ are 20.2 $\mathrm{kcal} / \mathrm{mol}$ and $9.5 \mathrm{kcal} / \mathrm{mol}$ respectively (step 5). The reason why this step is considerably less favorable thermodynamically than the first $\mathrm{H}$ transfer is related to the stability of $\mathrm{Ph}-\mathrm{N}(\mathrm{OH})_{2}$. This intermediate is anchored to the surface only through the aromatic ring since both $\mathrm{Ni}-\mathrm{O}$ bonds have been broken.

The optimized $\mathrm{N}-\mathrm{O}$ bond lengths in $\mathrm{Ph}-\mathrm{N}(\mathrm{OH})_{2}$ are $1.43 \AA$, indicating that both $\mathrm{N}-\mathrm{O}$ bonds have been activated and could be more easily broken in the next elementary step (step 6) to yield Ph-NOH (Figure 3.10). The calculated activation energy of step 6 is indeed much lower, $4.6 \mathrm{kcal} / \mathrm{mol}$, and the process is clearly exothermic, -34.0 $\mathrm{kcal} / \mathrm{mol}$, despite a strong $\mathrm{N}-\mathrm{O}$ being broken. The optimized $\mathrm{N}-\mathrm{O}$ and $\mathrm{Ni}-\mathrm{O}$ bond lengths in the transition state P-TS6 are 1.70 and $2.06 \AA$, respectively. The simultaneous formation of a stable $\mathrm{Ni}-\mathrm{O}$ bond as the $\mathrm{OH}$ group is released from $\mathrm{Ph}-\mathrm{N}(\mathrm{OH})_{2}$ overcompensates the energy required to dissociate the $\mathrm{N}-\mathrm{O}$ bond. This is not unexpected taking into account the oxophilic character of $\mathrm{Ni}$. In fact, the activation of the second $\mathrm{O}$ by hydrogen transfer is not even necessary.

The N-O bond of Ph-NOOH can be broken directly through the transition state $\mathrm{P}-\mathrm{TS} 7$ obtaining the same intermediate $\mathrm{Ph}-\mathrm{NOH}$. The calculated activation energy of this process is $9.2 \mathrm{kcal} / \mathrm{mol}$ (step 7), significantly lower than that of step 5. Similar to the previous $\mathrm{N}-\mathrm{OH}$ cleavage, this step is also very exothermic $(-26.2 \mathrm{kcal} / \mathrm{mol})$. There are again two possible elementary steps from Ph-NOH: 1) N-O bond breaking (step 8) leading to $\mathrm{Ph}-\mathrm{N}$ and an adsorbed hydroxyl or 2) $\mathrm{H}$ transfer to the $\mathrm{N}$ atom to obtain $\mathrm{Ph}-\mathrm{NHOH}$, according to step 9. Here again, the activation energy to break the $\mathrm{N}-\mathrm{O}$ bond $(4.4 \mathrm{kcal} / \mathrm{mol})$ is much lower than that needed to transfer a $\mathrm{H}$ atom to $\mathrm{N}$ $(24.4 \mathrm{kcal} / \mathrm{mol})$. Moreover, the former process is very exothermic $(-34.5 \mathrm{kcal} / \mathrm{mol})$ while the latter is almost thermoneutral $(-0.9 \mathrm{kcal} / \mathrm{mol})$. The global energy profile 
plotted in Figure 3.9 shows the higher stability of the $\mathrm{Ph}-\mathrm{N}$ intermediate, which can be explained by the large contribution to the energy of the Ni-OH bond formed. Two successive hydrogen transfers (steps 10 and 11) to the $\mathrm{N}$ atom will complete the mechanism and yield aniline adsorbed on the surface through the intermediate $\mathrm{Ph}$ $\mathrm{NH}$. Both of them involve activation energies of 23.1 and $23.5 \mathrm{kcal} / \mathrm{mol}$, similar to the $\mathrm{H}$ transfer to the $\mathrm{N}$ atom of step 9. Moreover, $\mathrm{Ph}-\mathrm{NH}$ can also be formed by direct dehydroxylation of $\mathrm{Ph}-\mathrm{NHOH}$ releasing $-39.3 \mathrm{kcal} / \mathrm{mol}$ and involving an activation energy of $10.4 \mathrm{kcal} / \mathrm{mol}$ (step 12).

So far, we have described pathways that lead to the formation of aniline, but nitrosobenzene does not participate in any of them. There should be at least one elementary step involving nitrosobenzene as intermediate since it has been detected experimentally and proposed as reaction intermediate in Ni nanoparticles ${ }^{45}$. We have already discussed that due to the oxophylic nature of $\mathrm{Ni}$ it was feasible to break the $\mathrm{N}-\mathrm{O}$ bonds in steps $6,7,8$ and 12 even without previously being activated by $\mathrm{H}$ transfer to the $\mathrm{O}$ atom in the case of step 7. Therefore, we also considered the formation of nitrosobenzene ( $\mathrm{Ph}-\mathrm{NO}$ ) by directly breaking one the two $\mathrm{N}-\mathrm{O}$ bonds from nitrobenzene. Interestingly, the calculated activation energy for this process (step 13), $7.1 \mathrm{kcal} / \mathrm{mol}$, is much lower than that found for the hydrogenation step 4, and the reaction is highly exothermic. The set of elementary steps following the formation of nitrosobenzene are similar to those described so far. Hydrogenation of either the $\mathrm{O}$ or the $\mathrm{N}$ atom of nitrosobenzene, steps 14 and 15, respectively, are slightly endothermic and involve activation energies of 17.0 and $19.7 \mathrm{kcal} / \mathrm{mol}$, while direct dissociation of the N-O bond yielding the previously described $\mathrm{Ph}-\mathrm{N}$ intermediate is clearly exothermic and involves a lower activation barrier of $14.0 \mathrm{kcal} / \mathrm{mol}$. Finally, steps 17 and 18 also constitute an example of how the mechanism can be split again in two directions from $\mathrm{Ph}-\mathrm{NHO}$ with either $\mathrm{H}$-transfer or N-O bond breaking.

\subsubsection{Normal pathways}

We have found that the activation energy of the rate-determining steps for the reduction of the nitro group with a parallel orientation is relatively high $(>20$ $\mathrm{kcal} / \mathrm{mol}$ ). Also, we saw that the hydrogenation of the vinyl group is faster, with activation energies of $\sim 14 \mathrm{kcal} / \mathrm{mol}$. The study of the mechanism of the reduction of 
3.7 Reduction of the nitro group on Ni(111). Direct Route.

nitrobenzene adsorbed in a normal orientation will help rationalize the performance of some Ni and Co based catalysts ${ }^{21}$.

We will use the same elementary steps described for the parallel pathways, except for the fact that in the normal orientation the aromatic ring is not interacting with the $\mathrm{Ni}(111)$ surface. The electronic energy profile is shown in Figure 3.11, and all optimized structures of the intermediates and transition states are depicted in Figures 3.12 and 3.13 .

Table 3.8 Calculated reaction ( $\left.E_{\text {reac }}\right)$ and activation ( $\left.E_{\text {act }}\right)$ energies for all elementary steps of the reduction of nitrobenzene on $\mathrm{Ni}(111)$ surface with normal geometries. All energies are given in $\mathrm{kcal} / \mathrm{mol}$.

\begin{tabular}{|c|c|c|c|}
\hline \multirow{2}{*}{ step } & \multirow{2}{*}{ Reaction } & \multicolumn{2}{|c|}{ Electronic Energy } \\
\hline & & $E_{\text {reac }}$ & $E_{\text {act }}$ \\
\hline 4 & $\mathrm{Ph}-\mathrm{NO}_{2}+\mathrm{H}^{*} \leftrightarrow \mathrm{Ph}-\mathrm{NOOH}(\mathrm{B})$ & -0.1 & 24.9 \\
\hline 5 & $\mathrm{Ph}-\mathrm{NOOH}^{*}+\mathrm{H}^{*} \leftrightarrow \mathrm{Ph}-\mathrm{N}(\mathrm{OH})_{2}{ }^{*}$ & 6.3 & 19.5 \\
\hline 6 & 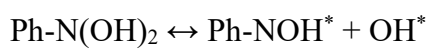 & -34.4 & 8.2 \\
\hline 7 & $\mathrm{Ph}-\mathrm{NOOH}^{*} \leftrightarrow \mathrm{Ph}-\mathrm{NOH}+\mathrm{O}^{*}$ & -29.7 & 4.6 \\
\hline 8 & $\mathrm{Ph}-\mathrm{NOH}^{*} \leftrightarrow \mathrm{Ph}-\mathrm{N}+\mathrm{OH}^{*}$ & -47.1 & 0.2 \\
\hline 9 & $\mathrm{Ph}-\mathrm{NOH}+\mathrm{H}^{*} \leftrightarrow \mathrm{Ph}^{-\mathrm{NHOH}^{*}}$ & 3.5 & 28.5 \\
\hline 10 & $\mathrm{Ph}-\mathrm{N}^{*}+\mathrm{H}^{*} \leftrightarrow \mathrm{Ph}-\mathrm{NH}^{*}$ & 12.1 & 25.8 \\
\hline 11 & $\mathrm{Ph}-\mathrm{NH}^{*}+\mathrm{H}^{*} \leftrightarrow \mathrm{Ph}-\mathrm{NH}_{2}{ }^{*}$ & -4.4 & 19.3 \\
\hline 12 & $\mathrm{Ph}-\mathrm{NHOH}^{*} \leftrightarrow \mathrm{Ph}-\mathrm{NH}^{*}+\mathrm{OH}^{*}$ & -38.5 & 8.0 \\
\hline 13 & $\mathrm{Ph}-\mathrm{NO}_{2}{ }^{*} \leftrightarrow \mathrm{Ph}-\mathrm{NO}^{*}+\mathrm{O}^{*}$ & -31.8 & 8.3 \\
\hline 14 & $\mathrm{Ph}-\mathrm{NO}^{*}+\mathrm{H}^{*} \leftrightarrow \mathrm{Ph}-\mathrm{NOH}^{*}$ & 2.0 & 22.3 \\
\hline 15 & $\mathrm{Ph}-\mathrm{NO}^{*}+\mathrm{H}^{*} \leftrightarrow \mathrm{Ph}-\mathrm{NHO}^{*}$ & 0.4 & 20.9 \\
\hline 16 & $\mathrm{Ph}-\mathrm{NO}^{*} \leftrightarrow \mathrm{Ph}-\mathrm{N}^{*}+\mathrm{O}^{*}$ & -46.7 & 4.3 \\
\hline 17 & 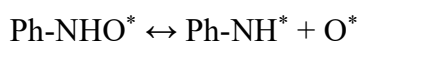 & -35.0 & 2.6 \\
\hline 18 & $\mathrm{Ph}-\mathrm{NHO}^{*}+\mathrm{H}^{*} \leftrightarrow \mathrm{Ph}_{-} \mathrm{NHOH}^{*}$ & 5.5 & 18.9 \\
\hline
\end{tabular}




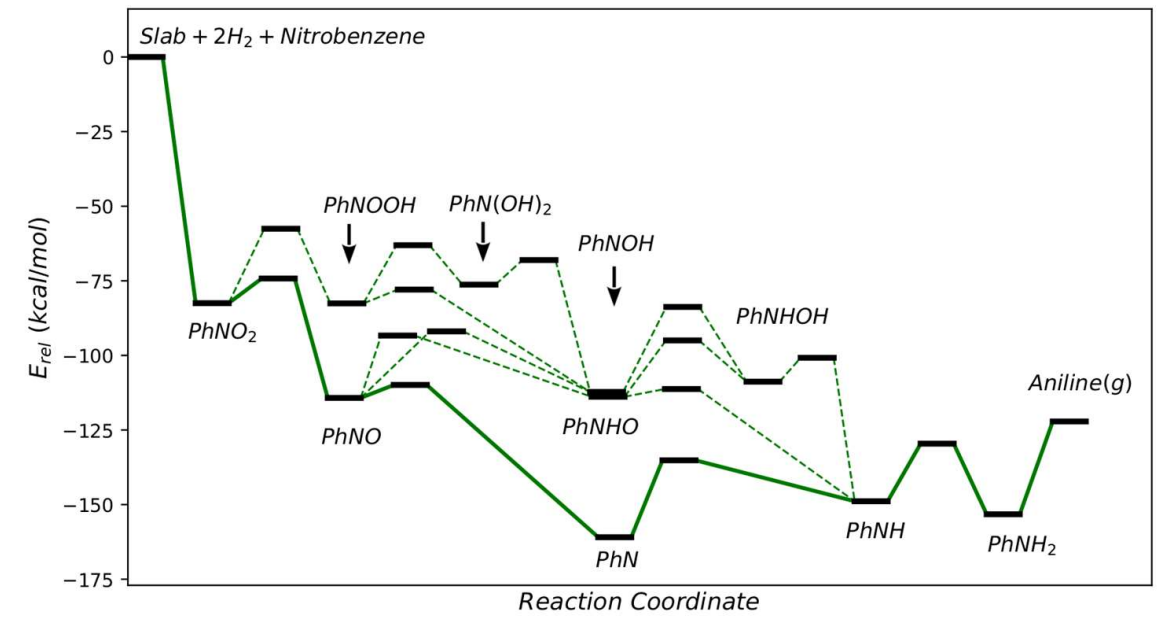

Figure 3.11 Calculated energy profile of the normal pathways for nitrobenzene reduction over $\mathrm{Ni}(111)$ surface. The zero energy level corresponds to the sum of the absolute energies of the Ni slab $+3 \mathrm{H}_{2}+$ Nitrobenzene. For clarity step 8 has been omitted.

Following the same order as in the parallel adsorption, we see in Table 3.8 that the activation energy for step 4 is $24.9 \mathrm{kcal} / \mathrm{mol}$. Ph-NOOH is significantly less stable than $\mathrm{Ph}-\mathrm{NO}_{2}$. Initially, there are two Ni-O bonds in the adsorbed $\mathrm{Ph}-\mathrm{NO}_{2}$ but after step 4 the only interaction of the molecule with the surface is through one $\mathrm{Ni}-\mathrm{O}$ bond. Therefore, before step 5 can take place, $\mathrm{Ph}-\mathrm{NOOH}$ has to rearrange to a more stable geometry with additional points of interaction with the surface. This is found in variant $\mathrm{B}$ of $\mathrm{Ph}-\mathrm{NOOH}$ (Figure 3.12) where the $\mathrm{N}$ atom is also anchored to a $\mathrm{Ni}$ atom with which the relative energy is significantly lowered by around $25.1 \mathrm{kcal} / \mathrm{mol}$. Another factor that contributes to the decrease of the energy is the orientation of the aromatic ring. Because the aromatic ring is closer to the surface, the contribution from the Van der Waals correction is $11.6 \mathrm{kcal} / \mathrm{mol}$ higher in $\mathrm{Ph}-\mathrm{NOOH}(\mathrm{B})$ than in $\mathrm{Ph}-\mathrm{NOOH}(\mathrm{A})$, that is, approximately half of the contribution.

The next logical step is a completely horizontal alignment of $\mathrm{Ph}-\mathrm{NOOH}$ (B) with respect to the surface. That would produce a further decrease in energy of 16 $\mathrm{kcal} / \mathrm{mol}$ and drive the intermediate to a parallel geometry. Thus, this step becomes a potential crossing point from the normal to the parallel mechanism. Although very likely on a clean surface, this is not expected to occur under reaction conditions 
because of the lack of empty sites to allow the formation of Ni-C bonds. Therefore, we proceeded to explore the following steps. After the second H-transfer (step 5), the most likely event involves the breaking of one of the $\mathrm{N}-\mathrm{O}$ bonds of $\mathrm{Ph}-\mathrm{N}(\mathrm{OH})_{2}$ (step 6). This is a very fast and exothermic process with an activation energy of 8.2 $\mathrm{kcal} / \mathrm{mol}$ and a reaction energy of $-34.4 \mathrm{kcal} / \mathrm{mol}$ (due to the formation of two Ni-O bonds).

Now, similar to the parallel route, the formation of nitrosobenzene occurs easily from nitrobenzene (step 13). The activation energy is only $8.3 \mathrm{kcal} / \mathrm{mol}$ and an important amount of energy $(\sim-31.8 \mathrm{kcal} / \mathrm{mol})$ is released. Further dissociation of the N-O bond in $\mathrm{Ph}-\mathrm{NO}$ yielding the $\mathrm{Ph}-\mathrm{N}$ intermediate is also favored in the normal orientation, since the activation energy for step 16 drops to $4.3 \mathrm{kcal} / \mathrm{mol}$ and the reaction is exothermic by $-43.7 \mathrm{kcal} / \mathrm{mol}$. The calculated activation energies for steps $7,8,12$ and 17 involving N-O bond breaking are also very low $(<5 \mathrm{kcal} / \mathrm{mol})$ in the normal pathway, while the activation barriers for hydrogen transfer steps are similar to those found in the parallel pathways, around $20 \mathrm{kcal} / \mathrm{mol}$. Notice, however, that the first $\mathrm{H}$ transfer to $\mathrm{Ph}-\mathrm{N}$ intermediate generating $\mathrm{Ph}-\mathrm{NH}$ is endothermic in the normal pathway $(12.1 \mathrm{kcal} / \mathrm{mol}$ versus $-5.9 \mathrm{kcal} / \mathrm{mol}$ in the parallel route), probably due to the aromatic ring having $\mathrm{H}$ atoms too close to the surface (Figure 3.12).

In conclusion, the same trend observed in the parallel route is also found here. The preferred pathway for the reduction of nitrobenzene on $\mathrm{Ni}(111)$ with both parallel and normal orientations seems to involve four steps: the formation of nitrosobenzene $\mathrm{Ph}-\mathrm{NO}$ as a primary reaction intermediate, its subsequent deoxygenation yielding a stable $\mathrm{Ph}-\mathrm{N}$ species, and two consecutive hydrogen transfers to $\mathrm{N}$ to finally produce aniline. The two last steps are the most demanding. It is also worth noticing that every time a $\mathrm{N}-\mathrm{O}$ bond is broken either an $\mathrm{O}$ atom or a $\mathrm{OH}$ group is released. These species occupy the same fcc and hcp hollow sites as H. Therefore, they have to be removed in order to avoid catalyst poisoning and guarantee continuous adsorption of $\mathrm{H}_{2}$ and nitroarene. The removal of $\mathrm{O}$ and $\mathrm{OH}$ can be achieved by reaction with the adsorbed $\mathrm{H}$ atoms, forming and subsequently desorbing $\mathrm{H}_{2} \mathrm{O}$. 


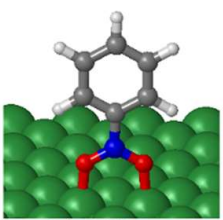

$\mathrm{Ph}-\mathrm{NO}_{2}$

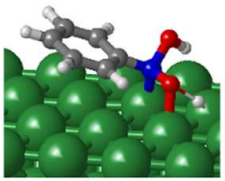

N-TS5
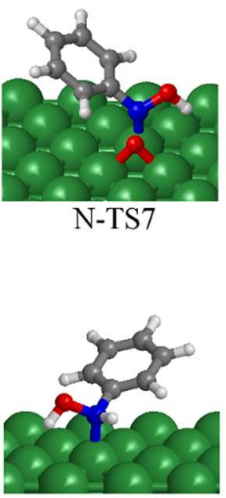

$\mathrm{Ph}-\mathrm{NHOH}$

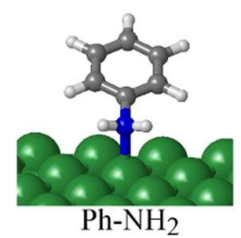

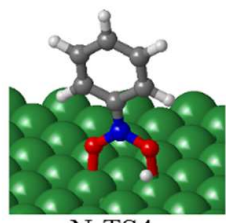

N-TS4

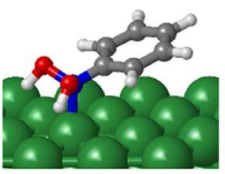

$\mathrm{Ph}-\mathrm{N}(\mathrm{OH})_{2}$
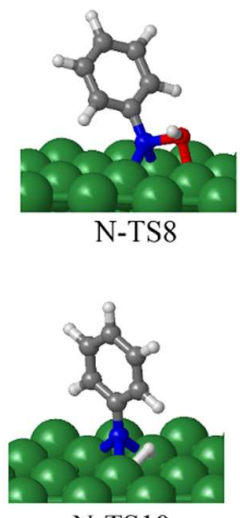

N-TS10

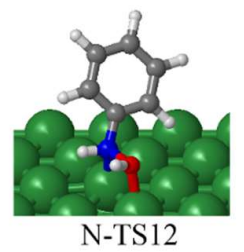

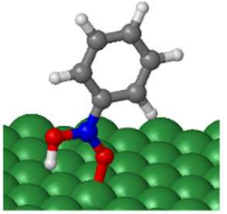

$\mathrm{Ph}-\mathrm{NOOH}$ (A)

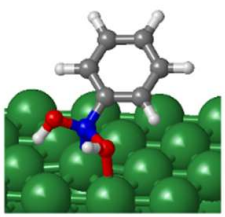

N-TS6
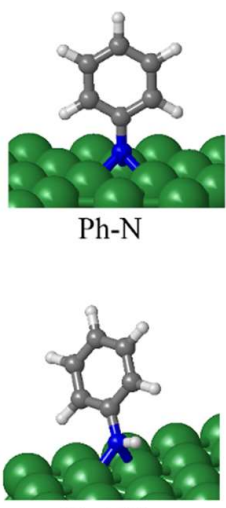

$\mathrm{Ph}-\mathrm{NH}$

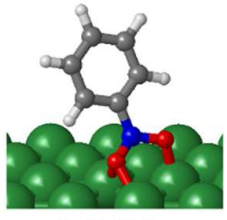

N-TS13

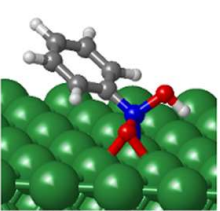

$\mathrm{Ph}-\mathrm{NOOH}(\mathrm{B})$

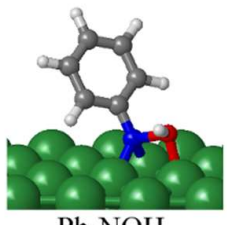

$\mathrm{Ph}-\mathrm{NOH}$
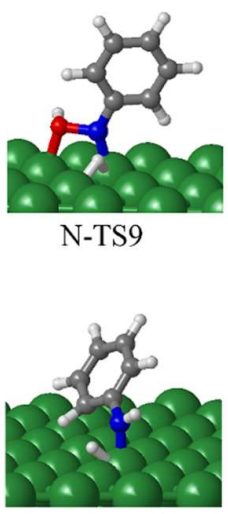

N-TS11

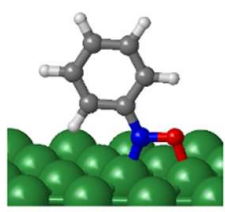

$\mathrm{Ph}-\mathrm{NO}$

Figure 3.12 Optimized geometries of minima and transition states involved in the normal pathway of nitrobenzene hydrogenation on $\mathrm{Ni}(111)$ surface. $\mathrm{Ni}, \mathrm{C}, \mathrm{N}, \mathrm{O}$ and $\mathrm{H}$ atoms are depicted as green, gray, blue, red and white balls. 


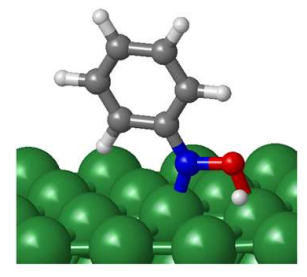

N-TS14

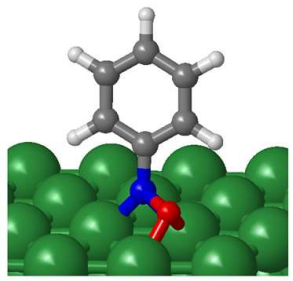

N-TS16

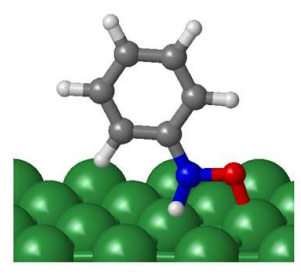

N-TS15

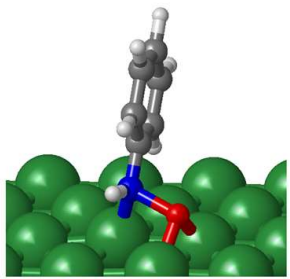

N-TS17

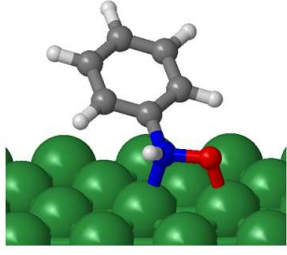

$\mathrm{Ph}-\mathrm{NHO}$

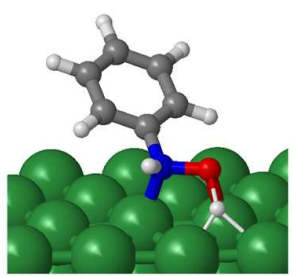

N-TS18

Figure 3.13 Optimized geometries of minima and transition states involved in the normal pathway of nitrobenzene hydrogenation on $\mathrm{Ni}(111)$ surface. $\mathrm{Ni}, \mathrm{C}, \mathrm{N}, \mathrm{O}$ and $\mathrm{H}$ atoms are depicted as green, gray, blue, red and white balls.

\subsection{Reduction of nitro group on Co(111). Direct route.}

The same reaction network used to study the reduction of nitrobenzene on the $\mathrm{Ni}(111)$ surface has been considered on Co(111) as well. For consistency, the order of the elementary steps is the same.

\subsubsection{Parallel pathways}

The calculated activation and reaction energies of all elementary steps are summarized in Table 3.9. The calculated energy profiles are shown in Figure 3.14. The optimized geometries of the intermediate and transition state structures involved in the parallel and normal pathways are depicted in Figure 3.15. 
Table 3.9 Calculated reaction $\left(E_{\text {reac }}\right)$ and activation $\left(E_{\text {act }}\right)$ energies for all elementary steps of the reduction of nitrobenzene on $\mathrm{Co}(111)$ surface with parallel geometries. All energies are given in $\mathrm{kcal} / \mathrm{mol}$.

\begin{tabular}{|c|c|c|c|}
\hline \multirow{2}{*}{ step } & \multirow{2}{*}{ Reaction } & \multicolumn{2}{|c|}{ Electronic Energy } \\
\hline & & $\mathrm{E}_{\text {reac }}$ & $E_{\text {act }}$ \\
\hline 4 & $\mathrm{Ph}-\mathrm{NO}_{2}+\mathrm{H}^{*} \leftrightarrow \mathrm{Ph}-\mathrm{NOOH}$ & 5.7 & 17.5 \\
\hline 5 & $\mathrm{Ph}-\mathrm{NOOH}^{*}+\mathrm{H}^{*} \leftrightarrow \mathrm{Ph}-\mathrm{N}(\mathrm{OH})_{2}{ }^{*}$ & 11.7 & 21.5 \\
\hline 6 & 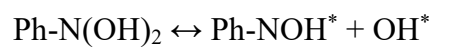 & -45.4 & 3.4 \\
\hline 7 & 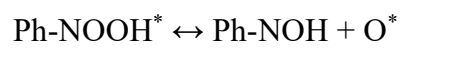 & -37.8 & 6.8 \\
\hline 8 & $\mathrm{Ph}-\mathrm{NOH}^{*} \leftrightarrow \mathrm{Ph}-\mathrm{N}+\mathrm{OH}^{*}$ & -52.1 & 1.6 \\
\hline 9 & $\mathrm{Ph}-\mathrm{NOH}+\mathrm{H}^{*} \leftrightarrow \mathrm{Ph}^{-\mathrm{NHOH}^{*}}$ & 2.4 & 26.7 \\
\hline 10 & $\mathrm{Ph}-\mathrm{N}^{*}+\mathrm{H}^{*} \leftrightarrow \mathrm{Ph}-\mathrm{NH}^{*}$ & 1.1 & 24.5 \\
\hline 11 & $\mathrm{Ph}-\mathrm{NH}^{*}+\mathrm{H}^{*} \leftrightarrow \mathrm{Ph}^{-\mathrm{NH}_{2}}{ }^{*}$ & 4.5 & 26.3 \\
\hline 12 & 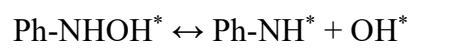 & -53.4 & 10.0 \\
\hline 13 & $\mathrm{Ph}-\mathrm{NO}_{2}{ }^{*} \leftrightarrow \mathrm{Ph}-\mathrm{NO}^{*}+\mathrm{O}^{*}$ & -45.1 & 5.2 \\
\hline 14 & $\mathrm{Ph}-\mathrm{NO}^{*}+\mathrm{H}^{*} \leftrightarrow \mathrm{Ph}-\mathrm{NOH}^{*}$ & 13.0 & 22.2 \\
\hline 15 & $\mathrm{Ph}-\mathrm{NO}^{*}+\mathrm{H}^{*} \leftrightarrow \mathrm{Ph}-\mathrm{NHO}^{*}$ & 5.6 & 22.5 \\
\hline 16 & $\mathrm{Ph}-\mathrm{NO}^{*} \leftrightarrow \mathrm{Ph}-\mathrm{N}^{*}+\mathrm{O}^{*}$ & -43.3 & 14.3 \\
\hline 17 & $\mathrm{Ph}-\mathrm{NHO}^{*} \leftrightarrow \mathrm{Ph}-\mathrm{NH}^{*}+\mathrm{O}^{*}$ & -47.8 & 6.9 \\
\hline 18 & $\mathrm{Ph}-\mathrm{NHO}^{*}+\mathrm{H}^{*} \leftrightarrow \mathrm{Ph}-\mathrm{NHOH}^{*}$ & 9.7 & 21.2 \\
\hline
\end{tabular}

The first obvious observation when looking at the energy profile in Figure 3.14 and the reaction and activation energies in Table 3.9 is that steps $6,7,8,12,13,16$ and 17 are very exothermic and have lower activation barriers, while the rest of steps are rather thermo-neutral and are much more highly activated. The energy profile is remarkably similar to that of nitrobenzene reduction on $\mathrm{Ni}(111)$. 


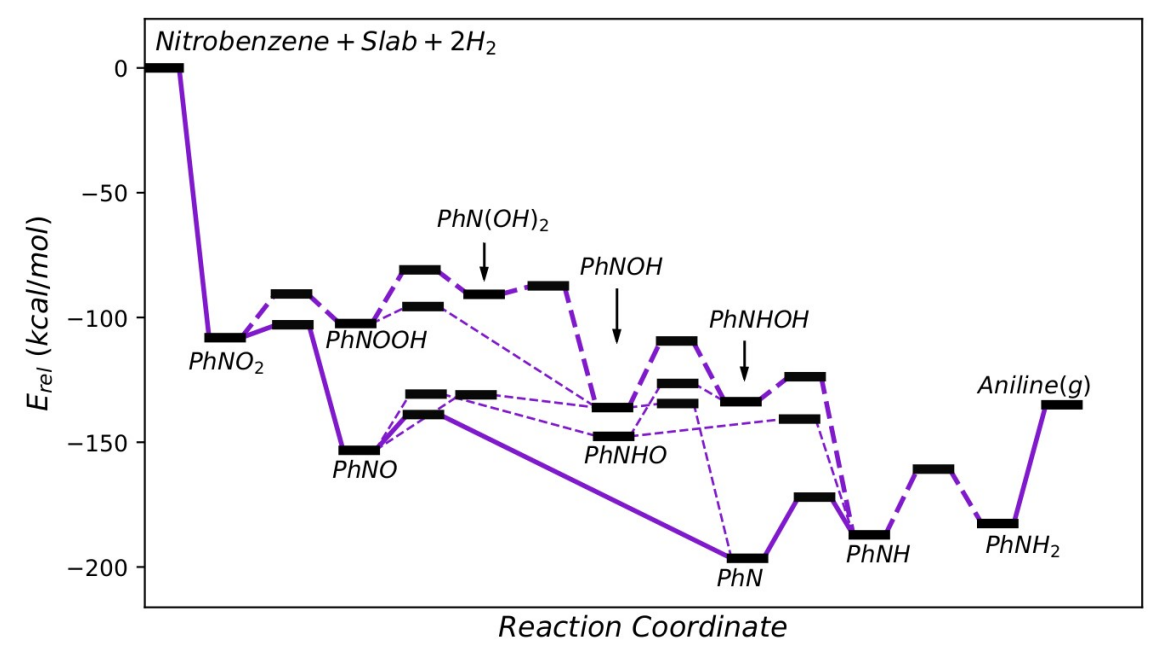

Figure 3.14. Calculated energy profile of the parallel pathways for nitrobenzene reduction over Co(111) surface. The zero energy level corresponds to the sum of the absolute energies of the Co slab $+3 \mathrm{H}_{2}+$ Nitrobenzene.

The mechanism starts with the formation of $\mathrm{Ph}-\mathrm{NOOH}$ with an activation energy of $17.5 \mathrm{kcal} / \mathrm{mol}$. In the transition state (P-TS4 in Figure 3.15) the aromatic ring remains anchored to three $\mathrm{Co}$ atoms but one of the $\mathrm{Co}-\mathrm{O}$ bonds has been completely broken while the $\mathrm{O}-\mathrm{H}$ bond being formed has a length of $1.43 \AA$. In Ph-NOOH the angle $\mathrm{Co}-\mathrm{N}-\mathrm{OH}$ is $123^{\circ}$. The other O-N-O, Co-N-C, Co-N-O, C-N-O angles lie between $101^{\circ}$ and $108^{\circ}$. Thus, $\mathrm{N}$ is trying to adopt a tetrahedral geometry but the repulsion caused by the $\mathrm{H}$ oriented toward the surface forces the $\mathrm{O}$ atoms to tilt away from the surface. In terms of relative energy, this intermediate is $5.5 \mathrm{kcal} / \mathrm{mol}$ less stable than $\mathrm{Ph}-\mathrm{NO}_{2}$.

Step 5 also consists of a hydrogen transfer to the second $\mathrm{O}$ atom of the $-\mathrm{NOOH}$ group. It requires an even higher activation energy, $21.5 \mathrm{kcal} / \mathrm{mol}$. Although the Co$\mathrm{O}$ bond is not completely broken its length increases from 1.96 to $2.16 \AA$ in the transition state (P-TS5 in Figure 3.15). Moreover, the Co-N distance is elongated by $0.11 \AA$. The two main bonds of the nitro group with the surface are thus destabilized. The resulting intermediate $\mathrm{Ph}-\mathrm{N}(\mathrm{OH})_{2}$ is bonded to the surface only through the aromatic ring and through a Co-N bond with a length of $2.1 \AA$ which might explain 
why it is $17.3 \mathrm{kcal} / \mathrm{mol}$ less stable than nitrobenzene in terms of relative energy. These results suggest that the $\mathrm{O}-\mathrm{H}$ and $\mathrm{Co}-\mathrm{N}$ bonds are less stable than $\mathrm{Co}-\mathrm{O}$ and Co-H bonds. Moreover, both $\mathrm{N}-\mathrm{O}$ bonds $(1.43 \AA)$ in $\mathrm{Ph}-\mathrm{NOOH}$ and $\mathrm{Ph}-\mathrm{N}(\mathrm{OH})_{2}$ are longer than the $\mathrm{N}-\mathrm{O}$ bonds in nitrobenzene $(1.33 \AA)$. That is, they have been somewhat activated.

From $\mathrm{Ph}-\mathrm{N}(\mathrm{OH})_{2}$, N-O cleavage is the only possible elementary reaction (step 6). The transition state is characterized by an elongated N-O bond, up to $1.69 \AA$, compensated by the partially formed Co-O bond with $2.11 \AA$ length. At the same time the $\mathrm{Co}-\mathrm{N}$ is shortened to $1.98 \AA$. Since the $\mathrm{Co}-\mathrm{O}$ interaction is strong, it is not surprising that the activation energy of this step is only $3.4 \mathrm{kcal} / \mathrm{mol}$. As a consequence of the strong $\mathrm{Co}-\mathrm{OH}$ interaction the relative energy of the product is very low, which makes it a very exothermic step. The $\mathrm{O}$ atom of the product intermediate $\mathrm{Ph}-\mathrm{NOH}$ is bonded to a Co atom at a distance of $2.22 \AA$. In this process the molecule is slightly displaced in order to maximize the Co-O interaction, implying again that this interaction determines to some extent the adsorption behavior of the intermediates.

At this point the mechanism bifurcates. On the one hand intermediate $\mathrm{Ph}-\mathrm{NOH}$ accepts an $\mathrm{H}$ atom leading to the slightly less stable phenylhydroxylamine (step 9) for which an energy barrier of $26.7 \mathrm{kcal} / \mathrm{mol}$ has to be surmounted. Because of the new $\mathrm{N}-\mathrm{H}$ bond formation, the $\mathrm{H}$ the $\mathrm{N}-\mathrm{O}$ bond is weakened with the consequent increase of its length up to $2.04 \AA$. The $\mathrm{Co}-\mathrm{OH}$ interaction is broken and now the Co-O distance is $3 \AA$. On the other hand, an $\mathrm{OH}$ group can be released from $\mathrm{Ph}-\mathrm{NOH}$ to the surface, resulting in the formation of $\mathrm{Ph}-\mathrm{N}$ (step 8). In relative energy terms, this is the most stable intermediate (Figure 3.14). However, the major contribution to the stability does not come from the energy of interaction between $\mathrm{Ph}-\mathrm{N}$ and the surface but rather because at this point two strong Co-O bonds have been formed ( $\mathrm{O}$ and $\mathrm{OH}$ released from previous intermediates). More details of these interactions are given in section 3.12. The path to aniline from $\mathrm{Ph}-\mathrm{N}$ consists of two relatively highly activated hydrogen transfers: both steps 10 and 11 require 25.6 and $27.3 \mathrm{kcal} / \mathrm{mol}$ respectively. The only fate of the phenylhydroxylamine formed in step 9 is to undergo a $\mathrm{N}-\mathrm{OH}$ bond breaking (step 12). As expected the activation energy is relatively low, $9.9 \mathrm{kcal} / \mathrm{mol}$. Finally, we have calculated the nitrosobenzene route without previous activation of nitrobenzene (steps 13 and 16) and we have not found any fundamental differences with the direct route discussed on $\mathrm{Ni}(111)$. 

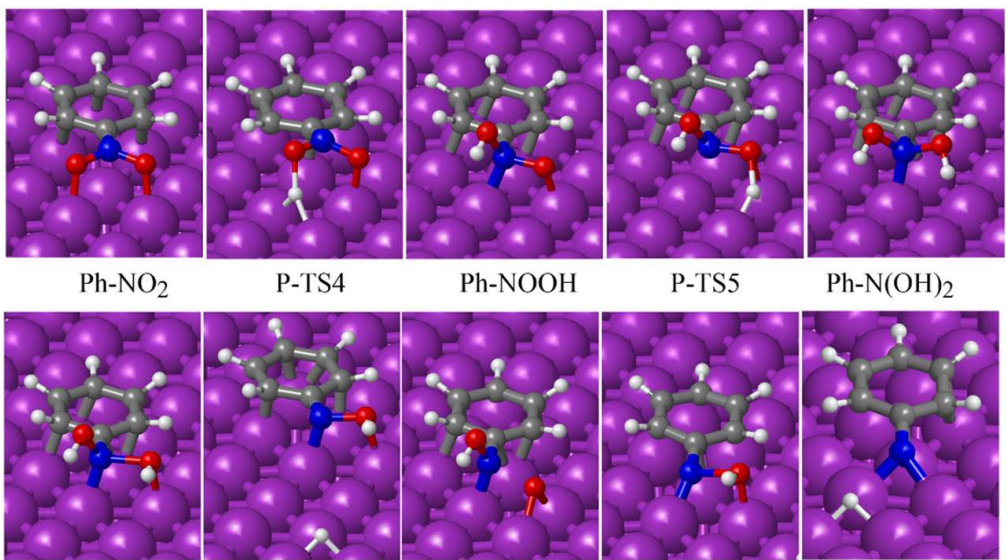

P-TS4

$\mathrm{Ph}-\mathrm{NOOH}$

P-TS5

$\mathrm{Ph}-\mathrm{N}(\mathrm{OH})_{2}$
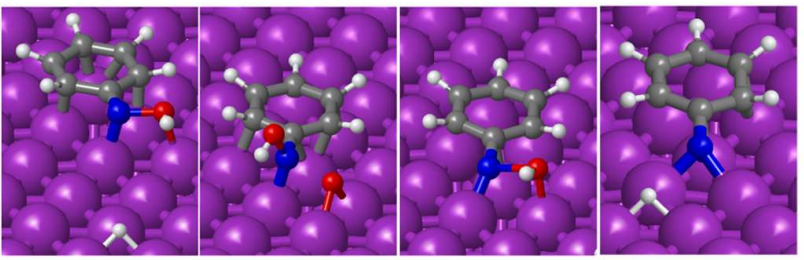

P-TS6

$\mathrm{Ph}-\mathrm{NOH}$

P-TS7

P-TS8

$\mathrm{Ph}-\mathrm{N}$
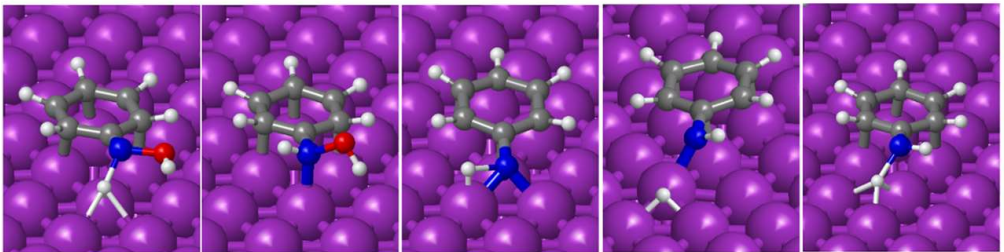

P-TS9

$\mathrm{Ph}-\mathrm{NHOH}$

P-TS10

$\mathrm{Ph}-\mathrm{HN}$

P-TS11
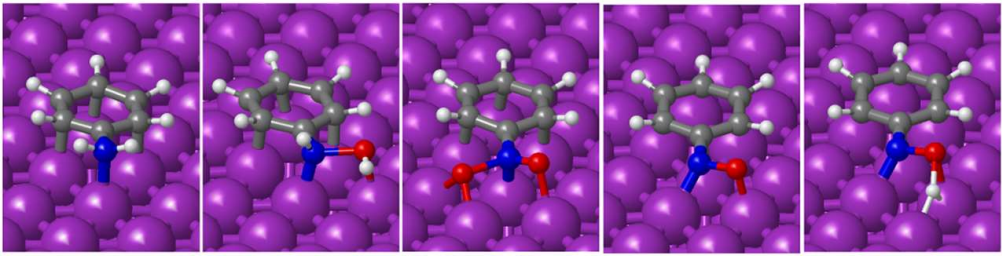

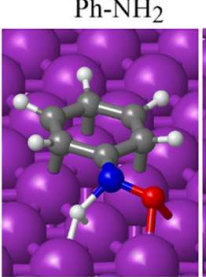

P-TS15

P-TS12

P-TS13

$\mathrm{Ph}-\mathrm{NO}$

P-TS14

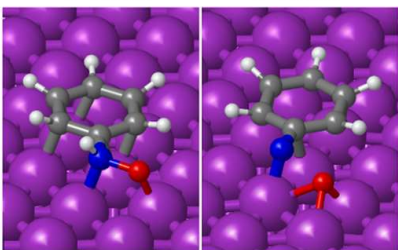

$\mathrm{Ph}-\mathrm{NHO}$

P-TS16

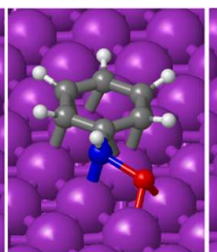

P-TS17

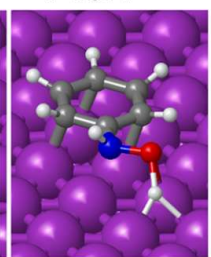

P-TS18

Figure 3.15 Optimized geometries of minima and transition states involved in the parallel pathway of nitrobenzene hydrogenation on $\mathrm{Co}(111)$ surface. $\mathrm{Co}, \mathrm{C}, \mathrm{N}, \mathrm{O}$ and $\mathrm{H}$ atoms are depicted as purple, gray, blue, red and white balls. 
It should be pointed out that all the products of hydrogen transfers are slightly less stable than the non-hydrogenated reactant. In other words, hydrogen transfer implies destabilizing one of the anchor points ( $\mathrm{N}$ or $\mathrm{O}$ atom) of the nitroaromatic with the surface and breaking a relatively stable $\mathrm{Co}-\mathrm{H}$ bond. Conversely, breaking the $\mathrm{N}-\mathrm{O}$ bonds implies the formation of a strong Co-O bond and the reduction of the coordination of the $\mathrm{N}$ atom forcing it to interact stronger with the Co atoms of the surface.

\subsubsection{Normal pathways}

Here we examine the mechanism where the nitroaromatic reactant and subsequent intermediates are adsorbed with a normal orientation, i.e. through the nitro group. The reaction and activation energies are summarized in Table 3.10. The electronic energy profile is displayed in Figure 3.16 and the optimized structures of the intermediates and transition states are shown in Figure 3.17. Results similar to those previously discussed for $\mathrm{Ni}$ are found.

Table 3.10 Calculated reaction $\left(E_{\text {reac }}\right)$ and activation $\left(E_{a c t}\right)$ energies for all elementary steps of the reduction of nitrobenzene on the $\mathrm{Co}(111)$ surface with normal geometries. All energies are given in $\mathrm{kcal} / \mathrm{mol}$.

\begin{tabular}{|c|c|c|c|}
\hline \multirow{2}{*}{ step } & \multirow{2}{*}{ Reaction } & \multicolumn{2}{|c|}{ Electronic Energy } \\
\hline & & $E_{\text {reac }}$ & $E_{\text {act }}$ \\
\hline 4 & $\mathrm{Ph}-\mathrm{NO}_{2}+\mathrm{H}^{*} \leftrightarrow \mathrm{Ph}-\mathrm{NOOH}(\mathrm{B})$ & 0.4 & 25.0 \\
\hline 5 & $\mathrm{Ph}-\mathrm{NOOH}^{*}+\mathrm{H}^{*} \leftrightarrow \mathrm{Ph}-\mathrm{N}(\mathrm{OH})_{2}{ }^{*}$ & 10.8 & 24.5 \\
\hline 6 & 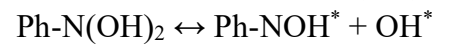 & -35.8 & 6.3 \\
\hline 7 & $\mathrm{Ph}-\mathrm{NOOH}^{*} \leftrightarrow \mathrm{Ph}-\mathrm{NOH}+\mathrm{O}^{*}$ & -29.1 & 4.4 \\
\hline 8 & $\mathrm{Ph}-\mathrm{NOH}^{*} \leftrightarrow \mathrm{Ph}-\mathrm{N}+\mathrm{OH}^{*}$ & -62.7 & 2.7 \\
\hline 9 & $\mathrm{Ph}-\mathrm{NOH}+\mathrm{H}^{*} \leftrightarrow \mathrm{Ph}^{-\mathrm{NHOH}^{*}}$ & -2.4 & 21.1 \\
\hline 10 & $\mathrm{Ph}-\mathrm{N}^{*}+\mathrm{H}^{*} \leftrightarrow \mathrm{Ph}-\mathrm{NH}^{*}$ & 10.7 & 25.6 \\
\hline 11 & $\mathrm{Ph}-\mathrm{NH}^{*}+\mathrm{H}^{*} \leftrightarrow \mathrm{Ph}-\mathrm{NH}_{2}{ }^{*}$ & 1.1 & 27.3 \\
\hline 12 & $\mathrm{Ph}-\mathrm{NHOH}^{*} \leftrightarrow \mathrm{Ph}-\mathrm{NH}^{*}+\mathrm{OH}^{*}$ & -49.6 & 7.9 \\
\hline 13 & $\mathrm{Ph}-\mathrm{NO}_{2}{ }^{*} \leftrightarrow \mathrm{Ph}-\mathrm{NO}^{*}+\mathrm{O}^{*}$ & -43.1 & 5.8 \\
\hline 14 & $\mathrm{Ph}-\mathrm{NO}^{*}+\mathrm{H}^{*} \leftrightarrow \mathrm{Ph}-\mathrm{NOH}^{*}$ & 14.3 & 24.5 \\
\hline 15 & $\mathrm{Ph}-\mathrm{NO}^{*}+\mathrm{H}^{*} \leftrightarrow \mathrm{Ph}-\mathrm{NHO}^{*}$ & -2.3 & 23.6 \\
\hline 16 & $\mathrm{Ph}-\mathrm{NO}^{*} \leftrightarrow \mathrm{Ph}-\mathrm{N}^{*}+\mathrm{O}^{*}$ & -52.6 & 8.1 \\
\hline 17 & $\mathrm{Ph}-\mathrm{NHO}^{*} \leftrightarrow \mathrm{Ph}-\mathrm{NH}^{*}+\mathrm{O}^{*}$ & -39.5 & 5.9 \\
\hline 18 & $\mathrm{Ph}-\mathrm{NHO}^{*}+\mathrm{H}^{*} \leftrightarrow \mathrm{Ph}-\mathrm{NHOH}^{*}$ & 14.3 & 27.5 \\
\hline
\end{tabular}




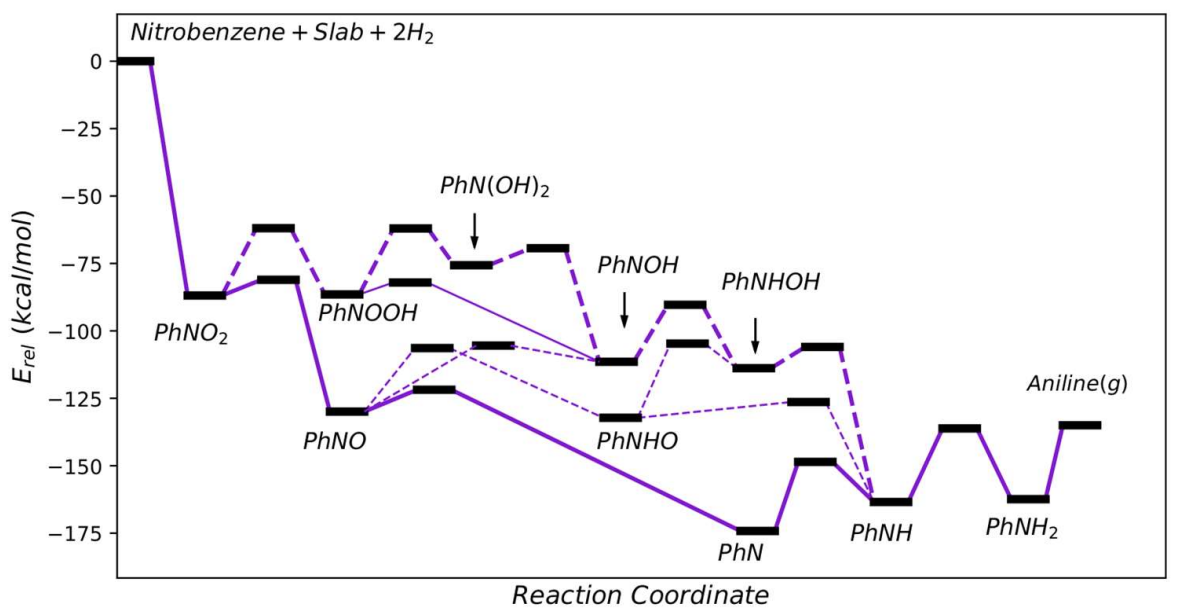

Figure 3.16. Calculated energy profile $\left(E_{\text {rel }}\right)$ of the normal pathways for nitrobenzene reduction over $\mathrm{Co}(111)$ surface. The zero energy level corresponds to the sum of the absolute energies of the Co slab $+3 \mathrm{H}_{2}+$ Nitrobenzene. For clarity step 8 has been omitted.

The first hydrogen transfer to nitrobenzene involves an activation energy of 25 $\mathrm{kcal} / \mathrm{mol}$. As a result of this reaction $\mathrm{Ph}-\mathrm{NOOH}(\mathrm{A})$ is formed, and similar to the same step on $\mathrm{Ni}(111)$, it is very unstable because it is only bonded to the Co(111) surface through one oxygen atom. Again, rearrangement of $\mathrm{Ph}-\mathrm{NOOH}(\mathrm{A})$ leads to a more favorable geometry, $\mathrm{Ph}-\mathrm{NOOH}(\mathrm{B})$, which is tilted significantly and the plane of the aromatic ring is almost aligned with the metal surface forming an angle of $\sim 23.4^{\circ}$ with it (Figure 3.17). Only the $\mathrm{N}$ atom is directly attached to a Co atom, with an optimized Co-N distance of $2.11 \AA$. The activation energy corresponding to the second H-transfer (step 5) is $24.5 \mathrm{kcal} / \mathrm{mol}$. The Co-N bond length increases from 2.05 to $2.13 \AA$, resulting again in a less stable intermediate, $\mathrm{Ph}-\mathrm{N}(\mathrm{OH})_{2}$. These last two intermediates will likely change to a horizontal orientation if the surface is partially clean. Nonetheless, the adsorbed $\mathrm{H}$ or $\mathrm{O}$ atoms would prevent this from occurring.

The subsequent formation of Ph-NOH (step 6) and Ph-N (step 8) follows from Ph$\mathrm{N}(\mathrm{OH})_{2}$ due to both $\mathrm{N}-\mathrm{O}$ breaking. In N-TS5 the $\mathrm{N}$ atom has an almost tetrahedral 
geometry while in N-TS8 (step 8) it has an almost planar geometry, but both activation energies are quite low, $<7 \mathrm{kcal} / \mathrm{mol}$. As in the parallel mechanism, $\mathrm{Ph}$ $\mathrm{NOH}$ could also follow a different path by reacting with one $\mathrm{H}$-atom to form phenylhydroxylamine (step 9) with an activation energy of above $20 \mathrm{kcal} / \mathrm{mol}$.

We will jump to step 12, which is again an $\mathrm{OH}$ release with the corresponding formation of $\mathrm{Ph}-\mathrm{NH}$, to compare it with the previous similar steps 6 and 8 . The barrier of step 12 is $7.9 \mathrm{kcal} / \mathrm{mol}$. It is actually higher than the barrier of steps 6 and 8. However, if we consider only the electronic energy (excluding dispersion forces) we see that the activation energies for the three steps are very similar, $3.2 \mathrm{kcal} / \mathrm{mol}$ (step 6), $2.5 \mathrm{kcal} / \mathrm{mol}$ (step 8) and $3.9 \mathrm{kcal} / \mathrm{mol}$ (step 12). The difference between step 6 and 8 is only $0.7 \mathrm{kcal} / \mathrm{mol}$ and between step 8 and 12 is only $0.6 \mathrm{kcal} / \mathrm{mol}$. The other seemingly important contribution comes from the dispersion forces due to the different orientation of the aromatic ring with respect to the surface. The more horizontal the greater the contribution. It would make the barrier increase or decrease depending on how differently the ring is oriented in the initial reactant and in the transition state. A final reaction between $\mathrm{Ph}-\mathrm{NH}$ and a $\mathrm{H}$-atom will lead to adsorbed aniline (step 11) by crossing a barrier of $27.2 \mathrm{kcal} / \mathrm{mol}$. Notice that this is the last step and all other elementary steps will ultimately lead to this last one. Therefore, because this high barrier cannot be avoided step 10 will be the rate-determining step.

Finally, we can see with a quick inspection of the energy profile in Figure 3.16 that, in the absence of $\mathrm{H}$, the system will be driven downwards to $\mathrm{Ph}-\mathrm{N}$. However, despite the whole reaction is exothermic, from $\mathrm{Ph}-\mathrm{N}$ the steps that depend on the concentration of H-atom lead in an uphill search for aniline. This trend is observed for the rest of steps: the hydrogenated product intermediates are less stable than the reactants. 

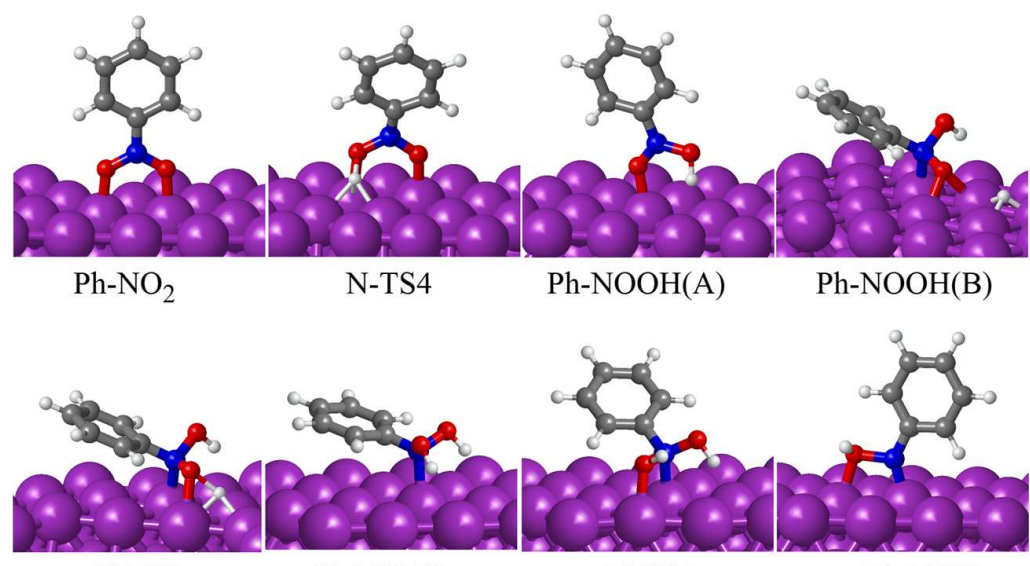

N-TS5

$\mathrm{Ph}-\mathrm{N}(\mathrm{OH})$,

N-TS6

$\mathrm{Ph}-\mathrm{NOH}$

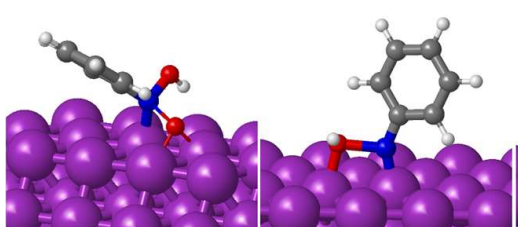

N-TS7

N-TS8
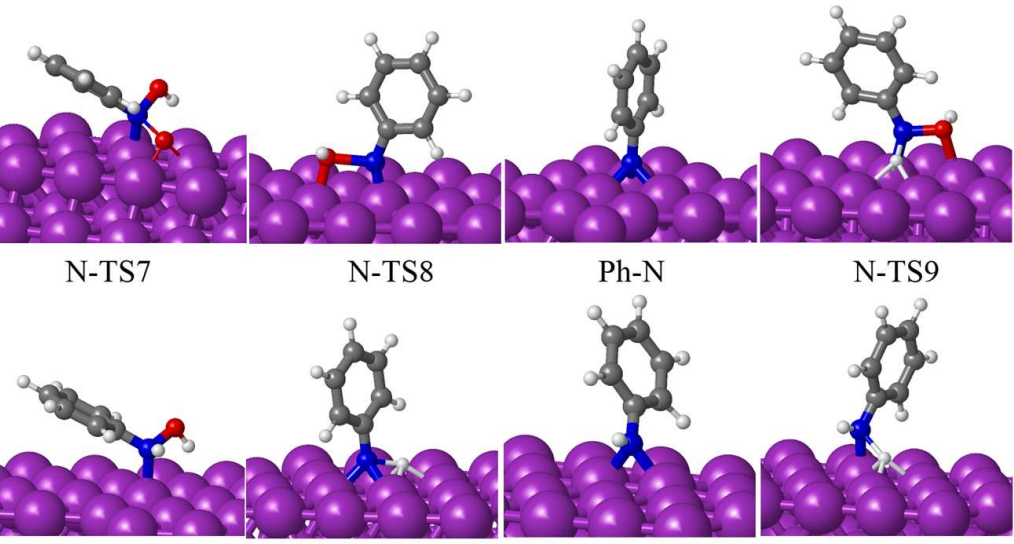

$\mathrm{Ph}-\mathrm{NHOH}$

N-TS10

$\mathrm{Ph}-\mathrm{N}$

N-TS9
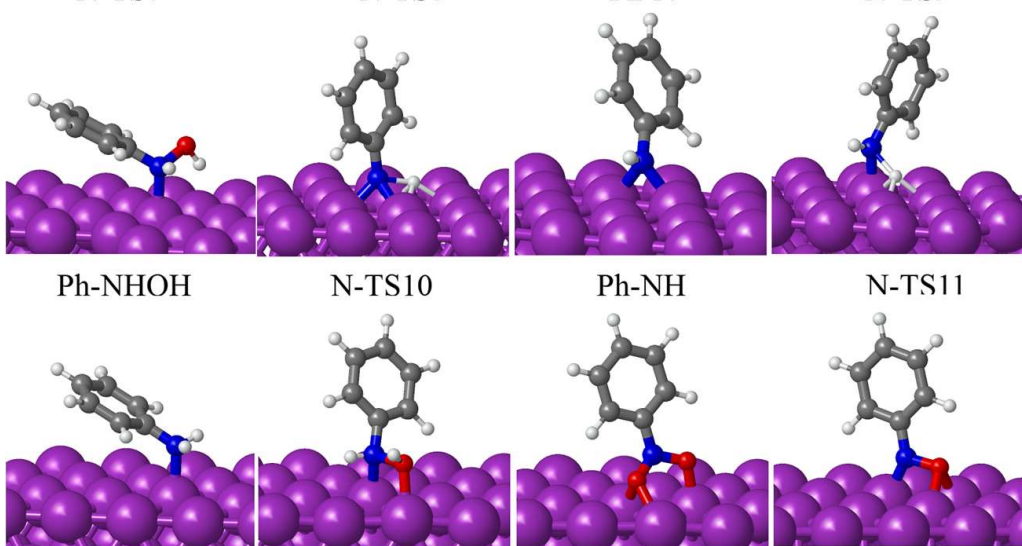

and

Ph-NH

N-TS 11
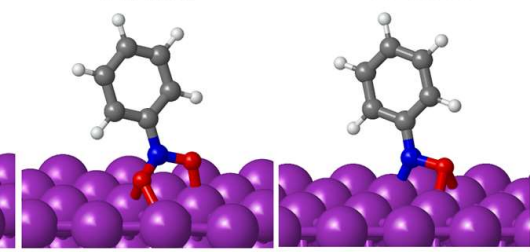

$\mathrm{Ph}-\mathrm{NH}_{2}$

N-TS12

N-TS13

$\mathrm{Ph}-\mathrm{NO}$

Figure 3.17 Optimized geometries of minima and transition states involved in the normal pathway of nitrobenzene hydrogenation on $\mathrm{Co}(111)$ surface. Co, C, N, O and $\mathrm{H}$ atoms are depicted as purple, gray, blue, red and white balls. 


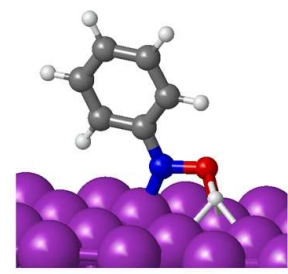

N-TS14

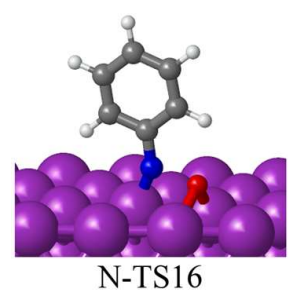

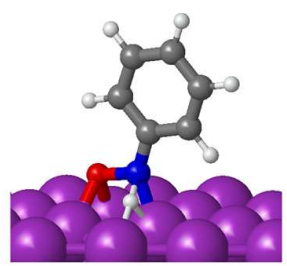

N-TS15

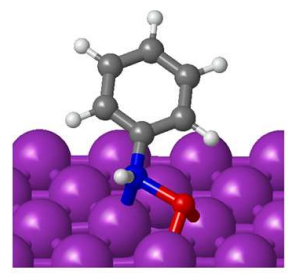

N-TS17

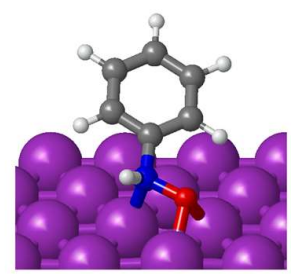

$\mathrm{Ph}-\mathrm{NHO}$

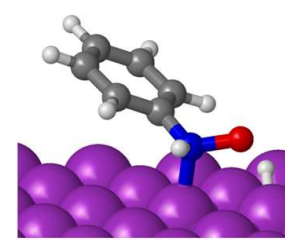

N-TS18

Figure 3.18 Optimized geometries of minima and transition states involved in the normal pathway of nitrobenzene hydrogenation on $\mathrm{Co}(111)$ surface. Co, C, N, O and $\mathrm{H}$ atoms are depicted as purple, gray, blue, red and white balls.

\subsection{Indirect or condensation route on $\mathrm{Ni}(111)$ and $\mathrm{Co}(111)$}

According to the mechanism proposed by Haber (Figure 3.2) the indirect route involves the formation of azoxybenzene (PhNO-NPh) through the condensation of nitrosobenzene ( $\mathrm{Ph}-\mathrm{NO})$ and phenylhydroxylamine (Ph-NHOH). Azoxybenzene is the precursor of azobenzene $(\mathrm{PhN}=\mathrm{NPh})$, whose hydrogenation leads to aniline $(\mathrm{Ph}-$ $\mathrm{NH}_{2}$ ), but no elementary steps are proposed for this process. A detailed mechanism that includes azoxybenzene and azobenzene requires the exploration of more than 20 possible elementary steps, which is by no means an uncostly endeavor. However, the study of the indirect route can be significantly reduced if we consider that according to the energy profiles in Figures 3.9, 3.11, 3.14 and 3.16 the most abundant species on $\mathrm{Ni}(111)$ and $\mathrm{Co}(111)$ surfaces are likely to be $\mathrm{PhNO}, \mathrm{Ph}-\mathrm{N}$ and $\mathrm{Ph}-\mathrm{NH}$ together with $\mathrm{H}, \mathrm{O}$, and $\mathrm{OH}$. Therefore, they will be the ones that should contribute the most to the condensation route. We have considered three elementary steps whose reaction 
and activation energies are summarized in Table 3.11, while the energy profiles and the optimized structures are shown in Figures 3.19 and 3.20.

Table 3.11 Calculated reaction $\left(E_{\text {reac }}\right)$ and activation $\left(E_{\text {act }}\right)$ energies of the indirect route on $\mathrm{Ni}(111)$ surface with parallel and normal geometries. All energies are given in $\mathrm{kcal} / \mathrm{mol}$.

\begin{tabular}{|c|c|c|c|c|c|}
\hline \multirow{2}{*}{ Step } & \multirow{2}{*}{ Reaction } & \multicolumn{2}{|c|}{ Parallel } & \multicolumn{2}{|c|}{ Normal } \\
\hline & & $E_{\text {reac }}$ & $E_{\text {act }}$ & $E_{\text {reac }}$ & $\mathrm{E}_{\text {act }}$ \\
\hline \multicolumn{6}{|c|}{$\mathrm{Ni}$} \\
\hline 19 & 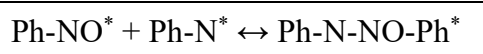 & 16.4 & 32.8 & 27.1 & 27.2 \\
\hline 20 & $\mathrm{Ph}-\mathrm{N}^{*}+\mathrm{Ph}-\mathrm{N}^{*} \leftrightarrow \mathrm{Ph}-\mathrm{N}-\mathrm{N}-\mathrm{Ph}^{*}$ & -0.7 & 38.1 & 41.9 & 47.2 \\
\hline 21 & $\mathrm{Ph}-\mathrm{N}-\mathrm{NO}-\mathrm{Ph}^{*} \leftrightarrow \mathrm{Ph}-\mathrm{N}-\mathrm{N}-\mathrm{Ph}^{*}+\mathrm{O}^{*}$ & -45.7 & 11.7 & -32.0 & 2.4 \\
\hline \multicolumn{6}{|c|}{ Co } \\
\hline 19 & $\mathrm{Ph}-\mathrm{NO}^{*}+\mathrm{Ph}-\mathrm{N}^{*} \leftrightarrow \mathrm{Ph}-\mathrm{N}-\mathrm{NO}-\mathrm{Ph}^{*}$ & 25.8 & 42.5 & 28.2 & 29.1 \\
\hline 20 & $\mathrm{Ph}-\mathrm{N}^{*}+\mathrm{Ph}-\mathrm{N}^{*} \leftrightarrow \mathrm{Ph}-\mathrm{N}-\mathrm{N}-\mathrm{Ph}^{*}$ & 18.5 & 47.4 & 40.8 & 45.7 \\
\hline 21 & $\mathrm{Ph}-\mathrm{N}-\mathrm{NO}-\mathrm{Ph}^{*} \leftrightarrow \mathrm{Ph}-\mathrm{N}-\mathrm{N}-\mathrm{Ph}^{*}+\mathrm{O}^{*}$ & -50.7 & 7.8 & -40.0 & 0.3 \\
\hline
\end{tabular}

The formation of azoxybenzene can occur by direct reaction between nitrosobenzene and Ph-N (step 19). On Ni(111), The optimized N-N bond length in the transition state structure with parallel orientation (P-TS19) is $1.97 \AA$. The high activation energy $(32.8 \mathrm{kcal} / \mathrm{mol})$ is related to the fact that the N-N bond is barely formed in the transition state. The aromatic rings, anchored to the surface, might prevent both $\mathrm{N}$ atoms from coming close enough to each other to form a chemical bond. Both $\mathrm{N}$ $\mathrm{N}$ and $\mathrm{N}-\mathrm{O}$ bond lengths are equal to $1.35 \AA$ in the adsorbed axozybenzene product, which is $16 \mathrm{kcal} / \mathrm{mol}$ less stable than the reactants, $\mathrm{Ph}-\mathrm{NO}+\mathrm{Ph}-\mathrm{N}$. This is by far the most endothermic step and the one with the highest $\mathrm{E}_{\text {act }}$ calculated up to now on $\mathrm{Ni}(111)$. In the normal geometry, the activation energy for the same step is lower, $27.1 \mathrm{kcal} / \mathrm{mol}$. The better stability of the N-TS19 is in part a consequence of the weaker interaction of the aromatic rings with the surface in the initial reactants, i.e the aromatic rings do not pose any constraint to the geometry of the transition state. In any case, the activation barrier is higher than that needed to form $\mathrm{Ph}-\mathrm{N}$ from $\mathrm{Ph}$ $\mathrm{NO}$ in step 13. Therefore, the coupling of $\mathrm{Ph}-\mathrm{NO}$ withPh-N is less probable. 
On $\mathrm{Co}(111)$, the formation of azoxybenzene through reaction between $\mathrm{Ph}-\mathrm{NO}$ and $\mathrm{Ph}-\mathrm{N}$ (step 19) is also very unfavorable due to the huge penalty of $50.3 \mathrm{kcal} / \mathrm{mol}$ calculated to reach the transition state. As in the case of $\mathrm{Ni}(111)$, the instability can be rationalized through the geometry of the transition state P-TS19 in which the distance of the N-N bond being formed is $2.41 \AA$. The reaction is very endothermic by $25.8 \mathrm{kcal} / \mathrm{mol}$ and thus azoxybenzene is thermodynamically less stable than $\mathrm{Ph}-\mathrm{NO}+\mathrm{Ph}-\mathrm{N}$ (Figure 3.21). The situation is different though in the case of normal orientation (Figure 3.21). Both aromatic rings no longer hinder the orientation of the $\mathrm{N}$ atoms so that they are able to come as close as $1.69 \AA$. The activation energy is $29.1 \mathrm{kcal} / \mathrm{mol}$, that is, about $23.1 \mathrm{kcal} / \mathrm{mol}$ lower than the parallel step. Still, it is higher than the $\mathrm{E}_{\text {act }}$ of the $\mathrm{H}$-transfer to $\mathrm{PhN}$ (step 10) so that the formation of the N$\mathrm{N}$ bond should not be considered probable on $\mathrm{Co}(111)$ either.

The association of two stable species Ph-N (step 20) can form azobenzene. We consider this step because $\mathrm{Ph}-\mathrm{N}$ is probably abundant on the surface, and it could compete with the $\mathrm{H}$ transfer to $\mathrm{Ph}-\mathrm{N}$ previously discussed (step 10). However, the activation energy for azobenzene formation in the parallel orientation is very high on $\mathrm{Ni}(111), 38.0 \mathrm{kcal} / \mathrm{mol}$, and even higher, $47 \mathrm{kcal} / \mathrm{mol}$, in the normal geometry. On the other hand, the azobenzene product is much more stable in the parallel adsorption geometry than in the normal conformation, due to the interaction of the two aromatic rings with the surface metal atoms (see Figure 3.20)

On Co(111), a barrier of $47.4 \mathrm{kcal} / \mathrm{mol}$ for the parallel geometry also renders step 20 as highly improbable. The reaction with a normal geometry has an equally high activation energy $45.7 \mathrm{kcal} / \mathrm{mol}$.

The last step considered in the condensation route is the deoxygenation of axozybenzene (step 21) which involves the dissociation of an N-O bond. Both normal and horizontal reactions are exothermic with very low activation barriers on $\mathrm{Ni}(111)$ and $\mathrm{Co}(111)$ (Figures 3.19 and 3.21); axozybenzene is unstable with respect to the deoxygenated intermediates. Thus, in the unlikely event that axozybenzene is generated it will probably evolve to azobenzene which will dissociate into two $\mathrm{Ph}-\mathrm{N}$. 

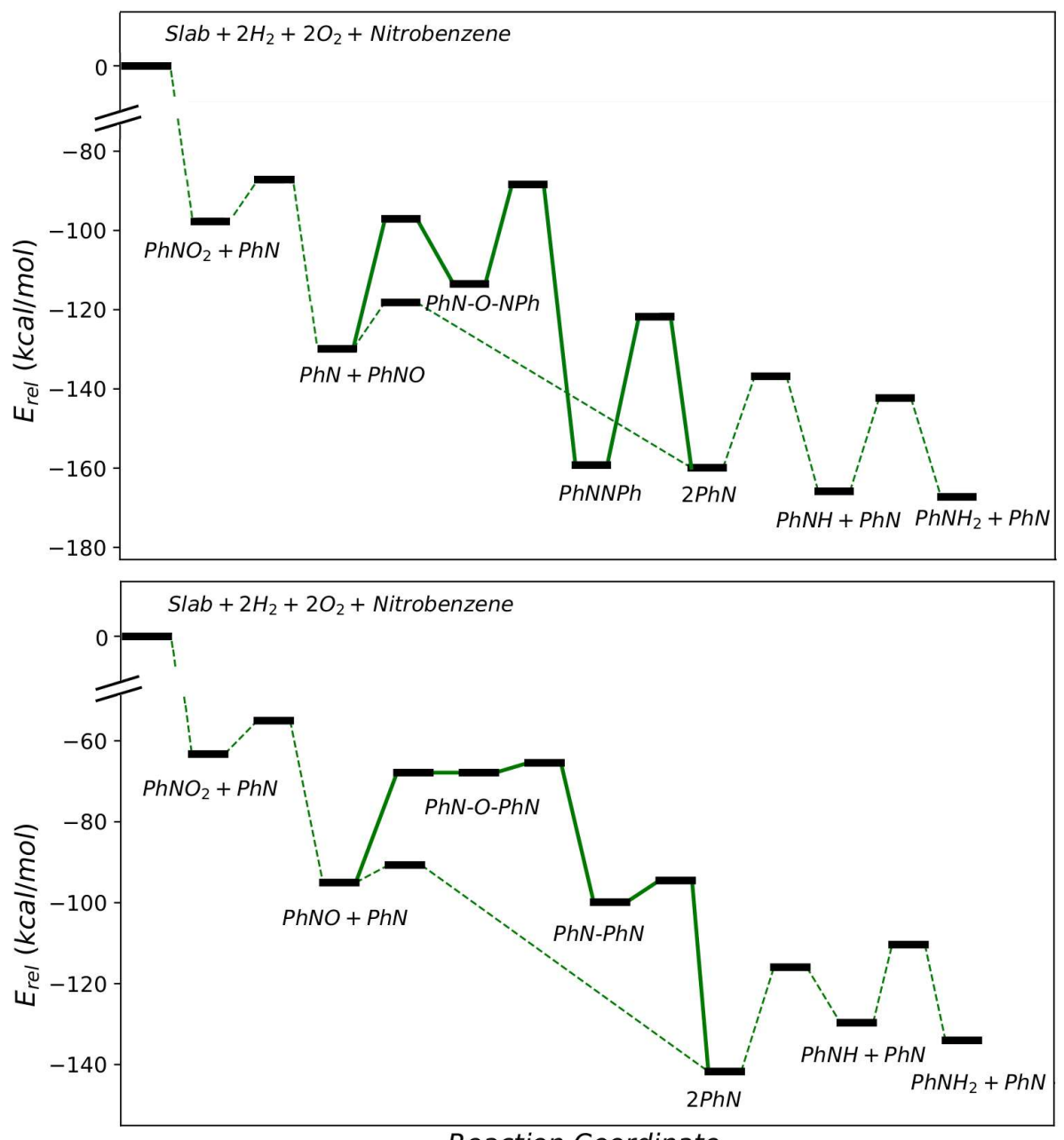

Reaction Coordinate

Figure 3.19 Calculated energy profile of steps 19, 20 and 21 (condensation) route on $\mathrm{Ni}(111)$ surface with parallel (top) and normal (bottom) orientations. The most favorable pathway found for the direct route is also shown (dotted line) for the sake of comparison. 


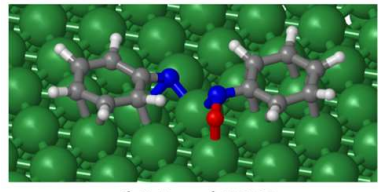

$\mathrm{phN}+\mathrm{phNO}$

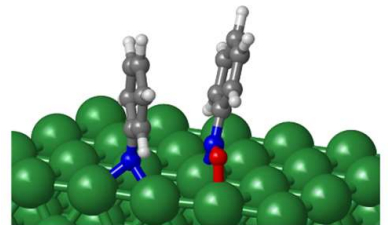

$\mathrm{phN}+\mathrm{phNO}$

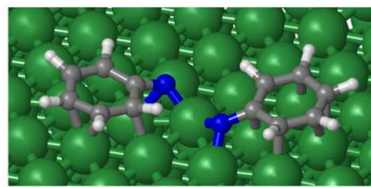

$\mathrm{phN}+\mathrm{phN}$

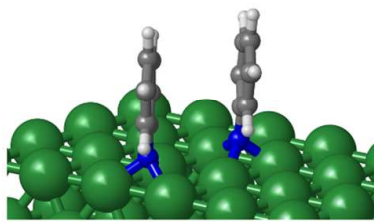

$\mathrm{phN}+\mathrm{phN}$

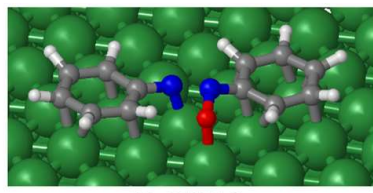

P-TS19

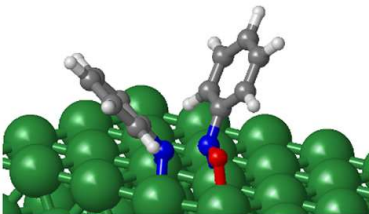

N-TS19

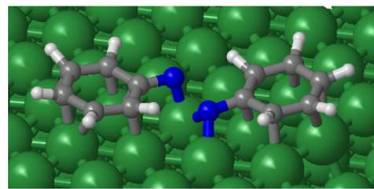

P-TS20

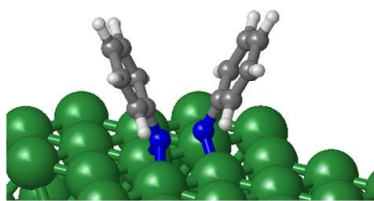

N-TS20

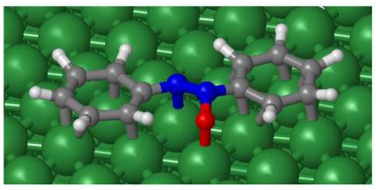

Azoxybenzene

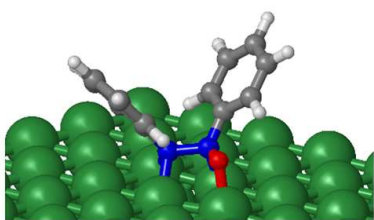

Azoxybenzene

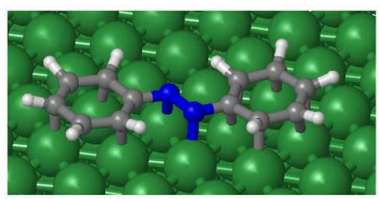

Azobenzene

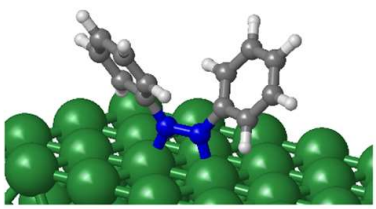

Azobenzene

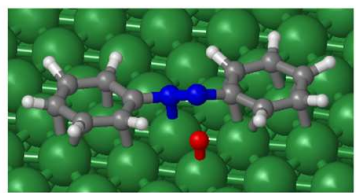

P-TS21

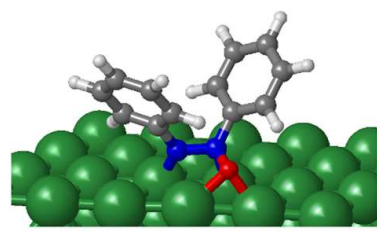

N-TS21

Figure 3.20 Optimized geometries of the transition states and product structures involved in the condensation route to form azoxy and azo compounds on $\mathrm{Ni}(111)$ surface with normal (N) and parallel (P) orientations. Ni, C, N, O and $\mathrm{H}$ atoms are depicted as green, gray, blue, red and white balls. 
3.9 Indirect or condensation route on $\mathrm{Ni}(111)$ and $\mathrm{Co}(111)$
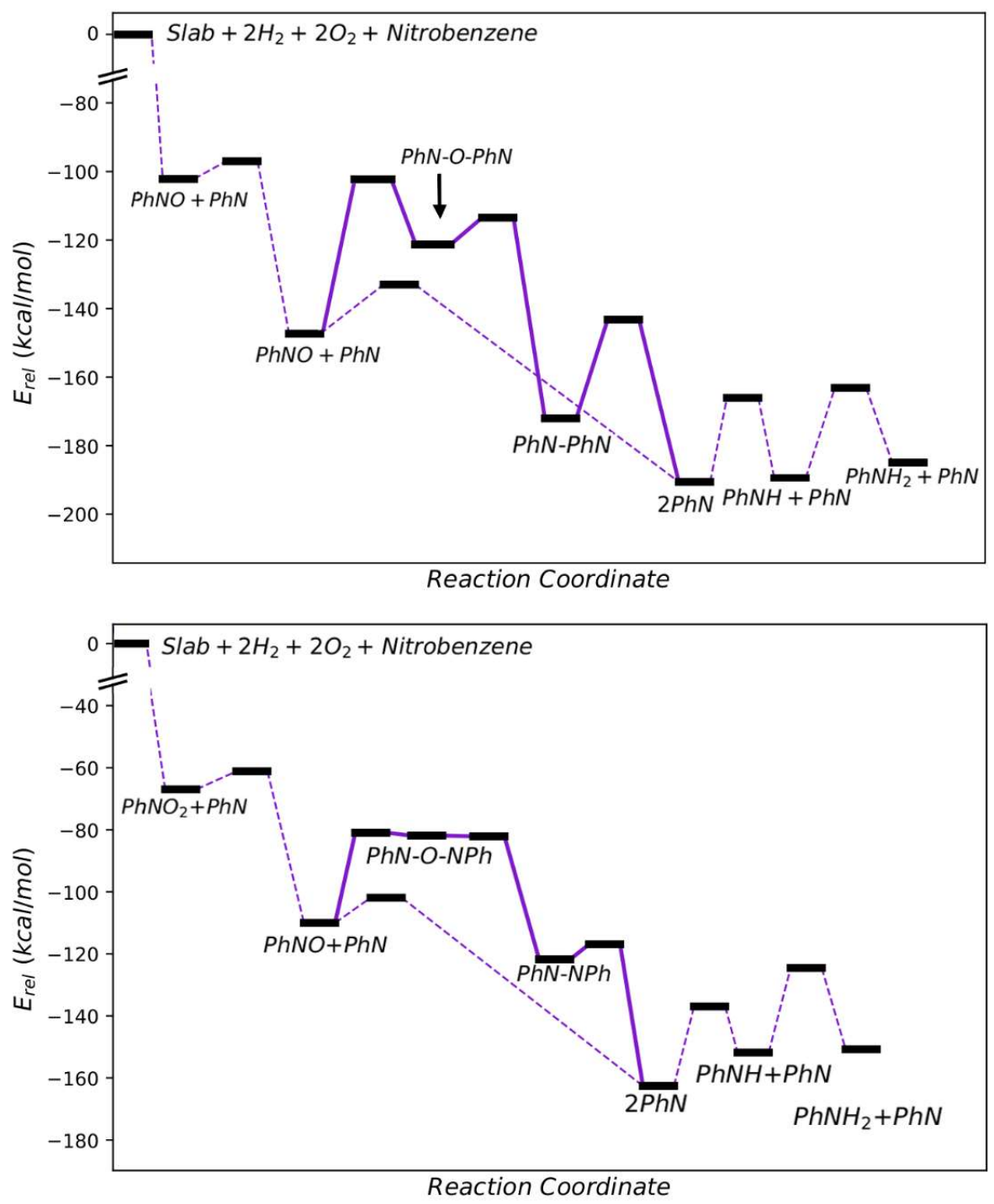

Figure 3.21 Calculated energy profile of steps 16, 17 and 18 (condensation) route on $\mathrm{Ni}(111)$ surface with parallel (top) and normal (bottom) geometries. The most favorable pathway found for the direct route is also shown for the sake of comparison. 


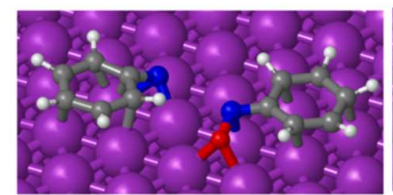

$\mathrm{phN}+\mathrm{phNO}$

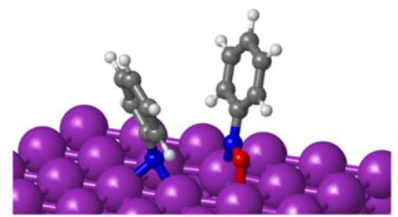

$\mathrm{phN}+\mathrm{phNO}$

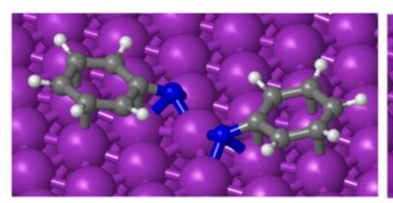

$\mathrm{phN}+\mathrm{phN}$

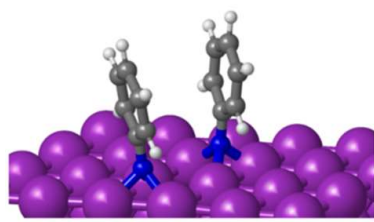

$\mathrm{phN}+\mathrm{phN}$

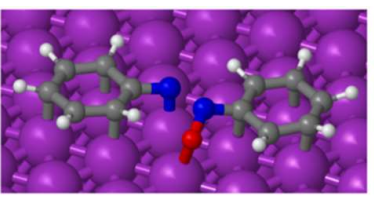

P-TS19

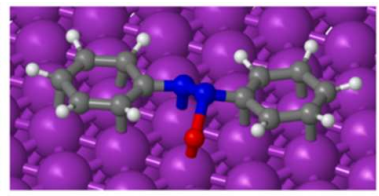

Azoxybenzene

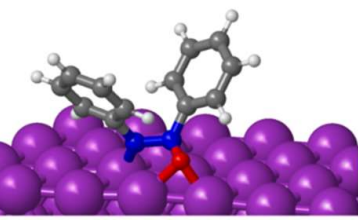

N-TS19

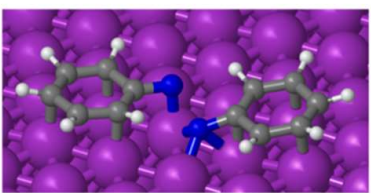

P-TS20

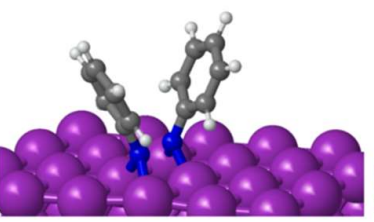

N-TS20

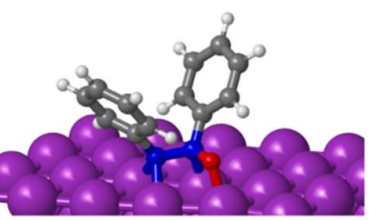

Azoxybenzene

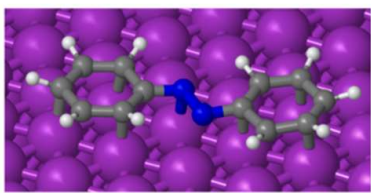

Azobenzene

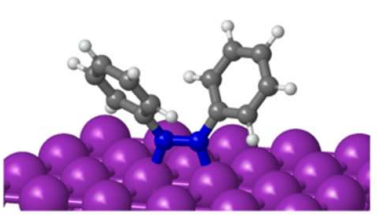

Azobenzene

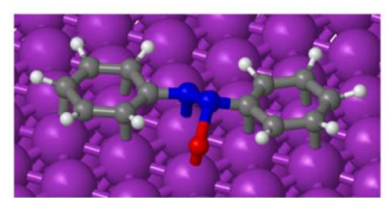

P-TS21

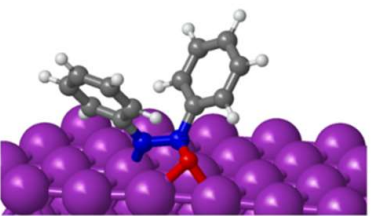

N-TS21

Figure 3.22 Optimized geometries of intermediates and transition states of the normal $(\mathrm{N})$ and parallel $(\mathrm{P})$ structures involved in the condensation route to form azoxy and azo compounds on $\mathrm{Co}(111)$ surface. $\mathrm{Co}, \mathrm{C}, \mathrm{N}, \mathrm{O}$ and $\mathrm{H}$ atoms are depicted as purple, gray, blue, red and white balls. 
High-energy penalties to bind two $\mathrm{N}$ atoms is not the only factor that blocks the indirect route. Another major problem, especially for the horizontally adsorbed species, is diffusion. Two nitroaromatic intermediates must meet each other on the surface with the right orientation. The simplest case for diffusion would be the normal Ph-N intermediate, since it is only bound to one hcp or fcc site through the $\mathrm{N}$ atom(monodentate). The diffusion barrier to hop to the next fcc site is $9.8 \mathrm{kcal} / \mathrm{mol}$ on $\mathrm{Ni}(111)$ and $7.31 \mathrm{kcal} / \mathrm{mol}$ on $\mathrm{Co}(111)$. That does not seem a too high barrier compared with that of hydrogen transfers but it is definitely slower than $\mathrm{H}$ diffusion. The latter is actually the fastest event of the whole reaction network. The calculated barrier is only $2.3 \mathrm{kcal} / \mathrm{mol}$ on $\mathrm{Ni}(111)$. For other multidentate intermediates diffusion is expected to be significantly slower or might not happen at all in the time scales of the reaction. The direct route is not supposed to be affected by the slow or even lack of diffusion of the multidentate species because, again, $\mathrm{H}$ diffusion is very fast. Hence, Ph-N would be hydrogenated with the abundant hydrogen atoms on the surface before it can encounter another Ph-N to bond to. Finally, the dissociation of the N-O bond of azoxybenzene also leads to the formation of azobenzene (step 21). We can conclude that the formation of N-N bonds and consequently the condensation route is very unlikely.

With these results, it is possible to classify all elementary steps in three groups. First, those involving $\mathrm{N}-\mathrm{O}$ bond breaking with the corresponding $\mathrm{M}-\mathrm{O}$ and $\mathrm{M}-\mathrm{N}$ bond formation. These steps require the lowest activation energies and are the most exothermic. Secondly, the hydrogen transfers, which involve M-H bond breaking and $\mathrm{N}-\mathrm{H}$ or $\mathrm{O}-\mathrm{H}$ bond formation. These steps require higher activation energies and are relatively thermo-neutral. Finally, those involving N-N bond formation. These steps have the highest activation energies and are very endothermic.

\subsection{Reduction of nitro group on $\mathrm{Cu}(111)$ and Pd (111)}

We have already seen that nitrostyrene does not adsorb parallel to the $\mathrm{Cu}(111)$ surface which, in addition, has a low ability to dissociate molecular $\mathrm{H}_{2}$. Consequently, only the normal orientation has been considered and only the most probable pathway, namely $\mathrm{Ph}-\mathrm{NO}_{2} \rightarrow \mathrm{Ph}-\mathrm{NO} \rightarrow \mathrm{Ph}-\mathrm{N} \rightarrow \mathrm{Ph}-\mathrm{NH} \rightarrow \mathrm{Ph}-\mathrm{NH}_{2}$ (steps 7, 8,10 and 13). For the sake of comparison and confirmation we have considered in our calculations this particular pathway on $\operatorname{Pd}(111)$ as well. On the one hand, we can compare this pathway on the four metals. On the other hand, Pd, as a noble metal, 
behaves differently from $\mathrm{Ni}$ and $\mathrm{Co}$ and hence we confirm its behavior with this pathway, which has not been considered before in the literature. We have also included the first $\mathrm{H}$-transfer to $\mathrm{Ph}-\mathrm{NO}_{2}$ (step 4). The reaction and activation energies are summarized in Table 3.12. The energy profiles of the four metals $(\mathrm{Ni}, \mathrm{Co}, \mathrm{Cu}$, Pd) are compared in Figure 3.23 and the optimized structures are shown in Figures 3.24 and 3.25 .

Table 3.12 Calculated reaction $\left(E_{\text {reac }}\right)$ and activation $\left(E_{\text {act }}\right)$ energies for all elementary steps of the reduction of nitrobenzene on $\mathrm{Cu}(111)$ and $\mathrm{Pd}(111)$ surfaces with normal geometries. All energies are given in $\mathrm{kcal} / \mathrm{mol}$.

\begin{tabular}{clcccc}
\hline \multirow{2}{*}{ step } & \multirow{2}{*}{ Reaction } & \multicolumn{2}{c}{$\mathrm{Cu}$} & \multicolumn{3}{c}{$\mathrm{Pd}$} \\
\cline { 3 - 6 } & & $\mathrm{E}_{\text {reac }}$ & $\mathrm{E}_{\text {act }}$ & $\mathrm{E}_{\text {reac }}$ & $\mathrm{E}_{\text {act }}$ \\
\hline 4 & $\mathrm{Ph}-\mathrm{NO}_{2}{ }^{*}+\mathrm{H}^{*} \leftrightarrow{\mathrm{Ph}-\mathrm{NOOH}^{*}}_{10}$ & 1.7 & 12.9 & 6.1 & 15.9 \\
11 & ${\mathrm{Ph}-\mathrm{N}^{*}+\mathrm{H}^{*} \leftrightarrow \mathrm{Ph}-\mathrm{NH}^{*}}$ & 0.8 & 19.8 & -14.5 & 17.0 \\
13 & $\mathrm{Ph}^{*}+\mathrm{NO}_{2}{ }^{*} \leftrightarrow \mathrm{H}^{*} \leftrightarrow \mathrm{Ph}-\mathrm{NH}_{2}{ }^{*}$ & -21.4 & 14.3 & -15.3 & 10.7 \\
16 & $\mathrm{Ph}^{*} \mathrm{NO}^{*} \leftrightarrow \mathrm{O}^{*}$ & -25.2 & 6.9 & 5.7 & 29.4 \\
\hline
\end{tabular}

When it comes to breaking the $\mathrm{N}-\mathrm{O}$ bond in $\mathrm{Ph}-\mathrm{NO}_{2}$ (step 13), $\mathrm{Cu}(111)$ behaves similar to $\mathrm{Ni}(111)$ and $\mathrm{Co}(111)$, exhibiting an equally low activation energy, 6.9 $\mathrm{kcal} / \mathrm{mol}$ and high exothermicity, $-25.2 \mathrm{kcal} / \mathrm{mol}$. On Pd(111), however, this step requires $29.4 \mathrm{kcal} / \mathrm{mol}$ to surpass the activation barrier. The same is true for the second $\mathrm{N}-\mathrm{O}$ cleavage, with an $\mathrm{E}_{\text {act }}$ of $7.5 \mathrm{kcal} / \mathrm{mol}$ on $\mathrm{Cu}(111)$, and of $30 \mathrm{kcal} / \mathrm{mol}$ on $\operatorname{Pd}(111)$. Therefore, the behaviour of $\mathrm{Cu}(111)$ as a non-noble metal is confirmed. That is, its affinity for $\mathrm{O}$ facilitates the dissociation of the $\mathrm{N}-\mathrm{O}$ bond of the nitro group as compared with the noble metals.

In contrast, the first hydrogen transfer to the nitro group has activation and reaction energies of 12.9 and $1.7 \mathrm{kcal} / \mathrm{mol}$, respectively. Interestingly, these values are similar to the activation and reaction energies obtained for the same step on $\operatorname{Pd}(111), 15.9$ and $6.1 \mathrm{kcal} / \mathrm{mol}$, and to the values reported on $\mathrm{Pt}(111)^{28}, 8$ and $1.4 \mathrm{kcal} / \mathrm{mol}$. Remember than on $\mathrm{Ni}(111)$ and $\mathrm{Co}(111)$ the $\mathrm{E}_{\text {act }}$ always lies in the range of $\sim 20-25$ $\mathrm{kcal} / \mathrm{mol}$. 
Inspection of the optimized geometries of $\mathrm{Ph}-\mathrm{NO}_{2}, \mathrm{~N}-\mathrm{TS} 4$ and $\mathrm{Ph}-\mathrm{NOOH}$ structures on $\mathrm{Cu}(111)$ shows that the aromatic ring is slightly bent toward the surface in the transition state and the product, as opposed to the reactant structure, $\mathrm{Ph}-\mathrm{NO}_{2}$. In the latter, the contribution of dispersion interactions (D3) is smaller by approximately 3.5 $\mathrm{kcal} / \mathrm{mol}$. Indeed, the activation and reaction energies of step 4 increases to 16.9 and $5.3 \mathrm{kcal} / \mathrm{mol}$ if only the electronic energy is taken into account.

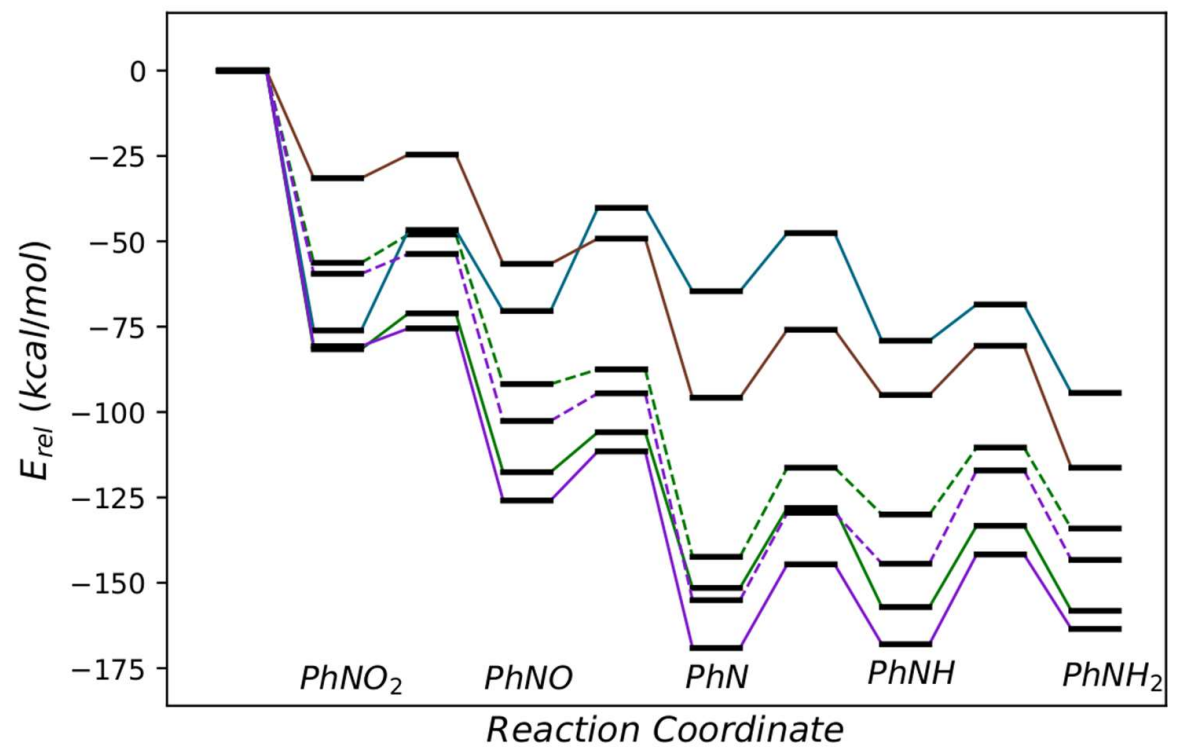

Figure 3.23 Calculated energy profile for the nitrobenzene hydrogenation through steps (9)(12) on $\mathrm{M}(111)$ surfaces, with $\mathrm{M}=\mathrm{Co}$ (purple), $\mathrm{Ni}$ (green), $\mathrm{Cu}$ (orange) and $\mathrm{Pd}$ (cyan). Full and dashed lines for $\mathrm{Co}(111)$ and $\mathrm{Ni}(111)$ correspond to parallel and normal pathways, respectively.

The subsequent hydrogenation steps to convert $\mathrm{Ph}-\mathrm{N}$ to aniline on $\mathrm{Cu}(111)$ are again more similar to $\mathrm{Pd}(111)$. Inspection of Table 3.12 shows that both activation energies are below $20 \mathrm{kcal} / \mathrm{mol}$. Step 10 is slightly endothermic but step 11 is clearly exothermic. Altogether, it seems clear that we can establish two extremes. On the one hand, noble metals have relatively high activation energies to break the N-O bonds and lower activation energies to form N-H and O-H bonds. On the other hand, non-noble metals have very low activation energies to break the $\mathrm{N}-\mathrm{O}$ bonds and 
relatively high activation energies to form $\mathrm{N}-\mathrm{H}$ and $\mathrm{O}-\mathrm{H}$ bonds. $\mathrm{Cu}(111)$ represents an intermediate situation between noble and non-noble metals. Even though the preferred pathway on $\mathrm{Cu}(111)$ seems to be the same as on $\mathrm{Ni}(111)$ and $\mathrm{Co}(111)$, the conversion of $\mathrm{Ph}-\mathrm{N}$ to aniline seems more favorable both kinetically and thermodynamically.

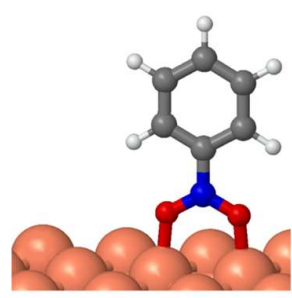

$\mathrm{Ph}-\mathrm{NO}_{2}$

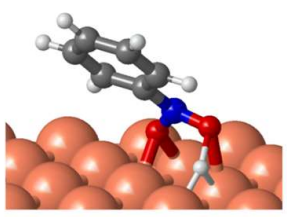

N-TS4

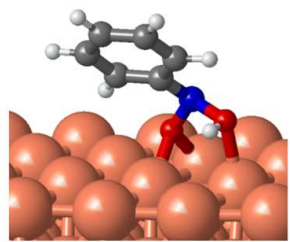

$\mathrm{Ph}-\mathrm{NOOH}$

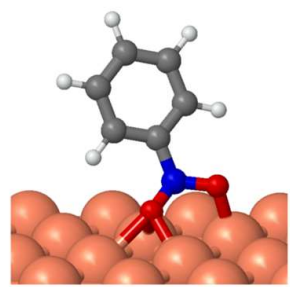

N-TS13

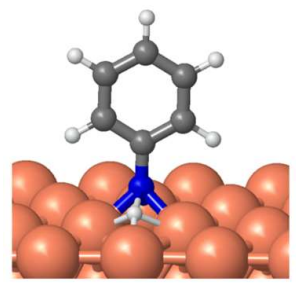

N-TS10

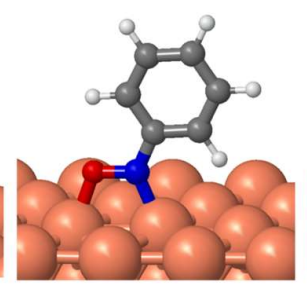

$\mathrm{Ph}-\mathrm{NO}_{2}$

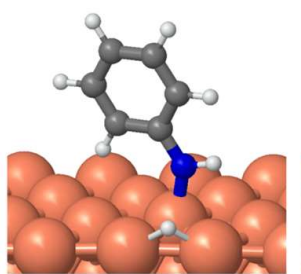

$\mathrm{Ph}-\mathrm{NH}$

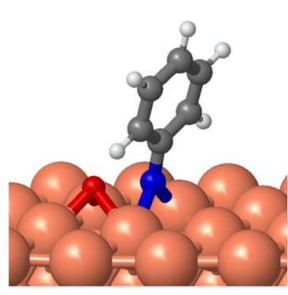

N-TS16

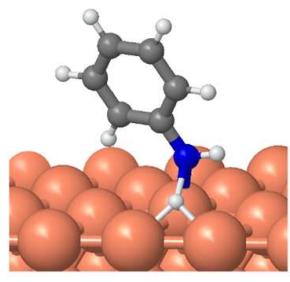

N-TS11

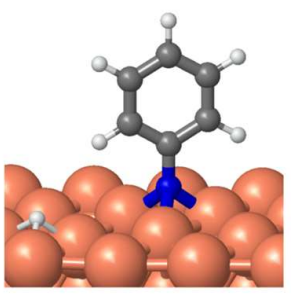

$\mathrm{Ph}-\mathrm{N}$

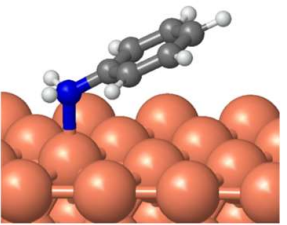

$\mathrm{Ph}-\mathrm{NH}_{2}$

Figure 3.24 Optimized geometries of structures involved in the mechanism of nitrobenzene hydrogenation on $\mathrm{Cu}(111)$ surface. $\mathrm{Cu}, \mathrm{C}, \mathrm{N}, \mathrm{O}$ and $\mathrm{H}$ atoms are depicted as orange, gray, blue, red and white balls. 


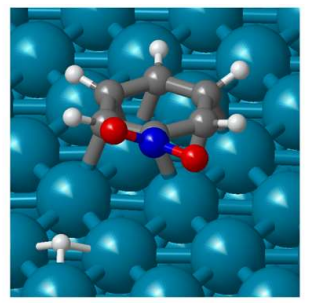

$\mathrm{Ph}-\mathrm{NO}_{2}$

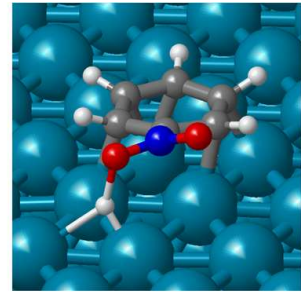

P-TS4

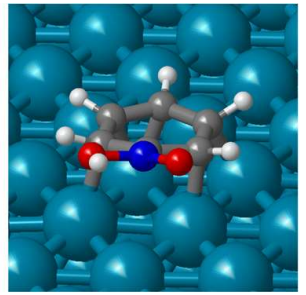

$\mathrm{Ph}-\mathrm{NOOH}$

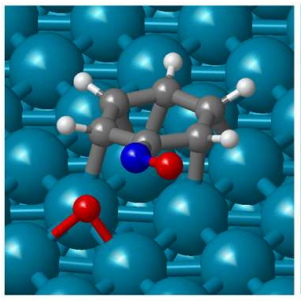

P-TS13

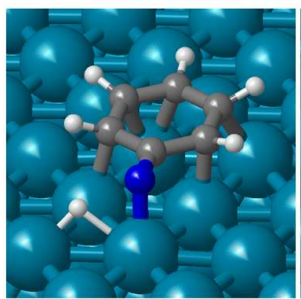

P-TS10

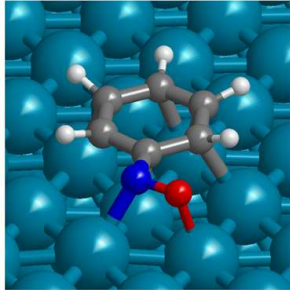

$\mathrm{Ph}-\mathrm{NO}$

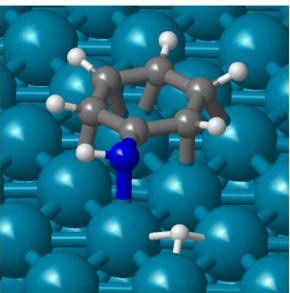

$\mathrm{Ph}-\mathrm{NH}$

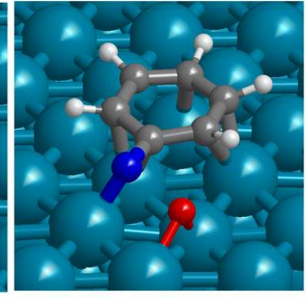

P-TS16

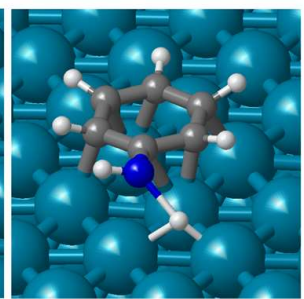

P-TS11

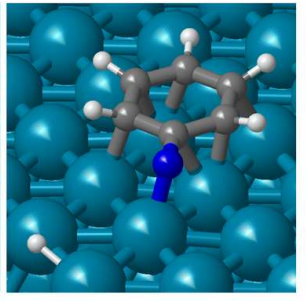

Ph-N

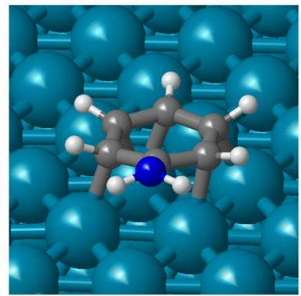

$\mathrm{Ph}-\mathrm{NH}_{2}$

Figure 3.25 Optimized geometries of structures involved in the mechanism of nitrobenzene hydrogenation on $\mathrm{Pd}(111)$ surface. $\mathrm{Pd}, \mathrm{C}, \mathrm{N}, \mathrm{O}$ and $\mathrm{H}$ atoms are depicted as cyan, gray, blue, red and white balls.

\subsection{Water formation}

The last step in the mechanism, necessary to recover the initial state of the metal and close the catalytic cycle, is the hydrogenation of the $\mathrm{O}$ atoms and $\mathrm{OH}$ groups that are released to the metal surface during the reaction. The following elementary steps:

$\mathrm{O}^{*}+\mathrm{H}^{*} \rightarrow \mathrm{OH}^{*}$

$\mathrm{OH}^{*}+\mathrm{H}^{*} \rightarrow \mathrm{H}_{2} \mathrm{O}^{*}$ 
have been now investigated on $\mathrm{Co}(111), \mathrm{Ni}(111), \mathrm{Cu}(111), \operatorname{Pd}(111)$ and $\operatorname{Pt}(111)$ surfaces. The optimized geometries of reactants, transition states and products involved are depicted in Figure 3.26 and the energy profiles are compared in Figure 3.27.

In all cases, $\mathrm{O}$ and $\mathrm{H}$ atoms are initially occupying three-fold hollow positions on the metal surface. In a first step, one $\mathrm{H}$ atom reacts with one $\mathrm{O}$ to form a hydroxyl group, with activation energies that slightly decrease from $23 \mathrm{kcal} / \mathrm{mol}$ on $\mathrm{Co}(111)$ and $\mathrm{Ni}(111)$ to $20 \mathrm{kcal} / \mathrm{mol}$ on $\mathrm{Cu}(111)$ and $\mathrm{Pd}(111)$, and to $18 \mathrm{kcal} / \mathrm{mol}$ on $\mathrm{Pt}(111)$. The optimized O-H bond lengths in TS22 are 1.30, 1.33 and $1.36 \AA$ in $\mathrm{Pd}(111), \mathrm{Co}(111)$ and $\mathrm{Ni}(111)$, respectively, while in $\mathrm{Cu}(111)$ and $\mathrm{Pt}(111)$ are longer, $1.55 \AA$ and 1.66 $\AA$ respectively. The stability of the resulting hydroxyl group and its location on the catalyst varies from one metal to another. Thus, the $\mathrm{OH}$ group is three-fold coordinated in $\mathrm{Co}(111), \mathrm{Ni}(111)$ and $\mathrm{Cu}(111)$, but moves to a bridge position in $\operatorname{Pd}(111)$ and is on top of just one metal atom in $\mathrm{Pt}(111)$. The reaction is slightly endothermic in $\mathrm{Co}(111)$ and $\mathrm{Ni}(111)$, and clearly exothermic on $\operatorname{Pt}(111)$ and especially on $\mathrm{Cu}(111)$. The second step, that is, formation of water by reaction of coadsorbed $\mathrm{H}$ and $\mathrm{OH}$, is thermodynamically favored and kinetically accessible on $\mathrm{Pt}(111)$ and $\mathrm{Pd}(111)$, and becomes much more difficult on the three non-noble metals considered, with activation energy barriers larger than $25 \mathrm{kcal} / \mathrm{mol}$ and endothermic reaction energies. The optimized geometries of the transition state TS23 structures are similar, with $\mathrm{O}-\mathrm{H}$ distances between 1.45 in $\mathrm{Cu}(111)$ and $1.69 \AA$ in $\mathrm{Pd}(111)$, and adsorbed water is always placed on top of a metal atom.

Regarding the implications of these results on the reaction mechanism, we must first consider that nitrobenzene hydrogenation on $\mathrm{Pd}(111)$ or $\mathrm{Pt}(111)$ only produces adsorbed hydroxyl groups that are easily removed via elementary step 23 . Therefore, clean or $\mathrm{H}$ covered surfaces can be expected for noble metal catalyzed reduction of nitroarenes. On the other hand, adsorbed oxygen atoms are obtained in large amounts on the three non-noble metals investigated, and the activation energies involved in their reaction with $\mathrm{H}$ to form water are at least as high as those obtained for hydrogenation of the $\mathrm{Ph}-\mathrm{N}$ intermediate to produce aniline. This means that the rate determining step of the global process is the removal of $\mathrm{Ph}-\mathrm{N}$ intermediates and adsorbed $\mathrm{O}$ atoms by reaction with $\mathrm{H}$, and consequently that the catalyst surface will be covered by $\mathrm{O}, \mathrm{OH}$ and $\mathrm{Ph}-\mathrm{N}$ species under reaction conditions, with the consequent implications on the geometry of adsorption of the nitroarene reactants. 


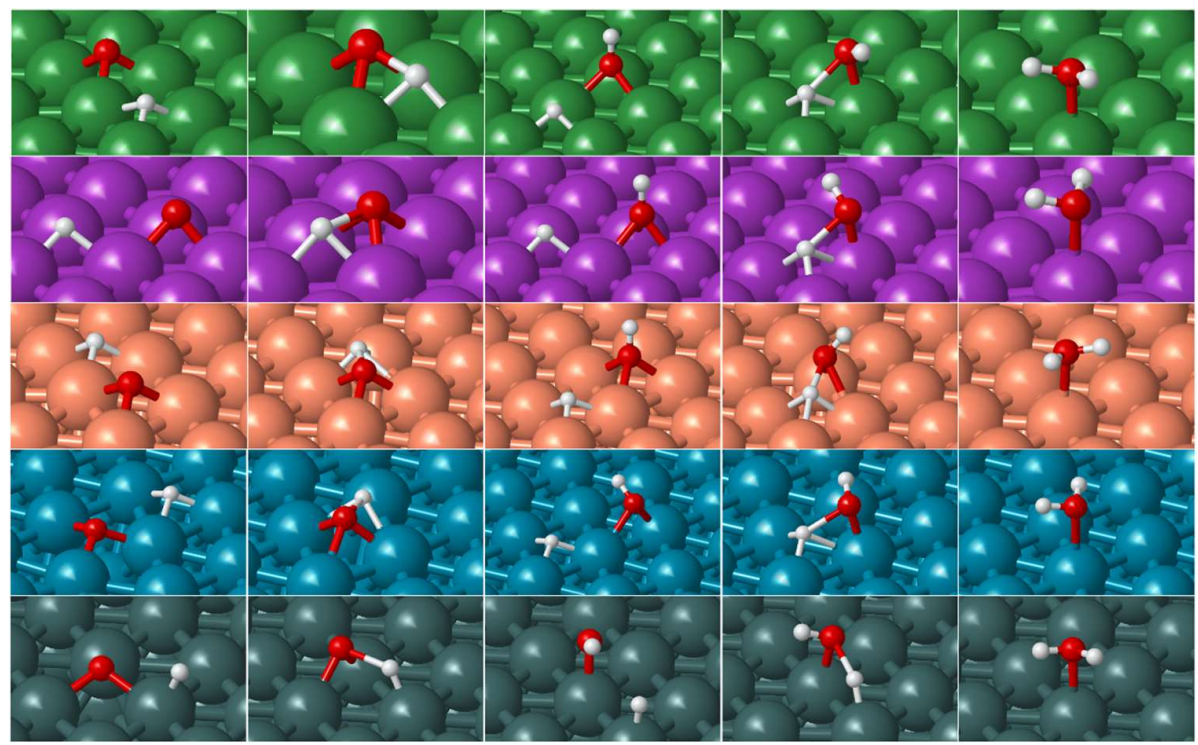

Figure 3.26 Optimized geometries of the structures involved in the formation of water from $\mathrm{O}$ and $\mathrm{H}$ atoms adsorbed on adsorbed on $\mathrm{M}(111)$ surfaces, with $\mathrm{M}=\mathrm{Co}$ (purple), Ni (green), $\mathrm{Cu}$ (orange), $\mathrm{Pd}$ (dark cyan), and Pt (dark green).

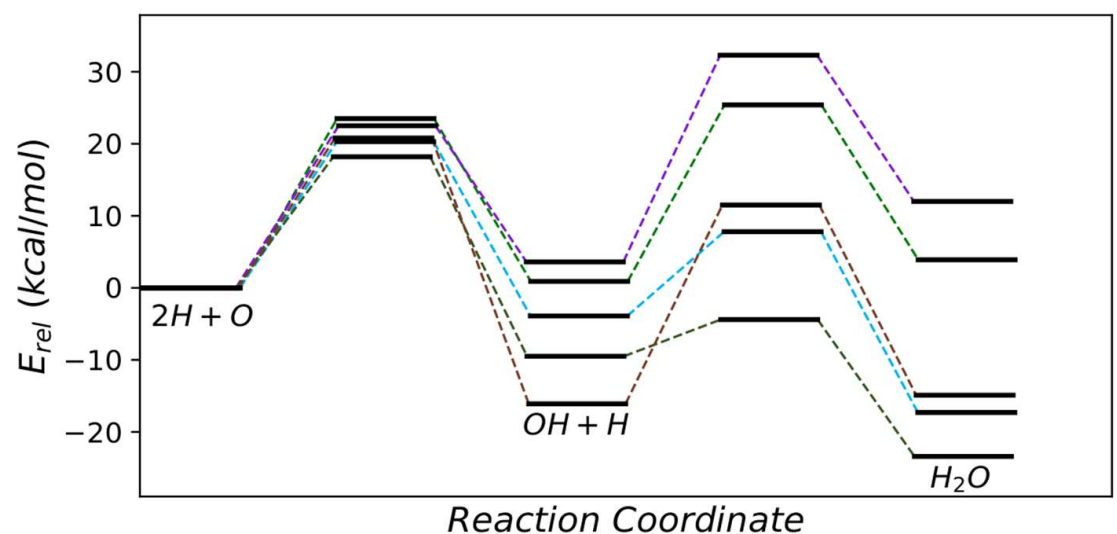

Figure 3.27. Calculated energy profile for the formation of water from $\mathrm{O}$ and $\mathrm{H}$ atoms adsorbed on $\mathrm{M}(111)$ surfaces, with $\mathrm{M}=\mathrm{Co}$ (purple), $\mathrm{Ni}$ (green), $\mathrm{Cu}$ (orange), $\mathrm{Pd}$ (dark cyan), and Pt (dark green). 


\subsection{Cu based bimetallic catalysts}

We have already seen that the slow hydrogenation of $\mathrm{O}, \mathrm{OH}$ and $\mathrm{Ph}-\mathrm{N}$ species will likely have a positive effect on the selectivity on $\mathrm{Ni}(111)$ and $\mathrm{Co}(111)$ because at high coverage nitrostyrene will be forced to adsorb perpendicular to the surface. Among the four metals $\mathrm{Cu}(111)$ is the best candidate to achieve nearly $100 \%$ of selectivity, because calculations suggest that there is no parallel chemisorption of nitrostyrene. However, high catalytic activity, which is another important factor when designing new catalysts, is achieved by lowering the activation energies of the most demanding steps.

In this regard, the relatively weak interaction of the $\mathrm{Cu}(111)$ surface (Figure 3.28) with atomic $\mathrm{H}$ constitutes a double-edge sword. On the one hand, it contributes to the lower activation energies of the hydrogenation of $\mathrm{Ph}-\mathrm{N}$ intermediate to produce aniline, steps (11) and (12), which should be reflected in a faster reaction rate. On the other hand, it contributes to the significantly high activation energies in the chemisorption of $\mathrm{H}_{2}$. This poor $\mathrm{H}_{2}$ activation, combined with the fact that $\mathrm{O}$ and $\mathrm{OH}$ removal is not particularly faster compared with $\mathrm{Ni}(111)$ and $\mathrm{Co}(111)$, suggests that the reaction will result in a rapid catalyst deactivation.

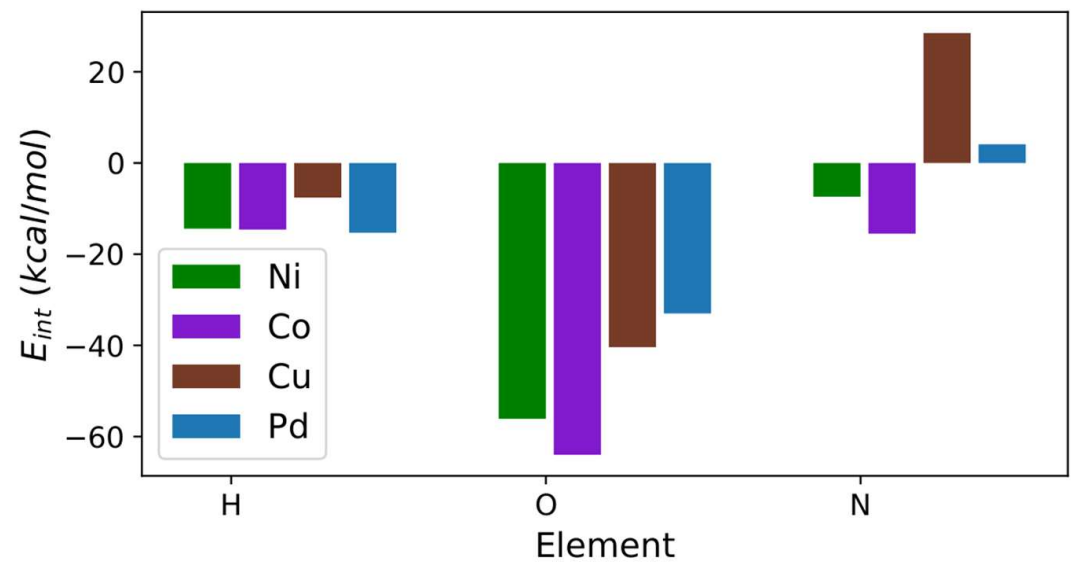

Figure 3.28. Comparison of a) interaction energies of $\mathrm{H}, \mathrm{O}$ and $\mathrm{N}$ atoms with $\mathrm{M}(111)$ surfaces. Values for $\mathrm{Co}, \mathrm{Ni}, \mathrm{Cu}$ and $\mathrm{Pd}$ are shown in purple, green, orange and cyan.

Figure 3.28 shows that among the four metals $\mathrm{Cu}(111)$ has the weakest interaction with $\mathrm{N}$ and with $\mathrm{H}$. The interaction with $\mathrm{O}$ follows the expected trend, $\mathrm{Co}>\mathrm{Ni}>\mathrm{Cu}$ 
$>$ Pd. Finally, the diffusion of atomic $\mathrm{H}$ on $\mathrm{Cu}(111)$ is as fast as on $\mathrm{Ni}(111)$ and $\mathrm{Co}(111)$. Taking all these factors into account, we can imagine that if somehow we are able to dissociate $\mathrm{H}_{2}$ on $\mathrm{Cu}(111)$ higher activities and selectivities than on $\mathrm{Ni}(111)$ and $\mathrm{Co}(111)$ should be obtained. One way to achieve this is by doping the $\mathrm{Cu}(111)$ surface with small amounts of $\mathrm{Ni}$. The activation of $\mathrm{H}_{2}$ could occur on the Ni sites while the rest of hydrogenation steps would proceed with activation energies similar to those calculated for $\mathrm{Cu}(111)$. The only problem is that the presence of $\mathrm{Ni}$ might increase the activation energies of the H-transfer steps. Figure 3.29 shows the calculated energy profile of the hydrogenation of nitrobenzene on the $\mathrm{Cu}(111)$ doped with one $\mathrm{Ni}$ atom. The reaction and activation energies are summarized in Table 3.13. It is clear from these values that doping the surface with one Ni atom does not change the kinetic parameters compared with those obtained without $\mathrm{Ni}$. The optimized minimum and transition states geometries are shown in Figure 3.30. In conclusion, bimetallic $\mathrm{Cu}-\mathrm{Ni}$ catalysts with small amounts of $\mathrm{Ni}$ are expected to attain higher selectivities and activities than monometalic $\mathrm{Ni}(111)$ or $\mathrm{Co}(111)$ based catalysts.

Table 3.13 Calculated reaction $\left(E_{\text {reac }}\right)$ and activation $\left(E_{\text {act }}\right)$ energies, for all elementary steps of the reduction of nitrobenzene with normal geometries on $\mathrm{Cu}(111)$ surface doped with one $\mathrm{Ni}$ atom. All energies are given in $\mathrm{kcal} / \mathrm{mol}$.

\begin{tabular}{clcc}
\hline step & Reaction & $\mathrm{E}_{\text {reac }}$ & $\mathrm{E}_{\text {act }}$ \\
\hline 7 & $\mathrm{Ph}-\mathrm{N}^{*}+\mathrm{H}^{*} \leftrightarrow \mathrm{Ph}-\mathrm{NH}^{*}$ & -5.1 & 15.9 \\
8 & $\mathrm{Ph}-\mathrm{NH}^{*}+\mathrm{H}^{*} \leftrightarrow \mathrm{Ph}-\mathrm{NH}_{2}{ }^{*}$ & -12.5 & 17.6 \\
10 & $\mathrm{Ph}_{-} \mathrm{NO}_{2}{ }^{*} \leftrightarrow \mathrm{Ph}-\mathrm{NO}^{*}+\mathrm{O}^{*}$ & -24.7 & 5.8 \\
13 & $\mathrm{Ph}^{*} \mathrm{NO}^{*} \leftrightarrow \mathrm{Ph}-\mathrm{N}^{*}+\mathrm{O}^{*}$ & -31.1 & 10.3 \\
\hline
\end{tabular}




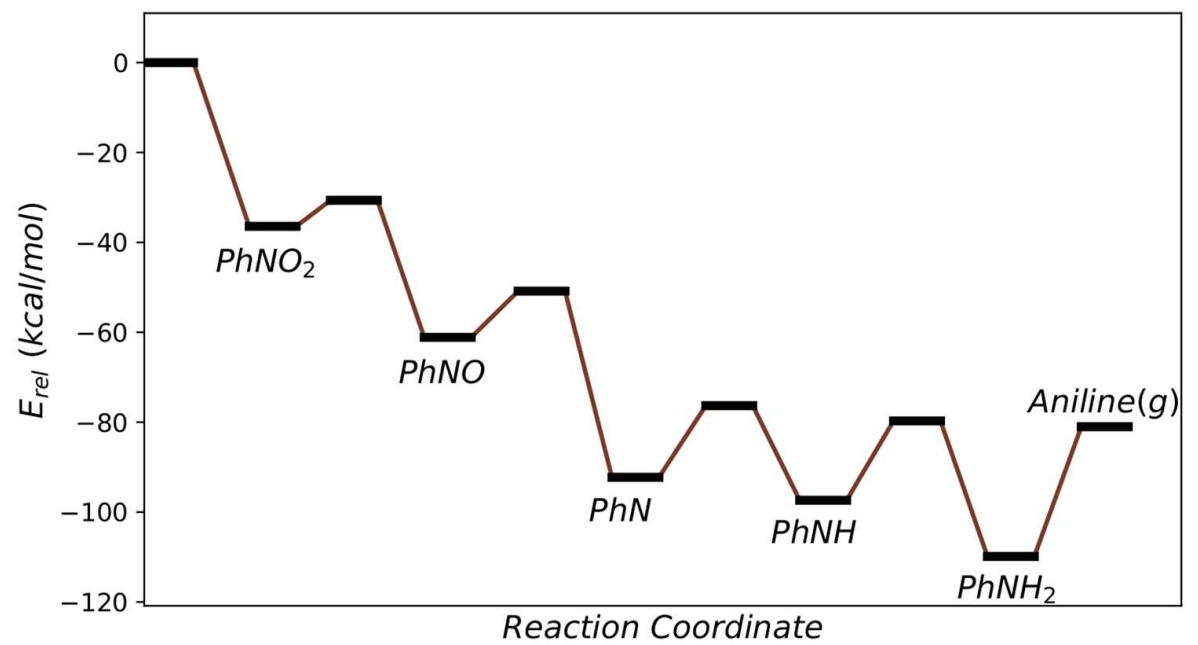

Figure 3.29 Calculated energy profile for the normal nitrobenzene hydrogenation through steps $9,8,10,13$ on $\mathrm{Cu}(111)$ surface doped with one $\mathrm{Ni}$ atom on the uppermost layer. 


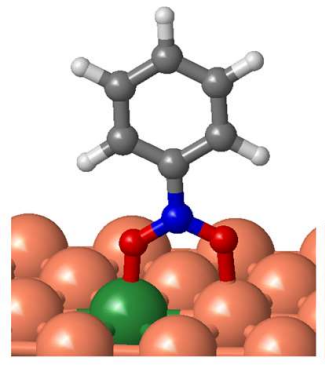

$\mathrm{Ph}-\mathrm{NO}_{2}$

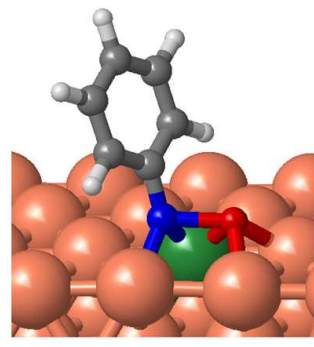

N-TS16

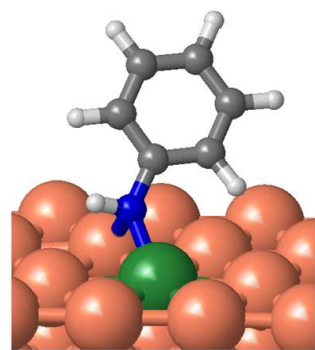

$\mathrm{Ph}-\mathrm{NH}$

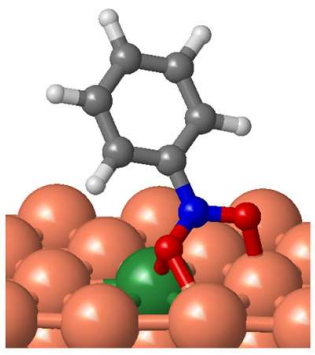

N-TS13

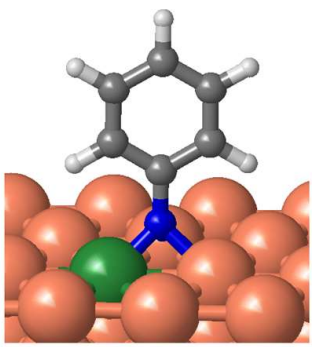

$\mathrm{Ph}-\mathrm{N}$

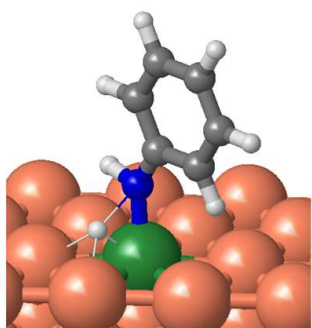

N-TS11

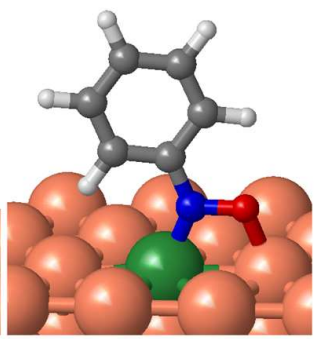

$\mathrm{Ph}-\mathrm{NO}$

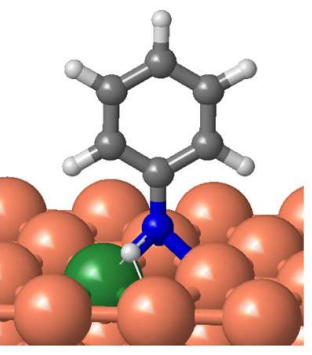

N-TS10

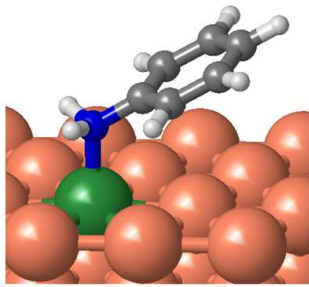

$\mathrm{Ph}-\mathrm{NH}_{2}$

Figure 3.30 Optimized geometries of structures involved in the mechanism of nitrobenzene hydrogenation on $\mathrm{Cu}(111)$ surface. $\mathrm{Cu}, \mathrm{C}, \mathrm{N}, \mathrm{O}$ and $\mathrm{H}$ atoms are depicted as orange, gray, blue, red and white balls. 


\subsection{Conclusions}

We have studied the reduction of nitrostyrene on different noble and non-noble metal (111) surfaces from which we can conclude that:

1) $\mathrm{Ni}(111)$ and $\mathrm{Co}(111)$ activate $\mathrm{H}_{2}$ very effectively while $\mathrm{Cu}(111)$ performs worse, especially at high coverage, and might not catalyze hydrogenation reactions at all.

2) The oxophylic character of $\mathrm{Ni}(111), \mathrm{Co}(111)$ and $\mathrm{Cu}(111)$ facilitates the breaking of $\mathrm{N}-\mathrm{O}$ bonds, due to the stabilizing effect on the transition states and products of the metal-O bond formed.

3) $\mathrm{H}$ transfers to either $\mathrm{O}$ or $\mathrm{N}$ atoms of the $-\mathrm{NO}_{2}$ group are significantly less favorable both kinetically and thermodynamically than $\mathrm{N}-\mathrm{O}$ bond breaking on $\mathrm{Ni}(111)$ and $\mathrm{Co}(111)$.

4) The significant difference between $\mathrm{H}$-transfer and $\mathrm{N}-\mathrm{O}$ bond breaking holds for $\mathrm{Ni}(111)$ and $\mathrm{Co}(111)$ independently of the adsorption geometry (normal or parallel) of the intermediates. Thus, it can be stated that the oxophylic nature of the metal determines the most favorable reaction pathway, independently of how the nitroaromatic adsorbs.

5) The condensation route is characterized by significantly higher activation energies and unstable intermediates compared with the direct route. Thus, the former route is not expected to contribute to the chemoselective reduction of nitroaromatics on non-noble metals (111) surfaces.

6) $\mathrm{Cu}(111)$ behaves as an intermediate case between noble and non-noble metals. Part of the reason why $\mathrm{H}$-transfers are more favorable kinetically than on $\mathrm{Ni}(111)$ and $\mathrm{Co}(111)$ is because the $\mathrm{Cu}-\mathrm{H}$ interaction is also less favorable than on $\mathrm{Ni}(111)$ and $\mathrm{Co}(111)$.

7) The most favorable pathway on non-noble metals consists of two N-O bond breaking steps followed by two H-transfers: $\mathrm{Ph}-\mathrm{NO}_{2} \rightarrow \mathrm{Ph}-\mathrm{NO} \rightarrow \mathrm{Ph}-\mathrm{N} \rightarrow$ 
$\mathrm{PhNH} \rightarrow \mathrm{PhNH}_{2}$. However, due to the complexity of the reaction network the preferred pathway might be dependent on the pressure of $\mathrm{H}_{2}$. On the one hand, high hydrogen pressures will force the surface to be highly covered with atomic $\mathrm{H}$ and due to lack of empty sites the $\mathrm{N}-\mathrm{O}$ breaking steps might be hindered and $\mathrm{H}$-transfer steps might become dominant. On the other hand, low $\mathrm{H}_{2}$ pressures will favor the $\mathrm{N}-\mathrm{O}$ bond breaking steps but the global reaction rate will drop. In any case, there will probably be a greater percentage of nitrostyrene molecules oriented normal to the surface at all coverages. This makes non-noble metals intrinsically more selective than noble metals like $\mathrm{Pd}(111)$ and $\mathrm{Pt}(111)$ where the molecule will have a greater tendency to adsorb parallel due to the better interaction of the aromatic ring compared with the weaker interaction of the nitro group with the surface.

8) $\mathrm{Cu}(111)$ exhibits two great advantages: 1) the hydrogenations steps to form aniline and remove the surface $\mathrm{O}$ and $\mathrm{OH}$ are less demanding than on $\mathrm{Ni}(111)$ and $\mathrm{Co}(111)$, so the global reaction is expected to be faster, 2) the parallel adsorption is not favorable, so the $\mathrm{Cu}(111)$ surface is expected to be intrinsically selective. Nonetheless, the great disadvantage is that $\mathrm{H}_{2}$ is not activated with which the reaction will not take place at all. Therefore, we propose a bimetallic catalyst based on $\mathrm{Cu}(111)$ with small amounts of $\mathrm{H}$ activating metal like $\mathrm{Ni}$. 


\subsection{References}

(1) Blaser, H.-U.; Steiner, H.; Studer, M. Selective Catalytic Hydrogenation of Functionalized Nitroarenes: An Update. ChemCatChem 2009, 1 (2), 210221. https://doi.org/10.1002/cctc.200900129.

(2) Tafesh, A. M.; Weiguny, J. A Review of the Selective Catalytic Reduction of Aromatic Nitro Compounds into Aromatic Amines, Isocyanates, Carbamates, and Ureas Using CO. Chem. Rev. 1996, 96 (6), 2035-2052. https://doi.org/10.1021/cr950083f.

(3) Takasaki, M.; Motoyama, Y.; Higashi, K.; Yoon, S.-H.; Mochida, I.; Nagashima, H. Chemoselective Hydrogenation of Nitroarenes with Carbon Nanofiber-Supported Platinum and Palladium Nanoparticles. Org. Lett. 2008, 10 (8), 1601-1604. https://doi.org/10.1021/ol800277a.

(4) Blaser, H.-U.; Malan, C.; Pugin, B.; Spindler, F.; Steiner, H.; Studer, M. Selective Hydrogenation for Fine Chemicals: Recent Trends and New Developments. Adv. Synth. Catal. 2003, 345 (1-2), 103-151. https://doi.org/10.1002/adsc.200390000.

(5) Siegrist, U.; Baumeister, P.; Blaser, H. U.; Studer, M. The Selective Hydrogenation of Functionalized Nitroarenes: New Catalytic Systems; Herkes, F. E., Ed.; Marcel Dekker: New York, 1998; Vol. 75.

(6) Boymans, E.; Boland, S.; Witte, P. T.; Müller, C.; Vogt, D. Chemoselective Hydrogenation of Functionalized Nitroarenes Using Supported Mo Promoted Pt Nanoparticles. ChemCatChem 2013, 5 (2), 431-434. https://doi.org/10.1002/cctc.201200437.

(7) Corma, A.; Serna, P.; Concepción, P.; Calvino, J. J. Transforming Nonselective into Chemoselective Metal Catalysts for the Hydrogenation of Substituted Nitroaromatics. J. Am. Chem. Soc. 2008, 130 (27), 8748-8753. https://doi.org/10.1021/ja800959g.

(8) Corma, A.; Serna, P. Chemoselective Hydrogenation of Nitro Compounds with Supported Gold Catalysts. SCIENCE 2006, 313 (5785), 332-334. https://doi.org/10.1126/science.1128383.

(9) Serna, P.; Concepción, P.; Corma, A. Design of Highly Active and Chemoselective Bimetallic Gold-Platinum Hydrogenation Catalysts through Kinetic and Isotopic Studies. J. Catal. 2009, 265 (1), 19-25. https://doi.org/10.1016/j.jcat.2009.04.004.

(10) Serna, P.; Boronat, M.; Corma, A. Tuning the Behavior of Au and Pt Catalysts for the Chemoselective Hydrogenation of Nitroaromatic Compounds. Top. Catal. 2011, 54 (5), 439-446. https://doi.org/10.1007/s11244-011-9668-z.

(11) Wei, H.; Liu, X.; Wang, A.; Zhang, L.; Qiao, B.; Yang, X.; Huang, Y.; Miao, 
S.; Liu, J.; Zhang, T. FeO x -Supported Platinum Single-Atom and PseudoSingle-Atom Catalysts for Chemoselective Hydrogenation of Functionalized Nitroarenes. Nat. Commun. 2014, 5 (1), 5634. https://doi.org/10.1038/ncomms6634.

(12) Boronat, M.; Corma, A. Origin of the Different Activity and Selectivity toward Hydrogenation of Single Metal $\mathrm{Au}$ and $\mathrm{Pt}$ on $\mathrm{TiO} 2$ and Bimetallic $\mathrm{Au}-\mathrm{Pt} / \mathrm{TiO} 2$ Catalysts. Langmuir 2010, 26 (21), 16607-16614. https://doi.org/10.1021/la101752a.

(13) Boronat, M.; Concepción, P.; Corma, A.; González, S.; Illas, F.; Serna, P. A Molecular Mechanism for the Chemoselective Hydrogenation of Substituted Nitroaromatics with Nanoparticles of Gold on TiO2 Catalysts: A Cooperative Effect between Gold and the Support. J. Am. Chem. Soc. 2007, 129 (51), 16230-16237. https://doi.org/10.1021/ja076721g.

(14) Vilé, G.; Almora-Barrios, N.; López, N.; Pérez-Ramírez, J. Structure and Reactivity of Supported Hybrid Platinum Nanoparticles for the Flow Hydrogenation of Functionalized Nitroaromatics. ACS Catal. 2015, 5 (6), 3767-3778. https://doi.org/10.1021/acscatal.5b00885.

(15) Westerhaus, F. A.; Jagadeesh, R. V.; Wienhöfer, G.; Pohl, M.-M.; Radnik, J.; Surkus, A.-E.; Rabeah, J.; Junge, K.; Junge, H.; Nielsen, M.; Brückner, A.; Beller, M. Heterogenized Cobalt Oxide Catalysts for Nitroarene Reduction by Pyrolysis of Molecularly Defined Complexes. Nat. Chem. 2013, 5 (6), 537-543. https://doi.org/10.1038/nchem.1645.

(16) Jagadeesh, R. V.; Surkus, A.-E.; Junge, H.; Pohl, M.-M.; Radnik, J.; Rabeah, J.; Huan, H.; Schünemann, V.; Brückner, A.; Beller, M. Nanoscale Fe2O3Based Catalysts for Selective Hydrogenation of Nitroarenes to Anilines. Science 2013, 342 (6162), 1073-1076. https://doi.org/10.1126/science.1242005.

(17) Wei, Z.; Wang, J.; Mao, S.; Su, D.; Jin, H.; Wang, Y.; Xu, F.; Li, H.; Wang, Y. In Situ-Generated Co0-Co3O4/N-Doped Carbon Nanotubes Hybrids as Efficient and Chemoselective Catalysts for Hydrogenation of Nitroarenes. $\begin{array}{lllll}\text { ACS Catal. } & \text { 2015, } & 5 & \text { (8), } & \text { 4783-4789. }\end{array}$ https://doi.org/10.1021/acscatal.5b00737.

(18) Beswick, O.; Parastaev, A.; Yuranov, I.; LaGrange, T.; Dyson, P. J.; KiwiMinsker, L. Highly Dispersed Cobalt Oxides Nanoparticles on Activated Carbon Fibres as Efficient Structured Catalysts for the Transfer Hydrogenation of M-Nitrostyrene. Catal. Today 2017, 279, 29-35. https://doi.org/10.1016/j.cattod.2016.06.043.

(19) Liu, L.; Concepción, P.; Corma, A. Non-Noble Metal Catalysts for Hydrogenation: A Facile Method for Preparing Co Nanoparticles Covered with Thin Layered Carbon. J. Catal. 2016, 340, $1-9$. 
https://doi.org/http://dx.doi.org/10.1016/j.jcat.2016.04.006.

(20) Ren, Y.; Wei, H.; Yin, G.; Zhang, L.; Wang, A.; Zhang, T. Oxygen Surface Groups of Activated Carbon Steer the Chemoselective Hydrogenation of Substituted Nitroarenes over Nickel Nanoparticles. Chem Commun 2017, 53 (12), 1969-1972. https://doi.org/10.1039/C6CC08505A.

(21) Liu, L.; Gao, F.; Concepción, P.; Corma, A. A New Strategy to Transform Mono and Bimetallic Non-Noble Metal Nanoparticles into Highly Active and Chemoselective Hydrogenation Catalysts. J. Catal. 2017, 350, 218 - 225. https://doi.org/http://dx.doi.org/10.1016/j.jcat.2017.03.014.

(22) Zhang, J.; Lu, G.; Cai, C. Chemoselective Transfer Hydrogenation of Nitroarenes by Highly Dispersed Ni-Co BMNPs. Catal. Commun. 2016, 84, 25 - 29. https://doi.org/http://dx.doi.org/10.1016/j.catcom.2016.05.023.

(23) Petkar, D. R.; Kadu, B. S.; Chikate, R. C. Highly Efficient and Chemoselective Transfer Hydrogenation of Nitroarenes at Room Temperature over Magnetically Separable Fe-Ni Bimetallic Nanoparticles. RSC Adv 2014, 4 (16), 8004-8010. https://doi.org/10.1039/C3RA45787G.

(24) Hahn, G.; Ewert, J.-K.; Denner, C.; Tilgner, D.; Kempe, R. A Reusable Mesoporous Nickel Nanocomposite Catalyst for the Selective Hydrogenation of Nitroarenes in the Presence of Sensitive Functional Groups. ChemCatChem 2016, 8 (15), 2461-2465. https://doi.org/10.1002/cctc.201600391.

(25) Haber, F. Gradual Electrolytic Reduction of Nitrobenzene with Limited Cathode Potential. Elektrochem 1898, 4, 506.

(26) A. Gelder, E.; David Jackson, S.; Martin Lok, C. The Hydrogenation of Nitrobenzene to Aniline : A New Mechanism. Chem. Commun. 2005, 0 (4), 522-524. https://doi.org/10.1039/B411603H.

(27) Corma, A.; Concepción, P.; Serna, P. A Different Reaction Pathway for the Reduction of Aromatic Nitro Compounds on Gold Catalysts. Angew. Chem. Int. Ed. 2007, 46 (38), 7266-7269. https://doi.org/10.1002/anie.200700823.

(28) Sheng, T.; Qi, Y.-J.; Lin, X.; Hu, P.; Sun, S.-G.; Lin, W.-F. Insights into the Mechanism of Nitrobenzene Reduction to Aniline over Pt Catalyst and the Significance of the Adsorption of Phenyl Group on Kinetics. Chem. Eng. J. 2016, 293, 337-344. https://doi.org/10.1016/j.cej.2016.02.066.

(29) Zhang, L.; Jiang, J.; Shi, W.; Xia, S.; Ni, Z.; Xiao, X. Insights into the Hydrogenation Mechanism of Nitrobenzene to Aniline on $\mathrm{Pd} 3 / \mathrm{Pt}(111)$ : A Density Functional Theory Study. RSC Adv. 2015, 5 (43), 34319-34326. https://doi.org/10.1039/C5RA02389K.

(30) Perdew, J. P.; Wang, Y. Accurate and Simple Analytic Representation of the Electron-Gas Correlation Energy. Phys. Rev. B 1992, 45 (23), 13244-13249. https://doi.org/10.1103/PhysRevB.45.13244. 
(31) Perdew, J. P.; Chevary, J. A.; Vosko, S. H.; Jackson, K. A.; Pederson, M. R.; Singh, D. J.; Fiolhais, C. Atoms, Molecules, Solids, and Surfaces: Applications of the Generalized Gradient Approximation for Exchange and Correlation. Phys. Rev. B 1992, 46 (11), 6671-6687. https://doi.org/10.1103/PhysRevB.46.6671.

(32) Kresse, G.; Furthmüller, J. Efficient Iterative Schemes for Ab Initio TotalEnergy Calculations Using a Plane-Wave Basis Set. Phys. Rev. B 1996, 54 (16), 11169-11186. https://doi.org/10.1103/PhysRevB.54.11169.

(33) Blöchl, P. E. Projector Augmented-Wave Method. Phys. Rev. B 1994, 50 (24), 17953-17979. https://doi.org/10.1103/PhysRevB.50.17953.

(34) Henkelman, G.; Jónsson, H. A Dimer Method for Finding Saddle Points on High Dimensional Potential Surfaces Using Only First Derivatives. J. Chem. Phys. 1999, 111 (15), 7010-7022. https://doi.org/10.1063/1.480097.

(35) Heyden, A.; Bell, A. T.; Keil, F. J. Efficient Methods for Finding Transition States in Chemical Reactions: Comparison of Improved Dimer Method and Partitioned Rational Function Optimization Method. J. Chem. Phys. 2005, 123 (22), 224101. https://doi.org/10.1063/1.2104507.

(36) Grimme, S. Semiempirical GGA-Type Density Functional Constructed with a Long-Range Dispersion Correction. J. Comput. Chem. 2006, 27 (15), 17871799. https://doi.org/10.1002/jcc.20495.

(37) Goerigk, L.; Grimme, S. A Thorough Benchmark of Density Functional Methods for General Main Group Thermochemistry, Kinetics, and Noncovalent Interactions. Phys. Chem. Chem. Phys. 2011, 13 (14), 66706688. https://doi.org/10.1039/C0CP02984J.

(38) Grimme, S.; Ehrlich, S.; Goerigk, L. Effect of the Damping Function in Dispersion Corrected Density Functional Theory. J. Comput. Chem. 2011, 32 (7), 1456-1465. https://doi.org/10.1002/jcc.21759.

(39) Davey, W. P. Precision Measurements of the Lattice Constants of Twelve Common Metals. Phys. Rev. 1925, 25 (6), 753-761. https://doi.org/10.1103/PhysRev.25.753.

(40) Aschroft, N. W.; Mermin, N. D. Solid State Physics; Holt, Rinehart and Winston: New York, 1976.

(41) van Helden, P.; van den Berg, J.-A.; Weststrate, C. J. Hydrogen Adsorption on Co Surfaces: A Density Functional Theory and Temperature Programmed Desorption Study. ACS Catal. 2012, 2 (6), 1097-1107. https://doi.org/10.1021/cs2006586.

(42) Saerens, S.; Sabbe, M. K.; Galvita, V. V.; Redekop, E. A.; Reyniers, M.-F.; Marin, G. B. The Positive Role of Hydrogen on the Dehydrogenation of Propane on $\operatorname{Pt}(111)$. ACS Catal. 2017, 7 (11), 7495-7508. https://doi.org/10.1021/acscatal.7b01584. 
(43) Chizallet, C.; Bonnard, G.; Krebs, E.; Bisson, L.; Thomazeau, C.; Raybaud, P. Thermodynamic Stability of Buta-1,3-Diene and But-1-Ene on Pd(111) and (100) Surfaces under H2 Pressure: A DFT Study. J. Phys. Chem. C 2011, 115 (24), 12135-12149. https://doi.org/10.1021/jp202811t.

(44) Corvaisier, F.; Schuurman, Y.; Fecant, A.; Thomazeau, C.; Raybaud, P.; Toulhoat, H.; Farrusseng, D. Periodic Trends in the Selective Hydrogenation of Styrene over Silica Supported Metal Catalysts. J. Catal. 2013, 307, 352361. https://doi.org/10.1016/j.jcat.2013.08.009.

(45) Rai, R. K.; Mahata, A.; Mukhopadhyay, S.; Gupta, S.; Li, P.-Z.; Nguyen, K. T.; Zhao, Y.; Pathak, B.; Singh, S. K. Room-Temperature Chemoselective Reduction of Nitro Groups Using Non-Noble Metal Nanocatalysts in Water. Inorg. Chem. 2014, 53 (6), 2904-2909. https://doi.org/10.1021/ic402674z. 


\section{Chapter 4}

\section{Insights on the $\mathrm{NH}_{3}-\mathrm{SCR}-\mathrm{NOx}$ reaction with $\mathrm{Cu}-\mathrm{CHA}$ catalysts using static DFT methods}

\subsection{Introduction}

Nitrogen oxides (NOx) are considered one of the main air pollutants responsible for acid rain and photochemical $\operatorname{smog}^{1,2}$. They are released mainly from stationary power plants and vehicle exhaust. Because $\mathrm{NO}_{2}$ is a traffic-related pollutant, its concentration can reach mean values of 10-45 ppb with peaks as high as $200 \mathrm{ppb}$ in urban areas. When they are present in such high concentrations they can cause serious problems in the environment and in human health, such as lung damage, shortness of breath and aggravation of the symptoms of respiratory diseases. Consequently, the environmental legislation has become more and more stringent, resulting in an increased interest in the development of new technologies to abate the NOx emissions. One of the most successful technologies to cope with this problem is the selective catalytic reduction (SCR) of NOx. It was first patented in the U.S. by Engelhard Corporation in $1957^{1}$ and it made reference to the reduction of NOx using $\mathrm{NH}_{3}\left(\mathrm{NH}_{3}-\mathrm{SCR}\right)$ as reducing agent ${ }^{3-7}$. Nowadays, it also indicates the use of hydrocarbons, HC (HC-SRC) $)^{8-14}$ or urea (SCR-urea) as reducing agent ${ }^{15,16}$.

The $\mathrm{NH}_{3}-\mathrm{SCR}$ consists in the selective reduction of $\mathrm{NOx}$ to $\mathrm{N}_{2}$ and $\mathrm{H}_{2} \mathrm{O}$ according to the following reactions:

$4 \mathrm{NO}+\mathrm{O}_{2}+4 \mathrm{NH}_{3} \rightarrow 4 \mathrm{~N}_{2}+6 \mathrm{H}_{2} \mathrm{O}$

$2 \mathrm{NO}+2 \mathrm{NO}_{2}+4 \mathrm{NH}_{3} \rightarrow 4 \mathrm{~N}_{2}+6 \mathrm{H}_{2} \mathrm{O}$ 
Reactions 1 and 2 are known as "standard $S C R$ " and "fast $S C R$ " respectively. Above $473 \mathrm{~K}$ and in the presence of equimolar quantities of $\mathrm{NO}$ and $\mathrm{NO}_{2}$, reaction 2 dominates the kinetics of the SCR process with reaction rates at least 10 times higher than the standard $\mathrm{SCR}^{1}$. Selectivity, in this context, is defined as the preferential reaction of the reducing agent with $\mathrm{NOx}$ and not with oxygen or other oxidizers present in the gas stream. For example, direct oxidation of $\mathrm{NH}_{3}$ with $\mathrm{O}_{2}$ according to reaction 3 is an undesired process. Reaction 3 is called selective catalytic oxidation of $\mathrm{NH}_{3}$.

$4 \mathrm{NH}_{3}+3 \mathrm{O}_{2} \rightarrow 2 \mathrm{~N}_{2}+6 \mathrm{H}_{2} \mathrm{O}$

The catalysts used in stationary power plants for the SCR are based mainly on $\mathrm{TiO}_{2}$ supported on $\mathrm{V}_{2} \mathrm{O}_{5}{ }^{2}$. However, the operating conditions of diesel-powered commercial vehicles make the application of these catalysts much more difficult. In this regard, the first breakthrough was the contribution from Iwamoto et al. ${ }^{17-19}$ in the mid-80s on $\mathrm{Cu}$-exchanged zeolites to directly convert $\mathrm{NO}$ into $\mathrm{N}_{2}$ and $\mathrm{O}_{2}$, and later in the selective catalytic reduction of $\mathrm{NOx}$ with $\mathrm{NH}_{3}$ or hydrocarbons (HC). The unique and stable activity for $\mathrm{NO}$ decomposition triggered the research over a variety of other zeolites, including the ZSM-5, Y and Beta zeolites, with promising activities for lean NOx reduction. Nonetheless, the insufficient long-term hydrothermal stabilities of these materials have delayed their application in mobile exhaust systems $^{20,21}$.

Recently, zeolites possessing the small-pore chabazite (CHA-type) structure, in particular Cu-SSZ-13 and Cu-SAPO-34, have been reported to exhibit both superior $\mathrm{NH}_{3}$-SCR activity, selectivity and hydrothermal stability ${ }^{22-28}$. Thanks to the extensive research carried out, some progress has been made in the understanding of the SCR process with $\mathrm{Cu}$-exchanged zeolites. It has been demonstrated that the redox pair $\mathrm{Cu}^{+} / \mathrm{Cu}^{2+}$ plays a key role in the SCR process ${ }^{29-31}$. In fact, the whole catalytic cycle can be split in an oxidation half cycle where $\mathrm{Cu}^{+}$is oxidized to $\mathrm{Cu}^{2+}$ and a reduction half cycle where $\mathrm{Cu}^{2+}$ is reduced back to $\mathrm{Cu}^{+}$. The SCR rate increases with the increase of the amount of exchanged $\mathrm{Cu}$ cations ${ }^{32}$. The oxidation of $\mathrm{NO}$ produces $\mathrm{NO}_{2}{ }^{-} / \mathrm{NO}_{3}{ }^{-}$species ${ }^{33,34}$ which in the presence of ammonia decompose into $\mathrm{H}_{2} \mathrm{O}$ and $\mathrm{N}_{2}{ }^{35}$. It is also known that both $\mathrm{NO}$ and $\mathrm{NH}_{3}$ are key for the reduction of $\mathrm{Cu}^{2+}$ to $\mathrm{Cu}^{+}$ 28,35-37. The reaction is of order 1 for $\mathrm{NO}$, order 0.5 for $\mathrm{O}_{2}$ and slightly negative for $\mathrm{NH}_{3}{ }^{20,38}$. However, the fact of the matter is that a consensus on the detailed catalytic 
mechanism that effectively accounts for the kinetics of the process has not been reached yet.

On the one hand, one important point of disagreement is the nature and location of the active sites. Some authors suggest that the active sites are isolated $\mathrm{Cu}$ ions in exchanged positions of the CHA framework. Two locations have been proposed for this mononuclear $\mathrm{Cu}$ species, namely the 6-membered $(6 r)$ and the 8-membered $(8 r)$ units $^{26,39-47}$ (see Figure 4.1). In addition, systems like $[\mathrm{Cu}-\mathrm{OH}]^{+}$, dimeric copper-oxo species $[\mathrm{Cu}-\mathrm{O}-\mathrm{Cu}]^{2+}$ and $\mathrm{CuOx}$ nanoclusters inside the channels or at the catalyst surface seem to be active for the SCR reaction as well ${ }^{39,48-52}$. Paolucci et al. ${ }^{44}$ identified two species: $\mathrm{Cu}^{2+}$ cations compensating $\mathrm{Al}$ pairs and $[\mathrm{Cu}-\mathrm{OH}]^{+}$ compensating an unpaired $\mathrm{Al}$ site, both sites exhibiting similar reactivities. $\mathrm{He}$ suggested that, below $473 \mathrm{~K}, \mathrm{NH}_{3}$ liberates copper from the framework masking the differences between the two $\mathrm{Cu}$ species. Lomachenko et al. ${ }^{29}$ concluded, on the basis of operando XANES, EXAFS, and vtc-XES, that the active sites at low temperature $(<500 \mathrm{~K})$ are mobile $\mathrm{Cu}^{+}\left(\mathrm{NH}_{3}\right)_{2}$ species but at high temperature $(>500 \mathrm{~K}) \mathrm{Cu}^{2+}$ ions are strongly coordinated to the framework oxygens accounting for more than $70 \%$ of total $\mathrm{Cu}$ sites. Most results indicate that the plane of the $6 r$ seems to be the most stable location for $\mathrm{Cu}^{+}$and $\mathrm{Cu}^{2+}$ cations. However, due to the wide range of synthesis conditions and post-synthesis treatments most catalysts have a mixture of all the aforementioned species ${ }^{49,53}$. That is, the nature of $\mathrm{Cu}$ species is highly dependent on the $\mathrm{Cu} / \mathrm{Al}$ and $\mathrm{Si} / \mathrm{Al}$ ratios, the distribution of framework $\mathrm{Al}$ sites (isolated or paired) and even on the reaction conditions.

On the other hand, the SCR mechanism does not seem to be clearer. The reduction half cycle is assumed to be fast. Most authors agree that $\mathrm{NO}$ and $\mathrm{NH}_{3}$ adsorb on $\mathrm{Cu}^{2+}$ and react to generate a Brønsted acid site and nitrosamine $\left(\mathrm{H}_{2} \mathrm{NNO}\right)^{35,37,54,55}$, which is easily decomposed into $\mathrm{N}_{2}$ and $\mathrm{H}_{2} \mathrm{O}$ through a push-pull mechanism ${ }^{54,56,57}$. As regards the oxidation half-cycle more effort has been made. Due to the higher rate of the SCR with equimolar quantities of $\mathrm{NO}$ and $\mathrm{NO}_{2}$, the $\mathrm{NO}$ oxidation with $\mathrm{O}_{2}$ has been considered as the rate-determining step of the mechanism ${ }^{58-61}$. Janssens et al. ${ }^{37}$ proposed that the reaction of $\mathrm{NO}$ with $\mathrm{O}_{2}$ on $\mathrm{Cu}^{+}$is the rate-determining step and produces $\mathrm{Cu}^{2+}-\mathrm{NO}_{3}{ }^{-}$, which later reacts with another $\mathrm{NO}$ to form $\mathrm{NO}_{2}$ and $\mathrm{Cu}^{2+}-\mathrm{NO}_{2}{ }^{-}$. In their scheme $\mathrm{NO}_{2}$ can also react directly with $\mathrm{Cu}^{+}$to form nitrites, which defines the fast SCR. They reported an apparent activation energy of $16.5 \mathrm{kcal} / \mathrm{mol}$ for the standard SCR. Other reports support nitrates as key intermediates in the SCR 
mechanism ${ }^{62}$. Conversely, Chen et al..$^{33}$ used isotope-exchange experiments to follow the oxidation state of $\mathrm{N}$ and concluded that nitrates are not formed in $\mathrm{Cu}-\mathrm{CHA}$ since the state of $\mathrm{N}$ changed from $2+$ to $3+$. Finally, it has also been proposed that the activation of the $\mathrm{O}_{2}$ at low temperature and low $\mathrm{Cu}$ content occurs through the formation of dimeric $\left(\mathrm{NH}_{3}\right)_{2}-\mathrm{Cu}-\mathrm{O}-\mathrm{O}-\mathrm{Cu}\left(\mathrm{NH}_{3}\right)_{2}$ species $^{32,63}$.

In the aforementioned studies IR spectroscopy has played a fundamental role because it is able to encode information about the chemical nature, interaction and geometry of the adsorbed species. Information about the surface adsorption sites (oxidation state, coordination, etc) are drawn indirectly by following the changes in the vibrational IR spectra of probe molecules adsorbed on those sites. Because of its small size, $\mathrm{NO}$ and $\mathrm{CO}$ are accessible to the surface sites, which makes them particularly useful as probe molecules. In addition, NO is very sensitive to the electronic state of the cation due to the unpaired electron in the antibonding $2 \pi$ orbital. For example, $\mathrm{NO}$ is known to be highly sensitive to $\mathrm{Cu}^{2+}$ sites $^{64-68}$ and $\mathrm{CO}$ has been used as probe molecule in IR to study the unsaturated $\mathrm{Cu}^{+}$species ${ }^{64,69,70}$. IR spectroscopy with $\mathrm{NO}$ as probe molecule has been employed to characterize samples of Cu-SSZ-13 and Cu-SAPO-34 synthesized in the Institute of Chemical Technology $(\text { ITQ })^{23,24}$. However, the assignation of IR bands is in some cases difficult and not conclusive.

In the following two chapters, we will try to shed some light on the complicated SCR process, specifically with $\mathrm{Cu}-\mathrm{CHA}$ catalysts. This chapter presents the results of the simulation of the IR spectroscopic properties of Cu-SSZ-13 and Cu-SAPO-34 using static DFT methods. We have combined these calculations with the experimental data available in the Institute of Chemical Technology to study the nature of exchange $\mathrm{Cu}$ active sites. We also explore in this chapter possible mechanisms for the oxidation and reduction half cycles of the SCR reaction.

\subsection{Methods and models}

Periodic density functional calculations were performed using the Perdew-Wang (PW91) exchange-correlation functional within the generalized gradient approach $(\mathrm{GGA})^{71,72}$, as implemented in the VASP $\operatorname{code}^{73}$. The valence density was expanded 
in a plane wave basis set with a kinetic energy cutoff of $450 \mathrm{eV}$, and the effect of the core electrons in the valence density was taken into account by means of the projected augmented wave (PAW) formalism ${ }^{74}$. Integration in the reciprocal space was performed at the $\Gamma$ k-point of the Brillouin zone. Electronic energies were converged to $10^{-6} \mathrm{eV}$ and geometries were optimized until forces on atoms were less than $0.01 \mathrm{eV} / \AA$. Transition states were obtained using the DIMER algorithm ${ }^{75,76}$. During geometry optimizations, the positions of all atoms in the system were allowed to relax without any restriction. Vibrational frequencies were calculated by diagonalizing the block Hessian matrix corresponding to the displacements of the $\mathrm{Cu}, \mathrm{N}, \mathrm{H}$, and $\mathrm{O}$ atoms not belonging to the catalyst framework.

We have considered four possible distributions of two $\mathrm{Al}$ atoms within the $\mathrm{CHA}$ unit cell specified in section 2.2. The 6RA, 6RB, 6RC and 8R distributions of $\mathrm{Al}$ in $\mathrm{Cu}$ SSZ-13, and the 6RA, 6RC and 8R Si positions in Cu-SAPO-34 shown in Figure 4.1

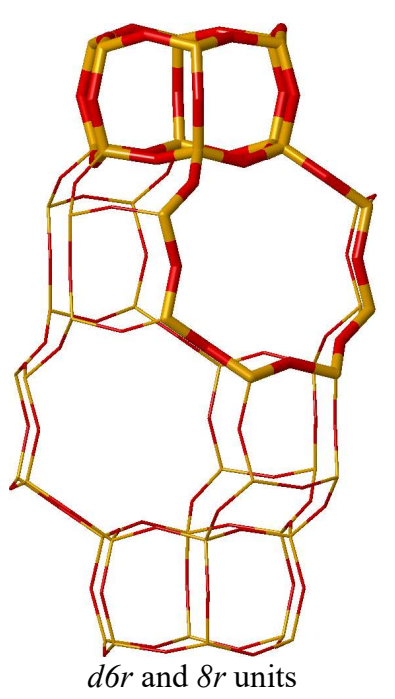

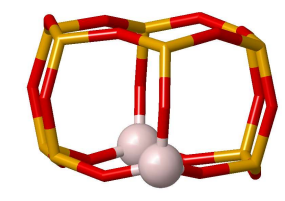

6RA

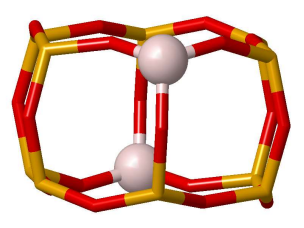

6RC

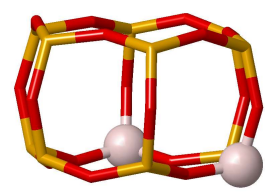

$6 \mathrm{RB}$

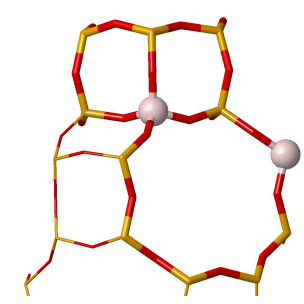

$8 \mathrm{R}$

Figure 4.1. CHA structure showing the $d 6 r$ and $8 r$ unis (left), and the four distributions of $\mathrm{Al}$ pairs (right). $\mathrm{Si}, \mathrm{O}$ and $\mathrm{Al}$ atoms are orange, red and thatch. The same distributions for $\mathrm{Si}$ pairs were used in $\mathrm{Cu}-\mathrm{SAPO}-34$. 


\subsection{Identification of $\mathrm{Cu}$ species in $\mathrm{Cu}-\mathrm{CHA}$ by IR spectroscopy and theoretical modeling}

\subsubsection{Stability of Cu species from DFT calculations}

The stability of the copper cations depends on the distribution of $\mathrm{Al}$ and $\mathrm{Si}$ atoms in Cu-SSZ-13 and Cu-SAPO-34 materials, respectively. In this section, we study the stability of the most relevant species. The optimized structures together with their relative energy referred to the most stable comparable system are shown in Figures 4.2 and 4.3 .

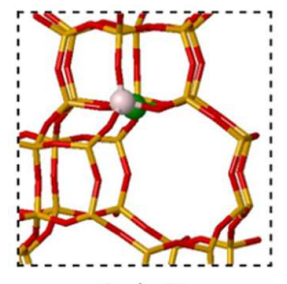

$\mathrm{Cu}^{+}-6 \mathrm{R}$

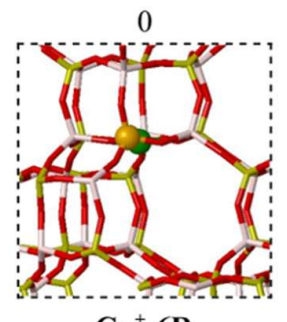

$\mathrm{Cu}^{+}-6 \mathrm{R}$

0

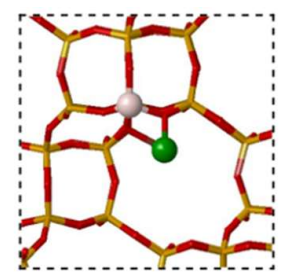

$\mathrm{Cu}^{+}-\mathbf{8 R}$

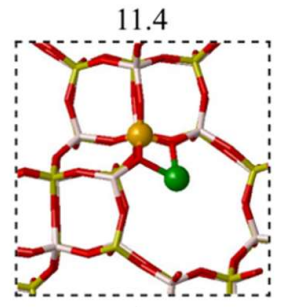

$\mathrm{Cu}^{+}-8 \mathrm{R}$

12.4

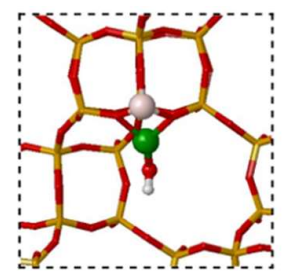

$\mathrm{Cu}^{2+}-\mathrm{OH}-6 \mathrm{R}$

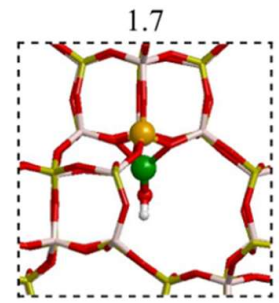

$\mathrm{Cu}^{2+}$-OH-6R

4.31

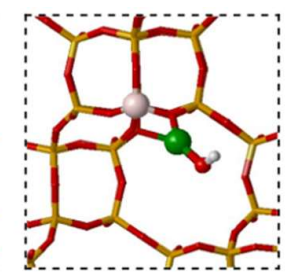

$\mathrm{Cu}^{2+}-\mathrm{OH}-8 \mathrm{R}$

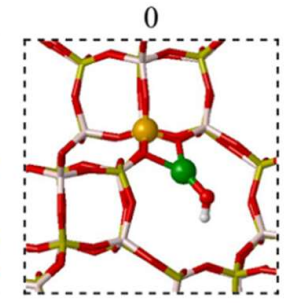

$\mathrm{Cu}^{2+}-\mathrm{OH}-8 \mathrm{R}$

0

Figure 4.2 Optimized structures of the different copper species of $\mathrm{Cu}-\mathrm{SSZ}-13$ with one $\mathrm{Al}$ (first row) and $\mathrm{Cu}-\mathrm{SAPO}-34$ with one $\mathrm{Si}$ (second row). $\mathrm{Si}, \mathrm{Al}, \mathrm{P}, \mathrm{O}$ and $\mathrm{Cu}$ atoms are depicted in orange, thatch, yellow, red and green, respectively. The relative stabilities of comparable systems are given in $\mathrm{kcal} / \mathrm{mol}$. 


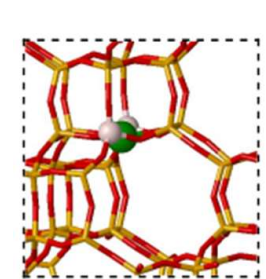

$\mathrm{Cu}^{2+}-6 \mathrm{RA}-6 \mathrm{R}$

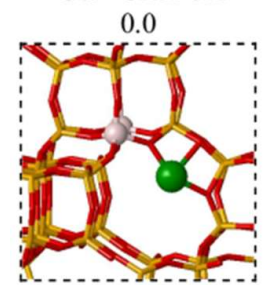

$\mathrm{Cu}^{2+}$-6RA-8R

43.4

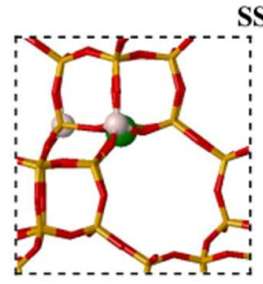

$\mathrm{Cu}^{2+}$-6RB-6R

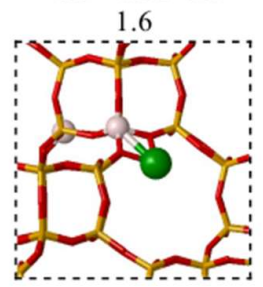

$\mathrm{Cu}^{2+}$-6RB-8R

38.6
SSZ-13

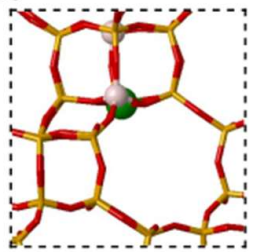

$\mathrm{Cu}^{2+}-6 \mathrm{RC}-6 \mathrm{R}$

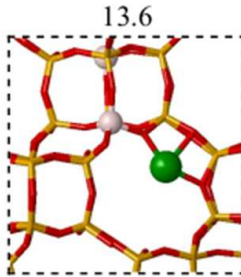

$\mathrm{Cu}^{2+}-6 \mathrm{RC}-8 \mathrm{R}$ 45.8

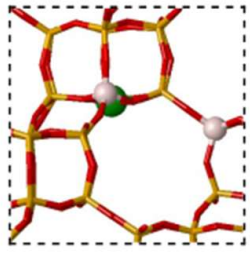

$\mathrm{Cu}^{2+}-8 \mathrm{R}-6 \mathrm{R}$

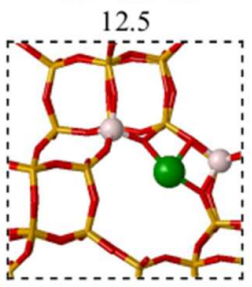

$\mathrm{Cu}^{2+}-8 \mathrm{R}-8 \mathrm{R}$

19.1

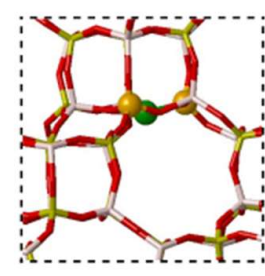

$\mathrm{Cu}^{2+}$-6RB-6R

0.0

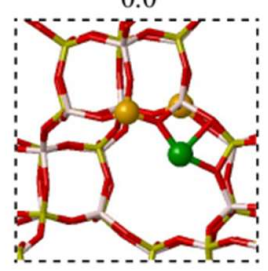

$\mathrm{Cu}^{2+}-6 \mathrm{RB}-8 \mathrm{R}$

35.2

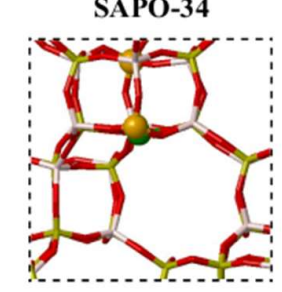

$\mathrm{Cu}^{2+}-6 \mathrm{RC}-6 \mathrm{R}$

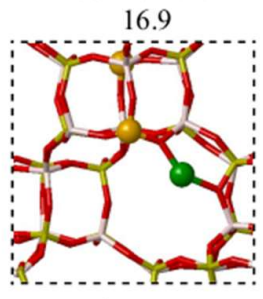

$\mathrm{Cu}^{2+}-6 \mathrm{RC}-8 \mathrm{R}$

39.2

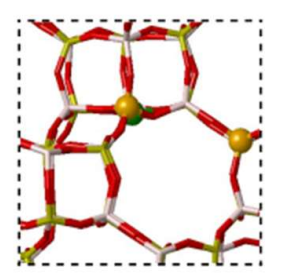

$\mathrm{Cu}^{2+}$-8R-6R

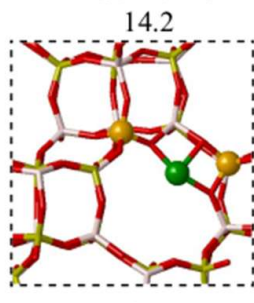

$\mathrm{Cu}^{2+}-8 \mathrm{R}-8 \mathrm{R}$

14.6

Figure 4.3 Optimized structures of the different copper species of Cu-SSZ-13 with $2 \mathrm{Al}$ (2 first rows) and SAPO-34 with $2 \mathrm{Si}$ (2 last rows). $\mathrm{Si}, \mathrm{Al}, \mathrm{P}, \mathrm{O}$ and $\mathrm{Cu}$ atoms are depicted in orange, thatch, yellow, red and green, respectively. The relative stabilities of comparable systems are given in $\mathrm{kcal} / \mathrm{mol}$. 
First, when one $\mathrm{Si}$ is substituted by one $\mathrm{Al}$ in the SSZ-13 framework, the negative charge can be compensated by one $\mathrm{Cu}^{+}$or $[\mathrm{Cu}(\mathrm{OH})]^{+}$species. These species can be located either in the $6 r$ of the chabazite $d 6 r$ unit $\left(\mathrm{Cu}^{+}-6 \mathrm{R}\right.$ and $\mathrm{Cu}^{2+}-\mathrm{OH}-6 \mathrm{R}$ models, see Figure 4.2) or in the $8 r$ in the large chabazite cage $\left(\mathrm{Cu}^{+}-8 \mathrm{R}\right.$ and $\mathrm{Cu}^{2+}-\mathrm{OH}-8 \mathrm{R}$ models, see Figure 4.2). The same holds for Cu-SAPO-34, except that in this case the negative charge is introduced by substitution of one $\mathrm{P}$ by one $\mathrm{Si}$. The position of $\mathrm{Cu}^{+}$in the $6 r$ is more stable by $11-12 \mathrm{kcal} / \mathrm{mol}$ than in the $8 r$ in both Cu-SSZ-13 and $\mathrm{Cu}-\mathrm{SAPO}-34$. As for $[\mathrm{Cu}(\mathrm{OH})]^{+}$the difference is smaller, being now the $8 r$ the most stable location.

Two Si or $\mathrm{P}$ atoms can also be replaced by $\mathrm{Al}$ or $\mathrm{Si}$ in $\mathrm{Cu}-\mathrm{SSZ}-13$ and $\mathrm{Cu}-\mathrm{SAPO}-34$ respectively. In these cases, more combinations are possible. The most important ones are depicted in Figure 4.1, namely 6RA, 6RB and 6RC (in the $d 6 r$ unit) and 8R (in the $8 r$ ). The most stable systems are those with the $\mathrm{Cu}^{2+}$ cation and the two substitutional $\mathrm{Al}$ (or $\mathrm{Si}$ ) atoms placed in the same $6 r$. The corresponding models are the $\mathrm{Cu}^{2+}-6 \mathrm{RA}-6 \mathrm{R}$ for $\mathrm{Cu}-\mathrm{SSZ}-13$ and $\mathrm{Cu}^{2+}-6 \mathrm{RB}-6 \mathrm{R}$ for $\mathrm{Cu}-\mathrm{SAPO}-34$, taken as reference and shown in Figure 4.3.

When the two $\mathrm{Al}$ (or $\mathrm{Si}$ ) are located in the same $6 r$ but the $\mathrm{Cu}^{2+}$ is located in the $8 r$ the relative energy is higher by more than $35 \mathrm{kcal} / \mathrm{mol}$ (Figure 4.3 ). This is the case for $\mathrm{Cu}^{2+}-6 \mathrm{RA}-8 \mathrm{R}$ and $\mathrm{Cu}^{2+}-6 \mathrm{RB}-8 \mathrm{R}$ models, which have therefore not been considered any more. A similar situation is observed when the two substitutional $\mathrm{Al}$ (or $\mathrm{Si}$ ) atoms are placed in two different $6 r$ of the same $d 6 r$ unit. Locating the $\mathrm{Cu}^{2+}$ cation in the $6 r$ is more stable by more than $20 \mathrm{kcal} / \mathrm{mol}$ than placing it in the $8 r$. Compare the models $\mathrm{Cu}^{2+}-6 \mathrm{RC}-6 \mathrm{R}$ and $\mathrm{Cu}^{2+}-6 \mathrm{RC}-8 \mathrm{R}$ in Figure 4.3 for both $\mathrm{Cu}-\mathrm{SSZ}$ 13 and $\mathrm{Cu}-\mathrm{SAPO}-34$. Finally, the two substitutional $\mathrm{Al}$ or $\mathrm{Si}$ can be placed in the $8 r$ together with the $\mathrm{Cu}^{2+}$ cation according to model $\mathrm{Cu}^{2+}-8 \mathrm{R}-8 \mathrm{R}$ or with the $\mathrm{Cu}^{2+}$ cation in the $6 r$ according to model $\mathrm{Cu}^{2+}-8 \mathrm{R}-6 \mathrm{R}$. The latter is $6.6 \mathrm{kcal} / \mathrm{mol}$ more stable than the former.

\subsubsection{Identification of the $\mathrm{Cu}$ species through IR analysis}

Different species have been proposed as possible active sites for the SCR-NH reaction in the $\mathrm{Cu}$-CHA catalysts. As mentioned in the introduction, the most relevant include $\mathrm{Cu}^{+}$and $\mathrm{Cu}^{2+}$ cations, $[\mathrm{Cu}-\mathrm{O}-\mathrm{Cu}]^{2+}$ dimers, hydroxylated $[\mathrm{Cu}-\mathrm{OH}]^{+}$ species and small $\mathrm{CuOx}$ nanoclusters. It is of paramount importance to identify 
4.3 Identification of $\mathrm{Cu}$ species in $\mathrm{Cu}$-CHA by IR spectroscopy and theoretical modeling

unambiguously these copper species in order to better understand the SCR mechanism, and the origin of its optimum performance. However, the true nature of the active sites is still not well understood due to the complexity of this catalytic system. We have also mentioned that the high sensitivity of small molecules like NO to the electronic properties of $\mathrm{Cu}^{+} / \mathrm{Cu}^{2+}$ cations is very useful to characterize these active centers through IR spectroscopy in solid catalysts like $\mathrm{Cu}$-CHA. In this section, we study the interaction of NO with the different copper species aiming to shed some light in the assignation of IR bands and in the nature of the active sites as well. To that end, we have built several models with the following copper states: $\mathrm{Cu}^{+},-\mathrm{Cu}^{2+},[\mathrm{Cu}(\mathrm{OH})]^{+}$and $[\mathrm{Cu}-\mathrm{O}-\mathrm{Cu}]^{2+}$ species in $\mathrm{Cu}-\mathrm{SSZ}-13$ and $\mathrm{Cu}-\mathrm{SAPO}-34$. The optimized structures of NO interacting with $\mathrm{Cu}^{+},[\mathrm{Cu}(\mathrm{OH})]^{+}$and $-\mathrm{Cu}^{2+}$ species in $\mathrm{Cu}-\mathrm{SSZ}-13$ zeolite as well as the interaction of $\mathrm{NO}$ with a $[\mathrm{Cu}-\mathrm{O}-\mathrm{Cu}]^{2+}$ dimer in $\mathrm{Cu}-\mathrm{SAPO}-34$ are depicted in Figures 4.4 and 4.5. The calculated $v \mathrm{NO}$ vibrational frequencies for all systems in $\mathrm{Cu}-\mathrm{SSZ}-13$ and $\mathrm{Cu}-\mathrm{SAPO}-34$ are summarized in Table 4.1 .

Table 4.1 Calculated and experimental $v \mathrm{NO}$ vibrational frequencies (in $\mathrm{cm}^{-1}$ ) for NO interacting with different models of $\mathrm{Cu}^{+},[\mathrm{Cu}(\mathrm{OH})]^{+}$and $\mathrm{Cu}^{2+}$ species and Brönsted acid sites in $\mathrm{Cu}-\mathrm{SSZ}-13$ and $\mathrm{Cu}-\mathrm{SAPO}-34$ catalysts, and with a $[\mathrm{Cu}-\mathrm{O}-\mathrm{Cu}]^{2+}$ dimer in $\mathrm{Cu}-\mathrm{SAPO}-34$. The experimental spectra are shown in Figure 4.6.

\begin{tabular}{lccccc}
\hline & \multicolumn{2}{c}{ Cu-SSZ-13 } & \multicolumn{2}{c}{ Cu-SAPO-34 } & Experimental $^{77}$ \\
& NO & 2NO & NO & 2NO & \\
\hline $\mathrm{Cu}^{+}-6 \mathrm{R}$ & 1804 & 1706,1820 & 1811 & 1720,1831 & \multirow{2}{*}{$1698,1753,1827,1820$} \\
$\mathrm{Cu}^{+}-8 \mathrm{R}$ & 1816 & 1716,1834 & 1821 & 1714,1828 & 1788 \\
$\mathrm{Cu}^{2+}-\mathrm{OH}-6 \mathrm{R}$ & 1795 & - & 1790 & - & \\
$\mathrm{Cu}^{2+}-\mathrm{OH}-8 \mathrm{R}$ & 1788 & - & 1798 & - & 1894 \\
$\mathrm{H} \mathrm{Brönsted}^{2}$ & 1891 & - & 1891 & - & \\
$\mathrm{Cu}^{2+}$-6RA & 1965 & 1801,1892 & - & - & 1924,1911 \\
$\mathrm{Cu}^{2+}-6 \mathrm{RB}$ & 1925 & - & 1907 & - & \\
$\mathrm{Cu}^{2+}-6 \mathrm{RC}$ & 1960 & 1801,1869 & 1968 & 1809,1888 & \\
$\mathrm{Cu}^{2+}-8 \mathrm{R}-6 \mathrm{R}$ & 1977 & 1813,1874 & 1943 & 1802,1868 & \\
$\mathrm{Cu}^{2+}-8 \mathrm{R}-8 \mathrm{R}$ & 1947 & - & 1940 & - & 1950,1943 \\
{$[\mathrm{Cu}-\mathrm{O}-\mathrm{Cu}]^{2+}$} & - & - & - & 1712,1887 & 1883 \\
\hline
\end{tabular}


The interaction of NO with the copper species considered produces stable adsorption states in all cases with adsorption energies ranging from -20 to $-46 \mathrm{kcal} / \mathrm{mol}$. For the mono-nitrosyl $\mathrm{Cu}^{+}(\mathrm{NO})$, the frequency corresponding to the NO stretching in $\mathrm{Cu}-$ SSZ-13 and Cu-SAPO-34 falls between $\sim 1805$ and $\sim 1820 \mathrm{~cm}^{-1}$.

As for di-nitrosyl complexes $\mathrm{Cu}^{+}(\mathrm{NO})_{2}$ in the $6 r$ and the $8 r$ the asymmetric stretching mode calculated lie at $1706 \mathrm{~cm}^{-1}$ and $1720 \mathrm{~cm}^{-1}$ in Cu-SSZ-13 and Cu-SAPO-34 respectively. The symmetric modes have frequencies of $1820 \mathrm{~cm}^{-1}$ for Cu-SSZ-13 and around $1830 \mathrm{~cm}^{-1}$ for Cu-SAPO-34. These values are in good agreement with the bands observed from experiments at 1827 and $1698 \mathrm{~cm}^{-1}$ for Cu-SSZ-13 and at 1820 and $1753 \mathrm{~cm}^{-1}$ for Cu-SAPO-34 at low dosing of NO. Furthermore, it is accepted that the $v \mathrm{NO}$ vibrational frequencies associated with isolated $\mathrm{Cu}^{+}$species appear in the $1700-1850 \mathrm{~cm}^{-1}$ range. These experimental frequencies correspond to mono and di-nitrosyl complexes in both $6 r$ and $8 r$ environments. 

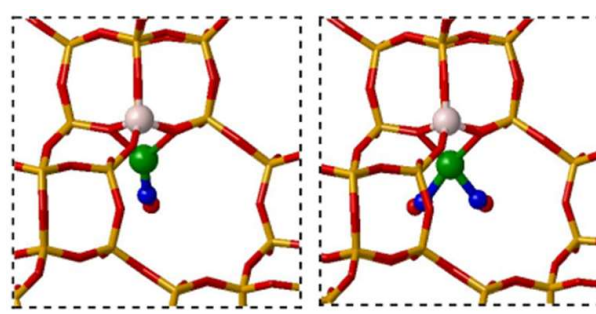

$\mathrm{Cu}^{+}-6 \mathrm{R}-\mathrm{NO}$

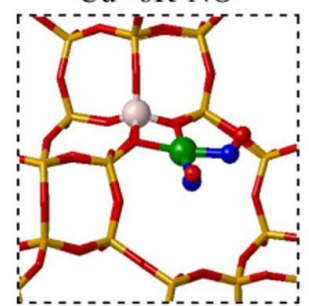

$$
\mathrm{Cu}^{+}-8 \mathrm{R}-2 \mathrm{NO}
$$
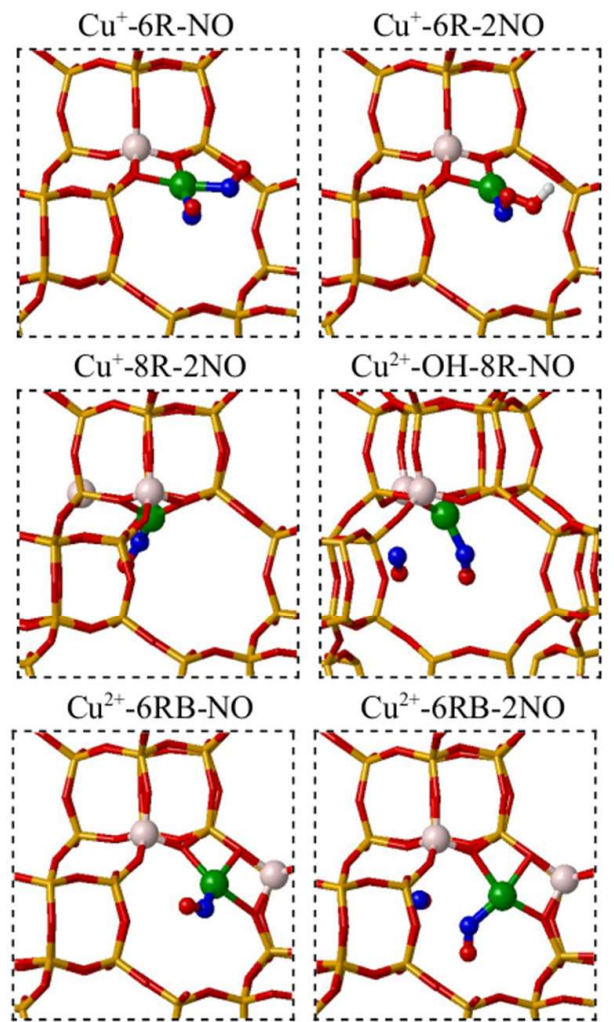

$\mathrm{Cu}^{2+}-8 \mathrm{R}-\mathrm{NO}$
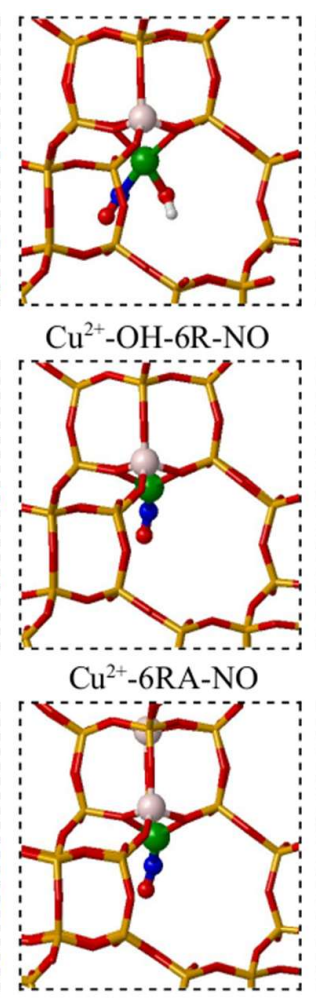

$\mathrm{Cu}^{2+}-6 \mathrm{RC}-\mathrm{NO}$

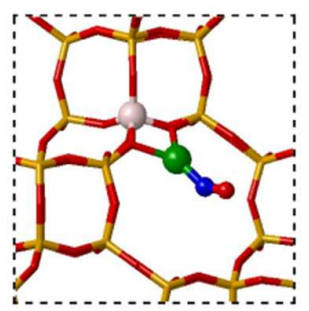

$\mathrm{Cu}^{+}-8 \mathrm{R}-\mathrm{NO}$

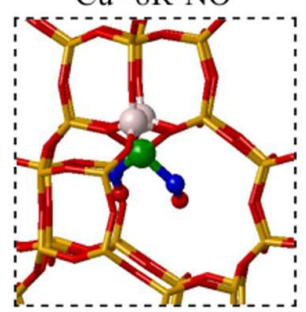

$\mathrm{Cu}^{2+}-6 \mathrm{RA}-2 \mathrm{NO}$

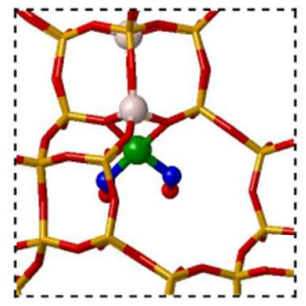

$\mathrm{Cu}^{2+}-6 \mathrm{RC}-2 \mathrm{NO}$

Figure 4.4 Optimized geometries of $\mathrm{NO}$ interacting with $\mathrm{Cu}^{+}$and $[\mathrm{Cu}(\mathrm{OH})]^{+}$species in $\mathrm{Cu}$ $\mathrm{SSZ}-13 . \mathrm{Si}, \mathrm{Al}, \mathrm{O}, \mathrm{Cu}, \mathrm{N}$ and $\mathrm{H}$ atoms are depicted in orange, thatch, red, green, blue and white, respectively. 


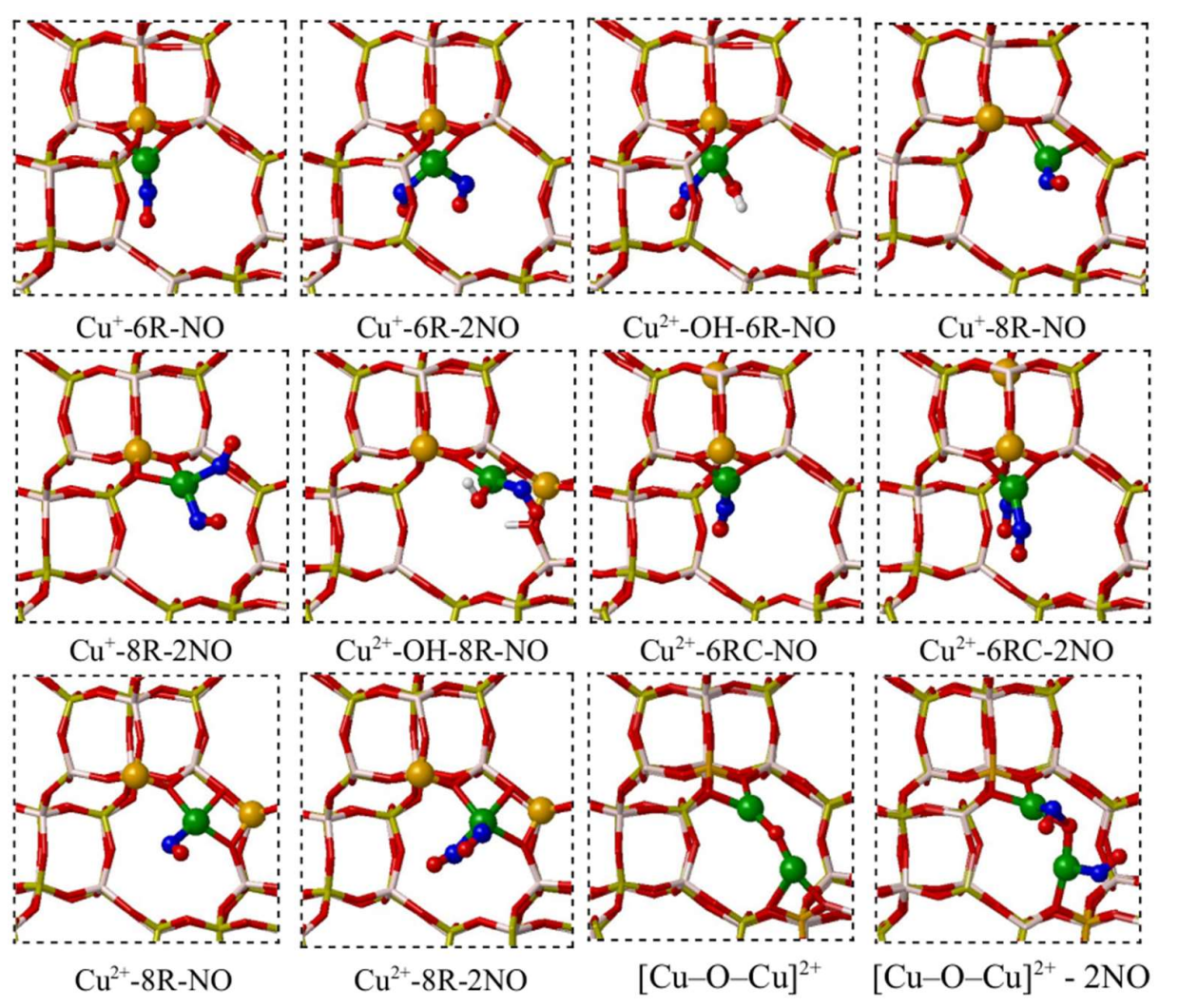

Figure 4.5 Optimized geometries of $\mathrm{NO}$ interacting with $\mathrm{Cu}^{+}$and $[\mathrm{Cu}(\mathrm{OH})]^{+}$species in $\mathrm{Cu}-$ SAPO-34. P, Si, Al, O, Cu, N and $\mathrm{H}$ atoms are depicted in yellow, orange, thatch, red, green, blue and white, respectively.

The interaction of $\mathrm{NO}$ with $[\mathrm{Cu}(\mathrm{OH})]^{+}$species is also very strong, and the computation of vibrational frequencies yields values between 1787 and $1798 \mathrm{~cm}^{-1}$. In the experimental IR spectrum of Cu-SSZ-13 (see Figure 4.6, top) a band at 1788 $\mathrm{cm}^{-1}$ is detected. This result is supported by an IR band at $3668 \mathrm{~cm}^{-1}$ due to the hydroxyl group attached to $\mathrm{Cu}^{2+}$. The calculated value of $\mathrm{O}-\mathrm{H}$ stretching frequency is $3656 \mathrm{~cm}^{-1}$ for $\mathrm{Cu}-\mathrm{SSZ}-13$ ( $\left(\mathrm{see} \mathrm{Cu}^{2+}-\mathrm{OH}-6 \mathrm{R}-\mathrm{NO}\right.$ in Figure 4.5). 

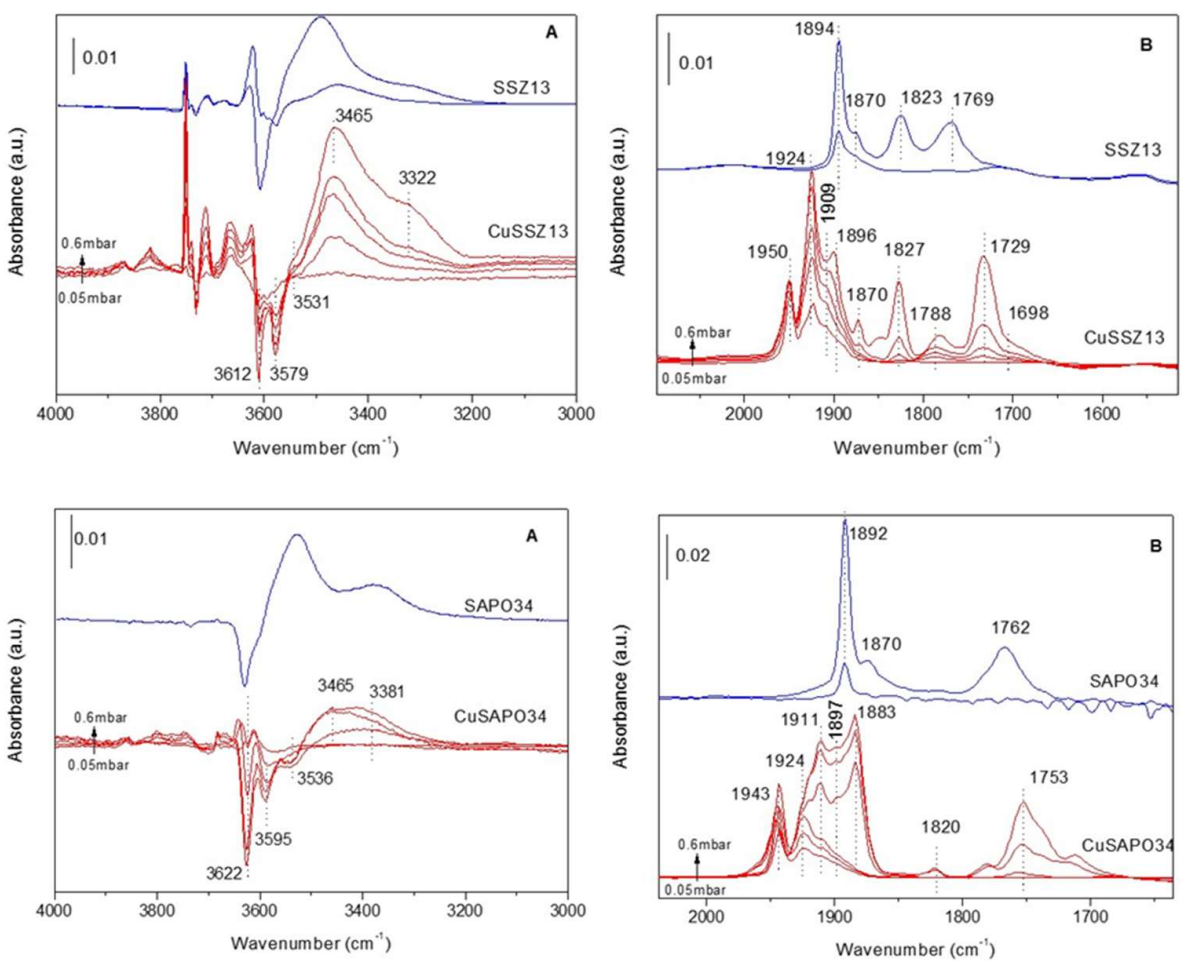

Figure 4.6. IR spectra in the $\mathrm{vOH}$ IR region (A) and $v \mathrm{NO}$ IR region (B) of NO adsorption at $-156^{\circ} \mathrm{C}$ and at increasing NO dosing (0.05-0.6mbar) on SSZ-13 (top, blue), Cu-SSZ-13 (top, red), SAPO-34 (bottom, blue) and Cu-SAPO-34 (bottom, red) samples.

On Brønsted acid sites, NO interacts very weakly and the calculated vibrational frequency corresponding to the NO stretching is $1891 \mathrm{~cm}^{-1}$ on both materials, not very different from that of the gas phase molecule $\left(1894 \mathrm{~cm}^{-1}\right)$. This is also in good agreement with the experimental frequency located at $1894 \mathrm{~cm}^{-1}$, observed in $\mathrm{H}$ SSZ-13 and supported by a shift in the $\mathrm{OH}$ region, around $3500 \mathrm{~cm}^{-1}$ (Figure 4.6, top).

Although less strongly, $\mathrm{NO}$ adsorbs also on $\mathrm{Cu}^{2+}$. However, due to the strong coordination of $\mathrm{Cu}^{2+}$ cation with three framework oxygen atoms when it is located 
either in the $6 \mathrm{Rb}-6 \mathrm{R}$ or in the $8 \mathrm{R}-8 \mathrm{R}$ positions (Figure 4.4), two NO molecules are not able to bind, and thus only the mono-nitrosyl complexes can be optimized for these two systems. The $v$ NO mode vibrates between 1925 and $1947 \mathrm{~cm}^{-1}$ on $\mathrm{Cu}$ SSZ-13 zeolite, and between quite similar values, 1907 and $1940 \mathrm{~cm}^{-1}$, on $\mathrm{Cu}-$ SAPO-34. These values match the experimental bands observed at 1924 and 1950 $\mathrm{cm}^{-1}$ for Cu-SSZ-13 and at 1911 and $1943 \mathrm{~cm}^{-1}$ for Cu-SAPO-34 in the spectrum of $\mathrm{NO}$ adsorption. (see figure 4.6, bottom).

Finally, dimeric $[\mathrm{Cu}-\mathrm{O}-\mathrm{Cu}]^{2+}$ species have been proposed in the literature and we have considered the interaction of NO with this species as well. The optimized structure for $\mathrm{Cu}-\mathrm{SAPO}-34$ is shown in Figure 4.5. In this case, the two $\mathrm{Si}$ atoms are placed in different $6 r$ units. The species $[\mathrm{Cu}-\mathrm{O}-\mathrm{Cu}]^{2+}$ located across the cavity help to stabilize this pair of negative charges. Other possible locations of the $[\mathrm{Cu}-\mathrm{O}-\mathrm{Cu}]^{2+}$ dimer were explored but they were between 5 and $25 \mathrm{kcal} / \mathrm{mol}$ less stable. In the optimized geometry resulting from the interaction of two $\mathrm{NO}$ molecules on each $\mathrm{Cu}$ atom of $[\mathrm{Cu}-\mathrm{O}-\mathrm{Cu}]^{2+}$ (Figure 4.5), one NO is adsorbed in a bridge mode bonded to both $\mathrm{Cu}$ and $\mathrm{O}$ atoms. The calculated vibrational frequency of the $v \mathrm{NO}$ mode is 1712 $\mathrm{cm}^{-1}$. The other NO molecule is bonded to the second $\mathrm{Cu}$ atom which is coordinated to only two $\mathrm{O}$ atoms with $\mathrm{O}-\mathrm{Cu}-\mathrm{O}$ angle close to $180^{\circ}$. The computed $v \mathrm{NO}$ vibrational frequency is $1887 \mathrm{~cm}^{-1}$, which could be associated with the band observed at $1883 \mathrm{~cm}^{-1}$ in the IR spectra of Cu-SAPO-34.

\subsection{Theoretical study of the reaction mechanism}

In this section, the mechanism of the NH3-SCR reaction on the Cu-SAPO-34 and $\mathrm{Cu}-\mathrm{SSZ}-13$ catalysts is investigated by means of periodic DFT calculations.

\subsubsection{Oxidation half-cycle: formation of nitrites and nitrates on $\mathrm{Cu}-\mathrm{CHA}$ catalysts}

There is experimental evidence that the oxidation of NO is significantly enhanced by the presence of exchange $\mathrm{Cu}$ cations ${ }^{32,78-80}$. There is also evidence for the formation of $\mathrm{Cu}^{2+} \mathrm{NO}_{\mathrm{x}}{ }^{-}$species when the catalyst is exposed to $\mathrm{NO}$ and $\mathrm{O}_{2}$ in the absence as well as in the presence of ammonia ${ }^{33,81}$. We delay the effect of ammonia to the next chapter and we study here how the oxidation of NO might occur on the 
$\mathrm{Cu}^{+}$site without $\mathrm{NH}_{3}$. Therefore, the initial state for the mechanistic study will be the most stable $\mathrm{Cu}^{+}$species identified in Section 4.3.1, plus $\mathrm{NO}$, plus $\mathrm{O}_{2}$. In this regard, the active site has been defined as a $\mathrm{Cu}^{+}$cation located in a $d 6 r$ unit containing two Si (or two Al) atoms, with a Brønsted acid site nearby to keep the system neutral. We have considered the most stable distributions of two $\mathrm{Si}$ and two $\mathrm{Al}$ from section 4.3.1, namely, the 6RB and 6RC for $\mathrm{Cu}-\mathrm{SAPO}-34$, and the 6RA, $6 \mathrm{RB}$ and $6 \mathrm{RC}$ for $\mathrm{Cu}-\mathrm{SSZ}-13$ (see Figure 4.1). Among these, the most stable model is the $6 \mathrm{RB}$ with two $\mathrm{Si}$ (or two $\mathrm{Al}$ ) atoms in the same $6 r$ ring for both materials, but the relative energies of all structures investigated (Table 4.2), and the activation barriers for all steps considered (Table 4.3) indicate that there are no significant differences in the mechanisms calculated with the five catalyst models. Therefore, the results presented and discussed are those related to the $6 \mathrm{RB}$ model of $\mathrm{Cu}-\mathrm{SAPO}-34$ and Cu-SSZ-13 catalysts unless otherwise stated. The calculated energy profiles over $\mathrm{Cu}-\mathrm{SAPO}-34$ and $\mathrm{Cu}-\mathrm{SSZ}-13$ are plotted in Figures 4.7 and 4.10 respectively, and the optimized geometries of all structures involved are depicted in Figures 4.8, 4.9, 4.11 and 4.12. 
Table 4.2 Relative stability (in $\mathrm{kcal} / \mathrm{mol}$ ) of all structures involved in the oxidation of $\mathrm{Cu}^{+}$ to $\mathrm{Cu}^{2+}$ by $\mathrm{NO}+\mathrm{O}_{2}$ with respect to the sum of the energies of $\mathrm{Cu}-\mathrm{CHA}$ catalyst $+2 \mathrm{NO}+\mathrm{O}_{2}$. The state of some molecules indicated with (g) means that such molecules are not coordinated to the copper cations. It does not mean an isolated gas phase molecule. See Figures 4.8, 1.9, 4.11 and 4.12 .

\begin{tabular}{|c|c|c|c|c|c|c|}
\hline \multirow{2}{*}{ Species } & & \multicolumn{3}{|c|}{$\mathrm{Cu}-\mathrm{SSZ}-13$} & \multicolumn{2}{|c|}{$\mathrm{Cu}-\mathrm{SAPO}-34$} \\
\hline & & 6RA & $6 \mathrm{RB}$ & $6 \mathrm{RC}$ & $6 \mathrm{RB}$ & $6 \mathrm{RC}$ \\
\hline \multirow[t]{2}{*}{$\mathrm{Cu}^{+}+\mathrm{NO}+\mathrm{O}_{2}$} & 1 & - & -26.3 & - & -27.0 & -33.0 \\
\hline & $\mathrm{TS}_{1 \rightarrow 2}$ & - & -4.0 & - & -4.3 & -10.1 \\
\hline $\mathrm{Cu}^{2+}+\mathrm{NO}_{3}^{-}$ & 2 & - & -76.0 & - & -76.7 & -80.8 \\
\hline $\mathrm{Cu}^{+}+\mathrm{NO}$ & 3 & -26.5 & -27.1 & -32.4 & -27.1 & -31.7 \\
\hline $\mathrm{Cu}^{+}+2 \mathrm{NO}$ & 4 & -45.3 & -45.7 & -50.9 & -46.8 & -51.0 \\
\hline \multirow[t]{2}{*}{$\mathrm{Cu}^{+}+2 \mathrm{NO}+\mathrm{O}_{2}$} & 5 & -56.3 & -56.3 & -61.9 & -57.1 & -62.0 \\
\hline & $\mathrm{TS}_{5 \rightarrow 6}$ & -41.9 & -43.3 & -48.8 & -43.5 & -48.8 \\
\hline \multirow[t]{2}{*}{$\mathrm{Cu}^{+}+2 \mathrm{NO}_{2}$} & 6 & -97.2 & -98.1 & -104.2 & -99.8 & -104.8 \\
\hline & $\mathrm{TS}_{5 \rightarrow 7}$ & -40.3 & -40.7 & -46.5 & -40.4 & -45.8 \\
\hline $\mathrm{Cu}^{2+}+\mathrm{NO}_{2}^{-}+\mathrm{NO}_{2}$ & 7 & -94.8 & -95.5 & -101.5 & -97.7 & -101.1 \\
\hline \multirow[t]{2}{*}{$\mathrm{Cu}^{2+}+\mathrm{NO}_{2}^{-}+\mathrm{NO}_{2}$} & 8 & -84.8 & -86.3 & -91.1 & -87.3 & -92.8 \\
\hline & $\mathrm{TS}_{8 \rightarrow 9}$ & -71.4 & -71.4 & -77.2 & -73.5 & -76.7 \\
\hline $\mathrm{Cu}^{2+}+\mathrm{NO}_{3}^{-}+\mathrm{NO}$ & 9 & -94.1 & -95.1 & -99.6 & -95.8 & -100.3 \\
\hline \multirow[t]{2}{*}{$\mathrm{Cu}^{2+}+\mathrm{NO}_{2}^{-}+\mathrm{NO}_{2}(\mathrm{~g})$} & 10 & - & -72.6 & - & -74.6 & - \\
\hline & $\mathrm{TS}_{10 \rightarrow 11}$ & - & -68.9 & - & -71.3 & - \\
\hline $\mathrm{Cu}^{2+}+\mathrm{NO}_{3}^{-}+\mathrm{NO}(\mathrm{g})$ & 11 & - & -76.0 & - & -82.0 & - \\
\hline \multirow[t]{2}{*}{$\mathrm{Cu}^{2+}+\mathrm{NO}_{2}^{-}+\mathrm{NO}_{2}+\mathrm{NO}_{2}(\mathrm{~g})$} & $12^{\mathrm{a}}$ & - & -95.5 & - & -97.7 & - \\
\hline & $\mathrm{TS}_{12 \rightarrow 13^{\mathrm{a}}}$ & - & -81.6 & - & -85.3 & - \\
\hline $\mathrm{Cu}^{2+}+\mathrm{NO}_{3}^{-}+\mathrm{NO}_{2}+\mathrm{NO}(\mathrm{g})$ & $13^{\mathrm{a}}$ & - & -90.5 & - & -93.8 & - \\
\hline
\end{tabular}

${ }^{\mathrm{a}} \mathrm{Calculated}$ with respect to the sum of the energies of $\mathrm{Cu}-\mathrm{CHA}$ catalyst $+2 \mathrm{NO}+\mathrm{O}_{2}+\mathrm{NO}_{2}$. 
Table 4.3 Electronic activation energies of the oxidation of $\mathrm{Cu}^{+}$to $\mathrm{Cu}^{2+}$ by $\mathrm{NO}+\mathrm{O}_{2}$. Energies are given in $\mathrm{kcal} / \mathrm{mol}$.

\begin{tabular}{ccccccc}
\hline & \multicolumn{3}{c|}{ SSZ-13 } & \multicolumn{2}{c}{ SAPO-34 } \\
\hline Steps & $6 \mathrm{RA}$ & $6 \mathrm{RB}$ & $6 \mathrm{RC}$ & $6 \mathrm{RB}$ & 6RC \\
\hline $\mathrm{TS}_{1 \rightarrow 2}$ & - & 22.3 & - & 22.7 & 22.8 \\
$\mathrm{TS}_{5 \rightarrow 6}$ & 14.3 & 13.0 & 13.1 & 13.6 & 13.2 \\
$\mathrm{TS}_{5 \rightarrow 7}$ & 16.0 & 15.6 & 15.4 & 16.7 & 16.1 \\
$\mathrm{TS}_{8 \rightarrow 9}$ & 13.4 & 15.0 & 13.9 & 13.8 & 16.1 \\
$\mathrm{TS}_{10 \rightarrow 11}$ & - & 3.7 & - & 3.2 & - \\
$\mathrm{TS}_{12 \rightarrow 13}$ & - & 13.9 & - & 12.5 & - \\
\hline
\end{tabular}

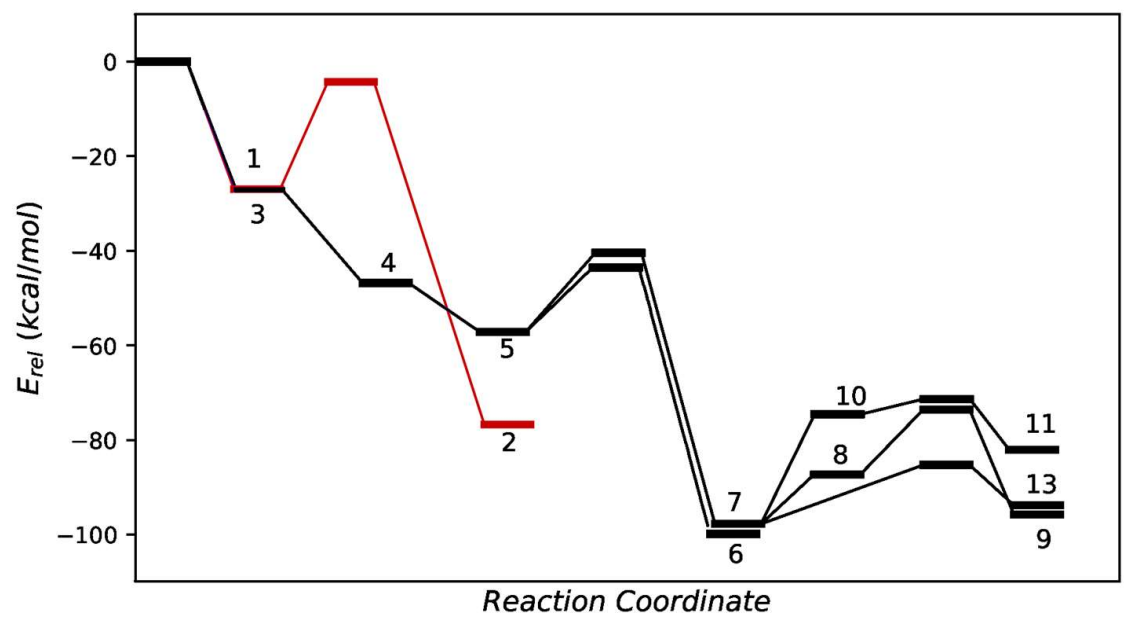

Figure 4.7 Calculated energy profile for all processes leading to the formation of nitrites and nitrates from $\mathrm{NO}+\mathrm{O}_{2}$ and from $\mathrm{NO}_{2}$ in Cu-SAPO-34 with $\mathrm{Cu}^{+}$in site $6 \mathrm{RB}$. The optimized structures involved are depicted in Figures 8 and 4.9. The path $1 \rightarrow 2$ has been represented in red for better visualization of minima 1 and 3 . 


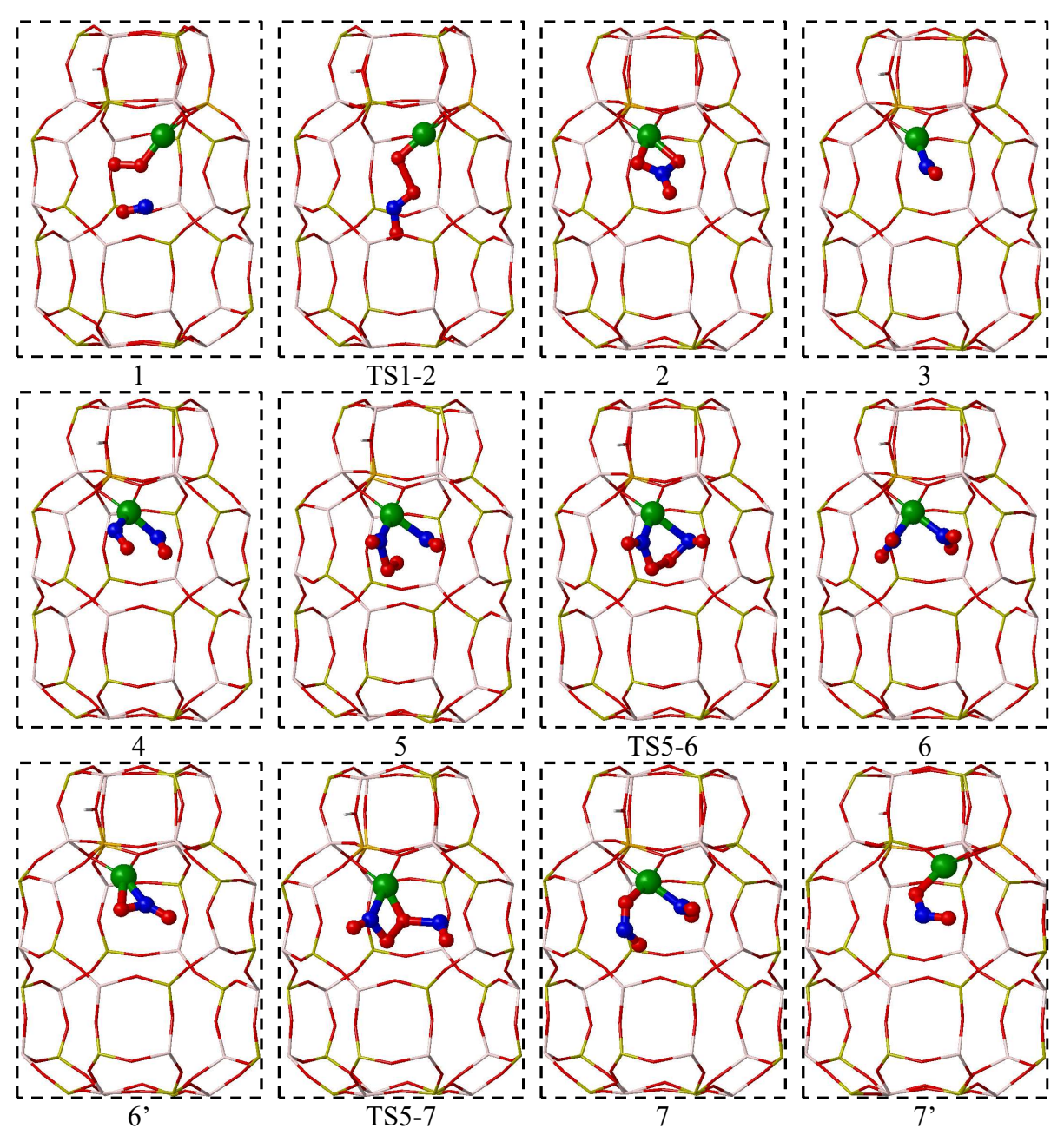

Figure 4.8 Optimized geometries of some $\mathrm{NO}$ and $\mathrm{O}_{2}$ adsorption complexes (1, 3, 4 and 5), and of the structures involved in the direct formation of nitrate (2) from co-adsorbed $\mathrm{NO}+\mathrm{O}_{2}$ in 6RB model of $\mathrm{Cu}-\mathrm{SAPO}-34$. Al, $\mathrm{P}, \mathrm{O}, \mathrm{Si}$, and $\mathrm{H}$ atoms in the framework depicted as thatch, yellow, red, orange and white sticks; $\mathrm{Cu}$ cations, $\mathrm{O}$ and $\mathrm{N}$ atoms in the reactant molecules depicted as green, red and blue balls. 

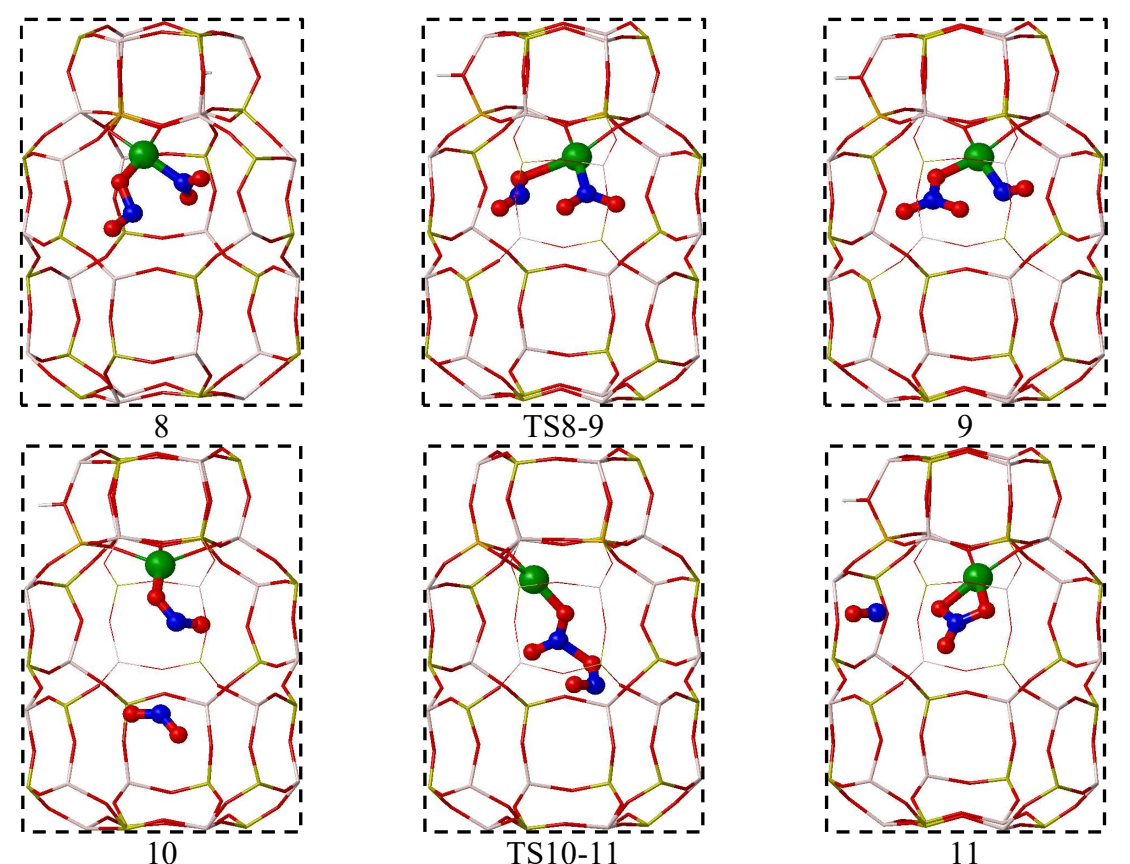

TS8-9
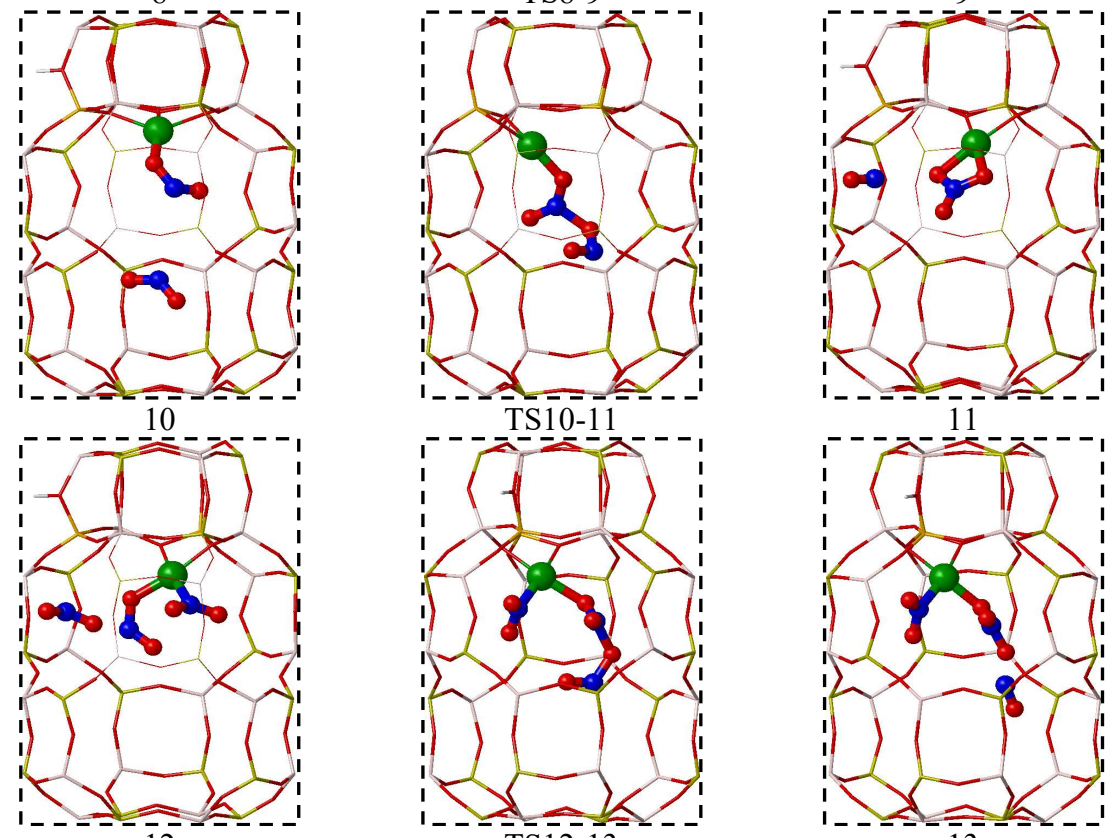

12

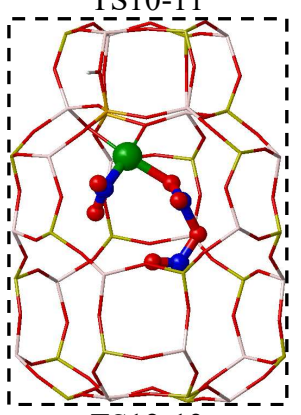

TS12-13

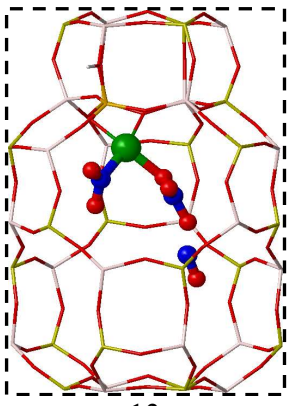

13

Figure 4.9 Optimized geometries of the structures involved in the formation of nitrites and nitrates in the 6RB model of Cu-SAPO-34. Al, P, O, Si, and $\mathrm{H}$ atoms in the framework depicted as thatch, yellow, red, orange and white sticks; $\mathrm{Cu}$ cations, $\mathrm{O}$ and $\mathrm{N}$ atoms in the reactant molecules depicted as green, red and blue balls.

The one-step formation of $\mathrm{Cu}^{2+} \mathrm{NO}_{3}{ }^{-}$by direct reaction of $\mathrm{Cu}^{+}$with $\mathrm{NO}$ and $\mathrm{O}_{2}$ is the first pathway considered (red pathway in Figure 4.7). The $\mathrm{O}_{2}$ molecule is first activated on $\mathrm{Cu}^{+}$and subsequently reacts with a NO molecule not 
directly bonded to the active site, breaking the 0-0 bond (TSl-2) and forming a bidentate nitrate complex. The calculated activation energy, $\sim 23 \mathrm{kcal} / \mathrm{mol}$, is similar to those previously reported on $\mathrm{Cu}-\mathrm{SSZ}-13(25-27 \mathrm{kcal} / \mathrm{mol})^{37,82}$ and the reaction is very exothermic. However, the interaction of $\mathrm{Cu}^{+}$with $\mathrm{O}_{2}$ is much weaker than with NO. The calculated adsorption energy of $\mathrm{O}_{2}$ on Cu-SAPO-34 and $\mathrm{Cu}-\mathrm{SSZ}-13$ are -9.63 and $-6.7 \mathrm{kcal} / \mathrm{mol}$, respectively, while the corresponding values obtained for NO are -27.1 and $-27.0 \mathrm{kcal} / \mathrm{mol}$ for mononitrosyl and -46.8 and -45.7 $\mathrm{kcal} / \mathrm{mol}$ for dinitrosyl complexes (structures 3 and 4 in Figures 4.8 and 4.11). These values suggest that the probability of formation of structure 1 is low in these catalysts.

Mao et al. ${ }^{54}$, reported a different pathway for the oxidation of $\mathrm{Cu}^{+}$to $\mathrm{Cu}^{2+}$ in $\mathrm{Cu}$ SAPO-34 where $\mathrm{NO}$ and $\mathrm{O}_{2}$ are first co-adsorbed on $\mathrm{Cu}^{+}$. However, the calculated activation energies lie around 20 and $24 \mathrm{kcal} / \mathrm{mol}$, which is somewhat high for a fast reaction at $298 \mathrm{~K}$.

Table 4.4 Calculated Adsorption Constants (Kads) of Reactant Molecules on Cu-SAPO34 and $\mathrm{Cu}-\mathrm{SSZ}-13$ at Different Temperatures. Numbered intermediates are shown in Figures 4.8 and 4.9

\begin{tabular}{lcccc}
\hline & \multicolumn{4}{c}{ Adsorption Constant, K ads } \\
\cline { 2 - 5 } & $\mathrm{T}=298 \mathrm{~K}$ & $\mathrm{~T}=373 \mathrm{~K}$ & $\mathrm{~T}=473 \mathrm{~K}$ & $\mathrm{~T}=623 \mathrm{~K}$ \\
\hline & \multicolumn{4}{c}{$\mathbf{C u}-\mathbf{S A P O}-\mathbf{3 4}$} \\
$\mathrm{Cu}^{+}+\mathrm{NO}+\mathrm{O}_{2} \rightarrow \mathbf{1}$ & $1.43 \times 10^{1}$ & $4.05 \times 10^{-3}$ & $5.92 \times 10^{-6}$ & $2.68 \times 10^{-8}$ \\
$\mathrm{Cu}^{+}+\mathrm{NO} \rightarrow \mathbf{3}$ & $1.14 \times 10^{11}$ & $3.01 \times 10^{7}$ & $3.86 \times 10^{4}$ & $1.39 \times 10^{2}$ \\
$\mathrm{Cu}^{+}+2 \mathrm{NO} \rightarrow \mathbf{4}$ & $1.57 \times 10^{15}$ & $5.33 \times 10^{8}$ & $2.71 \times 10^{3}$ & $7.60 \times 10^{-2}$ \\
$\mathrm{Cu}^{+}+2 \mathrm{NO}+\mathrm{O}_{2} \rightarrow \mathbf{5}$ & $9.03 \times 10^{12}$ & $1.48 \times 10^{5}$ & $6.91 \times 10^{-2}$ & $2.85 \times 10^{-7}$ \\
$\mathrm{Cu}^{+}+\mathrm{NO}_{2} \rightarrow \mathbf{6}^{\prime}$ & $4.74 \times 10^{8}$ & $1.82 \times 10^{3}$ & $3.15 \times 10^{-2}$ & $2.32 \times 10^{-6}$ \\
$\mathrm{Cu}^{+}+\mathrm{NO}_{2} \rightarrow \mathbf{7}^{\prime}$ & $7.52 \times 10^{6}$ & $2.27 \times 10^{2}$ & $6.31 \times 10^{-3}$ & $7.22 \times 10^{-7}$ \\
\hline & \multicolumn{4}{c}{$\mathbf{C u}-\mathbf{S S Z}-13$} \\
\\
$\mathrm{Cu}^{+}+\mathrm{NO}+\mathrm{O}_{2} \rightarrow \mathbf{1}$ & $8.66 \times 10^{0}$ & $3.01 \times 10^{-3}$ & $5.22 \times 10^{-6}$ & $2.76 \times 10^{-8}$ \\
$\mathrm{Cu}^{+}+\mathrm{NO} \rightarrow \mathbf{3}$ & $2.71 \times 10^{11}$ & $7.13 \times 10^{7}$ & $9.16 \times 10^{4}$ & $3.31 \times 10^{2}$ \\
$\mathrm{Cu}^{+}+2 \mathrm{NO} \rightarrow \mathbf{4}$ & $7.29 \times 10^{14}$ & $3.59 \times 10^{8}$ & $2.50 \times 10^{3}$ & $9.30 \times 10^{-2}$ \\
$\mathrm{Cu}^{+}+2 \mathrm{NO}+\mathrm{O}_{2} \rightarrow \mathbf{5}$ & $4.84 \times 10^{11}$ & $8.31 \times 10^{3}$ & $3.85 \times 10^{-3}$ & $1.48 \times 10^{-8}$ \\
$\mathrm{Cu}^{+}+\mathrm{NO}_{2} \rightarrow \mathbf{6}^{\prime}$ & $4.73 \times 10^{6}$ & $2.60 \times 10^{1}$ & $5.80 \times 10^{-4}$ & $5.04 \times 10^{-8}$ \\
$\mathrm{Cu}^{+}+\mathrm{NO}_{2} \rightarrow \mathbf{7}^{\prime}$ & $7.22 \times 10^{5}$ & $1.02 \times 10^{1}$ & $5.34 \times 10^{-4}$ & $1.08 \times 10^{-7}$ \\
\hline
\end{tabular}


4.4. Theoretical study of the reaction mechanism

Table 4.5 Calculated Kinetic Constants for Key Elementary Steps on Cu-SAPO-34 and CuSSZ-13 at Different Temperatures.

\begin{tabular}{lcccc}
\hline \multicolumn{5}{c}{ Kinetic Constant, kr } \\
\cline { 2 - 5 } & 298 & \multicolumn{5}{c}{473} & 623 \\
\hline \multicolumn{5}{c}{ Cu-SAPO-34 } \\
\hline $1 \rightarrow 2$ & $9.27 \times 10^{-4}$ & $1.55 \times 10^{0}$ & $7.95 \times 10^{2}$ & $2.19 \times 10^{5}$ \\
$5 \rightarrow 6$ & $2.15 \times 10^{2}$ & $1.49 \times 10^{4}$ & $4.97 \times 10^{5}$ & $1.10 \times 10^{7}$ \\
$5 \rightarrow 7$ & $4.84 \times 10^{1}$ & $9.23 \times 10^{3}$ & $7.40 \times 10^{5}$ & $3.64 \times 10^{7}$ \\
$8 \rightarrow 9$ & $3.98 \times 10^{3}$ & $5.82 \times 10^{5}$ & $4.02 \times 10^{7}$ & $1.91 \times 10^{9}$ \\
$10 \rightarrow 11$ & $2.08 \times 10^{-2}$ & $1.09 \times 10^{-1}$ & $3.11 \times 10^{-1}$ & $8.18 \times 10^{-1}$ \\
$12 \rightarrow 13$ & $5.60 \times 10^{-4}$ & $3.56 \times 10^{-6}$ & $1.28 \times 10^{-4}$ & $3.34 \times 10^{-3}$ \\
\hline & & Cu-SSZ-13 & \\
\hline $1 \rightarrow 2$ & $1.30 \times 10^{-4}$ & $1.53 \times 10^{-1}$ & $5.59 \times 10^{1}$ & $1.06 \times 10^{4}$ \\
$5 \rightarrow 6$ & $1.59 \times 10^{3}$ & $4.98 \times 10^{4}$ & $8.37 \times 10^{5}$ & $9.68 \times 10^{6}$ \\
$5 \rightarrow 7$ & $9.00 \times 10^{3}$ & $1.86 \times 10^{6}$ & $1.76 \times 10^{8}$ & $1.14 \times 10^{10}$ \\
$8 \rightarrow 9$ & $2.03 \times 10^{1}$ & $2.86 \times 10^{3}$ & $1.73 \times 10^{5}$ & $6.44 \times 10^{6}$ \\
$10 \rightarrow 11$ & $2.59 \times 10^{3}$ & $1.54 \times 10^{2}$ & $4.84 \times 10^{2}$ & $1.39 \times 10^{1}$ \\
$12 \rightarrow 13$ & $5.89 \times 10^{-10}$ & $8.09 \times 10^{-8}$ & $3.44 \times 10^{-6}$ & $9.75 \times 10^{-5}$ \\
\hline
\end{tabular}

A comparison of the adsorption constants Kads obtained from the calculated Gibbs free energies at increasing temperatures (Table 4.4) shows that two NO molecules will adsorb preferentially on $\mathrm{Cu}^{+}$over $\mathrm{O}_{2}$ in the entire range of temperature considered. Therefore, reaction paths involving the adsorption of $\mathrm{O}_{2}$ on $\mathrm{Cu}^{+}$sites have not been further considered but a different possibility has been explored starting from the dinitrosyl complex 4 (Figures 4.8 and 4.11). This pathway is described in detail for the 6RB model in $\mathrm{Cu}-\mathrm{SAPO}-34$, but energies and geometries for the other sites in $\mathrm{Cu}-\mathrm{SAPO}-34$ and $\mathrm{Cu}-\mathrm{SSZ}-13$ are given in Tables 4.2 and 4.3, and in Figures 4.11 and 4.12. After the dinitrosyl formation, $\mathrm{O}_{2}$ adsorbs close to the two NO 
molecules attached to $\mathrm{Cu}^{+}$with which it interacts very favorably. In this system, which is significantly more stable than structure $1, \mathrm{O}_{2}$ is oriented in such a way that each $\mathrm{O}$ atom is interacting with the $\mathrm{N}$ atom of each $\mathrm{NO}$ molecule. The optimized $\mathrm{N}$ $\mathrm{O}$ distances are $1.59 \AA$ and $1.90 \AA$. The activation of the $\mathrm{O}-\mathrm{O}$ bond is evident from the increase of the bond length to $1.34 \AA$, from $1.23 \AA$ in the gas phase.

From structure 5, there are two possible pathways leading to the formation of two $\mathrm{NO}_{2}$. Both transition states, TS5-6 and TS5-7, have similar activation energies, 13.6 and $16.7 \mathrm{kcal} / \mathrm{mol}$ respectively. In the transition state TS5-6 the O-O bond length increases to $1.63 \AA$ and two N-O bonds are formed with optimized distances of 1.39 and $1.41 \AA$. The result of the $\mathrm{O}-\mathrm{O}$ dissociation is the formation of two $\mathrm{NO}_{2}$ molecules bonded to $\mathrm{Cu}^{+}$through the $\mathrm{N}$ atoms (structure 6 in Figure 4.8). In TS5-7, as the 0-0 bond increases to $1.63 \AA$, one NO molecule moves away and the corresponding $\mathrm{Cu}-\mathrm{N}$ bond completely breaks. In this process, the 0 coming from the $\mathrm{O}_{2}$ simultaneously binds to $\mathrm{Cu}^{+}$and to the leaving NO, with optimized $\mathrm{Cu}-\mathrm{O}$ and $\mathrm{O}-\mathrm{N}$ distances of 1.85 and $1.69 \AA$, respectively. A nitrite-like fragment is formed together with a $\mathrm{NO}_{2}$ molecule coordinated through the $\mathrm{N}$ atom (structure 7 in Figure 4.8). The subsequent formation of nitrate requires the rotation of the nitrite fragment to a less stable intermediate (structure 8 in Figure 4.9) where the $\mathrm{N}$ atom of the nitrite is closer to the $\mathrm{O}$ atom of the $\mathrm{NO}_{2}$. From 8 , an $\mathrm{O}$ transfer through TS8-9 to the nitrite fragment is feasible now to produce nitrate co-adsorbed with $\mathrm{NO}$ on $\mathrm{Cu}^{2+}$ (structure 9 in Figure 4.9). This step requires only $13.9 \mathrm{kcal} / \mathrm{mol}$ but increases to $24 \mathrm{kcal} / \mathrm{mol}$ if we consider the most stable complex, 7 , as a starting point.

Apart from the fact that exchange cations accelerate the $\mathrm{NO}$ oxidation by $\mathrm{O}_{2}$, there is also evidence that the $\mathrm{H}$-form of several zeolites enhances the same reaction. Halasz et al. ${ }^{78}$ observed that in H-ZSM-5 the oxidation of NO was heterogeneously catalyzed at much faster rate than the gas phase reaction at $25{ }^{\circ} \mathrm{C}$ and $350{ }^{\circ} \mathrm{C}$ proposing that the BA-OH groups were responsible for this effect. Stevenson et al..$^{38}$ on the other hand proposed that the extra framework Al could also be the active sites. Others $^{83,84}$, claim that confinement effects accelerate the gas phase oxidation of NO to $\mathrm{NO}_{2}$ inside the zeolite cavities. It seems that $\mathrm{NO}_{2}$ could be formed in a variety of ways and we have also considered the formation of nitrates directly from $\mathrm{NO}_{2}$, which 
4.4. Theoretical study of the reaction mechanism

is actually expected to take place under fast $\mathrm{SCR}$ conditions. Thus, $\mathrm{Cu}^{2+} \mathrm{NO}_{2}{ }^{-}$ (structures $7^{\prime}$ in Figure 4.8) forms by direct adsorption of $\mathrm{NO}_{2}$ on $\mathrm{Cu}^{+}$. It further reacts with another $\mathrm{NO}_{2}$ (structure 10 in Figure 4.9) to form a bidentate $\mathrm{Cu}^{2+} \mathrm{NO}_{3}{ }^{-}$ species and non-bonded NO molecule (structure 11 in Figure 4.9) through transition state TS10-11. This process has an interestingly low activation energy of 3.1 $\mathrm{kcal} / \mathrm{mol}$. This reaction is also possible starting from the nitrite fragment co-adsorbed with $\mathrm{NO}_{2}$ on the $\mathrm{Cu}^{2+}$ cation. The initial optimized structure includes a third $\mathrm{NO}_{2}$ in the gas phase (structure 12 in Figure 4.9). Similar to the previous step, a monodentate nitrate species is formed along with a NO not adsorbed. In contrast, the activation energy is somewhat higher in this case, $12.4 \mathrm{kcal} / \mathrm{mol}$. These last two reactions (which correspond to an Eley-Rideal mechanism) have significantly lower activation energies than the pathways starting from dinitrosyl. However, the loss of entropy experienced by the gas phase $\mathrm{NO}_{2}$ when coming to the transition state will render this reaction less probable, especially at high temperatures. This is clearly reflected in the predicted reaction rates in Table 4.5.

So far, we have discussed several pathways for the formation of nitrates and nitrites on $\mathrm{Cu}-\mathrm{SAPO}-34$ (6RB). The same pathways have been computed on $\mathrm{Cu}-\mathrm{SAPO}-34$ (6RC) and on $\mathrm{Cu}-\mathrm{SSZ}-13$ with the three possible distributions of the Al pairs (6RA, $6 \mathrm{RB}$ and $6 \mathrm{RC}$ models) and no significant differences have been observed. Figure 4.11 and 4.12 show the optimized geometries obtained for the Cu-SSZ-13 catalyst with the 6RB model, which are almost identical to the ones on Cu-SAPO-34. The corresponding activation and reactions energies (Figure 4.10) are also very similar and the same conclusions can be drawn: there are several pathways to form $\mathrm{NO}_{2}$ and nitrites from $\mathrm{NO}+\mathrm{O}_{2}$ involving activation energy barriers lower than $17 \mathrm{kcal} / \mathrm{mol}$, as well as nitrates although with somewhat higher activations energies. The most probable routes involve the previous chemisorption of both $\mathrm{NO}$ molecules on $\mathrm{Cu}^{+}$ and the nearby and the physisorption of the $\mathrm{O}_{2}$ molecule.

It is true that an excess of $\mathrm{NH}_{3}$ will influence the formation of nitrates and the mechanism might be different. We will indeed study the effect of $\mathrm{NH}_{3}$ in the next chapter using molecular dynamics techniques. But the study of the formation of $\mathrm{NO}_{3}{ }^{-}$ $/ \mathrm{NO}_{2}{ }^{-}$species without ammonia is critical for a complete understanding of the SCR process. Nonetheless, after the formation of $\mathrm{NO}_{3}{ }^{-} / \mathrm{NO}_{2}{ }^{-}$species, the direct participation of $\mathrm{NH}_{3}$ and/or $\mathrm{NH}_{4}{ }^{+}$is necessary to form $\mathrm{NH}_{4} \mathrm{NO}_{2}$ and/or $\mathrm{NH}_{4} \mathrm{NO}_{3}$ 
which will decompose into $\mathrm{N}_{2}$ and water. The participation of $\mathrm{NH}_{3}$ is also necessary for the reduction half cycle. We will now continue with possible routes for the reduction of $\mathrm{Cu}^{2+}$ to $\mathrm{Cu}^{+}$with $\mathrm{NO}$ and $\mathrm{NH}_{3}$.

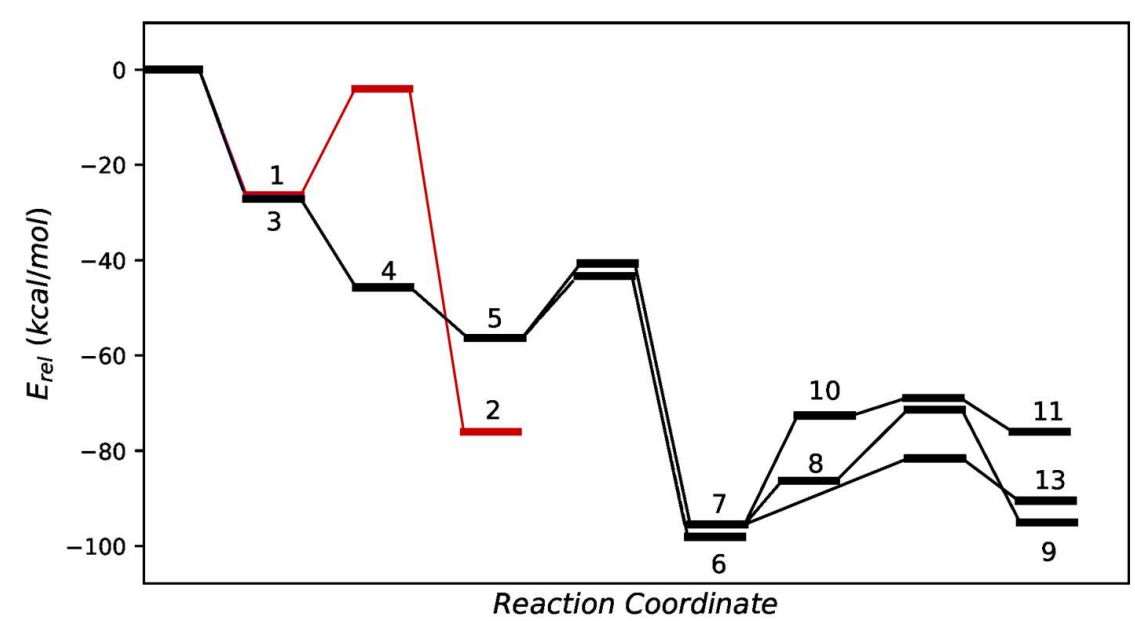

Figure 4.10 Calculated energy profile for all processes leading to formation of nitrites and nitrates from $\mathrm{NO}+\mathrm{O}_{2}$ and from $\mathrm{NO}_{2}$ in Cu-SSZ-13 with $\mathrm{Cu}^{+}$corresponding to model 6RB. The optimized structures involved are depicted in Figure 4.10 and Figure 4.11. The path $1 \rightarrow 2$ has been represented in red for better visualization of minima 1 and 3 . 
4.4. Theoretical study of the reaction mechanism
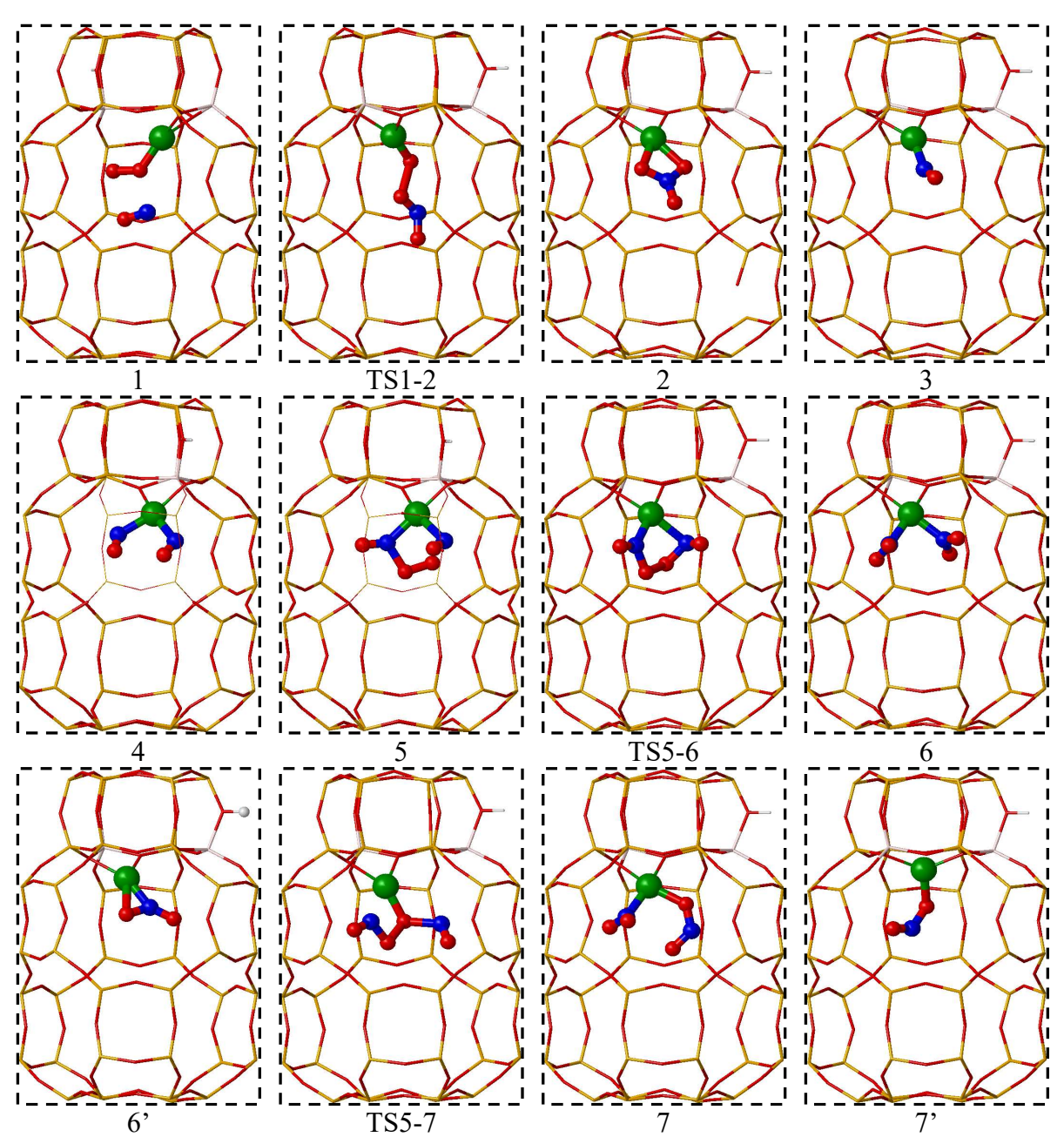

Figure 4.11 Optimized geometries of some $\mathrm{NO}$ and $\mathrm{O}_{2}$ adsorption complexes (1 to 7), and of the structures involved in the direct formation of nitrate (2) from co-adsorbed $\mathrm{NO}+\mathrm{O}_{2}$ in $6 \mathrm{RB}$ model of $\mathrm{Cu}-\mathrm{SSZ}-13 . \mathrm{Al}, \mathrm{O}, \mathrm{Si}$, and $\mathrm{H}$ atoms in the framework depicted as thatch, red, orange and white sticks; $\mathrm{Cu}$ cations, $\mathrm{O}$ and $\mathrm{N}$ atoms in the reactant molecules depicted as green, red and blue balls. 

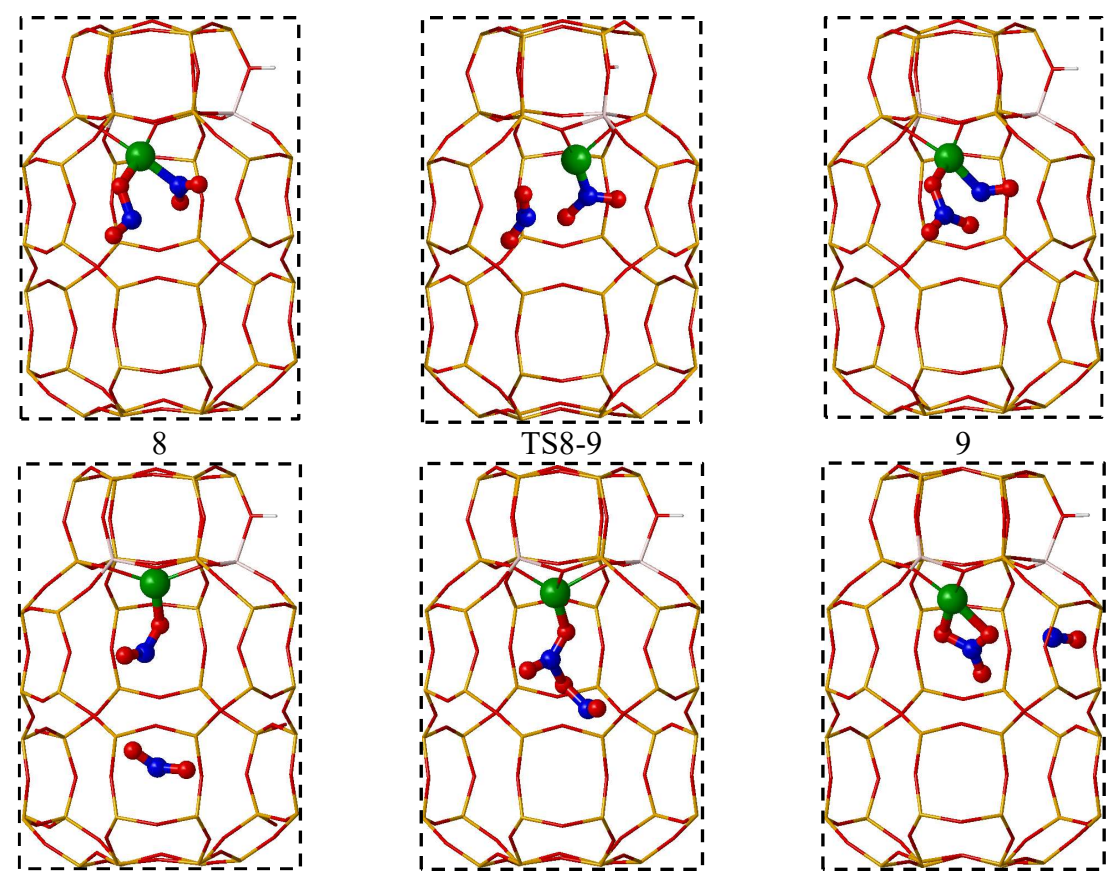

9

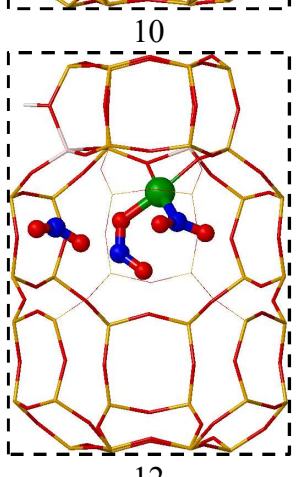

TS10-11
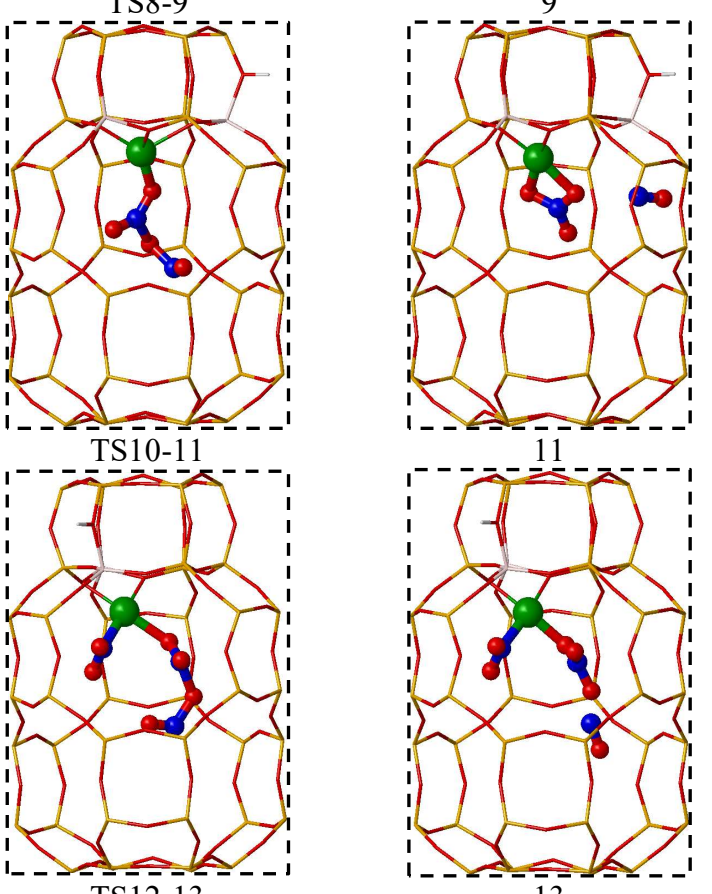

TS12-13

13

Figure 4.12 Optimized geometries of the structures involved in the formation of nitrites and nitrates from co-adsorbed $2 \mathrm{NO}+\mathrm{O}_{2}(5)$ in the $6 \mathrm{RB}$ model of $\mathrm{Cu}-\mathrm{SSZ}-13$. $\mathrm{Al}, \mathrm{O}, \mathrm{Si}$, and $\mathrm{H}$ atoms in the framework depicted as thatch, red, orange and white sticks; $\mathrm{Cu}$ cations, $\mathrm{O}$ and $\mathrm{N}$ atoms in the reactant molecules depicted as green, red and blue balls. 
4.4. Theoretical study of the reaction mechanism

\subsubsection{Reduction of $\mathrm{Cu}^{2+}$ to $\mathrm{Cu}^{+}$with $\mathrm{NO}$ and $\mathrm{NH}_{3}$}

As mentioned in the introduction, there is experimental evidence that the reduction of $\mathrm{Cu}^{2+}$ to $\mathrm{Cu}^{+}$, requires the simultaneous participation of $\mathrm{NO}$ and $\mathrm{NH}_{3}$. In this section, several pathways through which the reduction of $\mathrm{Cu}^{2+}$ to $\mathrm{Cu}^{+}$might take place have been investigated. These include reduction of $\mathrm{Cu}^{2+}$ with $\mathrm{NH}_{3}$ and $\mathrm{NO}$, and reduction of $\mathrm{Cu}^{2+}-\mathrm{OH}^{-}$with $\mathrm{NH}_{3}$ and $\mathrm{NO}$. The calculated relative energies are summarized in Table 4.2, the energy profiles on Cu-SAPO-34 and Cu-SSZ-13 are plotted in Figures 4.13 and 4.14, respectively, and the optimized structures are shown in Figures 4.15 and 4.16 for Cu-SAPO-34 model, and in Figures 4.17 and 4.18 for $\mathrm{Cu}-\mathrm{SSZ}-13$ models.

Table 4.6 Relative stability (in $\mathrm{kcal} / \mathrm{mol}$ ) of all structures of the model 6RB involved in the reduction of $\mathrm{Cu}^{2+}$ to $\mathrm{Cu}^{+}$by $\mathrm{NO}+\mathrm{NH}_{3}$ with respect to the sum of the energies of $\mathrm{Cu}-$ CHA catalyst $+\mathrm{NO}+\mathrm{NH}_{3}+\mathrm{H}_{2} \mathrm{O}$.

\begin{tabular}{cccc}
\hline Species & & $\mathrm{Cu}-\mathrm{SAPO}-34$ & $\mathrm{Cu}-\mathrm{SSZ}-13$ \\
\hline $\mathrm{Cu}^{2+}-\mathrm{NH}_{3}$ & 1 & -33.2 & -38.6 \\
& $\mathrm{TS}_{1-2}$ & 4.54 & 17.2 \\
$\mathrm{Cu}^{2+}-\mathrm{NH}_{2}{ }^{-}$ & 2 & -9.2 & -10.0 \\
$\mathrm{Cu}^{+}-\mathrm{H}_{2} \mathrm{NNO}$ & 3 & -42.0 & -42.0 \\
& $\mathrm{TS}_{3-4}$ & -7.2 & -11.5 \\
$\mathrm{Cu}^{+}-\mathrm{HNNOH}$ & 4 & -34.2 & -38.4 \\
$\mathrm{Cu}^{+}-\mathrm{HNNOH}$ & 5 & -41.9 & -47.7 \\
& $\mathrm{TS}_{5-6}$ & -12.4 & -14.2 \\
$\mathrm{Cu}^{+}-\mathrm{N}_{2}+\mathrm{H}_{2} \mathrm{O}(\mathrm{g})$ & 6 & -88.9 & -91.4 \\
$\mathrm{Cu}^{2+}-\mathrm{OH}$ & 7 & -5.7 & -5.2 \\
$\mathrm{Cu}^{2+}-\mathrm{OH}^{-}+\mathrm{NH}_{3}$ & 8 & -28.1 & -25.8 \\
& $\mathrm{TS}_{8-9}$ & 14.1 & 17.2 \\
$\mathrm{Cu}^{2+}-\mathrm{H}_{2} \mathrm{O}+\mathrm{NH}_{2}-$ & 9 & -18.3 & -6.3 \\
$\mathrm{Cu}^{2+}-\mathrm{OH}^{-}+\mathrm{NO}^{-}$ & 10 & -34.6 & -33.3 \\
$\mathrm{Cu}^{2+}-\mathrm{OH}^{-}+\mathrm{NH}_{3}+\mathrm{NO}$ & 11 & -47.2 & -43.5 \\
& $\mathrm{TS}_{11-12}$ & -44.0 & -40.4
\end{tabular}


Table 4.6 Relative stability (in $\mathrm{kcal} / \mathrm{mol}$ ) of all structures of the model $6 \mathrm{RB}$ involved in the reduction of $\mathrm{Cu}^{2+}$ to $\mathrm{Cu}^{+}$by $\mathrm{NO}+\mathrm{NH}_{3}$ with respect to the sum of the energies of $\mathrm{Cu}-$ CHA catalyst $+\mathrm{NO}+\mathrm{NH}_{3}+\mathrm{H}_{2} \mathrm{O}$.

\begin{tabular}{cccc}
\hline Species & & $\mathrm{Cu}-\mathrm{SAPO}-34$ & $\mathrm{Cu}-\mathrm{SSZ}-13$ \\
\hline $\mathrm{Cu}^{+}-\mathrm{ONNH}_{2}$ & 12 & -47.3 & -54.2 \\
& $\mathrm{TS}_{12-13}$ & -17.2 & -14.9 \\
$\mathrm{Cu}^{+}-\mathrm{NOHNH}$ & 13 & -42.1 & -45.9 \\
$\mathrm{Cu}^{2+}-\mathrm{NO}$ & 14 & -28.1 & -28.9 \\
$\mathrm{Cu}^{2+}-\mathrm{NO}-\mathrm{NH}_{3}$ & 15 & -46.4 & -45.4 \\
& $\mathrm{TS}_{15-12}$ & -39.7 & -30.3 \\
\hline
\end{tabular}

The interaction of $\mathrm{NH}_{3}$ with $\mathrm{Cu}^{2+}$ to form structure 1 is very favorable, with a calculated adsorption energy of $-33.2 \mathrm{kcal} / \mathrm{mol}$ (Table 4.6 and Figure 4.13 ). In the optimized structure, $\mathrm{Cu}^{2+}$ is coordinated to three framework oxygens in addition to $\mathrm{NH}_{3}$ (Figure 4.15). One $\mathrm{H}$ atom of $\mathrm{NH}_{3}$ is forming an $\mathrm{H}$-bond with one of the negatively charged oxygens $\left(\mathrm{AlO}_{4}^{-}\right)$of the $6 r$. The reduction of $\mathrm{Cu}^{2+}$ with $\mathrm{NH}_{3}$ proceeds through the $\mathrm{H}$-transfer from $\mathrm{NH}_{3}$ to $6 r$ through TS1-2 with an activation energy of $37.8 \mathrm{kcal} / \mathrm{mol}$, resulting in the formation of $\mathrm{Cu}^{2+}-\mathrm{NH}_{2}{ }^{-}$and a Brønsted site (structure 2 in Figure 4.15). The next step involves the formation of nitrosamine $\left(\mathrm{H}_{2} \mathrm{NNO}\right)$ which is carried out by the N-N coupling of a $\mathrm{NO}$ molecule with $\mathrm{Cu}^{2+}$ $\mathrm{NH}_{2}{ }^{-}$. The $\mathrm{Cu}-\mathrm{N}$ distances, 1.89 and $2.33 \AA$, show that $\mathrm{H}_{2} \mathrm{NNO}$ is bonded to $\mathrm{Cu}^{+}$ through both $\mathrm{N}$ atoms (structure 3 in Figure 4.15). Nitrosamine has been proposed as a key intermediate ${ }^{35}$ in the reduction half cycle. Notice that it has the right stoichiometry to decompose into $\mathrm{N}_{2}$ and $\mathrm{H}_{2} \mathrm{O}$. From the adsorbed nitrosamine $\left(\mathrm{Cu}^{+}-\mathrm{H}_{2} \mathrm{NNO}\right)$, this decomposition can be achieved through two H-transfers (TS3-4 and TS5-6) from $\mathrm{N}$ to the neighboring $\mathrm{O}$. After the first hydrogen transfer, the $\mathrm{HNNOH}$ intermediate (structure 4) must rearrange so that the $\mathrm{O}$ and $\mathrm{H}$ atoms have the right orientation for the second $\mathrm{H}$-transfer (compare structures 4 and 5 in Figure 4.15). These two last steps have equally high activation energies, $\sim 29 \mathrm{kcal} / \mathrm{mol}$, as the formation of $\left[\mathrm{Cu}-\mathrm{NH}_{2}\right]^{+}$. These results are in agreement with a similar pathway reported for $\mathrm{Cu}-\mathrm{SAPO}-18^{85}$. They also suggest that there is another 
4.4. Theoretical study of the reaction mechanism

pathway for the reduction of $\mathrm{Cu}^{2+}$ with lower activation energies because the reduction half cycle is not the rate-determining step.

The formation of $\mathrm{H}_{2} \mathrm{NNO}$ will always require that $\mathrm{NH}_{3}$ loses a proton at some step. Another scenario to achieve this is the adsorption of $\mathrm{NH}_{3}$ on the frequently proposed $\mathrm{Cu}^{2+}-\mathrm{OH}^{-}$species (structures 7 and 8 ). In this way, the hydroxyl could accept a $\mathrm{H}$ atom from $\mathrm{NH}_{3}$ and form water and $\mathrm{NH}_{2}{ }^{-}$adsorbed on $\mathrm{Cu}^{2+}$ (structure 9). However, the activation energy, $42.1 \mathrm{kcal} / \mathrm{mol}$, is even greater than the ones found so far. Moreover, the product $\mathrm{H}_{2} \mathrm{O}-\mathrm{Cu}^{2+}-\mathrm{NH}_{2}{ }^{-}$is $10 \mathrm{kcal} / \mathrm{mol}$ less stable compared with the reactants, which agrees with the fact that $\mathrm{NH}_{2}^{-}$is more basic than $\mathrm{OH}^{-}$.

The initial adsorption of $\mathrm{NO}$ on $\mathrm{Cu}^{2+}-\mathrm{OH}^{-}$, instead of $\mathrm{NH}_{3}$, constitutes an alternative pathway. The $\mathrm{OH}^{-}-\mathrm{Cu}^{2+}-\mathrm{NO}$ adduct (structure 10 in Figure 4.16) is slightly more stable than $\mathrm{OH}^{-}-\mathrm{Cu}^{2+}-\mathrm{NH}_{3}$ (structure 8 in Figure 4.15). An $\mathrm{NH}_{3}$ from gas phase approaches and forms a complex where one $\mathrm{H}$ is interacting strongly with the $\mathrm{O}$ atom of the $\mathrm{OH}^{-}$fragment (structure 11 in Figure 4.16). At the same time, the two $\mathrm{N}$ atoms are at $2.0 \AA$ from each other perfectly oriented to form a new N-N bond. Now, the formation of the N-N bond facilitates the N-H breaking and the simultaneous formation of $\mathrm{O}-\mathrm{H}$ through TS11-12. In spite of the complexity of this step, the activation energy is surprisingly low, only $3.1 \mathrm{kcal} / \mathrm{mol}$. As a result, a water molecule and nitrosamine $\left(\mathrm{H}_{2} \mathrm{NNO}\right)$ are formed (structure 12 in Figure 4.16). The nitrosamine formed in this pathway is adsorbed only through one $\mathrm{N}$ atom. However, it is more stable by $10.3 \mathrm{kcal} / \mathrm{mol}$ than the one absorbed through both $\mathrm{N}$ atoms (compare the relative stability of structure 3 and 12 in Figure 4.13). Since this structure is more stable we have calculated the activation energy required to decompose $\mathrm{H}_{2} \mathrm{NNO}$ with this geometry. Even though the intermediate resulting from the first H-transfer is more stable than its bidentate counterpart the activation energy of the transition state TS12-13 is still significantly high, $30.1 \mathrm{kcal} / \mathrm{mol}$. It can then be concluded that the decomposition of $\mathrm{H}_{2} \mathrm{NNO}$ does not occur on the $\mathrm{Cu}^{+}$site located in the $6 r$. 


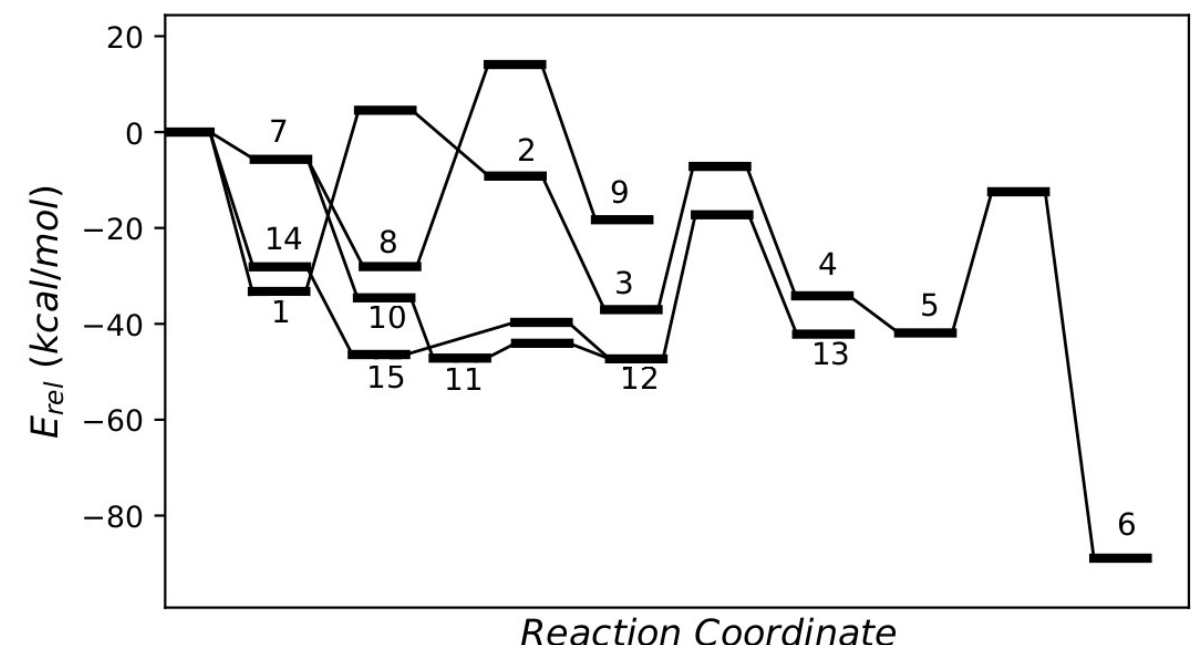

Figure 4.13 Calculated energy profile of the reduction of $\mathrm{Cu}^{2+}$ to $\mathrm{Cu}^{+}$on Cu-SAPO-34 with the $6 \mathrm{RB}$ model. Electronic energy is given in $\mathrm{kcal} / \mathrm{mol}$. Intermediates corresponding to minimum structures are labeled with numbers. The transition states are not labeled.

The N-N coupling between $\mathrm{NH}_{3}$ and $\mathrm{NO}$ can also occur on $\mathrm{Cu}^{2+}$ without the participation of $\mathrm{OH}^{-}$. It can proceed with an initial adsorption of $\mathrm{NO}$ on $\mathrm{Cu}^{2+}$, followed by adsorption of $\mathrm{NH}_{3}$ according to structure 15 . The orientation is again the right one to facilitate the N-N coupling. The N-N distance is $1.96 \AA$ and one N-H is interacting with the $\mathrm{AlO}_{4}{ }^{-}$through hydrogen bonding. Interestingly, this structure is only $2 \mathrm{kcal}$ less stable than the adsorption state where both $\mathrm{NH}_{3}$ and $\mathrm{NO}$ are coordinated to $\mathrm{Cu}^{2+}$ (compare structures 15 and 16 in Figure 4.16). The activation energy to extract a proton from $\mathrm{NH}_{3}$ and form $\mathrm{H}_{2} \mathrm{NNO}$ is again very low, $\sim 6$ $\mathrm{kcal} / \mathrm{mol}$.

In conclusion, the reduction half cycle of the SCR process seems to be aided by the exchange $\mathrm{Cu}^{2+}$ and $[\mathrm{Cu}-\mathrm{OH}]^{-}$species. The formation of the key intermediate nitrosamine on the active sites considered is favorable. Its decomposition is not likely to occur in the same active sites due to the high activation energies involved. Based on the literature we propose that nitrosamine desorbs from $\mathrm{Cu}^{+}$prior to its decomposition into $\mathrm{H}_{2} \mathrm{O}$ and $\mathrm{N}_{2}$. The decomposition of $\mathrm{H}_{2} \mathrm{NNO}$ on the Brønsted sites has been investigated theoretically by Bruggeman et al. ${ }^{56}$ and Mao et al. ${ }^{54}$ Both 
studies report low activation energies, thus supporting the hypothesis that $\mathrm{H}_{2} \mathrm{NNO}$ is readily decomposed in the Brønsted acid sites.

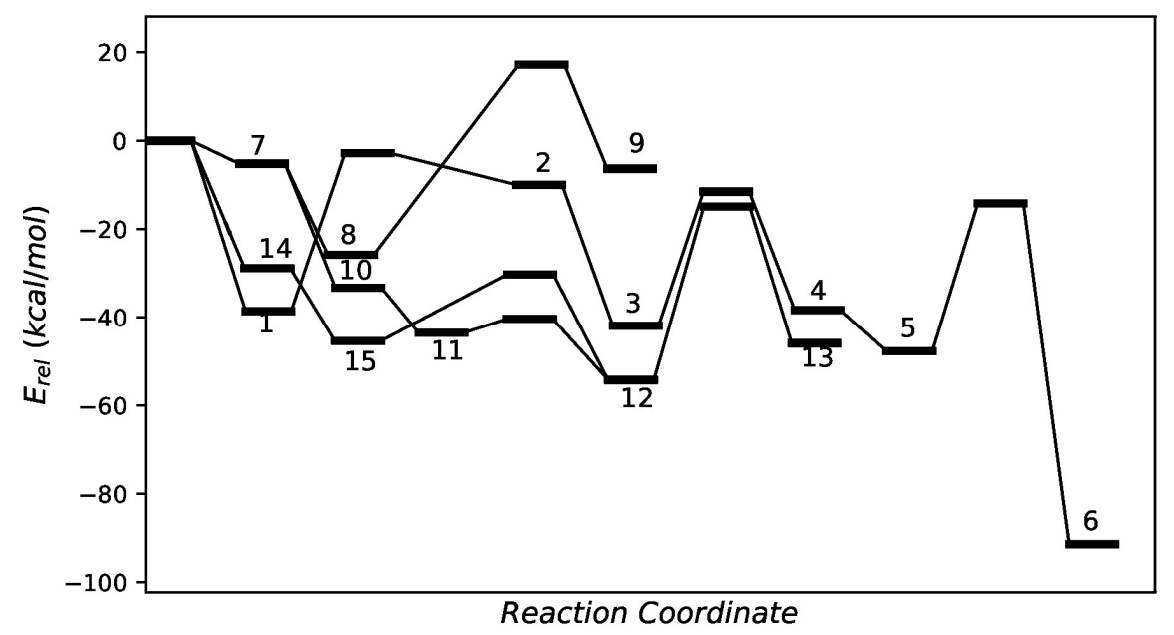

Figure 4.14 Calculated energy profile of the reduction of $\mathrm{Cu}^{2+}$ to $\mathrm{Cu}^{+}$on $\mathrm{Cu}-\mathrm{SSZ}-13$ with the $6 \mathrm{RB}$ model. Electronic energy is given in $\mathrm{kcal} / \mathrm{mol}$. Intermediates corresponding to minimum structures are labeled with numbers. The transition states are not labeled. 


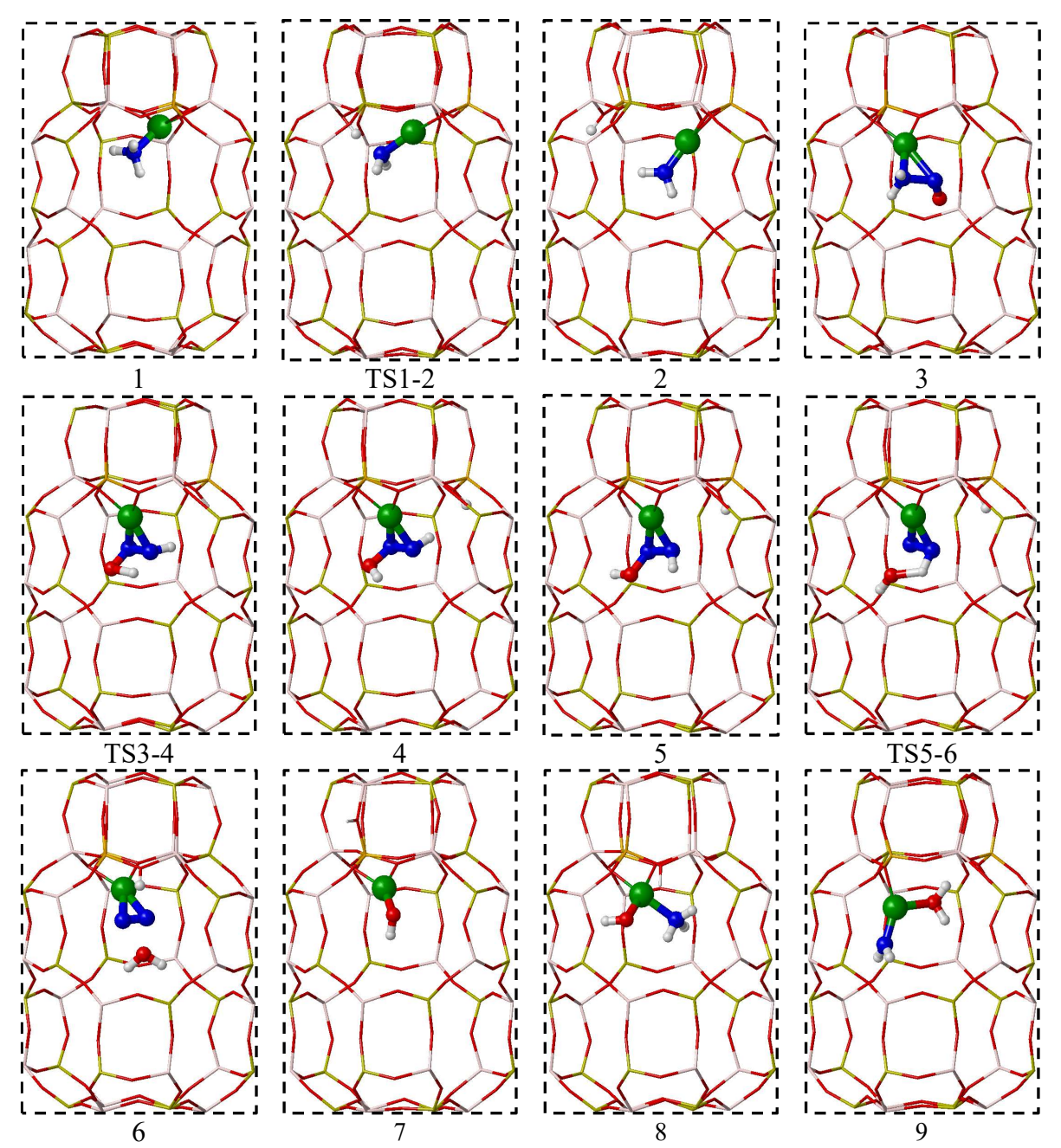

Figure 4.15. Optimized geometries of the structures involved in the reduction of $\mathrm{Cu}^{2+}$ to $\mathrm{Cu}^{+}$in the 6RB model of Cu-SAPO-34. Al, P, O, Si, and $\mathrm{H}$ atoms in the framework depicted as thatch, yellow, red, cyan and white sticks; $\mathrm{Cu}$ cations, $\mathrm{O}$ and $\mathrm{N}$ atoms in the reactant molecules depicted as green, red and blue balls. 

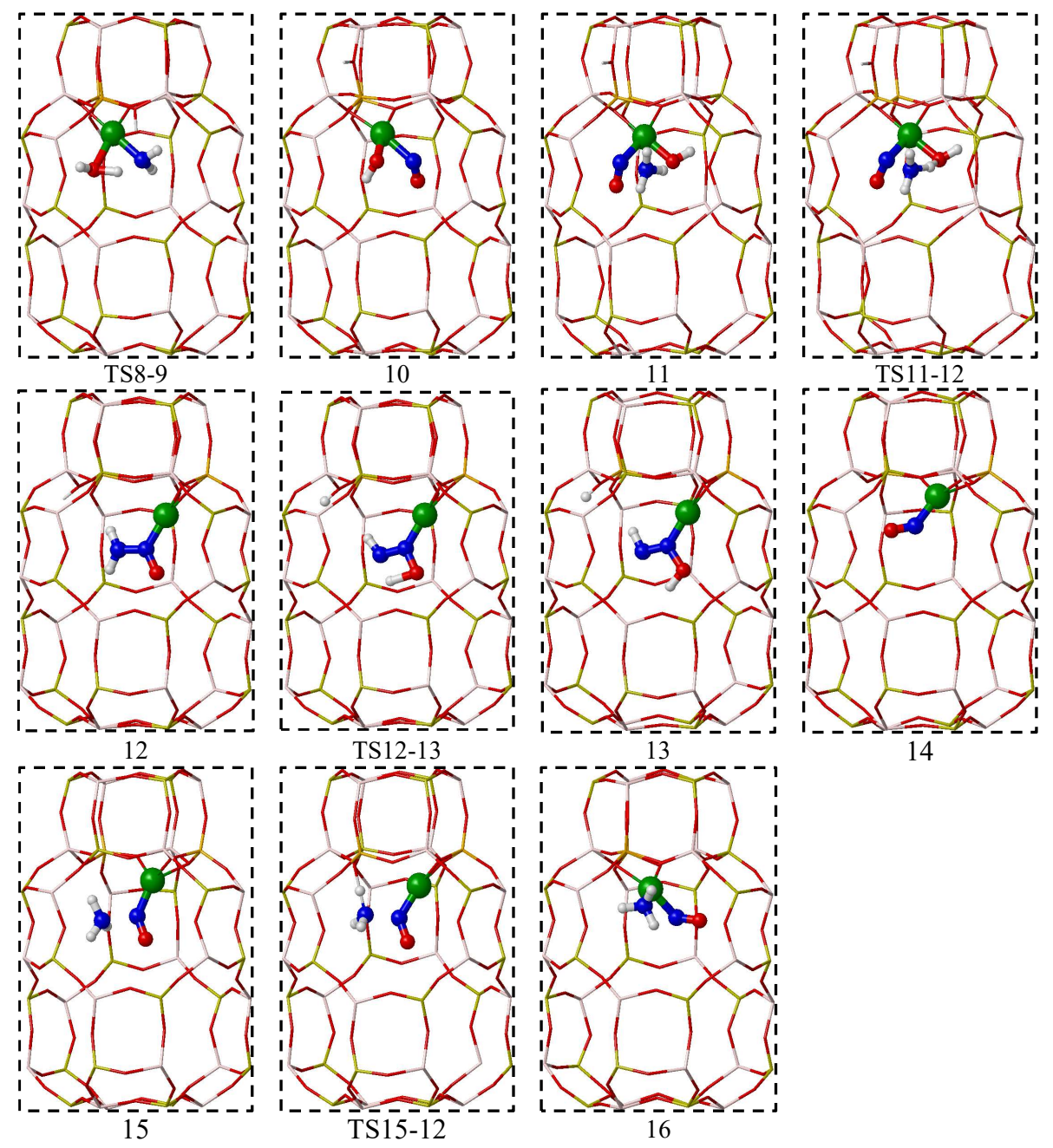

Figure 4.16. Optimized geometries of the structures involved in the reduction of $\mathrm{Cu}^{2+}$ to $\mathrm{Cu}^{+}$in the $6 \mathrm{RB}$ model of $\mathrm{Cu}-\mathrm{SAPO}-34$. Al, P, O, Si, and $\mathrm{H}$ atoms in the framework depicted as thatch, yellow, red, cyan and white sticks; $\mathrm{Cu}$ cations, $\mathrm{O}$ and $\mathrm{N}$ atoms in the reactant molecules depicted as green, red and blue balls. 

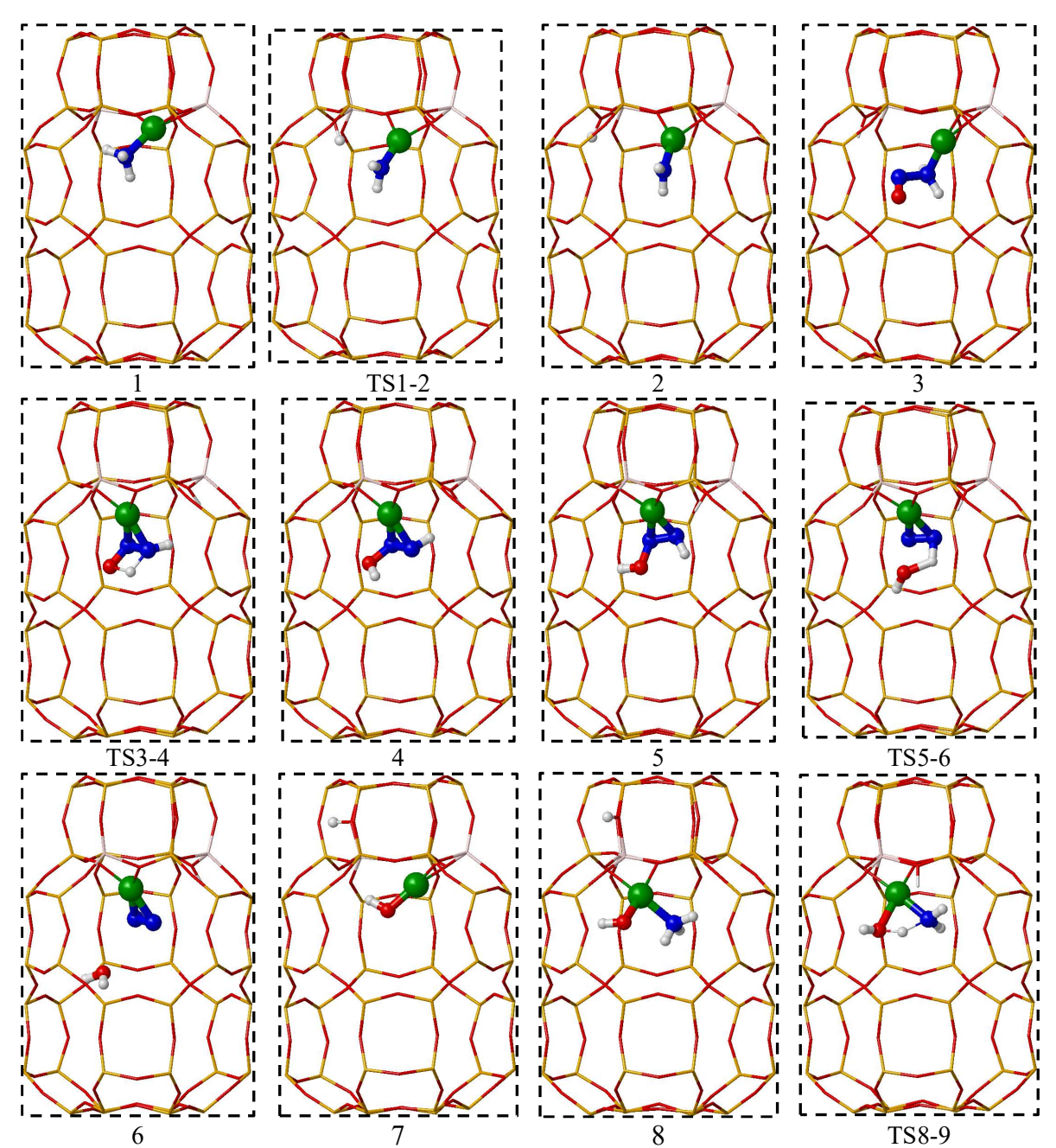

TS5-6

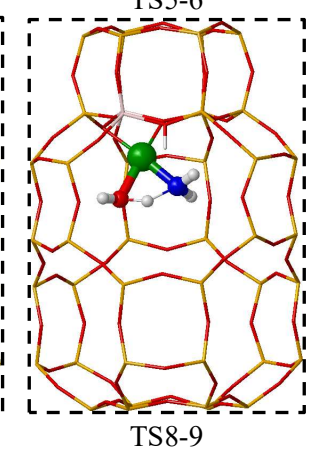

Figure 4.17. Optimized geometries of the structures involved in the reduction of $\mathrm{Cu}^{2+}$ to $\mathrm{Cu}^{+}$in the $6 \mathrm{RB}$ model of $\mathrm{Cu}-\mathrm{SSZ}-13 . \mathrm{Al}, \mathrm{P}, \mathrm{O}, \mathrm{Si}$, and $\mathrm{H}$ atoms in the framework depicted as thatch, yellow, red, cyan and white sticks; $\mathrm{Cu}$ cations, $\mathrm{O}$ and $\mathrm{N}$ atoms in the reactant molecules depicted as green, red and blue balls. 


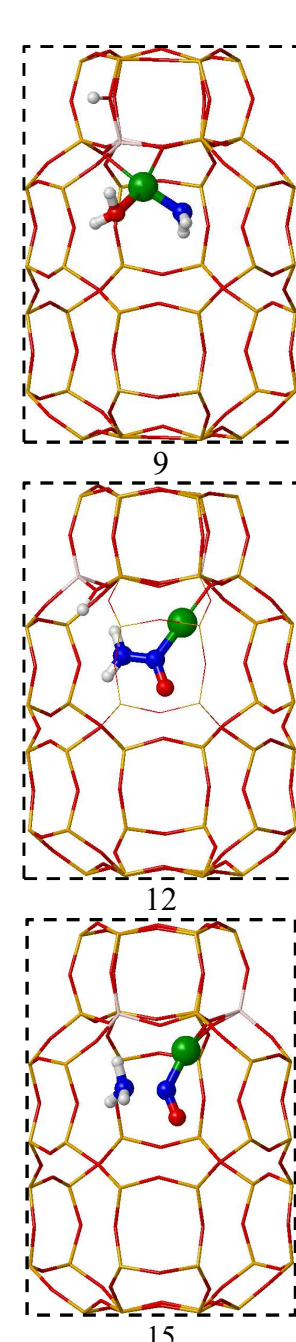

15

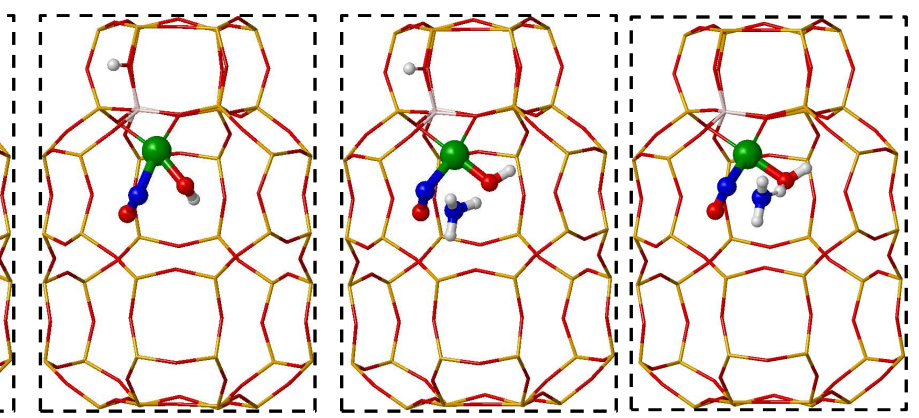

$\overline{0}-1$ L -1 TS $11-12$
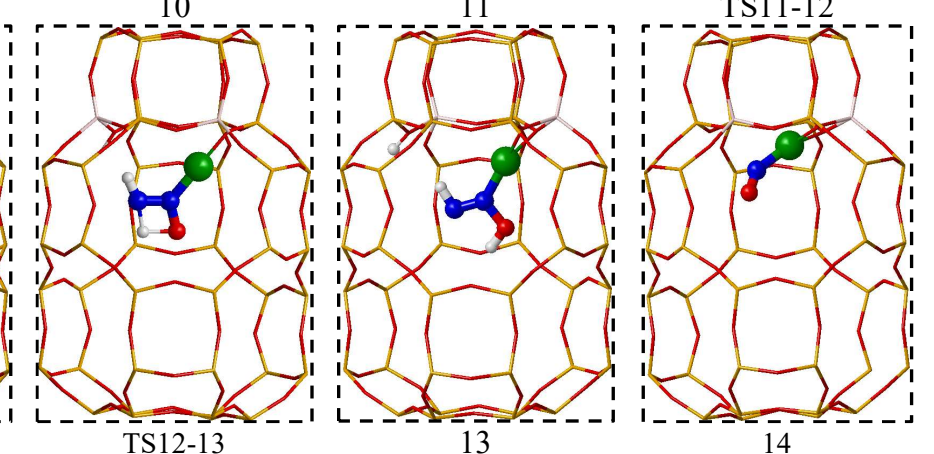

TS12-13

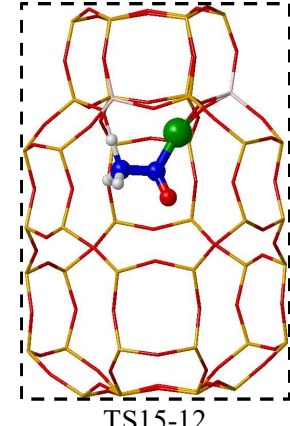

TS15-12

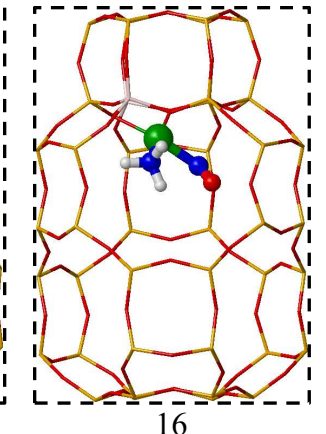

Figure 4.18. Optimized geometries of the structures involved in the reduction of $\mathrm{Cu}^{2+}$ to $\mathrm{Cu}^{+}$ in the $6 \mathrm{RB}$ model of $\mathrm{Cu}-\mathrm{SSZ}-13$. Al, $\mathrm{P}, \mathrm{O}, \mathrm{Si}$, and $\mathrm{H}$ atoms in the framework depicted as thatch, yellow, red, cyan and white sticks; $\mathrm{Cu}$ cations, $\mathrm{O}$ and $\mathrm{N}$ atoms in the reactant molecules depicted as green, red and blue balls. 


\subsection{Conclusions}

In this chapter we have used static DFT to help characterize the $\mathrm{Cu}-\mathrm{CHA}$ catalysts. First, we have built different models consistent with species proposed in the literature and concluded that the location of $\mathrm{Cu}^{+}$and $\mathrm{Cu}^{2+}$ in the plane of the $6 r$ is the most stable one for the $\mathrm{Al}$ (and $\mathrm{Si}$ ) pairs considered. However, in agreement with the literature we cannot postulate that this is the only existing state of $\mathrm{Cu}$ since isolated $\mathrm{Cu}^{2+}$ or $\mathrm{Cu}^{+}$ions located in $8 r$ positions, $[\mathrm{Cu}-\mathrm{OH}]^{+}$species, and $[\mathrm{Cu}-\mathrm{O}-\mathrm{Cu}]^{2+}$ dimers can coexist. The evidence comes from comparison of the calculated frequencies of NO vibrations with the experimental IR spectra using $\mathrm{NO}$ as probe molecule recorded on $\mathrm{Cu}-\mathrm{SSZ}-13$ and $\mathrm{Cu}-\mathrm{SAPO}-34$ samples synthesized in the Institute of Chemical Technology.

In this sense, the $[\mathrm{Cu}-\mathrm{OH}]^{+}$species have been well identified. The predicted IR bands at $\sim 1788-1798 \mathrm{~cm}^{-1}$ of the NO vibrations are consistent with the experiments. This species has been related however with an IR band at 1897 and $1895 \mathrm{~cm}^{-1} 86,87$. In disagreement with previous assignations, we associate the bands at 1897-1894 $\mathrm{cm}^{-1}$ to the interaction of NO with the Brønsted $\mathrm{H}$ by comparing the DFT-derived frequencies with spectra of the free and copper exchanged Cu-SSZ-13 and $\mathrm{Cu}-$ SAPO-34 samples. The dimeric copper oxo $[\mathrm{Cu}-\mathrm{O}-\mathrm{Cu}]^{2+}$ species was identified as well through the calculated vNO vibration at $1887 \mathrm{~cm}^{-1}$. This band has been associated with the IR band observed at $1882 \mathrm{~cm}^{-1}$ in the Cu-SAPO-34 sample, which has not been previously assigned in the literature.

Secondly, we can conclude that the oxidation of $\mathrm{Cu}^{+}$to $\mathrm{Cu}^{2+}$ can readily occur at low temperature mainly by reaction of $\mathrm{Cu}^{2+}$ dinitrosyl with $\mathrm{O}_{2}$ forming $\mathrm{NO}_{2}$ and nitrite in equilibrium with nitrates. This result supports the experimental evidence for the formation of nitrates and nitrites in the absence of $\mathrm{NH}_{3}$ and is in agreement with experimental reaction orders of dry $\mathrm{NO}$ oxidation on $\mathrm{Cu}-\mathrm{SSZ}-13$. It seems then, that $\mathrm{NH}_{3}$ does not directly participate in the oxidation of $\mathrm{Cu}^{+}$to $\mathrm{Cu}^{2+}$, although it might alter the state of the $\mathrm{Cu}^{+}$sites.

Finally, the DFT results on the reduction half cycle are in agreement with the experimental evidence that both $\mathrm{NO}$ and $\mathrm{NH}_{3}$ are key for the reduction of $\mathrm{Cu}^{2+}$ to $\mathrm{Cu}^{+}$. The proposed mechanism suggests that the formation of $\mathrm{H}_{2} \mathrm{NNO}$ occurs on $\mathrm{Cu}^{2+}$ 
through the $\mathrm{N}-\mathrm{N}$ coupling of $\mathrm{NO}$ and $\mathrm{NH}_{3}$ but its decomposition is more likely to occur on the Brønsted sites. 


\subsection{References}

(1) Centi, G.; Perathoner, S. Chapter 1 Introduction: State of the Art in the Development of Catalytic Processes for the Selective Catalytic Reduction of NOx into N2. In Studies in Surface Science and Catalysis; Granger, P., Pârvulescu, V. I., Eds.; Past and Present in DeNO Catalysis; Elsevier, 2007; Vol. 171, pp 1-23. https://doi.org/10.1016/S0167-2991(07)80202-3.

(2) Brandenberger, S.; Kroecher, O.; Tissler, A.; Althoff, R. The State of the Art in Selective Catalytic Reduction of NOx by Ammonia Using MetalExchanged Zeolite Catalysts. Catal. Rev.-Sci. Eng. 2008, 50 (4), 492-531. https://doi.org/10.1080/01614940802480122.

(3) NOJIRI, N.; SAKAI, Y.; WATANABE, Y. Two Catalytic Technologies of Much Influence on Progress in Chemical Process Development in Japan. Catal. $\quad$ Rev. 1995, $37 \quad 1), \quad$ 145-178. https://doi.org/10.1080/01614949508007093.

(4) Heck, R. M. Catalytic Abatement of Nitrogen Oxides-Stationary Applications. Catal. Today 1999, $53 \quad$ (4), 519-523. https://doi.org/10.1016/S0920-5861(99)00139-X.

(5) Nakajima, F.; Hamada, I. The State-of-the-Art Technology of NOx Control. Catal. Today 1996, 29 (1), 109-115. https://doi.org/10.1016/09205861(95)00288-X.

(6) Srivastava, R. K.; Hall, R. E.; Khan, S.; Culligan, K.; Lani, B. W. Nitrogen Oxides Emission Control Options for Coal-Fired Electric Utility Boilers. $J$. Air Waste Manag. Assoc. 2005, 55 (9), 1367-1388. https://doi.org/10.1080/10473289.2005.10464736.

(7) Yeh, S.; Rubin, E. S.; Taylor, M. R.; Hounshell, D. A. Technology Innovations and Experience Curves for Nitrogen Oxides Control Technologies. J. Air Waste Manag. Assoc. 2005, 55 (12), 1827-1838. https://doi.org/10.1080/10473289.2005.10464782.

(8) Amiridis, M. D.; Zhang, T.; Farrauto, R. J. Selective Catalytic Reduction of Nitric Oxide by Hydrocarbons. Appl. Catal. B Environ. 1996, 10 (1), 203227. https://doi.org/10.1016/0926-3373(96)00031-8.

(9) Hamada, H. Selective Reduction of NO by Hydrocarbons and Oxygenated Hydrocarbons over Metal Oxide Catalysts. Catal. Today 1994, 22 (1), 21-40. https://doi.org/10.1016/0920-5861(94)80090-1.

(10) Li, Y.; Armor, J. N. Catalytic Reduction of Nitrogen Oxides with Methane in the Presence of Excess Oxygen. Appl. Catal. B Environ. 1992, 1 (4), L31L40. https://doi.org/10.1016/0926-3373(92)80050-A.

(11) Fokema, M. D.; Ying, J. Y. The Selective Catalytic Reduction of Nitric Oxide with Methane Over Nonzeolitic Catalysts. Catal. Rev. 2001, 43 (1-2), 1-29. https://doi.org/10.1081/CR-100104385. 
(12) Shimizu, K.; Satsuma, A.; Hattori, T. Metal Oxide Catalysts for Selective Reduction of NOx by Hydrocarbons: Toward Molecular Basis for Catalyst Design. Catal. Surv. Jpn. 2001, 4 (2), 115-123. https://doi.org/10.1023/A:1011455304372.

(13) Kim, M. H.; Nam, I.-S. Water Tolerance of DeNOx SCR Catalysts Using Hydrocarbons: Findings, Improvements and Challenges. Korean J. Chem. Eng. 2001, 18 (5), 725-740. https://doi.org/10.1007/BF02706393.

(14) Kung, M. C.; Kung, H. H. Selective Lean NOxReduction over Metal Oxides. Top. Catal. 2004, 28 (1), 105-110. https://doi.org/10.1023/B:TOCA.0000024339.03914.fe.

(15) Koebel, M.; Elsener, M.; Kleemann, M. Urea-SCR: A Promising Technique to Reduce NOx Emissions from Automotive Diesel Engines. Catal. Today 2000, 59 (3), 335-345. https://doi.org/10.1016/S0920-5861(00)00299-6.

(16) Helden, R. van; Verbeek, R.; Willems, F.; Welle, R. van der. Optimization of Urea SCR DeNOx Systems for HD Diesel Engines; SAE Technical Paper 2004-01-0154; SAE International: Warrendale, PA, 2004. https://doi.org/10.4271/2004-01-0154.

(17) Iwamoto, M.; Furukawa, H.; Mine, Y.; Uemura, F.; Mikuriya, S.; Kagawa, S. Copper(II) Ion-Exchanged ZSM-5 Zeolites as Highly Active Catalysts for Direct and Continuous Decomposition of Nitrogen Monoxide. J. Chem. Soc. Chem. Commun. 1986, No. 16, 1272-1273. https://doi.org/10.1039/C39860001272.

(18) Iwamoto, M.; Yahiro, H.; Tanda, K.; Mizuno, N.; Mine, Y.; Kagawa, S. Removal of Nitrogen Monoxide through a Novel Catalytic Process. 1. Decomposition on Excessively Copper-Ion-Exchanged ZSM-5 Zeolites. $J$. Phys. Chem. 1991, 95 (9), 3727-3730. https://doi.org/10.1021/j100162a053.

(19) Yahiro, H.; Iwamoto, M. Copper Ion-Exchanged Zeolite Catalysts in DeNOx Reaction. Appl. Catal. Gen. 2001, 222 (1), 163-181. https://doi.org/10.1016/S0926-860X(01)00823-7.

(20) Beale, A. M.; Gao, F.; Lezcano-Gonzalez, I.; Peden, C. H. F.; Szanyi, J. Recent Advances in Automotive Catalysis for NOx Emission Control by Small-Pore Microporous Materials. Chem. Soc. Rev. 2015, 44 (20), 73717405. https://doi.org/10.1039/c5cs00108k.

(21) Wang, J.; Zhao, H.; Haller, G.; Li, Y. Recent Advances in the Selective Catalytic Reduction of NOx with NH3 on Cu-Chabazite Catalysts. Appl. Catal. B Environ. 2017, 202, 346-354. https://doi.org/10.1016/j.apcatb.2016.09.024.

(22) Kwak, J. H.; Tonkyn, R. G.; Kim, D. H.; Szanyi, J.; Peden, C. H. F. Excellent Activity and Selectivity of Cu-SSZ-13 in the Selective Catalytic Reduction 
of NOx with NH3. J. Catal. 2010, 275 (2), 187-190. https://doi.org/10.1016/j.jcat.2010.07.031.

(23) Martinez-Franco, R.; Moliner, M.; Concepcion, P.; Thogersen, J. R.; Corma, A. Synthesis, Characterization and Reactivity of High Hydrothermally Stable Cu-SAPO-34 Materials Prepared by "One-Pot" Processes. J. Catal. 2014, 314, 73-82. https://doi.org/10.1016/j.jcat.2014.03.018.

(24) Martínez-Franco, R.; Moliner, M.; Franch, C.; Kustov, A.; Corma, A. Rational Direct Synthesis Methodology of Very Active and Hydrothermally Stable Cu-SAPO-34 Molecular Sieves for the SCR of NOx. Appl. Catal. B Environ. 2012, 127, 273-280. https://doi.org/10.1016/j.apcatb.2012.08.034.

(25) Fickel, D. W.; D’Addio, E.; Lauterbach, J. A.; Lobo, R. F. The Ammonia Selective Catalytic Reduction Activity of Copper-Exchanged Small-Pore Zeolites. Appl. Catal. B Environ. 2011, 102 (3), 441-448. https://doi.org/10.1016/j.apcatb.2010.12.022.

(26) Deka, U.; Lezcano-Gonzalez, I.; Weckhuysen, B. M.; Beale, A. M. Local Environment and Nature of $\mathrm{Cu}$ Active Sites in Zeolite-Based Catalysts for the Selective Catalytic Reduction of NOx. ACS Catal. 2013, 3 (3), 413-427. https://doi.org/10.1021/cs300794s.

(27) Paolucci, C.; Di Iorio, J. R.; Ribeiro, F. H.; Gounder, R.; Schneider, W. F. Chapter One - Catalysis Science of NOx Selective Catalytic Reduction With Ammonia Over Cu-SSZ-13 and Cu-SAPO-34. In Advances in Catalysis; Song, C., Ed.; Academic Press, 2016; Vol. 59, pp 1-107. https://doi.org/10.1016/bs.acat.2016.10.002.

(28) Gao, F.; Kwak, J. H.; Szanyi, J.; Peden, C. H. F. Current Understanding of $\mathrm{Cu}$-Exchanged Chabazite Molecular Sieves for Use as Commercial Diesel Engine DeNO(x) Catalysts. Top. Catal. 2013, 56 (15), 1441-1459. https://doi.org/10.1007/s11244-013-0145-8.

(29) Lomachenko, K. A.; Borfecchia, E.; Negri, C.; Berlier, G.; Lamberti, C.; Beato, P.; Falsig, H.; Bordiga, S. The Cu-CHA DeNO(x) Catalyst in Action: Temperature-Dependent NH3-Assisted Selective Catalytic Reduction Monitored by Operando XAS and XES. J. Am. Chem. Soc. 2016, 138 (37), 12025-12028. https://doi.org/10.1021/jacs.6b06809.

(30) McEwen, J.-S.; Anggara, T.; Schneider, W. F.; Kispersky, V. F.; Miller, J. T.; Delgass, W. N.; Ribeiro, F. H. Integrated Operando X-Ray Absorption and DFT Characterization of $\mathrm{Cu}-\mathrm{SSZ}-13$ Exchange Sites during the Selective Catalytic Reduction of NOx with NH3. Catal. Today 2012, 184 (1), 129-144. https://doi.org/10.1016/j.cattod.2011.11.037.

(31) Praliaud, H.; Mikhailenko, S.; Chajar, Z.; Primet, M. Surface and Bulk Properties of $\mathrm{Cu}-\mathrm{ZSM}-5$ and $\mathrm{Cu} / \mathrm{Al} 2 \mathrm{O} 3$ Solids during Redox Treatments. 
Correlation with the Selective Reduction of Nitric Oxide by Hydrocarbons. Appl. Catal. B Environ. 1998, 16 (4), 359-374. https://doi.org/10.1016/S0926-3373(97)00093-3.

(32) Paolucci, C.; Khurana, I.; Parekh, A. A.; Li, S.; Shih, A. J.; Li, H.; Iorio, J. R. D.; Albarracin-Caballero, J. D.; Yezerets, A.; Miller, J. T.; Delgass, W. N.; Ribeiro, F. H.; Schneider, W. F.; Gounder, R. Dynamic Multinuclear Sites Formed by Mobilized Copper Ions in NOx Selective Catalytic Reduction. Science 2017, 357 (6354), 898-903. https://doi.org/10.1126/science.aan5630.

(33) Chen, H.-Y.; Wei, Z.; Kollar, M.; Gao, F.; Wang, Y.; Szanyi, J.; Peden, C. H. F. NO Oxidation on Zeolite Supported $\mathrm{Cu}$ Catalysts: Formation and Reactivity of Surface Nitrates. Catal. Today 2016, 267, 17-27. https://doi.org/10.1016/j.cattod.2015.11.039.

(34) Wang, D.; Zhang, L.; Kamasamudram, K.; Epling, W. S. In Situ-DRIFTS Study of Selective Catalytic Reduction of NOx by NH3 over Cu-Exchanged SAPO-34. Acs Catal. 2013, 3 (5), 871-881. https://doi.org/10.1021/cs300843k.

(35) Paolucci, C.; Verma, A. A.; Bates, S. A.; Kispersky, V. F.; Miller, J. T.; Gounder, R.; Delgass, W. N.; Ribeiro, F. H.; Schneider, W. F. Isolation of the Copper Redox Steps in the Standard Selective Catalytic Reduction on $\mathrm{Cu}-$ SSZ-13. Angew. Chem.-Int. Ed. 2014, 53 (44), 11828-11833. https://doi.org/10.1002/anie.201407030.

(36) Doronkin, D. E.; Casapu, M.; Günter, T.; Müller, O.; Frahm, R.; Grunwaldt, J.-D. Operando Spatially- and Time-Resolved XAS Study on Zeolite Catalysts for Selective Catalytic Reduction of NOx by NH3. J. Phys. Chem. C 2014, 118 (19), 10204-10212. https://doi.org/10.1021/jp5028433.

(37) Janssens, T. V. W.; Falsig, H.; Lundegaard, L. F.; Vennestrom, P. N. R.; Rasmussen, S. B.; Moses, P. G.; Giordanino, F.; Borfecchia, E.; Lomachenko, K. A.; Lamberti, C.; Bordiga, S.; Godiksen, A.; Mossin, S.; Beato, P. A Consistent Reaction Scheme for the Selective Catalytic Reduction of Nitrogen Oxides with Ammonia. Acs Catal. 2015, 5 (5), 2832-2845. https://doi.org/10.1021/cs501673g.

(38) Stevenson, S. A.; Vartuli, J. C.; Brooks, C. F. Kinetics of the Selective Catalytic Reduction of NO over HZSM-5. J. Catal. 2000, 190 (2), 228-239. https://doi.org/10.1006/jcat.1999.2747.

(39) Borfecchia, E.; Lomachenko, K. A.; Giordanino, F.; Falsig, H.; Beato, P.; Soldatov, A. V.; Bordiga, S.; Lamberti, C. Revisiting the Nature of Cu Sites in the Activated Cu-SSZ-13 Catalyst for SCR Reaction. Chem. Sci. 2015, 6 (1), 548-563. https://doi.org/10.1039/c4sc02907k.

(40) Kwak, J. H.; Varga, T.; Peden, C. H. F.; Gao, F.; Hanson, J. C.; Szanyi, J. 
Following the Movement of $\mathrm{Cu}$ Ions in a SSZ-13 Zeolite during Dehydration, Reduction and Adsorption: A Combined in Situ TP-XRD, XANES/DRIFTS Study. J. Catal. 2014, 314, 83-93. https://doi.org/10.1016/j.jcat.2014.03.003.

(41) Korhonen, S. T.; Fickel, D. W.; Lobo, R. F.; Weckhuysen, B. M.; Beale, A. M. Isolated Cu2+ Ions: Active Sites for Selective Catalytic Reduction of NO. Chem. Commun. 2011, 47 (2), 800-802. https://doi.org/10.1039/c0cc04218h.

(42) Beale, A. M.; Lezcano-Gonzalez, I.; Slawinksi, W. A.; Wragg, D. S. Correlation between $\mathrm{Cu}$ Ion Migration Behaviour and DeNO(x) Activity in $\mathrm{Cu}-\mathrm{SSZ}-13$ for the Standard NH3-SCR Reaction. Chem. Commun. 2016, 52 (36), 6170-6173. https://doi.org/10.1039/c6cc00513f.

(43) Kwak, J. H.; Zhu, H.; Lee, J. H.; Peden, C. H. F.; Szanyi, J. Two Different Cationic Positions in Cu-SSZ-13? Chem. Commun. 2012, 48 (39), 47584760. https://doi.org/10.1039/c2cc31184d.

(44) Paolucci, C.; Parekh, A. A.; Khurana, I.; Di Iorio, J. R.; Li, H.; Albarracin Caballero, J. D.; Shih, A. J.; Anggara, T.; Delgass, W. N.; Miller, J. T.; Ribeiro, F. H.; Gounder, R.; Schneider, W. F. Catalysis in a Cage: ConditionDependent Speciation and Dynamics of Exchanged Cu Cations in SSZ-13 Zeolites. J. Am. Chem. Soc. 2016, 138 (18), 6028-6048. https://doi.org/10.1021/jacs.6b02651.

(45) Fickel, D. W.; Lobo, R. F. Copper Coordination in Cu-SSZ-13 and Cu-SSZ16 Investigated by Variable-Temperature XRD. J. Phys. Chem. C 2010, 114 (3), 1633-1640. https://doi.org/10.1021/jp9105025.

(46) Gao, F.; Walter, E. D.; Karp, E. M.; Luo, J.; Tonkyn, R. G.; Kwak, J. H.; Szanyi, J.; Peden, C. H. F. Structure-Activity Relationships in NH3-SCR over Cu-SSZ-13 as Probed by Reaction Kinetics and EPR Studies. J. Catal. 2013, 300, 20-29. https://doi.org/10.1016/j.jcat.2012.12.020.

(47) Lezcano-Gonzalez, I.; Wragg, D. S.; Slawinski, W. A.; Hemelsoet, K.; Van Yperen-De Deyne, A.; Waroquier, M.; Van Speybroeck, V.; Beale, A. M. Determination of the Nature of the $\mathrm{Cu}$ Coordination Complexes Formed in the Presence of NO and NH3 within SSZ-13. J. Phys. Chem. C 2015, 119 (43), 24393-24403. https://doi.org/10.1021/acs.jpcc.5b06875.

(48) Lei, G. D.; Adelman, B. J.; Sárkány, J.; Sachtler, W. M. H. Identification of Copper(II) and Copper(I) and Their Interconversion in Cu/ZSM-5 De-NOx Catalysts. Appl. Catal. B Environ. 1995, 5 (3), 245-256. https://doi.org/10.1016/0926-3373(94)00043-3.

(49) Costa, P. D.; Modén, B.; D. Meitzner, G.; Ki Lee, D.; Iglesia, E. Spectroscopic and Chemical Characterization of Active and Inactive $\mathrm{Cu}$ Species in NO Decomposition Catalysts Based on Cu-ZSM5. Phys. Chem. Chem. Phys. 2002, 4 (18), 4590-4601. https://doi.org/10.1039/B203700A. 
(50) Andersen, C. W.; Bremholm, M.; Vennestrom, P. N. R.; Blichfeld, A. B.; Lundegaard, L. F.; Iversen, B. B. Location of $\mathrm{Cu} 2+$ in CHA Zeolite Investigated by X-Ray Diffraction Using the Rietveld/Maximum Entropy Method. Iucrj 2014, 382-386. https://doi.org/10.1107/s2052252514020181.

(51) Godiksen, A.; Stappen, F. N.; Vennestrøm, P. N. R.; Giordanino, F.; Rasmussen, S. B.; Lundegaard, L. F.; Mossin, S. Coordination Environment of Copper Sites in $\mathrm{Cu}-\mathrm{CHA}$ Zeolite Investigated by Electron Paramagnetic Resonance. J. Phys. Chem. C 2014, 118 (40), 23126-23138. https://doi.org/10.1021/jp5065616.

(52) Verma, A. A.; Bates, S. A.; Anggara, T.; Paolucci, C.; Parekh, A. A.; Kamasamudram, K.; Yezerets, A.; Miller, J. T.; Delgass, W. N.; Schneider, W. F.; Ribeiro, F. H. NO Oxidation: A Probe Reaction on Cu-SSZ-13. J. Catal. 2014, 312, 179-190. https://doi.org/10.1016/j.jcat.2014.01.017.

(53) Gao, F.; Washton, N. M.; Wang, Y.; Kollár, M.; Szanyi, J.; Peden, C. H. F. Effects of Si/Al Ratio on Cu/SSZ-13 NH3-SCR Catalysts: Implications for the Active Cu Species and the Roles of Brønsted Acidity. J. Catal. 2015, 331, 25-38. https://doi.org/10.1016/j.jcat.2015.08.004.

(54) Mao, Y.; Wang, Z.; Wang, H.-F.; Hu, P. Understanding Catalytic Reactions over Zeolites: A Density Functional Theory Study of Selective Catalytic Reduction of NOx by NH3 over Cu-SAPO-34. ACS Catal. 2016, 6 (11), 7882-7891. https://doi.org/10.1021/acscatal.6b01449.

(55) Rizzotto, V.; Chen, D.; Tabak, B. M.; Yang, J.-Y.; Ye, D.; Simon, U.; Chen, P. Spectroscopic Identification and Catalytic Relevance of $\mathrm{NH}_{4}^{+}$ Intermediates in Selective NOx Reduction over Cu-SSZ-13 Zeolites. Chemosphere 2020, 250, 126272. https://doi.org/10.1016/j.chemosphere.2020.126272.

(56) Bruggemann, T. C.; Keil, F. J. Theoretical Investigation of the Mechanism of the Selective Catalytic Reduction of Nitric Oxide with Ammonia on H-Form Zeolites. J. Phys. Chem. C 2008, 112 (44), 17378-17387. https://doi.org/10.1021/jp806674d.

(57) Li, J.; Li, S. New Insight into Selective Catalytic Reduction of Nitrogen Oxides by Ammonia over H-Form Zeolites: A Theoretical Study. Phys Chem Chem Phys 2007, 9 (25), 3304-3311. https://doi.org/10.1039/B700161D.

(58) Long, R. Q.; Yang, R. T. Reaction Mechanism of Selective Catalytic Reduction of NO with NH3 over Fe-ZSM-5 Catalyst. J. Catal. 2002, 207 (2), 224-231. https://doi.org/10.1006/jcat.2002.3528.

(59) Wallin, M.; Karlsson, C.-J.; Skoglundh, M.; Palmqvist, A. Selective Catalytic Reduction of NOx with NH3 over Zeolite H-ZSM-5: Influence of Transient 
Ammonia Supply. J. Catal. 2003, $218 \quad$ (2), 354-364. https://doi.org/10.1016/S0021-9517(03)00148-9.

(60) Devadas, M.; Kröcher, O.; Elsener, M.; Wokaun, A.; Mitrikas, G.; Söger, N.; Pfeifer, M.; Demel, Y.; Mussmann, L. Characterization and Catalytic Investigation of Fe-ZSM5 for Urea-SCR. Catal. Today 2007, 119 (1), 137144. https://doi.org/10.1016/j.cattod.2006.08.018.

(61) Metkar, P. S.; Balakotaiah, V.; Harold, M. P. Experimental and Kinetic Modeling Study of NO Oxidation: Comparison of $\mathrm{Fe}$ and $\mathrm{Cu}-\mathrm{Z}$ eolite Catalysts. Catal. Today 2012, 184 (1), 115-128. https://doi.org/10.1016/j.cattod.2011.11.032.

(62) Tyrsted, C.; Borfecchia, E.; Berlier, G.; Lomachenko, K. A.; Lamberti, C.; Bordiga, S.; Vennestrom, P. N. R.; Janssens, T. V. W.; Falsig, H.; Beato, P.; Puig-Molina, A. Nitrate-Nitrite Equilibrium in the Reaction of $\mathrm{NO}$ with a $\mathrm{Cu}-$ CHA Catalyst for NH3-SCR. Catal Sci Technol 2016, 6 (23), 8314-8324. https://doi.org/10.1039/C6CY01820C.

(63) Gao, F.; Mei, D.; Wang, Y.; Szanyi, J.; Peden, C. H. F. Selective Catalytic Reduction over Cu/SSZ-13: Linking Homo- and Heterogeneous Catalysis. $J$. Am. Chem. Soc. 2017, 139 (13), 4935-4942. https://doi.org/10.1021/jacs.7b01128.

(64) Lamberti, C.; Bordiga, S.; Salvalaggio, M.; Spoto, G.; Zecchina, A.; Geobaldo, F.; Vlaic, G.; Bellatreccia, M. XAFS, IR, and UV-Vis Study of the CuI Environment in CuI-ZSM-5. J. Phys. Chem. B 1997, 101 (3), 344360. https://doi.org/10.1021/jp9601577.

(65) Lamberti, C.; Groppo, E.; Spoto, G.; Bordiga, S.; Zecchina, A. Infrared Spectroscopy of Transient Surface Species. In Advances in Catalysis; Gates, B. C., Knözinger, H., Eds.; Academic Press, 2007; Vol. 51, pp 1-74. https://doi.org/10.1016/S0360-0564(06)51001-6.

(66) Lamberti, C.; Zecchina, A.; Groppo, E.; Bordiga, S. Probing the Surfaces of Heterogeneous Catalysts by in Situ IR Spectroscopy. Chem. Soc. Rev. 2010, 39 (12), 4951-5001. https://doi.org/10.1039/C0CS00117A.

(67) Spoto, G.; Zecchina, A.; Bordiga, S.; Ricchiardi, G.; Martra, G.; Leofanti, G.; Petrini, G. Cu(I)-ZSM-5 Zeolites Prepared by Reaction of H-ZSM-5 with Gaseous $\mathrm{CuCl}$ : Spectroscopic Characterization and Reactivity towards Carbon Monoxide and Nitric Oxide. Appl. Catal. B Environ. 1994, 3 (2), 151172. https://doi.org/10.1016/0926-3373(93)E0032-7.

(68) Prestipino, C.; Berlier, G.; Llabrés i Xamena, F. X.; Spoto, G.; Bordiga, S.; Zecchina, A.; Turnes Palomino, G.; Yamamoto, T.; Lamberti, C. An in Situ Temperature Dependent IR, EPR and High Resolution XANES Study on the NO/Cu+-ZSM-5 Interaction. Chem. Phys. Lett. 2002, 363 (3), 389-396. 
https://doi.org/10.1016/S0009-2614(02)01183-1.

(69) Lamberti, C.; Palomino, G. T.; Bordiga, S.; Berlier, G.; D’Acapito, F.; Zecchina, A. Structure of Homoleptic CuI(CO)3 Cations in CuI-Exchanged ZSM-5 Zeolite: An X-Ray Absorption Study. Angew. Chem. 2000, 112 (12), 2222-2225.https://doi.org/10.1002/15213757(20000616)112:12<2222::AID-ANGE2222>3.0.CO;2-2.

(70) Palomino, G. T.; Bordiga, S.; Zecchina, A.; Marra, G. L.; Lamberti, C. XRD, XAS, and IR Characterization of Copper-Exchanged Y Zeolite. J. Phys. Chem. B 2000, 104 (36), 8641-8651. https://doi.org/10.1021/jp000584r.

(71) Perdew, J. P.; Wang, Y. Accurate and Simple Analytic Representation of the Electron-Gas Correlation Energy. Phys. Rev. B 1992, 45 (23), 13244-13249. https://doi.org/10.1103/PhysRevB.45.13244.

(72) Perdew, J. P.; Chevary, J. A.; Vosko, S. H.; Jackson, K. A.; Pederson, M. R.; Singh, D. J.; Fiolhais, C. Atoms, Molecules, Solids, and Surfaces: Applications of the Generalized Gradient Approximation for Exchange and Correlation. Phys. Rev. B 1992, 46 (11), 6671-6687. https://doi.org/10.1103/PhysRevB.46.6671.

(73) Kresse, G.; Furthmüller, J. Efficient Iterative Schemes for Ab Initio TotalEnergy Calculations Using a Plane-Wave Basis Set. Phys. Rev. B 1996, 54 (16), 11169-11186. https://doi.org/10.1103/PhysRevB.54.11169.

(74) Blöchl, P. E. Projector Augmented-Wave Method. Phys. Rev. B 1994, 50 (24), 17953-17979. https://doi.org/10.1103/PhysRevB.50.17953.

(75) Henkelman, G.; Jónsson, H. A Dimer Method for Finding Saddle Points on High Dimensional Potential Surfaces Using Only First Derivatives. J. Chem. Phys. 1999, 111 (15), 7010-7022. https://doi.org/10.1063/1.480097.

(76) Heyden, A.; Bell, A. T.; Keil, F. J. Efficient Methods for Finding Transition States in Chemical Reactions: Comparison of Improved Dimer Method and Partitioned Rational Function Optimization Method. J. Chem. Phys. 2005, 123 (22), 224101. https://doi.org/10.1063/1.2104507.

(77) Concepción, P.; Boronat, M.; Millán, R.; Moliner, M.; Corma, A. Identification of Distinct Copper Species in Cu-CHA Samples Using NO as Probe Molecule. A Combined IR Spectroscopic and DFT Study. Top. Catal. 2017, 60 (19), 1653-1663. https://doi.org/10.1007/s11244-017-0844-7.

(78) Halasz, I.; Brenner, A.; Simon Ng, K. Y. Active Sites of H-ZSM5 Catalysts for the Oxidation of Nitric Oxide by Oxygen. Catal. Lett. 1995, 34 (1), 151161. https://doi.org/10.1007/BF00808331.

(79) SEIFERT, J.; EMIG, G. Untersuchung Der Katalytischen NO-Oxidation Als Zwischenstufe Der Rauchgasentstickung. Unters. Katalytischen NO-Oxid. Als Zwischenstufe Rauchgasentstickung 1989, 61 (7), 560-561. 
(80) Shelef, M.; Montreuil, C. N.; Jen, H. W. NO2 Formation over Cu-ZSM-5 and the Selective Catalytic Reduction of NO. Catal. Lett. 1994, 26 (3), 277-284. https://doi.org/10.1007/BF00810600.

(81) Moreno-González, M.; Millán, R.; Concepción, P.; Blasco, T.; Boronat, M. Spectroscopic Evidence and Density Functional Theory (DFT) Analysis of Low-Temperature Oxidation of $\mathrm{Cu}^{+}$to $\mathrm{Cu}^{2+} \mathrm{NO}_{x}$ in Cu-CHA Catalysts: Implications for the SCR-NO ${ }_{x}$ Reaction Mechanism. ACS Catal. 2019, 9 (4), 2725-2738. https://doi.org/10.1021/acscatal.8b04717.

(82) Falsig, H.; Vennestrøm, P. N. R.; Moses, P. G.; Janssens, T. V. W. Activation of Oxygen and $\mathrm{NO}$ in NH3-SCR over Cu-CHA Catalysts Evaluated by Density Functional Theory. Top. Catal. 2016, 59 (10), 861-865. https://doi.org/10.1007/s11244-016-0560-8.

(83) Artioli, N.; Lobo, R. F.; Iglesia, E. Catalysis by Confinement: Enthalpic Stabilization of NO Oxidation Transition States by Micropororous and Mesoporous Siliceous Materials. J. Phys. Chem. C 2013, 117 (40), 2066620674. https://doi.org/10.1021/jp406333d.

(84) Maestri, M.; Iglesia, E. First-Principles Theoretical Assessment of Catalysis by Confinement: $\mathrm{NO}-\mathrm{O}_{2}$ Reactions within Voids of Molecular Dimensions in Siliceous Crystalline Frameworks. Phys. Chem. Chem. Phys. 2018, 20 (23), 15725-15735. https://doi.org/10.1039/C8CP01615A.

(85) Li, Y.; Deng, J.; Song, W.; Liu, J.; Zhao, Z.; Gao, M.; Wei, Y.; Zhao, L. Nature of $\mathrm{Cu}$ Species in Cu-SAPO-18 Catalyst for NH3-SCR: Combination of Experiments and DFT Calculations. J. Phys. Chem. C 2016, 120 (27), 14669-14680. https://doi.org/10.1021/acs.jpcc.6b03464.

(86) Giordanino, F.; R. Vennestrøm, P. N.; F. Lundegaard, L.; N. Stappen, F.; Mossin, S.; Beato, P.; Bordiga, S.; Lamberti, C. Characterization of $\mathrm{Cu}-$ Exchanged SSZ-13: A Comparative FTIR, UV-Vis , and EPR Study with $\mathrm{Cu}-$ ZSM-5 and $\mathrm{Cu}-\beta$ with Similar $\mathrm{Si} / \mathrm{Al}$ and $\mathrm{Cu} / \mathrm{Al}$ Ratios. Dalton Trans. 2013, 42 (35), 12741-12761. https://doi.org/10.1039/C3DT50732G.

(87) Dedecek, J.; Sobalik, Z.; Tvaruazkova, Z.; Kaucky, D.; Wichterlova, B. Coordination of $\mathrm{Cu}$ Ions in High-Silica Zeolite Matrixes. $\mathrm{Cu}+$ Photoluminescence, IR of NO Adsorbed on $\mathrm{Cu} 2+$, and $\mathrm{Cu} 2+$ ESR Study. $J$. Phys. Chem. 1995, 99 (44), 16327-16337. https://doi.org/10.1021/j100044a020. 


\section{Chapter 5 Insights on the NH3-SCR-NOx reaction from Molecular Dynamics simulations}

\subsection{Introduction}

The results of the previous chapter correspond to the study of the $\mathrm{NH}_{3}$-SCR-NOx reaction with $\mathrm{Cu}-\mathrm{CHA}$ catalysts using static DFT in which we did not consider the effect of $\mathrm{NH}_{3}$ in the oxidation half cycle. Ammonia acts primarily as the reducing agent. However, its slightly negative apparent reaction order means that too much $\mathrm{NH}_{3}$ will actually inhibit the reaction. The role of $\mathrm{NH}_{3}$ in the $\mathrm{SCR}$ reaction seems to be more complex than that of the other reactants. Let us review with more detail the current understanding of the role of ammonia.

Lezcano et al. ${ }^{1}$ identified three adsorption states for $\mathrm{NH}_{3}$ in $\mathrm{Cu}-\mathrm{SSZ}-13$ by using a combination of in situ Fourier Transform Infrared Spectroscopy with DFT calculations and temperature programmed desorption of $\mathrm{NH}_{3}$. A desorption peak below $473 \mathrm{~K}$ was ascribed to ammonia adsorbed on extra-framework $\mathrm{Al}$, another peak around $\sim 573 \mathrm{~K}$ was associated to ammonia adsorbed on the $\mathrm{Cu}$ Lewis sites, forming the complex $\left[\mathrm{Cu}\left(\mathrm{NH}_{3}\right)_{4}\right]^{2+}$ and a third desorption peak above $673 \mathrm{~K}$ was attributed to the adsorption on the Brønsted acid sites. They concluded that $\mathrm{NH}_{3}$ adsorbed on the Lewis acid sites is indispensable for the SCR reaction over $\mathrm{Cu}-\mathrm{SSZ}-13$, while the Brønsted centers act primarily as $\mathrm{NH}_{3}$ storage sites. Wang et $\mathrm{al}^{2}$, observed that the SCR reaction on Cu-SAPO-34 involves the formation of $\mathrm{NH}_{4} \mathrm{NO}_{3}$ and its reduction by $\mathrm{NO}$ producing $\mathrm{N}_{2}$ at $\sim 373 \mathrm{~K}$. They concluded that $\mathrm{NH}_{4} \mathrm{NO}_{3}$ is formed in the Lewis sites and the Brønsted sites, acting as reservoirs, supply $\mathrm{NH}_{3}$ through migration to the Lewis sites. Other authors ${ }^{3}$ have proposed that 
this migration of $\mathrm{NH}_{3}$ from the Brønsted to the Lewis sites might be the ratedetermining step at low temperature in $\mathrm{Cu}-\mathrm{SAPO}-34$.

Lunsford and co-workers ${ }^{4-6}$ reported the formation of tetraamine-copper(II) complex inside the cavities of zeolite $\mathrm{Cu}-\mathrm{Y}$ in the presence of $\mathrm{NH}_{3}$ and $\mathrm{O}_{2}$ as well as with additional NO in the gas feed. The authors proposed this species as the active sites for the reduction of $\mathrm{NO}$ by $\mathrm{NH}_{3}$ in Y-type zeolites ${ }^{5}$. However, Delabi et al. ${ }^{7}$ based on UV-vis-NIR spectroscopy and static DFT calculations on cluster models concluded that the $\mathrm{Cu}^{2+}$ cation in this complex must still be coordinated to one or two framework oxygens. Similar amino-copper complexes have been identified as well in the Cu-SSZ-13 zeolite by combining IR spectroscopy, EPR and DFT methods ${ }^{1,8-10}$.

The $\mathrm{Cu}^{+}$cation has also been found to interact strongly with $\mathrm{NH}_{3}$. Paolucci et al. ${ }^{11-13}$ and Gao et al. ${ }^{14-16}$ have proposed that under low temperature SCR conditions $(<523 \mathrm{~K})$ ammonia liberates the $\mathrm{Cu}^{+}$ions from its coordination with the zeolite framework forming mobile $\mathrm{Cu}^{+}\left(\mathrm{NH}_{3}\right)_{2}$ species. These mobile amino-copper complexes are responsible for the activation of $\mathrm{O}_{2}$ through the formation of the transient dimeric species $\mathrm{Cu}^{+}\left(\mathrm{NH}_{3}\right)_{2}-\mathrm{O}-\mathrm{O}-\mathrm{Cu}^{+}\left(\mathrm{NH}_{3}\right)_{2}$. The diffusion of the monomeric $\mathrm{Cu}^{+}\left(\mathrm{NH}_{3}\right)_{2}$ species to the adjacent cavity through the $8 r$ window is the rate-determining step at low $\mathrm{Cu}$ loading $\left(<10^{-4} \mathrm{Cu} / \mathrm{A}^{3}\right)$. This is consistent with the quadratic dependence of the catalytic activity on the $\mathrm{Cu}$ density observed by these authors. They also remarked that the linear dependence observed at higher copper loadings is evidence that the diffusion of $\mathrm{Cu}^{+}\left(\mathrm{NH}_{3}\right)_{2}$ is no longer the rate-determining step. These copper-ammonia adducts are similar to those found in homogeneous catalysis. Their diffusion is limited because of the electrostatic interactions with the zeolite framework, and thus constitute an intermediate situation between homogeneous and heterogeneous catalysis ${ }^{11,14,17}$. Lomachenko et al. ${ }^{18}$ agreed with the former authors on the formation of mobile $\mathrm{NH}_{3}$-solvated $\mathrm{Cu}$ species in the lowtemperature regime. However, their findings suggest that, above $523 \mathrm{~K}, \mathrm{Cu}$ is mostly present as framework-coordinated $\mathrm{Cu}$ (II) species which are probably the active sites of the high-temperature regime. It means that the SCR reaction seems to occur through different routes at different temperatures. There are in fact two temperature regimes with different apparent activation energies ${ }^{15,16,19}$ (Figure 5.1). Table 5.1 summarizes the values of apparent activation energies at different temperature 
ranges and $\mathrm{Cu}$ loadings, reported in the literature for different $\mathrm{Cu}-\mathrm{CHA}$ catalysts. The apparent activation energy varies significantly with the $\mathrm{Cu}$ content and with the temperature.

It is clear from the above discussion that, in the SCR reaction, ammonia is not just the reducing agent but it also modifies significantly the environment and location of the $\mathrm{Cu}^{+}$and $\mathrm{Cu}^{2+}$ cations. The SCR is thus a very complex and dynamic network of elementary processes whose kinetic relevance is very sensitive to the temperature, $\mathrm{Cu}$ loadings and concentration of reactant molecules. In this chapter, we focus on the interaction of the SCR reactant molecules $\left(\mathrm{O}_{2}, \mathrm{NO}, \mathrm{NO}_{2}\right.$, and $\left.\mathrm{NH}_{3}\right)$ with the copper cations using molecular dynamics at different temperatures in Cu-SSZ-13 and $\mathrm{Cu}-\mathrm{SAPO}-34$.

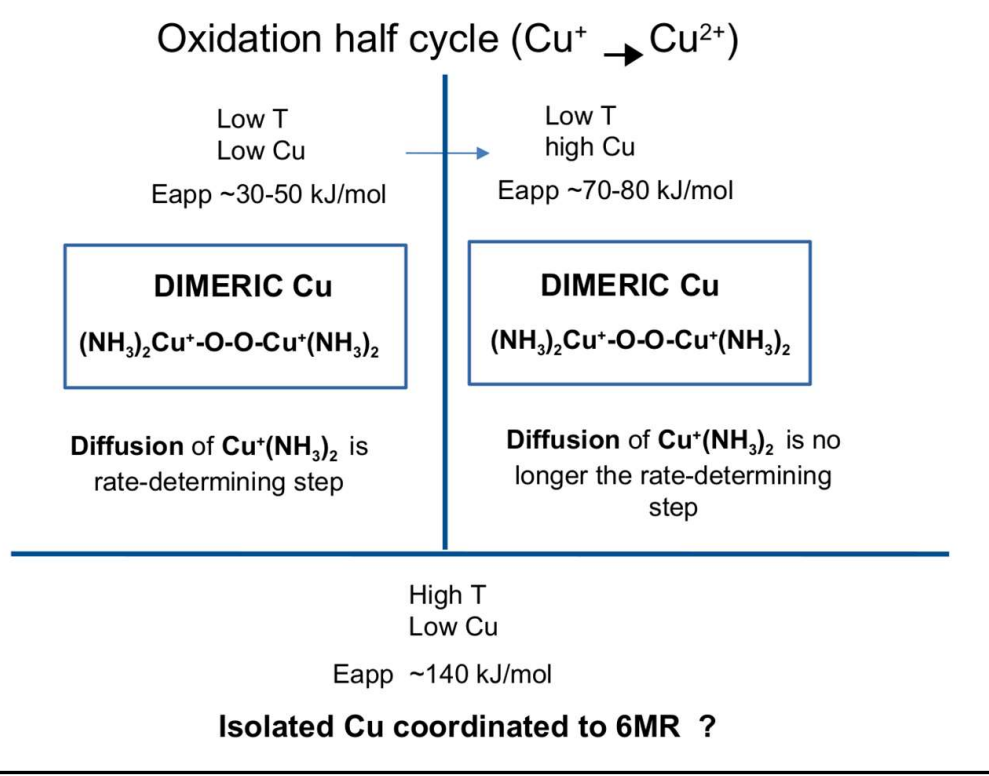

Figure 5.1 Scheme summarizing the mechanism dependence on the temperature and $\mathrm{Cu}$ loadings. 
Table 5.1 Apparent activation energies of different SCR-NOx catalysts with varying $\mathrm{Cu}$ loadings and at different temperature ranges.

\begin{tabular}{cccc}
\hline Catalyst & $\mathrm{Cu}(\% \mathrm{wt})$ & Temperature $(\mathrm{K})$ & Eapp $(\mathrm{kJ} / \mathrm{mol})$ \\
\hline $\mathrm{Cu}-\mathrm{SSZ}-13^{16}$ & 0.065 & $383-493$ & 43 \\
$\mathrm{Cu}-\mathrm{SSZ}-13^{16}$ & 5.15 & $370-470$ & 84 \\
$\mathrm{Cu}^{16} \mathrm{SZ}-13^{16}$ & $0.065-0.19$ & $600-700$ & 140 \\
$\mathrm{Cu}-\mathrm{SAPO}-34^{20}$ & $0.98-2.89$ & $373-473$ & 34 \\
$\mathrm{Cu}-\mathrm{SAPO}-34^{21}$ & 1.89 & $398-498$ & 32 \\
$\mathrm{Cu}-\mathrm{SSZ}-13^{11}$ & 0.3 & $\sim 473$ & 47 \\
\hline
\end{tabular}

\subsection{Methods and models}

In this chapter, we have used the model $6 \mathrm{RB}$ introduced in the previous chapter for $\mathrm{Cu}-\mathrm{SSZ}-13$ and $\mathrm{Cu}-\mathrm{SAPO}-34$. This model contains two substituting $\mathrm{Al}$ in the case of $\mathrm{Cu}-\mathrm{SSZ}-13$ and two subtituting $\mathrm{Si}$ in the case of $\mathrm{Cu}-\mathrm{SAPO}-34$. Both $\mathrm{Al}$ or $\mathrm{Si}$ are placed in the same $6 r$ (Figure 5.2). The negative charges introduced by this substitution are compensated by a proton and $\mathrm{C}^{+}$or by a $\mathrm{Cu}^{2+}$. The copper cations were always located in the plane of the $6 r$.

Static DFT calculations were performed at the revPBE ${ }^{22}$ level of theory with Grimme's D3 ${ }^{23}$ correction for long range interactions as implemented in VASP. The valence density was expanded in a plane wave basis set with a kinetic energy cutoff of $600 \mathrm{eV}$, and the effect of the core electrons in the valence density was taken into account by means of the projected augmented wave (PAW) formalism. In all calculations the Brillouin Zone was sampled at the gamma point. Electronic energies were converged to $10^{-7} \mathrm{eV}$ and geometries were optimized until forces on atoms were less than $0.01 \mathrm{eV} / \AA$. The positions of all atoms were allowed to relax in all geometry optimizations.

AIMD simulations were performed with the CP2K package at the revPBE-D3 level of theory. The Gaussian and plane waves (GPW) method was used with the TZVP basis set for all atoms except $\mathrm{Cu}$ which was described with the DZVP-MOLOPT basis set. A cutoff energy of 400 Ry was used for the auxiliary plane waves. Simulations in the NPT ensemble consisted in a production run of $50 \mathrm{ps}$ after $10 \mathrm{ps}$ 
5.3 Adsorption of reactant molecules on the copper cations with AIMD

of equilibration. Three temperatures were used, 298,523 and $673 \mathrm{~K}$, controlled by a Nosé-Hoover chain thermostat ${ }^{24}$ with three beads and a time constant of $300 \mathrm{fs}$. The pressure was set to $1 \mathrm{~atm}$ controlled with a Martyna-Tobias-Klein barostat ${ }^{25}$. The time step to integrate the equations of motion was set to $0.5 \mathrm{fs}$.

IR spectra of Cu-CHA catalysts exhibit some bands in the $800-1000 \mathrm{~cm}^{-1}$ region associated to the framework asymmetric T-O-T vibrations perturbed by the presence of coordinated cations. To assist in the assignation of such bands, vibrational frequencies were calculated using two approaches: static DFT and ab-initio molecular dynamics (AIMD).

On the one hand, the vibrational frequencies were determined for the optimized structures. To this end, the Hessian matrix is calculated using density functional perturbation theory (DFPT) ${ }^{26}$. In these calculations only the $\mathrm{Cu}$ atom, the adsorbed molecules and all atoms in the $d 6 r$ were included in the dynamical matrix. The intensities were derived from the Born effective charge tensor ${ }^{27}$. However, this approach does not provide information about the bandwidth so the spectra were built applying a Lorentzian line shape with full width at half-maximum (FWHM) of 10 $\mathrm{cm}^{-1}$.

On the other hand, as a consequence of the dynamic behavior of copper (see next sections) a static model might not fully describe the real system. Therefore, vibrational frequencies were also obtained from AIMD simulations. The spectra are computed as the Fourier transform of the dipole autocorrelation function. This approach allows to take into account anharmonicities and finite temperature effects $^{28}$. The dipole moments were calculated every 2 fs using the Berry phase approach on the simulations carried out at $298 \mathrm{~K}$ and $1 \mathrm{~atm}$.

\subsection{Adsorption of reactant molecules on the copper cations with AIMD}

We have studied the interaction of the SCR reactant molecules $\mathrm{NO}, \mathrm{NO}_{2}, \mathrm{O}_{2}$, and $\mathrm{NH}_{3}$ with the copper cations using ab-initio molecular dynamics (AIMD) aiming to gain more insight on the location and nature of the active sites under realistic reaction conditions. We have run AIMD simulations at 298, 523 and $623 \mathrm{~K}$ for Cu-SSZ-13, and at 298 and $523 \mathrm{~K}$ for $\mathrm{Cu}-\mathrm{SAPO}-34$. In order to have a quantitative notion of the 
mobility of copper when interacting with different molecules we have computed the root mean square deviations (RMSD) of $\mathrm{Cu}^{+}$and $\mathrm{Cu}^{2+}$ positions. We have also computed the average distance of the copper cations to the average plane of the $6 r$ $(\mathrm{Cu}-6 \mathrm{r})$ so as to have an idea of the extent of coordination of the copper cations with the oxygens of the $6 r$. See section 2.6.3 for details on the definition of $\mathrm{Cu}-6 \mathrm{r}$ distance.

When no molecules are adsorbed, both $\mathrm{Cu}^{+}$and $\mathrm{Cu}^{2+}$ cations are located in the plane of the $6 r$ forming three or four strong bonds with the framework oxygen atoms (Figure 5.2) in both $\mathrm{Cu}-\mathrm{SSZ}-13$ and $\mathrm{Cu}-\mathrm{SAPO}-34$. The average distance $\mathrm{Cu}-6 \mathrm{r}$ remains nearly constant for $\mathrm{Cu}^{+}$and $\mathrm{Cu}^{2+}$ in both materials, being larger for $\mathrm{Cu}^{+}$than for $\mathrm{Cu}^{2+}$ (Table 5.2). The RMSD is always larger for $\mathrm{Cu}^{+}$than for $\mathrm{Cu}^{2+}$ and increases with the increase of the temperature for both cations, although the increase is smaller for $\mathrm{Cu}^{2+}$. All these differences between $\mathrm{Cu}^{2+}$ and $\mathrm{Cu}^{+}$are a consequence of the stronger interaction of the divalent cation with the negatively charged $6 r$ oxygens.

Table 5.2 Average distance between $\mathrm{Cu}$ and the average plane of the $6 r(\mathrm{Cu}-6 \mathrm{r})$ and root mean square deviation (RMSD) obtained from AIMD simulations of $\mathrm{Cu}^{+}$and $\mathrm{Cu}^{2+}$ cations at 298, 523 and 673 K. Data obtained over 50 ps simulations.

\begin{tabular}{cccccccccccc}
\hline & \multicolumn{4}{c}{ Cu-SSZ-13 } & \multicolumn{4}{c}{ Cu-SAPO-34 } \\
\cline { 2 - 11 } Species & \multicolumn{3}{c}{ Cu-6r $(\AA)$} & \multicolumn{3}{c}{ RMSD $(\AA)$} & \multicolumn{2}{c}{ Cu-6r $(\AA)$} & \multicolumn{2}{c}{ RMSD $(\AA)$} \\
\cline { 2 - 11 } & $\mathbf{2 9 8}$ & $\mathbf{5 2 3}$ & $\mathbf{6 7 3}$ & $\mathbf{2 9 8}$ & $\mathbf{5 2 3}$ & $\mathbf{6 7 3}$ & $\mathbf{2 9 8}$ & $\mathbf{5 2 3}$ & $\mathbf{2 9 8}$ & $\mathbf{5 2 3}$ \\
\hline $\mathrm{Cu}^{+}$ & 0.30 & 0.31 & 0.32 & 0.30 & 0.44 & 0.50 & 0.51 & 0.48 & 0.30 & 0.47 \\
$\mathrm{Cu}^{2+}$ & 0.10 & 0.15 & 0.19 & 0.20 & 0.26 & 0.40 & 0.22 & 0.22 & 0.27 & 0.26 \\
\hline
\end{tabular}




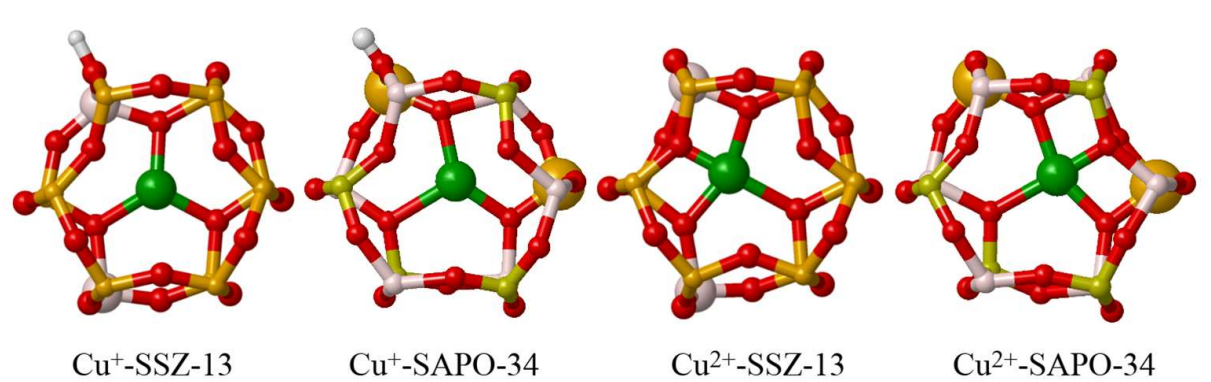

Figure 5.2 Snapshots of the NPT simulations at $298 \mathrm{~K}$ and $1 \mathrm{~atm}$ for $\mathrm{Cu}^{+}$and $\mathrm{Cu}^{2+}$ in $\mathrm{Cu}-\mathrm{SSZ}-13$ and $\mathrm{Cu}-\mathrm{SAPO}-34$. $\mathrm{Al}, \mathrm{Si}, \mathrm{P}, \mathrm{O}$ and $\mathrm{H}$ atoms are depicted as thatch, orange, yellow, red, white balls. $\mathrm{Cu}$ cations are shown as green balls.

\subsubsection{Interaction of the reactant molecules with $\mathrm{Cu}^{2+}$}

Since the interaction of $\mathrm{Cu}^{2+}$ with $\mathrm{O}_{2}, \mathrm{NO}$ and $\mathrm{NH}_{3}$ is very similar in $\mathrm{Cu}-\mathrm{SSZ}-13$ and $\mathrm{Cu}-\mathrm{SAPO}-34$ the following discussion applies to both materials. The average $\mathrm{Cu}-6 \mathrm{r}$ distance and the RMSD are summarized in Table 5.3. Snapshots of the trajectories of NPT simulations at $298 \mathrm{~K}$ and $1 \mathrm{~atm}$ are shown in Figures 5.3 and 5.4 for $\mathrm{Cu}-\mathrm{SSZ}-13$ and $\mathrm{Cu}-\mathrm{SAPO}-34$ respectively.

Table 5.3 Average distance between $\mathrm{Cu}$ and the $6 r$ plane $(\mathrm{Cu}-6 \mathrm{r})$ and root mean square deviation (RMSD) obtained from AIMD simulations of $\mathrm{Cu}^{2+}$ cations interacting with $\mathrm{O}_{2}$, $\mathrm{NO}, \mathrm{NO}_{2}$ and $\mathrm{NH}_{3}$ at 298,573 and $673 \mathrm{~K}$. Data obtained over 50 ps simulations.

\begin{tabular}{|c|c|c|c|c|c|c|c|c|c|c|}
\hline \multirow{3}{*}{ Species } & \multicolumn{6}{|c|}{ Cu-SSZ-13 } & \multicolumn{4}{|c|}{ Cu-SAPO } \\
\hline & \multicolumn{3}{|c|}{ Cu-6r $(\AA)$} & \multicolumn{3}{|c|}{ RMSD $(\AA)$} & \multicolumn{2}{|c|}{ Cu-6r ( $(\AA)$} & \multicolumn{2}{|c|}{ RMSD $(\AA)$} \\
\hline & $298 \mathrm{~K}$ & $523 \mathrm{~K}$ & $673 K$ & $298 \mathrm{~K}$ & $523 \mathrm{~K}$ & $673 K$ & $298 \mathrm{~K}$ & $523 \mathrm{~K}$ & $298 \mathrm{~K}$ & $523 \mathrm{~K}$ \\
\hline $\mathrm{Cu}^{2+}-\mathrm{O}_{2}$ & 0.11 & - & - & 0.19 & - & & 0.23 & - & 0.21 & - \\
\hline $\mathrm{Cu}^{2+}-\mathrm{NO}$ & & 0.24 & 0 . & 0.32 & 0.37 & .36 & 0.32 & 0.39 & 0.25 & 0.36 \\
\hline $\mathrm{Cu}^{2+}-2 \mathrm{NO}$ & 0.66 & 0.74 & 0.73 & 0.25 & 0.38 & 0.37 & 0.96 & 0.46 & 0.36 & 0.4 \\
\hline $\mathrm{Cu}^{2+}-\mathrm{ONO}^{-}-\mathrm{NO}_{2}$ & 2.58 & 1.67 & 1.97 & 0.24 & 0.38 & 0.69 & 1.12 & 1.14 & 0.26 & 0.43 \\
\hline $\mathrm{Cu}^{2+}-\mathrm{NO}-\mathrm{NO}_{3}^{-}$ & 1.91 & 2.24 & 2.24 & 0.23 & 0.45 & 0.64 & 1.07 & 1.23 & 0.36 & 0.53 \\
\hline $\mathrm{Cu}^{2+}-\mathrm{NH}_{3}$ & 0.65 & - & - & & - & - & 0.37 & 0.39 & 0.26 & 0.44 \\
\hline $\mathrm{Cu}^{2+}-2 \mathrm{NH}_{3}$ & 1.53 & 1.53 & 1.57 & 0.36 & 0.51 & 0.6 & 0.97 & 0.97 & 0.40 & 0.46 \\
\hline $\mathrm{Cu}^{2+}-\mathrm{NO}+\mathrm{NH}_{3}$ & 1.31 & 1.37 & 1.4 & 0.39 & 0.56 & 0.65 & - & - & - & - \\
\hline $\mathrm{Cu}^{2+}-4 \mathrm{NH}_{3}$ & 4.36 & 4.28 & 2.87 & 0.27 & 0.39 & 1.13 & 2.0 & 2.06 & 0.3 & 0.47 \\
\hline
\end{tabular}


One $\mathrm{O}_{2}$ molecule interacts very weakly with $\mathrm{Cu}^{2+}$ without the formation of chemical bonds (Figures 5.3a and 5.4a). The interaction of NO is stronger, but only one NO molecule binds to $\mathrm{Cu}^{2+}$, the second $\mathrm{NO}$ molecule drifts away from the copper cation (Figures $5.3 \mathrm{~b}$ and $5.3 \mathrm{c}, 5.4 \mathrm{~b}$ and $5.4 \mathrm{c}$ ). The system formed by nitrite and $\mathrm{NO}_{2}$ interacting with $\mathrm{Cu}^{2+}$ (Figures 5.3d and 5.4d), obtained through oxidation of $\mathrm{NO}$ with $\mathrm{O}_{2}$ (as described in section 4.5) is also stable. The nitrite species is monocoordianted to $\mathrm{Cu}^{2+}$ in $\mathrm{Cu}-\mathrm{SSZ}-13$ and $\mathrm{Cu}-\mathrm{SAPO}-34$ at $298 \mathrm{~K}$, but evolves differently in the two materials at higher temperature. In Cu-SSZ-13, the interaction of this nitrite species with $\mathrm{Cu}$ is weaker at $523 \mathrm{~K}$ and at $673 \mathrm{~K}$, it detaches from the $\mathrm{Cu}^{+}$site and remains in the cavity as a gas phase $\mathrm{NO}_{2}$ molecule (Figure 5.5a). In contrast, in Cu-SAPO-34 the nitrite remained coordinated to $\mathrm{Cu}^{2+}$ at $523 \mathrm{~K}$, although the $\mathrm{Cu}-\mathrm{O}$ bonds are somewhat more dynamic than at $298 \mathrm{~K}$. Thus, the original $\mathrm{Cu}-\mathrm{O} 1$ bond is broken and a new bond is formed between $\mathrm{Cu}^{2+}$ and the equivalent $\mathrm{O}_{2}$ atom of the nitrite anion (Figure 5.5c). A similar dynamic behavior is observed for $\mathrm{NO}_{3}{ }^{-}$co-adsorbed with $\mathrm{NO}$ (Figures 5.3e and 5.4e), in which an interchange of the $\mathrm{Cu}-\mathrm{O} 1$ and $\mathrm{Cu}-\mathrm{O} 2$ bonds occurs at $523 \mathrm{~K}$ in both materials (Figures $5.5 \mathrm{~b}$ and $5.5 \mathrm{~d}$ ).

In the case of ammonia, one and two molecules bind strongly to $\mathrm{Cu}^{2+}$, but their interaction is not strong enough to break its coordination with the framework oxygens and the $\mathrm{Cu}^{2+}$ cation remains always close to the $6 r$ plane (Figures 5.3f, 5.3g, $5.4 \mathrm{f}$ and 5.4g). Simulations with $\mathrm{Cu}^{2+}$ initially located in the plane of the $6 r$ and four uncoordinated $\mathrm{NH}_{3}$ molecules inside the cavity evolved to an adsorption state where $\mathrm{Cu}^{2+}$ is coordinated to three $\mathrm{NH}_{3}$ and one framework oxygen $(6 r)$ in square planar geometry (Figures $5.3 \mathrm{~h}$ and 5.4h). The $\mathrm{Cu}^{2+}$ cation is still coordinated to one $\mathrm{O}$ atom of the $6 r$ with an average $\mathrm{Cu}-\mathrm{O}$ bond length of $2.16 \AA$. The reason why the fourth $\mathrm{NH}_{3}$ did not bind to $\mathrm{Cu}^{2+}$ is probably because of its favorable hydrogen bond interaction with the other three $\mathrm{NH}_{3}$ molecules. In order to confirm whether four $\mathrm{NH}_{3}$ are enough to stabilize $\mathrm{Cu}^{2+}$ completely out of the $6 r$, we have simulated the same system with a different initial state. This time, $\mathrm{Cu}^{2+}$ is initially coordinated to the four $\mathrm{NH}_{3}$ molecules in the center of the cavity forming a square planar $\mathrm{Cu}^{2+}\left(\mathrm{NH}_{3}\right)_{4}$ complex (Figures 5.3i and 5.4i). This complex remained stable inside the cavity during the whole simulation time at the three temperatures. These results are in agreement with previously reported stability of the tetramine-copper (II) complex ${ }^{8,9}$. 


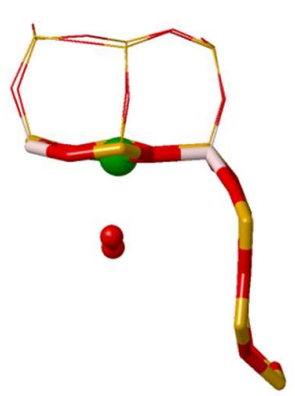

a) $\mathrm{O}_{2}$

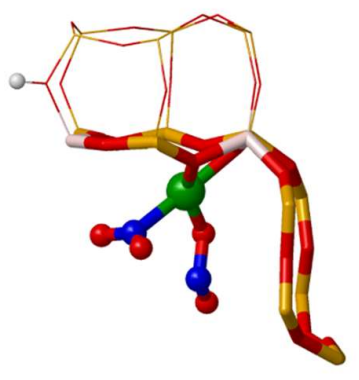

d) $\mathrm{ONO}^{-}-\mathrm{NO}_{2}$

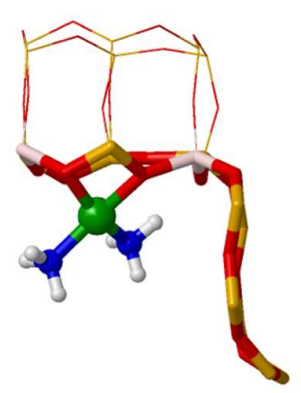

g) $2 \mathrm{NH}_{3}$

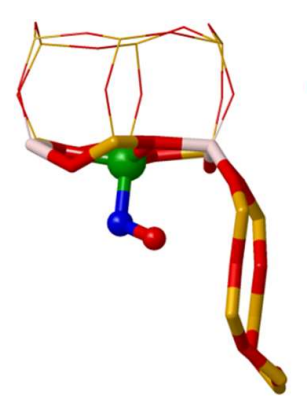

b) $\mathrm{NO}$

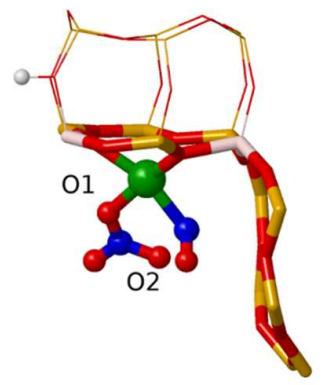

e) $\mathrm{NO}_{3}{ }^{-}-\mathrm{NO}$

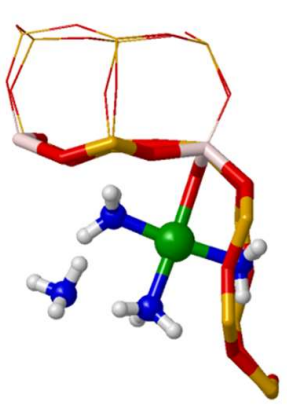

h) $4 \mathrm{NH}_{3}$

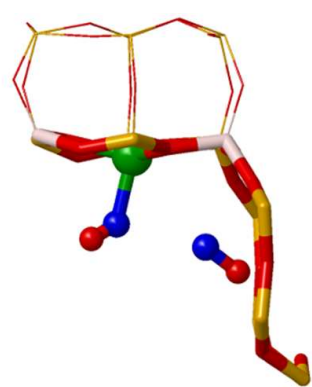

c) $2 \mathrm{NO}$

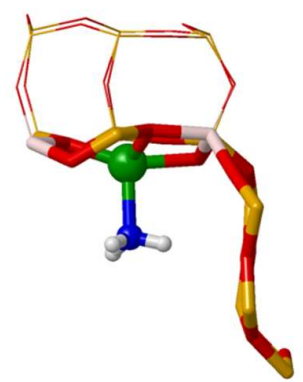

f) $\mathrm{NH}_{3}$

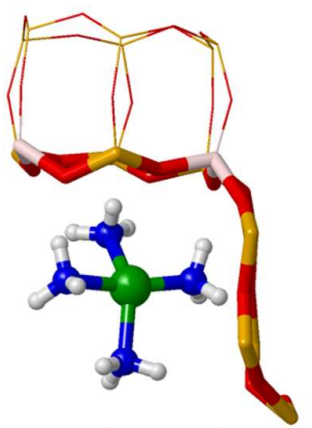

i) $4 \mathrm{NH}_{3}$

Figure 5.3 Simulation snapshots of the interaction of the $\mathrm{NH}_{3}-\mathrm{SCR}-\mathrm{NOx}$ reactant molecules with $\mathrm{Cu}^{2+}$ at $298 \mathrm{~K}$ in $\mathrm{Cu}-\mathrm{SSZ}-13 . \mathrm{Al}, \mathrm{O}$ and $\mathrm{Si}$ atoms in the framework depicted as thatch, red and orange sticks; $\mathrm{Cu}$ cations, $\mathrm{O}, \mathrm{N}$ and $\mathrm{H}$ atoms in the reactant molecules depicted as green, red, blue and white balls. 


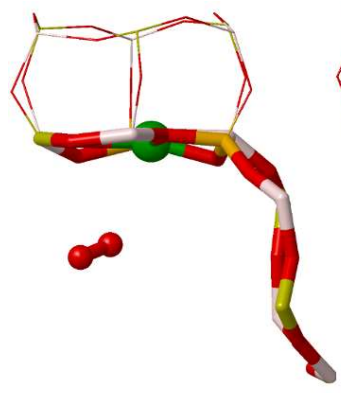

a) $\mathrm{O} 2$

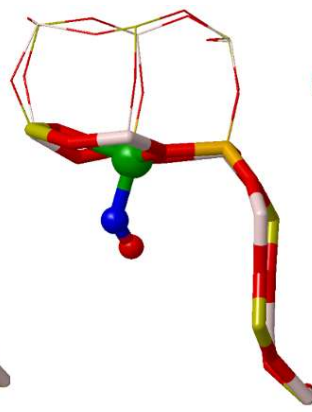

b) $\mathrm{NO}$

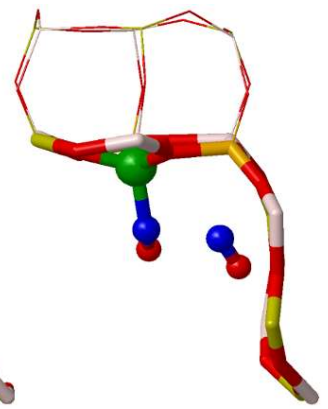

c) $2 \mathrm{NO}$

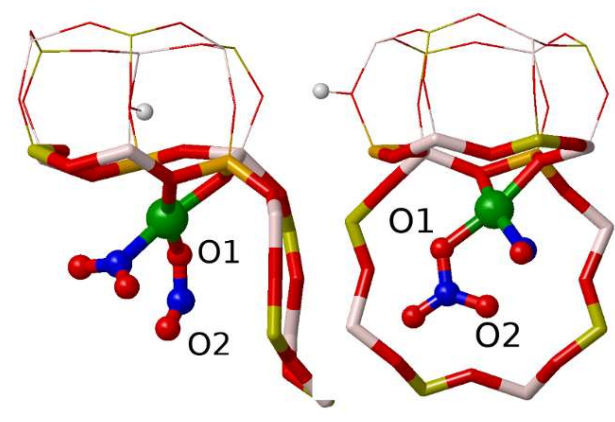

d) $\mathrm{ONO}^{-}-\mathrm{NO}_{2}$

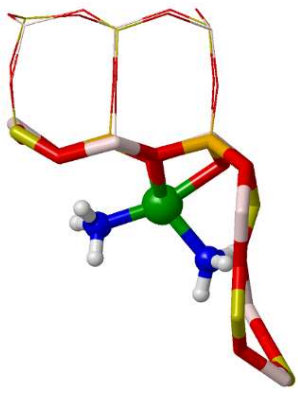

g) $2 \mathrm{NH}_{3}$ e) $\mathrm{NO}_{3}^{-}-\mathrm{NO}$

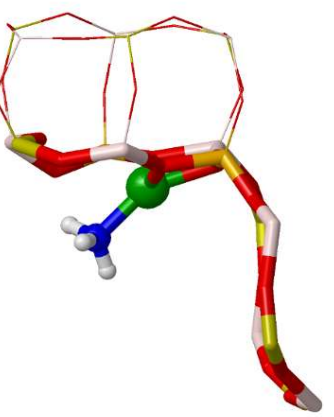

f) $\mathrm{NH}_{3}$

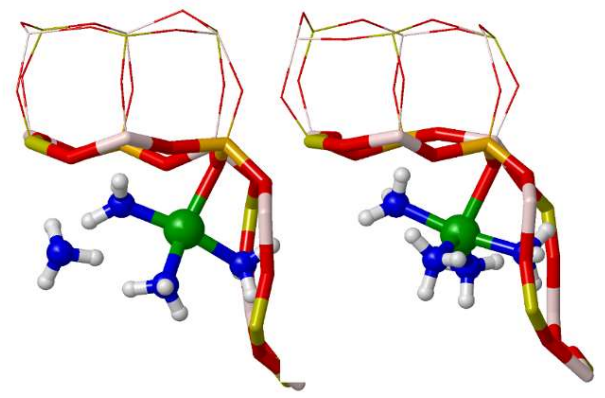

h) $4 \mathrm{NH}_{3}$

i) $4 \mathrm{NH}_{3}$

Figure 5.4 Simulation snapshots of the interaction of the SCR-NOx reactant molecules with $\mathrm{Cu}^{2+}$ at $298 \mathrm{~K}$ in $\mathrm{Cu}-\mathrm{SAPO}-34$. A1, P, O and $\mathrm{Si}$ atoms in the framework depicted as thatch, yellow, red and orange sticks; $\mathrm{Cu}$ cations, $\mathrm{O}, \mathrm{N}$ and $\mathrm{H}$ atoms in the reactant molecules depicted as green, red, blue and white balls. 

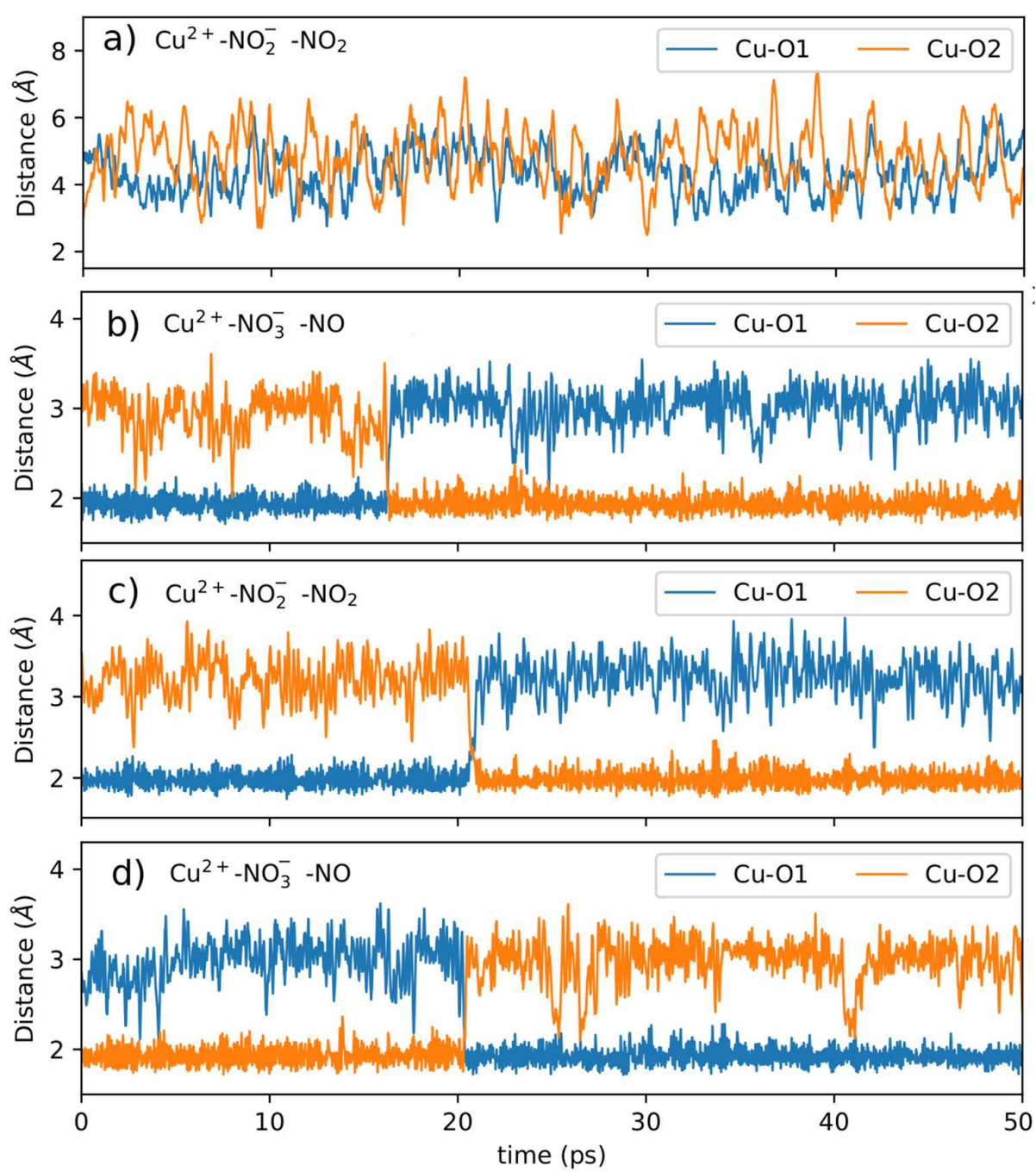

Figure 5.5 Time evolution of $\mathrm{Cu}-\mathrm{O} 1$ and $\mathrm{Cu}-\mathrm{O} 2$ distances during the AIMD simulations at $523 \mathrm{~K}$ of $\mathrm{Cu}^{2+}-\mathrm{NO}_{2}{ }^{-}-\mathrm{NO}_{2}\left(\mathrm{a}\right.$ and $\mathrm{c}$ ) and $\mathrm{Cu}^{2+}-\mathrm{NO}_{3}{ }^{-} \mathrm{NO}(\mathrm{b}$ and d) intermediates of $\mathrm{Cu}-\mathrm{SSZ}-13$ ( $\mathrm{a}$ and $\mathrm{b}$ ) and $\mathrm{Cu}-\mathrm{SAPO}-34$ (c and d). The oxygen atoms labelled $\mathrm{O} 1$ and $\mathrm{O} 2$ are shown in Figures $5.3 \mathrm{~d}, 5.3 \mathrm{e}$, and $5.4 \mathrm{~d}$ and $5.4 \mathrm{e}$. 
Finally, the average $\mathrm{Cu}-6 \mathrm{r}$ distance and the RMSD increase slightly with the increase of the temperature especially when two or more molecules are coordinated to $\mathrm{Cu}^{2+}$ (Table 5.3). The $\mathrm{Cu}^{2+}$ cation interacts favorably with the guest molecules, except $\mathrm{O}_{2}$, at the three temperatures in both materials which confirms the results of the previous chapter. Furthermore, it is found that at least four $\mathrm{NH}_{3}$ are necessary to stabilize the $\mathrm{Cu}^{2+}$ in the cavity with no coordination with the $6 r$ oxygen atoms.

\subsubsection{Interaction of the reactant molecules with $\mathrm{Cu}^{+}$}

The interaction of $\mathrm{Cu}^{+}$with one $\mathrm{NO}, \mathrm{NO}_{2}$, or $\mathrm{O}_{2}$ at $298 \mathrm{~K}$ results in an adsorption state where copper is coordinated to at least two $\mathrm{O}$ atoms of the $6 r$ and to the $\mathrm{N}$ or an $\mathrm{O}$ atom of the guest molecule (Figure 5.6). It is also slightly displaced down from the average plane of the $6 r$. The average $\mathrm{Cu}-6 \mathrm{r}$ distance ranges from 0.56 to $1.8 \AA$ and the RMSD from 0.22 to 0.52 in both zeolites at $298 \mathrm{~K}$ (Table 5.4). A second $\mathrm{O}_{2}$ molecule does not coordinate to $\mathrm{Cu}^{+}$, but remains interacting with the first $\mathrm{O}_{2}$ at 298 $\mathrm{K}$ (Figure 5.7a). Conversely, up to two $\mathrm{NO}$ or two $\mathrm{NO}_{2}$ molecules can strongly bind to $\mathrm{Cu}^{+}$causing an increase in the average $\mathrm{Cu}-6 \mathrm{r}$ distances and the RMSD of $\mathrm{Cu}^{+}$ positions compared with the adsorption of only one molecule (Table 5.4 and Figures $5.7 \mathrm{~b}$ and $5.7 \mathrm{c}$ ). The mobility (RMSD) of $\mathrm{Cu}^{+}$and the average $\mathrm{Cu}-6 \mathrm{r}$ distance also increase with the increase of the temperature in both materials (Table 5.4). Apart from the increase of the last two parameters no other difference was observed in the interaction of one and two molecules of $\mathrm{NO}$ and $\mathrm{NO}_{2}$ with $\mathrm{Cu}^{+}$. The adsorption is spontaneous at the three temperatures considered and $\mathrm{Cu}^{+}$remains coordinated to at least two oxygens of the $6 r$.

Table 5.4 Average distance between $\mathrm{Cu}$ and the $6 r$ plane $(\mathrm{Cu}-6 \mathrm{r})$ and root mean square deviation (RMSD) obtained from AIMD simulations of $\mathrm{Cu}^{+}$interacting with $\mathrm{O}_{2}, \mathrm{NO}, \mathrm{NO}_{2}$ and $\mathrm{NH}_{3}$ at 298, 573 and $673 \mathrm{~K}$. Data obtained over 50 ps simulations.

\begin{tabular}{|c|c|c|c|c|c|c|c|c|c|c|}
\hline \multirow{3}{*}{ Species } & \multicolumn{6}{|c|}{ Cu-SSZ-13 } & \multicolumn{4}{|c|}{ Cu-SAPO-34 } \\
\hline & \multicolumn{3}{|c|}{ Cu-6r (Å) } & \multicolumn{3}{|c|}{ RMSD (A) } & \multicolumn{2}{|c|}{ Cu-6r (Å) } & \multicolumn{2}{|c|}{ RMSD (§̊) } \\
\hline & 298 & 523 & 673 & 298 & 523 & 673 & 298 & 523 & 298 & 523 \\
\hline $\mathrm{Cu}^{+}-\mathrm{O}_{2}$ & 1.09 & - & - & 0.26 & - & - & 0.77 & - & 0.22 & - \\
\hline $\mathrm{Cu}^{+}-\mathrm{NO}$ & 1.82 & 1.98 & 2.01 & 0.52 & 0.45 & 0.51 & 0.83 & 0.86 & 0.32 & 0.47 \\
\hline $\mathrm{Cu}^{+}-2 \mathrm{NO}$ & 2.2 & 2.42 & 2.6 & 0.39 & 0.71 & 0.59 & 1.18 & 1.28 & 0.44 & 0.58 \\
\hline $\mathrm{Cu}^{+}-\mathrm{NO}_{2}$ & 1.6 & 1.67 & 1.92 & 0.29 & 0.38 & 0.6 & 0.53 & 0.65 & 0.28 & 0.44 \\
\hline $\mathrm{Cu}^{+}-2 \mathrm{NO}_{2}$ & 2.71 & 2.53 & 2.5 & 0.31 & 0.55 & 0.55 & 1.18 & 1.25 & 0.27 & 0.36 \\
\hline
\end{tabular}


5.3 Adsorption of reactant molecules on the copper cations with AIMD

Table 5.4 Average distance between $\mathrm{Cu}$ and the $6 r$ plane $(\mathrm{Cu}-6 \mathrm{r})$ and root mean square deviation (RMSD) obtained from AIMD simulations of $\mathrm{Cu}^{+}$interacting with $\mathrm{O}_{2}, \mathrm{NO}, \mathrm{NO}_{2}$ and $\mathrm{NH}_{3}$ at 298, 573 and $673 \mathrm{~K}$. Data obtained over 50 ps simulations.

\begin{tabular}{cccccccccccc}
\hline \multirow{2}{*}{ Species } & \multicolumn{4}{c}{ Cu-SSZ-13 } & \multicolumn{4}{c}{ Cu-SAPO-34 } \\
\cline { 2 - 12 } & \multicolumn{4}{c}{ Cu-6r $(\AA)$} & \multicolumn{3}{c}{ RMSD $(\AA)$} & \multicolumn{1}{c}{ Cu-6r $(\AA)$} & RMSD $(\AA)$ \\
\cline { 2 - 12 } & $\mathbf{2 9 8}$ & $\mathbf{5 2 3}$ & $\mathbf{6 7 3}$ & $\mathbf{2 9 8}$ & $\mathbf{5 2 3}$ & $\mathbf{6 7 3}$ & $\mathbf{2 9 8}$ & $\mathbf{5 2 3}$ & $\mathbf{2 9 8}$ & $\mathbf{5 2 3}$ \\
\hline $\mathrm{Cu}^{+}-\mathrm{NH}_{3}$ & 2.4 & 2.6 & 2.4 & 0.39 & 0.66 & 0.44 & 1.38 & 0.98 & 0.29 & 1.08 \\
$\mathrm{Cu}^{+}-2 \mathrm{NH}_{3}$ & 3.81 & 4.08 & 3.14 & 1.4 & 1.3 & 1.4 & 2.32 & 2.00 & 0.67 & 2.40 \\
$\mathrm{Cu}^{+}-4 \mathrm{NH}_{3}$ & 4.3 & 5.14 & 4.13 & 0.34 & 3.63 & 1.4 & 2.00 & 2.06 & 0.3 & 0.47 \\
$\mathrm{Cu}^{+}-\mathrm{NO}_{-} \mathrm{NH}_{3}$ & 2.07 & - & - & 0.44 & - & - & - & - & - & - \\
$\mathrm{Cu}^{+}-2 \mathrm{NO}-\mathrm{NH}_{3}$ & 2.21 & 2.3 & 2.58 & 0.36 & 0.51 & 0.97 & - & - & - & - \\
$\mathrm{Cu}^{+}-2 \mathrm{NO}-2 \mathrm{NH}_{3}$ & 3.87 & 6.1 & 4.01 & 0.52 & 1.1 & 1.04 & 3.13 & 3.25 & 0.5 & 0.7 \\
\hline
\end{tabular}
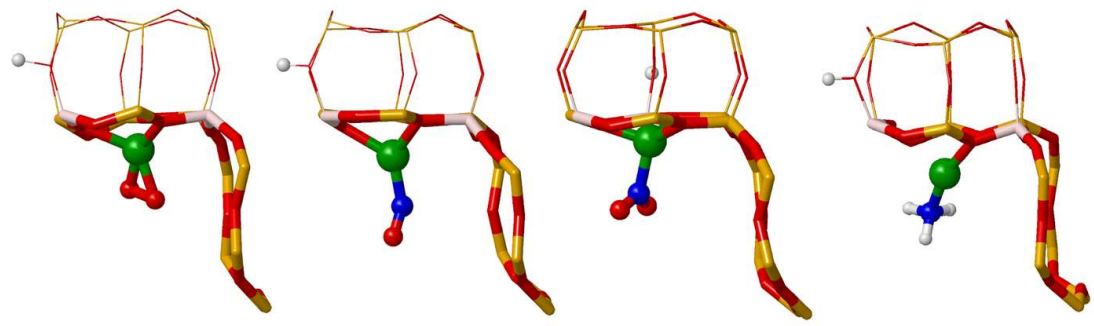

a) $\mathrm{O}_{2}$

b) $\mathrm{NO}$

c) $\mathrm{NO}_{2}$

d) $\mathrm{NH}_{3}$
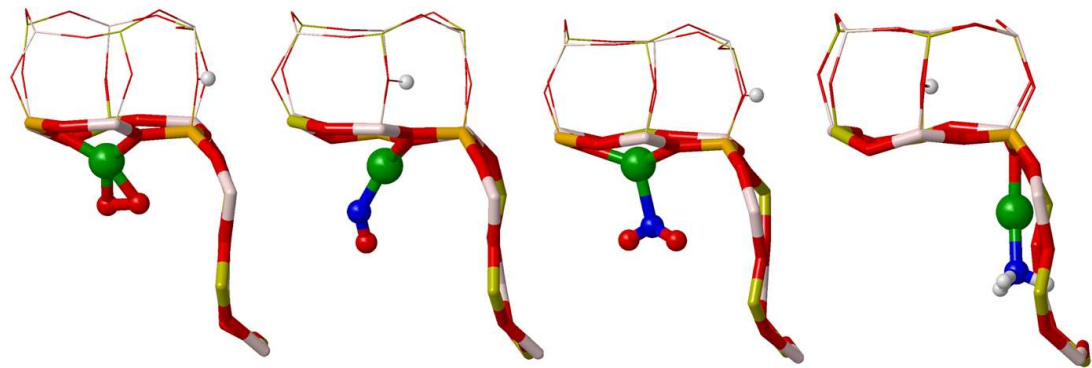
e) $\mathrm{O}_{2}$
f) $\mathrm{NO}$
g) $\mathrm{NO}_{2}$
h) $\mathrm{NH}_{3}$

Figure 5.6 Simulation snapshots of the interaction of one $\mathrm{O}_{2}, \mathrm{NO}, \mathrm{NO}_{2}$ and $\mathrm{NH}_{3}$ molecules with $\mathrm{Cu}^{+}$at $298 \mathrm{~K}$ in Cu-SSZ-13 (a-d) and in Cu-SAPO-34 (e-h). Al, P, Si, and $\mathrm{O}$ atoms in the framework depicted as thatch, yellow, orange and red sticks; $\mathrm{Cu}$ cations, $\mathrm{O}, \mathrm{N}$ and $\mathrm{H}$ atoms of the reactant molecules are depicted as green, red, blue and white balls. 


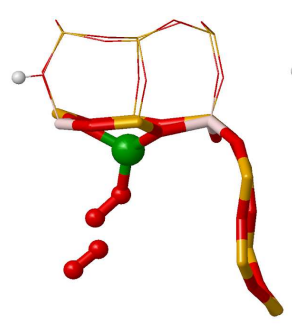

a) $2 \mathrm{O}_{2}$

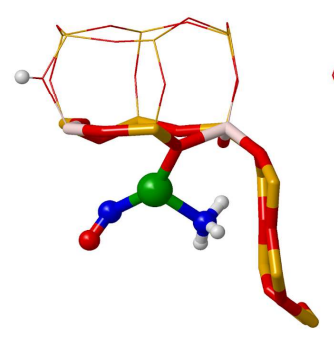

e) $\mathrm{NO}+\mathrm{NH}_{3}$

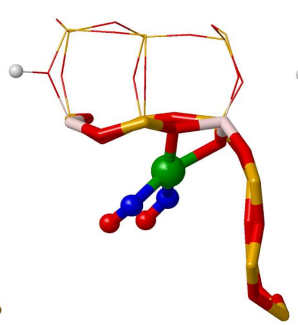

b) $2 \mathrm{NO}$

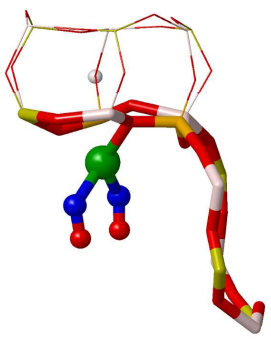

f) $2 \mathrm{NO}$

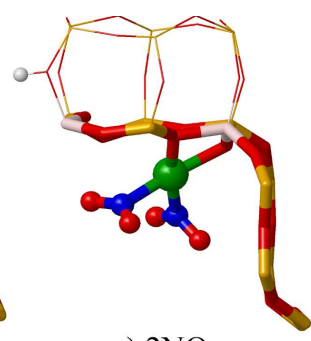

c) $2 \mathrm{NO}_{2}$

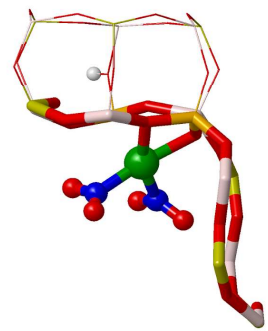

g) $2 \mathrm{NO}_{2}$

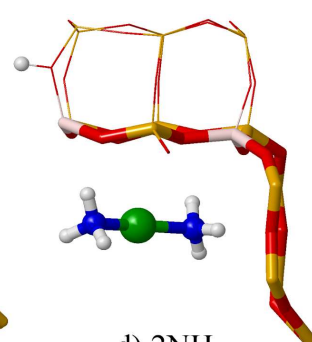

d) $2 \mathrm{NH}_{3}$

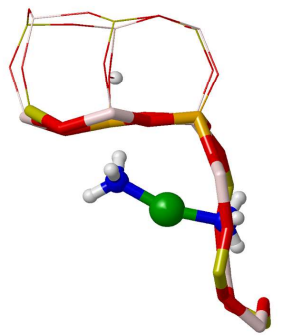

h) $2 \mathrm{NH}_{3}$

Figure 5.7 Simulation snapshots of the interaction of two $\mathrm{O}_{2}, \mathrm{NO}, \mathrm{NO}_{2}$ and $\mathrm{NH}_{3}$ molecules with $\mathrm{Cu}^{+}$at $298 \mathrm{~K}$ in $\mathrm{Cu}-\mathrm{SSZ}-13$ (a-e) and in $\mathrm{Cu}-\mathrm{SAPO}-34$ (f-h). Al, P, Si, and $\mathrm{O}$ atoms in the framework depicted as thatch, yellow, orange and red sticks; $\mathrm{Cu}$ cations, $\mathrm{O}, \mathrm{N}$ and $\mathrm{H}$ atoms of the reactant molecules are depicted as green, red, blue and white balls.

When one $\mathrm{NH}_{3}$ interacts with $\mathrm{Cu}^{+}$significantly displaces it toward the center of the cavity with average $\mathrm{Cu}-6 \mathrm{r}$ distances of $2.4 \AA$ and $1.38 \AA$ for $\mathrm{Cu}-\mathrm{SSZ}-13$ and $\mathrm{Cu}-\mathrm{SAPO}-34$, but the RMSDs are similar compared with $\mathrm{NO}, \mathrm{NO}_{2}$ and $\mathrm{O}_{2}$. The $\mathrm{Cu}^{+}$ is coordinated to only one $\mathrm{O}$ of the $6 r$ and to the $\mathrm{NH}_{3}$ molecule forming an almost linear complex (Figures 5.6d and 5.6h). The average angle N-Cu-O is $169.7^{\circ}$ and $169.0^{\circ}$ for $\mathrm{Cu}-\mathrm{SAPO}-34$ and for $\mathrm{Cu}-\mathrm{SSZ}-13$ respectively. These results indicate that the interaction of $\mathrm{Cu}^{+}$with $\mathrm{NH}_{3}$ is stronger than with $\mathrm{NO}, \mathrm{NO}_{2}$ and $\mathrm{O}_{2}$. The interaction with a second ammonia modifies the coordination of $\mathrm{Cu}^{+}$with the $6 r$ significantly in both materials. While $\mathrm{Cu}^{+}$maintains its coordination to the framework oxygens when interacting with one $\mathrm{NH}_{3}$, two $\mathrm{NH}_{3}$ are capable of pulling the $\mathrm{Cu}^{+}$cation out of $6 r$ and form the mobile linear complex $\left[\mathrm{Cu}\left(\mathrm{NH}_{3}\right)_{2}\right]^{+}($see $\mathrm{Cu}-6 \mathrm{r}$ distance in Table 5.4 and Figures 5.7d and 5.7h). At $298 \mathrm{~K}$, the complex forms as 
5.3 Adsorption of reactant molecules on the copper cations with AIMD

soon as the ammonia molecules approach $\mathrm{Cu}^{+}$and remains stable during the whole simulation time. The mobility of $\mathrm{Cu}^{+}$is significantly enhanced by the formation of this complex as evidenced by the higher RMSD values, 1.4 and 0.67 for Cu-SSZ-13 and Cu-SAPO-34 respectively. The strong effect of the interaction with two $\mathrm{NH}_{3}$ is also observed in the average $\mathrm{Cu}-6 \mathrm{r}$ distances, which are $\sim 1 \AA$ longer compared with the other molecules (Table 5.4). These results indicate that $\mathrm{Cu}^{+}$is no longer coordinated to the $6 r$ but is actually free to move inside the big CHA cage, which is clearly observed in the scatter plot in Figure 5.8.

Seeing that the two $\mathrm{NH}_{3}$ are able to pull $\mathrm{Cu}^{+}$out of the plane, we also tried the combination of one $\mathrm{NH}_{3}$ with one $\mathrm{NO}$ for $\mathrm{Cu}-\mathrm{SSZ}-13$. In this case, $\mathrm{Cu}^{+}$remained attached to one $\mathrm{O}$ atom of the $6 r$ at an average distance of $2.07 \AA$ (Table 5.4 and Figure 5.7e).

The effect of the temperature is more noticeable in the interaction of $\mathrm{Cu}^{+}$with $\mathrm{NH}_{3}$ in $\mathrm{Cu}-\mathrm{SAPO}-34$. At $523 \mathrm{~K}$, the adduct $\mathrm{Cu}^{+}-\mathrm{NH}_{3}$ crosses the $8 r$ to the adjacent cavity while $\mathrm{Cu}^{+}$is still coordinated to the framework. This is illustrated in the scatter plot of the $\mathrm{Cu}$ positions shown in Figure 5.8a. As a result of the interaction of two $\mathrm{NH}_{3}$ with $\mathrm{Cu}^{+}$at $523 \mathrm{~K}$, the diamine-copper(I) complex, $\mathrm{Cu}^{+}\left(\mathrm{NH}_{3}\right)_{2}$, also forms and stays at an average distance of $\sim 2.0 \AA$ from the average plane of the $6 r$. After $50 \mathrm{ps,}$ $\mathrm{Cu}^{+}\left(\mathrm{NH}_{3}\right)_{2}$ diffuses through the $8 r$ and remains between 0 and $1 \AA$ from the $6 r$ plane and close to the negatively charged framework oxygens (Figure $5.8 \mathrm{~b}$ ). 
a)

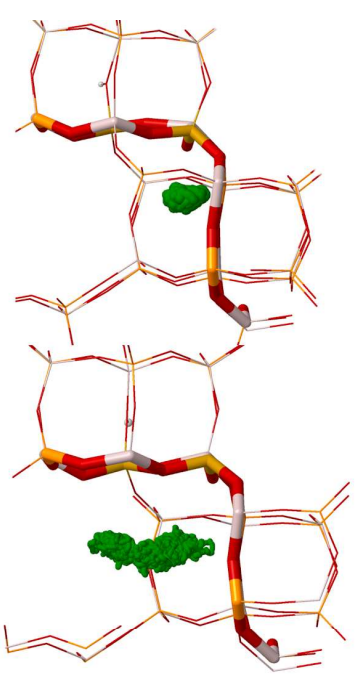

$298 \mathrm{~K}$

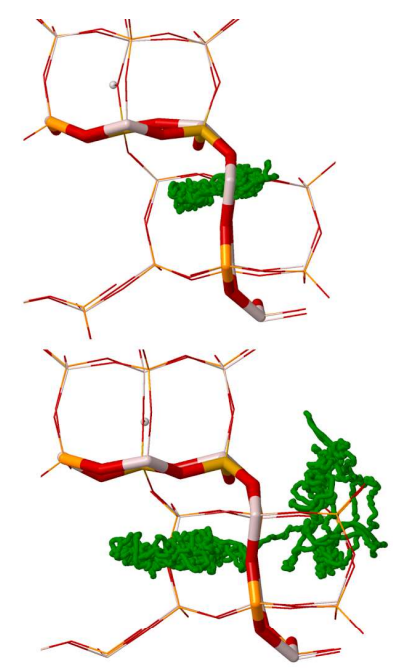

$523 \mathrm{~K}$

$\mathrm{Cu}-\mathrm{SAPO}-34$

c)

d)

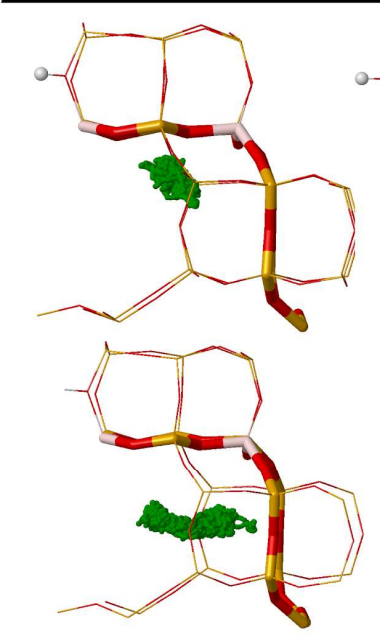

$298 \mathrm{~K}$
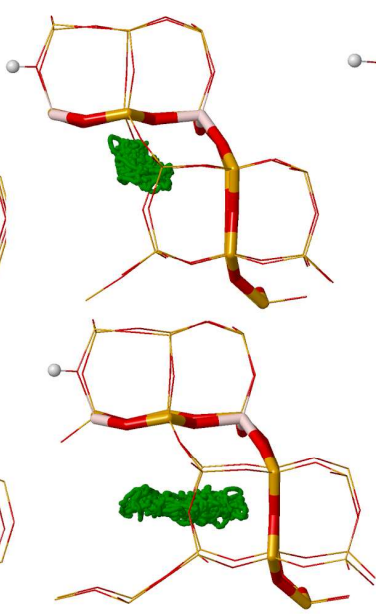

$523 \mathrm{~K}$
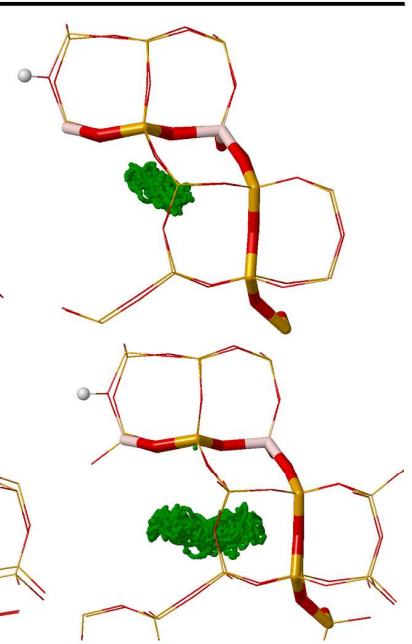

$673 \mathrm{~K}$

$\mathrm{Cu}-\mathrm{SSZ}-13$

Figure 5.8 Scatter plot of the position of $\mathrm{Cu}^{+}$(green) in the MD simulations for the interaction of $\mathrm{Cu}^{+}$with a) and c) one $\mathrm{NH}_{3}$, and b) and d) two $\mathrm{NH}_{3}$ at different temperatures. $\mathrm{Al}, \mathrm{P}, \mathrm{Si}$, and $\mathrm{O}$ atoms in the framework depicted as thatch, yellow, orange and red sticks. 
5.3 Adsorption of reactant molecules on the copper cations with AIMD

Similar enhancement of the mobility is observed in Cu-SSZ-13 for the interaction of one $\mathrm{NH}_{3}$ with $\mathrm{Cu}^{+}$. Conversely, the mobility seems to be nearly the same at the three temperatures when $\mathrm{Cu}^{+}\left(\mathrm{NH}_{3}\right)_{2}$ is formed. The spontaneous diffusion of $\mathrm{Cu}^{+}\left(\mathrm{NH}_{3}\right)_{2}$ was not observed in $\mathrm{Cu}-\mathrm{SSZ}-13$ (Figure 5.8c and Figure 5.8d) but the $\mathrm{Cu}-6 \mathrm{r}$ distance increases at $523 \mathrm{~K}$ and decreases at $400 \mathrm{~K}$ (Table 5.4). It confirms that the diaminecopper(I) complex is already detached from the zeolite framework and its motion is not constrained to the $6 r$. However, it also indicates that longer simulations are needed to sample regions far from the $6 r$ and thus obtain more consistent average values of $\mathrm{Cu}-6 \mathrm{r}$ distances at the three temperatures.

So far, we have seen the stronger interaction of $\mathrm{Cu}^{+}$with $\mathrm{NH}_{3}$ compared with the other reactant molecules. But it is not completely clear whether the $\mathrm{Cu}^{+}$cation is able to coordinate to more than two reactant molecules. To clarify this point, we studied the interaction of $\mathrm{Cu}^{+}$cation with a larger number of molecules. Thus, starting with $\mathrm{Cu}^{+}$in the $6 r$ we simulated the spontaneous adsorption of two $\mathrm{NO}$ and one $\mathrm{NH}_{3}$ in $\mathrm{Cu}-\mathrm{SSZ}-13$. In addition to two $\mathrm{NO}$ molecules, $\mathrm{Cu}^{+}$coordinates to an extra ammonia while still interacting with one $\mathrm{O}$ atom of the $6 r$, with average $\mathrm{Cu}-6 \mathrm{r}$ distances ranging from 2.21 to $2.58 \AA$ at the three temperatures (Table 5.4 and Figures 5.9a to $5.9 \mathrm{c})$. These $\mathrm{Cu}-6 \mathrm{r}$ distances indicate that the coordination of $\mathrm{Cu}^{+}$with the $6 r$ is significantly weakened. Simulations of similar systems were not performed for Cu-SAPO-34.

We have also considered the adsorption of two $\mathrm{NO}$ and two $\mathrm{NH}_{3}$ at 298, 523 and 673 $\mathrm{K}$ for $\mathrm{Cu}-\mathrm{SSZ}-13$, and at 298 and $523 \mathrm{~K}$ for $\mathrm{Cu}-\mathrm{SAPO}-34$ with the $\mathrm{Cu}^{+}$cation initially located in the $6 r$. When a second ammonia is included, the $\mathrm{Cu}^{+}$cation is completely detached from the $6 r$, which is indicated by $\mathrm{Cu}-6 \mathrm{r}$ distances larger than $3.0 \AA$ at the three temperatures and in both materials (Table 5.4). The species $\mathrm{Cu}^{+}\left(\mathrm{NH}_{3}\right)_{2}(\mathrm{NO})$ is formed at $298 \mathrm{~K}$ inside the cavity in Cu-SSZ-13 (Figure 5.9d), and the $\mathrm{Cu}^{+}\left(\mathrm{NH}_{3}\right)_{2}(\mathrm{NO})_{2}$ complex is formed at 523 and $673 \mathrm{~K}$ in Cu-SSZ-13, and at 298 and $523 \mathrm{~K}$ in $\mathrm{Cu}-\mathrm{SAPO}-34$ (Figures $5.9 \mathrm{e}$ to $5.9 \mathrm{~h}$ ). The diffusion to the neighboring cavity of such species was not observed. 


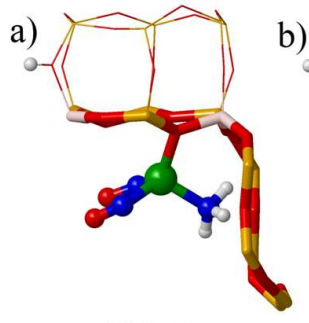

$298 \mathrm{~K}$ b)

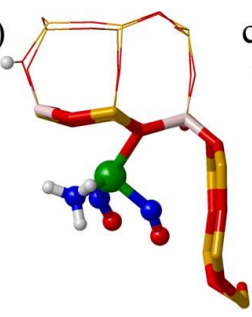

$523 \mathrm{~K}$ c)

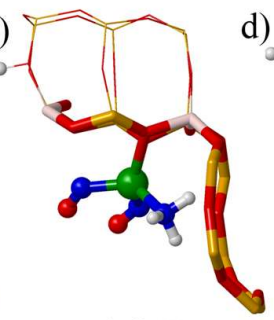

$673 \mathrm{~K}$ d)

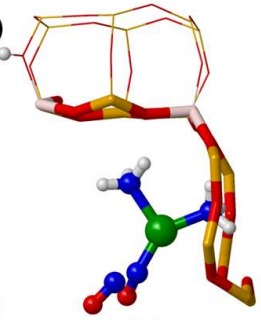

$298 \mathrm{~K}$ e)

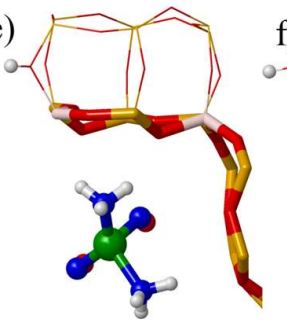

$523 \mathrm{~K}$

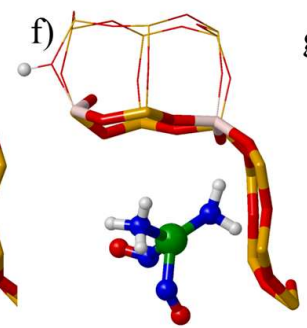

$673 \mathrm{~K}$ g)

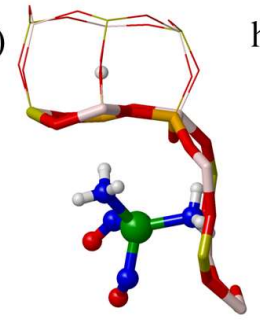

$298 \mathrm{~K}$ h)

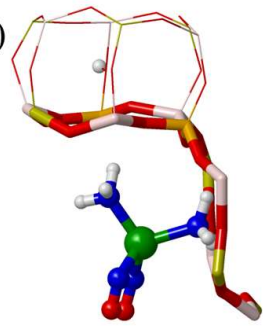

$523 \mathrm{~K}$

Figure 5.9 Simulation snapshots of the interaction of two $\mathrm{NO}$ and one $\mathrm{NH}_{3}$ with $\mathrm{Cu}^{+}$in $\mathrm{Cu}$ SSZ-13 (a, b and c), two NO and two $\mathrm{NH}_{3}$ with $\mathrm{Cu}^{+}$in $\mathrm{Cu}-\mathrm{SSZ}-13$ (d, e and f), and two NO and two $\mathrm{NH}_{3}$ with $\mathrm{Cu}^{+}$in Cu-SAPO-34 ( $\mathrm{g}$ and $\mathrm{h}$ ). The temperature is indicated below the corresponding snapshot. $\mathrm{Al}, \mathrm{P}, \mathrm{Si}$, and $\mathrm{O}$ atoms in the framework depicted as thatch, yellow, orange and red sticks; $\mathrm{Cu}$ cations, $\mathrm{O}, \mathrm{N}$ and $\mathrm{H}$ atoms of the reactant molecules are depicted as green, red, blue and white balls.

Finally, we have also studied the interaction of $\mathrm{Cu}^{+}$with four ammonia molecules in the center of the cavity in Cu-SSZ-13. The diamine-copper(I) complex is formed by the coordination of $\mathrm{Cu}^{+}$with two ammonia molecules and remains stable at the three temperatures considered. The other two ammonia molecules do not bind to $\mathrm{Cu}^{+}$but dynamically interact through hydrogen bonds with each other and with the two ammonia of the complex (Figure 5.10a). Figure 5.11 shows that at $298 \mathrm{~K}$ only two $\mathrm{NH}_{3}$ binds to $\mathrm{Cu}^{+}$with $\mathrm{Cu}-\mathrm{N}$ bond lengths of $\sim 2 \AA$. The other two ammonia remain at an average distance of $\sim 4.1 \AA$ from the $\mathrm{Cu}^{+}$cation. At $523 \mathrm{~K}$, the interaction $\mathrm{Cu}$ ammonia is more dynamic and at $\sim 14 \mathrm{ps}$ an ammonia initially not coordinated binds to $\mathrm{Cu}^{+}$(see $\mathrm{Cu}-\mathrm{N} 4$ distance in Figure 5.11) while one of the ammonia initially coordinated drifts away (see $\mathrm{Cu}-\mathrm{N} 3$ distance in Figure 5.11). Furthermore, the 
diamine-copper(I) complex diffuses spontaneously through the $8 r$ (Figure 5.10b). The dynamic character of the interaction increases even more at $673 \mathrm{~K}$. The $\mathrm{Cu}-\mathrm{N}$ distances represented in Figure 5.11 indicate that three ammonia $(\mathrm{Cu}-\mathrm{N} 1, \mathrm{Cu}-\mathrm{N} 3$, $\mathrm{Cu}-\mathrm{N} 4)$ are dynamically being part of the diamine-Cu complex. The distance $\mathrm{Cu}-\mathrm{N} 2$ increases after 10 ps because the $\mathrm{NH}_{3}$ approaches the Brønsted acid site and forms $\mathrm{NH}_{4}^{+}$.

a)

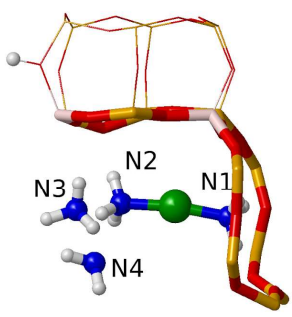

b)

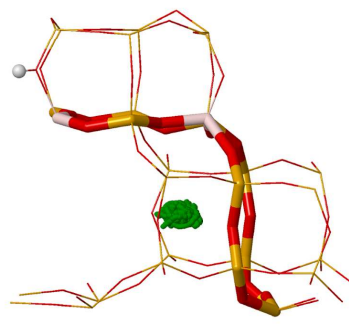

$298 \mathrm{~K}$
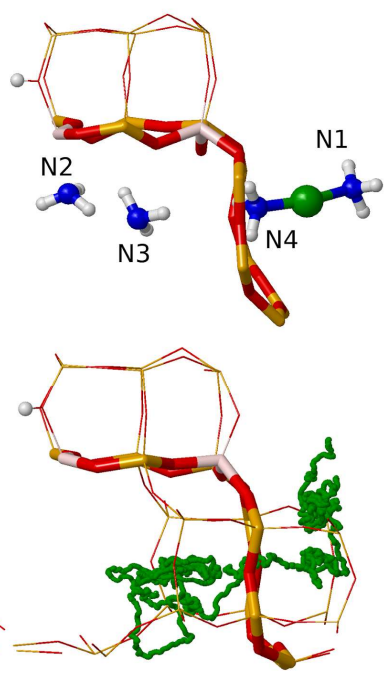

$523 \mathrm{~K}$
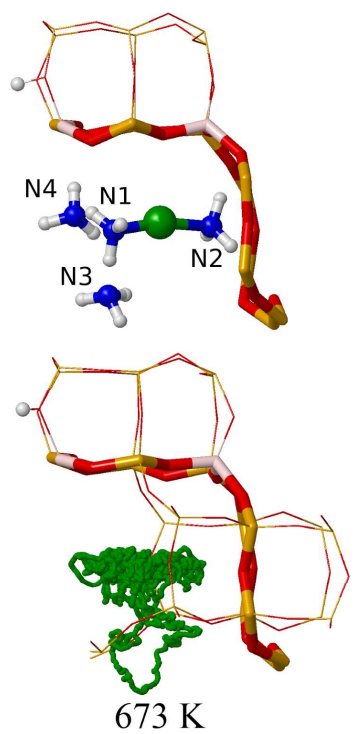

Figure 5.10 Snapshots (a) and scatter plot (b) of the interaction of four $\mathrm{NH}_{3}$ molecules with $\mathrm{Cu}^{+}$in $\mathrm{Cu}-\mathrm{SSZ}-13$ at 298, 523 and $673 \mathrm{~K}$. Al, O and Si atoms in the framework depicted as thatch, red and orange sticks; $\mathrm{Cu}$ cations, $\mathrm{O}, \mathrm{N}$ and $\mathrm{H}$ atoms in the reactant molecules depicted as green, red, blue and white balls. 
a)
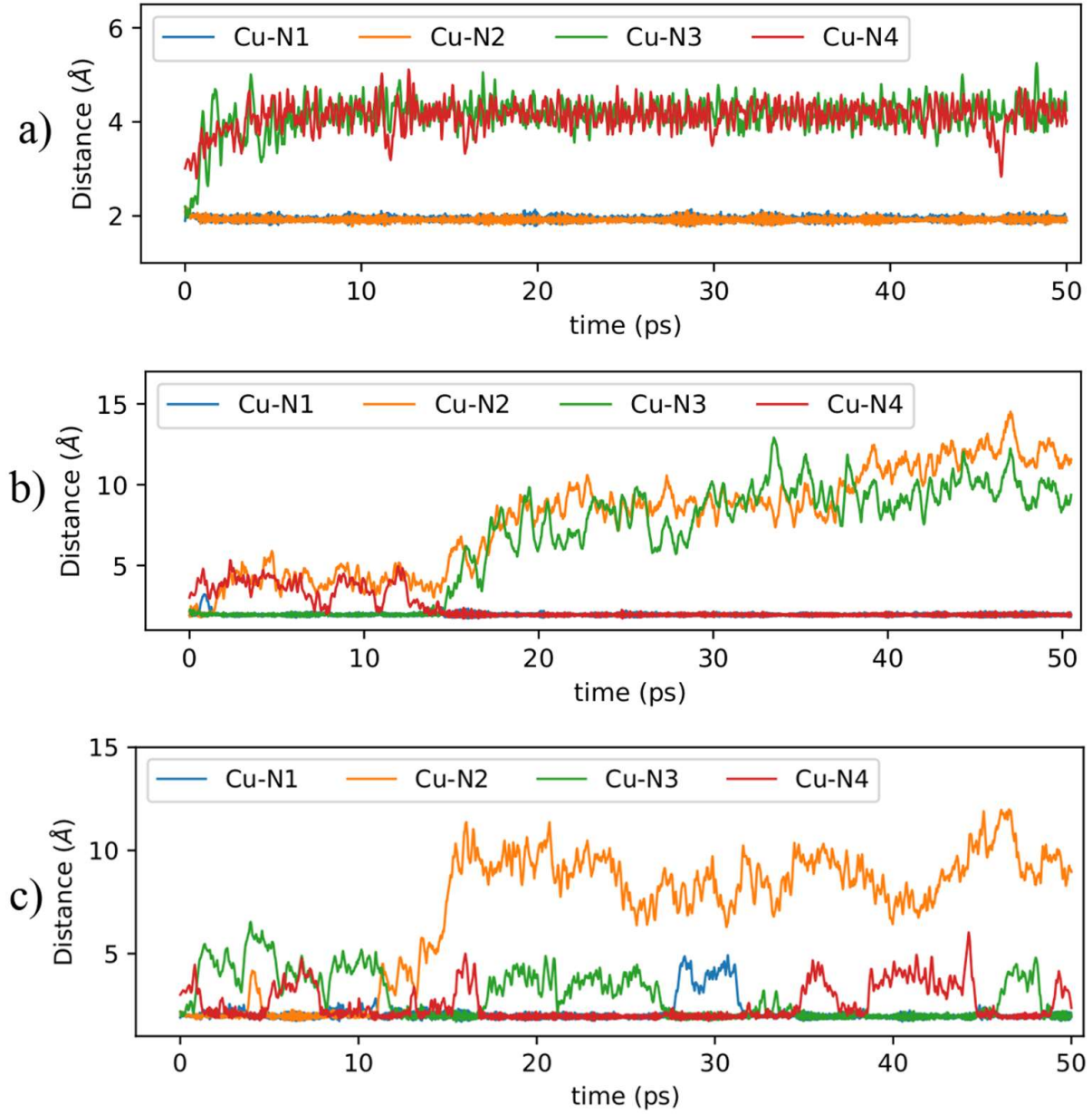

Figure 5.11 Time evolution of the distances between $\mathrm{Cu}^{+}$and the $\mathrm{N}$ atom of the four ammonia molecules. Simulations were performed at a) $298 \mathrm{~K}$, b) $523 \mathrm{~K}$ and c) $673 \mathrm{~K}$. The N atoms labelled as N1, N2, N3 and N4 are shown in Figure 5.10. 
5.4 Stability of $\mathrm{Cu}+$ complexes at low temperature with umbrella sampling

5.4 Stability of $\mathrm{Cu}^{+}$complexes at low temperature with umbrella sampling

We have seen in the previous section that when two $\mathrm{NH}_{3}$ molecules coordinate to $\mathrm{Cu}^{+}$the stable complex $\mathrm{Cu}^{+}\left(\mathrm{NH}_{3}\right)_{2}$ is formed. Furthermore, $\mathrm{NO}$ is still able to coordinate to $\mathrm{Cu}^{+}\left(\mathrm{NH}_{3}\right)_{2}$ and form other complexes. We have performed an umbrella sampling simulation to study the competitive interaction of two $\mathrm{NO}$ and two $\mathrm{NH}_{3}$ for $\mathrm{Cu}^{+}$. The simulation is biased so that it evolves from a state with the $\mathrm{Cu}^{+}$coordinated to two $\mathrm{NO}$ molecules to a state with the $\mathrm{Cu}^{+}$coordinated to only two $\mathrm{NH}_{3}$ molecules. Such a biased reaction path was achieved by defining a collective variable, CN3, that tracks the coordination of the four molecules to $\mathrm{Cu}^{+}$. To that end, we have used the following general definition of coordination number $(\mathrm{CN})$ :

$$
C N\left(r_{i}, r_{j}\right)=\sum_{i} \sum_{j} \frac{1-\left(\left|r_{i}-r_{j}\right| / d c\right)^{6}}{1-\left(\left|r_{i}-r_{j}\right| / d c\right)^{12}}
$$

Where $r_{i}$ and $r_{j}$ are the position vectors of species $i$ and $j$ and $d c$ is a cutoff distance. Two coordination numbers were defined, $C N 1$ that represents the coordination of the two $\mathrm{NO}$ molecules to the $\mathrm{Cu}^{+}$cation and $C N 2$ that represents the coordination of the two $\mathrm{NH}_{3}$ molecules also to $\mathrm{Cu}^{+}$, both with a cutoff distance $d c=3 \AA$ such that:

1) $C N 1 \rightarrow 2$ when the two NO molecules are located less than $3 \AA$ away from $\mathrm{Cu}^{+}$.

2) $C N 1 \rightarrow 1$ when one $\mathrm{NO}$ is close to $\mathrm{Cu}^{+}$by $3 \AA$ and the other is more than $3 \AA$ away.

3) $\mathrm{CN1} \rightarrow 0$ when both NO molecules are more than $3 \AA$ away from $\mathrm{Cu}^{+}$.

The same holds for $C N 2$ describing the coordination of the two $\mathrm{NH}_{3}$ molecules to $\mathrm{Cu}^{+}$. The distances of the $\mathrm{NO}$ or $\mathrm{NH}_{3}$ to $\mathrm{Cu}^{+}$used in equation 5.1 are calculated with the coordinates of the $\mathrm{N}$ atom of both molecules and the position of $\mathrm{Cu}$. CN3 was then defined as $C N 3=C N 2$ - CN1 so that it ranges from -2 to 2 for both $\mathrm{NO}$ and both $\mathrm{NH}_{3}$ coordinated to $\mathrm{Cu}$ respectively. See section 2.6 .3 for more details on the coordination numbers as collective variables.

Figure 5.12 shows Gibbs free energy as a function of $C N 3$ where three minima can be observed. The average $\mathrm{Cu}-\mathrm{N}$ distances for relevant states are summarized in Table 5.5. The most stable state (B) in the free energy profile is located at $\mathrm{CN} 3=-0.06$ 
which is characterized by the formation of the complex $\left[\mathrm{Cu}\left(\mathrm{NH}_{3}\right)_{2}(\mathrm{NO})_{2}\right]^{+}$. From Table 5.5 it can be seen that $\mathrm{Cu}^{+}$is found at an average distance of $3.6 \AA$ from the plane of the $6 r$ and is coordinated to the four molecules. Another minimum is found at $\mathrm{CN} 3=-0.80(\mathrm{~A})$. Two $\mathrm{NO}$ and one $\mathrm{NH}_{3}$ are coordinated to $\mathrm{Cu}^{+}$with average bond lengths of 1.92, 1.97, $2.07 \AA$ respectively. The second ammonia molecule is forming hydrogen bonds with the ammonia coordinated to $\mathrm{Cu}^{+}$and is located at $\sim 4 \AA$ from $\mathrm{Cu}^{+}$. Moreover, the average distance of the copper cation to the plane of the $6 r$ is $2.17 \AA$ (Table 5.5). In state $\mathrm{C}$, both $\mathrm{NH}_{3}$ are already coordinated to $\mathrm{Cu}^{+}$together with one $\mathrm{NO}$ forming the complex $\left[\mathrm{Cu}\left(\mathrm{NH}_{3}\right)_{2} \mathrm{NO}\right]^{+}$. States $\mathrm{A}$ and $\mathrm{C}$ are $\sim 20 \mathrm{~kJ} / \mathrm{mol}$ less stable than state $\mathrm{B}$. Notice that state $\mathrm{D}$, with the complex $\left[\mathrm{Cu}\left(\mathrm{NH}_{3}\right)_{2}\right]^{+}$not interacting with the two NO molecules, is $40 \mathrm{~kJ} / \mathrm{mol}$ less stable than state B and can not be clearly identified as a minimum in the profile.

In summary, according to the free energy profile, the interaction of $\left[\mathrm{Cu}\left(\mathrm{NH}_{3}\right)_{2}\right]^{+}$with two $\mathrm{NO}$ at $298 \mathrm{~K}$ will lead to the formation of $\left[\mathrm{Cu}\left(\mathrm{NH}_{3}\right)_{2} \mathrm{NO}\right]^{+}$in a non-activated step. The second $\mathrm{NO}$ will bind to $\left[\mathrm{Cu}\left(\mathrm{NH}_{3}\right)_{2} \mathrm{NO}\right]^{+}$through a transition state with activation barrier of $\sim 8 \mathrm{~kJ} / \mathrm{mol}$ forming the complex $\left[\mathrm{Cu}\left(\mathrm{NH}_{3}\right)_{2}(\mathrm{NO})_{2}\right]^{+}$.

Table 5.5 Average distance $(d)$ in angstroms between $\mathrm{Cu}^{+}$and the $\mathrm{N}$ atom of the two $\mathrm{NO}$ and two $\mathrm{NH}_{3}$ at relevant points along the collective variable $\mathrm{CN} 3$.

\begin{tabular}{ccccc}
\hline State & $\mathrm{A}$ & $\mathrm{B}$ & $\mathrm{C}$ & $\mathrm{D}$ \\
\hline $\mathrm{CN} 3$ & -0.80 & -0.06 & 0.60 & 1.80 \\
$d \mathrm{Cu}-\mathrm{N}(1)$ & 1.92 & 1.93 & 3.47 & 5.47 \\
$d \mathrm{Cu}-\mathrm{N}(2)$ & 1.97 & 1.93 & 1.90 & 5.41 \\
$d \mathrm{Cu}-\mathrm{N}(1)$ & 3.98 & 2.12 & 2.04 & 1.94 \\
$d \mathrm{Cu}-\mathrm{N}(2)$ & 2.07 & 2.10 & 2.02 & 1.95 \\
$d \mathrm{Cu}-6 \mathrm{r}$ & 2.17 & 3.61 & 3.82 & 3.16 \\
\hline
\end{tabular}




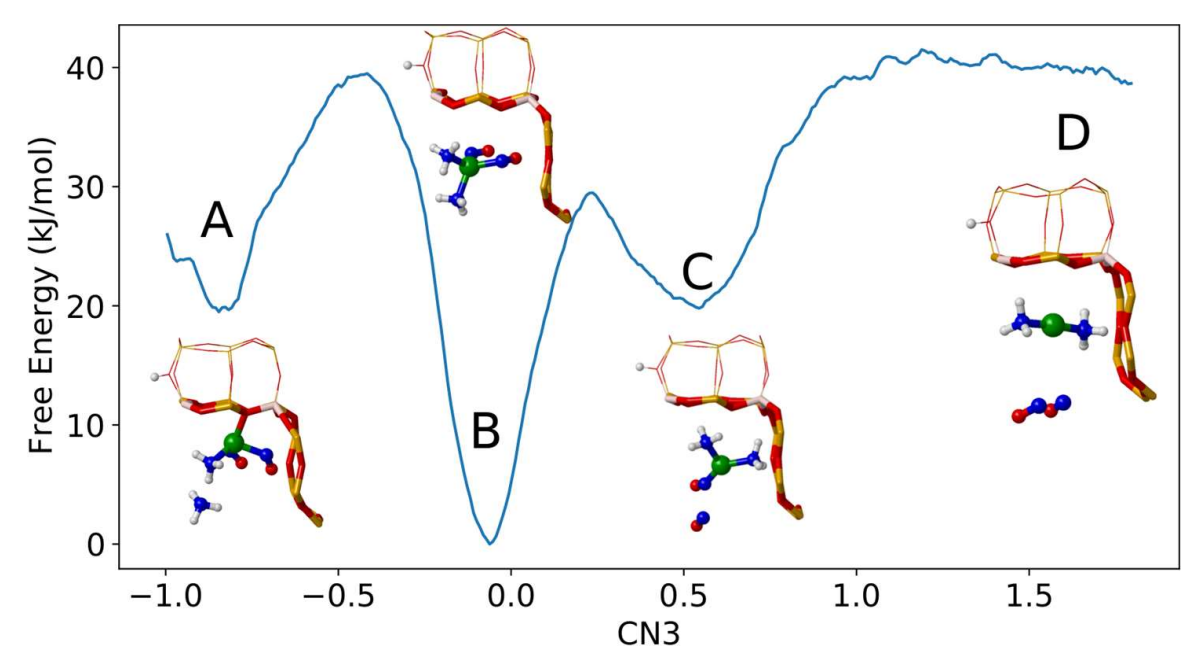

Figure 5.12 Free energy as a function of the collective variable $C N 3$ at $298 \mathrm{~K}$. Spheres in green, blue, gray and red represent $\mathrm{Cu}, \mathrm{N}, \mathrm{H}$, and $\mathrm{O}$ atoms respectively.

\subsection{Oxidation of $\mathrm{NO}$ to $\mathrm{NO}_{2}$}

We have considered first the interaction of two $\mathrm{NO}$ and one $\mathrm{O}_{2}$ with $\mathrm{Cu}^{+}$which was proposed as a relevant intermediate state in the oxidation of $\mathrm{NO}$ to $\mathrm{NO}_{2}$. At the three temperatures considered only one $\mathrm{NO}$ actually stays at bond distance from $\mathrm{Cu}^{+}$. The second $\mathrm{NO}$ moves rather far from $\mathrm{Cu}^{+}$at an average distance of $\sim 3.5 \AA$ but at 1.85 $\AA$ from one the $\mathrm{O}$ atoms of the $\mathrm{O}_{2}$ molecule (Figures 5.13a and 5.13d). 
Table 5.6 Average distance between $\mathrm{Cu}$ and the $6 r$ plane $(\mathrm{Cu}-6 \mathrm{r})$ and root mean square deviation (RMSD), both given in angstroms $(\AA)$, obtained from AIMD simulations of $\mathrm{Cu}^{+}$ interacting with $\mathrm{O}_{2}, \mathrm{NO}$, and $\mathrm{NH}_{3}$ at 298,573 and $673 \mathrm{~K}$. Data obtained over 50 ps simulations.

\begin{tabular}{cccccccccccc}
\hline \multirow{3}{*}{ Species } & \multicolumn{4}{c}{ SSZ-13 } & \multicolumn{4}{c}{ SAPO } \\
\cline { 2 - 12 } & \multicolumn{3}{c}{$\mathbf{C u}-6 r$} & \multicolumn{3}{c}{ RMSD } & \multicolumn{3}{c}{ Cu-6r } & \multicolumn{2}{c}{ RMSD } \\
\cline { 2 - 12 } & $\mathbf{2 9 8}$ & $\mathbf{5 2 3}$ & $\mathbf{6 7 3 K}$ & $\mathbf{2 9 8}$ & $\mathbf{5 2 3}$ & $\mathbf{6 7 3}$ & $\mathbf{2 9 8}$ & $\mathbf{5 2 3}$ & $\mathbf{2 9 8}$ & $\mathbf{5 2 3}$ \\
\hline $\mathrm{Cu}^{+}-2 \mathrm{NO}-\mathrm{O}_{2}$ & 1.16 & 1.39 & 1.35 & 0.33 & 0.52 & 0.64 & 0.79 & 0.97 & 0.24 & 0.47 \\
$\mathrm{Cu}^{+}-2 \mathrm{NO}+\mathrm{O}_{2}+\mathrm{NH}_{3}$ & 1.18 & - & 1.43 & 0.28 & - & 0.57 & - & - & - & - \\
\hline
\end{tabular}

a)

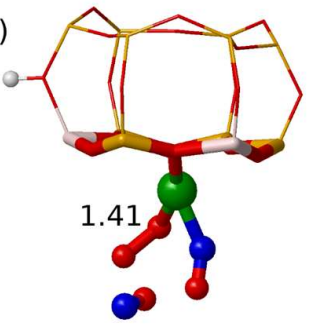

d)

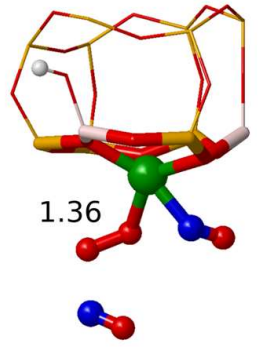

b)

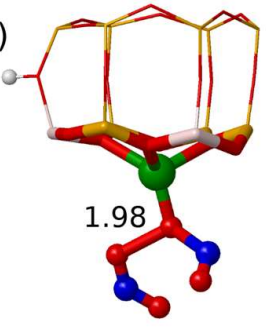

e)

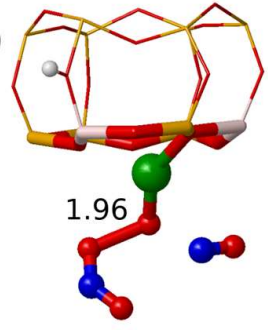

C)

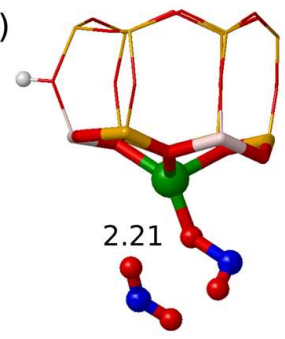

f)

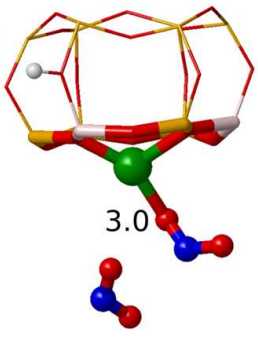

Figure 5.13 Snapshots of the spontaneous oxidation of $\mathrm{NO}$ to $\mathrm{NO}_{2}\left(2 \mathrm{NO}+\mathrm{O}_{2} \rightarrow 2 \mathrm{NO}_{2}\right)$ at $673 \mathrm{~K}$ in Cu-SSZ-13 without ammonia (a, b and c) and with ammonia (d, e and f). Al, O and $\mathrm{Si}$ atoms in the framework depicted as thatch, red and orange sticks; $\mathrm{Cu}$ cations, $\mathrm{O}, \mathrm{N}$ and $\mathrm{H}$ atoms in the reactant molecules depicted as green, red, blue and white balls. 
Interestingly, we observe at $673 \mathrm{~K}$ the spontaneous dissociation of the $\mathrm{O}-\mathrm{O}$ bond of the $\mathrm{O}_{2}$ molecule after 20 ps with the consequent formation of two $\mathrm{NO}_{2}$ molecules (Figures 5.13a, 5.13b and 5.13c). During the transition one $\mathrm{NO}$ and the $\mathrm{O}_{2}$ are still bonded to $\mathrm{Cu}^{+}$. The second $\mathrm{NO}$ approaches the $\mathrm{O}_{2}$ molecule, and the formation of a new $\mathrm{N}-\mathrm{O}$ bond and the O-O rupture occur simultaneously. Then, immediatly the second $\mathrm{N}-\mathrm{O}$ bond is formed. The reverse event did not happen and the two $\mathrm{NO}_{2}$ molecules formed remained attached to $\mathrm{Cu}^{+}$during the rest of the simulation.

The same event happened after $18 \mathrm{ps}$ in the presence of one $\mathrm{NH}_{3}$ inside the cavity (Figure 5.13d, 5.13e, and 5.13f). In this case, the $\mathrm{NH}_{3}$ molecule remained at an average distance of $8 \AA$ from $\mathrm{Cu}^{+}$during the entire simulation time, acting as a spectator while the formation of the two $\mathrm{NO}_{2}$ occurred. The transition is similar to the one without ammonia but the $\mathrm{NO}$ attached to $\mathrm{Cu}^{+}$moves away while the O-O bond is being broken. Then, the second $\mathrm{NO}_{2}$ is formed from $\mathrm{NO}$ and a $6 \mathrm{r}-\mathrm{Cu}^{+}-\mathrm{O}$ species. It means that indeed this process is very favorable, to the point of being spontaneous at $673 \mathrm{~K}$. This observation is in agreement with the mechanism proposed with static DFT in the previous chapter (section 4.5) where two NO molecules adsorbed on $\mathrm{Cu}^{+}$react with $\mathrm{O}_{2}$ to produce two $\mathrm{NO}_{2}$. However, by taking into account the dynamics of the system explicitly we can see that the $\mathrm{O}_{2}$ molecule is likely to be bonded to $\mathrm{Cu}^{+}$during the transition. The former spontaneous reaction was only observed for Cu-SSZ-13 at $673 \mathrm{~K}$. We have not performed similar simulations for SAPO-34 at $673 \mathrm{~K}$.

We have also run similar simulations constraining the mobility of $\mathrm{NH}_{3}$ inside the $\mathrm{Cu}-$ SSZ-13 cavity at a maximum distance of $6.5 \AA$ from $\mathrm{Cu}^{+}$in order to sample more configurations with $\mathrm{NH}_{3}$ close to $\mathrm{Cu}^{+}$. Figure 5.14a shows how $\mathrm{NH}_{3}$, in the presence of two $\mathrm{NO}$ and $\mathrm{O}_{2}$, is able to approach the $\mathrm{Cu}^{+}$cation and displace both $\mathrm{NO}$ molecules at $298 \mathrm{~K}$. The distance between $\mathrm{Cu}$ and the $\mathrm{N}$ atom of the ammonia molecule (Cu-N1) decreases to $2.0 \AA$ and the distances between $\mathrm{Cu}$ and the $\mathrm{N}$ atom of both $\mathrm{NO}$ molecules $(\mathrm{Cu}-\mathrm{N} 2$ and $\mathrm{Cu}-\mathrm{N} 3)$ increase after $\sim 50$ ps. At $523 \mathrm{~K}$, apart from the two $\mathrm{NO}$ and the $\mathrm{O}_{2}$ molecules being displaced from their coordination with $\mathrm{Cu}^{+}$, the formation of two $\mathrm{NO}_{2}$ molecules is observed at $\sim 22 \mathrm{ps}$, which is evidenced by the increase of the O-O bond length, O1-O2 (Figure 5.14b). Furthermore, notice how the distance $\mathrm{Cu}-\mathrm{N} 2$ decreases to $\sim 2.0 \AA$ at around $60 \mathrm{ps}$ indicating that one of the $\mathrm{NO}_{2}$ molecules coordinates to $\mathrm{Cu}^{+}$again after the reaction. In all simulations 
discussed so far in this section, the average $\mathrm{Cu}-6 \mathrm{r}$ distance and the RMSD increase with the increase of the temperature (Table 5.6) but the $\mathrm{Cu}^{+}$remains coordinated to two of the $6 r$ framework oxygens.

In summary, the above results evidence the ability of $\mathrm{NH}_{3}$ to coordinate spontaneously to $\mathrm{Cu}^{+}$even when $\mathrm{NO}$ and $\mathrm{O}_{2}$ are initially adsorbed. They also suggest that the oxidation of $\mathrm{NO}$ to $\mathrm{NO}_{2}$ is spontaneous at $673 \mathrm{~K}$ regardless of the presence or absence of ammonia and of the location of the $\mathrm{Cu}^{+}$. It is also confirmed that the strong interaction of $\mathrm{NH}_{3}$ with $\mathrm{Cu}^{+}$results in a significant higher mobility of the latter regardless of its initial state. Ammonia is able to spontaneously displace the NO and $\mathrm{O}_{2}$ molecules and eventually form the very stable diamine-copper(I) complex. Thus, when the catalyst is exposed to $\mathrm{NH}_{3}$ the $\mathrm{Cu}^{+}$sites will not be coordinated to the zeolite framework, but mobile inside the cavity as $\mathrm{Cu}^{+}\left(\mathrm{NH}_{3}\right)_{2}$. 
a)
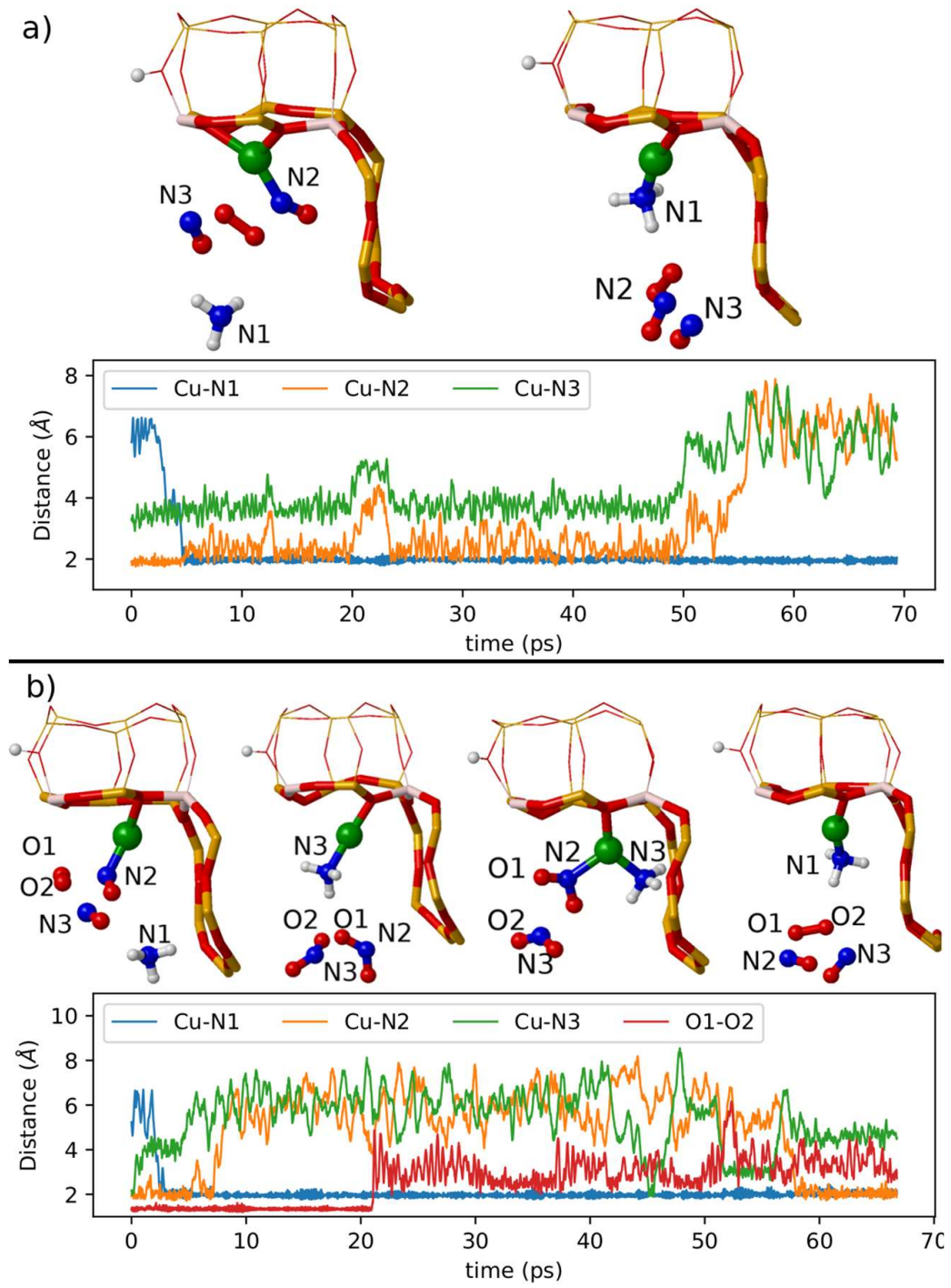

Figure 5.14 Snapshots of simulations of two $\mathrm{NO}$ and $\mathrm{O}_{2}$ coordinated to $\mathrm{Cu}^{+}$and one ammonia constrained to the vicinity of $\mathrm{Cu}^{+}$at $298 \mathrm{~K}$ (upper panel) and at $523 \mathrm{~K}$ (lower panel). $\mathrm{Al}, \mathrm{Si}$, and $\mathrm{O}$ atoms in the framework depicted as thatch, orange, and red sticks; $\mathrm{Cu}$ cations, $\mathrm{O}, \mathrm{N}$ and $\mathrm{H}$ atoms in the reactant molecules are depicted as green, red, blue and white balls. 


\subsubsection{Oxidation of $\mathrm{NO}$ to $\mathrm{NO}_{2}$ at low temperature with umbrella sampling}

The results presented so far indicate that the $\mathrm{Cu}^{+}$cations will not be coordinated to the $6 r$ in the presence of $\mathrm{NH}_{3}$ but rather forming stable complex species with $\mathrm{NH}_{3}$ and NO. In order to investigate in more detail the oxidation of $\mathrm{NO}$ with $\mathrm{O}_{2}$ under more realistic conditions we have considered a system made up of the stable $\left[\mathrm{Cu}\left(\mathrm{NH}_{3}\right)_{2}(\mathrm{NO})_{2}\right]^{+}$complex together with a $\mathrm{O}_{2}$ molecule inside the $\mathrm{CHA}$ cage (Cu-CHA). Another simulation without $\mathrm{Cu}$ and without $\mathrm{NH}_{3}(\mathrm{CHA})$ has been performed to find out whether or not the amine-copper(I) complex affects this reaction, both at $1 \mathrm{~atm}$ and $298 \mathrm{~K}$. Finally, we have also performed a third simulation (Gas Phase) consisting of two NO and one $\mathrm{O}_{2}$ molecule in a cubic simulation box of $15 \times 15 \times 15 \AA^{3}$. The pressure of this last simulation was equilibrated to $10 \mathrm{~atm}$, and the temperature to $298 \mathrm{~K}$.

We have performed umbrella sampling simulations biased along a collective variable (CN3) that represents the simultaneous $\mathrm{O}-\mathrm{O}$ bond dissociation of the $\mathrm{O}_{2}$ molecule and formation of two N-O bonds. Two coordination numbers CN1 and CN2 (see equation 5.1) have been defined. $\mathrm{CN} 1$ tracks the coordination of the $\mathrm{O}$ atoms of the $\mathrm{O}_{2}$ molecule with each other (Figure 5.15). CN2 tracks the coordination of the two $\mathrm{O}$ atoms of the $\mathrm{O}_{2}$ molecule with each $\mathrm{N}$ atom of the $\mathrm{NO}$ molecules (Figure 5.15). Finally, $\mathrm{CN} 3=\mathrm{CN} 2-\mathrm{CN} 1$, such that it ranges from -1 to 2 .
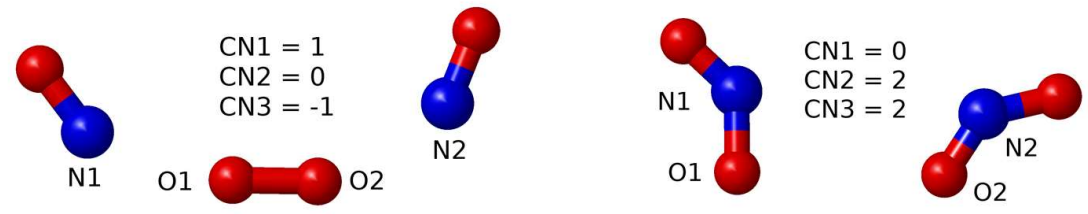

Figure 5.15 Representation of the coordination number CN1, CN2 and CN3 before (left panel) and after (right panel) the reaction. $\mathrm{CN} 3=\mathrm{CN} 2-\mathrm{CN} 1$.

Figure 5.16 shows the two free energy profiles of the aforementioned systems where the states labeled as $\mathrm{A}, \mathrm{B}$ and $\mathrm{C}$ correspond to the reactant, transition state and products respectively, and Figure 5.17 shows the snapshots of state B for the three systems. The values of $\mathrm{CN} 3$ for each state and system are summarized in Table 5.7. State $\mathrm{A}$ is located at $\mathrm{CN} 3=-0.20$ and at $\mathrm{CN} 3=-0.07$ for $\mathrm{Cu}-\mathrm{CHA}$ and $\mathrm{CHA}$ respectively. In the case of $\mathrm{Cu}-\mathrm{CHA}$, the two $\mathrm{NO}$ and $\mathrm{O}_{2}$ molecules are interacting 
with each other more strongly than with $\mathrm{Cu}^{+}$. On average, the three molecules are more than $2.6 \AA$ away from $\mathrm{Cu}^{+}$(see the distances $\mathrm{Cu}-\mathrm{N} 1, \mathrm{Cu}-\mathrm{N} 1, \mathrm{Cu}-\mathrm{O} 1$ and $\mathrm{Cu}-$ $\mathrm{O} 2$ in Table 5.7). However, a weak influence of $\mathrm{Cu}^{+}\left(\mathrm{NH}_{3}\right)_{2}$ on the state of the two $\mathrm{NO}$ and $\mathrm{O}_{2}$ is observed. The intermolecular distances N1-O1 and N2-O2 are slightly shorter for $\mathrm{Cu}-\mathrm{CHA}$ than for $\mathrm{CHA}$ and the bond length of $\mathrm{O}_{2}(\mathrm{O} 1-\mathrm{O} 2)$ is slightly longer.

Table 5.7 Average distance $(d)$ in angstroms between $\mathrm{Cu}^{+}$and the $\mathrm{N}$ atom of the two NO and two $\mathrm{NH}_{3}$ at relevant points along the collective variable $\mathrm{CN} 3$.

\begin{tabular}{ccccccccccc}
\hline \multicolumn{3}{c|}{ Cu-CHA } & \multicolumn{3}{c|}{ CHA } & \multicolumn{3}{c}{ Gas Phase } \\
\hline State & A & B & C & A & B & C & A & B & C \\
$\mathrm{CN} 3$ & -0.20 & 0.50 & 1.65 & -0.07 & 0.70 & 1.75 & -0.23 & 0.54 & 1.67 \\
$d \mathrm{~N} 1-\mathrm{O} 1$ & 1.73 & 1.51 & 1.24 & 1.82 & 1.53 & 1.21 & 1.86 & 1.47 & 1.21 \\
$d \mathrm{~N} 2-\mathrm{O} 2$ & 1.78 & 1.47 & 1.22 & 1.83 & 1.32 & 1.21 & 1.84 & 1.46 & 1.21 \\
$d \mathrm{O} 1-\mathrm{O} 2$ & 1.36 & 1.52 & 4.93 & 1.33 & 1.66 & 3.97 & 1.31 & 1.56 & 5.8 \\
$d \mathrm{Cu}-\mathrm{N} 1$ & 3.79 & 3.54 & 4.74 & - & - & - & - & - & - \\
$d \mathrm{Cu}-\mathrm{N} 2$ & 4.51 & 4.21 & 2.21 & - & - & - & - & - & - \\
$d \mathrm{Cu}-\mathrm{O} 1$ & 2.62 & 3.70 & 4.45 & - & - & - & - & - & - \\
$d \mathrm{Cu}-\mathrm{O} 2$ & 3.49 & 4.03 & 2.97 & - & - & - & - & - & - \\
$d \mathrm{Cu}-6 \mathrm{r}$ & 6.49 & 5.54 & 2.88 & - & - & - & - & - & - \\
\hline
\end{tabular}

State $\mathrm{B}$ (transition state) is located at $\mathrm{CN} 3=0.5$ and at $\mathrm{CN} 3=0.7$ for $\mathrm{Cu}-\mathrm{CHA}$ and CHA respectively. Again the two $\mathrm{NO}$ and $\mathrm{O}_{2}$ are located on average more than $3 \AA$ away from $\mathrm{Cu}^{+}$. However, the bond length of the $\mathrm{O}_{2}$ molecule $(\mathrm{O} 1-\mathrm{O} 2)$ is $\sim 0.1 \AA$ shorter when the diamine-copper(I) is present ( $\mathrm{Cu}-\mathrm{CHA})$ and the N2-O2 distance is $0.15 \AA$ longer. These changes are accompanied by a modest decrease of the activation energy to $25 \mathrm{~kJ} / \mathrm{mol}$ in $\mathrm{Cu}-\mathrm{CHA}$ from the $28 \mathrm{~kJ} / \mathrm{mol}$ calculated for CHA (Table 5.8). Finally, notice how the average $\mathrm{Cu}-6 \mathrm{r}$ gradually decreases along the $\mathrm{CN} 3$ coordinate from $6.49 \AA$ in state $\mathrm{A}$ to $2.88 \AA \AA$ in state $\mathrm{C}$ (Table 5.7). The presence of $\mathrm{NO}_{2}$ seems to be associated with a more constrained mobility of the $\mathrm{Cu}^{+}\left(\mathrm{NH}_{3}\right)_{2}$ close to the $6 r$. The same effect was observed for a similar system in section 5.3.3. 


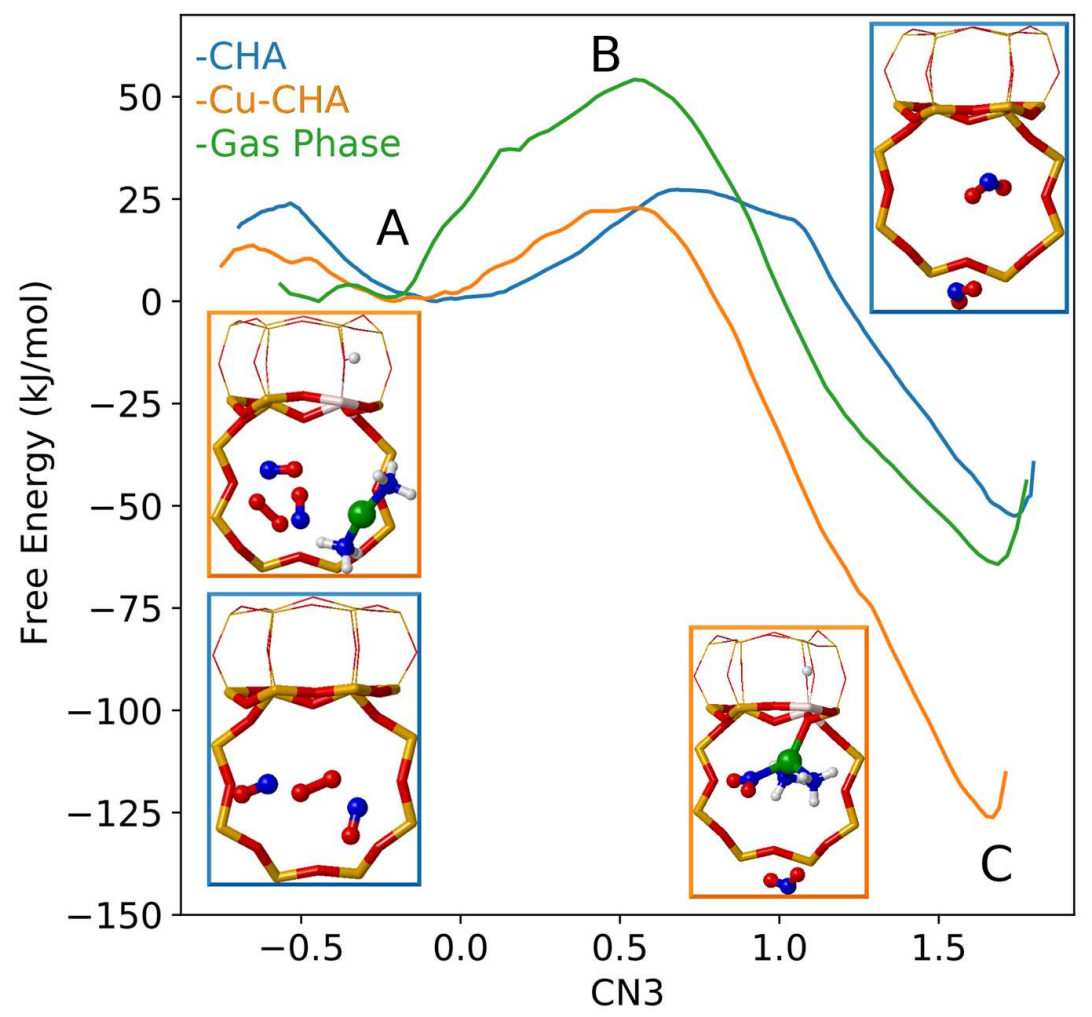

Figure 5.16 Free energy profile of the oxidation of $\mathrm{NO}$ with $\mathrm{O}_{2}$ at $298 \mathrm{~K}$. Spheres in green, blue, gray, red and orange represent $\mathrm{Cu}, \mathrm{N}, \mathrm{H}, \mathrm{O}$ and $\mathrm{Si}$ atoms respectively.

Comparison of the gas phase system with $\mathrm{Cu}-\mathrm{CHA}$ and $\mathrm{CHA}$ has to be carefully done because the conditions are slightly different. However, it can be clearly seen that the activation free energy, $54 \mathrm{~kJ} / \mathrm{mol}$, is higher than in the previous two cases. The decrease in the activation free energy in $\mathrm{Cu}-\mathrm{CHA}$ and CHA compared with the gas phase can be attributed to confinement effects. The entropic and enthalpic contributions to the free energy can not be calculated with these simulations but the accessible volume of the CHA framework ${ }^{29}$ is $\sim 413 \AA^{3}$ while the volume of the gas phase simulation box is $3375 \AA^{3}$. Thus, the entropy penalty from state A to B is higher in the gas phase than inside the zeolite. The rate constants of the three cases 
calculated from the activation free energies according to Eyring's equation (eq 2.63) are included in Table 5.8. The ratio between the rate constants of NO oxidation confined in the CHA zeolite (CHA) and unconfined (gas phase) is $3.8 \times 10^{4}$. This is in agreement with the experimental ratio between the rates of confined and unconfined reactions found by Maestri et al. ${ }^{30}$ in the CHA framework.

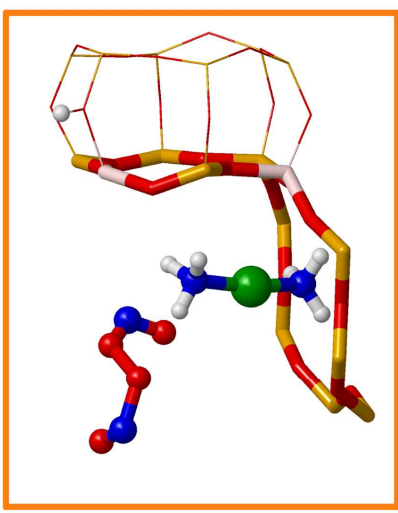

$\mathrm{Cu}-\mathrm{CHA}$

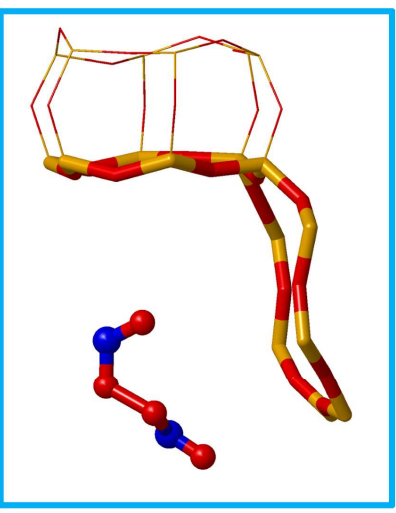

$\mathrm{CHA}$

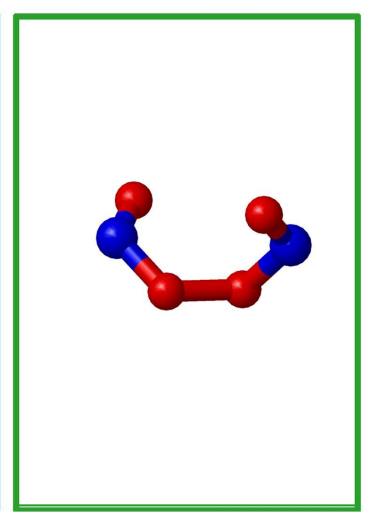

Gas Phase

Figure 5.17 Snapshots of the transition states in the oxidation of $\mathrm{NO}$ with $\mathrm{O}_{2}$ at 298 $\mathrm{K}$ for the three systems considered.

Table 5.8 Calculated activation free energy $\Delta \mathrm{G}_{\text {act }}$ and calculated rate constants $K r$ for the three umbrella sampling simulations at $298 \mathrm{~K}$.

\begin{tabular}{ccccc}
\hline & Cu-CHA & CHA & Gas Phase \\
\hline$\Delta \mathrm{G}_{\text {act }}(\mathrm{kJ} / \mathrm{mol})$ & 25 & 28 & 54 \\
$K r\left(\mathrm{~L}^{2} \cdot \mathrm{mol}^{-2} \cdot \mathrm{s}^{-1}\right)$ & $2.6 \times 10^{8}$ & $7.6 \times 10^{7}$ & $2.1 \times 10^{3}$ \\
\hline
\end{tabular}

In summary, these results indicate that the oxidation of NO inside the big CHA cage is relatively fast at $298 \mathrm{~K}$ and does not seem to be significantly enhanced by the diamine-copper(I) complex. Under these conditions, the formation of $\mathrm{NO}_{2}$ does not need a previous $\mathrm{O}_{2}$ activation by the $\mathrm{Cu}$ active sites. Finally, the reaction rate is significantly enhanced when it takes place inside the zeolite due to confinement effects. 


\subsection{Diffusion of amino complex}

In section 5.3, we saw that the motion of $\mathrm{Cu}^{+}$, enhanced by its interaction with $\mathrm{NH}_{3}$, is still constrained to the neighborhood of the $6 r$ because of the electrostatic attraction with the negatively charged framework $\mathrm{O}$ atoms directly attached to Al. In this section, we study the diffusion of the diamine-copper(I) complex from an initial cavity (A) to the neighbouring cavity (B) using umbrella sampling simulations at $423 \mathrm{~K}$. In order to describe the diffusion of $\mathrm{Cu}^{+}\left(\mathrm{NH}_{3}\right)_{2}$ through the $8 r$ toward the adjacent cavity we have used the collective variable $\xi$, defined as the projection of the $\mathrm{Cu}$ coordinate on the vector normal to the average plane of the $8 r$. See section 2.6.3 for more details. The free energy profiles of the diffusion of $\mathrm{Cu}^{+}\left(\mathrm{NH}_{3}\right)_{2}$ to the next cavity are shown in Figures 5.18a and 5.18b. The values of the activation free energies are summarized in Table 5.9 and the snapshots of minima and transition states are shown in Figures 5.19, 5.20 and 5.21.

Table 5.9 Activation free energies $\left(\Delta \mathrm{Gact}_{\mathrm{x}}\right)$ at the corresponding values of the collective variable $\left(\xi a c t_{x}\right)$, and reaction free energies $\left(\Delta \mathrm{Gr}_{1}\right)$ at the corresponding collective variable $\left(\xi \mathrm{r}_{1}\right)$ for the diffusion of $\mathrm{Cu}^{+}\left(\mathrm{NH}_{3}\right)_{2}$ through the $8 r$ window of $\mathrm{Cu}-\mathrm{SSZ}-13$ at $423 \mathrm{~K}$. Free energy is given in $\mathrm{kJ} / \mathrm{mol}$.

\begin{tabular}{ccccccccc}
\hline & Cavity A & Cavity B & $\Delta$ Gact $_{1}$ & $\Delta$ Gact $_{2}$ & $\xi$ act $_{1}$ & $\xi$ act $_{2}$ & $\Delta \mathrm{Gr}_{1}$ & $\xi \mathrm{r}_{1}$ \\
\hline a & $\mathrm{Cu}^{+}\left(\mathrm{NH}_{3}\right)_{2}$ & - & 17 & - & 0.35 & - & 1.5 & 2.4 \\
b & $\mathrm{Cu}^{+}\left(\mathrm{NH}_{3}\right)_{2}$ & $\mathrm{Cu}^{+}\left(\mathrm{NH}_{3}\right)_{2}$ & 28 & - & 0.30 & - & 7 & 2.45 \\
c & $\mathrm{Cu}^{+}\left(\mathrm{NH}_{3}\right)_{2}$ & $\mathrm{Cu}^{+}\left(\mathrm{NH}_{3}\right)_{2}+\mathrm{O}_{2}$ & 26 & - & 0.40 & - & 5 & 2.6 \\
d & $\mathrm{Cu}^{+}\left(\mathrm{NH}_{3}\right)_{2}$ & $\mathrm{Cu}^{+}\left(\mathrm{NH}_{3}\right)_{2}+2 \mathrm{NO}+\mathrm{O}_{2}$ & 24 & - & 0.2 & - & 0 & 2.85 \\
e & $\mathrm{Cu}^{+}\left(\mathrm{NH}_{3}\right)_{2}+\mathrm{H}_{2} \mathrm{O}$ & $\mathrm{Cu}^{+}\left(\mathrm{NH}_{3}\right)_{2}+\mathrm{H}_{2} \mathrm{O}$ & 22 & - & 0.67 & - & 10 & 2.30 \\
f & $\mathrm{Cu}^{+}\left(\mathrm{NH}_{3}\right)_{2}+\mathrm{NH}_{3}$ & $\mathrm{Cu}^{+}\left(\mathrm{NH}_{3}\right)_{2}+\mathrm{NH}_{3}$ & 28 & - & 0.90 & - & 15 & 2.40 \\
g & $\mathrm{Cu}^{+}\left(\mathrm{NH}_{3}\right)_{2}+2 \mathrm{NH}_{3}$ & $\mathrm{Cu}^{+}\left(\mathrm{NH}_{3}\right)_{2}$ & 48 & - & 1.04 & - & 36 & 2.30 \\
h & $\mathrm{Cu}^{+}\left(\mathrm{NH}_{3}\right)_{2}+2 \mathrm{NO}$ & $\mathrm{Cu}^{+}\left(\mathrm{NH}_{3}\right)_{2}$ & 48 & - & 0.50 & - & 34 & 2.40 \\
- & $\mathrm{Cu}^{+}\left(\mathrm{NH}_{3}\right)_{2}(1 \times 2 \times 1)$ & - & 22 & 76 & 0.80 & 8.4 & 14.4 & 2.08 \\
\hline
\end{tabular}



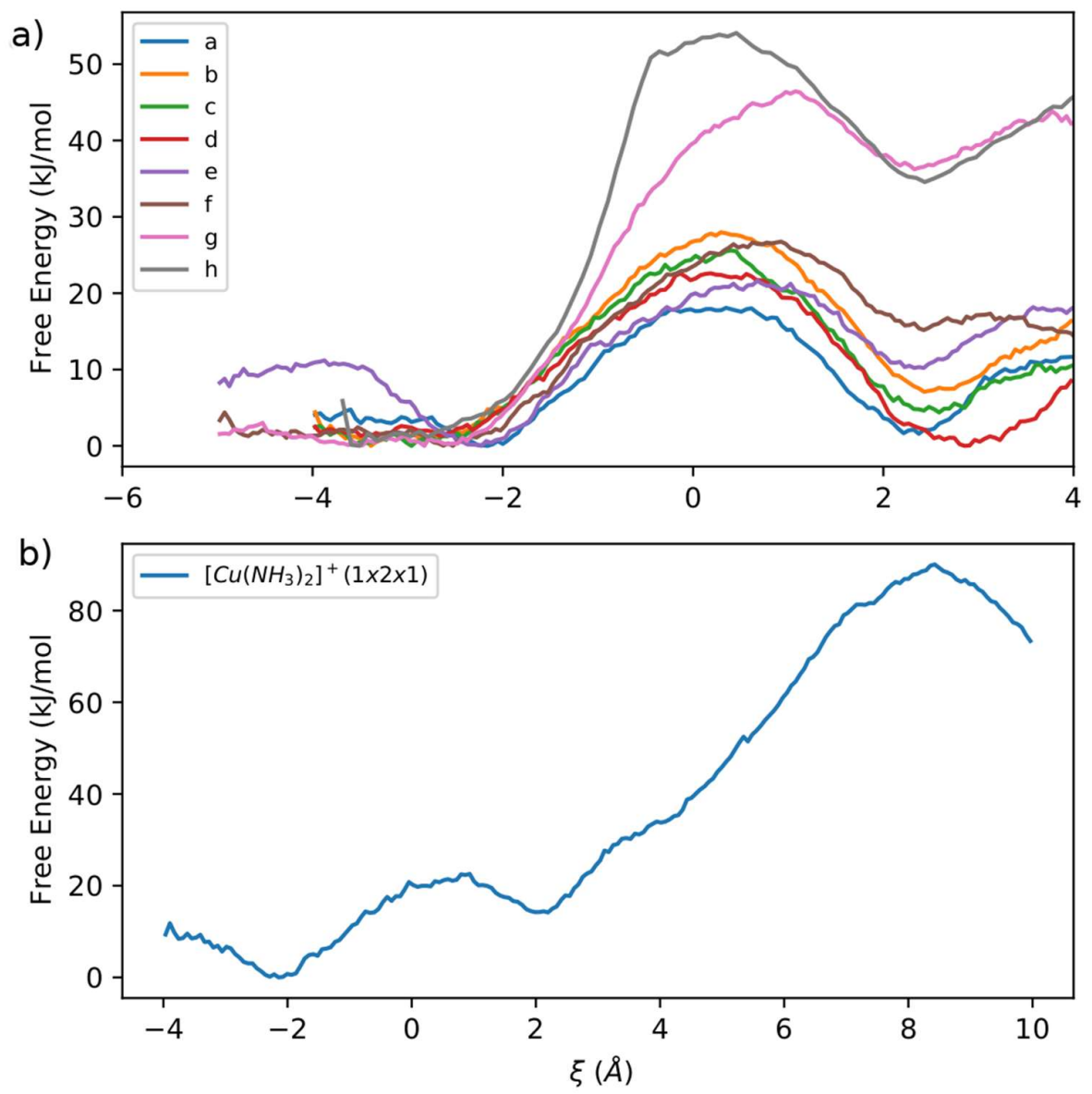

Figure 5.18 Free energy profiles of the diffusion of $\mathrm{Cu}^{+}\left(\mathrm{NH}_{3}\right)_{2}$ through the $8 r$ windows of SSZ-13 zeolite with umbrella sampling AIMD simulations at $423 \mathrm{~K}$. a) $\mathrm{Cu}^{+}\left(\mathrm{NH}_{3}\right)_{2}$ in cavity A and cavity B empty, b) $\mathrm{Cu}^{+}\left(\mathrm{NH}_{3}\right)_{2}$ in cavity $\mathrm{A}$ and $\mathrm{Cu}^{+}\left(\mathrm{NH}_{3}\right)_{2}$ in cavity $\left.\mathrm{B}, \mathrm{c}\right) \mathrm{Cu}^{+}\left(\mathrm{NH}_{3}\right)_{2}$ in cavity $\mathrm{A}$ and $\mathrm{Cu}^{+}\left(\mathrm{NH}_{3}\right)_{2}+\mathrm{O}_{2}$ in cavity $\mathrm{B}$, d) $\mathrm{Cu}^{+}\left(\mathrm{NH}_{3}\right)_{2}$ in cavity A and $\mathrm{Cu}^{+}\left(\mathrm{NH}_{3}\right)_{2}+2 \mathrm{NO}+$ $\mathrm{O}_{2}$ in cavity $\mathrm{B}$, e) $\mathrm{Cu}^{+}\left(\mathrm{NH}_{3}\right)_{2}+\mathrm{H}_{2} \mathrm{O}$ in cavity $\mathrm{A}$ and $\mathrm{Cu}^{+}\left(\mathrm{NH}_{3}\right)_{2}+\mathrm{H}_{2} \mathrm{O}$ in cavity $\mathrm{B}, \mathrm{f}$ ) $\mathrm{Cu}^{+}\left(\mathrm{NH}_{3}\right)_{2}+\mathrm{NH}_{3}$ in cavity $\mathrm{A}$ and $\mathrm{Cu}^{+}\left(\mathrm{NH}_{3}\right)_{2}+\mathrm{NH}_{3}$ in cavity $\left.\mathrm{B}, \mathrm{g}\right) \mathrm{Cu}^{+}\left(\mathrm{NH}_{3}\right)_{2}+2 \mathrm{NH}_{3}$ in cavity $\mathrm{A}$ and $\mathrm{Cu}^{+}\left(\mathrm{NH}_{3}\right)_{2}$ in cavity $\left.\mathrm{B}, \mathrm{h}\right) \mathrm{Cu}^{+}\left(\mathrm{NH}_{3}\right)_{2}+2 \mathrm{NO}$ in cavity $\mathrm{A}$ and $\mathrm{Cu}^{+}\left(\mathrm{NH}_{3}\right)_{2}$ in cavity $\mathrm{B}$. 
The diffusion of $\mathrm{Cu}^{+}\left(\mathrm{NH}_{3}\right)_{2}$ from an empty cavity to the adjacent empty cavity has a free energy penalty of only $17 \mathrm{~kJ} / \mathrm{mol}$ (Figures 5.18a and 5.19a). If there is another $\mathrm{Cu}^{+}\left(\mathrm{NH}_{3}\right)_{2}$ complex already formed in the adjacent cavity the activation energy to cross to the $8 r$ increases to $28 \mathrm{~kJ} / \mathrm{mol}$ (Figures 5.18a and 5.19b) and the final state is about $7 \mathrm{~kJ} / \mathrm{mol}$ less stable. This slight destabilization is probably related to both $\mathrm{Cu}^{+}$ being forced to be closer in the final state. Then, for the simulations with an additional $\mathrm{O}_{2}$ molecule in the adjacent cavity $\mathrm{B}$ (Figures 5.18a and 5.19c) or $2 \mathrm{NO}+\mathrm{O}_{2}$ (Figures 5.18a and 5.20a) the activation free energy slightly decreases to 26 and 24 $\mathrm{kJ} / \mathrm{mol}$ respectively. These molecules seem to have a slight positive effect in the activation free energy and final state stability probably because of the favorable interaction with $\mathrm{Cu}^{+}$. However, this effect is not significant. Thus, at $423 \mathrm{~K}$ the $\mathrm{Cu}^{+}\left(\mathrm{NH}_{3}\right)_{2}$ complex seems to be free to move between adjacent cavities.

We have introduced one extra water or ammonia molecule not coordinated to $\mathrm{Cu}^{+}$in each cavity (Figures 5.19b and 5.19c) for which activation free energies of 22 and $28 \mathrm{~kJ} / \mathrm{mol}$ respectively are calculated (Table 5.9 and Figure 5.18a). Compared with the system made up of only two $\mathrm{Cu}^{+}\left(\mathrm{NH}_{3}\right)_{2}$, one ammonia in each cavity does not seem to have any effect on the diffusion. However, if the two $\mathrm{NH}_{3}$ molecules are located in the first cavity then the stabilization of the $\mathrm{Cu}^{+}\left(\mathrm{NH}_{3}\right)_{2}$ in the first cavity is more evident and the activation free energy increases significantly to $48 \mathrm{~kJ} / \mathrm{mol}$. This increase in the activation energy is related to the strong $\mathrm{H}$-bonding between the two extra $\mathrm{NH}_{3}$ molecules and the two $\mathrm{NH}_{3}$ of the diamine-copper(I). The final state of the complex in the adjacent cavity B is also noticeably less stable compared with the initial state in cavity A. The same effect is observed when the two extra NO are present in the first cavity instead of $\mathrm{NH}_{3}$. The activation free energy increases to $\sim 48$ $\mathrm{kJ} / \mathrm{mol}$ and the final state (both complexes in the cavity B) is considerably less stable. In this case, the increase of the activation energy can be accounted for by considering the formation of the stable complex $\mathrm{Cu}^{+}\left(\mathrm{NH}_{3}\right)_{2}(\mathrm{NO})_{2}$ when two $\mathrm{NO}$ molecules interact with $\mathrm{Cu}^{+}\left(\mathrm{NH}_{3}\right)_{2}$ (section 5.4). Figure $5.21 \mathrm{~b}$ shows that the diffusion of $\mathrm{Cu}^{+}\left(\mathrm{NH}_{3}\right)_{2}$ implies the dissociation of the bonds between $\mathrm{Cu}$ and the two NO molecules. In other words, the bulky complex $\mathrm{Cu}^{+}\left(\mathrm{NH}_{3}\right)_{2}(\mathrm{NO})_{2}$ is not able to diffuse through the $8 r$.

Finally, we also simulated the diffusion of the diamine-copper(I) all the way to a third cavity (C) through the corresponding $8 r$ window (Figures 5.18a and 5.21c). To 
that end, we have used a $1 \times 2 \times 1$ simulation unit cell and no extra reactant molecules were considered. The activation free energy to cross the first $8 r$ is $22 \mathrm{~kJ} / \mathrm{mol}$, very similar to one found for $1 \times 1 \times 1$ unit cells. As the diamine-copper(I) moves away from the first $8 r$ the free energy increases continuously until a second maximum value of $90 \mathrm{~kJ} / \mathrm{mol}$ is found while crossing the second $8 r$. The high destabilization of the system as the diamine-copper(I) complex migrates far from cavity A is clearly associated to the weakening of the electrostatic interactions between the positively charged $\mathrm{Cu}^{+}\left(\mathrm{NH}_{3}\right)_{2}$ species and the negatively charged framework $\mathrm{O}$ atoms around the $\mathrm{Al}$ atom, which is located in the $8 r$ connecting cavities A and B (see Figure 5.21).

In summary, the diffusion of the stable $\mathrm{Cu}^{+}\left(\mathrm{NH}_{3}\right)_{2}$ species through the $8 r$ at $423 \mathrm{~K}$ is an activated process with relatively low activation energies. However, diffusion of this complex is apparently affected by the nature and distribution of reactant molecules in the neighboring cavities. In any case, because of the electrostatic interactions between $\mathrm{Cu}^{+}$and $\mathrm{AlO}_{4}^{-}$fragment of the $6 r$ it seems very unlikely that this species will diffuse to a third cavity so that its motions is constrained to neighboring cavities and to a maximum of 6-8 $\AA$ from the $\mathrm{Al}$ atom. 

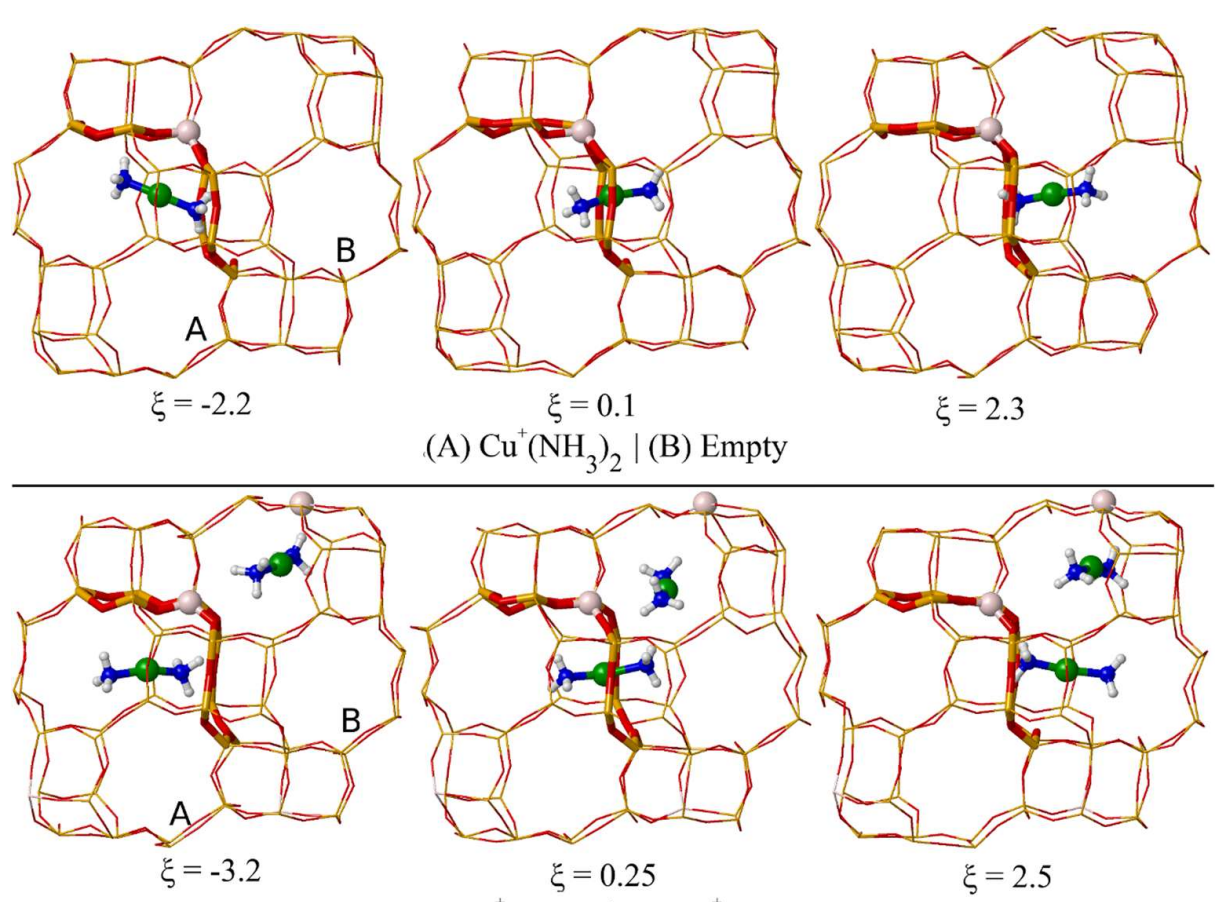

(A) $\mathrm{Cu}^{+}\left(\mathrm{NH}_{3}\right)_{2} \mid$ (B) $\mathrm{Cu}^{+}\left(\mathrm{NH}_{3}\right)_{2}$

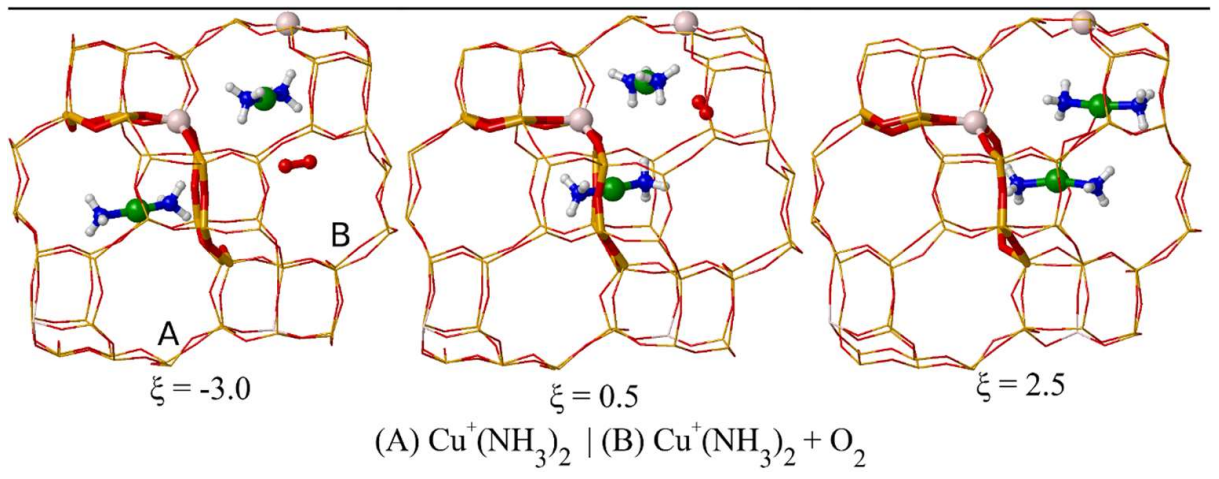

Figure 5.19 Snapshots of minima and transition states of diamine-copper (I) diffusion through the $8 r$ window of SSZ-13 at $423 \mathrm{~K}$ with umbrella sampling. The corresponding values of the collective variable are included below each snapshot. Si and $\mathrm{O}$ atoms in the framework depicted as yellow and red sticks; $\mathrm{Al}, \mathrm{Cu}, \mathrm{O}$ in molecules, $\mathrm{N}$ and $\mathrm{H}$ atoms depicted as thatch, green, red, blue and white balls. 


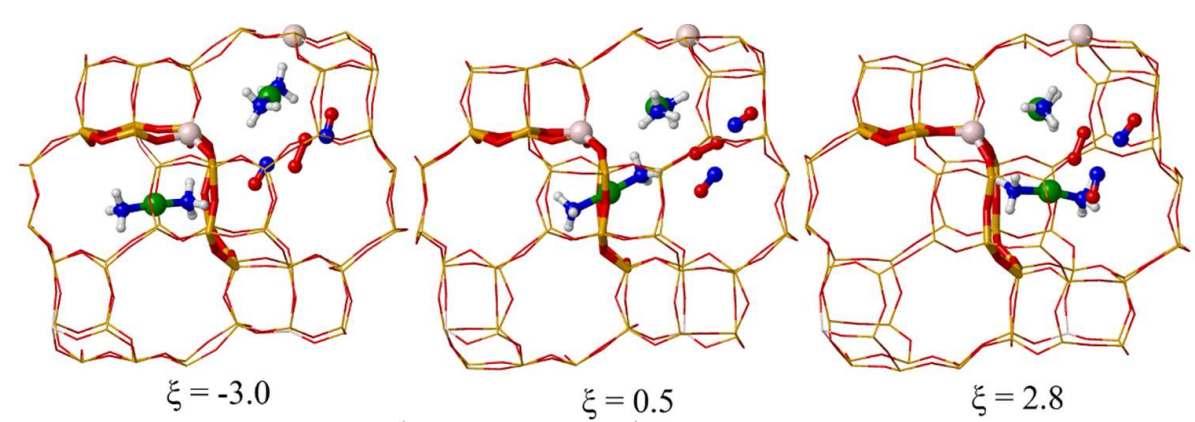

(A) $\mathrm{Cu}^{+}\left(\mathrm{NH}_{3}\right)_{2} \mid$ (B) $\mathrm{Cu}^{+}\left(\mathrm{NH}_{3}\right)_{2}+2 \mathrm{NO}+\mathrm{O}_{2}$

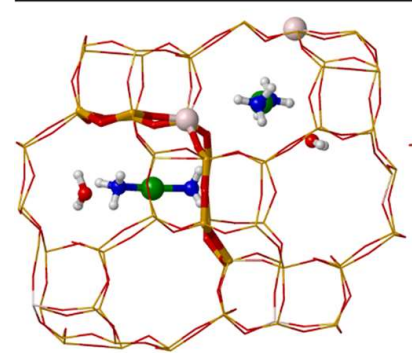

$\xi=-2.5$

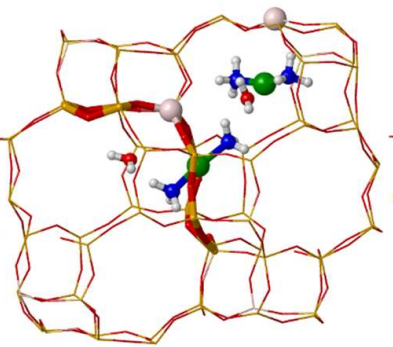

$\xi=0.7$

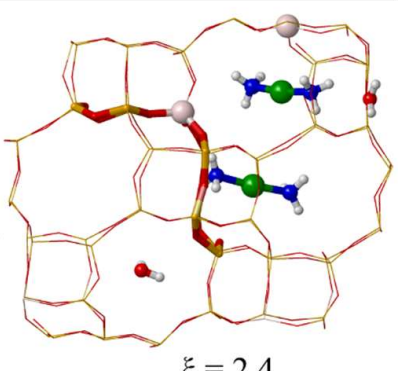

(A) $\mathrm{Cu}^{+}\left(\mathrm{NH}_{3}\right)_{2}+\mathrm{H}_{2} \mathrm{O} \mid$ (B) $\mathrm{Cu}^{+}\left(\mathrm{NH}_{3}\right)_{2}+\mathrm{H}_{2} \mathrm{O}$

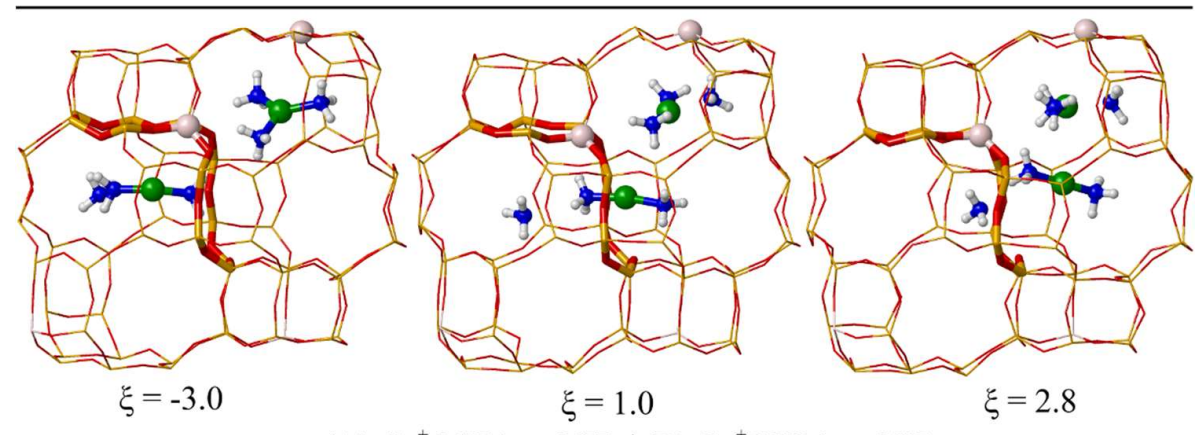

(A) $\mathrm{Cu}^{+}\left(\mathrm{NH}_{3}\right)_{2}+\mathrm{NH}_{3} \mid$ (B) $\mathrm{Cu}^{+}\left(\mathrm{NH}_{3}\right)_{2}+\mathrm{NH}_{3}$

Figure 5.20 Snapshots of minima and transition states of diamine-copper (I) diffusion through the $8 r$ at $423 \mathrm{~K}$ with umbrella sampling. The corresponding values of the collective variable are included below each snapshot. $\mathrm{Si}$ and $\mathrm{O}$ atoms in the framework depicted as yellow and red sticks; $\mathrm{Al}, \mathrm{Cu}, \mathrm{O}$ in molecules, $\mathrm{N}$ and $\mathrm{H}$ atoms depicted as thatch, green, red, blue and white balls. 


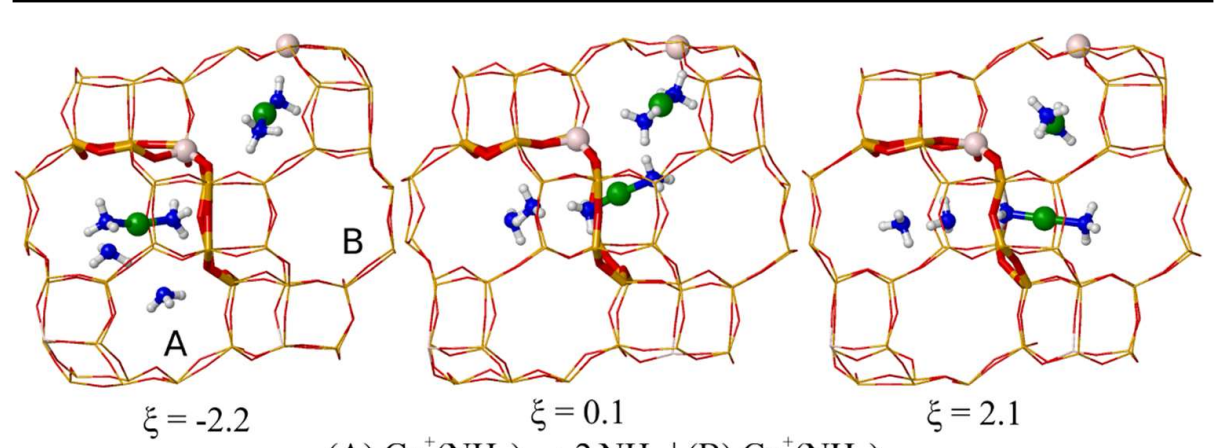

(A) $\mathrm{Cu}^{+}\left(\mathrm{NH}_{3}\right)_{2}+2 \mathrm{NH}_{3} \mid$ (B) $\mathrm{Cu}^{+}\left(\mathrm{NH}_{3}\right)_{2}$

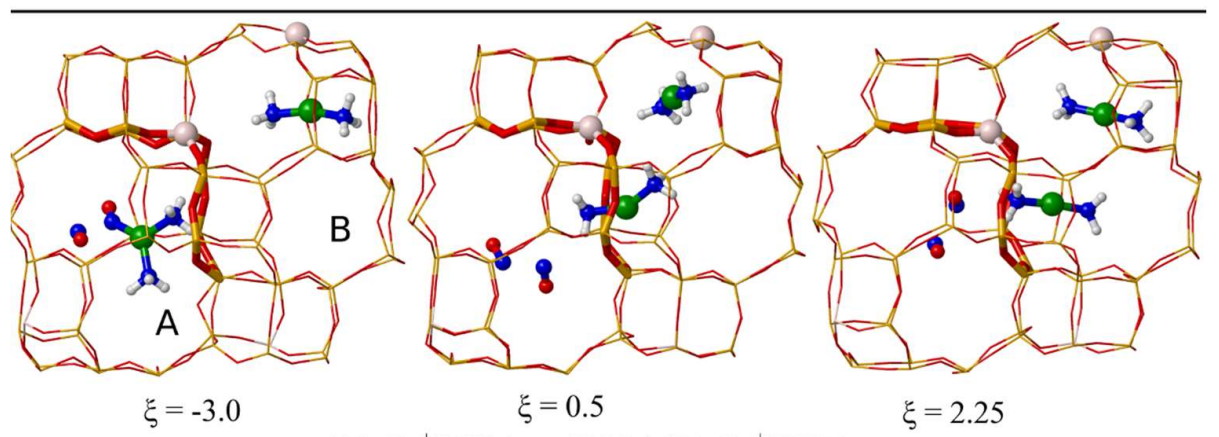

(A) $\mathrm{Cu}^{+}\left(\mathrm{NH}_{3}\right)_{2}+2 \mathrm{NO} \mid$ (B) $\mathrm{Cu}^{+}\left(\mathrm{NH}_{3}\right)_{2}$

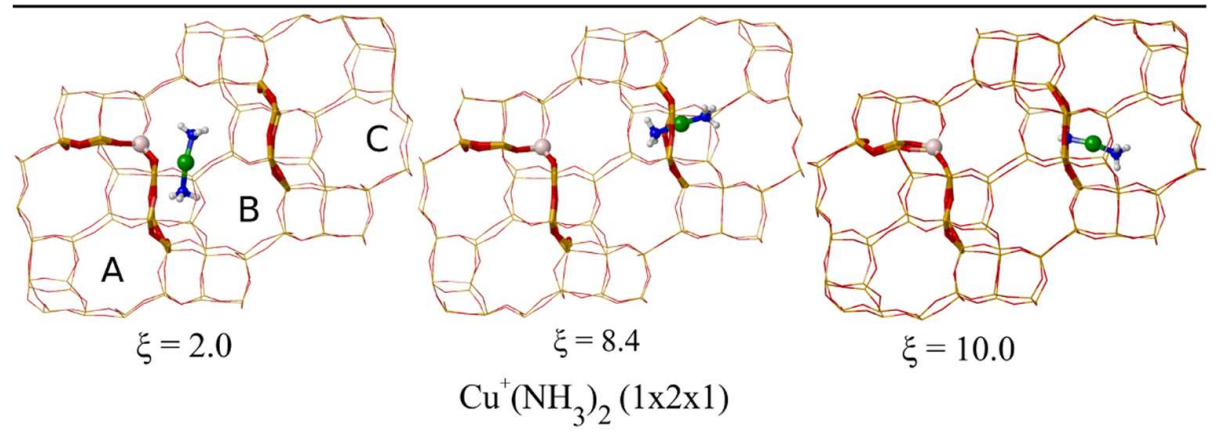

Figure 5.21 Snapshots of minima and transition states of diamine-copper (I) diffusion through the $8 r$ at $423 \mathrm{~K}$ with umbrella sampling. The corresponding values of the collective variable are included below each snapshot. $\mathrm{Si}$ and $\mathrm{O}$ atoms in the framework depicted as yellow and red sticks; $\mathrm{Al}, \mathrm{Cu}, \mathrm{O}$ in molecules, $\mathrm{N}$ and $\mathrm{H}$ atoms depicted as thatch, green, red, blue and white balls. 
5.7 IR analysis. Comparison of static DFT and AIMD computed frequencies

\subsection{IR analysis. Comparison of static DFT and AIMD computed frequencies}

The framework asymmetric T-O-T vibrations of the $\mathrm{Cu}-\mathrm{CHA}$ catalysts perturbed by the presence of copper cations give rise to bands in the $800-1000 \mathrm{~cm}^{-1}$ region of IR spectra $^{31-33}$. In this section, we show that these vibrations can be used as experimental evidence of the dynamic behavior of the copper cations under $\mathrm{NH}_{3}$-SCR-NOx reaction conditions. To that end, we have calculated the vibrational spectra of the most relevant structures of Cu-SAPO-34 investigated in sections 5.3 and 5.4, using the approach described in sections 2.6.8 and 5.2. We have compared the calculated vibrational frequencies with experimental spectra recorded with different reactant feeds and temperatures. The simulated spectra are depicted in Figures 5.22 to 5.26, and the calculated values are summarized in Table 5.10. We will limit the discussion to the IR region between $800-1000 \mathrm{~cm}^{-1}$.

Figure 5.22a shows the IR spectra of H-SAPO-34 and Cu-SAPO-34 activated either in vacuum at $723 \mathrm{~K}$ or in $\mathrm{O}_{2}$ at $623 \mathrm{~K}$. Apart from a small peak at $955 \mathrm{~cm}^{-1}$ corresponding to the bending mode of the Brønsted acid sites, no other bands are observed in the IR spectrum of H-SAPO-34. The spectra of H-SAPO-34 calculated with static DFT and AIMD (Figure 5.23a) are completely flat meaning that our models do not predict bands in the $800-1000 \mathrm{~cm}^{-1}$ region due to the framework T-O$\mathrm{T}$ vibrations in the absence of exchange copper cations, in agreement with the experiments.

The sample of Cu-SAPO-34 pre-activated in $\mathrm{O}_{2}$ at $623 \mathrm{~K}$ contains mostly $\mathrm{Cu}^{2+}$ cations $^{34,35}$. Its spectrum (Figure 5.22a, blue line) exhibits a peak at $964 \mathrm{~cm}^{-1}$ and a broad band at $\sim 885 \mathrm{~cm}^{-1}$. The incorporation of $\mathrm{Cu}^{2+}$ in the $6 r$ in the models results in new peaks between $800-1000 \mathrm{~cm}^{-1}$ in the calculated spectra with static DFT and AIMD (Figure 5.22c). The former features peaks at 960, 900, 874, $835 \mathrm{~cm}^{-1}$ and in the latter bands centered around 888,873 , and $833 \mathrm{~cm}^{-1}$ can be observed. Both in good agreement with experiments. The spectrum of the sample of $\mathrm{Cu}-\mathrm{SAPO}-34$ preactivated in vacuum at $723 \mathrm{~K}$ which mainly contains $\mathrm{Cu}^{+}$cations ${ }^{35,36}$ shows two bands centered at $\sim 850 \mathrm{~cm}^{-1}$ and $\sim 905 \mathrm{~cm}^{-1}$ (Figure 5.22a, red line). We obtained three peaks at 983,964 and $877 \mathrm{~cm}^{-1}$ with static DFT calculations for the model containing $\mathrm{Cu}^{+}$(Figure 5.22b). They are associated with the T-O-T vibrations where only the oxygen atoms coordinated to $\mathrm{Cu}^{+}$are involved. The AIMD spectrum is 
somewhat different. It shows a group of bands centered around $910 \mathrm{~cm}^{-1}$ and another band at $\sim 817 \mathrm{~cm}^{-1}$. The experimental band at $905 \mathrm{~cm}^{-1}$ is better predicted by the AIMD spectra while the one at $950 \mathrm{~cm}^{-1}$ is better described by the static calculations, which means that for this system with a significantly mobile $\mathrm{Cu}^{+}$cation the above methods reproduce only qualitatively the experimental spectrum.

Table 5.10. Vibrational frequencies (in $\mathrm{cm}^{-1}$ ) between 800 and $1000 \mathrm{~cm}^{-1}$ region calculated using two different methodological approaches.

\begin{tabular}{ccc}
\hline & Static DFT & AIMD \\
\hline $\mathrm{Cu}^{+}$ & $983,964,877$ & 910,817 \\
$\mathrm{Cu}^{2+}$ & $959,900,874,834$ & $888,876,833$ \\
$\mathrm{Cu}^{+}-\mathrm{O}_{2}$ & 985,816 & 862,850 \\
$\mathrm{Cu}^{+}-\mathrm{NO}$ & 972,894 & $920,888,855$ \\
$\mathrm{Cu}^{+}-2 \mathrm{NO}$ & 953,892 & - \\
$\mathrm{Cu}^{+}-\mathrm{NO}_{2}$ & 961,885 & 916,840 \\
$\mathrm{Cu}^{+}-2 \mathrm{NO}_{2}$ & $935,865,808$ & 888,812 \\
$\mathrm{Cu}^{+}-\mathrm{NH}_{3}$ & 969,905 & 920,835 \\
$\mathrm{Cu}^{+}-2 \mathrm{NH}_{3}$ & 984,964 & - \\
$\mathrm{Cu}^{2+}-\mathrm{ONO}^{-} \mathrm{NO}_{2}$ & $934,875,860$ & $932,895,820$ \\
$\mathrm{Cu}^{2+}-\mathrm{NO}_{3}-\mathrm{NO}^{2}$ & $993,968,873$ & $930,868,815$ \\
$\mathrm{Cu}^{2+}-\mathrm{NO}_{3}$ & 946,861 & 950 \\
$\mathrm{Cu}^{2+}-2 \mathrm{NO}^{2+}$ & $992,984,948,883$ & 900,868 \\
$\mathrm{Cu}^{2+}-2 \mathrm{NH}_{3}$ & $990,921,859$ & - \\
$\mathrm{Cu}^{2+}-4 \mathrm{NH}_{3}$ & 997 & \\
\hline
\end{tabular}




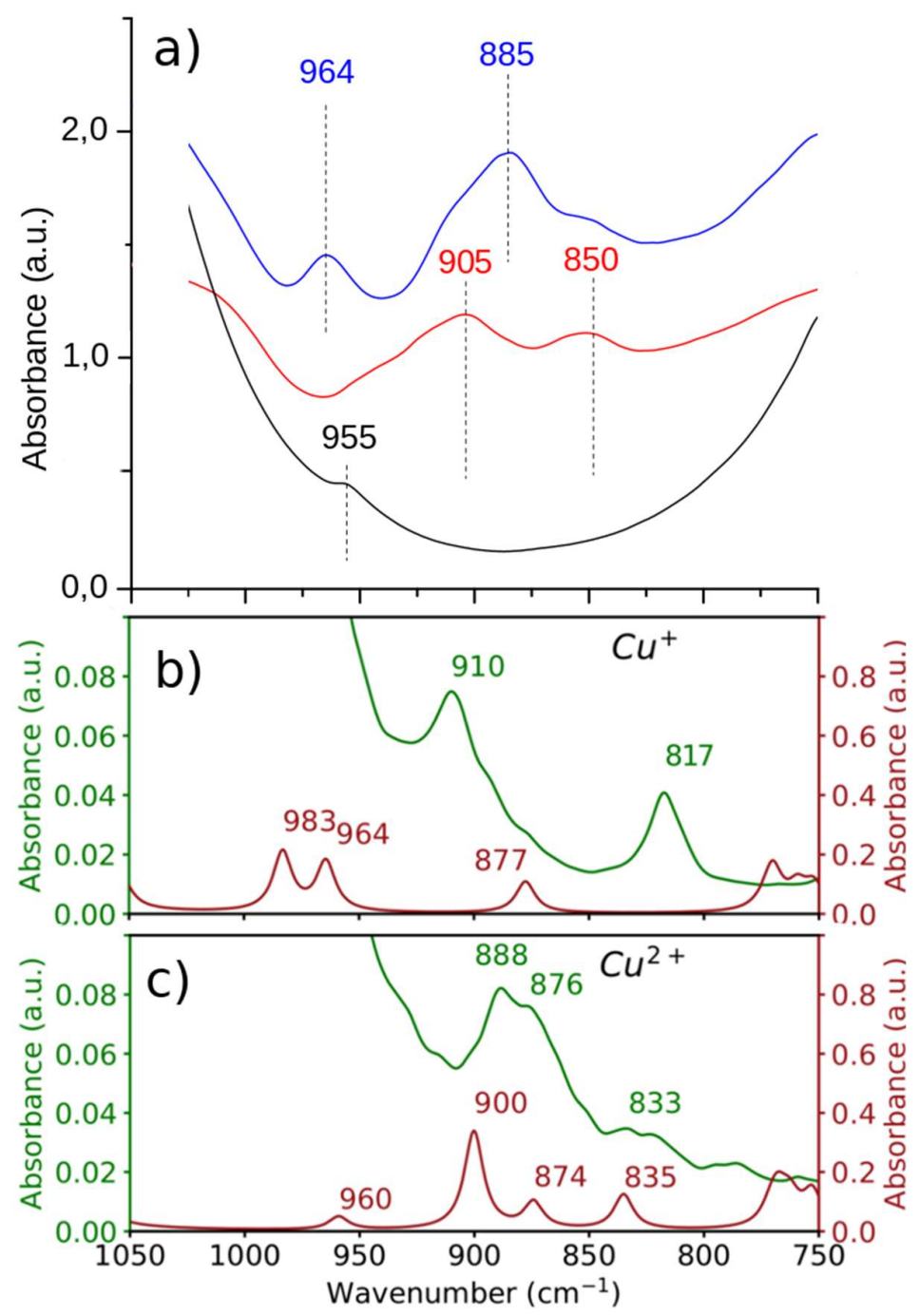

Figure 5.22 a) $v_{\text {asym }}(\mathrm{T}-\mathrm{O}-\mathrm{T})$ region of the FTIR spectra of H-SAPO-34 (black line) and CuSAPO-34 pre-activated in vacuum at $723 \mathrm{~K}$ (red line) or in $\mathrm{O}_{2}$ at $623 \mathrm{~K}$ followed by vacuum at $523 \mathrm{~K}$ (blue line). b) Vibrational frequencies in the same region calculated for $\mathrm{Cu}^{2+}$ and $\mathrm{Cu}^{+}$isolated cations in the $6 r$ of SAPO-34 using static DFT calculations (red lines) and AIMD simulations (green lines). 
Let us consider the effect of the interaction of the guest molecules with $\mathrm{Cu}^{+}$on the IR spectra. We have already discussed that $\mathrm{O}_{2}, \mathrm{NO}$ and $\mathrm{NO}_{2}$ bind to $\mathrm{Cu}^{+}$and slightly displace it to a maximum distance of $\sim 1.2 \AA$ from the plane of the $6 r$. As a consequence, the calculated frequencies in the $800-1000 \mathrm{~cm}^{-1}$ are modified when the copper cations interact with the reactant molecules (Table 5.10).

In general, there is relatively good qualitative agreement between the calculated spectra with AIMD and the static DFT. Both equally predict the presence or absence of bands in the $800-1000 \mathrm{~cm}^{-1}$ region. However, due to the differences of both methods the positions and number of the bands do not always match. Finite temperature effects are captured by the calculated AIMD spectra. Thus, the copper cations dynamically change their coordination with the framework oxygen atoms of the $6 r$ along the AIMD trajectories, while the frequencies calculated with static DFT arise from a model with fixed $\mathrm{Cu}-\mathrm{O}$ bonds. It can be seen that more complex spectra are obtained with AIMD (Figures 5.23 to 5.26). Moreover, the high mobility of the copper cations, especially $\mathrm{Cu}^{+}$, affects the relative intensity of the bands. For example, there are no clear bands in the calculated spectrum with AIMD in the interaction of two $\mathrm{NO}$ with $\mathrm{Cu}^{+}$(Figure 5.23). According to the calculated spectra shown in Figures 5.23 to 5.26 , bands around $\sim 850 \mathrm{~cm}^{-1}$ and $\sim 890 \mathrm{~cm}^{-1}$ should be visible when $\mathrm{NO}, \mathrm{NO}_{2}$, nitrites or nitrate species are adsorbed on $\mathrm{Cu}^{+}$and $\mathrm{Cu}^{2+}$. For the interaction of two ammonia molecules with $\mathrm{Cu}^{2+}$ features around $\sim 860 \mathrm{~cm}^{-1}$ and $\sim 900 \mathrm{~cm}^{-1}$ are observed in the AIMD spectrum. Conversely, the calculated spectra corresponding to the interaction of two $\mathrm{NH}_{3}$ with $\mathrm{Cu}^{+}$is very similar to that obtained for H-SAPO not exhibiting any clear band in the $800-1000 \mathrm{~cm}^{-1}$ region. The absence of bands in this case is explained by the absence of bonds between $\mathrm{Cu}^{+}$and $\mathrm{O}$ atoms of the $6 r$ when the mobile $\mathrm{Cu}^{+}\left(\mathrm{NH}_{3}\right)_{2}$ is formed.

Unfortunately, with these results no clear correlation is observed between the frequencies calculated for the asymmetric T-O-T lattice vibrations and either oxidation state of copper or adsorbed species, and therefore a direct assignment of each vibrational frequency to a particular species is not possible. Nonetheless, these data suggest that broad bands around $\sim 890 \mathrm{~cm}^{-1}$ could be associated to $\mathrm{Cu}^{2+}$ interacting with $\mathrm{NO}_{2}$, intermediates, and they confirm that the absence of bands in the $800-1000 \mathrm{~cm}^{-1}$ region of the IR spectra unambiguously indicates that the copper cations are no longer coordinated to the framework oxygen atoms. 
5.7 IR analysis. Comparison of static DFT and AIMD computed frequencies
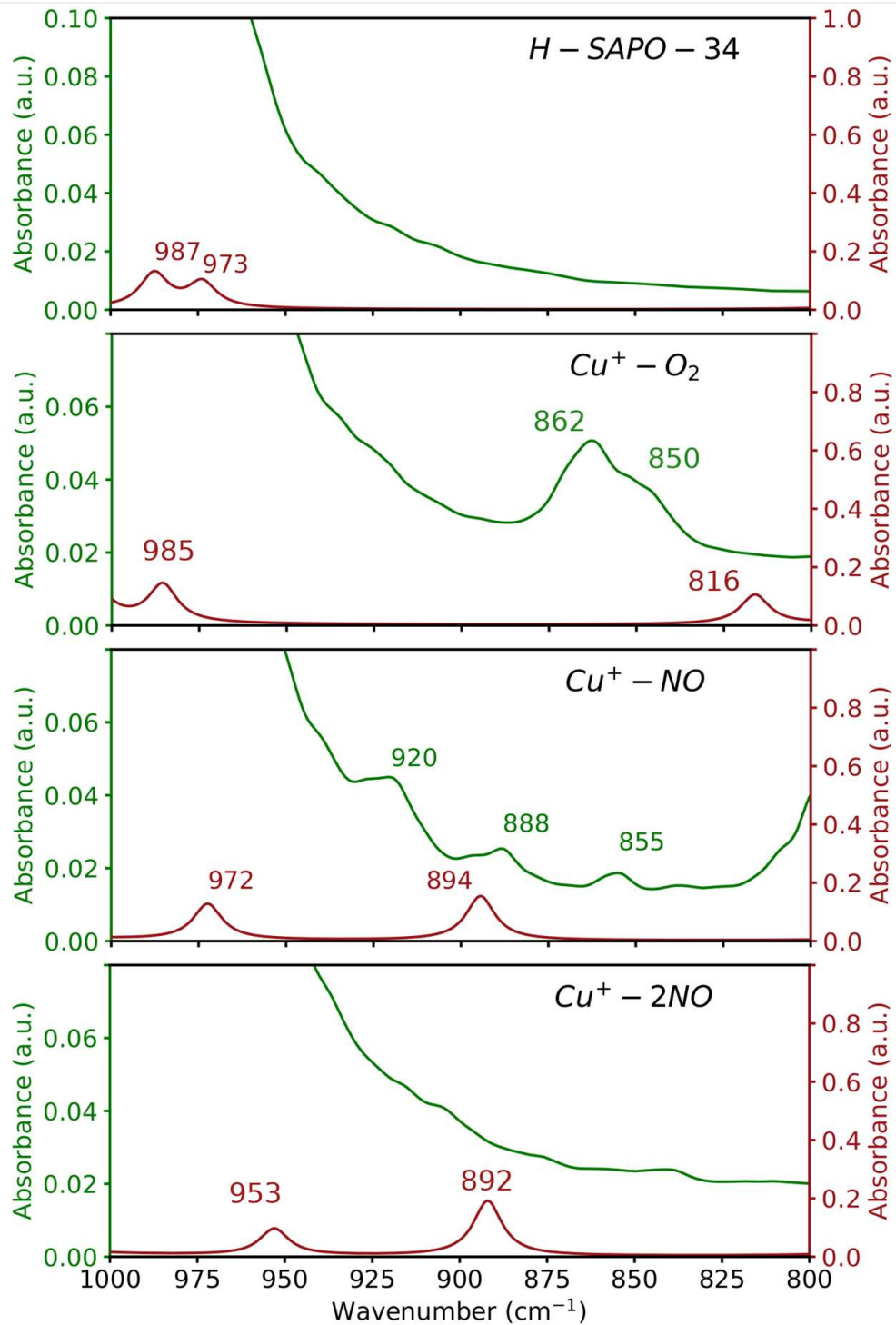

Figure 5.23 Simulated spectra in the $800-1000 \mathrm{~cm}^{-1}$ range calculated for $\mathrm{Cu}^{+}$and $\mathrm{Cu}^{2+}$ cations interacting with reactants and for some relevant intermediate species formed during the reaction using static DFT calculations (red lines) and AIMD simulations (green lines). 
5. Insights on the NH3-SCR-NOx reaction from Molecular Dynamics simulations
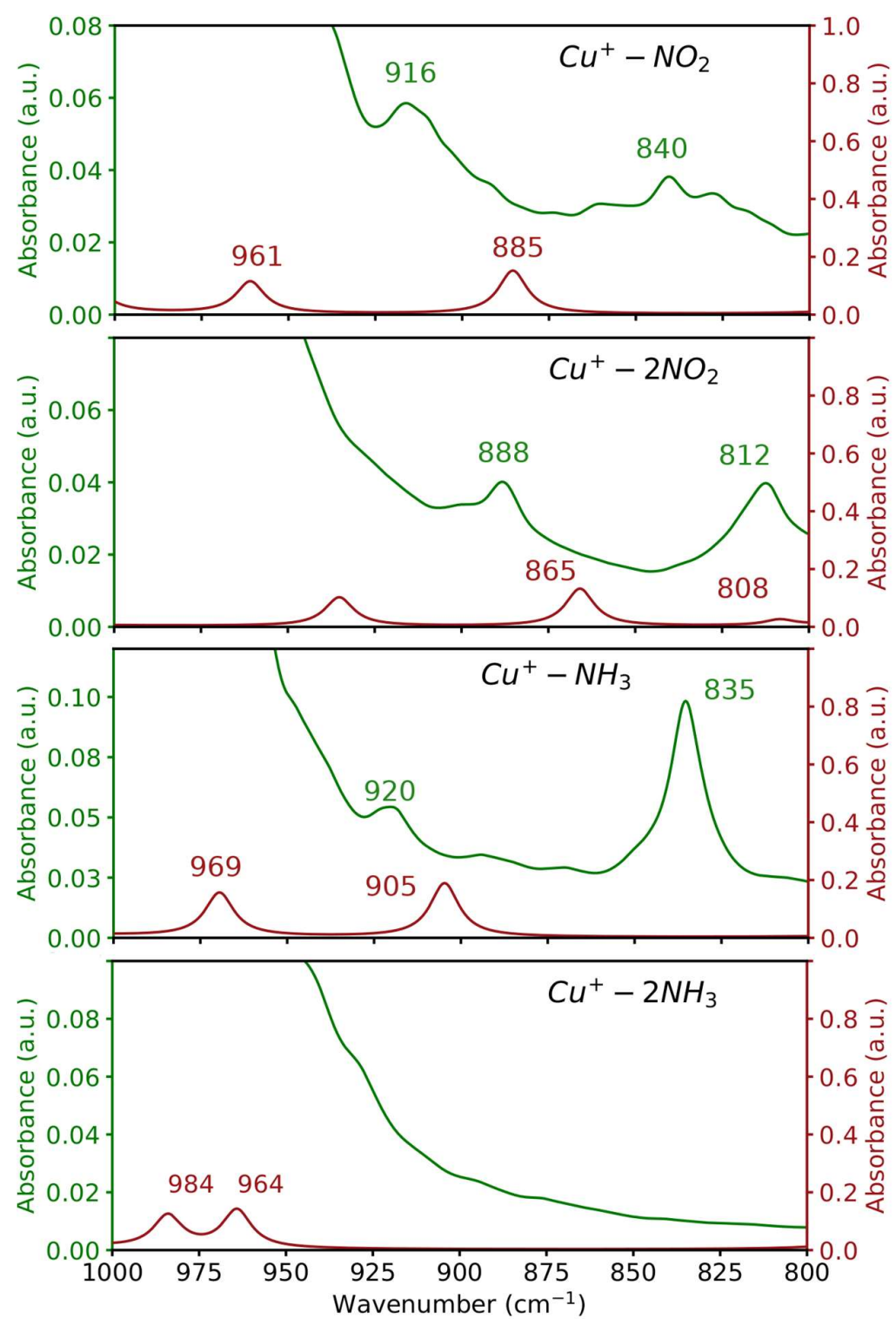

Figure 5.24 Simulated spectra in the $800-1000 \mathrm{~cm}^{-1}$ range calculated for $\mathrm{Cu}^{+}$and $\mathrm{Cu}^{2+}$ cations interacting with reactants and for some relevant intermediate species formed during the reaction using static DFT calculations (red lines) and AIMD simulations (green lines). 
5.7 IR analysis. Comparison of static DFT and AIMD computed frequencies
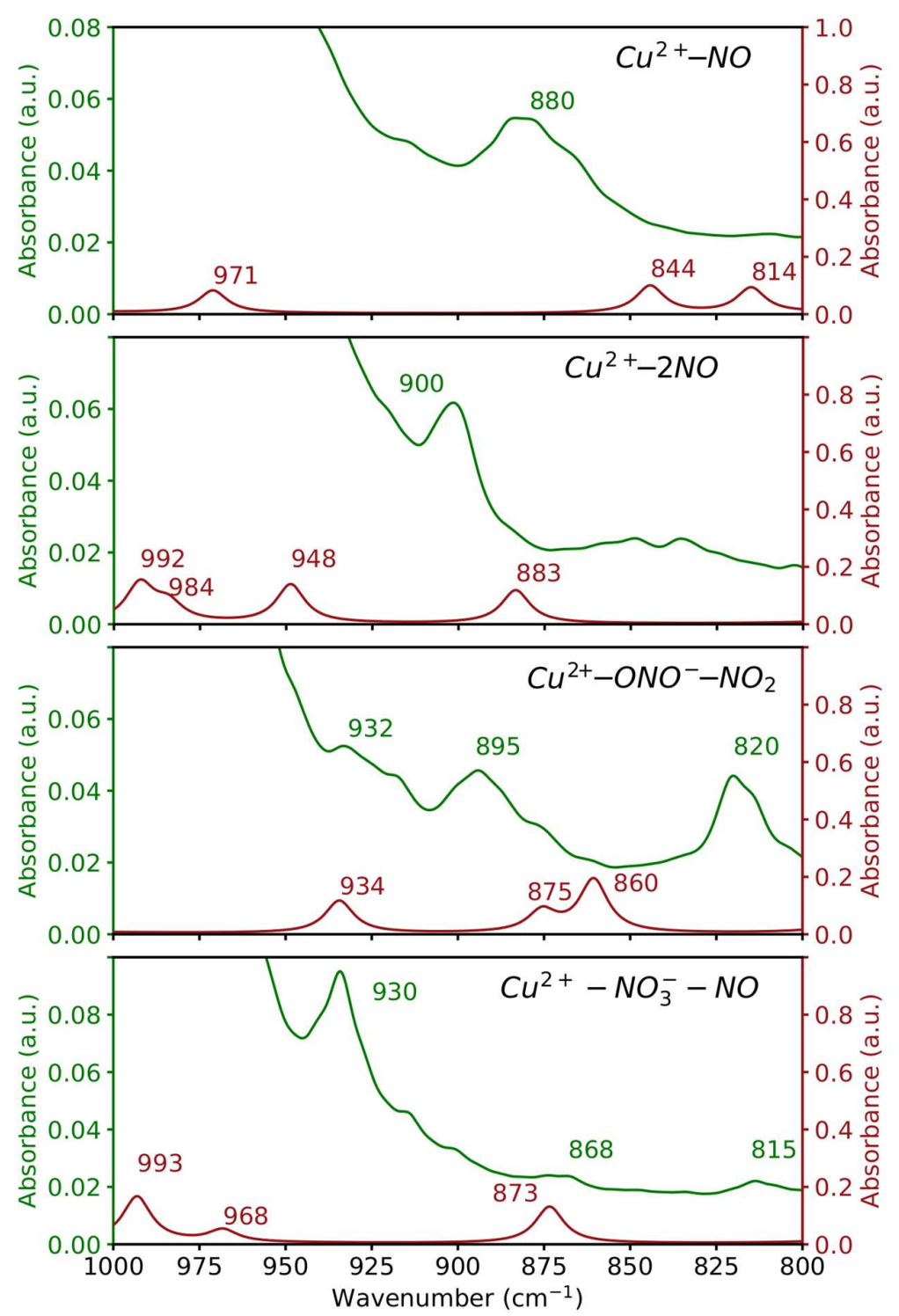

Figure 5.25 Simulated spectra in the $800-1000 \mathrm{~cm}^{-1}$ range calculated for $\mathrm{Cu}^{+}$and $\mathrm{Cu}^{2+}$ cations interacting with reactants and for some relevant intermediate species formed during the reaction using static DFT calculations (red lines) and AIMD simulations (green lines). 
5. Insights on the NH3-SCR-NOx reaction from Molecular Dynamics simulations

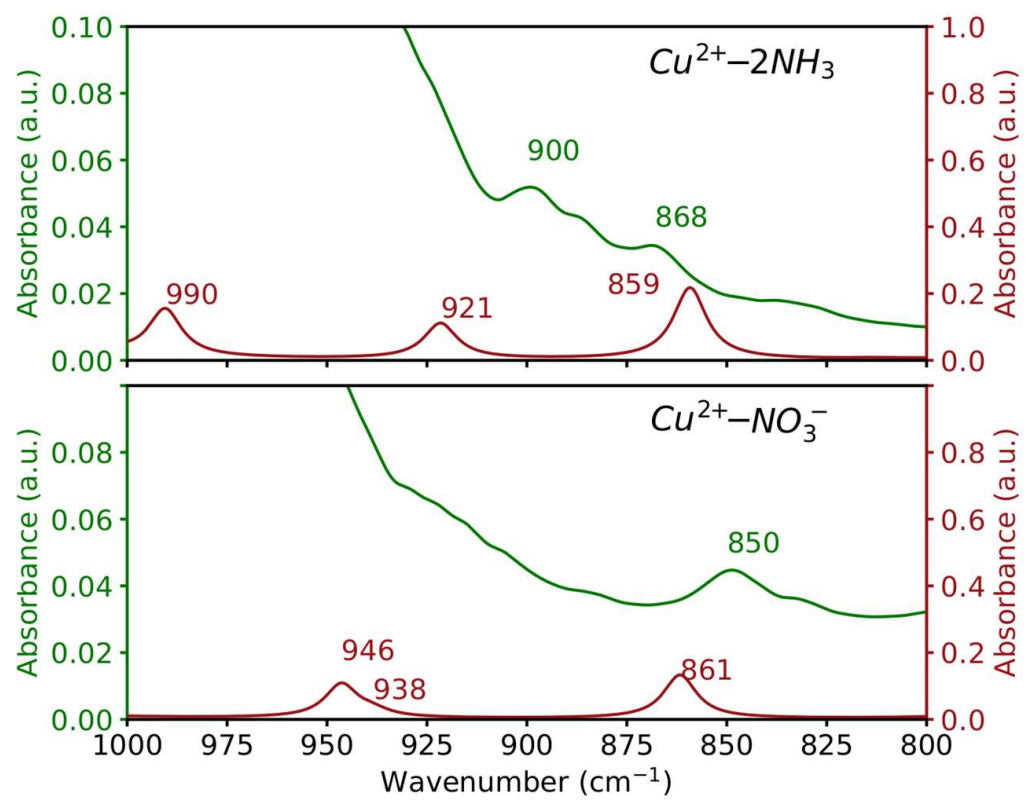

Figure 5.26 Simulated spectra in the $800-1000 \mathrm{~cm}^{-1}$ range calculated for $\mathrm{Cu}^{+}$and $\mathrm{Cu}^{2+}$ cations interacting with reactants and for some relevant intermediate species formed during the reaction using static DFT calculations (red lines) and AIMD simulations (green lines). 
5.8 Conclusions

\subsection{Conclusions}

In this chapter we have investigated the dynamic behaviour of the $\mathrm{Cu}(\mathrm{I})$ and $\mathrm{Cu}(\mathrm{II})$ cations in their interaction with the SCR reactant molecules at different temperatures in $\mathrm{Cu}-\mathrm{SSZ}-13$ and $\mathrm{Cu}-\mathrm{SAPO}-34$. The results of AIMD simulations show that the interaction of the copper cations with $\mathrm{NH}_{3}$ is stronger than with $\mathrm{O}_{2}, \mathrm{NO}$ and $\mathrm{NO}_{2}$.

The mobility of $\mathrm{Cu}^{+}$is highly enhanced by the interaction with $\mathrm{NH}_{3}$ to the point of being completely detached from their framework position in the $6 r$ forming the mobile diamino-copper(I) complex $\mathrm{Cu}^{+}\left(\mathrm{NH}_{3}\right)_{2}$. At least two $\mathrm{NH}_{3}$ molecules are necessary to detach $\mathrm{Cu}^{+}$. Four $\mathrm{NH}_{3}$ molecules are enough to stabilize the tetraaminecopper(II), $\mathrm{Cu}^{2+}\left(\mathrm{NH}_{3}\right)_{4}$ inside the cavity detached from the $6 r$ oxygens. Due to the strong electrostatic interaction with the negatively charged framework, the $\mathrm{Cu}^{2+}\left(\mathrm{NH}_{3}\right)_{4}$ complex is significantly less mobile than $\mathrm{Cu}^{+}\left(\mathrm{NH}_{3}\right)_{2}$. The NO molecule can interact with the diamine-copper(I) complex forming the stable complexes $\mathrm{Cu}^{+}\left(\mathrm{NH}_{3}\right)_{2}(\mathrm{NO})$ and $\mathrm{Cu}^{+}\left(\mathrm{NH}_{3}\right)_{2}(\mathrm{NO})_{2}$ at all the temperatures considered in both materials.

The high mobility of $\mathrm{Cu}^{+}\left(\mathrm{NH}_{3}\right)_{2}$ increases significantly with the temperature so that it is able to cross the $8 r$ window to the adjacent cavity spontaneously at $523 \mathrm{~K}$ in $\mathrm{Cu}-\mathrm{SSZ}-13$ and $\mathrm{Cu}-\mathrm{SAPO}-34$. At a lower temperature, $423 \mathrm{~K}$, the diffusion of $\mathrm{Cu}^{+}\left(\mathrm{NH}_{3}\right)_{2}$ has a relatively low activation free energy, $18 \mathrm{~kJ} / \mathrm{mol}$. This value is barely affected by the presence of $\mathrm{O}_{2}$, and another complex in the adjacent cavity so that under these conditions it is free to diffuse between adjacent cavities. However, the presence of other $\mathrm{NH}_{3}$, NO or water molecules could significantly hinder its diffusion. Furthermore, it is very unlikely that $\mathrm{Cu}^{+}\left(\mathrm{NH}_{3}\right)_{2}$ will diffuse further to a third cavity, its mobility is constrained to $\sim 4 \AA$ from the $\mathrm{AlO}_{4}{ }^{-}$site.

AIMD simulations also suggest that the oxidation of $\mathrm{NO}$ to $\mathrm{NO}_{2}$ inside the big CHA cage is a favorable process at $298 \mathrm{~K}$ and becomes spontaneous at $673 \mathrm{~K}$. The state and location of the $\mathrm{Cu}^{+}$cations seem to have little effect on the NO oxidation with $\mathrm{O}_{2}$ suggesting that the activation of $\mathrm{O}_{2}$ is not the rate-determining step of the SCR reaction.

The migration of $\mathrm{Cu}^{+}$away from the $6 r$ has been confirmed theoretically and experimentally by analysing the $800-1000 \mathrm{~cm}^{-1}$ region of the IR spectra of 
$\mathrm{Cu}-\mathrm{SAPO}-34$ exposed to different reactants and temperatures. Bands associated to the T-O-T framework vibrations perturbed by the $\mathrm{Cu}^{+}$and $\mathrm{Cu}^{2+}$ are observed in this region in the absence of $\mathrm{NH}_{3}$ or in an oxidizing atmosphere $\left(\mathrm{NO}+\mathrm{O}_{2}\right)$. The calculated vibrational modes cannot be associated unambiguously with the experimental bands. However, by tracking those bands during the SCR reaction it is possible to determine if the all copper cations are stabilized outside the $6 r$ (absence of bands) or if some $\mathrm{Cu}$ sites are still coordinated to the $6 r$ (bands are observed). The former would mean that all elementary steps involve mobile amino-copper complexes and the later would indicate that some elementary steps might be catalyzed by $\mathrm{Cu}$ species attached to the zeolite framework. 


\subsection{References}

(1) Lezcano-Gonzalez, I.; Deka, U.; Arstad, B.; Van Yperen-De Deyne, A.; Hemelsoet, K.; Waroquier, M.; Van Speybroeck, V.; Weckhuysen, B. M.; Beale, A. M. Determining the Storage, Availability and Reactivity of NH3 within Cu-Chabazite-Based Ammonia Selective Catalytic Reduction Systems. Phys. Chem. Chem. Phys. 2014, 16 (4), 1639-1650. https://doi.org/10.1039/c3cp54132k.

(2) Wang, D.; Zhang, L.; Kamasamudram, K.; Epling, W. S. In Situ-DRIFTS Study of Selective Catalytic Reduction of NOx by NH3 over Cu-Exchanged SAPO-34. Acs Catal. 2013, 3 (5), 871-881. https://doi.org/10.1021/cs300843k.

(3) Duan, Y.; Wang, J.; Yu, T.; Shen, M.; Wang, J. The Role and Activity of Various Adsorbed Ammonia Species on Cu/SAPO-34 Catalyst during PassiveSCR Process. RSC Adv 2015, 5 (19), $14103-14113$. https://doi.org/10.1039/C4RA13984D.

(4) Flentge, D. R.; Lunsford. J. H.; Jacobs, P. A.; Uytterhoeven, J. B. Spectroscopic Evidence for the Tetraamminecopper(II) Complex in a Y-Type Zeolite. J. Phys. Chem. 1975, 79 (4), 354-360. https://doi.org/10.1021/j100571a014.

(5) Williamson, W. B.; Flentge, D. R.; Lunsford, J. H. Ammonia Oxidation over $\mathrm{Cu}(\mathrm{II}) \mathrm{NaY}$ Zeolites. J. Catal. 1975, 37 (2), 258-266. https://doi.org/10.1016/0021-9517(75)90160-8.

(6) Deka, U.; Lezcano-Gonzalez, I.; Weckhuysen, B. M.; Beale, A. M. Local Environment and Nature of $\mathrm{Cu}$ Active Sites in Zeolite-Based Catalysts for the Selective Catalytic Reduction of NOx. ACS Catal. 2013, 3 (3), 413-427. https://doi.org/10.1021/cs300794s.

(7) Delabie, A.; Pierloot, K.; Groothaert, M. H.; Weckhuysen, B. M.; Schoonheydt, R. A. Study of the Coordination of $\mathrm{Cu} 2+$ in Zeolite Y: Interaction with Water and Ammonia. Microporous Mesoporous Mater. 2000, 37 (1), 209-222. https://doi.org/10.1016/S1387-1811(99)00267-X.

(8) Lezcano-Gonzalez, I.; Wragg, D. S.; Slawinski, W. A.; Hemelsoet, K.; Van Yperen-De Deyne, A.; Waroquier, M.; Van Speybroeck, V.; Beale, A. M. Determination of the Nature of the Cu Coordination Complexes Formed in the Presence of NO and NH3 within SSZ-13. J. Phys. Chem. C 2015, 119 (43), 24393-24403. https://doi.org/10.1021/acs.jpcc.5b06875.

(9) Moreno-Gonzalez, M.; Hueso, B.; Boronat, M.; Blasco, T.; Corma, A. Ammonia-Containing Species Formed in Cu-Chabazite As Per In Situ EPR, Solid-State NMR, and DFT Calculations. J. Phys. Chem. Lett. 2015, 6 (6), 1011-1017. https://doi.org/10.1021/acs.jpclett.5b00069.

(10) Kerkeni, B.; Berthout, D.; Berthomieu, D.; Doronkin, D. E.; Casapu, M.; 
Grunwaldt, J.-D.; Chizallet, C. Copper Coordination to Water and Ammonia in CuII-Exchanged SSZ-13: Atomistic Insights from DFT Calculations and in Situ XAS Experiments. J. Phys. Chem. C 2018, 122 (29), 16741-16755. https://doi.org/10.1021/acs.jpcc.8b03572.

(11) Paolucci, C.; Khurana, I.; Parekh, A. A.; Li, S.; Shih, A. J.; Li, H.; Iorio, J. R. D.; Albarracin-Caballero, J. D.; Yezerets, A.; Miller, J. T.; Delgass, W. N.; Ribeiro, F. H.; Schneider, W. F.; Gounder, R. Dynamic Multinuclear Sites Formed by Mobilized Copper Ions in NOx Selective Catalytic Reduction. Science 2017, 357 (6354), 898-903. https://doi.org/10.1126/science.aan5630.

(12) Paolucci, C.; Parekh, A. A.; Khurana, I.; Di Iorio, J. R.; Li, H.; Albarracin Caballero, J. D.; Shih, A. J.; Anggara, T.; Delgass, W. N.; Miller, J. T.; Ribeiro, F. H.; Gounder, R.; Schneider, W. F. Catalysis in a Cage: Condition-Dependent Speciation and Dynamics of Exchanged Cu Cations in SSZ-13 Zeolites. J. Am. Chem. Soc. 2016, 138 (18), 6028-6048. https://doi.org/10.1021/jacs.6b02651.

(13) Paolucci, C.; Verma, A. A.; Bates, S. A.; Kispersky, V. F.; Miller, J. T.; Gounder, R.; Delgass, W. N.; Ribeiro, F. H.; Schneider, W. F. Isolation of the Copper Redox Steps in the Standard Selective Catalytic Reduction on Cu-SSZ13. Angew. Chem.-Int. Ed. 2014, 53 (44), 11828-11833. https://doi.org/10.1002/anie.201407030.

(14) Gao, F.; Mei, D.; Wang, Y.; Szanyi, J.; Peden, C. H. F. Selective Catalytic Reduction over $\mathrm{Cu} / \mathrm{SSZ}-13$ : Linking Homo- and Heterogeneous Catalysis. $J$. Am. Chem. Soc. 2017, 139 (13), 4935-4942. https://doi.org/10.1021/jacs.7b01128.

(15) Gao, F.; Peden, C.; Gao, F.; Peden, C. H. F. Recent Progress in Atomic-Level Understanding of $\mathrm{Cu} / \mathrm{SSZ}-13$ Selective Catalytic Reduction Catalysts. Catalysts 2018, 8 (4), 140. https://doi.org/10.3390/catal8040140.

(16) Gao, F.; Walter, E. D.; Kollar, M.; Wang, Y.; Szanyi, J.; Peden, C. H. F. Understanding Ammonia Selective Catalytic Reduction Kinetics over Cu/SSZ13 from Motion of the $\mathrm{Cu}$ Ions. J. Catal. 2014, 319, 1-14. https://doi.org/10.1016/j.jcat.2014.08.010.

(17) Marberger, A.; Petrov, A. W.; Steiger, P.; Elsener, M.; Kröcher, O.; Nachtegaal, M.; Ferri, D. Time-Resolved Copper Speciation during Selective Catalytic Reduction of NO on Cu-SSZ-13. Nat. Catal. 2018, 1 (3), 221-227. https://doi.org/10.1038/s41929-018-0032-6.

(18) Lomachenko, K. A.; Borfecchia, E.; Negri, C.; Berlier, G.; Lamberti, C.; Beato, P.; Falsig, H.; Bordiga, S. The Cu-CHA DeNO(x) Catalyst in Action: Temperature-Dependent NH3-Assisted Selective Catalytic Reduction Monitored by Operando XAS and XES. J. Am. Chem. Soc. 2016, 138 (37), 12025-12028. https://doi.org/10.1021/jacs.6b06809. 
(19) Wang, J.; Zhao, H.; Haller, G.; Li, Y. Recent Advances in the Selective Catalytic Reduction of NOx with NH3 on Cu-Chabazite Catalysts. Appl. Catal. B Environ. 2017, 202, 346-354. https://doi.org/10.1016/j.apcatb.2016.09.024.

(20) Xue, J.; Wang, X.; Qi, G.; Wang, J.; Shen, M.; Li, W. Characterization of Copper Species over Cu/SAPO-34 in Selective Catalytic Reduction of NOx with Ammonia: Relationships between Active $\mathrm{Cu}$ Sites and de-NOx Performance at Low Temperature. J. Catal. 2013, 297, 56-64. https://doi.org/10.1016/j.jcat.2012.09.020.

(21) Fan, S.; Xue, J.; Yu, T.; Fan, D.; Hao, T.; Shen, M.; Li, W. The Effect of Synthesis Methods on $\mathrm{Cu}$ Species and Active Sites over Cu/SAPO-34 for NH3SCR Reaction. Catal. Sci. Technol. 2013, 3 (9), 2357-2364. https://doi.org/10.1039/C3CY00267E.

(22) Zhang, Y.; Yang, W. Comment on "Generalized Gradient Approximation Made Simple". Phys. Rev. Lett. 1998, 80 (4), 890-890. https://doi.org/10.1103/PhysRevLett.80.890.

(23) Grimme, S.; Antony, J.; Ehrlich, S.; Krieg, H. A Consistent and Accurate Ab Initio Parametrization of Density Functional Dispersion Correction (DFT-D) for the 94 Elements H-Pu. J. Chem. Phys. 2010, 132 (15), 154104. https://doi.org/10.1063/1.3382344.

(24) Hoover, W. G. Canonical Dynamics: Equilibrium Phase-Space Distributions. Phys. Rev. A 1985, $31 \quad$ (3), 1695-1697. https://doi.org/10.1103/PhysRevA.31.1695.

(25) Martyna, G. J.; Tobias, D. J.; Klein, M. L. Constant Pressure Molecular Dynamics Algorithms. J. Chem. Phys. 1994, 101 (5), 4177-4189. https://doi.org/10.1063/1.467468.

(26) Baroni, S.; de Gironcoli, S.; Dal Corso, A.; Giannozzi, P. Phonons and Related Crystal Properties from Density-Functional Perturbation Theory. Rev. Mod. Phys. 2001, 73 (2), 515-562. https://doi.org/10.1103/RevModPhys.73.515.

(27) King-Smith, R. D.; Vanderbilt, D. Theory of Polarization of Crystalline Solids. Phys. Rev. B 1993, 47 (3), 1651-1654. https://doi.org/10.1103/PhysRevB.47.1651.

(28) Thomas, M.; Brehm, M.; Fligg, R.; Vöhringer, P.; Kirchner, B. Computing Vibrational Spectra from Ab Initio Molecular Dynamics. Phys. Chem. Chem. Phys. 2013, 15 (18), 6608-6622. https://doi.org/10.1039/C3CP44302G.

(29) Baerlocher, C.; McCusker, L. B. Database of Zeolite Structures http://www.iza-structure.org/databases/ (accessed Jun 21, 2020).

(30) Maestri, M.; Iglesia, E. First-Principles Theoretical Assessment of Catalysis by Confinement: $\mathrm{NO}-\mathrm{O}_{2} \$$ Reactions within Voids of Molecular Dimensions in Siliceous Crystalline Frameworks. Phys. Chem. Chem. Phys. 2018, 20 (23), 15725-15735. https://doi.org/10.1039/C8CP01615A. 
(31) Kwak, J. H.; Varga, T.; Peden, C. H. F.; Gao, F.; Hanson, J. C.; Szanyi, J. Following the Movement of $\mathrm{Cu}$ Ions in a SSZ-13 Zeolite during Dehydration, Reduction and Adsorption: A Combined in Situ TP-XRD, XANES/DRIFTS Study. J. Catal. 2014, 314, 83-93. https://doi.org/10.1016/j.jcat.2014.03.003.

(32) Luo, J.; Gao, F.; Kamasamudram, K.; Currier, N.; Peden, C. H. F.; Yezerets, A. New Insights into Cu/SSZ-13 SCR Catalyst Acidity. Part I: Nature of Acidic Sites Probed by NH3 Titration. J. Catal. 2017, 348, 291-299. https://doi.org/10.1016/j.jcat.2017.02.025.

(33) Song, J.; Wang, Y.; Walter, E. D.; Washton, N. M.; Mei, D.; Kovarik, L.; Engelhard, M. H.; Prodinger, S.; Wang, Y.; Peden, C. H. F.; Gao, F. Toward Rational Design of Cu/SSZ-13 Selective Catalytic Reduction Catalysts: Implications from Atomic-Level Understanding of Hydrothermal Stability. $\begin{array}{lllll}\text { ACS Catal. } & \text { 2017, } & 7 & \text { (12), }\end{array}$ https://doi.org/10.1021/acscatal.7b03020.

(34) Borfecchia, E.; Lomachenko, K. A.; Giordanino, F.; Falsig, H.; Beato, P.; Soldatov, A. V.; Bordiga, S.; Lamberti, C. Revisiting the Nature of Cu Sites in the Activated Cu-SSZ-13 Catalyst for SCR Reaction. Chem. Sci. 2015, 6 (1), 548-563. https://doi.org/10.1039/c4sc02907k.

(35) Borfecchia, E.; Beato, P.; Svelle, S.; Olsbye, U.; Lamberti, C.; Bordiga, S. CuCHA - a Model System for Applied Selective Redox Catalysis. Chem. Soc. Rev. 2018, 47 (22), 8097-8133. https://doi.org/10.1039/C8CS00373D.

(36) Giordanino, F.; Borfecchia, E.; Lomachenko, K. A.; Lazzarini, A.; Agostini, G.; Gallo, E.; Soldatov, A. V.; Beato, P.; Bordiga, S.; Lamberti, C. Interaction of NH3 with Cu-SSZ-13 Catalyst: A Complementary FTIR, XANES, and XES Study. J. Phys. Chem. Lett. 2014, 5 (9), 1552-1559. https://doi.org/10.1021/jz500241m. 


\section{Chapter 6}

\section{Ab-initio simulation of NMR parameters: location of the $\mathrm{F}^{-}$in the as-made pure silica RTH}

\subsection{Introduction}

A new family of organic structure-directing agents (OSDA) based on tetraalkylphosphonium and phosphazene cations have been recently introduced in the synthesis of zeolites ${ }^{1-4}$. These P-containing cations show higher thermal stability than the N-containing counterparts allowing to perform the synthesis of zeolites at higher temperatures. Other advantages are related to the elucidation of the structure. As a result of the greater electron density of $\mathrm{P}$ atoms, they can be located more easily by means of X-ray diffraction (XRD). Furthermore, the ${ }^{31} \mathrm{P}$ nucleus $(\mathrm{I}=1 / 2)$, with a relative abundance of $100 \%$, is a better candidate for nuclear magnetic resonance spectroscopy (NMR) than the ${ }^{15} \mathrm{~N}$ nucleus $(\mathrm{I}=1 / 2)$ which has a relative abundance of $36 \%$.

When the zeolite synthesis is carried out in fluoride media the resulting crystals are free of connectivity defects, as opposed to the synthesis in $\mathrm{OH}^{-}$media which leads to zeolites with abundant amounts of silanols $\mathrm{s}^{5-7}$. Other advantages include the possibility of obtaining larger crystals ${ }^{8}$ and pure silica zeolites ${ }^{9-13}$ with low framework densities ${ }^{14}$. Several functions have been attributed to the fluoride ions: compensation of the positive charge of the OSDA ${ }^{15,16}$, increase of the solubility of silicate ions at neutral $\mathrm{pH}$ and catalysis of the formation of Si-O-Si bonds ${ }^{12}$. The fluoride ions have been found to be connected to the as-made zeolite frameworks, in particular to the $\mathrm{Si}$ atoms of 4-membered rings forming pentacoordinated $\mathrm{SiO}_{4 / 2} \mathrm{~F}^{-}$ 
units $^{15,17-22}$. These findings have led to the hypothesis that the fluoride anions have a structure-directing agent function as well, stabilizing small cages and 4-membered rings.

When it comes to determining the structure of crystalline solids, single-crystal X-ray diffraction (XRD) is the method of choice. However, there is a disadvantage when trying to localize the $\mathrm{F}$ atoms. $\mathrm{F}$ has, similar to $\mathrm{O}$, low electron density making it difficult to track by XRD. In fact, fluoride anions are isoelectronic with $\mathrm{OH}^{-}$which can be present as defects in zeolites. Another powerful technique for structure elucidation is Nuclear Magnetic resonance (NMR). In particular, the magic angle spinning (MAS) technique ${ }^{23,24}$ has facilitated the characterization of fluorine environments in solid materials. Using MAS-NMR alone Fyfe and co-workers ${ }^{19}$ determined the average location of fluoride covalently bonded to the Si9 site in the $\left[4^{1} 5^{2} 6^{2}\right]$ cage of the MFI framework. By combining solid state NMR and XRD they also elucidated the local structure of five-coordinated $\left[\mathrm{SiO}_{4 / 2} \mathrm{~F}\right]^{-}$units in the as-made zeolite with STF topology ${ }^{25}$ and showed that there is always some uncertainty in the location of fluoride by XRD.

Nonetheless, the interpretation of solid-state MAS-NMR spectra is very challenging and often ambiguous ${ }^{26}$. DFT calculations of NMR parameters have been proven a valuable tool to aid in the interpretation of NMR spectra of solids, especially after the development of the GIPAW method ${ }^{27-29}$. It has been successfully applied, hand in hand with MAS-NMR spectroscopy, to study zeolites structures ${ }^{29-43}$. For example, Rojas et al. ${ }^{44}$ combined these two techniques to show that the two ${ }^{19} \mathrm{~F}$ signals observed in the pure silica MFI synthesized in fluoride media with Bis(methylimidazolium) as OSDA was not a consequence of the fluorides sitting in different $\mathrm{TO}_{4}(\mathrm{~T}=\mathrm{Si})$ sites. Both fluorides are sitting in the $\mathrm{Si} 9$ position, but interact differently with the bicationic template.

Despite the advances in the last decades, the synthesis of zeolites is still an experimental field, where trial and error remains as the cornerstone for generating new zeolitic materials. Increasing our knowledge on the interactions that drive zeolite crystallization will lead us closer to a rational design of zeolites. In this sense, new ways of synthesis will probably be opened by understanding the role of $\mathrm{F}^{-}$in zeolite synthesis and, for that purpose, the structure elucidation of as-synthesized 
6.2 Methods

F-containing zeolites is a key step. Unfortunately, not much structural data is available regarding the location of fluoride ions in as-synthesized pure silica zeolites.

In our group, the RTH-type zeolite has been synthesized in fluoride media using triisopropyl-methyl-phosphonium as OSDA. From high resolution powder XRD the location of the OSDA can be determined accurately. With NMR, 16 crystallographic $\mathrm{SiO}_{4}$ positions and the existence of one type of $\mathrm{F}^{-}$in the as-made material have been identified. However, the location of the fluoride anion inside the zeolite cannot be unambiguously determined by using only these two techniques. In this chapter, we use ab initio simulations of ${ }^{19} \mathrm{~F}$ NMR chemical shifts to determine the location of the fluoride anion in the as-synthesized RTH framework and, from these calculations, a redefinition of the unit cell of the as-synthesized RTH framework is proposed and confirmed by XRD.

\subsection{Methods}

Periodic calculations were carried out with the VASP $\operatorname{code}^{45}$. Energy minimizations were performed with the Perdew-Burke-Ernzerhof generalised gradient approximation (PBE-GGA) ${ }^{46-49}$. In all calculations, the projected augmented wave $(\mathrm{PAW})^{50}$ pseudopotentials were used to describe the interaction of the valence electrons with the nuclei and core electrons. An energy cutoff of $600 \mathrm{eV}$ was used to expand the plane wave basis set. For zeolites and molecules, the Brillouin zone was sampled at the gamma point but for ionic compounds convergence of the energy with respect to the k-points was checked. Monkhorst-Pack grids of $8 \times 8 \times 1 k$-points were used for ionic fluorinated compounds. The structures were considered converged when the forces acting on atoms were lower than $0.01 \mathrm{eV} / \AA$. Dispersion energies were evaluated using the D3 Grimme's method ${ }^{51-53}$ with the Becke-Johnson damping ${ }^{54}$.

The optimization of the unit cell volume was performed as follows. A series of calculations with differing volumes is carried out for each structure, where the unit cell volume is fixed, and the atomic positions along with lattice parameters are relaxed. Then, the equilibrium volume is calculated by fitting the Birch-Murnaghan equation of state ${ }^{55}$ to the energy-volume curve. The atomic positions and the cell shape is finally optimized with the volume of the unit cell fixed at the equilibrium 
value. This procedure is computationally expensive but ensures the removal of the artifacts related to Pulay forces ${ }^{56,57}$.

The NMR absolute shielding tensors were computed with the GIPAW approach implemented in VASP. The same energy cutoff as in the optimizations was used but with a tighter convergence criterion of $10^{-10} \mathrm{eV}$ for the electronic energy. Two functionals were used for these calculations, the aforementioned PBE and the modified Becke-Johnson exchange potential (TB-mBJ) ${ }^{58,59}$. All NMR calculations were performed with optimized structures. In order to compare the calculated absolute shieldings $(\sigma)$ with experimental chemical shifts $(\delta)$ a linear regression model was constructed with a data set of 18 known fluorinated compounds.

\subsection{Elucidation of Fluoride location in the as-made RTH}

The simulation of NMR parameters in solids using periodic boundary conditions has only recently become possible with the introduction of the GIPAW method and has not been widely applied for zeolites, especially for ${ }^{19} \mathrm{~F}$ nuclei.

There are some reports highlighting the problems of GGA type functionals when predicting the ${ }^{19} \mathrm{~F}$ signal of ionic materials. Experimental isotropic chemical shifts and calculated isotropic shieldings usually correlate well but the slope of the linear fit deviates considerably from the theoretical value of $-1^{30,60-62}$. This behaviour is related to the underestimation of band gaps with GGA functionals ${ }^{59,63}$ as demonstrated by Laskowski et al. ${ }^{61,64}$ The modified Becke-Johnson exchange potential (TB-mBJ) shows a fairly correct relation between experiment and theory, at least for these ionic compounds.

As remarked in the introduction, zeolites synthesized in fluoride media are highly crystalline and with no connectivity defects and all fluoride anions are compensating the positive charge of the OSDA. The fluoride anion incorporated in the framework is convalently bonded to the $\mathrm{Si}$ site with bond lengths of $\sim 1.7-1.8 \AA^{16,34}$. For the sake of comparison we have calculated the NMR chemical shifts with PBE and with the modified Becke-Johnson exchange potential ${ }^{38,41}$. To the best of our knowledge calculations of the ${ }^{19} \mathrm{~F}$ chemical shift have not been performed in $\mathrm{F}$-containing zeolites using the TB-mBJ functional. 
6.3 Elucidation of Fluoride location in the as-made RTH

\subsubsection{Linear fit for the prediction of the ${ }^{19} \mathrm{~F}$ isotropic chemical shifts}

In order to predict accurately the ${ }^{19} \mathrm{~F}$ NMR chemical shifts, we first constructed a calibration curve. To this end, the experimental isotropic chemical shifts $\left(\delta_{\text {iso }}\right)$ of 18 fluorinated compounds were collected and correlated with the calculated absolute isotropic shieldings $\left(\sigma_{\text {iso }}\right)$. The experimental chemical shifts and calculated absolute shieldings are summarized in Table 6.1 and the correlation of both quantities is shown in Figure 6.1. The calculated absolute isotropic shieldings with both PBE and TB-mBJ functionals correlate well with the experimental values. The slope obtained with PBE is underestimated while TB-mBJ gives a value closer to -1 . These results are in agreement with the literature ${ }^{61}$ and indicate that the TB-mBJ functional should be used to scale the absolute isotropic shieldings to isotropic chemical shifts instead of PBE. Finally, notice also that the root mean square deviation (RMSD) of the regression is smaller for the TB-mBJ functional.

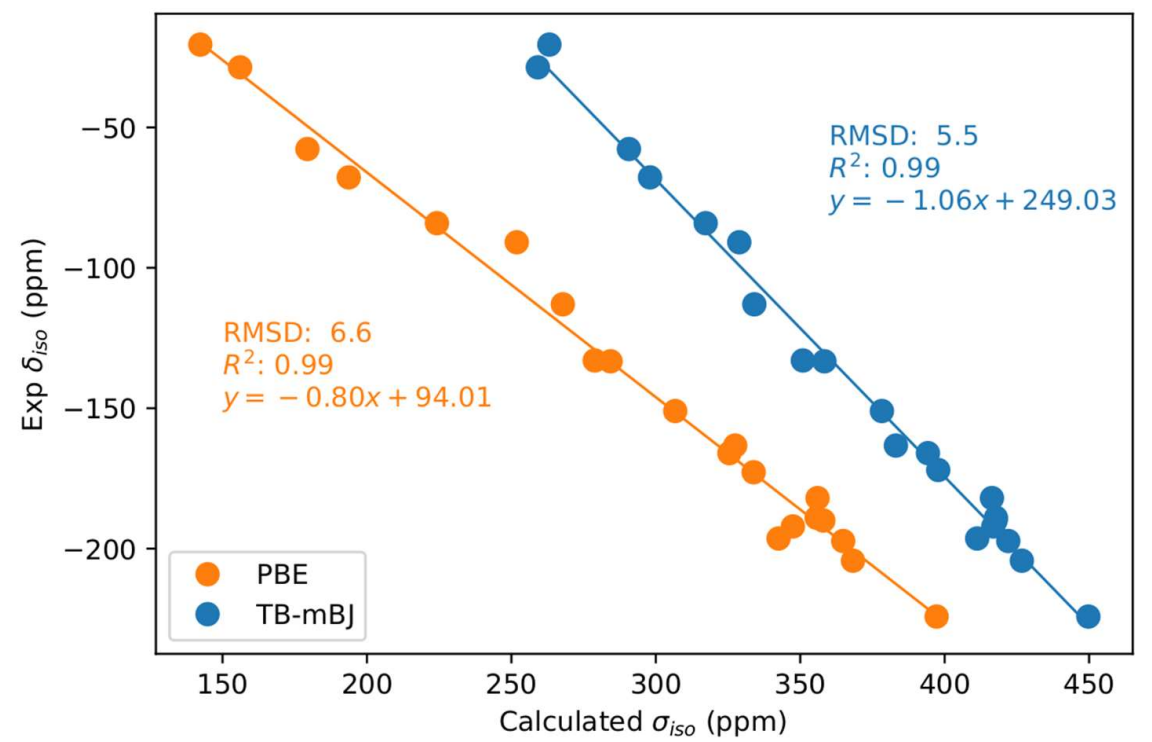

Figure 6.1 Correlation between measured isotropic chemical shifts $\left(\sigma_{\text {iso }}\right)$ and calculated NMR shielding $\left(\delta_{\text {iso }}\right)$ (in ppm) for ${ }^{19} \mathrm{~F}$ in fluorinated compounds with PBE (orange) and TB$\mathrm{mBJ}$ (blue) exchange potentials. All structures are optimized with PBE. The references for the experimental values of ${ }^{19} \mathrm{~F}$ are indicated in Table 6.1 . 
Table 6.1 Experimental ${ }^{19} \mathrm{~F} \delta_{\text {iso }}$, and ${ }^{19} \mathrm{~F} \sigma_{\text {iso }}$ calculated with PBE and the BJ-LDA exchangecorrelation potentials. For both functionals the GIPAW method was used. The linear regression fits are shown in Figure 6.1.

\begin{tabular}{cccc}
\hline $\begin{array}{c}\text { Fluorinated } \\
\text { compound }\end{array}$ & $\sigma_{\text {iso }}$ PBE & $\sigma_{\text {iso }}$ TB-mBJ & Experimental $\delta_{\text {iso }}$ \\
\hline $\mathrm{LiF}$ & 368.2 & 426.7 & $-204.3^{65}$ \\
$\mathrm{NaF}$ & 397.2 & 449.8 & $-224.2^{65}$ \\
$\mathrm{KF}$ & 284.3 & 358.4 & $-133.3^{65}$ \\
$\mathrm{RbF}$ & 251.9 & 328.9 & $-90.9^{65}$ \\
$\mathrm{RbF} . \mathrm{H}_{2} \mathrm{O}$ & 267.8 & 334.1 & $-113.0^{66}$ \\
$\mathrm{MgF}_{2}$ & 364.9 & 422.0 & $-197.3^{65}$ \\
$\mathrm{SrF}_{2}$ & 224.2 & 317.3 & $-108.0^{65}$ \\
$\mathrm{AlF}_{3}$ & 333.9 & 397.8 & $-172.0^{65}$ \\
$\mathrm{CdF}_{2}$ & 347.5 & 416.9 & $-192.1^{66}$ \\
$\mathrm{HgF}_{2}$ & 342.5 & 411.3 & $-196.4^{66}$ \\
$\mathrm{KF}_{2} 2 \mathrm{H}_{2} \mathrm{O}$ & 278.9 & 350.9 & $-133.0^{66}$ \\
$\mathrm{Na}_{3} \mathrm{AlF}_{6}$ & 355.7 & 417.8 & $-189.0^{66}$ \\
& 358.0 & 417.6 & $-190.0^{66}$ \\
$\mathrm{Na}_{5} \mathrm{Al}_{3} \mathrm{~F}_{14}$ & 325.5 & 394.2 & $-166.0^{66}$ \\
& 356.0 & 416.4 & -182.0 \\
& 358.0 & 417.6 & -190.0 \\
$\mathrm{Na}_{2} \mathrm{SiF}_{6}$ & 306.7 & 378.3 & $-151.0^{66}$ \\
$\alpha-\mathrm{PbF}_{2}$ & 179.4 & 290.7 & $-57.7^{66}$ \\
& 142.3 & 263.1 & -20.5 \\
$\mathrm{CF}_{3} \mathrm{Cl}$ & 156.1 & 259.2 & $-28.6^{67}$ \\
$\mathrm{CFCl}_{2} \mathrm{CFCl}_{2}$ & 193.7 & 298 & $-67.8^{67}$ \\
$\mathrm{SiF}_{4}$ & 327.5 & 383.1 & $-163.3^{67}$ \\
\hline
\end{tabular}


6.3 Elucidation of Fluoride location in the as-made RTH

\subsubsection{Simulation of the ${ }^{19} \mathrm{~F}$ chemical shift assuming a monoclinic unit cell}

The simulation of the ${ }^{19} \mathrm{~F}$ chemical shift is based on the following experimental information. The pure silica RTH framework is synthesized in fluoride media at 175 ${ }^{\circ} \mathrm{C}$ using tri-isopropyl-methyl-phosphonium as OSDA. The RTH zeolite is obtained after three days with no amorphous phase and with a $\mathrm{F}^{-} / \mathrm{OSDA}$ ratio of $\sim 1.0$, meaning that there are no defects in the final material. The pure silica RTH framework has 32 $\mathrm{Si}$ and $64 \mathrm{O}$ atoms per unit cell ${ }^{68}$ and there are two OSDA cations and two fluoride anions per unit cell.

The ${ }^{29} \mathrm{Si}$ MAS-NMR spectra exhibit 16 signals and in the ${ }^{19} \mathrm{~F}$ MAS-NMR spectra one signal is observed (Figure 6.2). This means that, according to the unit cell chemical composition, the $32 \mathrm{Si}$ atoms are grouped in 16 chemically equivalent pairs and that both fluorides are chemically equivalent. This experimental evidence suggests that we should find a model for which both fluoride anions have the same calculated absolute shielding and the 32 calculated Si shieldings can be grouped in 16 pairs. Thus, this piece of information provides a criterion to determine which model or subset of models agree with the experimental evidence. Other criteria include of course the relative stability and the predicted ${ }^{19} \mathrm{~F}$ chemical shift.

If the time of synthesis is increased to more than three days, the ${ }^{19} \mathrm{~F}$ signal shifts from $-71.9 \mathrm{ppm}$ to $-67.2 \mathrm{ppm}$ (Figure 6.2). It means that either the local environment of the fluoride changes or the fluoride migrates to a different Si position. Throughout this whole chapter we will refer to the sample with the ${ }^{19} \mathrm{~F}$ signal at $-71.9 \mathrm{ppm}$ as "sample I" and the one with the ${ }^{19} \mathrm{~F}$ signal at $-67.2 \mathrm{ppm}$ as "sample II". 

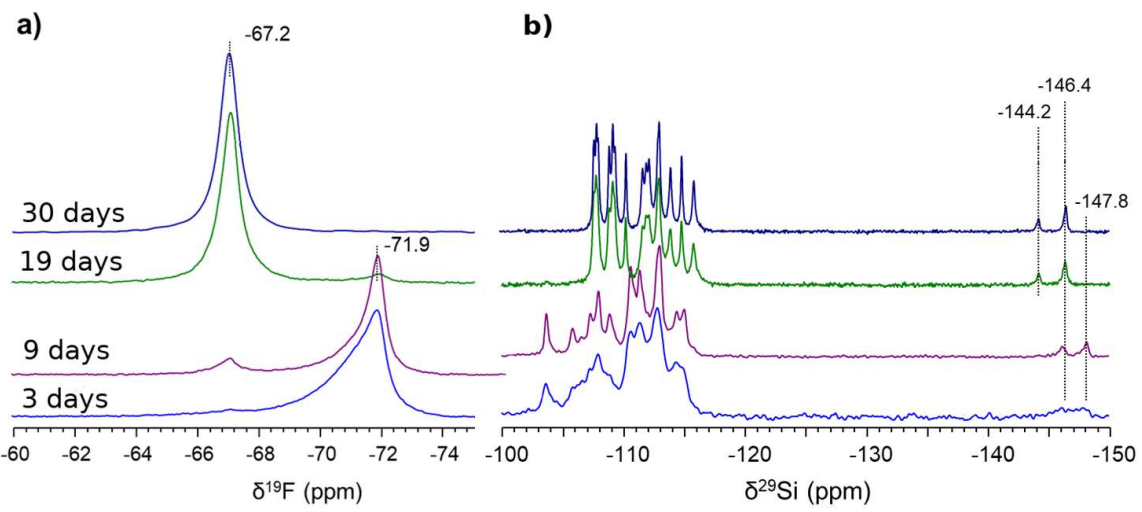

Figure 6.2 MAS-NMR spectra of the as-synthesized pure silica RTH framework. a) ${ }^{19} \mathrm{~F}$ and b) ${ }^{29} \mathrm{Si}$.

In order to simulate the ${ }^{19} \mathrm{~F}$ chemical shifts of the as-made $\mathrm{RTH}$ the initial coordinates of the $\mathrm{Si}, \mathrm{P}, \mathrm{O}$ and $\mathrm{C}$ atoms and the cell parameters were taken from the XRD structure. This piece of information is important because having a relatively accurate configuration of the OSDA significantly reduces the search for a minimum in the potential energy surface. The location of $\mathrm{F}^{-}$is however uncertain due its low electronic density. We have thus performed a series of calculations using models in which two $\mathrm{F}^{-}$anions have been placed in different tetrahedral sites $\left(\mathrm{TO}_{4}, \mathrm{~T}=\mathrm{Si}\right)$ together with the two compensating OSDA cations inside the cavity. All have the same chemical composition, $\mathrm{Si}_{32} \mathrm{O}_{64} \mathrm{P}_{2} \mathrm{C}_{20} \mathrm{H}_{47} \mathrm{~F}_{2}$.

Let us now comment on how the exact combinations were generated. According to the structure reported in the IZA database ${ }^{68}$ the calcined pure silica RTH has four nonequivalent $\mathrm{T}$ sites, namely $\mathrm{T} 1, \mathrm{~T} 2, \mathrm{~T} 3$ and $\mathrm{T} 4$, each with multiplicity 8 . Since the unit cell contains $32 \mathrm{Si}$ sites, there are, in principle, 496 possible ways of arranging the two fluorides in the $32 \mathrm{Si}$ sites (equation 6.1).

$m=\frac{n !}{(n-r) ! r !}$

However, it is obvious that two $\mathrm{F}^{-}$in two nonequivalent $\mathrm{T}$ sites will exhibit different chemical shifts i.e. a structure with one $\mathrm{F}^{-}$in a $\mathrm{T} 1$ site and the other in a $\mathrm{T} 2$ will result 
in two different values of absolute shielding. Thus, we explored all the possible models that could be built by placing the two fluoride ions in equivalent $\mathrm{TO}_{4}$ sites. Each $\mathrm{T}$ site has multiplicity 8 which, according to equation 6.1 , yields 28 possible combinations of two equivalent $\mathrm{T}$ sites. That would give a total of 112 combinations, that is, 28 for each of the four nonequivalent $\mathrm{T}$ sites. Notice that we have used the formula to calculate $m$ combinations of $r$ objects from a set of $n$ objects (equation 6.1) because the order does not matter. The two $\mathrm{F}^{-}$are equivalent and exchanging them produces the same chemical structure. In summary, for each of the four $\mathrm{TO}_{4}$ groups, the eight equivalent sites have been labelled with letters, A, B, C, D, E, F, G and $\mathrm{H}$, and combined in 28 pairs without repetition, namely, $\mathrm{AB}, \mathrm{AC}, \mathrm{AD}, \mathrm{AE}, \ldots$ GH. Figure 6.3 shows the labeled T sites. Each set with 28 structures is called a T group.

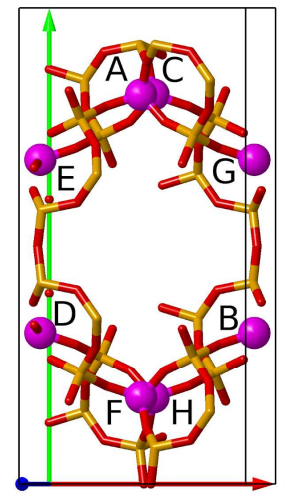

$\mathrm{T} 1$

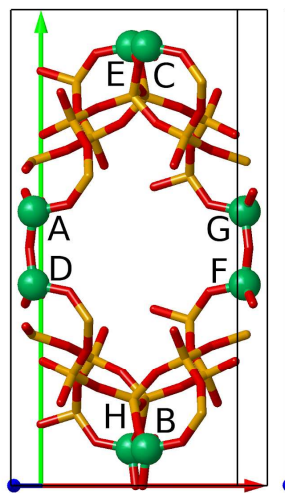

$\mathrm{T} 2$

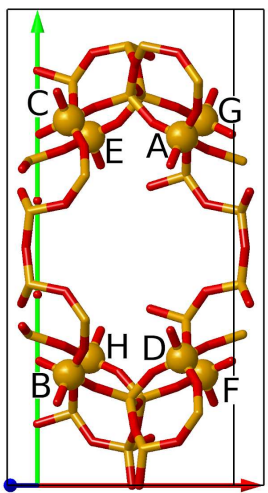

T3

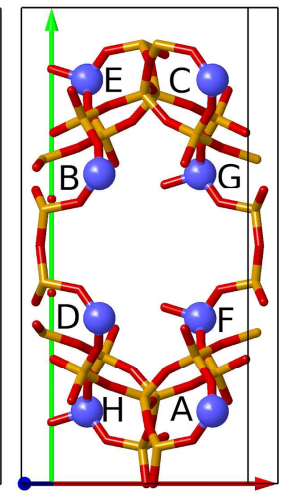

T4

Figure 6.3 Color representation of the labeled T crystallographic sites of the RTH framework according to IZA ${ }^{68}$. The 8 sites of every T group have been labelled with letters. T1, T2, T3 and T4 sites are depicted in pink, green, orange and blue respectively.

During the optimizations all atomic positions and the cell parameters were allowed to relax keeping the cell volume fixed. The orientation of the OSDA inside the cavity remained practically unchanged for all systems, indicating that the theoretical models are in agreement with the structure obtained from XDR. The relative energies of all the 112 structures with respect to the most stable one are presented in bar plots 
in Figure 6.4. We have also included the absolute difference between the calculated absolute shieldings of the two fluorides $\left(\Delta \sigma_{\text {iso }}\right)$ of each model.

An inspection of Figure 6.4 shows that when $\Delta \sigma_{\text {iso }} \gg>0$ the structure is significantly unstable. Some examples are the models $C G, C E$ and $D F$ of the T4 group. The opposite is not necessarily true. There are structures with very similar calculated ${ }^{19} \mathrm{~F}$ shielding also significantly unstable, such as $A F, A H, B E$ and $B G$.

Both fluorides of each of the models $A B, C D, E F$ and $G H$ of the four T groups (T1, $\mathrm{T} 2, \mathrm{~T} 3, \mathrm{~T} 4)$ have the same calculated isotropic shielding $\left(\Delta \sigma_{\text {iso }} \sim 0 \mathrm{ppm}\right)$. The calculated absolute ${ }^{29} \mathrm{Si}$ shieldings of these 16 structures are summarized in Tables B. 1 to B.4 in appendix B. The values of ${ }^{29} \mathrm{Si} \sigma_{\text {iso }}$ have been grouped in 16 pairs and it can be seen that both $\mathrm{Si}$ atoms of each pair have the same calculated value of $\sigma$. This is in agreement with the $16{ }^{29} \mathrm{Si} \delta_{\text {iso }}$ signals observed experimentally. It is of course a consequence of these pairs of atoms having the same chemical environment. More than 16 calculated ${ }^{29} \mathrm{Si} \sigma$ values are obtained for the rest of the models. We conclude that only these 16 structures are consistent with the experimental observations. 
6.3 Elucidation of Fluoride location in the as-made RTH
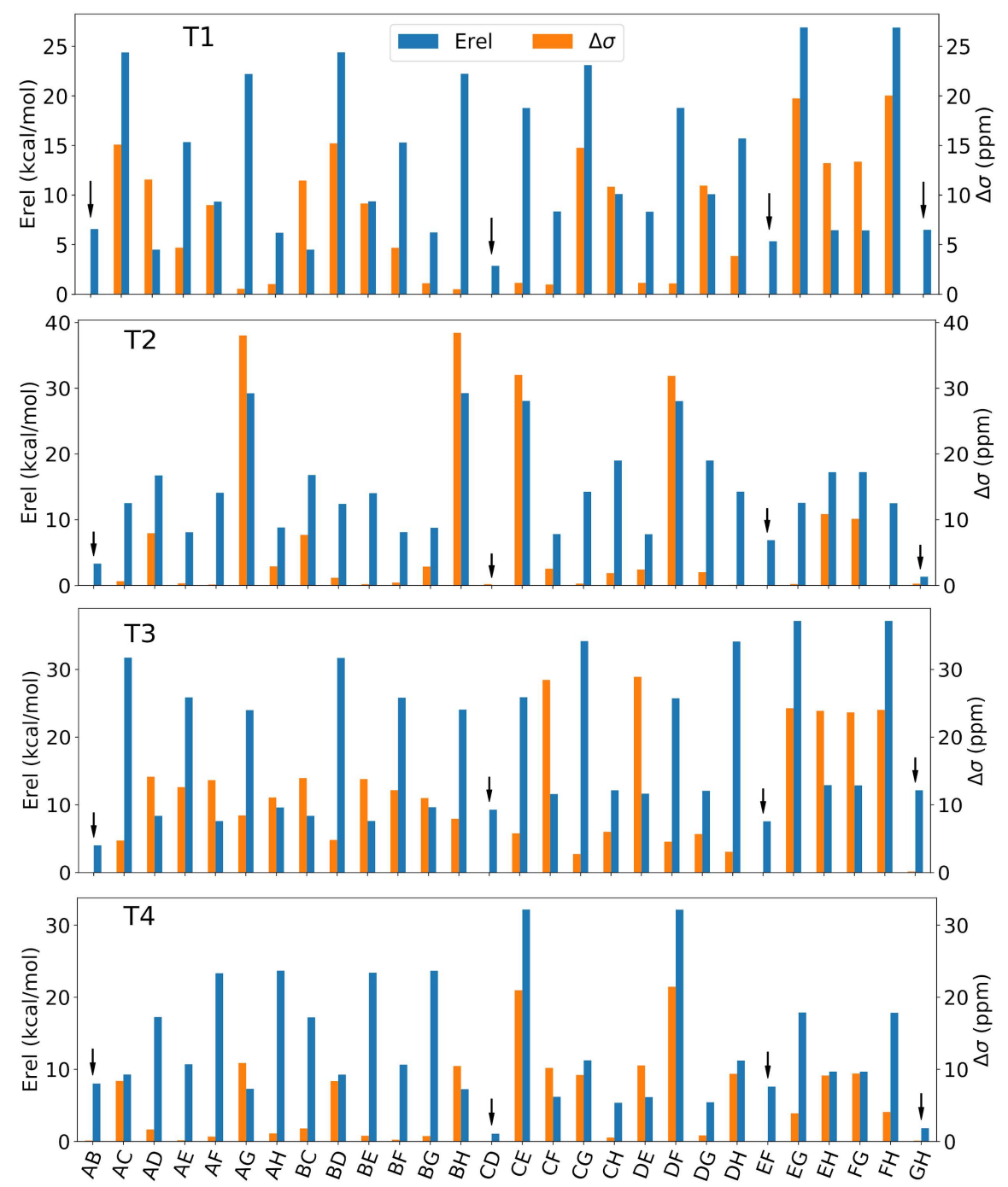

Figure 6.4 Relative energy (blue) in $\mathrm{kcal} / \mathrm{mol}$ with respect to the most stable structure (CD of T2) and absolute difference $\left(\Delta \sigma_{\text {iso }}\right)$ between the calculated shieldings of the two $\mathrm{F}^{-}$of every structure. Arrows mark the 16 structures with equivalent $\mathrm{F}^{-}$anions $\left(\Delta \sigma_{\text {iso }} \approx 0\right)$. 
Interestingly, these 16 structures share a common feature: all chemically equivalent pairs of atoms in each structure are related by the following symmetry operation $x+1 / 2, y+1 / 2, z$ (Figure 6.5). It means that, according to these calculations, when the two fluorides are sitting in any of these 16 positions, only two out of the eight symmetry operations of the $C 2 / m$ space group (calcined RTH) is actually detected by NMR, namely: $x+1 / 2, y+1 / 2, z$ and of course the identity operation $x, y, z$.

The next step is the realization that there is no space group with only these two symmetry operations: $\mathrm{x}, \mathrm{y}, \mathrm{z}$ and $\mathrm{x}+1 / 2, \mathrm{y}+1 / 2, \mathrm{z}$ (or equivalently $\mathrm{x}-1 / 2, \mathrm{y}-1 / 2, \mathrm{z}$ ). Among the 230 space groups, the only ones with two symmetry operations are P-1 ( $x, y, z$ and $-\mathrm{x},-\mathrm{y},-\mathrm{z}), \mathrm{P} 2(\mathrm{x}, \mathrm{y}, \mathrm{z}$ and $-\mathrm{x}, \mathrm{y},-\mathrm{z})$ and P21 (x, y, $\mathrm{z}$ and $-\mathrm{x}, \mathrm{y}+1 / 2,-\mathrm{z})$. Therefore, we can conclude that the as-made crystal structure belongs to a triclinic P-1 unit cell with half the number of atoms $(1 \mathrm{~F}, 16 \mathrm{Si}, 32 \mathrm{O}, 1 \mathrm{P})$ and where the aforementioned pairs belong to different unit cells. The symmetry operation $(x+1 / 2, y+1 / 2, z)$ is then a translation along one of the cell vectors. The unit cell must be redefined accordingly.

In Figure 6.5, the relative energy of the 112 structures is plotted versus the shortest F-F distance. The most stable models are among those for which the shortest F-F distance is maximized to $\sim 9-10 \AA$. The shortest F-F distance in all the aforementioned 16 structures is $\sim 9.6 \AA$. In the structures with chemically equivalent fluorides the distance between negative charges is maximized and as a result they are relatively stable. 

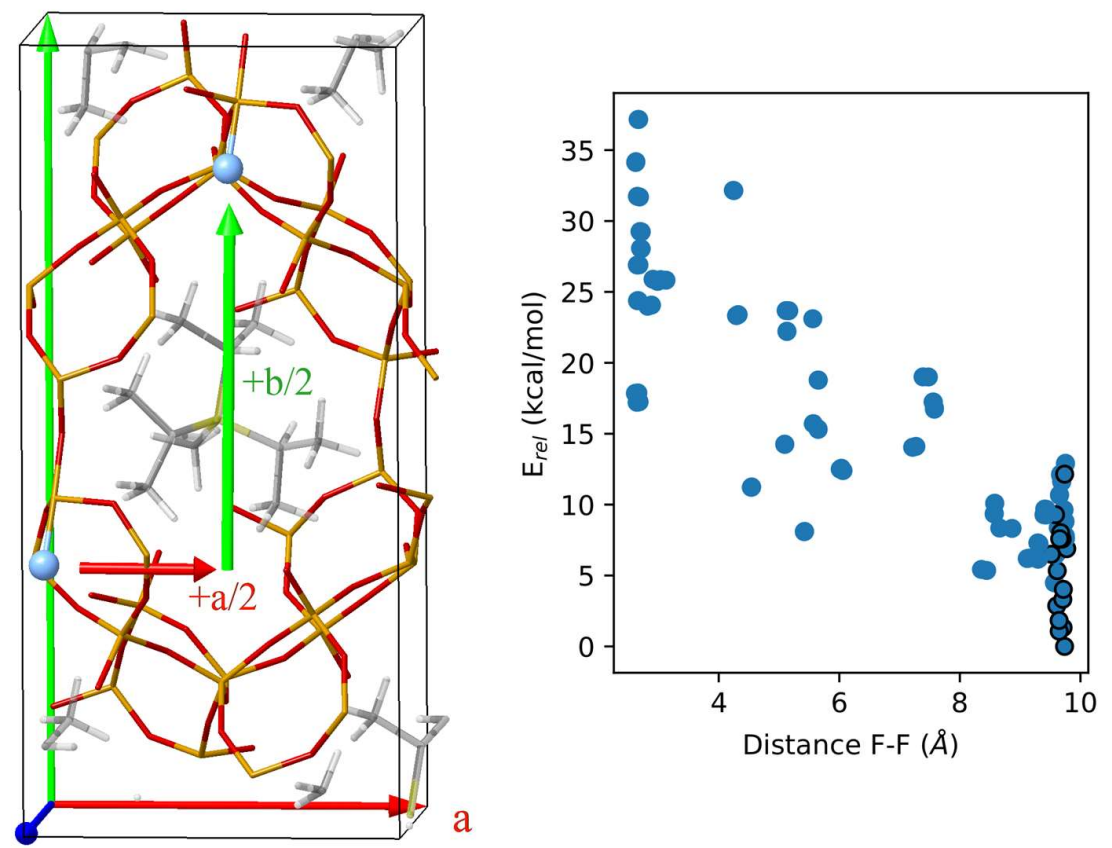

Figure 6.5 Representation of two equivalent fluoride anions (left panel). This particular model is the CD of the T2 group (Figure 6.3). Si, O, P, C, H, F are depicted in orange, red, yellow, gray, light gray and blue, respectively. Unit cell vectors $\mathrm{a}, \mathrm{b}$ and $\mathrm{c}$ are depicted in red, green and blue, respectively. The right panel shows the relative energy as a function of the shortest F-F distance. Circles corresponding to models with equivalent pairs of fluorides have a black border.

\subsubsection{Redefinition of the unit cell}

We have just seen that the symmetry operations found by simulating the chemical shifts of the as-made RTH does not correspond to any of the existing space groups and the unit cell must be redefined. The only possibility is to use a triclinic unit cell with 16 nonequivalent $\mathrm{Si}$ atoms, one $\mathrm{F}^{-}$anion and OSDA cation as well. Redefining the new triclinic unit cell implies the redefinition of the cell vectors. To this end, the RTH structure is repeated in the three (x, y, and $\mathrm{z}$ ) directions of space and the atoms related by just translations must be identified. Fortunately, the fact that the new unit cell contains only one $\mathrm{F}^{-}$makes this task simpler because all nearest $\mathrm{F}^{-}$are related by translation along the unit cell vectors. Thus, the new unit cell vectors can be defined 
by choosing one fluoride and connecting the three nearest fluorides (Figure 6.6). With this procedure, the vector that connects the previously found equivalent pairs defines one of the unit cell vectors, namely the $b$ vector. The other two vectors ( $a$ and $c$ ) coincide with the ones of the monoclinic unit cell. The atomic positions are, of course, equivalent to the positions belonging to the monoclinic unit cell used in the previous section. This is so because the same RTH topology must be generated by repetition of the unit cell along the three dimensions of space. Comparison of the periodically repeated structures of both the monoclinic and triclinic unit cell along the 3 dimensions shows that the same RTH topology is generated (Figure 6.6).

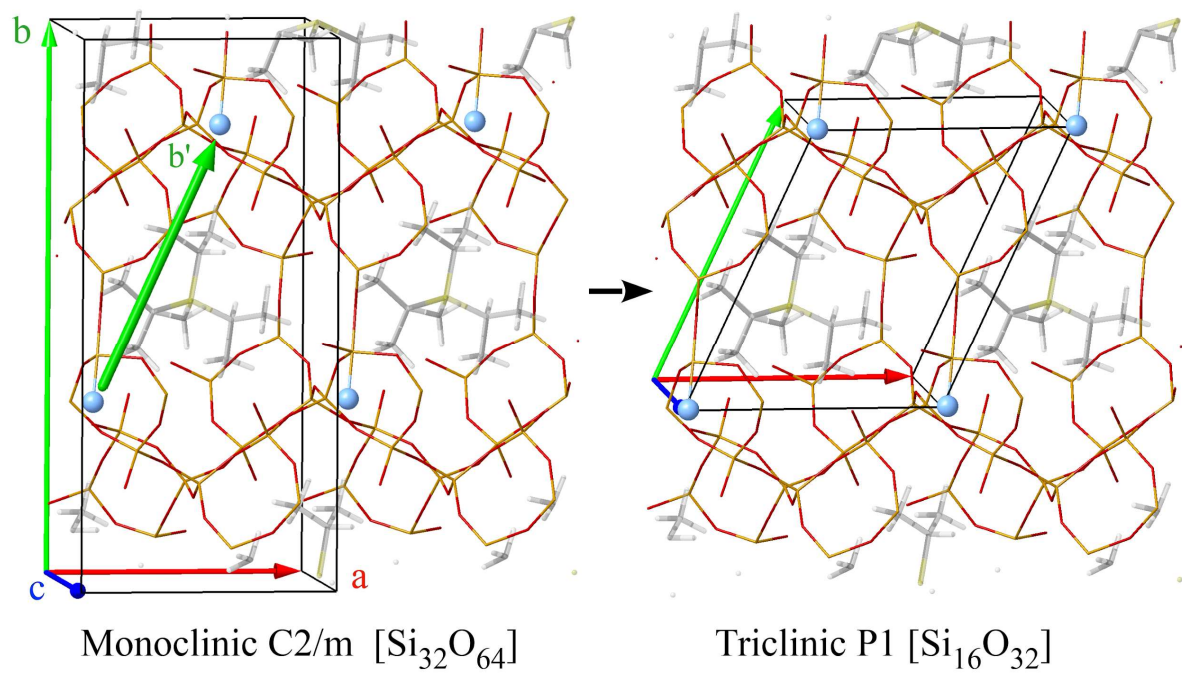

Figure 6.6 Redefinition of the as-made RTH unit cell. The vector, $b$ ', connecting the F pairs related by $(\mathrm{x}+1 / 2, \mathrm{y}+1 / 2, \mathrm{z})$ symmetry operation will now define the new $b$ vector. $\mathrm{Si}, \mathrm{O}, \mathrm{P}, \mathrm{C}$, $\mathrm{H}, \mathrm{F}$ are depicted in orange, red, yellow, gray, light gray, blue respectively. Unit cell vectors $\mathrm{a}, \mathrm{b}$ and $\mathrm{c}$ are depicted in red, green and blue respectively.

Another way to confirm that both cell definitions generate the same structure is to compute the energy per Si atom for both unit cells. The calculated values are -553.51 $\mathrm{kcal} / \mathrm{mol}$ and $-553.52 \mathrm{kcal} / \mathrm{mol}$, for the triclinic and the monoclinic respectively. This 
means that indeed both are the same structure. Finally, it has to be experimentally confirmed with XRD. The lattice parameters optimized with PBE were used as an initial approximation in the Rietveld refinement, which fits the cell parameters and atomic positions to the experimental diffraction pattern. Table 6.2 shows the theoretically proposed unit cell after optimization (atomic positions, cell volume and shape) and the ones obtained from XRD refinement corresponding to the samples I and II. The lattice parameters of the samples I and II are different and the unit cell volume of sample II is $\sim 5 \AA^{3}$ larger.

Table 6.2 Triclinic lattice parameters corresponding to the optimized with $\mathrm{PBE}+\mathrm{D} 3$ and those refined with XRD. Vector lengths are given in angstroms.

\begin{tabular}{cccccccc}
\hline & $\mathrm{a}$ & $\mathrm{b}$ & $\mathrm{c}$ & $\alpha$ & $\beta$ & $\gamma$ & $\mathrm{V}\left(\AA^{3}\right)$ \\
\hline Theory (PBE) & 9.753 & 11.471 & 9.729 & 86.32 & 95.78 & 115.84 & 974.4 \\
Sample I & 9.721 & 11.379 & 9.794 & 87.96 & 96.15 & 115.03 & 975.8 \\
Sample II & 9.767 & 11.514 & 9.690 & 87.33 & 96.34 & 115.12 & 980.6 \\
\hline
\end{tabular}

\subsubsection{Simulation of the ${ }^{19} \mathrm{~F}$ chemical shift with the triclinic unit cell}

The location of $\mathrm{F}^{-}$in the as-made RTH defined with the triclinic unit cell was further investigated through the simulation of the isotropic chemical shift of ${ }^{19} \mathrm{~F}$. The atomic positions of $\mathrm{Si}, \mathrm{P}$ and $\mathrm{C}$ atoms directly bonded to the $\mathrm{P}$ are again located accurately by XRD but the fluoride anion is much more difficult to locate because of its low electron density. There are now $16 \mathrm{Si}$ atoms in the triclinic unit cell, four T sites (T1, $\mathrm{T} 2, \mathrm{~T} 3$ and T4) with multiplicity 4, in addition to one fluoride anion, and one OSDA cation. The T3 and T4 sites make up the 4-membered rings that join two cages (Figure 6.7) and therefore the fluoride anion could be located in the T3 or T4 sites pointing to the center of any of the two cages, with which a total of 24 different arrangements for the $\mathrm{F}^{-}$anion are possible. 


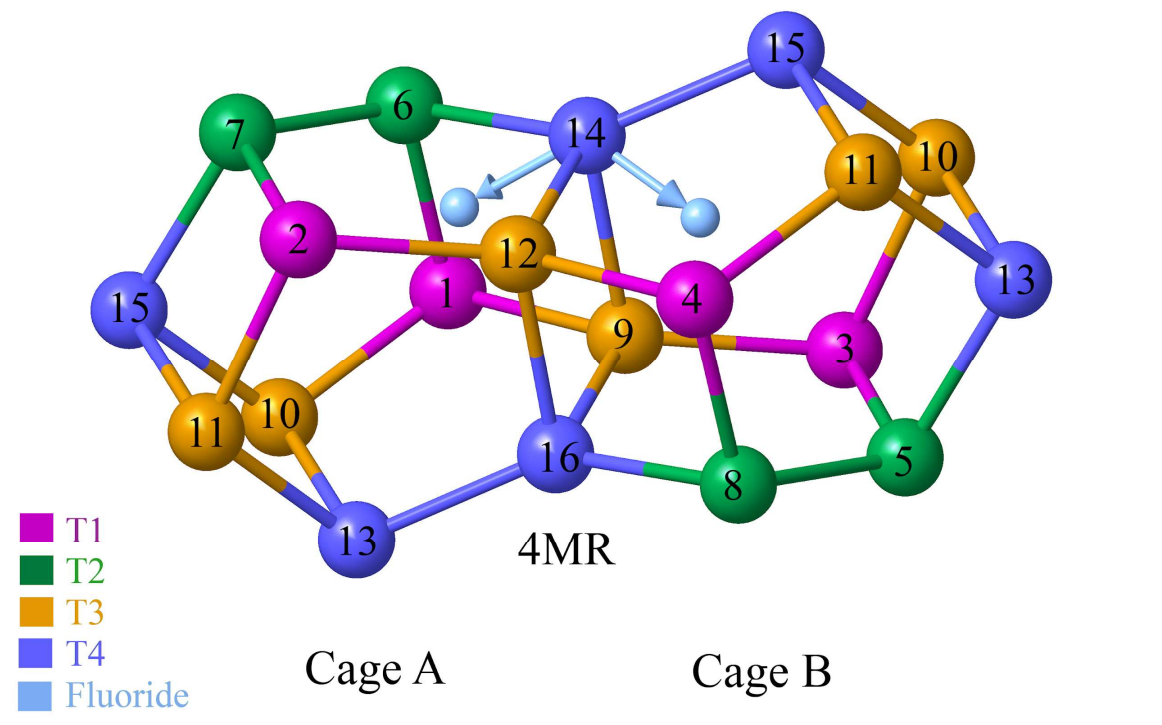

Figure 6.7 Illustration of the two possible orientations of the fluoride anion in a T4 site (position 14). The fluoride anion can be oriented toward the center of cage A or B. The same applies to positions $9,10,11,12,13$ and 15 . Cages are joined by 4-membered rings (4MR) made up of T3 and T4 sites. For visual aid of the cage B the periodic image of the positions $10,11,13$ and 15 are also represented.

The 24 models will be named according to the index of the $\mathrm{Si}$ atom where the $\mathrm{F}^{-}$is sitting, that is, from 1 to 16 . The duplicates of the T3 and T4 sites will be labeled $A$ and $B$ depending toward which cage the fluoride is pointing to (Figure 6.7). For each of the 24 arrangements the volume of the unit cell was optimized as explained in section 6.2 and the ${ }^{19} \mathrm{~F}$ absolute shielding was calculated for the 24 optimized structures. The Si-F bond length, the distance P-F, the volume of the unit cell, the predicted $\delta_{\text {iso, }}$ and the relative energy with respect to the most stable (position 6) are summarized in Table 6.3. 
6.3 Elucidation of Fluoride location in the as-made RTH

Table 6.3 Si-F bond lengths, P-F distance, unit cell volume, predicted isotropic chemical shift $\left(\delta_{\text {iso }}\right)$ of ${ }^{19} \mathrm{~F}$, and relative energy with respect to position 6 . The $\delta_{\text {iso }}$ were calculated using the regression equations shown in Figure 6.1 and taking as predictor variable the absolute isotropic shielding calculated with TB-mBJ and PBE functionals.

\begin{tabular}{|c|c|c|c|c|c|c|c|}
\hline \multirow{2}{*}{$\mathrm{Si}$} & \multirow{2}{*}{ Site } & \multirow{2}{*}{$\begin{array}{l}\text { Si-F bond } \\
(\AA)\end{array}$} & \multirow{2}{*}{$\begin{array}{c}\text { P-F distance } \\
(\AA)\end{array}$} & \multirow{2}{*}{$\begin{array}{c}\text { Unit Cell } \\
\text { volume }\left(\AA^{3}\right)\end{array}$} & \multicolumn{2}{|c|}{ Predicted $\delta_{\text {iso }}(\mathrm{ppm})$} & \multirow{2}{*}{$\begin{array}{c}\text { Erel } \\
(\mathrm{kcal} / \mathrm{mol})\end{array}$} \\
\hline & & & & & TB-mBJ & PBE & \\
\hline 1 & $\mathrm{~T} 1$ & 1.82 & 7.00 & 980.4 & -56.1 & -50.0 & 3.8 \\
\hline 2 & $\mathrm{~T} 1$ & 1.78 & 7.30 & 985.2 & -62.8 & -57.2 & 1.8 \\
\hline 3 & $\mathrm{~T} 1$ & 1.82 & 6.74 & 985.0 & -62.1 & -50.9 & 3.9 \\
\hline 4 & $\mathrm{~T} 1$ & 1.82 & 7.02 & 980.7 & -56.6 & -49.9 & 4.4 \\
\hline 5 & $\mathrm{~T} 2$ & 1.79 & 6.94 & 973.2 & -66.3 & -62.6 & 0.6 \\
\hline 6 & $\mathrm{~T} 2$ & 1.79 & 6.74 & 979.3 & -65.9 & -61.8 & 0.0 \\
\hline 7 & $\mathrm{~T} 2$ & 1.78 & 7.25 & 974.3 & -66.4 & -63.2 & 0.4 \\
\hline 8 & $\mathrm{~T} 2$ & 1.79 & 7.29 & 977.5 & -65.0 & -61.0 & 0.7 \\
\hline $9 \mathrm{~A}$ & $\mathrm{~T} 3$ & 1.80 & 6.88 & 981.0 & -58.1 & -55.0 & 2.4 \\
\hline 9B & $\mathrm{T} 3$ & 1.79 & 6.58 & 970.0 & -75.0 & -69.0 & 4.5 \\
\hline $10 \mathrm{~A}$ & $\mathrm{~T} 3$ & 1.77 & 6.68 & 973.4 & -73.0 & -69.8 & 5.3 \\
\hline 10B & $\mathrm{T} 3$ & 1.81 & 6.71 & 984.8 & -49.5 & -45.5 & 4.2 \\
\hline $11 \mathrm{~A}$ & $\mathrm{~T} 3$ & 1.81 & 7.13 & 989.4 & -50.6 & -46.7 & 3.9 \\
\hline $11 \mathrm{~B}$ & $\mathrm{~T} 3$ & 1.77 & 6.73 & 972.2 & -72.7 & -68.5 & 5.0 \\
\hline $12 \mathrm{~A}$ & $\mathrm{~T} 3$ & 1.77 & 6.66 & 971.0 & -73.0 & -70.0 & 5.0 \\
\hline $12 \mathrm{~B}$ & $\mathrm{~T} 3$ & 1.81 & 7.04 & 982.7 & -58.7 & -56.1 & 3.0 \\
\hline $13 \mathrm{~A}$ & $\mathrm{~T} 4$ & 1.77 & 6.79 & 972.1 & -71.9 & -67.2 & 4.3 \\
\hline $13 \mathrm{~B}$ & $\mathrm{~T} 4$ & 1.8 & 6.85 & 975.4 & -58.5 & -54.6 & 2.0 \\
\hline $14 \mathrm{~A}$ & $\mathrm{~T} 4$ & 1.79 & 6.79 & 976.0 & -58.5 & -54.8 & 0.4 \\
\hline 14B & $\mathrm{T} 4$ & 1.78 & 6.91 & 968.0 & -70.8 & -66.2 & 3.9 \\
\hline $15 \mathrm{~A}$ & $\mathrm{~T} 4$ & 1.80 & 7.03 & 976.2 & -57.8 & -54.0 & 2.2 \\
\hline $15 \mathrm{~B}$ & $\mathrm{~T} 4$ & 1.77 & 6.35 & 970.0 & -71.8 & -67.2 & 3.6 \\
\hline $16 \mathrm{~A}$ & $\mathrm{~T} 4$ & 1.77 & 6.52 & 967.8 & -70.4 & -65.4 & 5.2 \\
\hline $16 \mathrm{~B}$ & $\mathrm{~T} 4$ & 1.80 & 6.96 & 976.2 & -59.0 & -56.3 & 1.1 \\
\hline
\end{tabular}


Several observations can be made from inspection of Table 6.3. The Si-F bond lengths range from 1.77 to $1.82 \AA$ and the predicted $\delta_{\text {iso }}$ from -48 to $-73 \mathrm{ppm}$. In general, the ${ }^{19} \mathrm{~F} \delta_{\text {iso }}$ values calculated with PBE are systematically lower than the ones calculated with TB-mBJ by approximately 3-6 ppm. This is probably due to the different slopes of the regression equations obtained with the two functionals (Figure 6.1). It can also be seen that structures with relatively small unit cell volumes also have relatively short P-F distances.

Other relations are shown in Figure 6.8 where the ${ }^{19} \mathrm{~F}$ chemical shift is plotted versus the Si-F distance and compared together with the unit cell volume. Notice the significant correlation between the Si-F bond length and the chemical shift, the longer the Si-F bond the more negative the chemical shift is (Figure 6.8). The coefficient of determination shows that $\sim 80 \%$ of the chemical shift can be predicted with only the Si-F bond length.

The chemical shift also varies along with unit cell volume (Figure 6.8). Larger unit cell volumes $\left(>980 \AA^{3}\right)$ appear to be associated with less negative chemical shifts (> $-60 \mathrm{ppm})$. Conversely, the models with the smallest unit cell volumes, $\sim 970 \AA^{3}$, exhibit the more negative chemical shifts, $<-70 \mathrm{ppm}$. This agrees well with the experimental observations of samples I and II. Recall that the experimental ${ }^{19} \mathrm{~F}$ isotropic chemical shifts are $-67.2 \mathrm{ppm}$ for sample II with a unit cell volume of 980.6 $\AA^{3}$ and -71.9 ppm for sample I with a unit cell volume of $975.8 \AA^{3}$ (Figure 6.2 and Table 6.2). Also notice that the smallest volumes are predicted for the structures where the fluoride anion is located in T3 and T4 sites. 

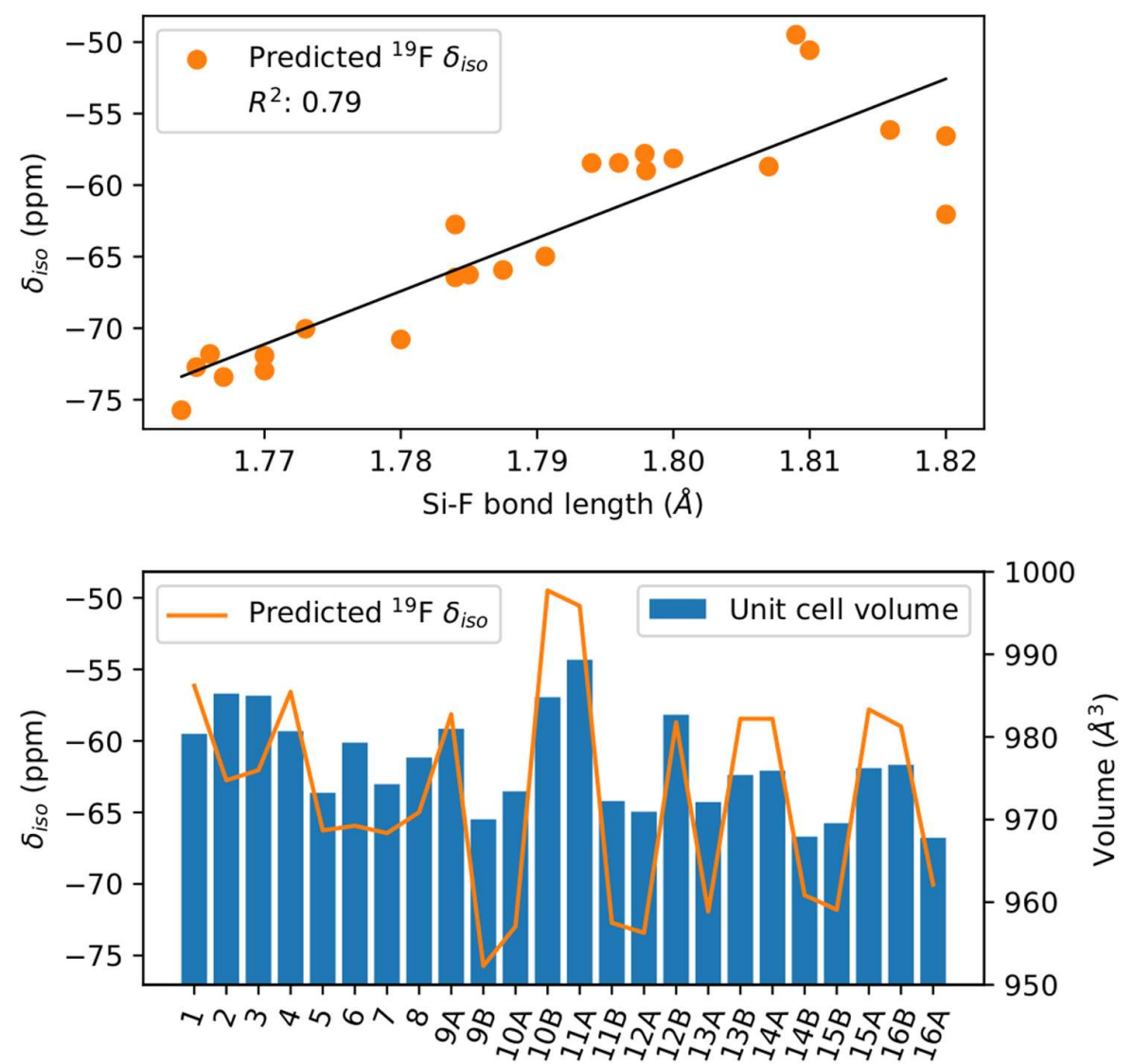

Figure 6.8 Correlation of the ${ }^{19} \mathrm{~F}$ chemical shift with Si-F bond length (upper panel) and unit cell volume (lower panel).

The prediction of the chemical shifts shows the different environments of the nonequivalent crystallographic sites. Values around -56 and $-62 \mathrm{ppm}$ are predicted for T1, -66 ppm for T2, $-50,-58$ and -73 ppm for T3, and -58 and $-71 \mathrm{ppm}$ for T4 sites. According to these results, the experimental signal observed at $-67.2 \mathrm{ppm}$ (sample II) can be assigned to a T2 site, in particular, the position 6 which is overall the most stable structure (Table 6.3), and the one for which the calculated ${ }^{29} \mathrm{Si}$ shieldings best correlate with the experimental ${ }^{29} \mathrm{Si}$ chemical shifts of sample II (Figure 6.9). The signal at $-71.8 \mathrm{ppm}$ can be attributed to the fluoride anion located in a T4 site, in particular, the position $16 \mathrm{~A}$ for which the calculated ${ }^{29} \mathrm{Si}$ shieldings 
best correlate with the experimental ${ }^{29} \mathrm{Si}$ chemical shifts of sample I (Figure 6.10). The calculated ${ }^{29} \mathrm{Si}$ chemical shifts of the 24 structures are included in appendix B. Finally, although position 6 is the most stable, there are not huge differences in the relative energies among all structures so that it is not surprising that two ${ }^{19} \mathrm{~F}$ chemical shifts can be observed experimentally.
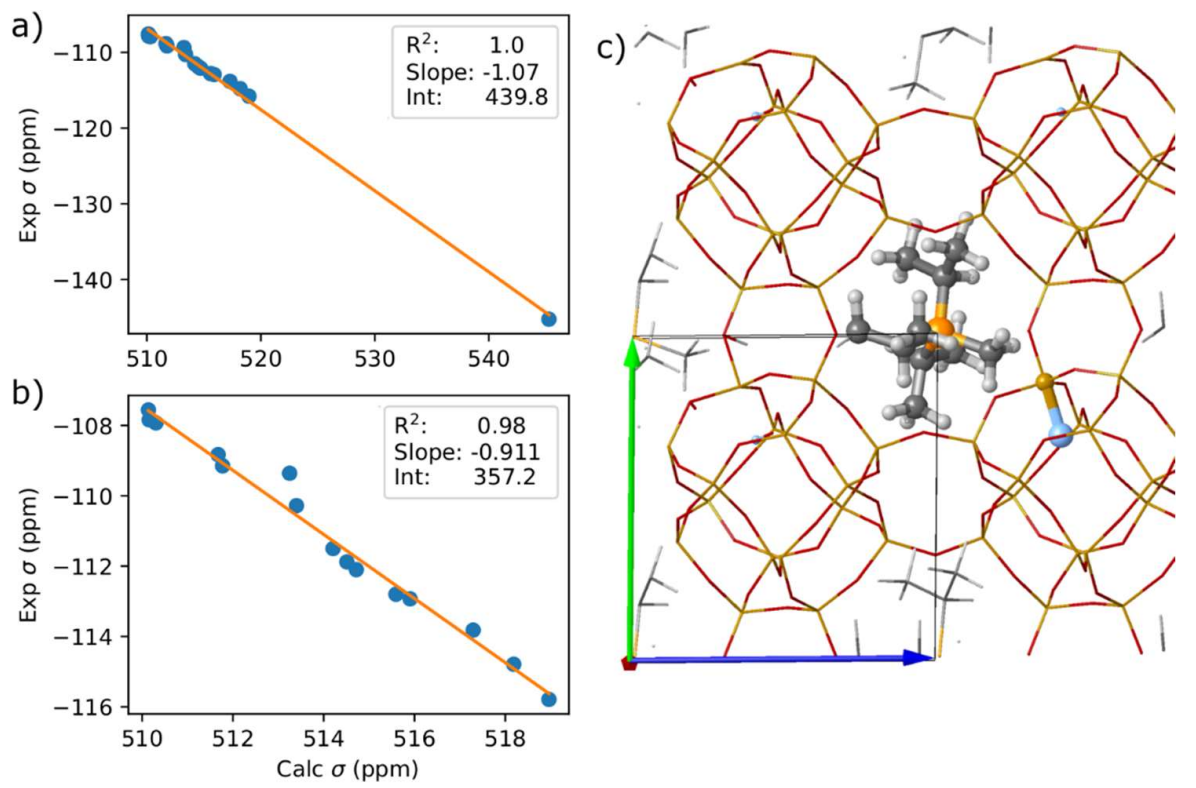

Figure 6.9 Correlation of the calculated ${ }^{29} \mathrm{Si}$ shieldings with the experimental ${ }^{29} \mathrm{Si}$ chemical shifts of sample I with the $16 \mathrm{Si}$ atoms (a) and leaving out the Si atom bonded to the fluoride (b). c) Optimized structure with the fluoride anion sitting on position 6 (T2 site). Only one OSDA and the nearest fluoride are represented with balls. Si, O, P, C, H, F are depicted in orange, red, yellow, gray, light gray and blue, respectively. 

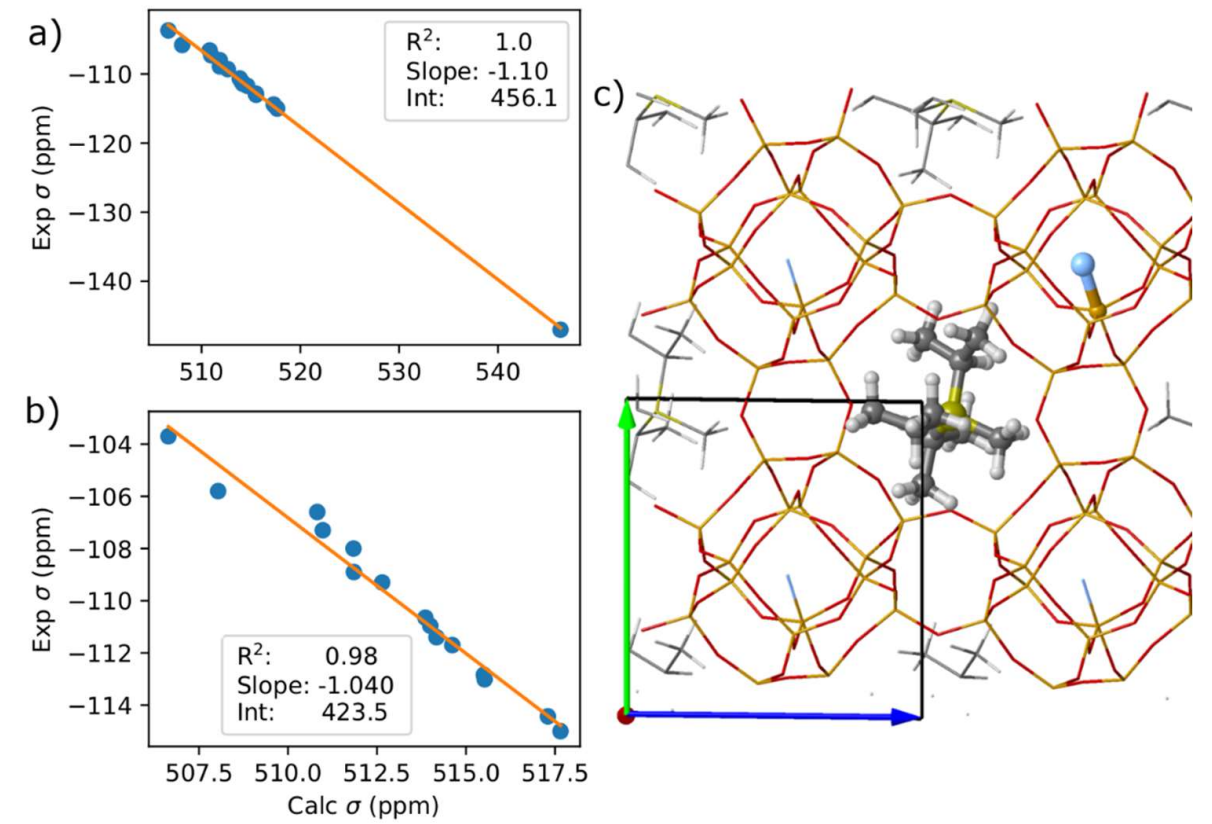

Figure 6.10 Correlation of the calculated ${ }^{29} \mathrm{Si}$ shieldings with the experimental ${ }^{29} \mathrm{Si}$ chemical shifts of sample II with the $16 \mathrm{Si}$ atoms (a) and leaving out the $\mathrm{Si}$ atom bonded to the fluoride (b). c) Optimized structure with the fluoride anion sitting on position 16A (T4 site). Only one OSDA and the nearest fluoride are represented with balls. Si, O, P, C, H, F are depicted in orange, red, yellow, gray, light gray, blue respectively. 


\subsection{Conclusions}

The study of the structure of the as-made RTH with DFT methods based on NMR and XDR experimental data allows us to come to the following conclusions:

The as-made RTH zeolite has $\mathrm{P}-1$ space group symmetry with the following chemical composition $\mathrm{Si}_{16} \mathrm{O}_{32} \mathrm{P}_{1} \mathrm{C}_{10} \mathrm{H}_{24} \mathrm{~F}$. The $\mathrm{PBE}$ functional systematically underestimates the value of the ${ }^{19} \mathrm{~F}$ chemical shift and more accurate predictions for F-containing zeolites are obtained with the modified Becke-Johnson exchange potential (TB-mBJ).

In the sample obtained after three days of synthesis the ${ }^{19} \mathrm{~F}$ signal at $-71.8 \mathrm{ppm}$ can be assigned to the fluoride anion located in a T4 site with a Si-F bond length of $\sim 1.77$ $\AA$. Furthermore, the ${ }^{19} \mathrm{~F}$ signal at $-67.2 \mathrm{ppm}$ observed in the sample obtained at longer synthesis times can be assigned to the fluoride anion located in a T2 site with a Si-F bond length of $\sim 1.79 \AA$. 


\subsection{References}

(1) Simancas, J.; Simancas, R.; Bereciartua, P. J.; Jorda, J. L.; Rey, F.; Corma, A.; Nicolopoulos, S.; Pratim Das, P.; Gemmi, M.; Mugnaioli, E. Ultrafast Electron Diffraction Tomography for Structure Determination of the New Zeolite ITQ-58. J. Am. Chem. Soc. 2016, 138 (32), 10116-10119.

(2) Rey, F.; Simancas, J. Beyond Nitrogen OSDAs. In Insights into the Chemistry of Organic Structure-Directing Agents in the Synthesis of Zeolitic Materials; Gómez-Hortigüela, L., Ed.; Structure and Bonding; Springer International Publishing: Cham, 2018; pp 103-138.

(3) Yun, Y.; Hernández, M.; Wan, W.; Zou, X.; L. Jordá, J.; Cantín, A.; Rey, F.; Corma, A. The First Zeolite with a Tri-Directional Extra-Large 14-Ring Pore System Derived Using a Phosphonium-Based Organic Molecule. Chem. Commun. 2015, 51 (36), 7602-7605.

(4) Martinez-Ortigosa, J.; Simancas, J.; Vidal-Moya, J. A.; Gaveau, P.; Rey, F.; Alonso, B.; Blasco, T. Host-Guest and Guest-Guest Interactions of P- and N-Containing Structure Directing Agents Entrapped inside MFI-Type Zeolite by Multinuclear NMR Spectroscopy. J. Phys. Chem. C 2019, 123 (36), 22324-22334.

(5) Chézeau, J.-M.; Delmotte, L.; Guth, J.-L.; Soulard, M. High-Resolution Solid-State 29Si and 13C n.m.r. on Highly Siliceous MFI-Type Zeolites Synthesized in Nonalkaline Fluoride Medium. Zeolites 1989, 9 (1), 78-80.

(6) Chézeau, J. M.; Delmotte, L.; Guth, J. L.; Gabelica, Z. Influence of Synthesis Conditions and Postsynthesis Treatments on the Nature and Quantity of Structural Defects in Highly Siliceous MFI Zeolites: A High-Resolution Solid-State 29Si n.m.r. Study. Zeolites 1991, 11 (6), 598-606.

(7) Davis, M. E. Zeolites from a Materials Chemistry Perspective. Chem. Mater. 2014, 26 (1), 239-245.

(8) Kuperman, A.; Nadimi, S.; Oliver, S.; Ozin, G. A.; Garcés, J. M.; Olken, M. M. Non-Aqueous Synthesis of Giant Crystals of Zeolites and Molecular.

(9) Villaescusa, L. A.; Barrett, P. A.; Camblor, M. A. ITQ-7: A New Pure Silica Polymorph with a Three-Dimensional System of Large Pore Channels. Angew. Chem. Int. Ed. 1999, 38 (13-14), 1997-2000.

(10) Camblor, M. A.; Corma, A.; Lightfoot, P.; Villaescusa, L. A.; Wright, P. A. Synthesis and Structure of ITQ-3, the First Pure Silica Polymorph with a Two-Dimensional System of Straight Eight-Ring Channels. Angew. Chem. Int. Ed. Engl. 1997, 36 (23), 2659-2661.

(11) Díaz-Cabañas, M.-J.; Barrett, P. A. Synthesis and Structure of Pure SiO 2 Chabazite: The SiO 2 Polymorph with the Lowest Framework Density. Chem. Commun. 1998, 0 (17), 1881-1882. 
(12) Barrett, P. A.; Camblor, M. A.; Corma, A.; Jones, R. H.; Villaescusa, L. A. Synthesis and Structure of As-Prepared ITQ-4, A Large Pore Pure Silica Zeolite: The Role and Location of Fluoride Anions and Organic Cations. $J$. Phys. Chem. B 1998, 102 (21), 4147-4155. https://doi.org/10.1021/jp980735e.

(13) Lemishko, T.; Simancas, J.; Hernández-Rodríguez, M.; Jiménez-Ruiz, M.; Sastre, G.; Rey, F. An INS Study of Entrapped Organic Cations within the Micropores of Zeolite RTH. Phys. Chem. Chem. Phys. 2016, 18 (26), 1724417252. https://doi.org/10.1039/C6CP00971A.

(14) Camblor, M. A.; Villaescusa, L. A.; Díaz-Cabañas, M. J. Synthesis of AllSilica and High-Silica Molecular Sieves in Fluoride Media. Top. Catal. 1999, 9 (1), 59-76. https://doi.org/10.1023/A:1019154304344.

(15) Villaescusa, L. A.; Wheatley, P. S.; Bull, I.; Lightfoot, P.; Morris, R. E. The Location and Ordering of Fluoride Ions in Pure Silica Zeolites with Framework Types IFR and STF; Implications for the Mechanism of Zeolite Synthesis in Fluoride Media. J. Am. Chem. Soc. 2001, 123 (36), 8797-8805. https://doi.org/10.1021/ja016113f.

(16) Bull, I.; Villaescusa, L. A.; Teat, S. J.; Camblor, M. A.; Wright, P. A.; Lightfoot, P.; Morris, R. E. Imposition of Polarity on a Centrosymmetric Zeolite Host: The Effect of Fluoride Ions on Template Ordering in Zeolite IFR. J. Am. Chem. Soc. 2000, 122 (29), 7128-7129. https://doi.org/10.1021/ja000885e.

(17) Koller, H.; Wölker, A.; Eckert, H.; Panz, C.; Behrens, P. Five-Coordinate Silicon in Zeolites: Probing SiO4/2F- Sites in Nonasil and ZSM-5 with 29Si Solid-State NMR Spectroscopy. Angew. Chem. Int. Ed. Engl. 1997, 36 (24), 2823-2825. https://doi.org/10.1002/anie.199728231.

(18) Koller, H.; Wölker, A.; Villaescusa, L. A.; Díaz-Cabañas, M. J.; Valencia, S.; Camblor, M. A. Five-Coordinate Silicon in High-Silica Zeolites. J. Am. Chem. Soc. 1999, 121 (14), 3368-3376. https://doi.org/10.1021/ja9840549.

(19) Fyfe, C. A.; Brouwer, D. H.; Lewis, A. R.; Chézeau, J.-M. Location of the Fluoride Ion in Tetrapropylammonium Fluoride Silicalite-1 Determined by ${ }^{1} \mathrm{H} /{ }^{19} \mathrm{~F} /{ }^{29} \mathrm{Si}$ Triple Resonance CP, REDOR, and TEDOR NMR Experiments. J. Am. Chem. Soc. 2001, 123 (28), 6882-6891. https://doi.org/10.1021/ja010532v.

(20) P. Attfield, M.; J. Weigel, S.; Taulelle, F.; K. Cheetham, A. Synthesis and Characterisation of a Linear [Cu(Pyr)2]2+ Complex in Siliceous Ferrierite. $J$. Mater. Chem. 2000, 10 (9), 2109-2113. https://doi.org/10.1039/B003005H.

(21) Goor, G. van de; Freyhardt, C. C.; Behrens, P. The Cobalticinium Cation $\left[\mathrm{CoIII}\left(\eta_{5}-\mathrm{C}_{5} \mathrm{H}_{5}\right)_{2}\right]^{+}$: A Metal-Organic Complex as a Novel Template for the 
Synthesis of Clathrasils. Z. Für Anorg. Allg. Chem. 1995, 621 (2), 311-322. https://doi.org/10.1002/zaac.19956210218.

(22) Camblor, M. A.; Díaz-Cabañas, M.-J.; Perez-Pariente, J.; Teat, S. J.; Clegg, W.; Shannon, I. J.; Lightfoot, P.; Wright, P. A.; Morris, R. E. SSZ-23: An Odd Zeolite with Pore Openings of Seven and Nine Tetrahedral Atoms. Angew. Chem. Int. Ed. 1998, 37 (15), 2122-2126. https://doi.org/10.1002/(SICI)1521-3773(19980817)37:15<2122::AIDANIE2122>3.0.CO;2-6.

(23) Andrew, E. R.; Eades, R. G. Possibilities for High-Resolution Nuclear Magnetic Resonance Spectra of Crystals. Discuss. Faraday Soc. 1962, 34, 38. https://doi.org/10.1039/df9623400038.

(24) Lowe, I. J. Free Induction Decays of Rotating Solids. Phys. Rev. Lett. 1959, 2 (7), 285-287. https://doi.org/10.1103/PhysRevLett.2.285.

(25) Fyfe, C. A.; Brouwer, D. H.; Lewis, A. R.; Villaescusa, L. A.; Morris, R. E. Combined Solid State NMR and X-Ray Diffraction Investigation of the Local Structure of the Five-Coordinate Silicon in Fluoride-Containing AsSynthesized STF Zeolite. J. Am. Chem. Soc. 2002, 124 (26), 7770-7778. https://doi.org/10.1021/ja012558s.

(26) Martineau, C.; Legein, C.; Buzaré, J.-Y.; Fayon, F. On the Assignment of 19F MAS NMR Spectra of Fluoroaluminates Using Through-Space Spectral Edition of 19F-27Al and 19F-19F Connectivities. Phys. Chem. Chem. Phys. 2009, 11 (6), 950-957. https://doi.org/10.1039/B812091A.

(27) Pickard, C. J.; Mauri, F. All-Electron Magnetic Response with Pseudopotentials: NMR Chemical Shifts. Phys. Rev. B 2001, 63 (24), 245101. https://doi.org/10.1103/PhysRevB.63.245101.

(28) Charpentier, T. The PAW/GIPAW Approach for Computing NMR Parameters: A New Dimension Added to NMR Study of Solids. Solid State Nucl. Magn. Reson. 2011, 40 (1), 1-20. https://doi.org/10.1016/j.ssnmr.2011.04.006.

(29) Dawson, D. M.; Moran, R. F.; Ashbrook, S. E. An NMR Crystallographic Investigation of the Relationships between the Crystal Structure and 29Si Isotropic Chemical Shift in Silica Zeolites. J. Phys. Chem. C 2017, 121 (28), 15198-15210. https://doi.org/10.1021/acs.jpcc.7b03730.

(30) Bonhomme, C.; Gervais, C.; Babonneau, F.; Coelho, C.; Pourpoint, F.; Azaïs, T.; Ashbrook, S. E.; Griffin, J. M.; Yates, J. R.; Mauri, F.; Pickard, C. J. FirstPrinciples Calculation of NMR Parameters Using the Gauge Including Projector Augmented Wave Method: A Chemist's Point of View. Chem. Rev. 2012, 112 (11), 5733-5779. https://doi.org/10.1021/cr300108a.

(31) Gervais, C.; Profeta, M.; Babonneau, F.; Pickard, C. J.; Mauri, F. Ab Initio Calculations of NMR Parameters of Highly Coordinated Oxygen Sites in 
Aluminosilicates. J. Phys. Chem. B 2004, 108 (35), 13249-13253. https://doi.org/10.1021/jp048462c.

(32) Brouwer, D. H. A Structure Refinement Strategy for NMR Crystallography: An Improved Crystal Structure of Silica-ZSM-12 Zeolite from 29Si Chemical Shift Tensors. J. Magn. Reson. 2008, 194 (1), 136-146. https://doi.org/10.1016/j.jmr.2008.06.020.

(33) Brouwer, D. H. NMR Crystallography of Zeolites: Refinement of an NMRSolved Crystal Structure Using Ab Initio Calculations of 29Si Chemical Shift Tensors. J. Am. Chem. Soc. 2008, 130 (20), 6306-6307. https://doi.org/10.1021/ja800227f.

(34) Brouwer, D. H.; Huizen, J. V. NMR Crystallography of Zeolites: How Far Can We Go without Diffraction Data? Magn. Reson. Chem. 2019, 57 (5), 167-175. https://doi.org/10.1002/mrc.4748.

(35) Brouwer, D. H.; Moudrakovski, I. L.; Darton, R. J.; Morris, R. E. Comparing Quantum-Chemical Calculation Methods for Structural Investigation of Zeolite Crystal Structures by Solid-State NMR Spectroscopy. Magn. Reson. Chem. 2010, 48 (S1), S113-S121. https://doi.org/10.1002/mrc.2642.

(36) Brouwer, D. H.; Darton, R. J.; Morris, R. E.; Levitt, M. H. A Solid-State NMR Method for Solution of Zeolite Crystal Structures. J. Am. Chem. Soc. 2005, 127 (29), 10365-10370. https://doi.org/10.1021/ja052306h.

(37) Cadars, S.; H. Brouwer, D.; F. Chmelka, B. Probing Local Structures of Siliceous Zeolite Frameworks by Solid-State NMR and First-Principles Calculations of $29 \mathrm{Si}-\mathrm{O}-29$ Si Scalar Couplings. Phys. Chem. Chem. Phys. 2009, 11 (11), 1825-1837. https://doi.org/10.1039/B815361B.

(38) Dib, E.; Mineva, T.; Veron, E.; Sarou-Kanian, V.; Fayon, F.; Alonso, B. ZSM-5 Zeolite: Complete Al Bond Connectivity and Implications on Structure Formation from Solid-State NMR and Quantum Chemistry Calculations. J. Phys. Chem. Lett. 2018, 9 (1), 19-24. https://doi.org/10.1021/acs.jpclett.7b03050.

(39) Brouwer, D. H.; Brouwer, C. C.; Mesa, S.; Semelhago, C. A.; Steckley, E. E.; Sun, M. P. Y.; Mikolajewski, J. G.; Baerlocher, C. Solid-State 29Si NMR Spectra of Pure Silica Zeolites for the International Zeolite Association Database of Zeolite Structures. Microporous Mesoporous Mater. 2020, 297, 110000. https://doi.org/10.1016/j.micromeso.2020.110000.

(40) Lu, P.; Gómez-Hortigüela, L.; Gao, Z.; Camblor, M. A. Synthesis of a Germanosilicate Zeolite HPM-12 Using a Short Imidazolium-Based Dication: Structure-Direction by Charge-to-Charge Distance Matching. Dalton Trans. 2019, 48 (48), $17752-17762$. https://doi.org/10.1039/C9DT04089G. 
(41) Lu, P.; Gómez-Hortigüela, L.; Xu, L.; Camblor, M. A. Synthesis of STW Zeolites Using Imidazolium-Based Dications of Varying Length. J. Mater. Chem. A 2018, 6 (4), 1485-1495. https://doi.org/10.1039/C7TA10002G.

(42) Bernardo-Maestro, B.; López-Arbeloa, F.; Pérez-Pariente, J.; GómezHortigüela, L. Comparison of the Structure-Directing Effect of Ephedrine and Pseudoephedrine during Crystallization of Nanoporous Aluminophosphates. Microporous Mesoporous Mater. 2017, 254, 211-224. https://doi.org/10.1016/j.micromeso.2017.04.008.

(43) Insights into the Chemistry of Organic Structure-Directing Agents in the Synthesis of Zeolitic Materials; Gómez-Hortigüela, L., Ed.; Structure and Bonding; Springer International Publishing: Cham, 2018; Vol. 175. https://doi.org/10.1007/978-3-319-74289-2.

(44) Rojas, A.; Gómez-Hortigüela, L.; Camblor, M. A. Zeolite Structure Direction by Simple Bis(Methylimidazolium) Cations: The Effect of the Spacer Length on Structure Direction and of the Imidazolium Ring Orientation on the 19F NMR Resonances. J. Am. Chem. Soc. 2012, 134 (8), 3845-3856. https://doi.org/10.1021/ja210703y.

(45) Kresse, G.; Furthmüller, J. Efficient Iterative Schemes for Ab Initio TotalEnergy Calculations Using a Plane-Wave Basis Set. Phys. Rev. B 1996, 54 (16), 11169-11186. https://doi.org/10.1103/PhysRevB.54.11169.

(46) Perdew, J. P.; Wang, Y. Accurate and Simple Analytic Representation of the Electron-Gas Correlation Energy. Phys. Rev. B 1992, 45 (23), 13244-13249. https://doi.org/10.1103/PhysRevB.45.13244.

(47) Perdew, J. P.; Chevary, J. A.; Vosko, S. H.; Jackson, K. A.; Pederson, M. R.; Singh, D. J.; Fiolhais, C. Atoms, Molecules, Solids, and Surfaces: Applications of the Generalized Gradient Approximation for Exchange and Correlation. Phys. Rev. B 1992, 46 (11), 6671-6687. https://doi.org/10.1103/PhysRevB.46.6671.

(48) Perdew, J. P.; Kurth, S.; Seidl, M. Exploring the Adiabatic Connection between Weak- and Strong-Interaction Limits in Density Functional Theory. Int. J. Mod. Phys. B 2001, 15 (10n11), 1672-1683. https://doi.org/10.1142/S0217979201006197.

(49) Perdew, J. P.; Burke, K.; Ernzerhof, M. Generalized Gradient Approximation Made Simple. Phys. Rev. Lett. 1996, 77 (18), 3865-3868. https://doi.org/10.1103/PhysRevLett.77.3865.

(50) Blöchl, P. E. Projector Augmented-Wave Method. Phys. Rev. B 1994, 50 (24), 17953-17979. https://doi.org/10.1103/PhysRevB.50.17953.

(51) Grimme, S. Semiempirical GGA-Type Density Functional Constructed with a Long-Range Dispersion Correction. J. Comput. Chem. 2006, 27 (15), 17871799. https://doi.org/10.1002/jcc.20495. 
(52) Grimme, S.; Antony, J.; Ehrlich, S.; Krieg, H. A Consistent and Accurate Ab Initio Parametrization of Density Functional Dispersion Correction (DFT-D) for the 94 Elements H-Pu. J. Chem. Phys. 2010, 132 (15), 154104. https://doi.org/10.1063/1.3382344.

(53) Goerigk, L.; Grimme, S. A Thorough Benchmark of Density Functional Methods for General Main Group Thermochemistry, Kinetics, and Noncovalent Interactions. Phys. Chem. Chem. Phys. 2011, 13 (14), 66706688. https://doi.org/10.1039/C0CP02984J.

(54) Grimme, S.; Ehrlich, S.; Goerigk, L. Effect of the Damping Function in Dispersion Corrected Density Functional Theory. J. Comput. Chem. 2011, 32 (7), 1456-1465. https://doi.org/10.1002/jcc.21759.

(55) Birch, F. Finite Elastic Strain of Cubic Crystals. Phys. Rev. 1947, 71 (11), 809-824. https://doi.org/10.1103/PhysRev.71.809.

(56) Jackson, A. J.; Skelton, J. M.; Hendon, C. H.; Butler, K. T.; Walsh, A. Crystal Structure Optimisation Using an Auxiliary Equation of State. J. Chem. Phys. 2015, 143 (18), 184101. https://doi.org/10.1063/1.4934716.

(57) Vinet, P.; Rose, J. H.; Ferrante, J.; Smith, J. R. Universal Features of the Equation of State of Solids. J. Phys. Condens. Matter 1989, 1 (11), 19411963. https://doi.org/10.1088/0953-8984/1/11/002.

(58) Becke, A. D.; Johnson, E. R. A Simple Effective Potential for Exchange. J. Chem. Phys. 2006, 124 (22), 221101. https://doi.org/10.1063/1.2213970.

(59) Tran, F.; Blaha, P. Accurate Band Gaps of Semiconductors and Insulators with a Semilocal Exchange-Correlation Potential. Phys. Rev. Lett. 2009, 102 (22), 226401. https://doi.org/10.1103/PhysRevLett.102.226401.

(60) Sadoc, A.; Biswal, M.; Body, M.; Legein, C.; Boucher, F.; Massiot, D.; Fayon, F. NMR Parameters in Column 13 Metal Fluoride Compounds (AlF3, GaF3, InF3 and TlF) from First Principle Calculations. Solid State Nucl. Magn. Reson. 2014, 59-60, 1-7. https://doi.org/10.1016/j.ssnmr.2014.01.001.

(61) Laskowski, R.; Blaha, P.; Tran, F. Assessment of DFT Functionals with NMR Chemical Shifts. Phys. Rev. B 2013, $87 \quad$ (19). https://doi.org/10.1103/PhysRevB.87.195130.

(62) Zheng, A.; Liu, S.-B.; Deng, F. 19F Chemical Shift of Crystalline Metal Fluorides: Theoretical Predictions Based on Periodic Structure Models. $J$. Phys. Chem. C 2009, 113 (33), 15018-15023. https://doi.org/10.1021/jp904454t.

(63) Jana, S.; Myneni, H.; Samal, P. Assessing The Band Gap Problem By Improving Upon The Semilocal Exchange Hole Potential. ArXiv170701071 Cond-Mat 2017. 
(64) Laskowski, R.; Blaha, P. Origin of NMR Shielding in Fluorides. Phys. Rev. B 2012, 85 (24). https://doi.org/10.1103/PhysRevB.85.245117.

(65) Sadoc, A.; Body, M.; Legein, C.; Biswal, M.; Fayon, F.; Rocquefelte, X.; Boucher, F. NMR Parameters in Alkali, Alkaline Earth and Rare Earth Fluorides from First Principle Calculations. Phys. Chem. Chem. Phys. 2011, 13 (41), 18539-18550. https://doi.org/10.1039/C1CP21253B.

(66) Griffin, J. M.; Yates, J. R.; Berry, A. J.; Wimperis, S.; Ashbrook, S. E. HighResolution 19F MAS NMR Spectroscopy: Structural Disorder and Unusual J Couplings in a Fluorinated Hydroxy-Silicate. J. Am. Chem. Soc. 2010, 132 (44), 15651-15660. https://doi.org/10.1021/ja105347q.

(67) Jr, W. R. D. Guide to Fluorine NMR for Organic Chemists; John Wiley \& Sons, 2016.

(68) Baerlocher, C.; McCusker, L. B. Database of Zeolite Structures http://www.iza-structure.org/databases/ (accessed Jun 21, 2020). 



\section{Conclusions}

In this work, we have successfully applied computational chemistry methods to the study of three heterogeneous catalytic systems. Specific conclusions are given at the end of the corresponding chapters, some comments, however, are dedicated here to more general conclusions.

In chapter 3, we studied the chemoselective hydrogenation of nitroarenes on the (111) surface of noble (Pd) and non-noble (Ni, Co and $\mathrm{Cu}$ ) metals. Noble and nonnoble metals exhibit opposite trends for some steps of the mechanism of reduction of the nitro group to form an amino group. $\mathrm{N}-\mathrm{O}$ bond dissociations are the most demanding steps for noble metals, but the most favorable steps for non-noble metals. Conversely, $\mathrm{H}$-transfers to the either $\mathrm{O}$ or $\mathrm{N}$ atoms of the reactant molecule are relatively favorable steps on noble metals, but energetically demanding on non-noble metals. As a result of these trends in bond forming and bond breaking steps, the reaction mechanism on noble and non-noble metals differs, and on $\mathrm{Ni}(111)$ and $\mathrm{Co}(111)$ it proceeds through the direct formation of a nitrosobenzene intermediate: $\mathrm{Ph}-\mathrm{NO}_{2} \rightarrow \mathrm{Ph}-\mathrm{NO} \rightarrow \mathrm{Ph}-\mathrm{N} \rightarrow \mathrm{Ph}-\mathrm{NH} \rightarrow \mathrm{Ph}-\mathrm{NH}_{2}$., The behavior of $\mathrm{Cu}(111)$ surface lies in between noble and non-noble metals, with relatively low activation energies for the two types of elementary steps. The $\mathrm{Cu}(111)$ surface has the additional advantage of interacting with 3-nitrostyrene only through the nitro group, so that competitive hydrogenation of the $\mathrm{C}=\mathrm{C}$ bond is avoided. As a result of these findings, we have been able to propose a presumably more efficient bimetallic catalyst based on $\mathrm{Cu}$, promoted with an $\mathrm{H}_{2}$-activating metal such as $\mathrm{Ni}$.

The second reaction studied in this work, the $\mathrm{NH}_{3}$-SCR-NOx, is more challenging than the aforementioned one due to the more complicated structure of the catalyst and to the highly dynamic nature of the active sites. However, several important insights have been drawn. To the best of our knowledge, this is the first time that free energy methods of molecular dynamics have been applied systematically to study the interaction of the active sites with all the reactant molecules, at different temperatures. We have identified the nature of the active sites under realistic conditions and found processes that might be relevant during the SCR reaction. Our calculations show that in the presence of $\mathrm{NH}_{3}$, the $\mathrm{Cu}^{+}$and probably $\mathrm{Cu}^{2+}$ cations 
(the latter under excess of $\mathrm{NH}_{3}$ ) will not be attached to the $6 r$ but forming stable mobile amino complexes. Our results also show that the detachment of the $\mathrm{Cu}^{+}$and $\mathrm{Cu}^{2+}$ cations from the $6 r$ is related to the absence of bands in the $800-1000 \mathrm{~cm}^{-1}$ region of the IR spectrum. Thus, we propose that the analysis of this IR region during the SCR reaction will provide additional relevant insights on the reaction mechanism and on the dynamic nature of the catalytically active sites.

The last topic is the simulation of the ${ }^{19} \mathrm{~F}$ chemical shift of the as-synthesized RTH framework in fluoride medium. The analysis of the ${ }^{19} \mathrm{~F} \delta_{\text {iso }}$ allowed us to detect the symmetry of the synthesized material, which was also confirmed by XRD. In a second stage, we have studied the localization of the $\mathrm{F}^{-}$anion in the different $\mathrm{T}$ sitting positions. Based on the relative stability and the prediction of the ${ }^{19} \mathrm{~F}$ chemical shift, we have been able to determine the location of the fluoride anion in the final material.

In general, we have gained a better understanding of the studied catalytic systems, through the application of computational chemistry methods. This work lays the foundation on which new experiments can be devised in the search for more efficient catalysts and proves that computational chemistry has an undeniable role in the rational design of heterogeneous catalysts. 


\section{Summary}

In this work, we have studied two heterogeneous catalytic reactions and the localization of the fluoride anion in the as-made RTH framework, synthesized in fluoride medium. Since the various topics of this thesis are not related to each other, a general introduction comprising the whole subject is not provided but rather each topic is introduced separately in its corresponding chapter. The structure of this manuscript is outlined below.

In chapter one, we provide a summary of the basic concepts related to heterogeneous catalysis that will appear in the discussion of the results presented in chapters 3 to 6 . A brief description of the two types of catalytic systems studied, namely, metal surfaces and zeolites is also given. This chapter ends with some general comments on the motivation for this work.

Computational chemistry has been used as the fundamental tool throughout this work. Therefore, the theoretical models and methods thereof are explained in chapter two. In the first section, a general description of the models for metal surfaces and zeolites is given. Section two lays out the fundamentals of Density Functional Theory (DFT) that constitutes the basis of the computational methods applied. In this section, some basic notions of the Hartree-Fock method serve as preamble for DFT after which more practical aspects are touched, such as the concept of Potential Energy Surface (PES) and the techniques for its exploration. Aspects of infrared (IR) properties calculation is also included. This chapter closes with an overview of nuclear magnetic resonance (NMR) and its theoretical treatment in DFT.

The first results, included in chapter 3, correspond to the chemoselective reduction of nitrostyrene on different metal surfaces, i.e, $\mathrm{Ni}(111), \mathrm{Co}(111), \mathrm{Cu}(111)$ and $\operatorname{Pd}(111)$. Until very recently, the reduction of the nitro group was explained on the basis of the general mechanism proposed by Haber in 1898 where the reaction can follow two routes, the direct and condensation route. We have explored the relevant elementary steps of both routes and found that because of the oxophilic nature of $\mathrm{Ni}$ and $\mathrm{Co}$, the steps involving the dissociation of $\mathrm{N}-\mathrm{O}$ bonds and formation of metal-O 
bonds are significantly favored compared with the other steps on both metal surfaces. In addition, the most demanding steps in terms of energy involve the formation of $\mathrm{N}-\mathrm{H}$ bonds. These findings are in contrast to those of noble metals such as $\mathrm{Pt}$ and $\mathrm{Pd}$, where the opposite behavior is observed. The behavior of $\mathrm{Cu}(111)$ lies in between the aforementioned cases, and also no chemical bonds between the carbon atoms of the aromatic ring of nitrostyrene and the $\mathrm{Cu}(111)$ surface is formed. For this reason, it might be an ideal candidate to achieve nearly $100 \%$ selectivity. However, the $\mathrm{Cu}(111)$ surface does not seem to activate the $\mathrm{H}_{2}$ molecule. In this regard, we propose a bimetallic $\mathrm{Cu}$-based catalyst whose surface is doped with atoms of a $\mathrm{H}_{2}$-activating metal, such as Ni or Pd.

On another matter, we have also investigated the selective catalytic reduction of nitrogen oxides (SCR-NOx) and the main results are presented in the following two chapters, 4 and 5. By using static DFT methods, we found pathways for the oxidation of $\mathrm{NO}$ to $\mathrm{NO}_{2}$, nitrites and nitrates with relatively low activation energies. We also found, in agreement with experimental reports, that the reduction of $\mathrm{Cu}^{2+}$ to $\mathrm{Cu}^{+}$ requires the simultaneous participation of $\mathrm{NO}$ and $\mathrm{NH}_{3}$. Later, molecular dynamics simulations allowed us to assess the influence of $\mathrm{NH}_{3}$. The strong interaction of $\mathrm{NH}_{3}$ with the $\mathrm{Cu}^{+}$cation is evidenced by its ability to detach $\mathrm{Cu}^{+}$from the zeolite framework and form the mobile linear complex $\left[\mathrm{Cu}\left(\mathrm{NH}_{3}\right)_{2}\right]^{+} . \mathrm{Cu}^{+}$is no longer coordinated to the zeolite framework in the presence of two $\mathrm{NH}_{3}$ molecules. This observation and the fact that the T-O-T vibrations of the framework produce bands in the $800-1000 \mathrm{~cm}^{-1}$ region of the IR spectrum when perturbed by the coordination of $\mathrm{Cu}^{+}$and $\mathrm{Cu}^{2+}$ cations, indicate that it is possible to track the location of these cations experimentally. The analysis of the vibrational fingerprint of several models with $\mathrm{Cu}^{+}$and $\mathrm{Cu}^{2+}$ attached to the 6-membered ring or solvated by $\mathrm{NH}_{3}$ unambiguously indicate that bands in the $800-1000 \mathrm{~cm}^{-1}$ regions should be observed when both copper cations are bonded to the framework oxygens.

Finally, we have also studied NMR properties of the as-made pure silica RTH framework, aiming at locating the compensating fluoride anion. The calculation of the ${ }^{19} \mathrm{~F}$ chemical shift in different $\mathrm{T}$ sites and comparison with the experimental NMR spectra shows that the as-made RTH belongs to the P-1 space group with $16 \mathrm{Si}, 32$ $\mathrm{O}$ atoms, one fluoride anion and one OSDA cation. These results have been confirmed experimentally by XRD. In addition, we have assigned the experimental 
signal of ${ }^{19} \mathrm{~F}$ at $-67.2 \mathrm{ppm}$ to the fluoride anion in a $\mathrm{T} 2$ site, which in turn is the most stable location found, and the signal of $-71.8 \mathrm{ppm}$ to a fluoride anion sitting in a T4 site. 



\section{Resumen}

En este trabajo estudiamos dos reacciones catalíticas relevantes para la industria y la localización del anión fluoruro en la zeolita RTH, sintetizada en medio fluoruro. Debido a que los tres temas de esta tesis no están directamente relacionados entre sí, cada tema es introducido de manera independiente en su capítulo correspondiente. A continuación se explica la estructura de esta tesis.

El capítulo 1 sirve como introducción general a los conceptos básicos de catálisis heterogénea que aparecen en la discusión de los resultados. También se ha incluido una descripción de los dos tipos de catalizadores estudiados, a saber, superficies de metales y zeolitas. Este capítulo termina con algunos comentarios acerca de la motivación de este trabajo.

La química computacional ha sido la herramienta fundamental usada en la presente tesis. La primera parte del capítulo 2 describe brevemente los modelos usados para las superficies de metales y para las zeolitas. La segunda sección presenta los fundamentos de la teoría del funcional de la densidad (DFT, por sus siglas en inglés), la cual constituye la base de los métodos aplicados. Además, se incluyen algunos aspectos del cálculo de frecuencias infrarrojas (IR) y algunos comentarios sobre la teoría de resonancia magnética nuclear (RMN) y su descripción con DFT en materiales cristalinos.

El capítulo 3 es el primer capítulo de resultados, donde se estudia la reducción quimioselectiva del nitroestireno en las superficies $\mathrm{Ni}(111), \mathrm{Co}(111), \mathrm{Cu}(111)$ y Pd(111). El mecanismo generalmente aceptado de esta reacción está basado en el esquema propuesto por Haber en 1898, en el que la reacción puede transcurrir por dos rutas, la directa y la de condensación. En este capítulo exploramos ambas rutas, y observamos que la ruptura de los enlaces $\mathrm{N}-\mathrm{O}$ y la consecuente formación de enlaces metal-O está más favorecida que la formación de enlaces $\mathrm{N}-\mathrm{H}$ en las superficies $\mathrm{Ni}(111)$ y $\mathrm{Co}(111)$, debido al carácter oxofílico de ambos metales. Las etapas más lentas involucran la formación de enlaces N-H. En las superficies de metales nobles como $\mathrm{Pt}(111)$ y $\mathrm{Pd}(111)$ se observa el comportamiento contrario. $\mathrm{La}$ superficie $\mathrm{Cu}(111)$ es un caso intermedio comparado con los metales nobles y no 
nobles. Además, el nitroestireno interactúa con los átomos de $\mathrm{Cu}$ de la superficie solo a través de grupo nitro, con lo cual es un candidato ideal para alcanzar selectividades cerca del 100\%. Sin embargo, la superficie $\mathrm{Cu}(111)$ no es capaz de activar la molécula de $\mathrm{H}_{2}$. En este sentido, proponemos un catalizador bimetálico basado en $\mathrm{Cu}$, dopado con otro metal capaz de activar al $\mathrm{H}_{2}$, tales como el Pd o el Ni.

En los capítulos 4 y 5 se ha estudiado la reducción catalítica selectiva de los óxidos de nitrógeno (SCR, en inglés) con amoníaco. Usando métodos de DFT, hemos encontrado rutas para la oxidación de $\mathrm{NO}$ a $\mathrm{NO}_{2}$, nitritos y nitratos con energías de activación relativamente bajas. También, hemos encontrado que la reducción de $\mathrm{Cu}^{2+}$ $\mathrm{a} \mathrm{Cu}^{+}$requiere la participación simultánea de $\mathrm{NO}$ y $\mathrm{NH}_{3}$. Posteriormente, hemos estudiado la influencia del $\mathrm{NH}_{3}$ en este sistema con métodos de dinámica molecular. $\mathrm{El} \mathrm{NH}_{3}$ interacciona fuertemente con el $\mathrm{Cu}^{+}$de forma que dos moléculas de este gas son suficientes para romper la coordinación del catión $\mathrm{Cu}^{+}$con los oxígenos del anillo $6 r$, y formar el complejo lineal $\left[\mathrm{Cu}\left(\mathrm{NH}_{3}\right)_{2}\right]^{+}$. Además, los cationes $\mathrm{Cu}^{2+}$ pueden ser estabilizados fuera de la red mediante la formación del complejo tetraamincobre(II). Debido a la presencia de los cationes $\mathrm{Cu}^{+}$y $\mathrm{Cu}^{2+}$ coordinados a la red de la zeolita, aparecen bandas en la región entre $800-1000 \mathrm{~cm}^{-1}$ del espectro infrarrojo. El análisis de las frecuencias IR de varios modelos con $\mathrm{Cu}^{+}$y $\mathrm{Cu}^{2+}$ coordinados al anillo $6 r$, o formando complejos con amoniaco indica que cuando los cationes $\mathrm{Cu}^{+}$y $\mathrm{Cu}^{2+}$ están coordinados a los oxígenos del anillo $6 r$ aparecen vibraciones entre 830 y $960 \mathrm{~cm}^{-1}$. Frecuencias en esta zona también se obtienen en los casos en que $\mathrm{NO}, \mathrm{NO}_{2}, \mathrm{O}_{2}$ y combinaciones de dos de ellos están adsorbidos en $\mathrm{Cu}^{+}$y $\mathrm{Cu}^{2+}$. Sin embargo, cuando los cationes $\mathrm{Cu}^{+}$y $\mathrm{Cu}^{2+}$ están fuera del anillo (no hay enlaces entre los cationes de cobre y los oxígenos del anillo $6 r$ ) no se obtienen vibraciones de IR en esta región del espectro. Estos resultados indican que con el seguimiento del espectro IR durante la reacción SCR es posible determinar si los cationes $\mathrm{Cu}^{+}$y $\mathrm{Cu}^{2+}$ están coordinados o no al anillo de $6 r$ en las etapas de oxidación y reducción.

Por último, hemos simulado el desplazamiento químico de ${ }^{19} \mathrm{~F}, \delta_{\text {iso }}$, en la zeolita sintetizada RTH. El análisis del $\delta_{\text {iso }}$ de los distintos modelos utilizados nos ha permitido reconocer la simetría del material sintetizado, el cual pertenece al grupo espacial P1 y la nueva celda unidad ha sido confirmada experimentalmente por 
difracción de rayos X. Finalmente, hemos asignado la señal experimental que aparece en el espectro de ${ }^{19} \mathrm{~F}$ a $-67.2 \mathrm{ppm}$, al F- localizado en un sitio T2, el cual es a su vez la posición más estable. Además, la señal a -71.8 ppm se ha asignado al anión $\mathrm{F}^{-}$localizado en un sitio $\mathrm{T} 4$. 



\section{Resum}

En aquest treball estudiem dues reaccions catalítiques rellevants per a la indústria i la localització de l'anió fluorur en la zeolita RTH, sintetitzada al mig fluorur. Pel fet que els tres temes d'aquesta tesi no estan directament relacionats entre si, cada tema és introduït de manera independent en el seu capítol corresponent. A continuació s'explica l'estructura d'aquesta tesi.

El capítol 1 serveix com a introducció general als conceptes bàsics de catàlisi heterogènia que apareixen en la discussió dels resultats. També s'ha inclòs una descripció dels dos tipus de catalitzadors estudiats, a saber, superfícies de metalls i zeolites. Aquest capítol acaba amb alguns comentaris sobre la motivació d'aquest treball.

La química computacional ha sigut l'eina fonamental usada en la present tesi. La primera part del capítol 2 descriu breument els models usats per a la superfícies de metalls i per a les zeolites. La segona secció presenta els fonaments de la teoria del funcional de la densitat (DFT, per la seua sigles en anglés), la qual constitueix la base dels mètodes aplicats. A més, s'inclouen alguns aspectes del càlcul de freqüències infraroges (IR) i alguns comentaris sobre la teoria de ressonància magnètica nuclear $(\mathrm{RMN})$ i la seua descripció amb DFT en materials cristal·lins.

El capítol 3 és el primer capítol de resultats, on s'estudia la reducció quimioselectiva del nitroestireno en les superfícies $\mathrm{Ni}(111), \mathrm{Co}(111), \mathrm{Cu}(111)$ i $\mathrm{Pd}(111)$. El mecanisme generalment acceptat d'aquesta reacció està basat en l'esquema proposat per Haver-hi en 1898, en el qual la reacció pot transcórrer per dues rutes, la directa i la de condensació. En aquest capítol explorem totes dues rutes, i observem que la ruptura dels enllaços $\mathrm{N}-\mathrm{O}$ i la conseqüent formació d'enllaços metall-O està més afavorida que la formació d'enllaços N-H en les superfícies Ni(111) i Co(111), a causa del caràcter oxofílico de tots dos metalls. Les etapes més lentes involucren la formació d'enllaços N-H. En les superfícies de metalls nobles com Pt(111) i Pd(111) s'observa el comportament contrari. La superfície $\mathrm{Cu}(111)$ és un cas intermedi comparat amb els metalls nobles i no nobles. A més, el nitroestireno interactua amb els àtoms de $\mathrm{Cu}$ de la superfície sol a través de grup nitre, amb la qual cosa és un 
candidat ideal per a aconseguir selectivitats prop del $100 \%$. No obstant això, la superfície $\mathrm{Cu}(111)$ no és capaç d'activar la molècula d' $\mathrm{H}_{2}$. En aquest sentit, proposem un catalitzador bimetàl lic basat en $\mathrm{Cu}$, dopat amb un altre metall capaç d'activar a $\mathrm{l}^{\prime} \mathrm{H}_{2}$, com ara el Pd o el Ni.

En els capítols 4 i 5 hem estudiat la reducció catalítica selectiva dels òxids de nitrogen (SCR, en anglés) amb amoníac. Usant mètodes de DFT, hem trobat rutes per a l'oxidació de $\mathrm{NO}$ a $\mathrm{NO}_{2}$, nitrits i nitrats amb energies d'activació relativament baixes. També, hem trobat que la reducció de $\mathrm{Cu}^{2+} \mathrm{a} \mathrm{Cu}^{+}$requereix la participació simultània de $\mathrm{NO}$ i $\mathrm{NH}_{3}$. Posteriorment, hem estudiat la influència del $\mathrm{NH}_{3}$ en aquest sistema amb mètodes de dinàmica molecular. $\mathrm{El} \mathrm{NH}_{3}$ interacciona fortament amb el $\mathrm{Cu}^{+}$de manera que dues molècules d'aquest gas són suficients per a trencar la coordinació del catió $\mathrm{Cu}^{+}$amb els oxígens de l'anell $6 r$, i formar el complex lineal $\left[\mathrm{Cu}\left(\mathrm{NH}_{3}\right)_{2}\right]^{+}$. A més, els cations $\mathrm{Cu}^{2+}$ poden ser estabilitzats fora de la xarxa mitjançant la formació del complex tetraamincobre(II). A causa de la presència dels cations $\mathrm{Cu}^{+} \mathrm{i} \mathrm{Cu}^{2+}$ coordinats a la xarxa de la zeolita, apareixen bandes a la regió entre 800-1000 $\mathrm{cm}^{-1}$ de l'espectre infraroig. L'anàlisi de les freqüències IR de diversos models amb $\mathrm{Cu}^{+} \mathrm{i} \mathrm{Cu}^{2+}$ coordinats a l'anell $6 r$, o formant complexos amb amoníac indica que quan els cations $\mathrm{Cu}^{+} \mathrm{iCu}^{2+}$ estan coordinats als oxígens de l'anell $6 r$ apareixen vibracions entre 830 i $960 \mathrm{~cm}^{-1}$. Freqüències en aquesta zona també s'obtenen en els casos en què $\mathrm{NO}, \mathrm{NO}_{2}, \mathrm{O}_{2}$ i combinacions de dues d'ells estan adsorbidos en $\mathrm{Cu}^{+} \mathrm{i} \mathrm{Cu}^{2+}$. No obstant això, quan els cations $\mathrm{Cu}^{+} \mathrm{i} \mathrm{Cu}^{2+}$ estan fora de l'anell (no hi ha enllaços entre els cations de coure i els oxígens de l'anell 6r) no s'obtenen vibracions d'IR en aquesta regió de l'espectre. Aquests resultats indiquen que amb el seguiment de l'espectre IR durant la reacció SCR és possible determinar si els cations $\mathrm{Cu}^{+} \mathrm{i} \mathrm{Cu}^{2+}$ estan coordinats o no a l'anell de $6 r$ en les etapes d'oxidació i reducció.

Finalment, hem simulat el desplaçament químic de ${ }^{19} \mathrm{~F}, \delta_{\text {iso }}$, en la zeolita sintetitzada RTH. L'anàlisi del $\delta_{\text {iso }}$ dels diferents models utilitzats ens ha permés reconéixer la simetria del material sintetitzat, el qual pertany al grup espacial P1 i la nova cel·la unitat ha sigut confirmada experimentalment per difracció de raigs X. Finalment, hem assignat el senyal experimental que apareix en l'espectre de ${ }^{19} \mathrm{~F}$ a $-67.2 \mathrm{ppm}$, al $\mathrm{F}^{-}$localitzat en un lloc T2, el qual és al seu torn la posició més estable. A més, el senyal a -71.8 ppm s'ha assignat a l'anió $\mathrm{F}^{-}$localitzat en un lloc T4. 


\section{Appendix A}

\section{Reaction and activation free energies of the chemoselective hydrogenation of nitrobenzene}

Table A.1 Calculated reaction and activation free energies $\left(\Delta \mathrm{G}_{\text {reac }}\right.$ and $\left.\Delta \mathrm{G}_{\text {act }}\right)$ at $393 \mathrm{~K}$ for all elementary steps of the reduction of nitrobenzene on the $\mathrm{Ni}(111)$ surface with parallel $(\mathrm{P})$ and normal $(\mathrm{N})$ geometries. All energies are given in $\mathrm{kcal} / \mathrm{mol}$.

\begin{tabular}{|c|c|c|c|c|c|}
\hline \multirow{2}{*}{ step } & \multirow{2}{*}{ Reaction } & \multicolumn{2}{|c|}{ Parallel } & \multicolumn{2}{|c|}{ Normal } \\
\hline & & $\Delta \mathrm{G}_{\text {reac }}$ & $\Delta \mathrm{G}_{\text {act }}$ & $\Delta \mathrm{G}_{\text {reac }}$ & $\Delta \mathrm{G}_{\text {act }}$ \\
\hline 4 & $\mathrm{Ph}-\mathrm{NO}_{2}+\mathrm{H}^{*} \leftrightarrow \mathrm{Ph}-\mathrm{NOOH}$ & 2.8 & 17.6 & 1.8 & 22.7 \\
\hline 5 & $\mathrm{Ph}-\mathrm{NOOH}^{*}+\mathrm{H}^{*} \leftrightarrow \mathrm{Ph}-\mathrm{N}(\mathrm{OH})_{2}$ & 11.8 & 18.8 & $\mathrm{R}$ & 8.1 \\
\hline 6 & $\mathrm{Ph}-\mathrm{N}(\mathrm{OH})_{2} \leftrightarrow \mathrm{Ph}-\mathrm{NOH}^{*}+\mathrm{OH}^{*}$ & -35.4 & 4.5 & -35.0 & 8.8 \\
\hline 7 & $\mathrm{Ph}-\mathrm{NOOH}^{*} \leftrightarrow \mathrm{Ph}-\mathrm{NOH}+\mathrm{O}^{*}$ & -26.6 & 8.3 & -29.9 & 4.0 \\
\hline 8 & $\mathrm{Ph}-\mathrm{NOH}^{*} \leftrightarrow \mathrm{Ph}-\mathrm{N}+\mathrm{OH}^{*}$ & -35.7 & 3.6 & -47.0 & 0.3 \\
\hline 9 & $\mathrm{Ph}-\mathrm{NOH}+\mathrm{H}^{*} \leftrightarrow \mathrm{Ph}^{-\mathrm{NHOH}^{*}}$ & 2.4 & 22.5 & 6.8 & 27.1 \\
\hline 10 & $\mathrm{Ph}-\mathrm{N}^{*}+\mathrm{H}^{*} \leftrightarrow \mathrm{Ph}-\mathrm{NH}^{*}$ & -2.9 & 21.2 & 14.2 & 24.0 \\
\hline 11 & $\mathrm{Ph}-\mathrm{NH}^{*}+\mathrm{H}^{*} \leftrightarrow \mathrm{Ph}-\mathrm{NH}_{2}{ }^{*}$ & 2.5 & 21.8 & -0.8 & 16.7 \\
\hline 12 & $\mathrm{Ph}-\mathrm{NHOH}^{*} \leftrightarrow \mathrm{Ph}-\mathrm{NH}^{*}+\mathrm{OH}^{*}$ & -41.0 & 9.5 & -39.6 & 7.7 \\
\hline 13 & $\mathrm{Ph}-\mathrm{NO}_{2} \leftrightarrow \mathrm{Ph}-\mathrm{NO}^{*}+\mathrm{O}^{*}$ & -32.6 & 9.2 & -32.8 & 7.6 \\
\hline 14 & $\mathrm{Ph}-\mathrm{NO}^{*}+\mathrm{H}^{*} \leftrightarrow \mathrm{Ph}-\mathrm{NOH}^{*}$ & 8.7 & 15.7 & 4.7 & 20.9 \\
\hline 15 & $\mathrm{Ph}-\mathrm{NO}^{*}+\mathrm{H}^{*} \leftrightarrow \mathrm{Ph}-\mathrm{NHO}^{*}$ & 2.6 & 18.5 & 4.2 & 20.4 \\
\hline 16 & $\mathrm{Ph}-\mathrm{NO}^{*} \leftrightarrow \mathrm{Ph}-\mathrm{N}^{*}+\mathrm{O}^{*}$ & -30.3 & 10.8 & -45.5 & 4.2 \\
\hline 17 & $\mathrm{Ph}-\mathrm{NHO}^{*} \leftrightarrow \mathrm{Ph}-\mathrm{NH}^{*}+\mathrm{O}^{*}$ & -35.8 & 7.1 & -35.6 & 2.0 \\
\hline 18 & $\mathrm{Ph}-\mathrm{NHO}^{*}+\mathrm{H}^{*} \leftrightarrow \mathrm{Ph}^{-\mathrm{NHOH}^{*}}$ & 8.5 & 16.8 & 7.3 & 18.0 \\
\hline
\end{tabular}


Appendix A

Table A.2 Calculated reaction and activation free energies $\left(\Delta \mathrm{G}_{\text {reac }}\right.$ and $\left.\Delta \mathrm{G}_{\text {act }}\right)$ at $393 \mathrm{~K}$ for all elementary steps of the reduction of nitrobenzene on the $\operatorname{Co}(111)$ surface with parallel and normal geometries. All energies are given in $\mathrm{kcal} / \mathrm{mol}$.

\begin{tabular}{|c|c|c|c|c|c|}
\hline \multirow{2}{*}{ step } & \multirow{2}{*}{ Reaction } & \multicolumn{2}{|c|}{ Parallel } & \multicolumn{2}{|c|}{ Normal } \\
\hline & & $\Delta \mathrm{G}_{\text {reac }}$ & $\Delta \mathrm{G}_{\text {act }}$ & $\Delta \mathrm{G}_{\text {reac }}$ & $\Delta \mathrm{G}_{\text {act }}$ \\
\hline 1 & $\mathrm{Ph}-\mathrm{NO}_{2}+\mathrm{H}^{*} \leftrightarrow \mathrm{Ph}-\mathrm{NOOH}$ & 7.6 & 15.37 & 2.8 & 23.1 \\
\hline 2 & $\mathrm{Ph}-\mathrm{NOOH}^{*}+\mathrm{H}^{*} \leftrightarrow \mathrm{Ph}-\mathrm{N}(\mathrm{OH})_{2}{ }^{*}$ & 14.0 & 20.1 & 12.7 & 23.3 \\
\hline 3 & 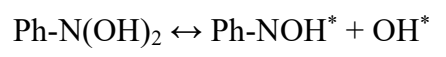 & -45.5 & 3.4 & -36.6 & 6.7 \\
\hline 4 & $\mathrm{Ph}-\mathrm{NOOH}^{*} \leftrightarrow \mathrm{Ph}-\mathrm{NOH}+\mathrm{O}^{*}$ & -37.4 & 6.1 & -29.9 & 3.84 \\
\hline 5 & $\mathrm{Ph}-\mathrm{NOH}^{*} \leftrightarrow \mathrm{Ph}-\mathrm{N}+\mathrm{OH}^{*}$ & 53.2 & 0.1 & -62.8 & 2.1 \\
\hline 6 & $\mathrm{Ph}-\mathrm{NOH}+\mathrm{H}^{*} \leftrightarrow \mathrm{Ph}-\mathrm{NHOH}^{*}$ & 4.6 & 24.0 & -1.2 & 20.3 \\
\hline 7 & $\mathrm{Ph}-\mathrm{N}^{*}+\mathrm{H}^{*} \leftrightarrow \mathrm{Ph}-\mathrm{NH}^{*}$ & 3.6 & 23.6 & 13.4 & 24.1 \\
\hline 8 & $\mathrm{Ph}-\mathrm{NH}^{*}+\mathrm{H}^{*} \leftrightarrow \mathrm{Ph}-\mathrm{NH}_{2}{ }^{*}$ & 7.8 & 24.9 & 4.1 & 25.3 \\
\hline 9 & $\mathrm{Ph}-\mathrm{NHOH}^{*} \leftrightarrow \mathrm{Ph}-\mathrm{NH}^{*}+\mathrm{OH}^{*}$ & -54.2 & 9.6 & -5.6 & 7.1 \\
\hline 10 & $\mathrm{Ph}-\mathrm{NO}_{2}{ }^{*} \leftrightarrow \mathrm{Ph}-\mathrm{NO}^{*}+\mathrm{O}^{*}$ & -45.8 & 4.8 & -43.6 & 5.2 \\
\hline 11 & $\mathrm{Ph}-\mathrm{NO}^{*}+\mathrm{H}^{*} \leftrightarrow \mathrm{Ph}-\mathrm{NOH}^{*}$ & 16.0 & 20.5 & 16.4 & 22.8 \\
\hline 12 & $\mathrm{Ph}-\mathrm{NO}^{*}+\mathrm{H}^{*} \leftrightarrow \mathrm{Ph}-\mathrm{NHO}^{*}$ & 9.1 & 21.2 & 1.9 & 22.1 \\
\hline 13 & $\mathrm{Ph}-\mathrm{NO}^{*} \leftrightarrow \mathrm{Ph}-\mathrm{N}^{*}+\mathrm{O}^{*}$ & -43.3 & 12.9 & -52.4 & 5.6 \\
\hline 14 & 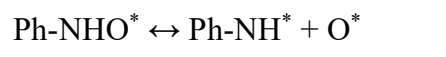 & -48.8 & 5.3 & -40.8 & 4.1 \\
\hline 15 & $\mathrm{Ph}-\mathrm{NHO}^{*}+\mathrm{H}^{*} \leftrightarrow \mathrm{Ph}-\mathrm{NHOH}^{*}$ & 11.4 & 19.6 & 15.7 & 24.9 \\
\hline
\end{tabular}


Table A.3 Calculated reaction and activation free energies $\left(\Delta \mathrm{G}_{\text {reac }}\right.$ and $\left.\Delta \mathrm{G}_{\text {act }}\right)$ at $393 \mathrm{~K}$ for all elementary steps of the reduction of nitrobenzene with vertical geometries on $\mathrm{Cu}(111)$ surface doped with one $\mathrm{Ni}$ atom. All energies are given in $\mathrm{kcal} / \mathrm{mol}$.

\begin{tabular}{clcc}
\hline \multirow{2}{*}{ step } & \multicolumn{1}{c}{ Reaction } & \multicolumn{2}{c}{$\mathrm{Cu}$} \\
\cline { 3 - 4 } & & $\Delta \mathrm{G}_{\text {reac }}$ & $\Delta \mathrm{G}_{\text {act }}$ \\
\hline 7 & $\mathrm{Ph}-\mathrm{N}^{*}+\mathrm{H}^{*} \leftrightarrow \mathrm{Ph}-\mathrm{NH}^{*}$ & -2.7 & 14.9 \\
8 & $\mathrm{Ph} \mathrm{NH}^{*}+\mathrm{H}^{*} \leftrightarrow \mathrm{Ph}-\mathrm{NH}_{2}{ }^{*}$ & -8.5 & 8.5 \\
10 & $\mathrm{Ph}_{-\mathrm{NO}_{2}}{ }^{*} \leftrightarrow \mathrm{Ph}-\mathrm{NO}^{*}+\mathrm{O}^{*}$ & -24.0 & 8.6 \\
13 & ${\mathrm{Ph}-\mathrm{NO}^{*} \leftrightarrow \mathrm{Ph}-\mathrm{N}^{*}+\mathrm{O}^{*}}$ & -28.9 & 11.6 \\
\hline
\end{tabular}





\section{Appendix B \\ Calculated absolute ${ }^{29} \mathrm{Si}$ shieldings of the RTH framework}

B.1 Calculated absolute ${ }^{29} \mathrm{Si}$ isotropic shieldings for the RTH framework with a monoclinic unit cell

The calculated ${ }^{29} \mathrm{Si} \sigma_{\text {iso }}$ for the RTH framework, with a monoclinic unit cell, are summarized in Tables B.1 to B.4. These tables include the absolute ${ }^{29} \mathrm{Si} \sigma_{\text {iso }}$ of the 16 optimized models (AC, AD, etc) for which the ${ }^{19} \mathrm{~F} \sigma_{\text {iso }}$ of the both fluoride anions are equal (Figure 6.4). The location of both fluoride anions in each model is explained in section 6.3.2. The 32 calculated ${ }^{29} \mathrm{Si}$ shieldings are included in 16 entries to show that these 16 models predict $16{ }^{29} \mathrm{Si}$ signals. 
Appendix B

Table B.1 Calculated absolute shieldings $\left({ }^{29} \mathrm{Si} \sigma_{\text {iso }}\right)$ of the $32 \mathrm{Si}$ atoms of the RTH with combinations of two $\mathrm{F}$ atoms in $\mathrm{T} 1$ sites.

\begin{tabular}{ccc|cc|cc|cc}
\hline \multicolumn{2}{c}{ AB } & \multicolumn{2}{c|}{ CD } & \multicolumn{2}{c|}{ EF } & \multicolumn{2}{c}{ GH } \\
\hline 1 & 436.61 & 436.6 & 436.88 & 436.85 & 436.51 & 436.5 & 435.74 & 435.73 \\
2 & 438.78 & 438.75 & 437.6 & 437.59 & 437.96 & 437.95 & 436.46 & 436.45 \\
3 & 439.77 & 439.77 & 439.18 & 439.15 & 440.07 & 440.06 & 438.04 & 438.04 \\
4 & 440.84 & 440.81 & 439.9 & 439.88 & 440.31 & 440.28 & 438.42 & 438.41 \\
5 & 440.99 & 440.93 & 440.27 & 440.23 & 440.41 & 440.41 & 439.78 & 439.78 \\
6 & 441.12 & 441.11 & 440.38 & 440.36 & 440.43 & 440.43 & 440.33 & 440.32 \\
7 & 441.65 & 441.58 & 440.94 & 440.89 & 440.87 & 440.84 & 441.22 & 441.22 \\
8 & 442.28 & 442.26 & 440.97 & 440.95 & 441.25 & 441.24 & 441.65 & 441.65 \\
9 & 442.39 & 442.36 & 441.06 & 441.05 & 441.27 & 441.25 & 441.92 & 441.92 \\
10 & 442.49 & 442.48 & 442.23 & 442.23 & 442.15 & 442.11 & 442.39 & 442.39 \\
11 & 442.55 & 442.5 & 442.35 & 442.29 & 443.07 & 443.06 & 443.3 & 443.3 \\
12 & 442.89 & 442.87 & 442.51 & 442.49 & 443.12 & 443.09 & 443.57 & 443.57 \\
13 & 444.75 & 444.72 & 443.84 & 443.77 & 443.29 & 443.25 & 443.74 & 443.71 \\
14 & 448.31 & 448.28 & 447.11 & 447.04 & 447.35 & 447.33 & 447.02 & 447.01 \\
15 & 449.29 & 449.25 & 447.59 & 447.51 & 447.67 & 447.65 & 447.12 & 447.11 \\
16 & 462.85 & 462.84 & 462.54 & 462.52 & 461.5 & 461.48 & 459.88 & 459.86 \\
\hline & & & & & & & & \\
\hline
\end{tabular}


Appendix B

Table B.2 Calculated absolute shieldings $\left({ }^{29} \mathrm{Si} \sigma_{\text {iso }}\right)$ of the $32 \mathrm{Si}$ atoms of the RTH with combinations of two $\mathrm{F}$ atoms in $\mathrm{T} 2$ sites.

\begin{tabular}{|c|c|c|c|c|c|c|c|c|}
\hline & \multicolumn{2}{|c|}{$\mathbf{A B}$} & \multicolumn{2}{|c|}{ CD } & \multicolumn{2}{|c|}{ EF } & \multicolumn{2}{|c|}{ GH } \\
\hline 1 & 432.91 & 432.91 & 434.47 & 434.45 & 434.13 & 434.09 & 434 & 433.98 \\
\hline 2 & 434.91 & 434.90 & 435.37 & 435.37 & 435.12 & 435.12 & 434.98 & 434.91 \\
\hline 3 & 437.01 & 436.98 & 437.41 & 437.38 & 436.84 & 436.83 & 437.49 & 437.46 \\
\hline 4 & 437.54 & 437.48 & 437.45 & 437.42 & 437.24 & 437.23 & 438.66 & 438.66 \\
\hline 5 & 438.71 & 438.65 & 439.33 & 439.27 & 439.31 & 439.3 & 438.87 & 438.83 \\
\hline 6 & 438.77 & 438.76 & 439.59 & 439.52 & 441.87 & 441.87 & 439 & 438.9 \\
\hline 7 & 440.09 & 440.05 & 439.93 & 439.83 & 442.29 & 442.29 & 439.37 & 439.28 \\
\hline 8 & 441.03 & 441.03 & 440.57 & 440.4 & 442.44 & 442.41 & 440.18 & 440.13 \\
\hline 9 & 441.50 & 441.49 & 441.5 & 441.43 & 442.76 & 442.74 & 440.21 & 440.19 \\
\hline 10 & 442.50 & 442.36 & 441.52 & 441.51 & 443.1 & 443.08 & 440.78 & 440.77 \\
\hline 11 & 443.87 & 443.86 & 443.8 & 443.76 & 443.42 & 443.4 & 444.12 & 444.09 \\
\hline 12 & 444.01 & 444.00 & 444.35 & 444.29 & 443.73 & 443.72 & 444.53 & 444.47 \\
\hline 13 & 444.93 & 444.86 & 444.95 & 444.94 & 444.13 & 444.12 & 445.22 & 445.19 \\
\hline 14 & 445.87 & 445.83 & 446.17 & 446.17 & 445.71 & 445.71 & 445.24 & 445.24 \\
\hline 15 & 446.02 & 446.02 & 446.79 & 446.77 & 445.94 & 445.93 & 446.69 & 446.68 \\
\hline 16 & 466.17 & 466.17 & 465.74 & 465.7 & 466.92 & 466.92 & 465.57 & 465.54 \\
\hline
\end{tabular}


Appendix B

Table B.3 Calculated absolute shieldings $\left({ }^{29} \mathrm{Si} \sigma_{\text {iso }}\right)$ of the $32 \mathrm{Si}$ atoms of the RTH with combinations of two $\mathrm{F}$ atoms in $\mathrm{T} 3$ sites.

\begin{tabular}{|c|c|c|c|c|c|c|c|c|}
\hline & \multicolumn{2}{|c|}{$\mathbf{A B}$} & \multicolumn{2}{|c|}{ CD } & \multicolumn{2}{|c|}{ EF } & \multicolumn{2}{|c|}{ GH } \\
\hline 1 & 432.19 & 432.19 & 434.06 & 434.03 & 429.95 & 429.94 & 434.57 & 434.53 \\
\hline 2 & 433.66 & 433.66 & 434.27 & 434.18 & 434.57 & 434.57 & 436.7 & 436.58 \\
\hline 3 & 437.04 & 436.97 & 434.59 & 434.54 & 437.79 & 437.76 & 436.92 & 436.82 \\
\hline 4 & 437.91 & 437.86 & 436.96 & 436.94 & 438.35 & 438.31 & 437.15 & 437.11 \\
\hline 5 & 437.96 & 437.93 & 438.46 & 438.41 & 438.52 & 438.44 & 438.15 & 438.08 \\
\hline 6 & 438.12 & 438.08 & 438.94 & 438.9 & 439.11 & 439.05 & 439.16 & 439.05 \\
\hline 7 & 438.22 & 438.2 & 439.15 & 439.08 & 439.97 & 439.96 & 440.46 & 440.43 \\
\hline 8 & 438.31 & 438.31 & 439.23 & 439.16 & 440.18 & 440.16 & 440.83 & 440.8 \\
\hline 9 & 439.16 & 439.13 & 439.49 & 439.36 & 441.57 & 441.55 & 440.93 & 440.91 \\
\hline 10 & 439.77 & 439.77 & 440.35 & 440.34 & 441.67 & 441.61 & 441.85 & 441.82 \\
\hline 11 & 442.83 & 442.79 & 442.8 & 442.77 & 441.69 & 441.68 & 442.22 & 442.18 \\
\hline 12 & 443.57 & 443.54 & 443.92 & 443.89 & 442.3 & 442.27 & 444.24 & 444.09 \\
\hline 13 & 445.43 & 445.4 & 446.13 & 446.07 & 444.55 & 444.54 & 445.27 & 445.24 \\
\hline 14 & 446.74 & 446.73 & 446.42 & 446.41 & 446.67 & 446.65 & 445.54 & 445.49 \\
\hline 15 & 448.49 & 448.49 & 447.74 & 447.73 & 448.26 & 448.19 & 447.64 & 447.61 \\
\hline 16 & 464.93 & 464.91 & 469.23 & 469.14 & 466.22 & 466.2 & 469.27 & 469.22 \\
\hline
\end{tabular}


Appendix B

Table B.4 Calculated absolute shieldings $\left({ }^{29} \mathrm{Si} \sigma_{\text {iso }}\right)$ of the $32 \mathrm{Si}$ atoms of the RTH with combinations of two $\mathrm{F}$ atoms in $\mathrm{T} 4$ sites.

\begin{tabular}{ccc|ccccccc}
\hline & \multicolumn{2}{c}{ AB } & \multicolumn{2}{c|}{ CD } & \multicolumn{2}{c|}{ EF } & \multicolumn{2}{c}{ GH } \\
\hline 1 & 430.94 & 430.9 & 433.25 & 433.16 & 432.79 & 432.6 & 433.33 & 433.19 \\
2 & 434.38 & 434.35 & 434.48 & 434.42 & 435.43 & 435.42 & 435.15 & 435.1 \\
3 & 436.97 & 436.97 & 436.36 & 436.31 & 436.30 & 436.06 & 436.88 & 436.83 \\
4 & 438.56 & 438.49 & 437.37 & 437.35 & 437.23 & 437.07 & 438.22 & 438.21 \\
5 & 438.87 & 438.81 & 439.34 & 439.28 & 437.83 & 437.74 & 438.38 & 438.35 \\
6 & 439.28 & 439.26 & 439.73 & 439.71 & 439.04 & 438.93 & 439.66 & 439.63 \\
7 & 439.75 & 439.73 & 440.19 & 440.1 & 439.64 & 439.51 & 439.86 & 439.78 \\
8 & 440.21 & 440.19 & 440.27 & 440.24 & 440.06 & 439.97 & 440.07 & 439.95 \\
9 & 440.39 & 440.35 & 440.81 & 440.7 & 440.32 & 440.28 & 440.8 & 440.79 \\
10 & 441.29 & 441.28 & 440.93 & 440.83 & 440.45 & 440.39 & 440.89 & 440.81 \\
11 & 441.65 & 441.63 & 441.89 & 441.88 & 442.33 & 442.22 & 441.94 & 441.87 \\
12 & 443.69 & 443.67 & 442.78 & 442.77 & 443.75 & 443.71 & 442.16 & 442.1 \\
13 & 444.99 & 444.96 & 443.89 & 443.82 & 443.88 & 443.82 & 443.41 & 443.37 \\
14 & 445.23 & 445.19 & 444.28 & 444.19 & 444.6 & 444.39 & 444.44 & 444.36 \\
15 & 445.64 & 445.6 & 445.8 & 445.78 & 446.66 & 446.55 & 445.35 & 445.23 \\
\hline 16 & 469.41 & 469.4 & 464.29 & 464.27 & 468.79 & 468.54 & 464.19 & 464.13 \\
\hline
\end{tabular}


Appendix B

\section{B.2 Calculated absolute ${ }^{29} \mathrm{Si}$ isotropic shieldings for the RTH framework with a triclinic unit cell}

The experimental isotropic ${ }^{29} \mathrm{Si}$ chemical shifts $\left(\delta_{\text {iso }}\right)$ of the samples I and II, and calculated absolute isotropic ${ }^{29} \mathrm{Si}$ shieldings $\left(\sigma_{\text {iso }}\right)$ of the 24 RTH optimized models are summarized in Tables B.5 to B.7. These models have $16 \mathrm{Si}, 32 \mathrm{O}$ atoms, one fluoride anion and one OSDA cation. The curve fitting of the calculated $\sigma_{\text {iso }}$ vs the experimental $\delta_{\text {iso }}$, of the models that best predict the experimental ${ }^{19} \mathrm{~F} \delta_{\text {iso }}(\mathrm{T} 2, \mathrm{~T} 3$ and T4), are shown in Figure B. 1 to Figure B.3.

Table B.5 Experimental isotropic ${ }^{29} \mathrm{Si}$ chemical shifts $\left(\delta_{\text {iso }}\right)$ of the samples I and II, and calculated absolute isotropic ${ }^{29} \mathrm{Si}$ shieldings $\left(\sigma_{\text {iso }}\right)$ of the RTH optimized models with a triclinic unit cell and the fluoride anion located in T1 and T2 sites.

\begin{tabular}{|c|c|c|c|c|c|c|c|c|c|}
\hline \multicolumn{2}{|c|}{${ }^{29} \mathrm{Si} \delta_{\text {iso }}(\mathrm{ppm})$} & \multicolumn{8}{|c|}{${ }^{29} \mathrm{Si} \sigma_{\text {iso }}(\mathrm{ppm})$} \\
\hline \multirow{2}{*}{ I } & \multirow{2}{*}{ II } & \multicolumn{4}{|c|}{$\mathrm{T} 1$} & \multicolumn{4}{|c|}{$\mathrm{T} 2$} \\
\hline & & 1 & 2 & 3 & 4 & 5 & 6 & 7 & 8 \\
\hline-103.70 & -107.56 & 498.83 & 498.77 & 509.15 & 497.79 & 496.72 & 510.14 & 508.96 & 497.11 \\
\hline-105.80 & -107.84 & 499.77 & 499.95 & 509.7 & 499.29 & 496.74 & 510.16 & 509.12 & 497.31 \\
\hline-106.60 & -107.93 & 499.8 & 500.18 & 509.8 & 499.56 & 498.54 & 510.30 & 510.04 & 498.26 \\
\hline-107.3 & -108.83 & 499.86 & 500.27 & 511.17 & 499.58 & 499.03 & 511.67 & 511.21 & 499.09 \\
\hline-108.00 & -109.15 & 500.41 & 500.44 & 512.6 & 499.7 & 499.1 & 511.77 & 511.38 & 499.45 \\
\hline-108.90 & -109.36 & 500.89 & 500.49 & 512.9 & 500.87 & 499.46 & 513.25 & 512.33 & 500.09 \\
\hline-109.30 & -110.28 & 501.34 & 501.05 & 513.8 & 501.65 & 500.07 & 513.40 & 512.99 & 501.22 \\
\hline-110.65 & -111.50 & 502.03 & 501.23 & 513.86 & 501.69 & 500.73 & 514.21 & 513.08 & 501.23 \\
\hline-110.96 & -111.88 & 502.5 & 502.58 & 514.01 & 502.03 & 501.00 & 514.51 & 513.37 & 501.29 \\
\hline-111.40 & -112.10 & 503 & 502.67 & 514.1 & 503.18 & 501.78 & 514.72 & 513.78 & 501.43 \\
\hline-111.70 & -112.80 & 503.36 & 502.82 & 515.12 & 503.18 & 502.37 & 515.59 & 514.67 & 502.63 \\
\hline-112.84 & -112.93 & 503.9 & 502.84 & 516.12 & 503.74 & 503.68 & 515.90 & 515.97 & 503.59 \\
\hline-113.01 & -113.82 & 504.99 & 503.47 & 516.93 & 504.68 & 504.37 & 517.29 & 516.57 & 504.75 \\
\hline
\end{tabular}


Table B.5 Experimental isotropic ${ }^{29} \mathrm{Si}$ chemical shifts $\left(\delta_{\text {iso }}\right)$ of the samples I and II, and calculated absolute isotropic ${ }^{29} \mathrm{Si}$ shieldings $\left(\sigma_{\text {iso }}\right)$ of the RTH optimized models with a triclinic unit cell and the fluoride anion located in T1 and T2 sites.

\begin{tabular}{|c|c|c|c|c|c|c|c|c|c|}
\hline \multicolumn{2}{|c|}{${ }^{29} \mathrm{Si} \delta_{\text {iso }}(\mathrm{ppm})$} & \multicolumn{8}{|c|}{${ }^{29} \mathrm{Si} \sigma_{\text {iso }}(\mathrm{ppm})$} \\
\hline \multirow{2}{*}{ I } & \multirow{2}{*}{ II } & \multicolumn{4}{|c|}{$\mathrm{T} 1$} & \multicolumn{4}{|c|}{$\mathrm{T} 2$} \\
\hline & & 1 & 2 & 3 & 4 & 5 & 6 & 7 & 8 \\
\hline-114.43 & -114.79 & 506.44 & 506.37 & 518.45 & 506.6 & 505.17 & 518.19 & 517.72 & 505.68 \\
\hline-115.00 & -115.79 & 507.14 & 506.81 & 519.00 & 506.95 & 506.02 & 518.96 & 518.07 & 506.17 \\
\hline-147.00 & -145.25 & 524.52 & 527.46 & 537.67 & 523.9 & 530.37 & 545.27 & 544.25 & 530.59 \\
\hline
\end{tabular}

Table B.6 Calculated absolute isotropic ${ }^{29} \mathrm{Si}$ shieldings $\left(\sigma_{\text {iso }}\right)$ of the RTH optimized models with a triclinic unit cell and the fluoride anion located in T3 sites.

\begin{tabular}{|c|c|c|c|c|c|c|c|}
\hline $9 \mathrm{~A}$ & $9 B$ & $10 \mathrm{~A}$ & 10B & $11 \mathrm{~A}$ & $11 \mathrm{~B}$ & $12 \mathrm{~A}$ & $12 \mathrm{~B}$ \\
\hline 494.06 & 510.38 & 494.88 & 509.58 & 495.78 & 508.79 & 494.61 & 507.21 \\
\hline 497.68 & 511.09 & 497.35 & 509.67 & 496.76 & 509.4 & 496.79 & 510.54 \\
\hline 498.42 & 512.29 & 497.67 & 510.27 & 498.48 & 509.83 & 497.58 & 510.75 \\
\hline 498.71 & 512.63 & 498.01 & 510.34 & 498.79 & 510.14 & 498.15 & 510.83 \\
\hline 499.16 & 512.68 & 498.45 & 511.17 & 500.35 & 510.54 & 498.25 & 511.33 \\
\hline 499.39 & 512.77 & 499.58 & 512.33 & 500.57 & 510.74 & 499.51 & 511.35 \\
\hline 499.85 & 512.79 & 499.9 & 512.83 & 500.57 & 511.04 & 499.85 & 512.32 \\
\hline 500.46 & 512.9 & 500.08 & 512.99 & 500.65 & 512.22 & 500.06 & 512.34 \\
\hline 500.79 & 512.92 & 500.33 & 513.42 & 501.18 & 512.56 & 500.61 & 512.50 \\
\hline 501.30 & 513.05 & 501.08 & 513.52 & 501.2 & 513.16 & 500.91 & 513.05 \\
\hline
\end{tabular}


Appendix B

Table B.6 Calculated absolute isotropic ${ }^{29} \mathrm{Si}$ shieldings $\left(\sigma_{\text {iso }}\right)$ of the RTH optimized models with a triclinic unit cell and the fluoride anion located in $\mathrm{T} 3$ sites.

\begin{tabular}{cccccccc}
\hline $9 \mathrm{~A}$ & $9 \mathrm{~B}$ & $10 \mathrm{~A}$ & $10 \mathrm{~B}$ & $11 \mathrm{~A}$ & $11 \mathrm{~B}$ & $12 \mathrm{~A}$ & $12 \mathrm{~B}$ \\
502.23 & 513.4 & 502.58 & 514.41 & 502.15 & 514.73 & 502.57 & 513.59 \\
502.64 & 516.58 & 503.76 & 515.1 & 503.24 & 514.84 & 504.24 & 513.82 \\
504.61 & 516.63 & 504.89 & 515.87 & 503.47 & 516.06 & 504.75 & 516.69 \\
506.05 & 518.26 & 506.06 & 520.31 & 507.32 & 518.29 & 506.64 & 517.92 \\
508.00 & 519.48 & 507.07 & 520.58 & 508.16 & 519.01 & 507.65 & 520.08 \\
530.31 & 547.98 & 535.29 & 545.14 & 530.41 & 549.2 & 534.79 & 544.63 \\
\hline
\end{tabular}

Table B.7 Calculated absolute isotropic ${ }^{29} \mathrm{Si}$ shieldings $\left(\sigma_{\text {iso }}\right)$ of the RTH optimized models with a triclinic unit cell and the fluoride anion located in T4 sites.

\begin{tabular}{cccccccc}
\hline $13 \mathrm{~A}$ & $13 \mathrm{~B}$ & $14 \mathrm{~A}$ & $14 \mathrm{~B}$ & $15 \mathrm{~A}$ & $15 \mathrm{~B}$ & $16 \mathrm{~A}$ & $16 \mathrm{~B}$ \\
\hline 494.99 & 507.24 & 496.49 & 508.07 & 509.27 & 506.50 & 506.64 & 495.86 \\
495.85 & 508.15 & 497.38 & 509.03 & 510.67 & 507.28 & 508.04 & 497.25 \\
499.26 & 509.67 & 497.83 & 512.26 & 512.61 & 510.03 & 510.82 & 498.00 \\
499.28 & 510.68 & 498.93 & 512.97 & 512.86 & 511.65 & 510.98 & 498.73 \\
499.62 & 511.55 & 499.27 & 513.27 & 513.31 & 511.80 & 511.84 & 499.07 \\
499.79 & 511.71 & 499.87 & 513.4 & 513.73 & 511.99 & 511.85 & 499.88 \\
499.82 & 512.11 & 500.30 & 513.42 & 514.46 & 512.34 & 512.65 & 499.99 \\
500.00 & 512.92 & 500.79 & 513.57 & 515.21 & 512.76 & 513.86 & 500.98 \\
501.03 & 513.81 & 500.86 & 513.84 & 515.91 & 513.75 & 514.00 & 501.24
\end{tabular}


Table B.7 Calculated absolute isotropic ${ }^{29} \mathrm{Si}$ shieldings $\left(\sigma_{\text {iso }}\right)$ of the RTH optimized models with a triclinic unit cell and the fluoride anion located in T4 sites.

\begin{tabular}{cccccccc}
\hline $13 \mathrm{~A}$ & $13 \mathrm{~B}$ & $14 \mathrm{~A}$ & $14 \mathrm{~B}$ & $15 \mathrm{~A}$ & $15 \mathrm{~B}$ & $16 \mathrm{~A}$ & $16 \mathrm{~B}$ \\
\hline 501.55 & 513.85 & 501.14 & 514.1 & 515.91 & 514.22 & 514.17 & 501.6 \\
501.55 & 515.79 & 501.15 & 514.44 & 516.19 & 514.54 & 514.62 & 501.74 \\
502.7 & 516.24 & 503.68 & 514.57 & 516.32 & 515.22 & 515.50 & 503.19 \\
504.48 & 516.40 & 504.27 & 516.31 & 517.17 & 516.47 & 515.52 & 503.6 \\
504.88 & 517.12 & 504.38 & 518.08 & 518.57 & 516.63 & 517.30 & 504.49 \\
505.05 & 517.59 & 505.85 & 520.06 & 518.84 & 517.33 & 517.65 & 505.48 \\
532.53 & 543.50 & 528.58 & 544.45 & 542.21 & 546.92 & 546.33 & 528.52 \\
\hline
\end{tabular}

In Figures B.1 to B.3, the calculated $\sigma_{\text {iso }}$ of each model is plotted vs the experimental $\delta_{\text {iso }}$ of both samples (I and II). In general, the models with the fluoride located in a T2 site correlate better with the experimental $\delta_{\text {iso }}$ of sample II, in agreement with prediction of the ${ }^{19} \mathrm{~F} \delta_{\text {iso. Notice how the }}{ }^{29} \mathrm{Si}$ signal at $-103.70 \mathrm{ppm}$ (sample I) deviates from the regression. This signal is a distinctive feature between of the sample II (Figure 6.2). The models with the fluoride anion located in T4 sites correlate better with the experimental $\delta_{\text {iso }}$ of sample I, in particular, the model 16A,

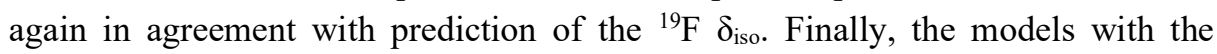
fluoride anion located in T3 sites perform worse than those in T4 sites. 


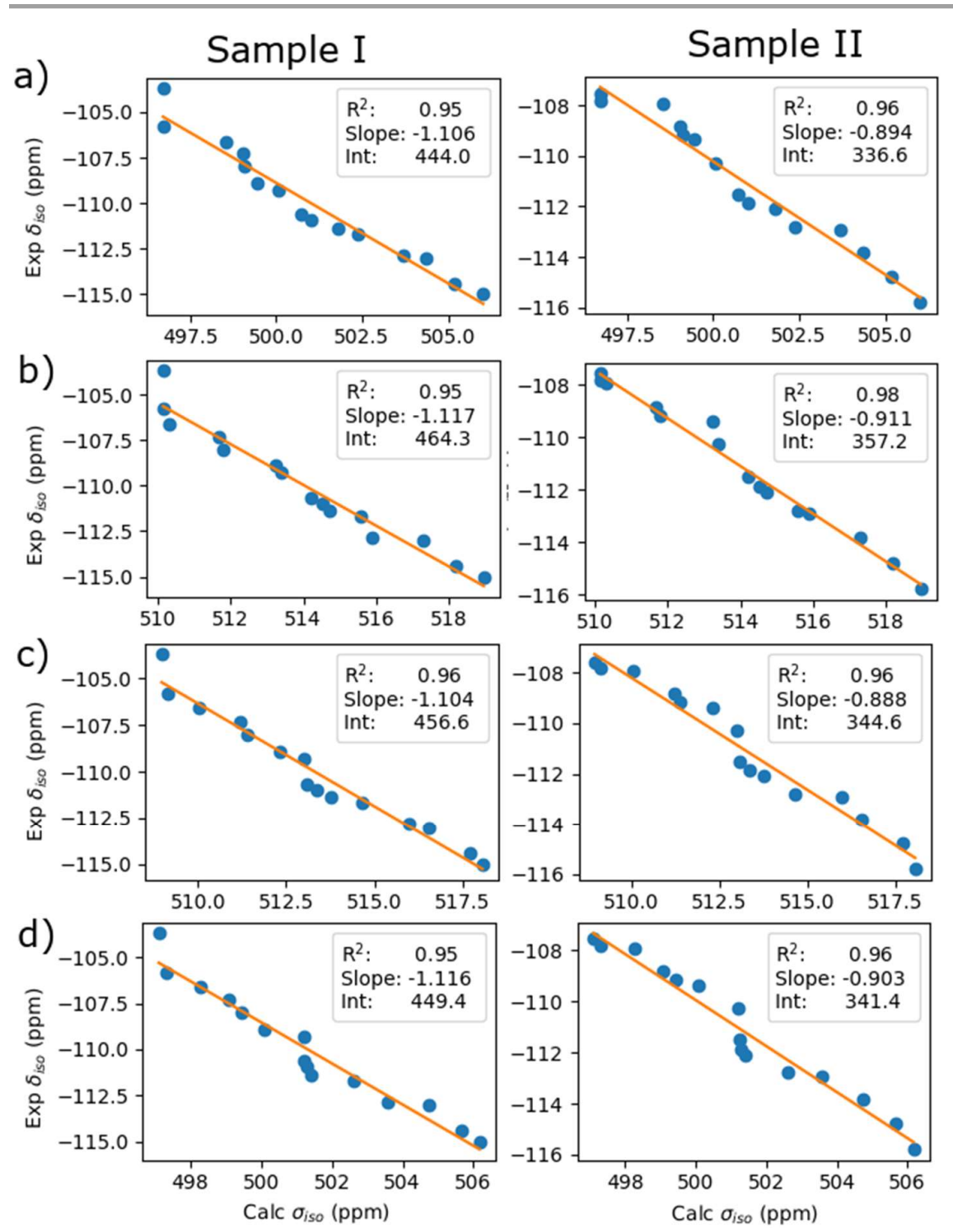

Figure B. 1 Correlation of the calculated ${ }^{29} \mathrm{Si} \sigma_{\text {iso }}$ of the models 5 (a), 6 (b), 7 (c) and 8 (d) with the experimental ${ }^{29} \mathrm{Si} \delta_{\text {iso }}$ of samples I and II. In the four models the fluoride anion is located in T2 sites. Notice how the distinctive ${ }^{29} \mathrm{Si}$ signal at $-103.70 \mathrm{ppm}$ is not well predicted with these models. They correlation is better with the signals of sample II. 
Appendix B
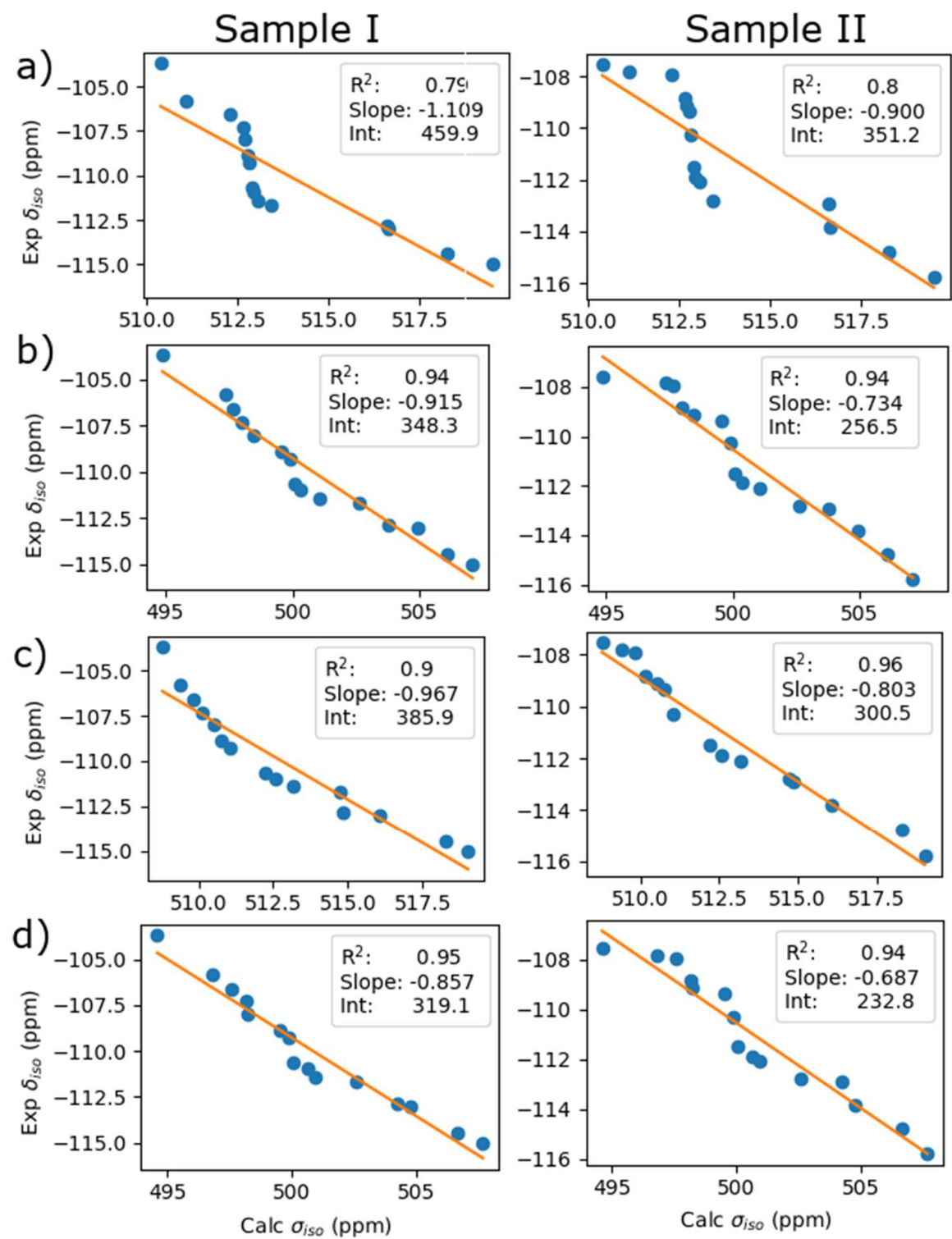

Figure B. 2 Correlation of the calculated ${ }^{29} \mathrm{Si} \sigma_{\text {iso }}$ of the models $9 \mathrm{~B}(\mathrm{a}), 10 \mathrm{~A}(\mathrm{~b}), 11 \mathrm{~B}(\mathrm{c})$ and $12 \mathrm{~A}$ (d) with the experimental ${ }^{29} \mathrm{Si} \delta_{\text {iso }}$ of samples I and II. In the four models the fluoride anion is located in T3 sites. 

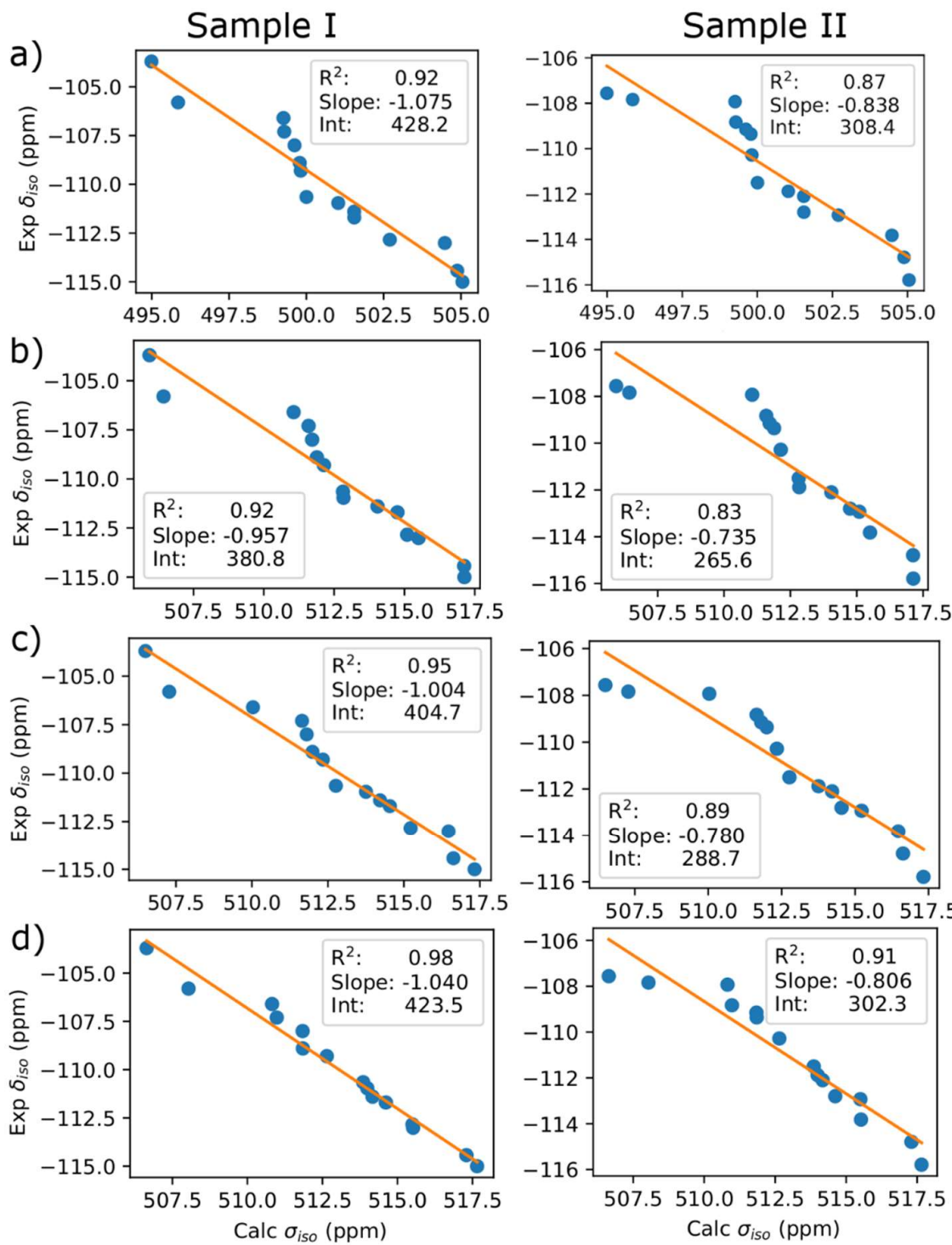

Figure B.3 Correlation of the calculated ${ }^{29} \mathrm{Si} \sigma_{\text {iso }}$ of the models 13A (a), 14B (b), 15B (c) and $16 \mathrm{~A}$ (d) with the experimental ${ }^{29} \mathrm{Si} \delta_{\text {iso }}$ of samples I and II. In the four models the fluoride anion is located in T4 sites. Notice how the distinctive ${ }^{29} \mathrm{Si}$ signal at $-103.70 \mathrm{ppm}$ well predicted with these models. They correlation is better with the signals of sample I. 